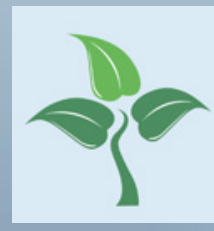

\title{
sustainability
}

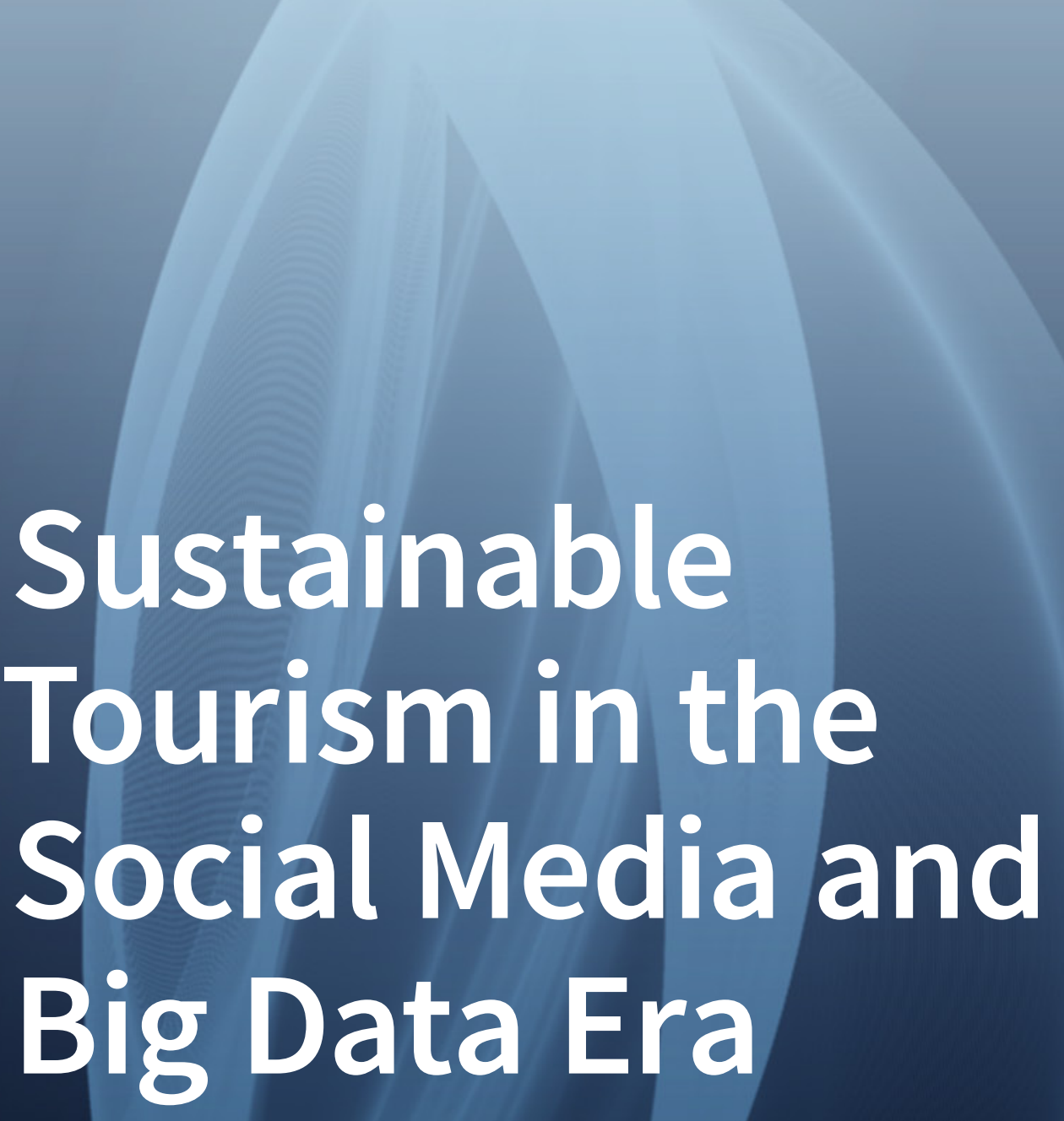


Sustainable Tourism in the Social Media and Big Data Era 



\section{Sustainable Tourism in the Social Media and Big Data Era}

Editors

Yoonjae Nam

So Young Bae 


\section{Editors}

Yoonjae Nam

Kyung Hee University

Korea
So Young Bae

Kyung Hee University

Korea

Editorial Office

MDPI

St. Alban-Anlage 66

4052 Basel, Switzerland

This is a reprint of articles from the Special Issue published online in the open access journal Sustainability (ISSN 2071-1050) (available at: https://www.mdpi.com/journal/sustainability/ special_issues/smbd).

For citation purposes, cite each article independently as indicated on the article page online and as indicated below:

LastName, A.A.; LastName, B.B.; LastName, C.C. Article Title. Journal Name Year, Article Number, Page Range.

ISBN 978-3-03943-324-7 (Hbk)

ISBN 978-3-03943-325-4 (PDF)

(C) 2020 by the authors. Articles in this book are Open Access and distributed under the Creative Commons Attribution (CC BY) license, which allows users to download, copy and build upon published articles, as long as the author and publisher are properly credited, which ensures maximum dissemination and a wider impact of our publications.

The book as a whole is distributed by MDPI under the terms and conditions of the Creative Commons license CC BY-NC-ND. 


\section{Contents}

About the Editors $\ldots \ldots \ldots \ldots \ldots \ldots \ldots \ldots \ldots \ldots \ldots$ vii

Preface to "Sustainable Tourism in the Social Media and Big Data Era" . . . . . . . . . ix

Pam Lee, William Cannon Hunter and Namho Chung

Smart Tourism City: Developments and Transformations

Reprinted from: Sustainability 2020, 12, 3958, doi:10.3390/su12103958 . . . . . . . . . . . . 1

Insu Hong and Changsok Yoo

Analyzing Spatial Variance of Airbnb Pricing Determinants Using Multiscale GWR Approach

Reprinted from: Sustainability 2020, 12, 4710, doi:10.3390/su12114710 . . . . . . . . . . . 17

Mengyi Lin, Fu-Yuan Li and Haibin Zhou

A Research on the Combination of Oblique Photography and Mobile Applications Based on the Sustainable Development of Tourism

Reprinted from: Sustainability 2020, 12, 3501, doi:10.3390/su12093501 . . . . . . . . . . . . . 35

Bidur Devkota, Hiroyuki Miyazaki, Apichon Witayangkurn and Sohee Minsun Kim

Using Volunteered Geographic Information and Nighttime Light Remote Sensing Data to

Identify Tourism Areas of Interest

Reprinted from: Sustainability 2019, 11, 4718, doi:10.3390/su11174718 . . . . . . . . . . . . 55

Carlos de las Heras-Pedrosa, Elena Millan-Celis, Patricia P. Iglesias-Sánchez

and Carmen Jambrino-Maldonado

Importance of Social Media in the Image Formation of Tourist Destinations from the Stakeholders' Perspective

Reprinted from: Sustainability 2020, 12, 4092, doi:10.3390/su12104092 . . . . . . . . . . . . . . 85

Yeajin Joo, Hwayoon Seok and Yoonjae Nam

The Moderating Effect of Social Media Use on Sustainable Rural Tourism: A Theory of Planned

Behavior Model

Reprinted from: Sustainability 2020, 12, 4095, doi:10.3390/su12104095 . . . . . . . . . . . . . 113

Eun Ju Seo, Jin-Woo Park and Yu Jin Choi

The Effect of Social Media Usage Characteristics on e-WOM, Trust, and Brand Equity:

Focusing on Users of Airline Social Media

Reprinted from: Sustainability 2020, 12, 1691, doi:10.3390/su12041691 . . . . . . . . . . . . . 127

\section{Hyung Min Lee, Jinwoo Park and Yoonjae Nam}

A Sustainable Solution to Overtourism in the Social Media Era: An Exploratory Analysis on the

Roles and Functions of Place-Visitor Relationship (PVR)

Reprinted from: Sustainability 2020, 12, 3043, doi:10.3390/su12073043 . . . . . . . . . . . . . 145

Jongwon Won, Jong Yoon Lee and Jong Woo Jun

Influences of SNS (Social Network Service) Uses and Musical Consumption on City Branding:

A Focus on Broadway, New York and the West End, London

Reprinted from: Sustainability 2020, 12, 3856, doi:10.3390/su12093856 . . . . . . . . . . . . . 161

Hun Kim and Byenghee Chang

A Study on the Effects of Crowdfunding Values on the Intention to Visit Local Festivals:

Focusing on Mediating Effects of Perceived Risk and e-WOM

Reprinted from: Sustainability 2020, 12, 3264, doi:10.3390/su12083264 . . . . . . . . . . . . . 171 


\section{Bo-Kyeong Kim and Kyoung-Ok Kim}

Relationship between Viewing Motivation, Presence, Viewing Satisfaction, and Attitude toward Tourism Destinations Based on TV Travel Reality Variety Programs

Reprinted from: Sustainability 2020, 12, 4614, doi:10.3390/su12114614 . . . . . . . . . . . . . . 191

Ian Sutherland, Youngseok Sim, Seul Ki Lee, Jaemun Byun and Kiattipoom Kiatkawsin

Topic Modeling of Online Accommodation Reviews via Latent Dirichlet Allocation

Reprinted from: Sustainability 2020, 12, 1821, doi:10.3390/su12051821 . . . . . . . . . . . . . . 205

\section{Jie Zhao and Zhixiang Peng}

Shared Short-Term Rentals for Sustainable Tourism in the Social-Network Age: The Impact of Online Reviews on Users' Purchase Decisions

Reprinted from: Sustainability 2019, 11, 4064, doi:10.3390/su11154064 . . . . . . . . . . . . . 221

\section{Hwayoon Seok, Yeajin Joo and Yoonjae Nam}

An Analysis of the Sustainable Tourism Value of Graffiti Tours through Social Media: Focusing on TripAdvisor Reviews of Graffiti Tours in Bogota, Colombia

Reprinted from: Sustainability 2020, 12, 4426, doi:10.3390/su12114426 . . . . . . . . . . . . . . 241

So Young Bae and Ju Hyoung Han

Considering Cultural Consonance in Trustworthiness of Online Hotel Reviews among Generation Y for Sustainable Tourism: An Extended TAM Model

Reprinted from: Sustainability 2020, 12, 2942, doi:10.3390/su12072942 . . . . . . . . . . . . . . 261 


\section{About the Editors}

Yoonjae Nam (Ph.D.) is an associate professor in the Department of Culture, Tourism, and Content at Kyung Hee University, Seoul, South Korea. He is interested in current digital media, particularly corporate communication, the diffusion of media content, the culture and tourism industry, and social networks. He has published papers in Sustainability, Information Processing \& Management, Technological Forecasting \& Social Change, the International Journal of Information Management, and the International Journal of Advertising, among others.

So Young Bae (Ph.D.) is an assistant professor in the Department of Culture, Tourism, and Content at Kyung Hee University, Seoul, South Korea. She has a background in tourism, leisure, and cultural anthropology, and her work primarily focuses on cultural tourism, travel culture, leisure behaviors, and quality of life, based on fieldwork in these areas. She has conducted research in an intercultural context, including a cross-cultural comparison of online leisure behaviors between Korean and Taiwanese mothers, experiences of international participants staying at a temple in Korea, and the travel motivations and patterns of international students. Her recent work examined "untact" tourism behaviors, highlighting the paradigm shift in travel culture in response to the unprecedented challenges of the COVID-19 pandemic. 



\section{Preface to "Sustainable Tourism in the Social Media and Big Data Era"}

Sustainability has been discussed as a crucial topic in the tourism industry. Considering the substantial impacts of tourism on global society, it is crucial to develop tourism management strategies based on social, cultural, economic, and environmental aspects of sustainability. In the era of the fourth industrial revolution, social media and big data have brought new opportunities to support the practice of sustainable tourism by connecting ideas, products, and people, as well as developing new business models and strategies. This Special Issue seeks to expand upon the innovative and advanced understanding of tourism, social media, and big data and offer new multidisciplinary insights into the industry. To encourage scientific debate on major current trends in social media and big data research primarily focusing on sustainable tourism, this Special Issue offers wide-ranging topics and research questions with regard to smart tourism cities, social media impacts, online reviews, and tourist behaviors, and it represents a call to action for scholars to engage with broader social issues. We would like to express our appreciation to all the contributors to this edition for their creative, intellectual work. This edition covers all areas of the sustainability debate, including 15 selected papers based on the following core ideas: smart tourism and big data, social media in the tourism industry, and online reviews and tourist behaviors.

We are grateful to the editorial staff at MDPI for making this edition possible. We welcome any enquiries or comments relating to this Special Issue, and we invite you to contribute to our next editions relating to the tourism industry, social media, big data, and sustainability.

Yoonjae Nam, So Young Bae Editors 



\title{
Smart Tourism City: Developments and Transformations
}

\author{
Pam Lee ${ }^{1}$, William Cannon Hunter ${ }^{2}$ and Namho Chung ${ }^{2, *}$ \\ 1 College of Hotel \& Tourism Management, Kyung Hee University, 26 Kyungheedae-ro, Dongdaemun-gu, \\ Seoul 02447, Korea; pampam@khu.ac.kr \\ 2 Smart Tourism Education Platform, Kyung Hee University, 26 Kyungheedae-ro, Dongdaemun-gu, \\ Seoul 02447, Korea; primalamerica@yahoo.com \\ * Correspondence: nhchung@khu.ac.kr; Tel.: +82-2-961-2353; Fax: +82-2-961-9557
}

Received: 13 April 2020; Accepted: 10 May 2020; Published: 12 May 2020

\begin{abstract}
Cities and tourism entities invest massive resources into smart system initiatives as information technologies are a key factor for a city's destination competitiveness. Moreover cities around the world are increasingly recognizing the smart tourism city concept and related strategies as means of optimizing sustainable environments. Particularly for cities facing emerging issues of residents' negative perceptions towards tourism, smart tourism city empowers a city to rise to this challenge by creating urban spaces that residents and visitors can enjoy together. However, smart tourism city research initiatives still fail to address the full spectrum of related and potential developments. This study presents a conceptual approach to defining smart tourism city: the smart city and its components are defined and contrasted with smart tourism and its components. The resulting convergence-smart tourism city—is then examined in light of a number of pioneering examples of smart tourism cities and its vital roles in the age of sustainable development. The main purpose of this study is to show the interests of locals and tourists context and the roles of 'smart' government leadership to researchers and practitioners.
\end{abstract}

Keywords: smart tourism city; smart tourism; smart city; sustainable development; COVID-19

\section{Introduction}

The heyday of information communication technologies (ICT) arising out of the digital and computer revolution have transformed entire market systems, moving them toward greater diversity and vigor. ICT's role is steeply imperative, given the pervasiveness of smartphones and related technology convergence in every field. ICT becomes progressively more ubiquitous because of its 'anytime anywhere' accessibility. Presently, ICTs have been reaching their pinnacle with the advent of the fourth industrial revolution and the proliferation of the Internet of Things (IoT).

ICTs, including IoT-enabled devices and sensors are changing our daily life as they also play a significant role in urban development, such as resource utilization, economy and sustainable development. The acceleration of urbanization causes population concentrations, poor environments and severe challenges to the cities that will be met with increasingly sophisticated smart systems. Cities find out about solutions to urbanization by being coupled with smart technology and building smart cities. The word 'smart' refers to "technological, economic and social developments enriched by ICT revolutions that bank on sensors, data, new ways of connectivity and exchange of information" [1]. Forward-thinking cities are making significant investments in the design and development of these smart cities (initially called digital cities and intelligent cities). With the tremendous innovation in existing information technology, cities are finally able to create more connected and intelligent designs. A smart city is thereby defined as a high-tech, intensively connected city that uses advanced new 
technologies to create a sustainable metropolis, innovative commerce and enriched quality of life for its citizens [2].

From the industrial and public sector perspectives, the most important benefit associated with advanced technology is the exponential increase in information, which interacts with the biologic world on a daily basis and enables the industry to predict consumers' cultural interests [3]. A smart city also focuses on social and cultural life and it may enhance urban social interactions [4]. Given that tourism is highly related to cultural phenomena [5], the notion of 'smart' has been adapted to the context of tourism. As a new buzzword, 'smart technology' has been significantly changing the way visitors think about browsing websites; smart phones and mobile applications are exploited for making various travel decisions, such as those pertaining to transportation, accommodation and activities available at a desired tourism destination [5]. Tourism activities based on smart technology, smart tourism, is a social phenomenon arising from the incorporation of ICT with the tourism experience [6]. The smart tourism experience represents technology-mediated experiences that combine personalization, context-awareness, and real-time monitoring [7].

The fourth industrial revolution has bolstered development in every industry including tourism; the tourism industry as many cities that tap into tourism competitiveness bank on smart city infrastructures and focus on enhancing the city's competitiveness. Examining the smart city phenomenon in the tourism context is crucial, particularly regarding its influence on the travel experience and tourists' decision-making process. Besides economic benefits, the tourism industry also affects the destination city's society and cultural environment [8] and the residents' perceptions of tourism affect the destination city's enthusiasm towards tourism business. Popular tourism destination cities face environmental damage and residents' complaints caused by tourist overcrowding. Moreover a correlation between sustainable urban development and residents' happiness is proven as a significant factor for the urban sustainability [9]. Accordingly, stakeholders in the tourism industry regard the smart tourism city, convergence of smart city and smart tourism, as an important means to enhance the quality of life of visitors and residents alike. Smart tourism cities are willing to invest massive resources to implement and sustain smart tourism systems that work to solve over-tourism problems, protect citizens, and offer a better living environment [10]. In this context, a smart tourism city is an innovative tourist destination that guarantees sustainable development that facilitates [11] and enhances visitors' interaction with experiences at the destination and eventually improves the residents' quality of life.

However with technology being embedded within the cities environment, the smart tourism city concept represents new challenges to enhance their sustainability, smart tourism city research still fails to fully cover smart tourism city developments and their current status. The aim of this study is to provide definitional clarity and comprehensive approach to the anatomy of smart tourism city as a convergence of a smart city and smart tourism: which elements are critical for the development of a smart tourism city. This study presents insights regarding smart tourism cities' roles in sustainable development. It is therefore the ultimate aim of this study to provoke researchers in associated fields and inspire practitioners to perform smart tourism city practice.

\section{Convergence of Smart City and Smart Tourism}

\subsection{Smart City}

With acceleration of urbanization, a number of cities face challenges to design and develop a better city for living. Urban communities endeavor to mitigate urbanization challenges and ensure a quality living environment through more efficient asset and resource management. As ICTs have a central role to play in the quest for industry development, cities find out about solutions to urbanization by being coupled with technology. Many city governments are seeking to infuse technology into every aspect of city operation to create more connected and intelligent designs. A smart city, new approaches to urban planning and living, is a high-tech, intensively connected city that uses advanced new smart technologies [12] to create a greener metropolis, innovative commerce, and enriched quality of life for 
its citizens [2]. Smart city ideas absorbed urban policies such as digital city, green city, knowledge city, therefore it is a complex approach and requires long-term perspectives. Smart city is also referred to as an intelligent city as the information flow is analyzed and translated throughout the IT, social and business infrastructures to enhance the intelligence of the city [13]. As a part of information flow, it is essential to collect citizen's opinions about live-ability in cities and create the public value [14]. Cardullo and Kitchin [15] highlighted the 'citizen-centric' measuring citizen inclusion, participation and empowerment in smart city initiatives in Dublin, Ireland. The result shows that how stewardship, civic paternalism, and a neoliberal conception of citizenship, source of 'citizen-centric', prioritizes consumption choice and individual autonomy within a framework of state.

The concept of the smart city is to optimize infrastructures in order to ideally ensure quality of citizens' life [2]: transportation, water and power supply, waste management, IT connectivity, efficient urban mobility, e-governance and citizen participation. Therefore, the factors defining the smart city were identified as hard and soft domains, including eight areas of sub-domains, such as mobility, building, health care, entertainment, education, public safety, environment and economy [12,16]; six key domains, including governance, economy, environment, mobility, living and people [17]; four pillars of key components, including energy, environment, industry, living and service [18]. An integrated model is shown in Figure 1, below.

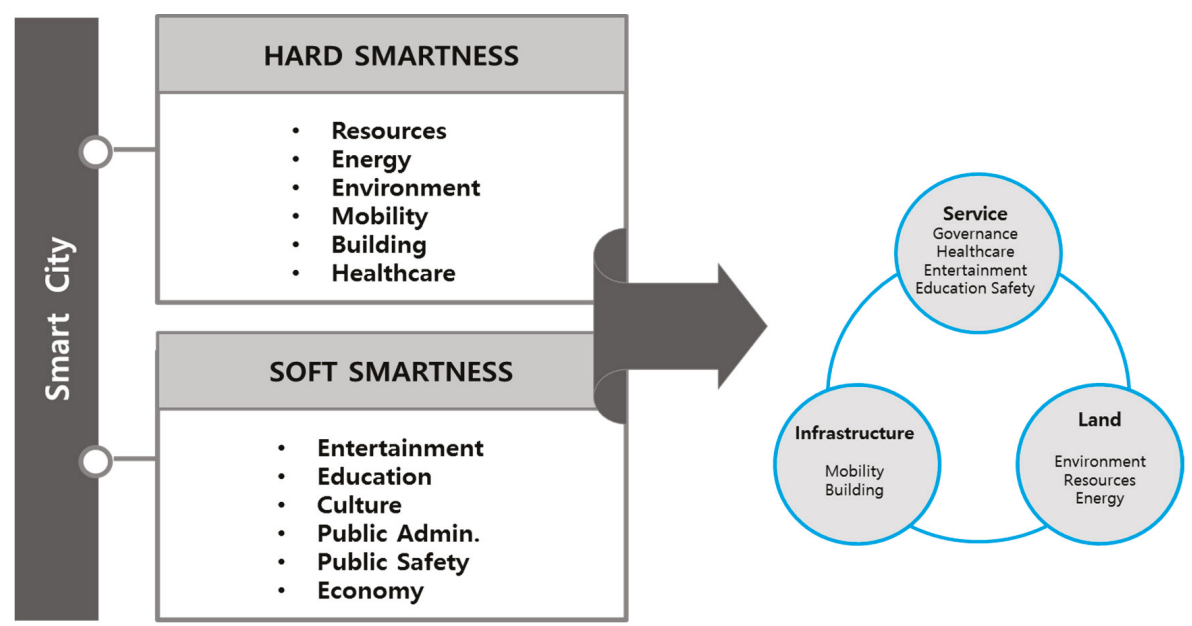

Figure 1. Components of a smart city.

Figure 1 illustrates three main domains of the smart city: service, infrastructure and land, with their corresponding sub-domains. The service domain covers health care, entertainment, education, safety and quality of life in the urban space. Cultural heritage management and human capital management are also included [12]. The infrastructure domain includes transport and mobility features, such as city logistics, info mobility and people mobility, which concerns ICT utilization for intelligent transportation products and management. The land domain involves urban infrastructure, which refers to the interactive management of building services and housing quality with ICT. Social issues that address the digital divide, social relations and ICT connectivity [19] are addressed with other challenges from the perspective of e-governance [20].

The design of a smart city depends on various local context factors, including geographic location and population density [12]. Each city has its own priorities regarding the sectors in which to develop smart tourism initiatives. In 2014, Singapore started making an extensive effort to build a smart nation program by collecting data on urban daily life. Based on its findings, the government is now deploying systems that monitor the city's crowdedness, cleanliness of public spaces and traffic flow. 
The collected data are incorporated into an online platform called "Virtual Singapore," which works to inform government policy and potential disaster risk management. It also informs the decision-making processes of residents and private enterprises by managing data in real time. In Dubai, the government launched the "Smart Dubai Initiative," created 22 government entities, and developed 50 smart services for functions related to electric bills, vehicle registration, locations of ATMs, reporting violations, tracking visa status and monitoring taxi services. The Seoul Metropolitan Government has spent the past three years developing an advanced IoT ecosystem with interlinked smart devices. The city government recently announced another five-year (2018-2022) urban plan which includes 14 public services in five areas, with a budget of \$108 million. In 2015, Estonia government also launched the world's first crowdfunding initiative designed to develop a smart city to create new green technologies. This initiative raised $40 \%$ of the total required amount within three days.

The participation and cooperation of governments, implementing businesses and the public are vital in smart cities. Especially governments must understand the elements of a smart city and carry out roles as an ecosystem builder, strategic planner, policymaker and investor.

\subsection{Smart Tourism}

Tourism is a social, cultural and economic phenomenon that involves the movement of people to places outside their usual environment for personal or professional purposes [5], and the main purpose of traveling is for cultural immersion. The beginning of the 1990s cultural tourism became one of the alternative forms of tourism, opposed to mass tourism [21]. Tourists demonstrate a proactive approach and create experiences actively while traveling. Thus, tourists' heterogeneous preferences have been drawing attention from destinations and the destination cities endeavor to provide customized high-quality travel experiences. Ever since the Internet is influence the distribution of tourism information and sales, tourism industry is seeking for successful e-tourism strategies by developing Web sites, electronic commerce and related governance [22]. And now cities are aggressively pushing the new tourism agenda forward with the tremendous development of smart technology.

Smart tourism represents the convergence of ICT and tourism and denotes the transformation of tourism through technology. It indicates a new tourism braced by integrated efforts at a destination to collect and analyze data extracted from diverse sources in combination with the use of advanced information technologies to transform travel experiences to make them more enriched, efficient and sustainable [1]. In this regards smart tourism is a social phenomenon arising from the incorporation of ICT with the tourism experience [6]. Moreover, smart technology has been significantly changing the way visitors make diverse travels decisions, such as those pertaining to transportation, accommodation and activities available at a desired tourism destination [5]. Smart tourism identifies three travel phases: pre-travel, travel and post-travel [7] wherein tourists' expectations and behavior may change. During the pre-travel (planning) phase, tourists decide where to go, how to get there, and where to stay. During the travel (onsite) phase, tourists decide where and what to eat or what activities to engage in. During the post-travel (evaluation) phase, tourists express varying degrees of satisfaction which they share in travel reviews. Further advanced smart devices enable tourists to obtain real-time travel-related information and enhance visitors' ability to acquire information [23]. Accordingly, tourists take more initiative in creating customized travel experiences [24]. The smart experience refers to technology-mediated tourism experiences and their enrichment via personalization, uniqueness and real-time monitoring [7]. The smart experience refers to technology-mediated tourism experiences and their enrichment via personalization, uniqueness, and real-time monitoring [7]; the overall experience has progressed from e-tourism (managing data) to smart tourism (maximizing interest).

Wang, Xiang and Fesenmaier [25] identified increase in tourists' creativity through applications such as the camera and more flexibility in the travel activities which are more personally tailored. Li, Hu, Huang and Duan [26] conducted characteristics comparison study of both traditional tourist information services and those incorporated in smart tourism against Chinese market. And they found that smart tourism information service has significant influence on tourist destinations, enterprises and 
also tourists themselves. From the industry perspective hotels use ICT in their operation to increase productivity, reduce expenses, and improve the service quality and it brings sustainability in profit and better guest satisfaction. Abdul, Bakhtiar, Syaquif, Kamaruddin and Ahmad [27] identified hotels find ICT applications essential for effective strategy operation, accordingly the divisions for reservation, room management, accounting, and telecommunication utilize ICTs to alleviate the workload. On the contrary, other studies elucidate the perspective of customers using hotel service related applications. The customers' adoption of hotel reservation mobile apps made by hospitality corporations is examined. And the findings indicated the applications' requirements such as high information quality, good system quality, easy-of-use layout and low usage fees [25]. Gretzel, Sigala, Xiang and Koo [1] proposed three components of smart tourism: smart destination, smart experience and smart business. Smart cities (the smart destination) provide mobility, resource allocation and availability and sustainable quality of life to their residents, and further facilitate tourism with integrated smart surroundings, enhancing the experience of visitors (smart experience). Smart business refers to the complex business ecosystem of dynamically interconnected stakeholders and the exchange and co-creation of touristic resources.

Figure 2 illustrate these three components based on another three layers of data-related factors: a smart information layer that indicates data collection; a smart exchange layer that aids interconnectivity and a smart processing layer related to the analysis, visualization, integration and intelligent use of data [28].

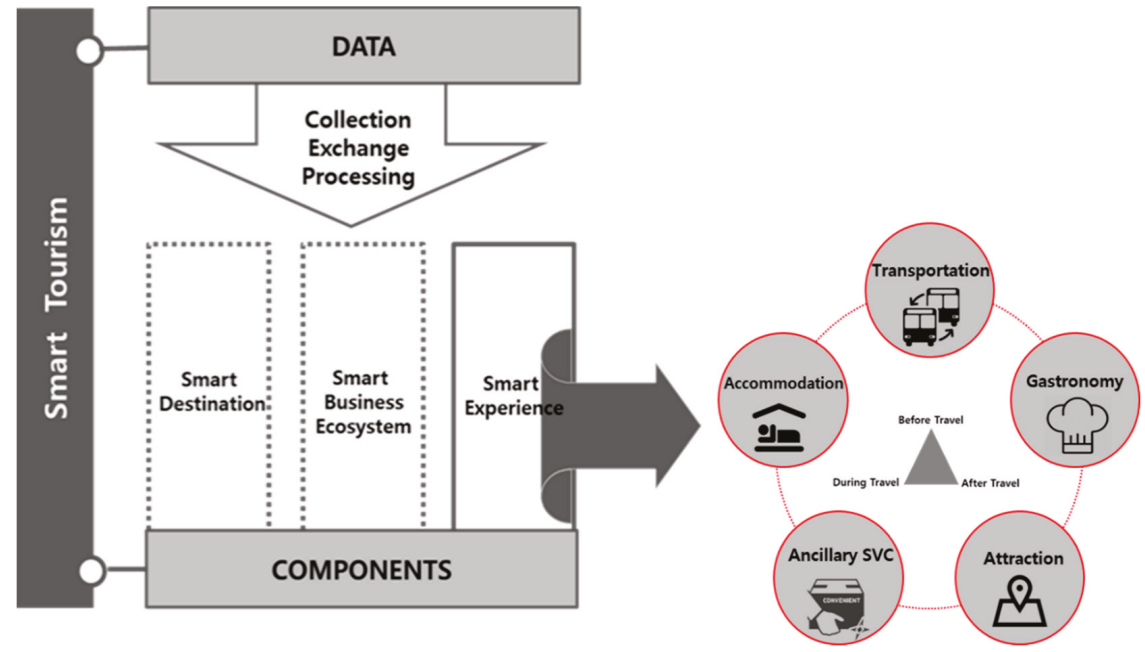

Figure 2. Components of smart tourism.

As mentioned earlier, in smart tourism, visitors not only consume data from their tourism experiences, but also enable the creation and illustration of data. Potential data for smart tourism is identified in several ways. There are six main tourist-related elements of smart cities: smart mobility, smart government, smart economy, smart people, smart living and smart environment [29]. There are also the six As of tourism-attractions, accessibility, amenities, available packages, activities, and ancillary services-which are the attributes that eventually generate profit and benefits for the destination by offering valuable experiences to tourists [29]. Figure 2 also summarizes the essential components of smart tourism: transportation, accommodation, gastronomy, attraction and ancillary service. The smart tourism experience is based on a concrete smart business ecosystem at a destination that works through data sharing among stakeholders. This refined model reflects the more nuanced and individual smart travel experience and eliminates certain potential data factors described previously, such as 'available 
package,' and adds others, such as 'gastronomy.' Gastronomic tourism increasingly influences travel motivation and behavior through memorable food and drink experiences [30].

Buonincontri and Micera [31] analyzed two best smart tourism practices cities, Venice and Salzburg, and revealed that providing ICT based smart tourism services is enhancing the interaction with tourists and increasing their active participation and ultimately improving co-creation of tourism experiences. Particularly in the time of global dispersion of COVID-19 pandemic, with offline cultural activities and tourism affected by the stay-at-home quarantine order, smart tourism has its potential to rise to this challenge by creating smart tourism products exploiting AR (augmented reality) [32,33], VR (virtual reality) services [34]. There is no doubt that under social uncertainty and crisis, tourism development may be different from those noted in times of prosperity [35]. And Smart tourism approach may offer new paradigm and ecosystem to the stakeholders. Korean government has just launched new project to encourage travel startups expanding their business domain through exploiting advanced ICT benefits. The following section will explore the city offering smart tourism experiences, smart tourism city, in more detail.

\subsection{Smart Tourism City}

The fourth industrial revolution has bolstered development in the tourism industry, and many cities tap into their tourism competitiveness by developing a smart tourism ecosystem based on existing smart city digital technology infrastructures. These developments in tourism have worked to catalyze the idea of building smart tourism cities. If so, is it true that tourism becomes smarter when tourism meet smart city? Making a movement to a smart city, visitors are guaranteed to indulge smart tourism experiences? Examining the smart city phenomenon in the tourism context is definitely crucial, particularly regarding its influence on the travel experience and its citizens' quality life. In this context, a smart tourism city is defined as an innovative tourist destination that guarantees sustainable development that facilitates [11] and enhances visitors' interaction with experiences at the destination and eventually improves the residents' quality of life. Majority of researchers use the term 'destination' rather than 'city.' Both concepts are based on integrated ICT infrastructures, but the concept of 'smart tourism cities' focuses more on its residents, whereas smart tourism destinations more emphasize the enhancement of tourists' experiences [36].

Buhalis and Amaranggana [16] emphasized that smart tourism city is required to enhance tourism experience through more personalized products/services to meet each of visitor's unique preferences. Using of big data could be the solution for providing right services that suit users' preference at the right time. However emphatically interconnecting with stakeholders becomes also critical for understanding the unique preferences of visitors and the competitiveness of cities. Smart cities embraced the five main stakeholders in tourism: governments, tourism organizations, local residents, tourists and environments [16]. Collaboration between stakeholders and a user-friendly platform based on connected infrastructure ensures the enhancement of the quality of life for locals, enriches the experiences of tourists, boosts private businesses, and increases governments' competitiveness in terms of smart personal experiences, occupations and the highest value for the environment. Services offered by smart tourism cities have to be useful throughout the travel phase as they facilitate tourists' ability to organize schedules, peruse information about the city and check local transportation. Thus, a smart tourism city requires the physical infrastructure, technology, a resource database, and the city's basic conditions to promote tourism development [37]. Most importantly, a smart tourism city provides intelligent services to visitors in terms of transportation, gastronomy, accommodation, ancillary services and attractions throughout three phases: the pre-travel (planning) phase, the travel (onsite) phase and the post-travel phase (evaluation). All those services are realized on the basis of the main domains of the smart city infrastructure: service, land and infrastructure.

Figure 3, below, depicts the components of the smart tourism city as an interpretative framework of symbolic components, based on the literature presented in the previous sections. The proposed framework provides a clearer explanation of the origin of the smart tourism city. 


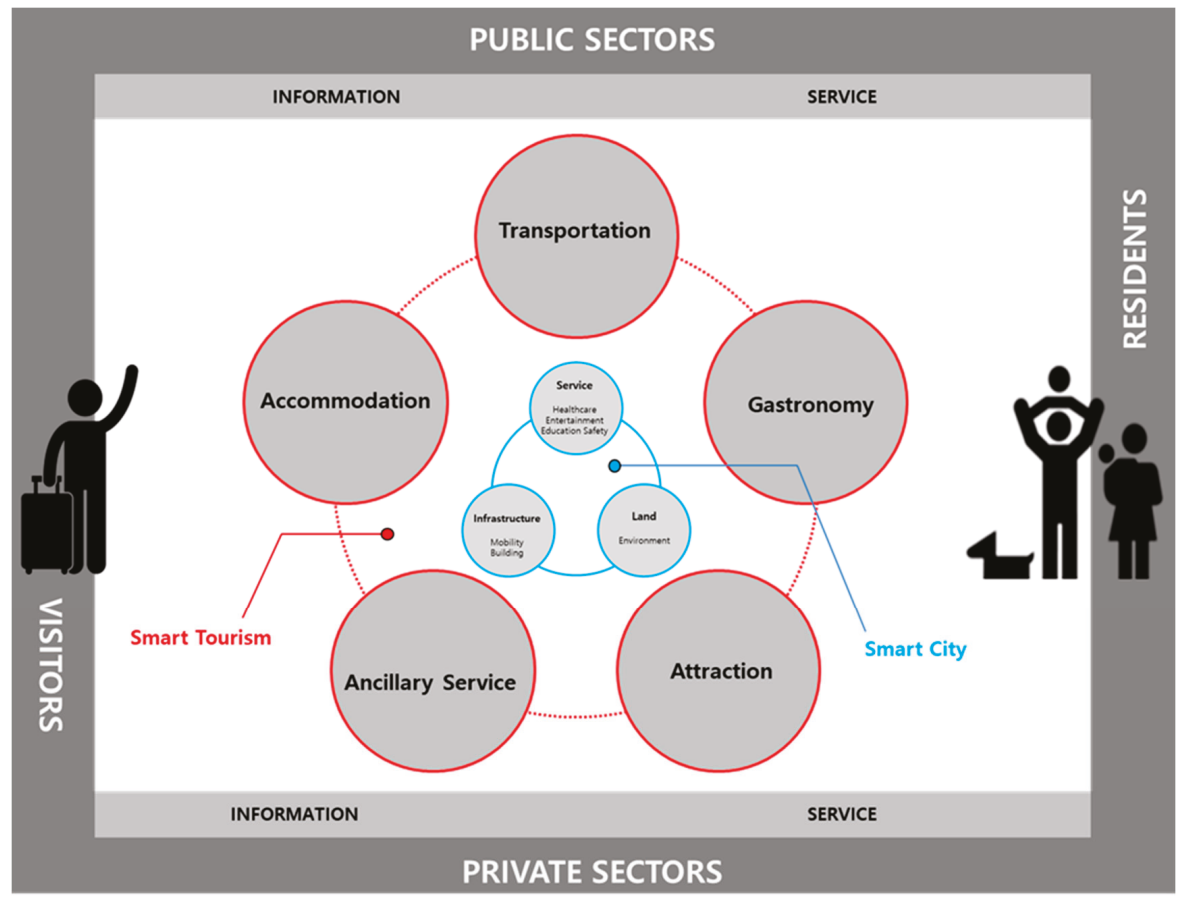

Figure 3. Components of a smart tourism city.

As shown in Figure 3, organic relationships that present information and service transactions as a result of data sharing between the public and private sectors. And visitors and residents are playing a significant role in providing and receiving that information (see outside frame). The connected circles, five smart tourism components, embrace the three components of the smart city, showing the integrated and coordinated implementation of the technological components to enhance the smart tourism experience during all three traveling phases. The active participation of both visitors and residents by sharing their on-site experiences is highly crucial during the smart experience process. In this regard, on the basis of the smart cities' dimensions (namely, smart governance, smart economy, smart environment, smart mobility, smart living and smart people), smart tourism cities are realized in accordance with the destination's components, providing AR/VR services, vehicle tracking systems, multi-lingual applications, near field communication tags and registering complaints systems, etc.

Venice, one of most popular destinations in Europe, introduced an application named "VeneziaUnica", which helps tourists experience direct interaction with the destination supply system, inducing their active participation and facilitating experience sharing. Specifically, the application enables users to directly contact service providers and their websites and share opinions and photos with other users. Salzburg, a historical, artistic and cultural destination in Austria, realized its "Master Plan 2025-Smart City Salzburg" which is related to livability, intelligent networking, sustainable mobility and open collaboration. An application, "Salzburger Mittagsplaner" was introduced to empower its users to collect information on service providers (restaurants, pubs, bistros and cafes), including menus, prices and locations, and allows them to choose seats and decide what to eat in advance. Afterward, users may share opinions and their preferences. To promote tourism in Korea, the Korea Tourism Organization (KTO) provides "VisitKorea," an application, that offers the latest travel information, categorized information (shopping, accommodation, dining and more), customized travel plans and discount coupons. Seoul, as a smart tourism city, offers a variety of smart attractions 
that offer experience connectivity (see Table 1). Also, the Korean government has just launched new project to create a smart tourism city to support travel startups expanding their business domain based on ICT and provide advanced contents and infrastructures to the residents and visitors.

Table 1. Experience connectivity in smart tourism city.

\begin{tabular}{|c|c|c|c|c|}
\hline Attractions & Smart Tech. Tools & Locations & Elements & Smart Experience Enhancement \\
\hline $\begin{array}{l}\text { BOUT: Uber } \\
\text { Boat (Finland) }\end{array}$ & Application & On-site & Transportation & $\begin{array}{l}\text { The licensed owners, independent } \\
\text { entrepreneurs, carrying passengers on a } \\
\text { commercial basis. } \\
\text { - Active participation: Improve access to } \\
\text { islands and waterfronts in the Helsinki } \\
\text { metropolitan area } \\
\text { - Interaction: For-profit P2P platform for } \\
\text { on-demand boat rides }\end{array}$ \\
\hline $\begin{array}{c}\text { VeneziaUnica } \\
\text { (Italy) }\end{array}$ & Application & $\begin{array}{c}\text { On-site } \\
\text { Post-travel }\end{array}$ & $\begin{array}{c}\text { Service Activity } \\
\text { Attraction }\end{array}$ & $\begin{array}{l}\text { - Active participation: Directly contact } \\
\text { service providers and their websites } \\
\text { - Interaction: Share opinions and photos } \\
\text { with other users }\end{array}$ \\
\hline $\begin{array}{l}\text { Salzburger } \\
\text { Mittagsplaner } \\
\text { (Austria) }\end{array}$ & Application & $\begin{array}{l}\text { Pre-travel } \\
\text { On-site } \\
\text { Post-travel }\end{array}$ & $\begin{array}{c}\text { Service Activity } \\
\text { Gastronomy }\end{array}$ & $\begin{array}{l}\text { - Active participation: Empowering users } \\
\text { to collect information on food and } \\
\text { beverage service providers } \\
\text { - Interaction: Links to providers' websites } \\
\text { and share opinions }\end{array}$ \\
\hline K-live (Korea) & Hologram & On-site & $\begin{array}{l}\text { Activity } \\
\text { Ancillary } \\
\text { Service }\end{array}$ & $\begin{array}{l}\text { - Active participation: Digital attractions } \\
\text { such as photo box, star lounge, secret } \\
\text { window and three-dimensional } \\
\text { representations of K-pop } \\
\text { stars' performances } \\
\text { - } \quad \begin{array}{l}\text { Interaction: Directly contacts service } \\
\text { providers and books tickets }\end{array}\end{array}$ \\
\hline $\begin{array}{l}\text { City of Love } \\
\text { (Korea) }\end{array}$ & $\begin{array}{l}\text { Games and } \\
\text { Application }\end{array}$ & On-site & $\begin{array}{c}\text { Attraction } \\
\text { Transportation } \\
\text { Activity }\end{array}$ & $\begin{array}{l}\text { - Active participation: 'Escape' game } \\
\text { playing at Seoulro-7017, with mission } \\
\text { rewards and opportunities to upload } \\
\text { photos using a geo-reference system } \\
\text { - Interaction: Links to nearby retail shops } \\
\text { and tourism attractions }\end{array}$ \\
\hline
\end{tabular}

Although smart tourism city sheds light on destination city's competitiveness, but the journey to sustainability may be long and there would be no shortcut. The concept and paradigm of smart tourism cities should not overlook the multitude of collateral aspects such as privacy or data storage [38]. In fact, a smartphone is the pivot of all smart systems since users' data are stored mostly via smartphones and the city monitor the physical world in real time and provide intelligent services to both residents and visitors mostly via smartphones [18]. Nevertheless, collecting data from smartphones may cause the security and privacy problem for cities such as data over-collection: smartphones apps collect users' data exceeding its initial purpose [39]. Therefore, the cities are recommended to take a prudent approach in its operating smart tourism city services. For instance securing a network with a large 
attack surface, properly utilizing artificial intelligence [40], identifying at stake privacy concerns associated with specific technologies, checking suitability with EU data protection regulation, and developing a specific city policy accordingly [41] are the indisputable ways of ensuring privacy. There is no doubt that tourists' experiences mediated by ICT are decisive in destination cities depending on how the city is ready with appropriate policies and actions which encourage tourists share and use smart technologies to enhance experiences [42].

\section{Smart Tourism City Roles}

\subsection{Sustainability Problems}

Tourism destination is a place where offering tourism infrastructures and package of tourism services [43] and focuses on tourism related services and infrastructures to meet visitors' expectations. Ritchie and Crouch [44] assessed tourism destination competitiveness through categorizing it into supporting factors, core resources and attractions, destination management and determinants. Since the tourism business reflects current and future economic, social and environmental impacts, addressing the needs of visitors, the industry, the environment and host communities [45]. Thus, tourism must be sustainable in environmental, economic and socio-cultural dimensions. The model of Vargas [46] is the most well-known conceptual model of destination competitiveness in tourism literature and has been the starting point for many other research studies 'sustainable' destination competitiveness. Cucculelli and Goffi [47] extends the Richie and Crouch [44] model and examined a set of sustainability indicators that factors directly referring to sustainability have a positive impact on all the competitiveness indicators on their study of "destinations of excellence" based on the model of destination competitiveness.

In terms of sustainability, cities face over-tourism and inclusive tourism issues. Tourism growth can be a potential cause of environmental damage and sociocultural problems [48]. With environmental damage and sociocultural problems caused by too many tourists, inhabitants experience routine-life-disturbance, tourists would no longer be welcome. Over-tourism occurs when too many tourists overwhelm a destination, displacing the balance from positive effects to a situation in which tourism becomes unsustainable. Originally burdened with problems in globalization, rapid urbanization, competitiveness and sustainable development, popular tourism cities are now facing tourism growth that challenges existing carrying capacity. The combination of budget flights and home-sharing sites has contributed significantly to over-tourism: Budget airlines enable more people to reach tourism destinations: home-sharing sites, Airbnb, provide accommodation in the middle of local community so that tourists can experience local life in communities.

Over-tourism manifests itself differently in each destination but is based on the common themes of alienated local residents, degraded tourist experience, overloaded infrastructure, damage to nature and threats to culture and heritage [49]. These problems manifest themselves in a variety of ways. In Barcelona, unlicensed rentals caused an incident between a landlord and his tenant for illegally listing a flat on Airbnb. Moreover, as Airbnb has become ever more commercialized, it has caused problems for the housing market and lives of local people. The demand for apartments from visitors means local people are pushed out. Therefore, locals fear being priced out of the city. The city of Lucerne is a very popular destination for Asian tourists is now facing complaints from the locals regarding traffic congestion in the town, which they allege is caused by tourist buses dropping off visitors. The problem involves overcrowding on the streets and too many people trying to gain access to key tourist spots in town and the lack of infrastructure around it. In a town with just 80,000 inhabitants, locals are starting to complain and issue warnings that the city must figure out a way to ensure sustainable tourism development. Challenges include reducing resource costs and creating market differentiation prior to developing the market further. Venice, too, has a problem with over-tourism in that the influx of over 30 million tourists a year is threatening its authentic ambience. As a solution, the Venice City government has restricted tourists to a few of the attractions and introduced a prohibition on new tourist accommodations as part of its self-preservation concerns about over-tourism. Amsterdammer 
were happy on the day the city removed the iconic "I Amsterdam" letters from the Museumplein in its efforts to 'chase away' tourist after suffering from too many tourists. Bukchon Hanok Village in Seoul is one of the most popular attractions for visitors as it is a 600-year-old historic village featuring traditional Korean houses. More than 10,000 visitors visit Bukchon Hanok Village from 6 am onward every day, which causes tourism fatigue. Accordingly the Seoul government presented a guidebook on how to walk around Bukchon Hanok Village and specifically requests visitors not to make any loud noises, take pictures of residents or look into people's houses.

Practicing inclusive tourism is also an issue for cities' sustainable development. As tourism in the socio-cultural context must particularly contribute to inter-cultural understanding and tolerance, United Nations World Tourism Organization (UNWTO) [45] highlights tourism should be a driver of inclusive development. "inclusive tourism" is generally referred to as "disabled tourism' or "accessible tourism," which is about making travel easy for all people; that is, inclusive tourism involves a set of services and facilities that offer people, including the disabled, pregnant women, children in prams and seniors, accessibility that facilitates mobility, vision, hearing and the cognitive dimensions of access. Darcy and Dickson [50] asserted that accessibility in tourism is a social right that concerns all citizens. Such service includes emotional support, so that technology does not bear the entire burden, and various options are made available, which instills confidence in all people, empowering them to indulge in new experiences.

\subsection{Smart Tourism City Solutions}

Over-tourism may also bring with it low levels of hostility or, in some places, there may be extreme cases of violence. In this regard, UNWTO [5] has warned that people will not travel to places where they are made to feel unwelcome and that many jobs are at stake in such locations. However, bans and restrictions are not ideal solutions to over-tourism. City governments must still maximize the positive impacts of tourism because $11 \%$ of global consumption involves tourists as consumers.

With COVID-19 outbreak, the world stays home, there is no tourism. Some cities like Amsterdam still said that they want their only for Amsterdammers: only quality tourism, no more massive tourists in their city. Whereas some cites reliant on travel and tourism for survival since tourism is one of the biggest employers. Those cities face survival concerns, as one of the biggest and fastest growing sectors, tourism, has been considered as a vulnerable industry under the pandemic. How can we make tourism generate ideal benefit to all of us? No matter what is a city's approach, the impacts of tourism are diverse. Further over-tourism is generally observed in specific parts of the city at a certain time which means it would be a residents' perception regardless intensity of travel activities [51]. However, the solution for dealing with over-tourism issues has to be diverse depending on local context and has a sustainable approach in the long-term [38].

A long-term solution regarding the demands from locals and destinations for more sustainable tourism practices is to promote travel during off-peak seasons, which will help reduce the negative impacts of over-tourism. In fact, not all tourism destinations are crowded with tourists, and many cities are keen to have more tourists. And ICT could be one of solution and there is a need for an increasing use of "smart" technology to design smart tourism city to rise to this challenge [52] in spite of technological or smart solutions alone will not solve over-tourism [51]. As smart sustainability is regarded as a new perspective in the sustainable tourism debate, Perles and Ivars [53] identified the elements of smartness and sustainability which benefit from synergetic approach: monitoring systems, real-time management, public-private cooperation, and open innovation are closely related elements.

In this context, the smart tourism city, a tourism destination based on an extremely developed technological infrastructure and enable tourists to communicate and interact with their environment, offers opportunities to address these challenges with its systematic collaboration among diverse stakeholders, advanced infrastructure and user-friendly platform in a connected city. A smart tourism city would not only solve urban problems and provide citizens with a better living environment [25], but would also enable visitors to explore new destinations and indulge in local products and services 
at the right time by having real time available and infrastructure to control. Thus, under the smart tourism city, governments take a more novel approach to tourism that could mitigate the negative effects using aggregated and analyzed data from each stakeholder.

Pearce [38] identified five specific themes needed for limiting over-tourism and offering sustainable quality of life for residents and unique experiences for visitors: smart preparation, smart guest, smart traveler, smart user of technology and smart immersion. "Smart traveler" and "smart technology user" are the solutions based on smart tourism city factors. First, "smart traveler" is about key phases of the tourists' mobility efforts: getting there, getting around and leaving destinations. Using data offered to tourists may help smooth the local traffic flow. Second, "smart technology user" is about the tourists' abilities to manage their time and reduce their impacts. Using online live streams of notices regarding peak visiting times at sites, mobile guides and access to services reduces frustrations for the tourists themselves and the residents. After all governments and local authorities must look for ways to control tourist numbers and it is essential to work closely between public sectors, locals and tourism companies and share gathered "data", respectively based on extremely developed technological infrastructure. The City of Barcelona, recognizing that the city was facing a severe over-tourism problem, used data aggregates based on cutting-edge technology and developed an apartment rental-detection program, a novel solution designed to fight illegal tourist apartment rentals. This user-friendly platform provides information to residents and visitors and increases the quality of life for both parties.

As a smart tourism city provides increasingly customized and enriched tourism experiences to people, such a city should concern itself with its role in inclusive sustainable tourism and transform its accessibility to include all people, irrespective of their gender, age or physical status. ICT solutions, such as beacons, which add intelligence to the identification and location of close objects, could be developed into inclusive devices in this regard. A few cities have transformed themselves into smart tourism city to contribute to inclusivity in the context of sustainable development: Vancouver, Hamburg and Copenhagen have taken initiatives and developed more accessible cities to enhance their inhabitants' quality of life as well as attract more varied visitors. Vancouver is collaborating with experts to ensure universal design and accessibility best practices in its region by 2024 . The city government announced collaboration between the public and private sectors on projects such as monitoring, based on information technology of tourism experiences, to identify how universally accessible they are, which, in turn, will help private businesses and communities apply for or access funding and grants to enhance the degree of inclusivity.

In sum, cities are facing a new challenge for its sustainability and inclusiveness and technology-based social infrastructure, smart tourism city, combined with creativity and innovation is the answer for designing sustainable environment for both residents and visitors.

\section{Conclusions and Implications}

In the past 10 years, the ICT revolution has offered cities the opportunity to solve urbanization issues by altering living environment of citizens, industries and the governing mode of cities. This entire environmental restructuring caused by ICTs, which has offered vast amounts of data, has altered the patterns of the tourism industry as well. In addition, with tremendous impacts of tourism, cities use advanced living environment to obtain its competitiveness in tourism market. In this context, a smart tourism city, convergence of smart city and smart tourism, is an innovative tourist destination that guarantees sustainable development that facilitates [11] and enhances visitors' interaction with experiences at the destination, tourism quality over quantity, and eventually improves the residents' quality of life. The concept of the smart tourism city is based on data sharing between the public and private sectors; visitors and residents are playing a significant role in providing and receiving that information. The active participation of both visitors and residents by sharing their on-site experiences is highly crucial during the smart experience process. The components of smart city are combined with the elements of smart city (service, infrastructure, land) and smart tourism (transportation, accommodation, gastronomy, attraction and ancillary service). The integrated and coordinated 
implementation of the smart city elements is to enhance the smart tourism experience during all three traveling phases (before-during-after). The development of a smart tourism city depends highly on its local context factors. In fact, a tourism city in which the tourists are of central importance also has the ultimate goal of enhancing the city's competitiveness [54]. More important, the smart tourism city's competitiveness can be increased by government leadership based on environmental quality [55], and cities must identify their strong spots that can accommodate massive numbers of visitors, and the spots they want to remain untapped.

On the other hand in terms of sustainable development, cities face over-tourism and inclusive tourism issues. Last few years, too many tourists overwhelm a destination, thereby displacing the balance from positive effects to one where tourism becomes unsustainable. Many popular destinations are suffering from over-tourism, which hinders residents' daily routine. Smart tourism city is likewise one of powerful solution to the challenges posed by sustainable development issues such as over-tourism and rapid urbanization and can serve as a dynamic means to enhance local economies via tourism. Smart tourism city with an extremely developed technological infrastructure enables tourists to communicate and interact with their environment by monitoring and controlling overflow crowd with its systematic collaboration among diverse stakeholders, advanced infrastructure and user-friendly platform in a connected city. Moreover as new technologies have contributed to making the tourism experience more accessible and rewarding for "everyone," smart tourism cities must include not only geographic fair development principles, but also social inclusiveness for the population as a whole, which is the principle that human rights must be afforded to everyone without exception. Smart tourism city development should benefit all and displace none and transform the domain beyond the traditional mindset of tourism. Of course, the ICT revolution alone is not the answer for sustainable development [46] since the concept of smart city is highly complex and interdependent [56]. Moreover, smart city framework is a much stronger focus on advanced technology than sustainability framework. However smart city encompass aspects of the sustainability challenge by developing smart solutions for sustainability and promoting citizen participation. Therefore, it is imperative to measure the impact of smart city in relation to sustainability: contribution towards environmental, economic or social sustainability [57].

The role of a smart tourism city is not limited. Particularly smart tourism city is even more focused in terms of standardization protocols for increased data sharing in the event of COVID-19 outbreak [58,59]. People are canceling their travel plans and staying at home, smart tourism city may consider creating timely customized AR/VR contents which was initially for natural preservation and enhancing tourists' experiences. Thus, smart tourism city development requires the informed participation of each stakeholder to shape the city efficiently and gain sustainable competitiveness in this dynamic tourism market [45].

This study underlines the city government's approach, which is based on the following key aspects: (1) Rational or based on prioritizing the residents' and tourists' enterprise, for which the city must develop a tourism infrastructure that offers vigorous technological connectivity among related entities; and (2) Emotional, that is, the city must involve itself in the full cycle of tourism consumption. Thus, its "every" resident will be protected from overcrowding tourist problems, and its tourists will be guaranteed personalized, inclusive-travel experiences. Furthermore, its industries will be motivated to take the initiative to share and create the information needed. Finally, we need to ask what makes a city "smart" and which smart tourism cities do we design for the sustainable development. To answer this question, the ideal approach is to shift the focus from technologies to the people.

This study provides an overview of the smart tourism city and helps academia and industry practitioners identify the main components of smart tourism city and its contexts. While in recent years obvious progress has been made in smart tourism destination research, but there is research gap in smart tourism "city" which focuses more on its residents. Further by identifying of smart tourism city roles, this study provides long term solution to design sustainable city where embracing residents' and tourists' quality of life and experience. While this study contributed to a better understanding of 
anatomy of smart tourism city, limitations remain. First, the components of smart tourism city model were designed focusing on limited factors in the previous studies. Second, it has lack of theoretical findings, but it sought to emphasize the current issues in tourism, sustainability and proposed smart tourism city as one of powerful solution. However further research is needed to strengthen the empirical and theoretical contributions.

Author Contributions: P.L. performed the literature review and wrote the paper. W.C.H. performed writing-review and editing. N.C. designed the paper and revised the paper. All authors have read and agreed to the published version of the manuscript.

Funding: This work was supported by the Ministry of Education of the Republic of Korea and the National Research Foundation of Korea (NRF-2019S1A3A2098438).

Conflicts of Interest: The authors declare no conflict of interest. The founding sponsors had no role in the design of the study; in the collection, analyses, or interpretation of data; in the writing of the manuscript, and in the decision to publish the results.

\section{References}

1. Gretzel, U.; Sigala, M.; Xiang, Z.; Koo, C. Smart tourism: Foundations and developments. Electr. Mark. 2015, 25, 179-188. [CrossRef]

2. Bakıc1, T.; Almirall, E.; Wareham, J. A Smart City Initiative: The Case of Barcelona. J. Knowl. Econ. 2012, 2, 1-14. [CrossRef]

3. Gretzel, U.; Werthner, H.; Koo, C.; Lamsfus, C. Conceptual foundations for understanding smart tourism ecosystems. Comput. Hum. Behav. 2015, 50, 558-563. [CrossRef]

4. Christopoulou, E.; Ringas, D.; Garofalakis, J. The vision of the sociable smart city. In International Conference on Distributed, Ambient, and Pervasive Interactions; Springer: Cham, Germany, 2014; pp. 545-554.

5. UNWTO. 2015. Available online: http://media.unwto.org/en/content/understanding-tourism-basic-glossary (accessed on 12 May 2020).

6. Hunter, W.C.; Chung, N.; Gretzel, U.; Koo, C. Constructivist research in smart tourism. APJIS 2015, 25, 105-120. [CrossRef]

7. Buhalis, D.; Amaranggana, A. Smart tourism destinations enhancing tourism experience through personalization of services. In Information and Communication Technologies in Tourism; Springer: Cham, Germany, 2015; pp. 377-389.

8. Okumus, F.; Kar, M.; Bilim, Y.; Ozturk, A.B.; Ozer, O.; Çaliskan, U. The relationship between local residents' perceptions of tourism and their happiness: A case of Kusadasi, Turkey. Tour. Rev. 2015. [CrossRef]

9. Cloutier, S.; Larson, L.; Jambeck, J. Are sustainable cities "happy" cities? Associations between sustainable development and human well-being in urban areas of the United States. Environ. Dev. Sustain. 2014, 16, 633-647. [CrossRef]

10. Yin, C.; Xiong, Z.; Chen, H.; Wang, J.; Cooper, D.; David, B. A literature survey on smart cities. Sci. China Inf. Sci. 2015, 58, 1-18. [CrossRef]

11. Bifulco, F.; Tregua, M.; Amitrano, C.C.; D'Auria, A. ICT and sustainability in smart cities management. Int. J. Public Sec. Manag. 2016, 29, 132-147. [CrossRef]

12. Neirotti, P.; De Marco, A.; Cagliano, A.C.; Mangano, G.; Scorrano, F. Current trends in Smart City initiatives: Some stylised facts. Cities 2014, 38, 25-36. [CrossRef]

13. Harrison, B.; Eckman, R.; Hamilton, P.; Hartswick, J.; Kalagnanam, P.J.; Williams, P. Foundations for Smarter Cities. IBM J. Res. Dev. 2010, 54, 1-16. [CrossRef]

14. Benevolo, C.; Dameri, R.P.; D'Auria, B. Smart mobility in smart city. In Empowering Organizations; Springer: Cham, Germany, 2016; Volume 11, pp. 13-28.

15. Cardullo, P.; Kitchin, R. Being a 'citizen'in the smart city: Up and down the scaffold of smart citizen participation in Dublin, Ireland. Geo J. 2019, 84, 1-13.

16. Buhalis, D.; Amaranggana, A. Smart tourism destinations. In Information and Communication Technologies in Tourism 2014; Springer: Cham, Germany, 2013; pp. 553-564.

17. Zubizarreta, I.; Seravalli, A.; Arrizabalaga, S. Smart City Concept: What It Is and What It Should Be. J. Urban Plan. Dev. 2016, 142, 04015005. [CrossRef] 
18. Zhang, K.; Ni, J.; Yang, K.; Liang, X.; Ren, J.; Shen, X.S. Security and privacy in smart city applications: Challenges and solutions. IEEE Commun. Mag. 2017, 55, 122-129. [CrossRef]

19. Glebova, I.S.; Yasnitskaya, Y.S.; Maklakova, N.V. Assessment of cities in Russia according to the concept of "smart city" in the context of the application of information and communication technologies. Mediterr. J. Soc. Sci. 2014, 5, 55-60. [CrossRef]

20. Khansari, N.; Mostashari, A.; Mansouri, M. Impacting sustainable behavior and planning in smart city. Int. J. Sustain. Dev. 2014, 1, 46-61. [CrossRef]

21. Jovicic, D. Cultural tourism in the context of relations between mass and alternative tourism. Curr. Issues Tour. 2016, 19, 605-612. [CrossRef]

22. Kim, C.W. E-tourism. In Innovation and Growth; OECD: Paris, France, 2006; pp. 135-146.

23. Chung, N.; Han, H.; Koo, C. A Comparative Analysis of Usage Motivation and Tourism Information Search Behavior in Online Travel Community Using Elaboration Likelihood Model. J. Tour. Sci. 2013, 37, $219-240$.

24. Lee, B.C.; Byun, H.J. The Impact of Online Review on Purchasing Behavior: A Case of Hotel and Resort. Tour. Leis. Res. 2014, 26, 59-79.

25. Wang, D.; Park, S.; Fesenmaier, D.R. The role of smartphones in mediating the touristic experience. J. Travel Res. 2012, 51, 371-387. [CrossRef]

26. Li, Y.; Hu, C.; Huang, C.; Duan, L. The concept of smart tourism in the context of tourism information services. Tour. Manag. 2017, 58, 293-300. [CrossRef]

27. Abdul Aziz, A.; Bakhtiar, S.; Faeez, M.; Kamaruddin, M.S.Y.; Ahmad, N.A. Information and communication technology application's usage in hotel industry. JTHCA 2012, 4, 34-48.

28. Tu, Q.; Liu, A. Framework of smart tourism research and related progress in China. In Proceedings of the International Conference on Management and Engineering (CME) 2014, Shanghai, China, 24-25 May 2014; DEStech Publications, Inc.: Lancaster, PA, USA; pp. 140-146.

29. Boes, K.; Buhalis, D.; Inversini, A. Smart tourism destinations: Ecosystems for tourism destination competitiveness. Int. J. Tour. Cities 2016, 2, 108-124. [CrossRef]

30. Ottenbacher, M.C.; Harrington, R.J. A case study of a culinary tourism campaign in Germany: Implications for strategy making and successful implementation. J. Hosp. Tour. Res. 2013, 37, 3-28. [CrossRef]

31. Buonincontri, P.; Micera, R. The experience co-creation in smart tourism destinations: A multiple case analysis of European destinations. Inf. Technol. Tour. 2016, 16, 285-315. [CrossRef]

32. Jung, T.; Chung, N.; Leue, M.C. The determinants of recommendations to use augmented reality technologies: The case of a Korean theme park. Tour. Manag. 2015, 49, 75-86. [CrossRef]

33. Chung, N.; Han, H.; Joun, Y. Tourists' intention to visit a destination: The role of augmented reality (AR) application for a heritage site. Comput. Hum. Behav. 2015, 50, 588-599. [CrossRef]

34. Lee, H.; Jung, T.H.; tom Dieck, M.C.; Chung, N. Experiencing immersive virtual reality in museums. Inf. Manag. 2020, 103229. [CrossRef]

35. Garau-Vadell, J.B.; Gutierrez-Taño, D.; Diaz-Armas, R. Economic crisis and residents' perception of the impacts of tourism in mass tourism destinations. J. Dest. Mark. Manag. 2018, 7, 68-75. [CrossRef]

36. Neuhofer, B.; Buhalis, D.; Ladkin, A. Conceptualising technology enhanced destination experiences. J. Dest. Mark. Manag. 2012, 1, 36-46. [CrossRef]

37. Qin, Y. Analysis of Key Elements for Smart Tourist City Construction with G1-Entrophy Methods. Rev. Fac. Ing. 2017, 32, 759-763.

38. Pearce, P.L. Limiting Overtourism; The Desirable New Behaviours of the Smart Tourist. In The Tourism Intelligent Forum; University of Alicante: Palma, Spain, 2018.

39. Li, Y.; Dai, W.; Ming, Z.; Qiu, M. Privacy protection for preventing data over-collection in smart city. IEEE Transac. Comput. 2015, 65, 1339-1350. [CrossRef]

40. Braun, T.; Fung, B.C.; Iqbal, F.; Shah, B. Security and privacy challenges in smart cities. Sustain. Cities Soc. 2018, 39, 499-507. [CrossRef]

41. Van Zoonen, L. Privacy concerns in smart cities. Gov. Inf. Q. 2016, 33, 472-480. [CrossRef]

42. Femenía, F.; Perles, J.F.; IVars, J.A. Smart destinations and tech-savvy millennial tourists: Hype versus reality. Tour. Rev. 2019, 74, 63-81. [CrossRef]

43. Hu, Y.; Ritchie, J.B. Measuring destination attractiveness: A contextual approach. JTR 1993, 32, $25-34$.

44. Ritchie, J.R.B.; Crouch, G.I. The competitive destination, a sustainable perspective. Tour. Manag. 2000, 21, 1-7. 
45. UNWTO. Making Tourism More Sustainable. A Guide for Policy Makers; UNEP and UNWTO: Madrid, Spain, 2005.

46. Vargas Sánchez, A. Exploring the concept of smart tourist destination. Enlightening Tourism. Pathmak. J. 2016, 6, 178-196.

47. Cucculelli, M.; Goffi, G. Does sustainability enhance tourism destination competitiveness? Evidence from Italian Destinations of Excellence. J. Clean. Prod. 2016, 111, 370-382. [CrossRef]

48. Goffi, G.; Cucculelli, M.; Masiero, L. Fostering tourism destination competitiveness in developing countries: The role of sustainability. J. Clean. Prod. 2019, 209, 101-115. [CrossRef]

49. McKinsey \& Company. Report of Coping with Success: Managing Overcrowding in Tourism Destinations; McKinsey \& Company: New York, NY, USA, 2017.

50. Darcy, S.; Dickson, T. A whole-of-life approach to tourism: The case for accessible tourism experiences. J. Hosp. Tour. Manag. 2009, 16, 32-44. [CrossRef]

51. Koens, K.; Postma, A.; Papp, B. Is overtourism overused? Understanding the impact of tourism in a city context. Sustainability 2008, 10, 4384. [CrossRef]

52. Ivars-Baidal, J.; García Hernández, M.; Mendoza de Miguel, S. Integrating Over-tourism in the Smart Tourism Cities Agenda. e-Rev. Tour. Res. 2019, 17, 122-139.

53. Perles, J.F.; IVars, J.A. Smart sustainability: A new perspective in the sustainable tourism debate. J. Reg. Res. 2018, 42, 151-170.

54. Fyall, A. Destination Management: Challenges and Opportunities. Dest. Market. Manag. 2011, 340. [CrossRef]

55. Crouch, G.I. Destination competitiveness: An analysis of determinant attributes. J. Travel Res. 2011, 50, 27-45. [CrossRef]

56. Basiri, M.; Azim, A.Z.; Farrokhi, M. Smart city solution for sustainable urban development. European. J. Sustain. Dev. 2017, 6, 71-84.

57. Ahvenniemi, H.; Huovila, A.; Pinto-Seppä, I.; Airaksinen, M. What are the differences between sustainable and smart cities? Cities 2017, 60, 234-245. [CrossRef]

58. Allam, Z.; Jones, D.S. On the coronavirus (COVID-19) outbreak and the smart city network: Universal data sharing standards coupled with artificial intelligence (AI) to benefit urban health monitoring and management. Healthcare 2020, 8, 46. [CrossRef]

59. Um, T.; Chung, N. Does smart tourism technology matter? Lessons from three smart tourism cities in South Korea. Asia Pac. J. Tour. Res. 2019. [CrossRef]

(C) 2020 by the authors. Licensee MDPI, Basel, Switzerland. This article is an open access article distributed under the terms and conditions of the Creative Commons Attribution (CC BY) license (http://creativecommons.org/licenses/by/4.0/). 

Article

\title{
Analyzing Spatial Variance of Airbnb Pricing Determinants Using Multiscale GWR Approach
}

\author{
Insu Hong ${ }^{1}$ and Changsok Yoo ${ }^{2, *}$ \\ 1 Department of Geology and Geography, West Virginia University, Morgantown, WV 26506, USA; \\ insu.hong@mail.wvu.edu \\ 2 Department of Culture, Tourism \& Content, Kyung Hee University, Seoul 02447, Korea \\ * Correspondence: csyoo@khu.ac.kr; Tel.: +82-2-961-0425
}

Received: 30 April 2020; Accepted: 4 June 2020; Published: 9 June 2020

\begin{abstract}
A sharing economy accommodation service like Airbnb, which provides trust between strangers to connect them for profiting from underutilized assets, was born and has thrived thanks to the innovations in the platform technology. Due to the unique structure of Airbnb, the pricing strategies of hosts are very different from the conventional hospitality industry. However, existing Airbnb pricing studies have limitations considering the varying scale of operation among hosts, spatial variances in pricing strategies, and crucial geographic information for estimating the influence of the pricing variables, as well as ignoring inter-city variances. In this research, we explored the spatially heterogeneous relationship between price and pricing variables using an innovative spatial approach, Multiscale Geographically Weighted Regression (MGWR). Analysis results for Airbnb listing in Log Angeles and New York in the US showed the effectiveness of MGWR regarding estimating the influence of pricing variables spatially. By revealing spatially heterogeneous and dependent relationships, this research fills gaps in Airbnb pricing research and deepens the understanding of the pricing strategies of the hosts.
\end{abstract}

Keywords: spatial variance; multiscale GWR; sharing economy; Airbnb

\section{Introduction}

Pricing has become one of the most effective tools for customer satisfaction and promotion of demand [1]. Pricing strategy has been widely used in the tourism and hospitality industry, and become a field of revenue management, applied to airlines, hotels, restaurants, golf, casinos, and theme parks [2-5]. With the advancement in tourism information services that enable customers to compare prices, the strategic importance of pricing is tremendous now more than ever $[2,6]$.

Tourism products are experience goods that users have strong heterogeneity in their value recognition, which makes revenue management for the hospitality industry focus on price differentiation [7]. Numerous studies have investigated strategies for pricing and enhancing profit margin in the hospitality industry, focusing on hotels [8]. However, sharing economy accommodation has distinctive attributes compared to hotels, mainly due to its residential housing-based service. These unique features make it challenging to apply the conventional understanding of the hospitality industry on the value recognition of users [9-12]. For example, the star rating system based on user reviews has limited influence on the price of Airbnb listings, unlike the hotel industry, while indirect signals such as the service duration and personal information of the hosts are considered as alternative sources for the trust and significant influence on the price [9,13].

However, existing Airbnb pricing studies have limitations considering spatial variances and crucial geographic information for estimating the influence of the pricing variables. It is well known that the influence of pricing variables may have local variations, indicating different dynamics among factors across space $[14,15]$. To explicitly reflect this spatial heterogeneity, the geographically weighted 
regression (GWR) has been utilized for pricing research into hotels and Airbnb hosts [16] and the provisions of accommodations in the sharing economy [17-20]. An issue here is that GWR does not consider a varying scale of spatial heterogeneity, treating all variables as having an identical scale of operation [21]. Pricing variables are spatial processes that determine the price, and each of them will operate under a different scale, from local to global. However, to date, no research effort has been made that considers this multiscale regression approach for Airbnb pricing analysis.

The second issue is negligence on a crucial decision criterion for accommodation: distance to tourism destinations [14]. Since tourists are the most frequent customers of Airbnb [6], the distance criterion needs to be included in Airbnb pricing research. A few previous studies have considered the distance variable in their pricing model. However, they used surrogate points such as a city center and highway exits for tourism destinations [22,23], which is not an accurate representation. A tourist will consider accessibility to multiple tourism destinations to make accommodation decisions. Therefore, distance to multiple tourism destinations must be explicitly considered in the Airbnb pricing research.

Furthermore, the possibility of spatial heterogeneity in pricing decisions among different areas has not been explored in the previous Airbnb research, such as inter-city differences. It is highly likely that different regions will show varying spatial dynamics of pricing decisions, as each of them have unique characteristics. Analyzing big data that cover multiple cities and comparing the results will reveal such inter-city level spatial heterogeneity, as well as intra-city level spatial variance in each location. Such a comparison will help us understand the underlying causes of varying patterns of pricing strategies more effectively. However, to date, the authors have not found Airbnb pricing research that made a comparison of pricing strategies among places.

In this research, we try to answer these research questions to fill the gaps mentioned above in the analysis of the sharing economy and the interaction between tourist attractions and accommodation, using big data: (1) what does relationship between price and pricing variables look like if we consider spatial heterogeneity in multiscale?; and (2) How does the distance of tourist attractions affect the price? We utilize a novel spatial approach that enables us to reveal more complex relationships and spatial patterns among pricing determinants and Airbnb listing price. We use the Multiscale GWR (MGWR) model for estimating the influence of pricing variables on varying scales. Using MGWR, we compare the influence of pricing variables in two major tourist destinations in the US, Los Angeles and New York. Additional geographic information, distances to popular tourism destinations, poverty ratio, and Airbnb listing density are also included as Airbnb pricing variables to reflect the influence of external factors. The structure of this research is as follows. We will review the previous studies related to pricing research in shared economy accommodation and attempts to understand spatial heterogeneity in pricing strategies. Study areas and the research method will be introduced. We then present the MGWR analysis results for the influence of the pricing variables in LA and New York, which will be followed by discussions and concluding remarks.

\section{Background and Literature Review}

\subsection{Sharing Economy, Tourism, and Sustainability}

The sharing economy is a social phenomenon from the convergence of mobile technology and social media platforms [24]. There are many definitions of the sharing economy by academics $[25,26]$. However, the sharing economy can be narrowly defined as a business or online platform which provides temporary access to underutilized physical assets for a fee or for free without a transfer of ownership [27].

Although the sharing economy uses the word "sharing," the core of the sharing economy is not charity but a decentralized commerce platform connecting under-utilized assets and potential customers [28]. The sharing economy coordinates the acquisition and distribution of goods for a fee or other compensation, which is called "collaborative consumption" in academia [29]. 
Because the most valuable under-utilized assets for people are a house and a car, sharing economy activities have flourished, especially for accommodation and transportation services in the tourism industry [30]. The sharing economy services in the tourism industry not only provide the cost leadership based on the idling capacity of resources but also suggest a new way of life among the local community through interactive communication [31-33]. The emerging trend of fully independent tourists accelerated the growth of the sharing economy in the tourism industry [34,35].

Regarding sustainability, the emerging sharing economy in the tourism industry has a higher possibility of this than the traditional industry. Through the utilization of under-utilized assets, the sharing economy can increase the amount of service without additional construction or acquisition, which would decrease the environmental footprint of the industry [31]. Several studies have shown that consumers of the sharing economy have a higher interest in the local community and the environment, so that both consumers and suppliers lead to a reduced consumption of energy and water and reduced waste generation [30,33]. This change in consumption activity ultimately contributes to the reduction in greenhouse gas emissions and is considered to be an effective response to climate change [25].

Furthermore, the sharing economy increases the employment rate of the local community [36], as well as the profits of the community [37]. For example, Airbnb's message to tourists, "travelers to your neighborhood," encourages them to have new experiences via sharing the same space with the local community members, which makes the Airbnb tourists engage more in economic activities in the local areas, like eating at a local restaurant, than other tourists [37]. On the supply side, the gains in the sharing economy operations help to vitalize the local economy, as those benefits are transferred to hosts who operate at the local scene [37-39]. Consumers of the sharing economy are also given the opportunity to experience meaningful social experiences within the community, away from the traditional tourism industry's consumption system [25]. The human capital of the community that provides idle assets to the sharing economy serves as a bridge between these opportunities and helps consumers to respect the culture of the community and to resolve social imbalances $[32,40]$.

\subsection{Pricing Research in Hospitality Industry}

Pricing strategy is a crucial tool in the accommodation business for sales optimization [41]. The tourism services and accommodations are highly perishable; therefore, pricing has been used as a strategic tool to promote demand for the specified time [1]. Pricing strategy in the tourism industry has incorporated heterogeneity within hospitality services and grown as a discipline referred to as revenue management $[2,5,42]$. With readily available pricing comparison tools for the customers based on innovative mobile technologies and tourism information systems, the importance of revenue management has been increased more than ever $[2,6]$. Understanding how customers recognize the value of the various attributes of the accommodation has become crucial for successful revenue management [4].

However, a direct survey to customers is not a viable option due to the numerousness of hotel service attributes. Naturally, the indirect inference of the decision-making criteria of consumers has become the standard practice, such as conjoint analysis and the hedonic pricing model [14]. The hedonic pricing model (HPM) is based on the characteristics theory, which assumes that the utilities of customers are structured not from a good itself but from attributes within the good [43]. Typically, a single good has multiple attributes by its nature, and customers try to make the optimal selection of the attributes to maximize their utilities under the budget constraints [44]. Observation of market price can reveal a unit value of each attribute within the given good, and this is referred to as the hedonic price. The highly differentiated market usually shows a better performance from measuring the hedonic price [45].

Thus, the hospitality industry, where the most sophisticated price and product differentiation is used under high competition, frequently uses the HPM to recognize the value of various attributes in the hotel service. Previous studies have found that star ratings, location, reputation, amenities, cleanliness, room attributes, and facilities are vital attributes for the hotel price [23,46-49]. 
It is generally accepted that the most critical attributes for the hotel pricing are the star ratings and location $[14,47]$. Star ratings can be perceived as an index signal evaluated by an independent organization that does not benefit from the biased report of hotel quality and experience. This makes the star rating system strongly influential to customers and hotels as well [13]. Empirical studies also showed that star ratings of hotels have high explanatory power on the hotel price [23,50,51].

Hotel location and the surrounding environment are additional key drivers of product differentiation in the revenue management of hotels. For example, hotel customers willingly pay more for a room with a better view [52]. The hotel industry has been aware of such consumer behavior regarding location, and this has been reflected in the hotel price [46]. Empirical studies have revealed that sea views $[14,46,51]$, city center $[23,51,53]$, and transportation center $[47,50,54]$ are the most-considered location factors for hotel pricing.

Despite the importance of location in the hospitality industry, previous studies in tourism have failed to capture spatial variations properly in the HPM. In many cases, researchers assumed that each region has homogeneous geographical characteristics to control the heterogeneity among regions. From this perspective, every hedonic pricing study for each city has unique value and meaning [14]. However, even in a small city, various geographical characteristics affect the pricing of hotels in different ways. To reflect this, strategies that indirectly include heterogeneity have been developed, such as converting spatial heterogeneity to dichotomous variables $[46,53]$ and including simple Euclidean distance information as a variable [23,54]. However, these strategies oversimplify the spatial heterogeneity or even distort it, failing to reflect spatial variance in the results.

\subsection{Pricing Model in the Sharing Economy}

Airbnb listings are based on residential housing in the local communities, and their characteristics are extremely different from those of the hotels. The difference between Airbnb listings and hotels come from not only real estate, but also service, amenities, the personality of hosts, and local communities. Thus, accumulated knowledge of revenue management in the hospitality industry cannot be applied directly to the sharing economy accommodations [9].

Most pricing studies on Airbnb commonly pointed out that the standardization of business is the key difference between Airbnb and hotels. In the case of hotels, the shape and format of services are quite similar among hotels due to the standardization, so that consumers can easily recognize or compare the values of the service to these industrial service standards. Unlike hotels, each listing in Airbnb has unique characteristics that are hard to compare. However, Airbnb's user interface provides standardized information of listings to customers. In this way, customers recognize the value of listings through the user interface of the platform [13].

Acknowledging these differences, pricing studies on Airbnb have recently started using HPM [9, $13,16,55,56]$. This revealed that trust-related information and the attributes of a given property have a statistically significant impact on the price, while amenities and other service factors showed mixed or even contradicting results. The customer review has been considered as the most indicative information on the quality of Airbnb listings. However, the interactivity of the trust mechanism in Airbnb has distorted the reviews under social pressure from the hosts [57].

Although the reviews of customers are still meaningful indicators of the price $[9,13]$, customers have started to look for other signals to eliminate the biases. The empirical studies found that the status of the host, the service length of the listing, and the super host badge are now utilized for customers to measure the quality and value of a listing [6,56].

The attributes of the given property, and the number, size and types of rooms also showed statistically significant effects on the price of Airbnb listings through product differentiation like hotels. However, amenities and other service factors did not show consistent results. Sometimes, they showed contradictory results $[55,56]$. 


\subsection{Limitations of Previous Hedonic Pricing Research for Sharing Economy Accommodation}

The fundamental assumption of GWR (or any other spatial analysis methods) is Tobler's First Law of Geography: "Everything is related to everything else. However, near things are more related than distant things" [58]. If we translate this for Airbnb pricing, the decision-making processes of Airbnb hosts are related, not independent, so the pricing strategy of one host will influence (and be influenced by) other hosts of nearby listings. However, nearby hosts' strategies will be more influential to each other than distant hosts' strategies. There will also be a threshold distance where the degree of this interaction diminishes to an irrelevant level, which would be the scale of operation. With it, the differences in the attributes of listings and surrounding environment create spatial variances in pricing strategies, resulting in heterogeneous patterns of the influence of each pricing variable, instead of the uniform one across the region, as depicted in OLS(Ordinary Least Squares) analysis results.

GWR captures spatial heterogeneity in pricing strategies by conducting a localized estimation of the influence of the pricing variables. The core idea of GWR is directly capturing geographical variance in regression estimates across space [59]. Unlike OLS, it computes localized regression coefficients of given explanatory variables for a given location, only using its neighboring locations, to show the varying influence of pricing variables. The neighborhood is defined by search bandwidth, a reflection of the concept of the scale of operation. Airbnb pricing research has adopted the concept of locally varying relationships among variables, introducing GWR to the research $[16,19,20]$.

However, the scale of operation is likely to vary for each pricing variable, depending on its nature [21,52]. For example, management variables such as cancellation policy and superhost status are likely to show a similar tendency at the regional or global level. Other variables, such as the number of bedrooms, guests, and distance to tourism destinations, would be the more localized scale of operation, as the influence of them would be more sensitive to the local environment. A critical limitation of GWR is that it applies an identical scale of operation for all the explanatory variables, ignoring the possibility of varying scales [21]. If we assume that all the pricing variables have an identical scale of operation, it will result in creating false spatial variances in global variables. Differences in the scale of operation among local variables will be ignored as well.

Another issue is a lack of or flawed consideration of the proximity to local tourism destinations. Considering that most customers of Airbnb are tourists, proximity to major tourism destinations is the key factor for pricing decisions. However, it has been completely neglected in most previous studies [9,55]. The few studies that attempted to consider it they have used surrogate locations, such as a city center and highway exits, instead of using actual locations [16,23,51]. These are inaccurate representations of tourism destinations, as they are not necessarily located in the city center or near the highway. Considering that most tourists would like to visit multiple destinations, measuring the distance to a single destination is not an effective way to reflect tourists' influence of the location on price [60].

Previous studies only focus on analyzing pricing strategy in a single city [14]. However, considering the unique characteristics of each city, we will likely see significantly different patterns in pricing strategies among cities. These different patterns may be the product of external factors, such as the size, demographics, and employment structure of the given city. Therefore, to improve our understanding of Airbnb pricing decisions, comparison studies among cities need to be conducted.

To analyze the influence of pricing variables on Airbnb price accurately, a different regression model needs to be utilized to reflect the different scale of operation. Additional pricing variables also need to be included to reflect the geographical characteristics of the given areas.

\section{Method, Data, and Study Area}

\subsection{Study Area and Data}

We chose two famous and large tourist destinations in the United States, Los Angeles and New York, that have a large number of Airbnb listings and distinctive characteristics. They are located 
on the opposite side of North America, showing very different demographics, population densities, landscapes, and climate.

Airbnb listing data were collected from 'Inside Airbnb' (http://insideairbnb.com), which is an independent Airbnb data collection website, providing updated and cleaned listing data around the world every month. The most recent listing datasets available at the time of our data collection effort (September 2019) of New York and LA were collected from Inside Airbnb websites. Additionally, listings in Jersey City were included as a part of the New York dataset, considering its proximity and easy access to New York. For the New York area, a total of 51,385 Airbnb listings were collected. For LA, Inside Airbnb collected 45,044 Airbnb listings for the same month.

We defined the price of Airbnb listing as the listed price plus cleaning fee of each listing. The cleaning fee is a "one-time fee charged by a host to cover the cost of cleaning" after each stay. The amount of the fee was widely varying for each host, even for the listings with similar conditions and locations. As this fee will be included in the final price, it should be included in the price variable. However, we excluded listings that charged a cleaning fee higher than the listed price, as this price abuse possibly distorts the analysis results. After this filtering, a total of 44,291 records were remained, 20,422 and 23,869 for LA and New York, respectively. Figure 1 depicts the general distribution of Airbnb listing in the two cities with price information, while Table 1 shows descriptive statistics of the price.
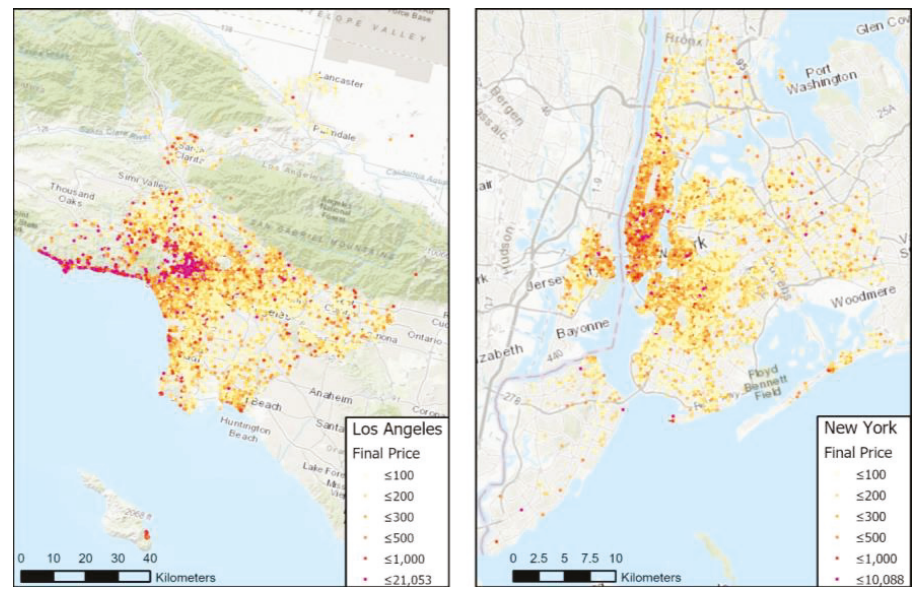

Figure 1. Airbnb and price distribution in Los Angeles (left) and New York (right).

Table 1. Price distribution (US dollar).

\begin{tabular}{ccc}
\hline & LA & NY \\
\hline Mean & 209.9 & 207.9 \\
Median & 167 & 150 \\
Min & 10 & 10 \\
Max & 21,053 & 10,888 \\
Standard deviation & 623.3 & 313.9 \\
\hline
\end{tabular}

Based on the previous research, we selected nine pricing variables, with three additional variables (Table 2). We grouped variables in four categories: listing functions, attributes of host, reputation, and geographical characteristics. Listing functions include the number of bathrooms, bedrooms, and guests, rental type, and cancellation policy, representing the attributes of each Airbnb listing. The rent type variable was included to represent different accommodation types that are unique to Airbnb. The attributes of the host are also crucial, so we have adopted duration and superhost status 
variables. The influence of feedback from other tourists (reputation) was considered through rating and reviews variables. The last category is geographical characteristics, which are density, poverty ratio, and distance index. Density was included to consider the influence of the existence of other competitors on the accommodation price. The poverty ratio from the 2016 American Community Survey was used to indirectly reflect the impact of the housing price of a given Airbnb listing and its neighborhood, since individual housing price data were not available.

Table 2. Selected pricing variables.

\begin{tabular}{|c|c|c|}
\hline \multicolumn{2}{|c|}{ Variable } & Explanation \\
\hline Attributes of host & $\begin{array}{l}\text { duration } \\
\text { superhost }\end{array}$ & $\begin{array}{c}\text { Duration of a host in Airbnb } \\
\text { Superhost status of a host }\end{array}$ \\
\hline Geographical characteristics & $\begin{array}{l}\text { density } \\
\text { proverty_r } \\
\text { dist_index }\end{array}$ & $\begin{array}{l}\text { The number of other Airbnb listings within } 1 \mathrm{~km} \text { range } \\
\text { The ratio of households under the federal poverty line } \\
\text { (census blockgroup) } \\
\text { Combined distance index to } 30 \text { popular tourism destinations in a city } \\
\text { (sum of inversed distance) }\end{array}$ \\
\hline
\end{tabular}

The distance variable was included to reflect tourists' consideration for accessibility to popular tourism destinations, which was measured using straight line distance, as travel time in these large cities is very sensitive to traffic conditions. It has been proved that a straight distance can be used as a surrogate for network travel distance in urban areas with a dense grid road network [61]. The second consideration was measuring the distance to multiple destinations. We picked the top 30 popular tourism destinations of each city, based on Tripadvisor's 'Things to do' ranking. In LA, to reflect the unique characteristics of the area, we have included ten beaches on the list. We then derived a distance index for each listing by adding inverse distances to the selected destinations. The inverse distance represents the distance decay effect in the accessibility.

\subsection{Multiscale Geographically Weighted Regression}

A regression model estimates relationships between explanatory and response variables. Conventional models like OLS assume globally identical relationships in a given region. Therefore, they estimate the relationships, expressed as correlation coefficients, using the entire cases with the same level of weight. If we apply this logic to the Airbnb pricing strategy, we would assume that all the hosts in the region use the same logic and criteria to determine their price, independently to each other. However, this is not entirely true. First, the decision criteria will vary, responding to the surrounding environment. For example, Airbnb listings located in an area with higher housing prices are likely to be more sensitive to the number of bedrooms and bathrooms, compared to lower housing price areas. Furthermore, spatial dependency may exist in the hosts' decision-making process. They will consider the pricing behavior of nearby hosts. The surrounding environment of Airbnb listings in proximity would also be similar, resulting in a similar pricing strategy. To estimate relationships between pricing variables and Airbnb price, we should consider spatial non-stationary processes that determine the influence of pricing variables on the outcome, as well as their spatial dependency.

To accept spatial non-stationary processes and use it for regression analysis, the scale of operation of processes must be analyzed [21]. The scale of operation can be defined as a threshold distance that represents an area where a spatial process works similarly. A spatial process will work differently for every location, in varying degrees of change [21]. However, estimating correlation coefficients for a location based on only one case is not acceptable, as it only uses a single sample. Instead, we can expand the scope of analysis to other cases that are assumed to have a very similar spatial process [21]. 
This scope is the scale of operation of the given spatial process. For a local process, this will vary significantly across the region, as it has a limited scale of operation. If a process has a large enough scale of operation to cover the entire given region, we call it a global process, which has an ignoble level of spatial heterogeneity. To estimate the relationship for the process, only cases within its scale of operation should be used, as we assume spatial non-stationary of processes [21]. Since we assume spatial heterogeneity, this step needs to be repeated for each case in the given region [21,62].

Another crucial concept is spatial dependency [21,62]. Socio-economic decision-making processes like Airbnb pricing decisions cannot happen entirely independent to each other. Decision-makers will influence each other either directly or indirectly, and similarity in the surrounding environment will also add another layer of resemblance. This spatial dependency, as stated in Tobler's First Law of Geography [63], has a diminishing impact, along with distance [62]. To consider this regression analysis for spatial non-stationary processes, distance-decayed weight needs to be applied for analysis, even for those cases within the scale of operation [62]. Closely located cases will have more influence than cases located near the boundary of the scale of operation. It should be noted that, however, this does not mean that such a method would be able to identify the reasons behind the spatial dependency. This approach focuses on estimating relationships under the influence of the spatial dependency, revealing the degree of spatial dependency. Still, it can be used as an exploratory tool for further analysis to find hidden variables or processes behind the spatial dependency.

Based on this logic, GWR was developed to estimate locally varying coefficients for the explanatory variables for each sample location, $i$, with intercept and residual [62]. The expression of the GWR model is like the following

$$
y_{i}=\sum_{j=0}^{m} \beta_{j}\left(\mu_{i}, v_{i}\right) x_{i j}+\epsilon_{i}, i=1, \ldots, n
$$

where:

$y_{i}$ : dependent variable at location $i$;

$x_{i j}$ : jth explanatory variable at location $i$;

$\beta_{j}\left(\mu_{i}, v_{i}\right)$ : jth local correlation coefficient at location $\left(\mu_{i}, v_{i}\right)$;

$\epsilon_{i}$ : error term at location $i$.

As mentioned previously, to estimate a unique set of coefficients using the given case and nearby cases (neighbors), a distance-based non-zero weight function will be applied for them. The weight function can be a Gaussian function or a kernel function. Other cases located beyond the bandwidth will get zero weight.

Then how does GWR estimate the scale of operation? Under ideal circumstances, we would know the actual scale of the operation of the given spatial process. However, this is not the case for most research, requiring an approach based on the distribution of the given data. GWR uses least-squares cross-validation and goodness of fit measures to estimate the distance that minimizes the sum of residuals, considering distance decay effect. In other words, it searches the distance that can maximize the similarity of the spatial process.

For each location $i$, GWR derives a set of unique parameters using weighted least squares. The weight is determined by distance from the given location, usually based on a kernel function. Only neighboring locations, defined as either the locations within a given distance threshold (fixed) or the given number of the nearest locations regardless of the distance (adaptive), will be given non-zero weight. This is expressed as in matrix form

$$
\hat{\boldsymbol{\beta}}(i)=\left[\boldsymbol{X}^{\prime} \boldsymbol{W}(i) \boldsymbol{X}\right]^{-1} \boldsymbol{X}^{\prime} \boldsymbol{W}(i) \boldsymbol{y}
$$

where $\hat{\boldsymbol{\beta}}(i)$ denotes a $j \times 1$ vector of estimated coefficients, $X$ represents $n \times j$ matrix of the explanatory variables, $\boldsymbol{W}(i)$ means diagonal weight matrix, and $y$ is a $n \times 1$ vector for the dependent variable. 
However, as stated earlier, applying an identical scale of operation for every explanatory variable can be a wrong idea. To consider the mixed effect of a local and global variable simultaneously, GWR model was modified as follows, referred to as semi-parametric GWR, or SGWR [64]

$$
y_{i}=\sum_{j=1}^{k_{a}} a_{j} x_{i j}(a)+\sum_{l=1}^{k_{b}} b_{l}\left(\mu_{i}, v_{i}\right) x_{i l}(b)+\epsilon_{i}, i=1, \ldots, n
$$

This model estimates coefficients of global variables and local variables separately, limiting spatial variance only for local explanatory variables. In simple terms, SGWR computes general linear regression for global variables, while it produces local estimates only for variables designated as local.

However, SGWR has two limiting issues. Firstly, making the distinction between local and global variables could be arbitrary or extremely time-consuming. Arbitrary classification possibly results in erroneous estimation. Alternatively, the local-global variable test is possible by altering a variable's category one at a time, followed by the goodness-of-fit test for evaluation. However, this can be a very time-consuming procedure, as all possible combinations need to be evaluated. Furthermore, even if the local-global distinction can be made easily, SGWR only allows two scales of interaction: global or fixed-scale local.

To overcome this limitation, Fotheringham et al. (2017) propose another variation of GWR, multiscale GWR (MGWR) [21]. The fundamental idea of MGWR is using different search bandwidth, the scale of operation, for each explanatory variable to estimate its coefficient. Bandwidths are determined by the data rather than relying on external factors.

$$
y_{i}=\sum_{j=0}^{m} \beta_{b w j}\left(\mu_{i}, v_{i}\right) x_{i j}+\epsilon_{i}, i=1, \ldots, n
$$

Here, $b_{w j}$ indicates the scale of operation (bandwidth) of the $j$ th explanatory variable. To determine the bandwidth, MGWR requires a different approach than GWR and SGWR as it needs to derive multiple different bandwidths for given variables at the same time, so it uses a back-fitting approach. This back-fitting is initialized from ordinary GWR or OLS estimation, and tests goodness-of-fit for each variable to find the most suitable bandwidth to reflect the scale of operation indicated in the given dataset.

\section{Results}

\subsection{Traditional Hedonic Pricing Model}

Before the analysis of MGWR, we conducted the traditional HPM-analysis-based OLS method, and summarized the reultd in Table 3. In the listing functions group, the number of guests, bedrooms, and bathrooms have positive relationships with the price, while sharing a property (private room or shared room) lowers the price. The tendency of the listing functions is similar to previous research results in both cities $[9,56]$. However, the influence of the cancellation policy is different in LA and New York. In New York, the strict cancellation policy has a positive effect on the price compared to a moderate one, while a flexible policy has no impact. In LA, the influence of cancellation policies was precisely the opposite. Previous studies also showed similarly mixed results: a strict policy may have a positive impact $[13,56]$ or may not have any significant influence, while flexible policies impact negatively [9]. Local characteristics may determine the relationship between the cancellation policy and the price. 
Table 3. OLS results.

\begin{tabular}{|c|c|c|c|c|c|c|}
\hline \multirow[b]{2}{*}{ Variables } & \multicolumn{3}{|c|}{ LA } & \multicolumn{3}{|c|}{ New York } \\
\hline & Coefficient & SD & T-Value & Coefficient & SD & T-Value \\
\hline guests & $0.053^{* * *}$ & 0.01 & 5.54 & $0.200 * * *$ & 0.009 & 21.917 \\
\hline bedrooms & $0.157^{* * *}$ & 0.01 & 15.722 & $0.078^{* * *}$ & 0.008 & 9.533 \\
\hline bathrooms & $0.387^{* * *}$ & 0.008 & 48.006 & $0.124^{* * *}$ & 0.007 & 18.685 \\
\hline shared & $-0.080 * * *$ & 0.007 & -12.08 & $-0.100^{* * * *}$ & 0.007 & -14.057 \\
\hline strict & -0.007 & 0.007 & -1.015 & $0.020 * * *$ & 0.007 & 2.725 \\
\hline flexible & $-0.034^{* * *}$ & 0.007 & -4.695 & -0.001 & 0.008 & -0.096 \\
\hline superhost & $0.025^{* * *}$ & 0.006 & 4.053 & $0.030 * * *$ & 0.006 & 4.773 \\
\hline duration & $0.017^{* * *}$ & 0.006 & 2.994 & $0.020^{* * *}$ & 0.006 & 3.228 \\
\hline rating & $-0.115^{* * *}$ & 0.006 & -18.766 & $-0.049^{* * *}$ & 0.006 & -7.762 \\
\hline reviews & $-0.015^{* *}$ & 0.006 & -2.461 & $-0.041^{* * *}$ & 0.006 & -6.366 \\
\hline density & -0.008 & 0.008 & -1.09 & $0.020 * * *$ & 0.007 & 3.038 \\
\hline poverty_r & $-0.055^{* * *}$ & 0.006 & -9.413 & $-0.031^{* * *}$ & 0.006 & -5.134 \\
\hline \multirow[t]{2}{*}{ dist_index } & $0.028^{* * *}$ & 0.008 & 4.363 & $0.195^{* * *}$ & 0.007 & 27.841 \\
\hline & \multicolumn{3}{|c|}{$\mathrm{R}^{2}=0.368$} & \multicolumn{3}{|c|}{$\mathrm{R}^{2}=0.205$} \\
\hline
\end{tabular}

The OLS results revealed that web-based reputation variables have a negative influence on the price. This seems to contradict the previous theories about the influence of reputation. However, it has been theoretically and empirically confirmed that the customers have less faith in the review ratings of the Airbnb listings, as the rating score can be determined by any customer, unlike ratings for hotels evaluated by a third party [13]. The number of reviews also showed the same tendency as in previous studies, impacting negatively on the price. On the other hand, superhost status and service duration have a positive impact on the price. In the signal theory, the customers try to find alternatives (superhost, durations) for the neutralized signals (rating, the number of reviews) to make decisions [13]. Our finding agrees with the signal theory.

In the geographic information group, distance to the tourism destinations and poverty ratio follows the conventional pricing mechanism. The positive coefficient shows a similar tendency to the analysis in the existing HPMs $[6,14]$. LA has a relatively higher coefficient for the distance. This is a reflection of the geographical characteristics of the two cities, as the destinations in the New York area are clustered to each other compared to LA destinations, and most accommodations are located close to those destinations. The larger size of the LA area is also assumed to influence the pricing decisions regarding distance, which was reflected in the different scale bars that appeared in figures.

However, the relationship between the density of nearby Airbnb listings and the price showed almost opposite results compared to the traditional understanding of the competition and price in the economics, as it turned out to be utterly irrelevant in the LA area while showing a positive influence in New York. After all, Airbnb accommodation is not a business that provides identical goods at the same location. Therefore, it can be interpreted as Airbnb listings being more sensitive to demand based on geographical location, and higher density is an indication of higher demand, which corresponds to the results in Chen and Xie (2017) [9].

The implication of the negative relationship between the review rating and the price in the OLS-based HPM needs to be discussed more in detail, which also appeared in the previous studies $[13,16]$. We assume that it is a result of spatial spurious regression, based on our findings like high t-values (LA: -18.766, NY: -7.762) with relatively lower R2 values, as well as the existence of spatial autocorrelation in both cities as depicted in Figure 2 (high-high and low-low clusters). Spatial spurious regression is a regression that shows false evidence of the correlation between two independent non-stationary variables due to random walk in error terms. Methods that can consider spatial non-stationarity are required to analyze such datasets with spatial spurious regression [65], such as GWR and its variations. 

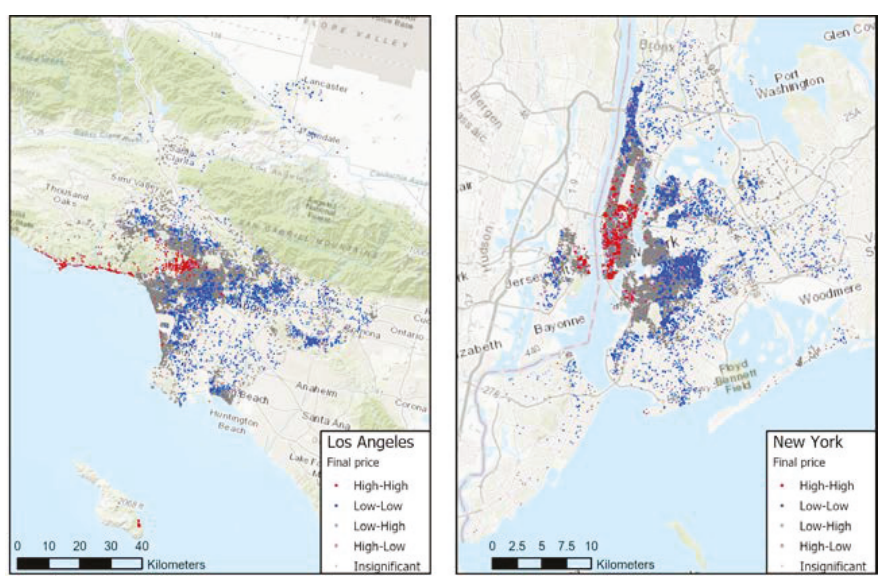

Figure 2. Local Moran's I results for the price.

\subsection{MGWR Analysis Results}

MGWR estimation results are presented in Tables 4 and 5 , which only include statistically significant cases. As each case has unique correlation coefficients for the explanatory variables, their mean, standard deviation, median, minimum, and maximum values were presented to summarize tendency in each city. Bandwidth, or the scale of the interaction of each variable, were also included. The ratio of significance represents the percent of statistically significant cases for each variable. The explanatory power of MGWR is noticeably higher than OLS, which indicates that MGWR successfully considers localized spatial influence in the price of Airbnb listings.

Table 4. Multiscale Geographically Weighed Regression (MGWR) results of LA area.

\begin{tabular}{cccccccc}
\hline Variables & Bandwidth $(\mathbf{m})$ & Mean & Std & Min & Median & Max & Ratio of Significance (\%) \\
\hline guests & 45 & 0.264 & 0.540 & -3.216 & 0.300 & 2.384 & 9.3 \\
bedrooms & 1321 & 0.100 & 0.039 & 0.043 & 0.093 & 0.324 & 90.9 \\
bathrooms & 250 & 0.289 & 0.141 & -0.225 & 0.253 & 0.931 & 61.0 \\
shared & 3663 & -0.064 & 0.015 & -0.181 & -0.063 & -0.026 & 99.8 \\
strict & 264 & -0.097 & 0.210 & -0.798 & -0.126 & 0.346 & 5.9 \\
flexible & 20,427 & -0.011 & 0.000 & -0.012 & -0.011 & -0.010 & 93.9 \\
superhost & 3452 & 0.042 & 0.047 & -0.048 & 0.051 & 0.097 & 2.8 \\
duration & 20,428 & $\mathrm{~N} / \mathrm{A}$ & $\mathrm{N} / \mathrm{A}$ & $\mathrm{N} / \mathrm{A}$ & $\mathrm{N} / \mathrm{A}$ & $\mathrm{N} / \mathrm{A}$ & 0.0 \\
rating & 43 & -0.503 & 0.465 & -2.763 & -0.368 & 0.909 & 6.7 \\
reviews & 20,441 & -0.015 & 0.000 & -0.016 & -0.015 & -0.015 & 100.0 \\
density & 670 & 0.068 & 0.155 & -0.196 & 0.076 & 1.237 & 48.2 \\
poverty_r & 1869 & -0.014 & 0.071 & -0.089 & -0.041 & 0.295 & 26.1 \\
dist_index & 15,732 & 0.019 & 0.004 & 0.014 & 0.019 & 0.031 & 22.7 \\
& & & \multicolumn{7}{c}{$\mathrm{R}^{2}=0.705$} & & \\
\hline
\end{tabular}

Regarding the scale of operation of the pricing variables, MGWR results revealed that (1) the scale of operation varies widely among variables; and (2) even for the identical variable, the scale of operation differs between cities. Based on the bandwidth and the statistical distribution of the coefficients across each city, we classified the pricing variables as local, regional, and global variables. In LA, one cancellation policy dummy (flexible), the number of reviews, and the distance to tourism destinations were identified as global variables. In New York, the strict dummy variable appeared to be regional, and superhost, poverty ratio, and Airbnb density were identified as global variables in addition to the above three variables. These global variables can be interpreted in two ways: (1) Airbnb hosts in each city use similar pricing strategies regarding these variables, and/or (2) the hosts are 
influenced by other competitors' pricing strategy related to these variables' city-wide scale. The spatial distribution of correlation coefficients of the flexible cancellation policy in the two cities in Figure 3 shows the global characteristic of the given variable. In LA, the flexible policy resulted in a slight price drop of $1 \%$, with less than $0.1 \%$ variance across the entire area. A similar observation has been made in the New York area, though the influence on the price was a little stronger than the LA area.

Table 5. MGWR results of New York area.

\begin{tabular}{cccccccc}
\hline Variables & $\begin{array}{c}\text { Bandwidth } \\
(\mathbf{m})\end{array}$ & Mean & Std & Min & Median & Max & $\begin{array}{c}\text { Ratio of Significance } \\
(\%)\end{array}$ \\
\hline guests & 44 & 0.443 & 0.548 & -4.674 & 0.377 & 4.351 & 14.6 \\
bedrooms & 1391 & 0.097 & 0.050 & -0.063 & 0.088 & 0.348 & 64.1 \\
bathrooms & 110 & 0.339 & 0.347 & -2.116 & 0.294 & 2.637 & 20.3 \\
shared & 81 & -0.192 & 0.460 & -1.409 & -0.242 & 3.926 & 11.4 \\
strict & 18,305 & 0.025 & 0.008 & 0.014 & 0.024 & 0.037 & 91.0 \\
flexible & 23,868 & -0.014 & 0.000 & -0.014 & -0.014 & -0.013 & 100.0 \\
superhost & 23,868 & 0.024 & 0.000 & 0.024 & 0.024 & 0.025 & 100.0 \\
duration & 45 & 0.002 & 1.273 & -7.844 & 0.219 & 4.715 & 3.2 \\
rating & 44 & -0.340 & 0.744 & -4.392 & -0.330 & 3.515 & 4.9 \\
reviews & 23,845 & -0.030 & 0.001 & -0.032 & -0.030 & -0.027 & 100.0 \\
density & 23,868 & 0.049 & 0.000 & 0.049 & 0.049 & 0.050 & 100.0 \\
poverty_r & 23,868 & -0.016 & 0.000 & -0.017 & -0.016 & -0.015 & 100.0 \\
dist_index & 20,393 & 0.115 & 0.004 & 0.110 & 0.114 & 0.132 & 100.0 \\
& & & & $R^{2}=0.598$ & & \\
\hline
\end{tabular}
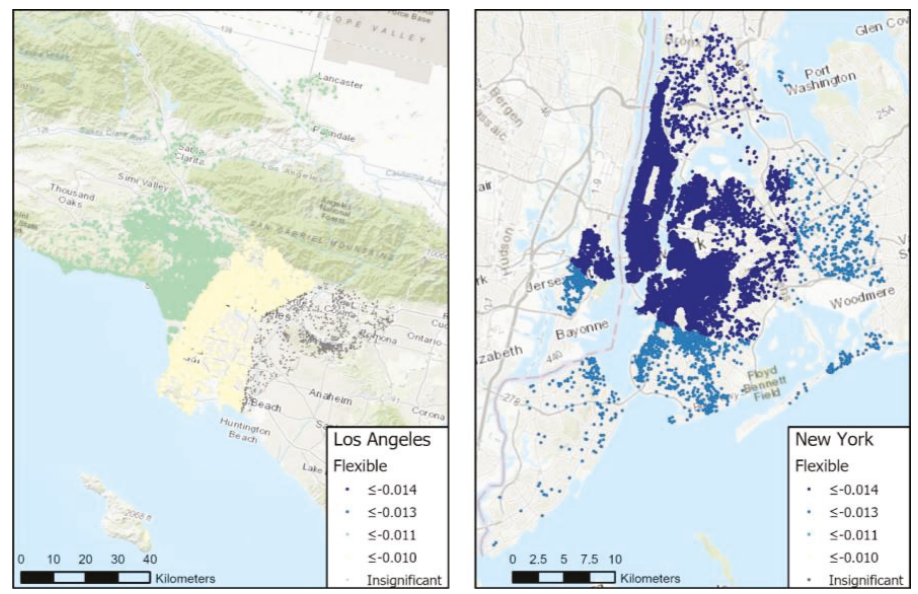

Figure 3. MGWR result for cancellation policy (flexible over moderate).

The distribution of the coefficients for the number of bedrooms shows the difference between local and global variables clearly (Figure 4). The bandwidth of the bedroom is about $1.3 \mathrm{~km}$ in both cities, which reveals that the hosts in the two cities tend to refer only the neighboring competitor's strategy regarding the pricing with bedrooms. In the New York area, the stronger influence of the bedrooms that appeared in Harlem and lower Brooklyn areas can be partially explained by the poverty ratio. Figure 5 shows bivariate cluster analysis results between the poverty ratio and the correlation coefficient of bedroom variable, where high-high points are clustered in those two areas. However, in the LA area, such a relationship was not observed (Figure 5). Instead, Airbnb listings more sensitive to the number of bedrooms are clustered in the Hollywood area (central cluster) and Malibu area (western beach area), where the poverty rate is relatively low (low-high clusters). Therefore, this could be a result of higher housing prices. 

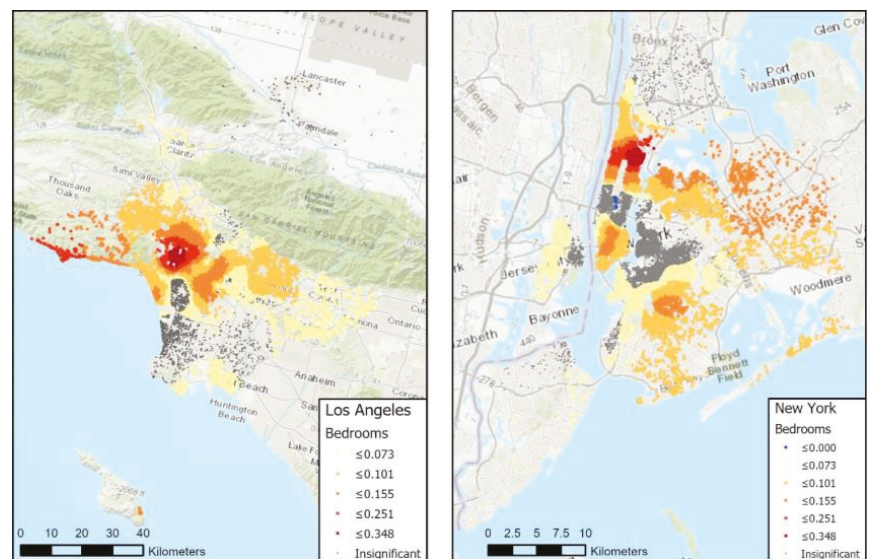

Figure 4. MGWR result for bedroom.
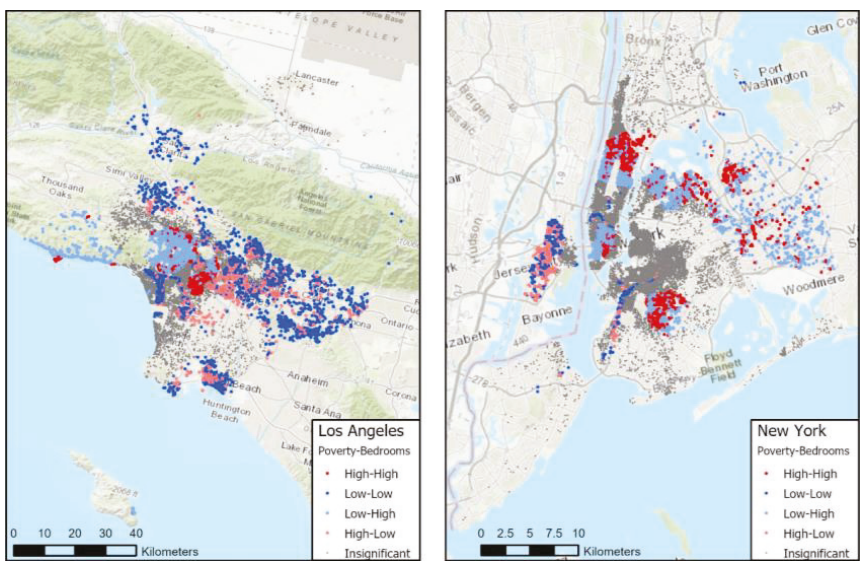

Figure 5. Bivariate cluster analysis for poverty rate and the number of bedrooms.

However, the influence of the pricing variables has inter-city differences, not just intra-city variances. For example, the density of Airbnb listings has a globalized scale of operation in the New York area, increasing by $10 \%$ in the final price with a rising one-standard deviation (Figure 6). On the other hand, the density is a local variable with much narrower bandwidth $(670 \mathrm{~m})$ in LA. This is consistent with the conclusions of previous studies, where the price characteristics of accommodations vary significantly from city to city $[14,53]$.

The most noteworthy aspect of this study is the relationship between review rating and Airbnb price. In OLS results, they have a negative relationship with significantly higher t-values (LA: -18.766 , NY: -7.762). However, MGWR revealed a different pattern (Figure 7). The review rating was not statistically significant in most of the listings in the two cities. In New York, no distinctive pattern exists, and less than $5 \%$ of Airbnb listings in New York show statistically valid relationship between the review rating and price. This confirms that the observed effect of the review rating in the OLS-based HPM was incorrect, which was the result of spatial spurious regression. Spatial non-stationary should be considered using MGWR to prevent such erroneous estimation. In the case of LA, a negative relationship exists in Hollywood and Malibu areas. This is consistent with the previous Airbnb research, as well as hotel practice $[13,56]$. There have been two possibilities suggested for this anomaly in the Airbnb pricing [16]. Firstly, biased and inconsistent customer review ratings in the Airbnb 
platform. It has been discovered that the ratings in Airbnb tend to be higher than other accommodation platforms [35], which can lead to such different relationships between the price and the review ratings in Airbnb. Another possibility is the unique nature of customer satisfaction in Airbnb, as the customers mostly value its low price. In the previous Airbnb research, the low price has been recognized as the core value of Airbnb, along with cultural exchange. This causes a higher correlation between the low price and the satisfaction of Airbnb, as a customer likely to give higher ratings more easily for a low-priced listing, since the expectation for other factors would be low. However, for the high-priced listings, an opposite tendency would exist, as it is possible that a customer has higher expectations that are likely to result in disappointment regarding other services. Figure 8 shows bivariate cluster analysis results for the price and review rating for the LA area, which suggests the potential influence of the expectation-based negative relationship in LA (high-low clusters).
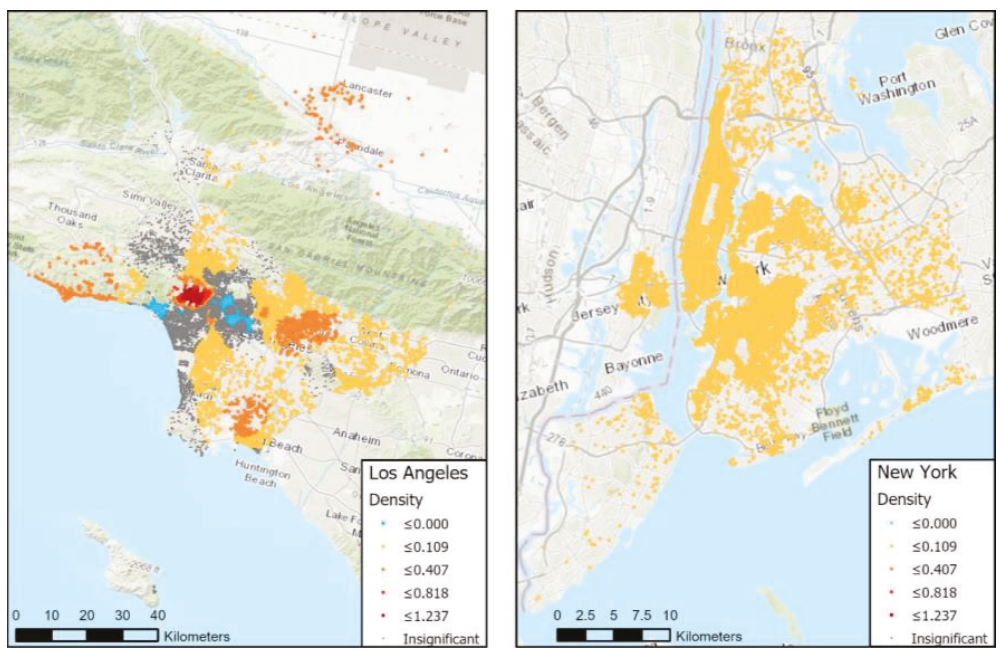

Figure 6. MGWR result for density.
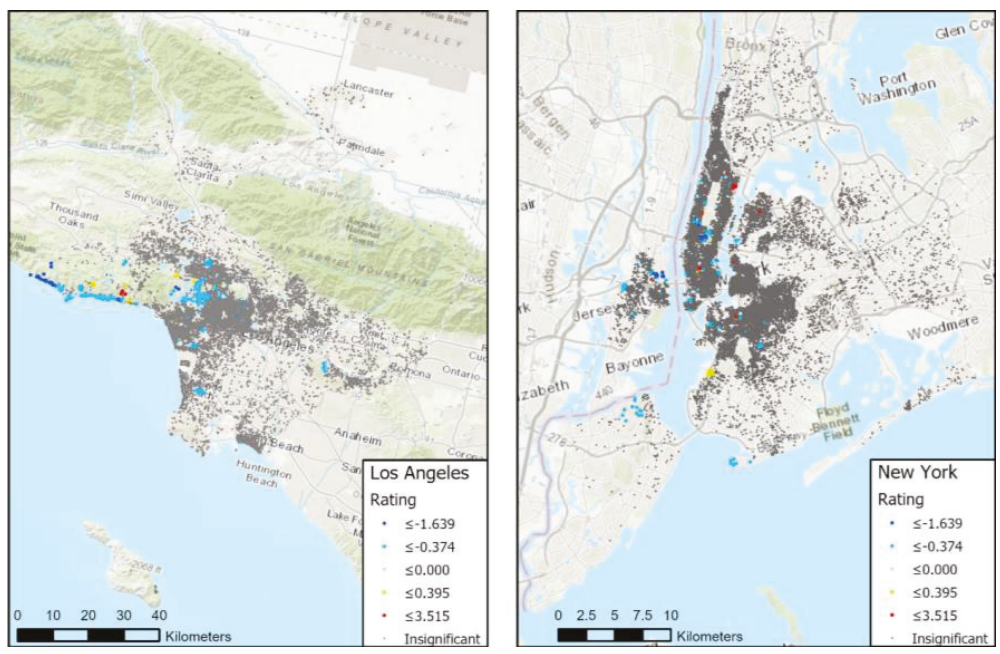

Figure 7. MGWR results for review rating. 


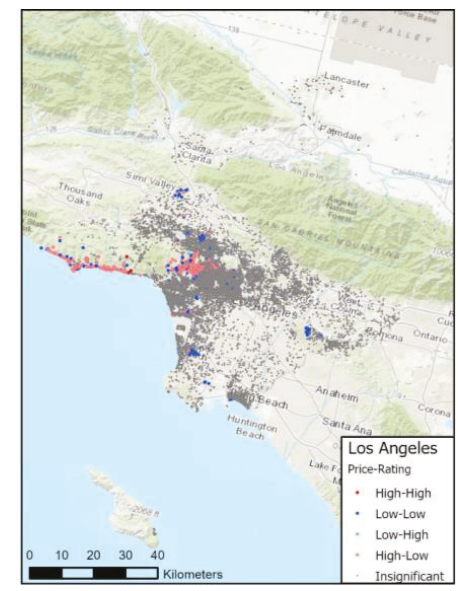

Figure 8. Bivariate cluster analysis of price and rating in LA.

\section{Discussions and Conclusions}

In this research, we analyzed the pricing determinants of Airbnb listings in LA and New York, using OLS and MGWR HPMs. OLS-based results presented similar results to the previous Airbnb studies regarding the influence of listing functions, attributes of the host, reputation variables, and geographical characteristics. The results also showed that the explanatory powers of the identical pricing variables were different between locations, and distinctive geographical properties explain this. However, OLS was not able to reveal the reasons behind such differences, since it cannot include spatial variance in the model. The opposite estimation in the review rating to the previous studies could not be explained with OLS results. On the contrary, MGWR was able to reveal that the spatial variance (the scale of operation) of pricing variables varies within a city, as well as between cities, due to their geographical characteristics. Unlike the OLS, MGWR estimated differing spatial influences for the variables. From this, MGWR provided the groundwork to analyze how geographical location affects the influence of the pricing variables, as well as geographic visualization of the results. Additionally, we presented that the traditional OLS-based approach can mislead a non-significant relationship to a significant one due to its ignorance of spatial variance.

Based on the MGWR results, we demonstrated the importance of the spatial variance and effectiveness of MGWR for pricing research, providing a deepened understanding of the pricing strategies in Airbnb listings. From a theoretical perspective, this research presented the risk of spatial spurious regression in the OLS-based HPM by comparing it with MGWR results. We also proved that MGWR is a useful tool to account for spatial variance in pricing strategies.

Practically, this research showed that pricing models were different among cities and even within a city, with visualization of the results over the maps. Using the MGWR method, this research proposes a practical approach for analyzing spatially varying relationships between pricing determinants and prices for different cities and regions. In addition, in constructing the price policy, the model provides guidelines for practitioners to effectively distinguish the influence of various factors related to price through the model. By theoretically explaining the relationship between price and review rating, which has not been adequately explained in the previous price research, we have empirically demonstrated a methodology to optimize the price of Airbnb and other sharing economy services.

This study suggested a method to effectively analyze the relationship between the price and the geographical characteristics of tourist destinations, but did not cover detailed characteristics of the region. Subsequent studies are expected to expand on how the diversity of a tourist attraction affects 
price and service characteristics. There is also a need to look more socially and economically into why regional differences in influence arise.

It should be noted that this research has several limitations. First, the estimated pricing strategies and the scale of operation of the variables cannot be generalized, since this research analyzed only two cities. We will need more cities to analyze how the geographical characteristics of locations affect the pricing determinants. Second, distance measurements to tourism destinations need to be updated to reflect various transportation modes for tourists and actual travel time considering traffic and road conditions. Fourth, the housing price of individual houses that host Airbnb listings needs to be included as a pricing variable to evaluate the influence of the housing price directly and more accurately. Lastly, monthly or seasonal variations in the price and the pricing strategy need to be considered since the price varies with time.

Author Contributions: Conceptualization, I.H. and C.Y.; Data Collection: I.H.; Method: I.H.; Analysis: I.H., and C.Y.; writing and editing: I.H. and C.Y.; supervision of the research: C.Y. All authors have read and agreed to the published version of the manuscript.

Funding: This research received no external funding.

Conflicts of Interest: The autors declare no conflict of interes.

\section{References}

1. Kimes, S.E.; Chase, R.B. The strategic levers of yield management. J. Serv. Res. 1998, 1, 156-166. [CrossRef]

2. Abrate, G.; Fraquelli, G.; Viglia, G. Dynamic pricing strategies: Evidence from European hotels. Int. J. Hosp. Manag. 2012, 31, 160-168. [CrossRef]

3. Guillet, B.D.; Mohammed, I. Revenue management research in hospitality and tourism. Int. J. Contemp. Hosp. Manag. 2015, 27, 526-560. [CrossRef]

4. Wirtz, J.; Kimes, S.E.; Theng, J.H.P.; Patterson, P. Revenue management: Resolving potential customer conflicts. J. Revenue Pricing Manag. 2003, 2, 216-226. [CrossRef]

5. Wirtz, J.; Kimes, S.E. The moderating role of familiarity in fairness perceptions of revenue management pricing. J. Serv. Res. 2007, 9, 229-240. [CrossRef]

6. Gibbs, C.; Guttentag, D.; Gretzel, U.; Morton, J.; Goodwill, A. Pricing in the sharing economy: A hedonic pricing model applied to Airbnb listings. J. Travel Tour. Mark. 2018, 35, 46-56. [CrossRef]

7. Vives, A.; Jacob, M.; Payeras, M. Revenue management and price optimization techniques in the hotel sector: A critical literature review. Tour. Econ. 2018, 24, 720-752. [CrossRef]

8. Chen, C.; Schwartz, Z. Room rate patterns and customers' propensity to book a hotel room. J. Hosp. Tour. Res. 2008, 32, 287-306. [CrossRef]

9. Chen, Y.; Xie, K. Consumer valuation of Airbnb listings: A hedonic pricing approach. Int. J. Contemp. Hosp. Manag. 2017, 29, 2405-2424. [CrossRef]

10. Ikkala, T.; Lampinen, A. Monetizing Network Hospitality: Hospitality and Sociability in the Context of Airbnb. In Proceedings of the 18th ACM Conference on Computer Supported Cooperative Work \& Social Computing, Vancouver, CB, Canada, 14-18 March 2015; pp. 1033-1044.

11. Ceptureanu, E.G.; Ceptureanu, S.I.; Herteliu, C.; Cerqueti, R. Sustainable consumption behaviours in P2P accommodation platforms: An exploratory study. Soft Comput. 2020, 1-8. [CrossRef]

12. Liang, L.J.; Choi, H.C.; Joppe, M. Understanding repurchase intention of Airbnb consumers: Perceived authenticity, electronic word-of-mouth, and price sensitivity. J. Travel Tour. Mark. 2018, 35, 73-89. [CrossRef]

13. Teubner, T.; Hawlitschek, F.; Dann, D. Price determinants on airbnb: How reputation pays off in the sharing economy. J. Self Gov. Manag. Econ. 2017, 5. [CrossRef]

14. Soler, I.P.; Gemar, G.; Correia, M.B.; Serra, F. Algarve hotel price determinants: A hedonic pricing model. Tour. Manag. 2019, 70, 311-321. [CrossRef]

15. Adamiak, C.; Szyda, B.; Dubownik, A.; García-Álvarez, D. Airbnb Offer in Spain-Spatial Analysis of the Pattern and Determinants of Its Distribution. ISPRS Int. J. Geo Inf. 2019, 8, 155. [CrossRef]

16. Zhang, Z.; Chen, R.; Han, L.; Yang, L. Key factors affecting the price of Airbnb listings: A geographically weighted approach. Sustainability 2017, 9, 1635. [CrossRef] 
17. Lagonigro, R.; Martori, J.C.; Apparicio, P. Understanding Airbnb spatial distribution in a southern European city: The case of Barcelona. Appl. Geogr. 2020, 115, 102136. [CrossRef]

18. Shabrina, Z.; Buyuklieva, B.; Ming, M.N.K. Airbnb, hotels, and saturation of the food industry: A multi-scale GWR approach. arXiv 2019, arXiv:1905.12543.

19. Xu, F.; Hu, M.; La, L.; Wang, J.; Huang, C. The influence of neighbourhood environment on Airbnb: A geographically weighed regression analysis. Tour. Geogr. 2019. [CrossRef]

20. Xu, Y.-H.; Pennington-Gray, L.; Kim, J. The sharing economy: A geographically weighted regression approach to examine crime and the shared lodging sector. J. Travel Res. 2019, 68, 1193-1208. [CrossRef]

21. Fotheringham, A.S.; Yang, W.; Kang, W. Multiscale geographically weighted regression (MGWR). Ann. Am. Assoc. Geogr. 2017, 107, 1247-1265. [CrossRef]

22. Hung, W.-T.; Shang, J.-K.; Wang, F.-C. Pricing determinants in the hotel industry: Quantile regression analysis. Int. J. Hosp. Manag. 2010, 29, 378-384. [CrossRef]

23. Pawlicz, A.; Napierala, T. The determinants of hotel room rates: An analysis of the hotel industry in Warsaw, Poland. Int. J. Contemp. Hosp. Manag. 2017, 29, 571-588. [CrossRef]

24. Yi, J.; Yuan, G.; Yoo, C. The effect of the perceived risk on the adoption of the sharing economy in the tourism industry: The case of Airbnb. Inf. Process. Manag. 2020, 57, 102108. [CrossRef]

25. Curtis, S.K.; Lehner, M. Defining the sharing economy for sustainability. Sustainability 2019, $11,567$. [CrossRef]

26. Plewnia, F.; Guenther, E. Mapping the sharing economy for sustainability research. Manag. Decis. 2018. [CrossRef]

27. Frenken, K.; Schor, J. Putting the sharing economy into perspective. Environ. Innov. Soc. Transit. 2017, 23, 3-10. [CrossRef]

28. Acquier, A.; Daudigeos, T.; Pinkse, J. Promises and paradoxes of the sharing economy: An organizing framework. Technol. Forecast. Soc. Chang. 2017, 125, 1-10. [CrossRef]

29. Belk, R. You are what you can access: Sharing and collaborative consumption online. J. Bus. Res. 2014, 67, 1595-1600. [CrossRef]

30. Sung, E.; Kim, H.; Lee, D. Why do people consume and provide sharing economy accommodation?-A sustainability perspective. Sustainability 2018, 10, 2072. [CrossRef]

31. Botsman, R.; Rogers, R. What's Mine is Yours: The Rise of Collaborative Consumption; Harper Business: New York, NY, USA, 2010.

32. Guttentag, D. Airbnb: Disruptive innovation and the rise of an informal tourism accommodation sector. Curr. Issues Tour. 2015, 18, 1192-1217. [CrossRef]

33. Tussyadiah, I.P. An Exploratory Study on Drivers and Deterrents of Collaborative Consumption in Travel. In Information and Communication Technologies in Tourism 2015; Springer: Cham, Switzerland, 2015; pp. 817-830.

34. Sacks, D. The sharing economy. Fast Co. 2011, 155, 88-131.

35. Zervas, G.; Proserpio, D.; Byers, J.W. The rise of the sharing economy: Estimating the impact of Airbnb on the hotel industry. J. Mark. Res. 2017, 54, 687-705. [CrossRef]

36. Fang, B.; Ye, Q.; Law, R. Effect of sharing economy on tourism industry employment. Ann. Tour. Res. 2016, 57, 264-267. [CrossRef]

37. Midgett, C.; Bendickson, J.S.; Muldoon, J.; Solomon, S.J. The sharing economy and sustainability: A case for Airbnb. Small Bus. Inst. J. 2018, 13, 51-71.

38. Lyon, J. 'Buy Local' Campaigns can Actually Supercharge Sales; CNN Money: Atlanta, GA, USA, 2014; Available online: https://money.cnn.com/2014/03/28/smallbusiness/buy-local/ (accessed on 8 June 2020).

39. Weisul, K. Consumers Buy into "Buy Local"; Bloomberg Businessweek Magazine: New York, NY, USA, 2010; Available online: https://www.bloomberg.com/news/articles/2010-02-18/consumers-buy-into-buy-local (accessed on 8 June 2020).

40. Martin, C.J.; Upham, P.; Budd, L. Commercial orientation in grassroots social innovation: Insights from the sharing economy. Ecol. Econ. 2015, 118, 240-251. [CrossRef]

41. Yoo, M.; Lee, S.; Bai, B. Hospitality marketing research from 2000 to 2009: Topics, methods, and trends. Int. J. Contemp. Hosp. Manag. 2011, 23, 517-532. [CrossRef]

42. Kimes, S.E.; Wirtz, J. Has revenue management become acceptable? Findings from an international study on the perceived fairness of rate fences. J. Serv. Res. 2003, 6, 125-135. [CrossRef] 
43. Thrane, C. Hedonic price models and sun-and-beach package tours: The Norwegian case. J. Travel Res. 2005, 43, 302-308. [CrossRef]

44. Lancaster, K.J. A new approach to consumer theory. J. Polit. Econ. 1966, 74, 132-157. [CrossRef]

45. Rosen, S. Hedonic prices and implicit markets: Product differentiation in pure competition. J. Polit. Econ. 1974, 82, 34-55. [CrossRef]

46. Fleischer, A. A room with a view-A valuation of the Mediterranean Sea view. Tour. Manag. 2012, 33, 598-602. [CrossRef]

47. Lee, S.K.; Jang, S. Room rates of US airport hotels: Examining the dual effects of proximities. J. Travel Res. 2011, 50, 186-197.

48. Schamel, G. Weekend vs. midweek stays: Modelling hotel room rates in a small market. Int. J. Hosp. Manag. 2012, 31, 1113-1118. [CrossRef]

49. Zhang, Z.; Ye, Q.; Law, R. Determinants of hotel room price: An exploration of travelers' hierarchy of accommodation needs. Int. J. Contemp. Hosp. Manag. 2011, 23, 972-981. [CrossRef]

50. Abrate, G.; Capriello, A.; Fraquelli, G. When quality signals talk: Evidence from the Turin hotel industry. Tour. Manag. 2011, 32, 912-921. [CrossRef]

51. Espinet, J.M.; Saez, M.; Coenders, G.; Fluvià, M. Effect on prices of the attributes of holiday hotels: A hedonic prices approach. Tour. Econ. 2003, 9, 165-177. [CrossRef]

52. Latinopoulos, D. Using a spatial hedonic analysis to evaluate the effect of sea view on hotel prices. Tour. Manag. 2018, 65, 87-99. [CrossRef]

53. Chen, C.-F.; Rothschild, R. An application of hedonic pricing analysis to the case of hotel rooms in Taipei. Tour. Econ. 2010, 16, 685-694. [CrossRef]

54. Thrane, C. Examining the determinants of room rates for hotels in capital cities: The Oslo experience. J. Revenue Pricing Manag. 2007, 5, 315-323. [CrossRef]

55. Chattopadhyay, M.; Mitra, S.K. Do airbnb host listing attributes influence room pricing homogenously? Int. J. Hosp. Manag. 2019, 81, 54-64. [CrossRef]

56. Wang, D.; Nicolau, J.L. Price determinants of sharing economy based accommodation rental: A study of listings from 33 cities on Airbnb.com. Int. J. Hosp. Manag. 2017, 62, 120-131. [CrossRef]

57. Zervas, G.; Proserpio, D.; Byers, J. A first look at online reputation on Airbnb, where every stay is above average. SSRN 2015. [CrossRef]

58. Tobler, W.R. Geographical filters and their inverses. Geogr. Anal. 1969, 1, 234-253. [CrossRef]

59. Brunsdon, C.; Fotheringham, S.; Charlton, M. Geographically weighted regression. J. R. Stat. Soc. Ser. D (Stat.) 1998, 47, 431-443. [CrossRef]

60. Zhang, H.; Zhang, J.; Lu, S.; Cheng, S.; Zhang, J. Modeling hotel room price with geographically weighted regression. Int. J. Hosp. Manag. 2011, 30, 1036-1043. [CrossRef]

61. Cudnik, M.T.; Yao, J.; Zive, D.; Newgard, C.; Murray, A.T. Surrogate markers of transport distance for out-of-hospital cardiac arrest patients. Prehosp. Emerg. Care 2012, 16, 266-272. [CrossRef]

62. Fotheringham, A.S.; Charlton, M.E.; Brunsdon, C. Geographically weighted regression: A natural evolution of the expansion method for spatial data analysis. Environ. Plan. A 1998, 30, 1905-1927. [CrossRef]

63. Tobler, W.R. A computer movie simulating urban growth in the Detroit region. Econ. Geogr. 1970, 46, $234-240$. [CrossRef]

64. Brunsdon, C.; Fotheringham, A.S.; Charlton, M. Some notes on parametric significance tests for geographically weighted regression. J. Reg. Sci. 1999, 39, 497-524. [CrossRef]

65. Lee, L.-F.; Yu, J. Spatial Nonstationarity and Spurious Regression: The Case with a Row-normalized Spatial Weights Matrix. Spat. Econ. Anal. 2009, 4,301-327. [CrossRef]

(C) 2020 by the authors. Licensee MDPI, Basel, Switzerland. This article is an open access article distributed under the terms and conditions of the Creative Commons Attribution (CC BY) license (http://creativecommons.org/licenses/by/4.0/). 
Article

\title{
A Research on the Combination of Oblique Photography and Mobile Applications Based on the Sustainable Development of Tourism
}

\author{
Mengyi Lin ${ }^{1}$, Fu-Yuan $\mathrm{Li}^{2, *}$ and Haibin $\mathrm{Zhou}^{3}$ \\ 1 The Graduate Institute of Design Science, Tatung University, Taipei 104, Taiwan; d10517014@ms.ttu.edu.tw \\ 2 Department of Industrial Design, Tatung University, Taipei 104, Taiwan \\ 3 Academy of Art Design, Fujian Business University, Fuzhou 350012, China; bingo353416@fjbu.edu.cn \\ * Correspondence: richardlee@gm.ttu.edu.tw; Tel.: +886-931-216524
}

Received: 22 March 2020; Accepted: 21 April 2020; Published: 25 April 2020

\begin{abstract}
Tourism is one of the world's fastest driving forces of economic development, playing an important role in achieving sustainable development goals. In modern society, mobile social media is a communication and decision-making platform for users and a source of big data information about travel. Obtaining and analyzing travel data can provide customer-oriented information about travel destinations and comprehensive services for both tourism operators and tourists. It has a positive impact on the sustainable development of society, economy, environment, and humanities. Starting with theoretical analysis and empirical research, this study combines social media and oblique photography, conducts a case study of the Pingtan comprehensive experimental area in China, and develops an app about online travelling to provide corresponding information for consumers' decisions. This study also discusses the potential value of the app, i.e., assisting the development of smart travel in city, achieving sustainable development of tourism, and contributing to tourism globally.
\end{abstract}

Keywords: sustainable tourism; oblique photography; mobile applications; social media

\section{Introduction}

Global tourism has shown a rapid growth trend, which has been affected by the emergence of digitization and internet service. The continuous growth of the tourism industry has made it a major industry in the world economy. It has also drawn great attention from researchers, especially in the field of sustainable tourism [1]. The United Nation (UN) member states proposed the 2030 Agenda for Sustainable Development in 2015, pointing out a shared blueprint of peace and prosperity for the present and the future of human beings and the planet [2]. Sustainable tourism has been marked as having a potentially valuable role in achieving "sustainable development goals": "Tourism has the potential to contribute directly or indirectly to all goals. In particular, it has been included as targets in Goals 8, 12, and 14 on inclusive and sustainable economic growth, sustainable consumption/production (SCP), and the sustainable use of oceans and marine resources, respectively." In 2019, the contribution of China's tourism to its GDP was 10.94 trillion-yuan, accounting for $11.05 \%$ of the total GDP. Tourism directly and indirectly employed 79.87 million people, accounting for $10.31 \%$ of the total employed population of the country. [3] China is also striving to become a smart and sustainable tourist destination. In fact, the sustainability of tourist destinations has become a key factor for improving competitiveness [4]. Therefore, how to carry out the marketing plan to improve the core competitiveness of tourist destinations and at the same time balance the ecology of tourist destinations to achieve sustainable and coordinated development becomes particularly important. In recent years, the rapid development of smart technologies and informatization of tourism have assisted operators 
to make full use of technology to inform tourists about tourist destinations (especially available services), making tourist destinations smart and sustainable, providing tourists with high-quality tourism services, benefiting the tourism industry, and improving the quality of life for local people [5].

Previous surveys show that tourism is a complicated industry. Statistics, information, and knowledge from or about tourists are the essential basis of the competition and innovation on tourist destination [6,7]. They enable people to obtain detailed information about tourism, including the general understanding of local people, such as their preferences, feelings, and attitudes. These data are useful for creating tourism services that better meet the demands of tourists [8]. With the expansion of online travel applications (apps), consumer data will be enriched and easier to access, the information will be timely and accurately touched, and the capacity of data analysis will be improved to support the analysis of market behavior and consumer behavior motivation. Thus far, there are only a few papers about how tourist destination managers and tourism organizations can take advantage of the massive information generated by tourists in the travel experience to achieve a more effective process of value creation, especially those in information access and between valuable and uncertain targeting managers and entrepreneurs [9-13]. Although several tycoons in the tourism industry, such as Ctrip and Qunar.com, have issued multiple reports on tourism consumption each year to provide direction for the research of tourism market demand and tourists' consumption concept, it is difficult for managers to create a large database to analyze a specific tourist destination. Most tourism apps feature a comprehensive collection of tourism information throughout the country and provide standardized tourism services, while tourists are increasingly interested in various commodities and diverse services featured with local characteristics of a certain tourist destination. In the past, tourism operators have been required to offer an all-solve and inclusive plan that combines massive products/services for travel experience [14]. Therefore, a tourist destination must depend on mainstream apps for online travel to produce refined services or they can develop of a new app, which brings difficulties in the research and analysis on destination's information and is unable to provide inclusive services for tourism operators. Firstly, they have to rely on mainstream apps, lacking in flexibility of data collection for customized tourism services; secondly, mainstream apps limit the access to big data in the data analysis of some specific area; thirdly, independently developed apps are faced with high cost of development, popularization, and maintenance. If we can use an existing app, coupled with new technology, the "all-around" services and data collection and analysis of tourist destinations will be of great help to the process of transforming the information of tourist destinations into value.

Nowadays, tourism operators still have not found a proper solution to problems that emerge in data acquisition and analysis of one single tourist destination. This paper focuses on whether we can rely on an existing mobile social media to develop a tool that can not only meet tourist demand on online travel services but also help small- and medium-sized tourism operations provide convenient data analysis for business decisions through a platform of their own. This paper focus on a new useful technological model aimed at improving tourist destination marketing by conducting a case study on the Pingtan comprehensive experimental area in China and proposing an online travel mini program that will focus on consumer behavior and decisions. Moreover, we will show the tourism operators how to use the backstage information provided by this mini program to improve the attractiveness of a tourist destination so as to enhance its competitiveness and consumer satisfaction, as well as to receive guidance for a more precise marketing strategy. This study combines oblique photography, a new type of three-dimensional space measurement, with present social media. Oblique photography technology has been mostly used in homeland security, urban management, disaster assessment, environmental monitoring, real estate, flood risk simulation, aboveground tree biomass estimation, archaeology, engineering construction, and live navigation [15-18]. Such combination realizes information exchange based on the necessity of information and the way of information release, seeking convenient interaction and deep connection between the Internet and tourism and providing reference cases on the sustainable development of tourist destinations. Applying the oblique photography technology to a current app to achieve information exchange, combining the necessity of information with the way of information 
releasing, and seeking for the convenient interaction and the deep connection between Internet and tourism, we present a reference case for other tourism social media, which assists the sustainable development of tourist destinations.

\section{Literature Review}

\subsection{Sustainable and Smart Tourist Destination}

The concept of sustainable development is crucial for tourism both today and in the future [19]. Early in 2005, the United Nations environment program (UNEP) and the World Tourism Organization (WTO) ([20], p. 12) have defined sustainable tourism as "tourism that takes full account of its current and future economic, social and environmental impacts, addressing the needs of visitors, the industry, the environment, and host communities".

Sustainable tourism is derived and developed from the concept of sustainable development and can be defined as a tourism marketing process in which the balance between the high-quality tourist experience of tourists and the high-quality life of local communities is sought and valued [21,22]. In order to make progress, sustainable tourism requires active participation of stakeholders [23]. The sustainable tourism business model is only feasible when marketers, tourists, and local communities cooperate strategically and build up mutually beneficial relationships [24]. With the increasing maturity of mobile technology, online travel service providers provide users with a considerable number of applications which meet users' demands in various aspects through high-quality services and offer tourists better travel experiences. At an international seminar organized by the World Tourism Organization (UNWTO), Buhalis pointed out that information and communication technologies constitute "electronic tools that help company's operations and strategic management, enabling them to manage information, functions, and processes and establish interactive communication to achieve their mission and goals" [25].

Tourists use smart tourism technologies in various stages of making their travel decisions [26]. The findings indicate that smart technologies can enable social media and social networking sites to contribute to sustainable tourism within the smart tourism paradigm [27]. The concept of a smart tourist destination is the result of applying the smart city approach to tourist destinations [28]. The ultimate goal of smart tourist destinations is to improve user travel experience, increase a tourist destinations competitiveness, improve consumer satisfaction, and gain long-term sustainable attention from consumers [29].

In recent years, there have been more individual tourists and people are more inclined to arrange trips according to personal preferences. For example, individuals choose destinations, make travel plans, plan travel routes, and book air tickets and hotels online. The purpose of smart tourism is to make the tour transparent, three-dimensional, and interactive through digitalization, so that tourists can feel and enjoy the charm of a destination. It posts real-time tourism resources, tourism economy, tourism activities, and other information related to tourists to facilitate information needs. Visitors can immediately see and use information, as well as adjust and arrange work/travel plans in a timely manner, which realizes real-time interaction between tourists and the Internet, and makes travel simpler. Smart tourism provides tourists with customized tours and better services and improve tourists' travel experiences, so that tourists can fully experience the benefits of smart tourism during their travels. Smart tourist destinations work together with smart healthcare, smart transportation, and smart public safety to promote the construction and development of smart cities. On the one hand, smart tourism can promote the development of related industries, bring about an increase in urban income, promote the steady growth of the regional economy, and create economic benefits for the city. On the other hand, smart tourism can expand employment, promote the construction of a harmonious society, and enhance the city's popularity and attractiveness, bring social value to the city, and promote the sustainable development of society. 


\subsection{The Choice of Mobile Apps}

In recent years, the emergence of smart tourism and the informatization of tourism have also developed rapidly. Tourism-related websites, social media, and smartphones have been popularized among tourists and the number of them is constantly increasing. According to the 2018 global digital travel sales report, convenient mobile payment options are driving online booking, with global digital travel sales up 10.4\% to \$694 billion in 2018 compared with 2017 [30]. New digital 2020 reports-published in partnership with We Are Social and Hootsuite-show that digital, mobile, and social media have become an indispensable part of everyday life for people all over the world. More than 4.5 billion people use internet in 2020, while social media users are over 3.8 billion; $92 \%$ of the world's internet users are now connected via mobile devices. Mobile apps now account for 10 out of every 11 minutes we spend using mobile devices, with web browsing only responsible for $9 \%$ of our mobile time. China is truly mobile-first, with $95 \%$ of the people accessing the internet via their smartphones [31].

Besides, the growth of tourism investment and tourist numbers has stimulated the surge of tourism app users. Tourists use mobile devices to plan, purchase, and improve their travel experience [32,33]. It is a crucial support for smart tourism destinations that make travel more functional and practical [34]. According to the results of the research survey [35], PC-side traffic in China's online tourism industry stabilized in 2017, with the overall growth rate of online tourism app users reaching a compound growth rate of $32.1 \%$ over the next few years. Therefore, the development of China's online tourism and consumption patterns have changed. At the same time, tourism users have also switched from the PC side to the mobile side, and mobile social media has become a popular platform for users to make travel plans. Past research has shown that travel information circulated and sought in social media had a major impact on tourists' decision-making and behavior [36]. Social media not only allows tourists to search for information about travel destinations but also allows them to make evaluations online (such as reviews, photos, and videos) related to these places [37,38]. Social media is now regarded as one of the main sources of big data on tourism and provides huge opportunities for a more decisive process [39]. Seo, Park, and Choi [40] found that the personality and informational characteristics from social media usage had statistically significant effects on electronic word-of-mouth (E-WOM). People who use social media frequently receive unexpected suggestions or recommendations depending on their previous interactions, preferences, and likes [41]. The earlier research conducted by Foux [42] reveals that consumers believe social media is a more reliable information source than the promotion and marketing activities sponsored by enterprises which are the traditional method to popularize commodities and services. Tourist destinations should promote the app as the preferred method for buying online and developing an appropriate strategy [43]. It will help tourists make travel decisions through evaluating the experience of previous tourists. Thus, dialogues with tourists and product promotions should not only be conducted face-to-face at the destination but also online with new technologies and exclusive channels [27]. That means the marketing of the tourist destination not only needs provide inclusive solutions in the app before travel and during travel but also needs maintain the constant connection between tourists and the tourist destination after travel, such as the recommendation and promotion to others by former tourists, a second visit to the destination and sending returned costumers gifts and souvenirs with local features, which requires the tourist destination app be closely and constantly connected with tourists who may receive the information about the tourist destination at any time.

If each tourist destination can develop an app that can provide an inclusive approach to accurate and careful services, the question is that apart from the cost of developing an app and the storage of smartphones, most smartphone users do not like too many apps in their phone. Data show that by the end of October 2018, 4.46 million mobile apps have been monitored in the Chinese market [44]. However, according to the 2018 Q3 report, the total number of apps installed per capita on mobile phones in China is only 50 [45]. The users' preference for less apps increases the difficulty of keeping a tourist destination app in their phone and further increases the difficulty of keeping visitor's 
connected with the tourist destination. On 9 January 2017, the mini program released by WeChat solved this problem.

WeChat is a free application program implemented by China Tencent in 2011, providing the instant messaging service by a smart terminal. It integrates the function of other applications into an all-around service platform including social network communication, mobile payment, e-commercial, and public service, etc. The latest news of Tencent released in March 2018 shows that the usage rate of WeChat among Chinese netizens is up to $94.5 \%$; the number of daily global active users exceeds 1 billion [46]. Moreover, WeChat is based on one-to-one close communication between friends and acquaintances from mobile phone contacts and QQ friends, concentrating on information that shares strong relationship chains. This is an important reason why this study chose WeChat as the research object social media. The function of the mini program in WeChat is a new way to connect users and services, and it can be accessed and shared within WeChat with user experiences [47]. Through the mini program provided by WeChat, various services are offered, including notification, online mall shopping, offline code scanning, and subscription association. Tourists can easily enter and quit official accounts and mini programs, which not only optimize user experience but also is convenient to transfer those mini program users into brand followers. The convenience of the mini program has strengthened the connection between the tourist destination's brand and consumers, creating constant opportunities for the follow-up services. Therefore, on the base of the advantage of massive and highly close-connected users, each WeChat mini program can correspond to one single tourist destination brand, offering potential opportunities to construct a new business ecosystem.

Mini programs are a sort of "light app", because they are connected with dozens of WeChat scene access ports whose number increases, i.e., "used-then-go" and "conveniently to share". Users do not need to install any apps. Instead, they only need to scan or search in WeChat before enjoying the same app functions. Mini programs do not take too much storage or space on mobile phones. After using them, users can find the records stored in "recent used mini program". Even though the mini program was deleted unintentionally, the users can still find it immediately through texts or audios in the search box on the WeChat homepage. It offers an open platform for premium services (Figure 1). Nevertheless, in terms of a user's increasing demands for the richness of content and quality, the mini program, as a light application, is not able to guarantee requirements such as interaction and vision. This paper puts forward the plan of combining oblique photography with mini program to tackle the obstacles above.

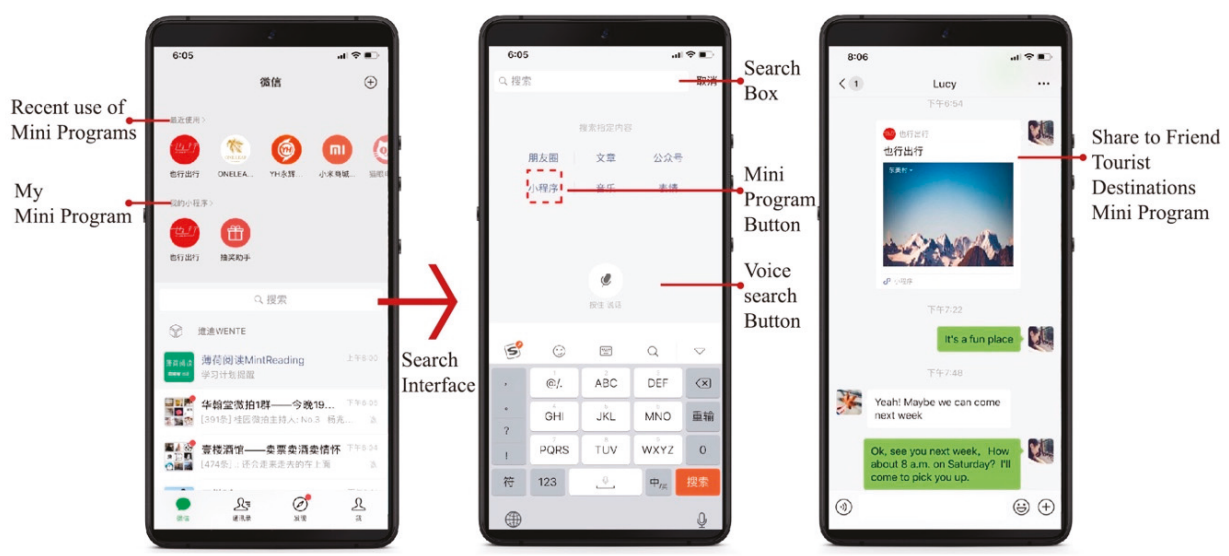

Figure 1. Finding and sharing mini programs in WeChat.

Furthermore, it provides enterprises with publicity, featuring low cost and high transformation rates, providing users with the best carrier for data collection of online travel about tourist destinations. Besides the edges of WeChat, the announced rules in its official design guideline of developing mini 
programs provide a set of instructions for web design and mini programs, which provides useful resources for developers and proposes visual standards on interface design including font, font color, button, and chart [47], bringing great convenience to developers. Meanwhile, compared with various apps, the integration of the user interface (UI) of WeChat mini programs and operation procedures improves user's usability.

\subsection{The Oblique Photography Technology in Tourism Applications}

The oblique photography technology can simultaneously collect target images from five different angles including a vertical angle and four slope angles to capture the detailed and high definition images of a building's texture from the top and sides. It not only can reflect the real condition of object surface and accurately acquire the texture information of images but also has advanced techniques such as locating, integrating, and modeling [48]. The oblique photography mainly relies on unmanned aerial vehicle (UAV) aerial image for high accuracy and three-dimensional quality, which has the features of flexibility, efficiency, low cost, multiple angles, vast scale, high definition, and easy online publishing due to a small amount of data. Much of the previous oblique photography research concentrated on areas such as emergency command, homeland security, city management, disaster evaluation, environment monitor, real estate, flood risk simulation, aboveground tree biomass estimation, archeology, engineering construction, and real view navigation [15-18]. Though vast applied researches have been conducted on oblique photography technology and its advantages shown in other fields have been discussed, few papers concentrate on the oblique photography in tourism, especially from the perspective of analyzing it with the assistance of data and social media through which the massive data produced by the tourist travel terminal can efficiently bring value to tourist destinations [15-18,49,50]. Big data generated by users, as well as real-time analysis and data synchronization, have become the main driving forces of the value creation process of smart destinations [29]. On the basis of satisfying consumers' demands and bringing the utmost benefits for operators with deep-in researches and objective analysis, we find oblique photography combined with the tourism industry can be transferred into commercial value, which can be regarded as an output port.

Proper management, repair, and maintenance of tourism resources have an extremely important role in tourism development and are key to sustainable development of tourism resources, which can facilitate tourists' visit so as to improve their satisfaction and travel experience, Moreover, they show great significance in promoting tourism consumption, social development, and economic growth. In the process of displaying tourist destinations in the past, most operators collected various scenes and features of the destination and then selected representative scenes with video explanations, celebrity interviews, and other materials. However, such approaches do not fully display the original features of the land. With the help of oblique photography measurement technology, this problem can be effectively solved. At the same time, three-dimensional images produced by oblique photography show significant advantages in the development and management of tourism destinations, which offers the perspective of planners and tourists so that users can fully observe different stages during the planning and construction of a tourist destination. In addition, oblique photography can comprehensively measure three-dimensional information, such as the original features and historical buildings, providing accurate and clear data for the subsequent maintenance and repair of the destination.

In summary, the application of oblique photography technology in scenic spots will improve the entertainment and enrichment of scenic spots, and the final three-dimensional (3D) geographic information frame will provide a base for the subsequent construction and planning of the tourism destinations, especially on scientific protection, reasonable exploitation, effective operation, scientific management, and planned development and introduction to new amusement programs, which will avoid mistakes caused by the lack of geographic information. This research puts forward that oblique photography can be applied in tourism, and with some software including Inpho, Pix4Dmapper and Street Factory, the real scene 3D modeling of the tourist destination will be available, which 
demonstrates the surrounding, the outside looking, location, height of buildings in the tourist destination, and provides an effective method of basic information acquisition for tourist destinations. For the use of multiple angle's photography measurements and the outcome of large-scale mapping, users are provided with enriched geographic information and details about the surroundings of their destinations, as well as friendlier user experiences and local services. For data usage, a data vectorization technique, ArcGIS (Geographic Information System) is used to decode and interpret scenic spots, streets, buildings, and sceneries. Through the advanced location technique, the exact geography, humanity, and marketing information can be embedded, and the 3D scene map and route design will be shown, expanding the application field's scenic spots.

Against the above background, the innovation of this study is to propose that it is feasible to apply oblique photography to mobile social media and combine it with tourism. Oblique photography has a dependent system that bridges the cloud server, which shares the commonality of the mobile set. Thus, social media only needs to build and adjust to the corresponding webpage to present the real 3D map of the destination, which builds a deep interaction between tourist destinations and consumers from the mobile network terminal, realizing the functions of visitors punching the clock in the scenic spots online and long-distance visits of scenic spots. Before traveling, tourists can have a general understanding of destinations through mini-programs and quickly make travel decisions and reasonable arrangements for routes. Using mini programs during travel helps people notice places that are unintentionally ignored at most tourist destinations, providing real-time local public service needs and route recommendations. After traveling, tourists can purchase souvenirs related to travel destinations in the mini program mall and comment on the destination. Some will recommend it to friends, thereby transforming users into word-of-mouth advertisers and improve the popularity of the tourist destination. Therefore, consumers will be steadily connected with their tourist destination and business links will be triggered. Meanwhile, it will enrich the experience of the tourist destination and exploit a territory to the ways of data capture and information flow for tourism. In the future, researchers will further develop oblique photography and mobile technology, aiming to provide tourists with higher quality services, promote the planning of tourist destinations, and trigger further progress in tourism, which supports the urban construction and economic growth to achieve the goal of sustainable development of tourism.

\section{Materials and Methods}

In order to find out whether social media combined with oblique photography smart technology can contribute to the sustainable tourism industry in the smart tourism paradigm, this study launches a project where oblique photography technology and social media are combined to develop a mini program, which is a one-stop tool for making travel plans that meets relevant stakeholder demands. These stakeholders include tourists, tourist destination operators, suppliers, and local residents. Using the Tokai Wonderland attractions in the Pingtan comprehensive experimental area as a sample, this study uses empirical research methods to develop a light application that combines social media with oblique photography to improve online travel services and demonstrates the thinking process of mini program development. The Pingtan comprehensive experimental area is located in the east of Fujian Province; the Tokai Wonderland attractions are on the east side by the sea, covering an area of 7.5 square kilometers. Due to its geological structure and long-term seawater and aeolian sand erosion, it has been turned into a magnificent and mysterious sea eroded landform. Furhter, it was nominated as a Haitan National Scenic Area on 10 January 1994 [51]. In this study, large-scale modeling work has been done in the Tokai Wonderland attractions and two nearby villages. By oblique photography technology, the scanning speed is accelerated and the cost of artificial modeling is reduced. The team only spent one day on field scanning and five days on 3D data reconstruction on the computer. In total, we spent six days restoring complex three-dimensional scenes of the Tokai Wonderland. Finally, the corresponding webpage is constructed and adjusted, and the real 3D map of Tokai Wonderland is presented on a WeChat mini program service (Figure 2). 

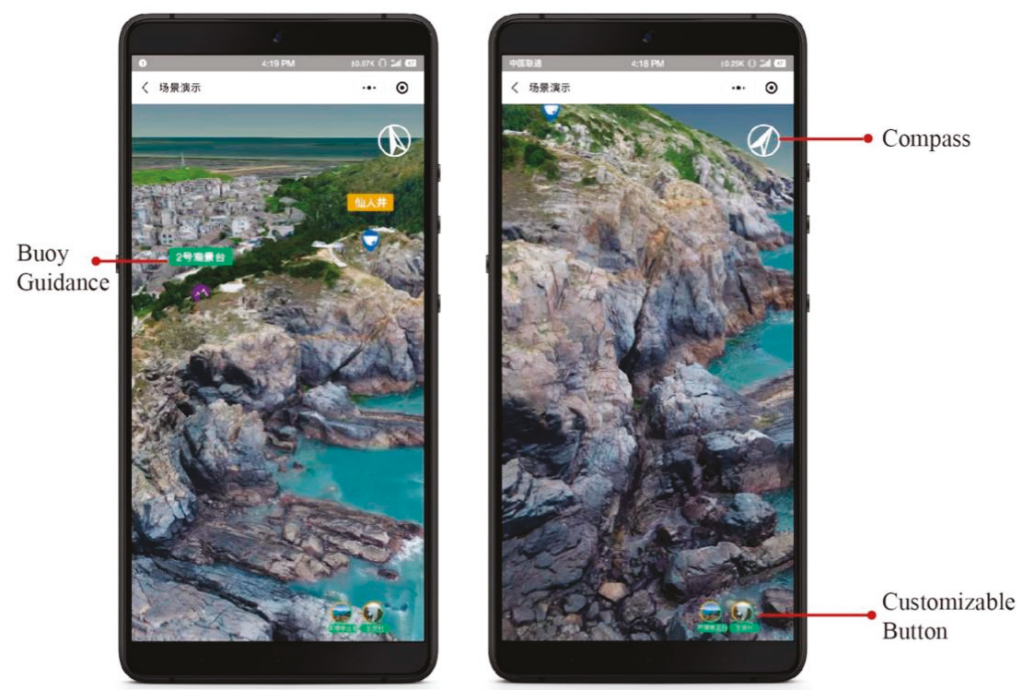

Figure 2. In a WeChat mini program, the three-dimensional (3D) reality restoration of Tokai Wonderland attractions are displayed based on oblique photography.

Schiffman, Kanuk, and Wisenblit [52] defined consumer behavior as the behaviors that appear in the period when a consumer searches, purchases, uses, evaluates, and deals with services and products provided by operators who believe that customers expect there products and services. In the past, consumers did not actively seek information from the market but rathee received help from exterior agents who will filtered information, selected products, gave advice, and even represented consumers to make decisions [53]. Based on Solomon's research, Stone [54] confirms that travel agents are an essential part of travel decision-making. Nevertheless, the wide use of mobile devices, the fluency and smoothness of network connection, the improvement of travel apps, and the convenience of mobile payment have stimulated the growth of social network services. Social media as an information source for travelers plays an important role. Apps are now major assets with regard to booking tickets and hotels, as well as searching for travel tips. In the tourism industry, social media is a unique marketing and communication tool for marketing personnel, because they can directly interact with tourists to affect decision-making $[55,56]$. Meanwhile, social media tremendously affects how customers search and share information, even after they have decided where they are going. Thus, when developing the mini program, we should include the function of a travel agent's decision-making throughout the entire process. Figures 3 and 4 show the mind maps of the process of developing this new mini program.

Figure 3 shows that consumers at the first stage of "need recognition" evoke the demand for traveling. The second stage is "information search", where users search the conditions and information of their desired destination on the mini program to make an "alternative evaluation". The third stage is "choice decision". Through the functions of the mini program, users decide whether to visit the tourist destination and then book tickets and accommodations. "During Trip" is the fourth stage, wherein the mini program can provide instant information and assist interaction at the tourist destination. The fifth stage is the "post-trip evaluation", which includes users using the mini program to share and evaluate the trip and purchase local products. The mini program penetrates the entire travel service circle and plays an important role in decision-making. What is more, with the function of electronic word of mouth (e-WOM), it completes a closed loop in the travel decision-making process. The role of e-WOM is similar to advertisements that cause potential tourists to achieve a virtuous cycle of tourism economic growth for tourism purposes. 


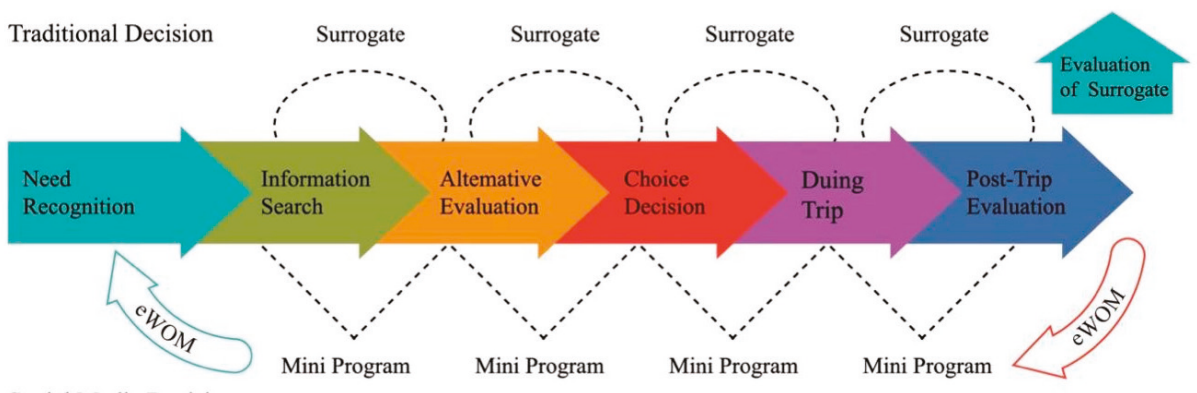

Social Media Decision

Figure 3. The simplified model of mini programs replacing surrogates in the decision-making process (Solomon, 1986). E-WOM: electronic word of mouth.

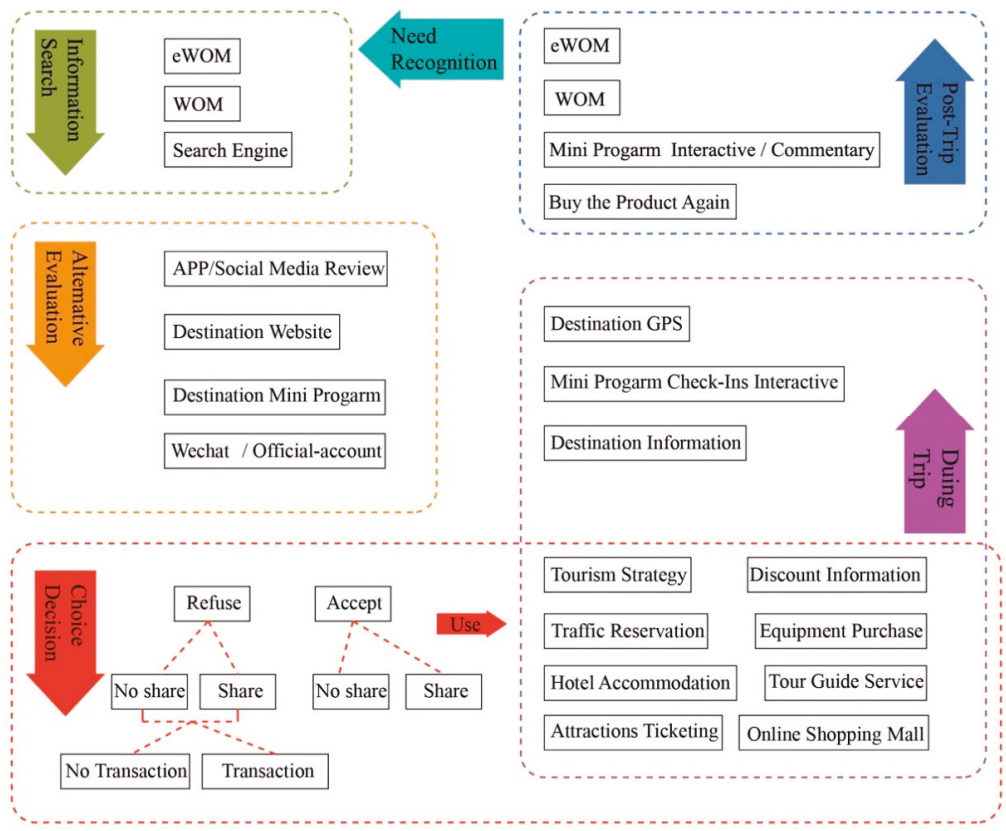

Figure 4. The mind map of a consumer when using the mini program.

Previous research has discerned that some tourists decide their travel destination according to comments written by those who have already visited [57-59]. Naturally, a good travel experience will produce word of mouth that will create a tremendous influence via the spread of social media and travel review websites [60]. According to Penguin Intelligence, tourist users are used to preparing travel schedules on travel apps in advance and the one-stop service is preferred by users in which travel tips, accommodation reservation, and transportation reservation are the most popular, followed by equipment purchase, scenic spot ticket booking, discount information, and pick up service [61]. Those who are content with these services are most likely to not only visit again but become faithful customers and recommend the corresponding products to others. This is incredibly important for face-to-face interaction during the trip and comments after-sales service can influence the decisions of potential customers in the earlier service stage [62,63].

Clatworthy [64] defines a service supplier interacting with clients from various aspects. At the a tourist destination, products are mainly service and include various contact points before, during, 
and after travel. Trying to satisfy clients' expectations is vital to improve their satisfaction, which needs to continuously be measured [65]. Therefore, we list all possible interactions and contact points between tourists/potential tourists and the mini program (Figure 3). This can be referred to as a basis for the design of function modules in mini programs.

In Figure 4, it is clear that, after the "demand recognition" stage before the trip, potential tourists during the "information search" are most likely to know the tourist destination through e-WOM, recommendations from relatives, or mini program searches. During "alternative evaluation", tourists resort to travel apps, social media reviews, websites, mini programs, WeChat, or its subscriptions to decide whether to go. Now, however, potential tourists can experience reality demonstrations through the 3D view provided by oblique photography technology. At the stage of "choice decision", tourists may decide to set off or instantly recommend the destination to others. Then, they are likely to prepare for the trip using the mini program. Such preparations include searching for travel tips, reserving hotels, and learning traffic information. Those who decide not to go will probably share the mini program to others. What is superior to the traditional ways of promotion is that consumers may use the mini program's online shopping mall and WeChat payment to buy local products online, which creates a new sales method for the destination's specialties and an opportunity for the destination to advertise itself. For the "during the trip" stage, tourists can use the mini program to search for information and acquire travel tips, which, unlike the traditional Global Position System (GPS), enables tourists to experience a $3 \mathrm{D}$ view and punch the clock when they arrive at the spot marked in the mini program. This promotes the close interaction between tourists and the tourist destination. During the "post-trip evaluation" stage, tourists may share the mini program to others, make comments in the mini program, and buy local products again through the mini program's online shopping mall. These functions invisibly influence customer's demand recognition and further contribute to a closed loop of consumer decisions.

Figures 5 and 6 are demonstrations of the Tokai Wonderland attractions of the Pingtan comprehensive experimental area in Wechat. What we need to do is to find the contact points in the choice decision process and figure out how to optimize the functional module of the destination to offer a better online experience, raise consumer satisfaction, win more positive e-WOMs, and achieve sustainable development of the destination.

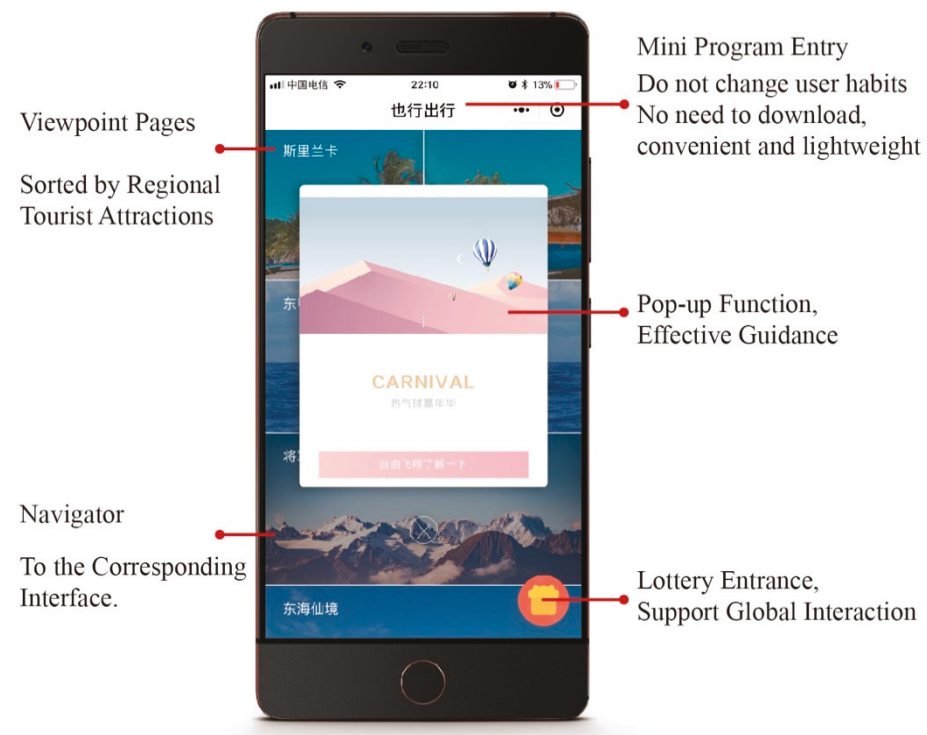

Figure 5. The homepage of tourism service in the WeChat mini program. 


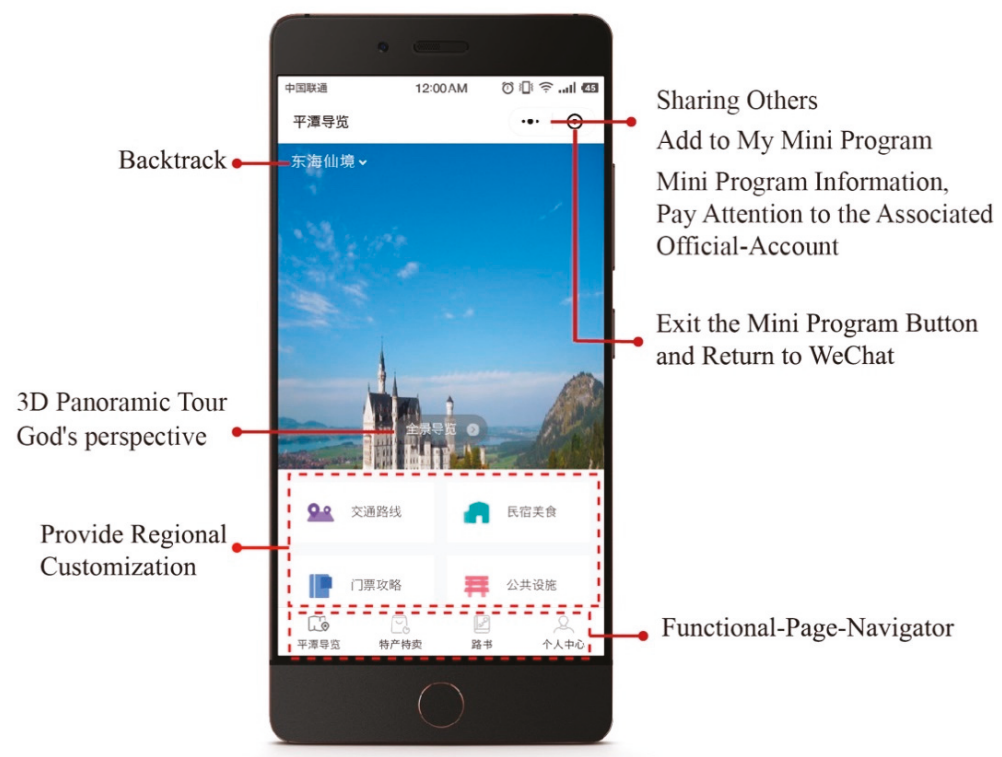

Figure 6. The sub page's column navigation of the Tokai Wonderland attractions in the WeChat mini program.

Figure 7 demonstrates 3D images of a tourist destination, so that non-WeChat users and domestic and foreign tourists who prefer using a PC can access the web through a link. Since this study mainly discusses the innovative use of combining oblique photography technology with social media on mobile devices, the computer version will not be discussed in detail.
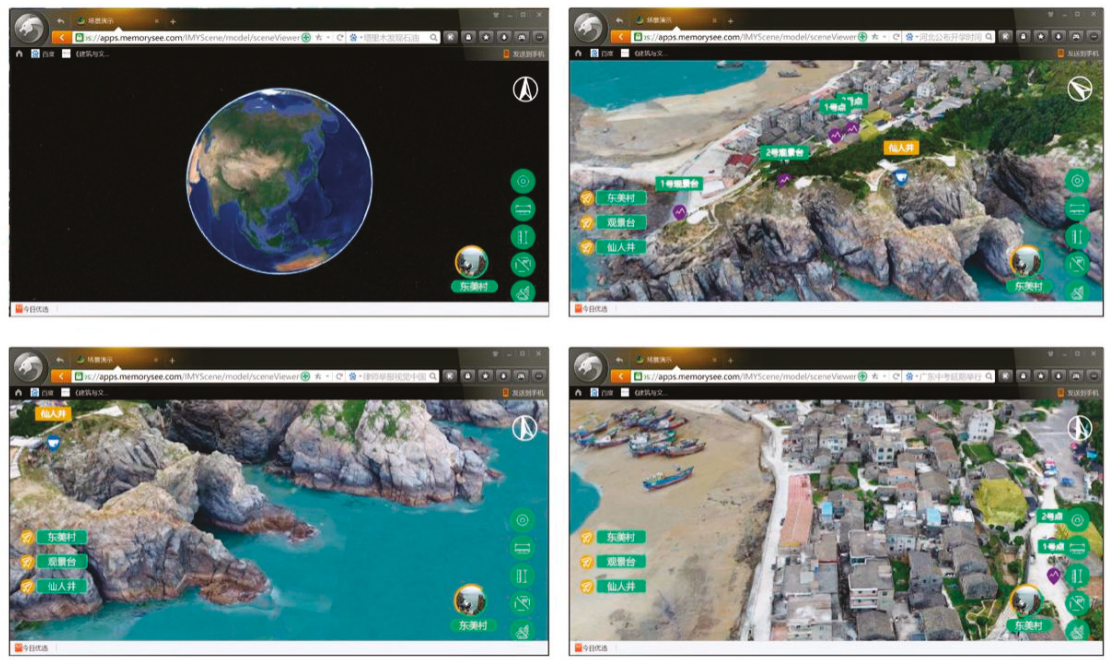

Figure 7. The computer version of 3D images of the Tokai Wonderland attractions in the Pingtan comprehensive experimental area. 


\section{Results and Discussion}

This study combines social media applications with oblique photography technology on mobile devices and realizes the use of three-dimensional scenes of travel destinations in online travel applications. The travel application of social media combined with oblique photography technology allows tourists to interact more actively with destinations and collect more targeted information, which plays a very important role in marketing tourist destinations. Various stakeholders (e.g., communities, government agencies, suppliers, tourist destination managers, non-profit organizations, tourists, etc.) will benefit from the data collected on this mini program. In 2000, Gordon Philips [66] proposed that the concept of smart tourism is a comprehensive, long-term, and sustainable method to plan, develop, operate, and market products and tourism businesses. In fact, the big data generated by user-generated content and the real-time analysis and synchronization of these data have become the driving force of the smart destination value creation process $[29,34]$. They can analyze tourism profiles with more details, including the sociodemographic profiles and users' preferences, feelings, and attitudes. These data are useful for creating tourism services that better meet the demands of tourists [8]. In addition, factual information about a tourist destination retained by oblique photography brings stable resources, culture, environment, and civic life quality, including life safety and tourist property protection. Through the Internet, data collection, feedback, and decision-making optimization, the entire system of stakeholders operates jointly, bringing benefits to the sustainable development of tourist destinations. The following parts will focus on the benefits of these data for tourists, tourist destination marketers/planners, and government agencies.

\subsection{For Tourists}

Compared with the application's interface adopting traditional panoramic photography, oblique photographs show the real scene in an overall view on one page and thus no secondary page is needed. Tourists are able to zoom in and out in the demonstration page. With markers and page modules, it distinctly shows the regional service distribution. With such functions, tourists can easily reserve services from the tourist destination and shop online at any time. These functions can be presented on one page, meaning it contains overall information on the destination. Oblique photography enables users to observe the sites from multiple angles. The overall view directly represents the real situations of the tourist destination, because this technique can capture the map of dozens of buildings, shaping it into a terrain model that could generate a sight shock. In the field of tourism, tourists do not merely have relations with the scenic spot but are connected with the place out of their basic demands on traveling. In the 3D vision, apart from the scenic spots, users can also have an overview of surrounding areas because the mini program clearly shows the location of hotels, nearby scenic spots, ATMs, medical care centers, and public facilities.

The 3D model based on oblique photography can add many dimensions to information. Not only has it replaced the traditional scenic spot map but it instantly locates tourists and recommends routes according to live scenes. In addition, different types of tourists, such as free travel, group tourists, elderly tourists, or parent-child tourists, can quickly and accurately decide the travel route from the $3 \mathrm{D}$ reality images. In route recommendation, the interaction button on the main page can be motivated, which is a surprise entrance to many interactive activities, allowing users to to know activities and achieve original goals orientationally. Furthermore, we are able to construct different modular designs for different tourist destinations and choose different marketing tools and ways of interacting. Mini programs provide services such as navigation, tour guides, shopping guide sharing, public service, and real-time travel route recommendation, which appropriately designs the travel routes and makes travel plans for tourists in advance to save their time so that tourists can quickly get familiar with the surrounding. Based on the mini program's online payment function, it offers one-stop and inclusive services for tourists, thus improving their satisfaction degree, promoting tourism consumption, and stimulating tourism economic growth. 


\subsection{For Tourist Destination Managers/Planners}

For tourist destination managers, the biggest advantage of mini programs from an information perspective is that the data are preserved by operators. In order to enable mini program developers and operators to easily view mini program information, many data analysis platforms have emerged, especially for mini program data analysis (Table 1). The tools of these data analysis platforms include the following analytical layers in order to better understand users and optimize the mini program to contribute to the transformation of data into value:

1. Application analysis contains live data, historical trend, web page analysis, etc., from which we can know how long users stay on the interface, user preferences, and the last web page the user visited. According to the statistics of a users' page staying time, the fit points between users' preference and the importance of popularization will be found to adjust the ways of information, releasing management concepts so as to ensure a second visit and leave a chance to collect more information from these users next time.

2. Environment analysis is the analysis of regions, terminals, network, mobile types, etc., which helps operators understand the mini program's operating environment.

3. User exploration analysis concludes user tracking, user role, new and regular users, users' retention rate, user activation, user turnover, and the inverse flow rate of users, which can be used by operators to target the objective user group. According to the distribution of user's browsing time, it targets the golden time to update the latest information of destination activities for the faithful clients and vast customer groups so as to reach a high information-receiving rate. According to the user analytics, it can ensure major users visit the tourist destination. Therefore, we can adjust the scene in the mini program according to their age, gender, and education background to improve the ways of meeting user demands and strengthen the relationship between the mini program and its users.

4. User behavior analysis includes the list of events, event details, funnel plot analysis, etc., which can precisely show the concrete reason for user loss. By analyzing the number of users in every link, we can find out the part that leads to user loss and warn the operators to take timely actions to deal with abnormal statistics.

5. Usage analysis covers the analysis of sharing, pull-to-refresh, bottom reaching, time of using period, etc., which can quickly give feedback so as to adjust unreasonable page modules and even the way of displaying information. Based on most users' browsing habits, it helps adjust the contents of the subordinate page so that the sub-subordinate page can effectively lead users to interfaces which may trigger their desire to visit or to shop.

6. Business analysis of e-commerce consists of transaction analysis, repurchase rate analysis, etc., which can analyze the acceptability of new products so as to produce more popular ones, improve those that are not so popular, or upgrade services.

7. Source analysis consists of channel analysis or data sources. The mini program has many entrances and each entrance has a corresponding scene ID number. Thus, the operators can decide the ratio of different ads according to the statistics from different scene ID number.

8. The heat map shows the number of page views of each interface and the preference of clicks, from which we can immediately find out whether the current frame of interfaces is appropriate or whether the interfaces are too compact. If so, we can timely add the missing information to increase user's involvement and target conversion rate.

9. Mini program user analysis can be a reference for our media, the WeChat subscriptions about the tourist destination is connected with the mini program. It can combine various media terminals to access efficient information to upgrade the modes of business operation and provide proper discounts for sponsored content. The heat map of a tourist's preference can help design customer-oriented activities of the tourist destination. 
10. According to the data of past visits to the mini program, operators can effectively predict the number of tourists in the scenic spots of the tourist destination, improve the quality of management, arrange staff, facilities, and performances, improving the configuration of relevant scenic resources.

11. The data collection from tourist evaluation, complaints, and suggestions can strengthen the effective contact and interaction between tourists and tourist destination operators who are able to collect real-time feedback to improve the level of customized services and make more appropriate plans for tourist destinations.

Table 1. Analysis platforms for mini programs.

\begin{tabular}{|c|c|}
\hline Analysis Tools for Mini Programs & Functions \\
\hline $\begin{array}{l}\text { Tencent mobile analysis } \\
\text { WeChat mini program's analytics }\end{array}$ & $\begin{array}{c}\text { Application analysis, environment analysis, user } \\
\text { analysis, custom events, source analysis, usage } \\
\text { analysis, etc. }\end{array}$ \\
\hline Baidu analytics & $\begin{array}{l}\text { Custom analysis, user analysis, using behavior, } \\
\text { thermodynamic diagram, channel analysis, start-up } \\
\text { source, retention analysis, conversion analysis, etc. }\end{array}$ \\
\hline TalkingData & $\begin{array}{c}\text { Data overview, user exploration, user's behavior } \\
\text { analysis, e-commerce business analysis, statistics } \\
\text { exploration, etc. }\end{array}$ \\
\hline ALaDing mini program analytic tool & $\begin{array}{l}\text { Data overview, scene analysis, } \mathrm{QR} \text { code channel, } \mathrm{AD} \\
\text { monitoring, sharing analysis, page analysis, events } \\
\text { and conversion analysis, user sorting, personas, etc. }\end{array}$ \\
\hline WeChat public platform & $\begin{array}{l}\text { Overview, real-time statistics, visiting analysis, source } \\
\text { analysis, user analysis, custom analysis }\end{array}$ \\
\hline $\begin{array}{l}\text { Mini program data analysis helper (official mini } \\
\text { program tools) }\end{array}$ & $\begin{array}{l}\text { Data overview, visiting basic analysis, real-time } \\
\text { analysis and personas, etc. }\end{array}$ \\
\hline Hot app mini program analytics & $\begin{array}{c}\text { Statistical profile, QR scan statistics, user analysis, } \\
\text { sharing analytics, events statistics, event } \\
\text { management, user's feedback, etc. }\end{array}$ \\
\hline Sensors data & $\begin{array}{l}\text { Event analysis, funnel plot analysis, retention } \\
\text { analysis, distribution analysis, user paths, heat map } \\
\text { of web page, attributive analysis of users, etc. }\end{array}$ \\
\hline Growing IO statistical analysis of mini program & $\begin{array}{l}\text { The visible board of core products, product purchase } \\
\text { conversion; the visible board of core indicators in } \\
\text { investment, user attribute, the core indicators of } \\
\text { market and customer acquisition, the heat map of } \\
\text { operation analysis visible board, conversion rate, etc. }\end{array}$ \\
\hline
\end{tabular}

With the development of the Internet, the efficient acquisition of user information has become the most valuable market resource. Operators do not need to rely on a tycoon's app or invest in the development of a new app. Instead, operators can collect data precisely and transfer valuable information so as to acquire the orientations of operation, development, and optimization of comprehensive and inclusive services. Such combination of oblique photography and mini program's big data acquisition saves the operators a large amount of cost, for they do not need to develop a new app or invest in its maintenance; the mini program provides the operators with user statistics. In addition, the results presented by the mini program's background data can effectively improve the management level of tourist destination operators, promote the innovative development of tourism products and services, and provide suggestions to help planners make long-term plans to balance the interests among various stakeholders, promoting the sustainable development of tourist destinations. 


\subsection{For Government Agencies}

Based on the application of combining oblique photography technology with WeChat mini programs, the 3D geographic information of the restored tourist destination can provide a geographic basis for subsequent construction, planning, protection, development, and management. The mini program's information sharing platform can help government agencies find out problems more easily through smart office, smart supervision, smart service, and smart decision-making, improving the management level, coordination, and service ability of various functional departments for tourist destinations. Through the real-time positioning of tourists in tourist destinations and the heat map feedback on travel routes and public service demands, it can be seen that:

1. It helps the government to play the guiding role in the early stage of tourism development and construction, making appropriate plans and designs for the tourism resources.

2. It helps government agencies carry out comprehensive diagnoses and orderly management of construction through the organization and management mechanism, so that tourism public services including life services, hospitals, transportation, tourist centers, and other resources.

3. It helps government agencies play a leading role in terms of capital and acquiring a global perspective on the capital investment through tourism popularization.

4. It helps government agencies quickly respond to the structural adjustment of the local tourism industry, prompting it to further expand, develop, transform, and upgrade.

5. It helps government agencies realize smart scheduling, improve the efficiency of various management activities of tourist destinations, and promote healthy and sustainable development for the tourism industry.

Currently, the developing function of the mini program is being improved. The operators of each mini program can independently develop and add new functions according to their own demands. Therefore, through the Internet, the operators can resort to highly efficient media platform to take full advantage of the interaction function of the mini program, for the tourism industry is featured with sharing information, and is thus more likely to discover new ways to stimulate consumption and increase interest growth rate. Combination with oblique photography is a positive attempt for interactive conversion.

\section{Conclusions and Limitations}

Social media is an increasingly important partner to the tourism industry. Making full use of mini programs and embedding oblique photography technology has become a new element and new selling point for the tourism industry. This study analyzes and regards oblique photography as an important technical method of smart tourism. A newly emerged technology of 3D space measurement is characterized by its flexibility, low cost, multiple angles, large range, and high definition, providing efficient technical means for basic data acquisition of tourist destinations without costing manpower, material resources, and financial resources for repeated measurements. Using oblique photography technology to obtain 2D and 3D data at once reflects the secondary, location, height, and other data of the tourist destination. The 3D model enhances the vividness and provides reliable basic data for the coordinated development of proper protection, appropriate development, effective management, and proper management of tourist destinations.

There is no doubt that the combination of oblique photography and mini program provides comprehensive service and more convenience for regional tourism. Not only does it interact with tourists perfectly in each service link but it provides a way to access statistical information and reverse information, thus connecting operators with consumers. In this study, the unresolved problems in the literature review are fixed. The application of oblique photography technology is moved from the PC to mobile. This paper clarifies the contribution of oblique photography to online travel services, making up for the vacancy of the real-time interactive experience of tourists in other small-scale tourism applications. Taking the Tohai Wonderland attractions in the Pingtan comprehensive experimental 
area as an example according to the mind map of tourists using mini programs, this paper conducts a case study on the application of oblique photography to a mini program. The background data analysis of the mini program can guide the development and operation of a tourist destination business, providing provide operators with the value conversion of a user profile analysis. This case provides a reference for other social media tycoons (i.e., Facebook, Instagram, LinkedIn, Line, Twitter, etc.) and apps for online travel such as Ctrip and Booking, for it is based on a large number of users. With the emerging technology and oblique photography, there is a new online interaction between users and tourist destinations.

However, oblique photography has its limitations. Successful and smooth high-definition image display can be affected by a network's bandwidth. With the popularization of 5G (the 5th generation wireless systems), this problem will be solved soon. This study also conducts an in-depth analysis of relative stakeholders such as tourists, tourist destination managers, and government agencies. In future research, we will apply case demonstrations to the practical use of specific tourist destinations, collect real statistics to verify the effectiveness and feasibility of such combinations, and optimize the tool set. We will demonstrate and explain how to analyze the data in real conditions and reshape it as the target big data by trying to use the research results as a reference for stakeholders so as to fully promote the sustainable development of tourism.

Author Contributions: This research conceptualization by M.L. and F.-Y.L.; methodology by F.L.; investigation by M.L. and H.Z.; resources by H.Z.; writing—original draft preparation by M.L.; writing-review and editing by F.-Y.L., M.L. and H.Z.; visualization by M.L. All authors have read and agreed to the published version of the manuscript.

Funding: This research received no external funding.

Conflicts of Interest: The authors declare no conflict of interest.

\section{References}

1. Serrano, L.; Sianes, A.; Ariza-Montes, A. Using Bibliometric Methods to Shed Light on the Concept of Sustainable Tourism. Sustainability 2019, 11, 6964. [CrossRef]

2. Nations, U. Transforming Our World: The 2030 Agenda for Sustainable Development; United Nations General Assembly: New York, NY, USA, 2015.

3. China Tourism Academy. Basic Situation of Tourism Market in 2019. Available online: http://www.ctaweb. org/html/2020-3/2020-3-10-16-48-64712.html (accessed on 8 April 2020).

4. Moral, M.M.; Alles, M.T.F.; Franco, M.J.S. Attitudes of rural accommodation managers towards the development of sustainable tourism. Cuad. Tur. 2018, 41, 707-711.

5. Cuesta-Valiño, P.; Bolifa, F.; Núñez-Barriopedro, E. Sustainable, Smart and Muslim-Friendly Tourist Destinations. Sustainability 2020, 12, 1778. [CrossRef]

6. Jafari, J. The scientification of tourism. In Hosts and Guests Revisited: Tourism Issues of the 21st Century; Smith, V., Brent, M., Eds.; Cognizant Communication Corporation: New York, NY, USA, 2001; pp. $28-41$.

7. Hjalager, A.M.; Nordin, S. User-driven innovation in tourism-A review of methodologies. J. Qual. Assur. Hosp. Tour. 2011, 12, 289-315. [CrossRef]

8. Del Chiappa, G.; Baggio, R. Knowledge transfer in smart tourism destinations: Analyzing the effects of a network structure. J. Destin. Mark. Manag. 2015, 4, 145-150. [CrossRef]

9. Encalada, L.; Boavida-Portugal, I.; Cardoso Ferreira, C.; Rocha, J. Identifying Tourist Places of Interest Based on Digital Imprints: Towards a Sustainable Smart City. Sustainability 2017, 9, 2317. [CrossRef]

10. Bustamante, A.; Sebastia, L.; Onaindia, E. Can Tourist Attractions Boost Other Activities Around? A Data Analysis through Social Networks. Sensors 2019, 19, 2612. [CrossRef] [PubMed]

11. Malik, K. Optimal Travel Route Recommendation Mechanism Based on Neural Networks and Particle Swarm Optimization for Efficient Tourism Using Tourist Vehicular Data. Sustainability 2019, 11, 3357. [CrossRef] 
12. Li, Q.; Li, S.; Zhang, S.; Hu, J.; Hu, J. A Review of Text Corpus-Based Tourism Big Data Mining. Appl. Sci. 2019, 9, 3300. [CrossRef]

13. Ting, L.; Jian, H.X.; Crowe-Delaney, L. Tourism Information Diffusion through SNSs: A Theoretical Investigation. Sustainability 2020, 12, 1731.

14. Buhalis, D.; Law, R. Progress in information technology and tourism management: 20 years on and 10 years after the Internet-The state of eTourism research. Tour. Manag. 2008, 29, 609-623. [CrossRef]

15. Yang, G.D.; Wang, M.S. The Tilt Photographic Measuration Technique and Expectation. Geomat. Spat. Inf. Technol. 2016, 39, 13-18.

16. Wu, Y.; Peng, F.; Peng, Y.; Kong, X.; Liang, H.; Li, Q. Dynamic 3D Simulation of Flood Risk Based on the Integration of Spatio-Temporal GIS and Hydrodynamic Models. ISPRS Int. J. Geo-Inf. 2019, 8, 520. [CrossRef]

17. Lin, J.; Wang, M.; Ma, M.; Lin, Y. Aboveground Tree Biomass Estimation of Sparse Subalpine Coniferous Forest with UAV Oblique Photography. Remote Sens. 2018, 10, 1849. [CrossRef]

18. Verhoeven, G.; Taelman, D.; Vermeulen, F. Computer vision-based orthophoto mapping of complex archaeological sites: The ancient quarry of itaranha (Portugal-Spain). Archaeometry 2012, 54, 1114-1129. [CrossRef]

19. Saarinen, J. Critical Sustainability: Setting the Limits to Growth and Responsibility in Tourism. Sustainability 2013, 6, 1-17. [CrossRef]

20. United Nations Environment Programme (UNEP). Making Tourism More Sustainable: A Guide for Policy Makers; UNEP: Paris, France, 2005.

21. McIntyre, G. Sustainable Tourism Development: Guide for Local Planners; World Tourism Organization: Madrid, Spain, 1993.

22. Choi, H.C.; Sirakaya, E. Measuring residents' attitude toward sustainable tourism: Development of sustainable tourism attitude scale. J. Travel Res. 2005, 43, 380-394. [CrossRef]

23. Waligo, V.M.; Clarke, J.; Hawkins, R. Implementing sustainable tourism: A multi-stakeholder involvement management framework. Tour. Manag. 2013, 36, 342-353. [CrossRef]

24. Clarke, J. A framework of approaches to sustainable tourism. J. Sustain. Tour. 1997, 5, 224-233. [CrossRef]

25. Buhalis, D. Tendencias y retos de turismo electrónico en la era de las redes sociales. Tur. y Nuevas Tecnol.-Semin. Téc. San José Costa Rica 2013, 14, 5-17.

26. Ho, C.-T.B.; Gebsombut, N. Communication Factors Affecting Tourist Adoption of Social Network Sites. Sustainability 2019, 11, 4198. [CrossRef]

27. Shen, S.; Sotiriadis, M.; Zhou, Q. Could Smart Tourists Be Sustainable and Responsible as Well? The Contribution of Social Networking Sites to Improving Their Sustainable and Responsible Behavior. Sustainability 2020, 12, 1470. [CrossRef]

28. Baggio, R.; Cooper, C. Knowledge transfer in a tourism destination: The effects of a network structure. Ser. Ind. J. 2015, 4, 145-150. [CrossRef]

29. Vecchio, P.D.; Mele, G.; Ndou, V.; Secundo, G. Creating value from Social Big Data: Implications for Smart Tourism Destinations. Inf. Process. Manag. 2018, 54, 847-860. [CrossRef]

30. eMarketer. Global Digital Travel Sales 2018. Available online: https://www.emarketer.com/content/globaldigital-travel-sales-2018 (accessed on 12 September 2019).

31. We Are Social Hootsuite. Dilgital 2020: Global Dilgital Overviwe. Available online: https://datareportal.com/ reports/digital-2020-global-digital-overview? (accessed on 8 April 2020).

32. Wang, D.; Xiang, Z.; Fesenmaier, D.R. Smartphone use in everyday life and travel. J. Travel Res. 2016, 55, 52-63. [CrossRef]

33. Garcia-Madariaga, J.; Virto, N.R.; López, M.F.B.; Manzano, J.A. Optimizing website quality: The case of two superstar museum websites. Int. J. Cult. Tour. Hosp. Res. 2019, 13, 16-36. [CrossRef]

34. Dickinson, J.E.; Ghali, K.; Cherrett, T.; Speed, C.; Davies, N.; Norgate, S. Tourism and the smartphone app: Capabilities, emerging practice and scope in the travel domain. Curr. Issues Tour. 2014, 1, 84-101. [CrossRef]

35. iResearch. China Online Travel Platform User Insight Report 2018. Available online: https://www.iresearch. com.cn/Detail/report?id=3225\&isfree $=0$ (accessed on 25 August 2019).

36. Hudson, S.; Thal, K. The impact of social media on the consumer decision process: Implications for tourism marketing. J. Travel Tour. Mark. 2013, 30, 156-160. [CrossRef]

37. Leung, D.; Law, R.; Van Hoof, H.; Buhalis, D. Social media in tourism and hospitality: A literature review. J. Travel Tour. Mark. 2013, 30, 3-22. [CrossRef] 
38. Sigala, M.; Christou, E.; Gretzel, U. Social Media in Travel, Tourism and Hospitality: Theory, Practice and Cases; Ashgate Publishing Limited: Surrey, UK, 2012.

39. Miah, S.J.; Vu, H.Q.; Gammack, J.; McGrath, M. A Big Data analytics method for tourist behaviour analysis. Inf. Manag. 2016, 54, 771-785. [CrossRef]

40. Seo, E.J.; Park, J.-W.; Choi, Y.J. The Effect of Social Media Usage Characteristics on e-WOM, Trust, and Brand Equity: Focusing on Users of Airline Social Media. Sustainability 2020, 12, 1691. [CrossRef]

41. Zimaitis, I.; Degutis, M.; Urbonavicius, S. Social Media Use and Paranoia: Factors That Matter in Online Shopping. Sustainability 2020, 12, 904. [CrossRef]

42. Foux, G. Consumer-generated media: Get your customers involved. Brand Strat. 2006, 8, 38-39.

43. Gretzel, U. Intelligent systems in tourism. A Social Science Perspective. Ann. Tour. Res. 2011, 38, 757-779. [CrossRef]

44. Minstry of Industry and Infoemation Technology of the People's Repulic of China. Internet and Related Services Remain Stable and Stable in January-October 2018. Available online: http://www.miit.gov.cn/ n1146312/n1146904/n1648355/c6531993/content.html (accessed on 25 November 2019).

45. JIGUANG. 2018 Q3 Mobile Internet Industry Data Report. Retrieved 26 November 2018. Available online: https://www.jiguang.cn/reports/350 (accessed on 26 November 2019).

46. Wen, Z.; Geng, X.; Ye, Y. Does the use of Wechat lead to subjective well-being?: The effect of use intensity and motivations. Cyber Psychol. Behav. Soc. Netw. 2016, 19, 587-592. [CrossRef]

47. WeChat Public Platform. Available online: https://mp.weixin.qq.com/cgi-bin/wx (accessed on 1 November 2019).

48. Li, A.F.; Zeng, Z.X.; Wu, X.M. The Analysis of the Development of Oblique Photography Technique in China. Geomat. Spat. Inf. Technol. 2014, 37, 57-59.

49. Xue, X.; Wu, C.; Sun, Z.; Wu, Y.; Xiong, N. Vegetation Greening for Winter Oblique Photography Using Cycle-Consistence Adversarial Networks. Symmetry 2018, 10, 294. [CrossRef]

50. Harwin, S.; Lucieer, A.; Osborn, J. The Impact of the Calibration Method on the Accuracy of Point Clouds Derived Using Unmanned Aerial Vehicle Multi-View Stereopsis. Remote Sens. 2015, 7, 11933-11953. [CrossRef]

51. Lin, H.; Shen, X.D. Pingtan County Local Chronicles Compilation Committee. In Pingtan County Records, 1st ed.; Fang Zhi Publishing House: Beijing, China, 2010; pp. 667-671.

52. Schiffman, L.G.; Kanuk, L.L.; Wisenblit, J. Consumer Behaviour, 10th ed.; Prentice Hall: Upper Saddle River, NJ, USA, 2010.

53. Solomon, M.R. The missing link: Surrogate consumers in the marketing chain. J. Mark. 1986, 50, 208-218. [CrossRef]

54. Stone, M.J. Deciding not to choose: Delegation to social surrogates in tourism decisions. Tour. Manag. 2016, 57, 168-179. [CrossRef]

55. Noti, E. Web 2.0 and its influence in the tourism sector. Eur. Sci. J. 2013, 9, 115-123.

56. Királ'ová, A.; Pavlíčeka, A. Development of social media strategies in tourism destination. Procedia Soc. Behav. Sci. 2015, 175, 358-366. [CrossRef]

57. Chevalier, J.A.; Mayzlin, D. The Effect of Word of Mouth on Sales: Online Book Reviews. J. Mark. Res. 2006, 43, 345-354. [CrossRef]

58. Duan, W.; Gu, B.; Whinston, A.B. Do online reviews matter?-An empirical investigation of panel data. Decis. Support Syst. 2008, 45, 1007-1016. [CrossRef]

59. Forman, C.; Ghose, A.; Wiesenfeld, B. Examining the Relationship between Reviews and Sales: The Role of Reviewer Identity Disclosure in Electronic Markets. Inf. Syst. Res. 2008, 19, 291-313. [CrossRef]

60. Stickdorn, M. Tourism and service design thinking: Who learns from whom? Touchpoint 2012, 4, 58-61.

61. Penguin Intelligence. China Tourism APP Market Full Data Report. Available online: http://www.199it.com/ archives/519529.html (accessed on 25 November 2018).

62. Geser, G.; Markus, M. Prosumer im Tourismus; ITD-Verlag: Hamburg, Germany, 2008.

63. Egger, R. Grundlagen des Etourism-Informations- und Kommunikations-Technologien im Tourismus; Shaker Verlag: Aachen, Germany, 2005.

64. Clatworthy, S. Bridging the gap between brand strategy and customer experience. Manag. Serv. Qual. Int. J. 2012, 22, 108-127. [CrossRef] 
65. Stickdorn, M.; Zehrer, A. Service design in tourism: Customer experience driven destination management. In Proceedings of the First Nordic Conference on Service Design and Service Innovation, DeThinking Service-ReThinking-Design, Oslo, Norway, 24-26 November 2009; Linköping University Electronic Press: Linköping, Sweden, 2012.

66. Li, Y.; Hu, C.; Huang, C.; Duan, L. The concept of smart tourism in the context of tourism information services. Tour. Manag. 2016, 58, 293-300. [CrossRef]

(C) 2020 by the authors. Licensee MDPI, Basel, Switzerland. This article is an open access article distributed under the terms and conditions of the Creative Commons Attribution (CC BY) license (http://creativecommons.org/licenses/by/4.0/). 



\title{
Using Volunteered Geographic Information and Nighttime Light Remote Sensing Data to Identify Tourism Areas of Interest
}

\author{
Bidur Devkota ${ }^{1, *}$, Hiroyuki Miyazaki ${ }^{1,2}$, Apichon Witayangkurn ${ }^{1,2}$ and Sohee Minsun Kim ${ }^{3}$ \\ 1 School of Engineering and Technology, Department of Information and Communication Technologies, \\ Asian Institute of Technology, Post Box No 4, Pathumthani 12120, Thailand \\ 2 Center for Spatial Information Science, Tokyo University, Chiba 277-8568, Japan \\ 3 School of Environment, Resources, and Development, Department of Development and Sustainability, \\ Asian Institute of Technology, Post Box No 4, Pathumthani 12120, Thailand \\ * Correspondence: devkota.npl@gmail.com
}

Received: 25 July 2019; Accepted: 23 August 2019; Published: 29 August 2019

\begin{abstract}
Easy, economical, and near-real-time identification of tourism areas of interest is useful for tourism planning and management. Numerous studies have been accomplished to analyze and evaluate the tourism conditions of a place using free and near-real-time data sources such as social media. This study demonstrates the potential of volunteered geographic information, mainly Twitter and OpenStreetMap, for discovering tourism areas of interest. Active tweet clusters generated using Density-Based Spatial Clustering of Applications with Noise (DBSCAN) clustering algorithm and building footprint information are used to identify touristic places that ensure the availability of basic essential facilities for travelers. Furthermore, an investigation is made to examine the usefulness of nighttime light remotely sensed data to recognize such tourism areas. The study successfully discovered important tourism areas in urban and remote regions in Nepal which have relatively low social media penetration. The effectiveness of the proposed framework is examined using the F1 measure. The accuracy assessment showed F1 score of 0.72 and 0.74 in the selected regions. Hence, the outcomes of this study can provide a valuable reference for various stakeholders such as tourism planners, urban planners, and so on.
\end{abstract}

Keywords: social media; Twitter; tourism; volunteered geographic information; OpenStreetMap; nighttime light remote sensing

\section{Introduction}

Tourism is a key economic sector and plays a crucial role in expanding economic opportunities for a place. The United Nations reports that the tourism industry contributes up to $40 \%$ of the Gross Domestic Product (GDP) of developing countries [1]. World Travel and Tourism Council states that the tourism sector contributed to $10.4 \%$ of global GDP and created employment for 313 million people (i.e., 9.9\% of global employment) in 2017 [2]. Also, the United Nations Sustainable Development Goals have identified tourism as one of the important tools to attain sustainable economic growth [3]. With the increase in the use of modern information and communication technologies, the global promotion of remote destinations has become easier resulting in the growth of international tourism. To raise the tourism industry even further, cost efficient and up-to-date identification and dissemination of information on tourist attractions is necessary. This will help the industry stakeholders update their knowledge of popular as well as obscure attractions. Hence, informed service providers can better manage available resources and informed visitors can optimize their itineraries for better travel experience. 
Over recent years, various problems in tourism have stimulated researchers. The traditional approach uses conventional techniques such as interviews, focus groups and questionnaire surveys $[4,5]$. Even if the various knowledge, methodologies, and techniques are available these methods are often slow, costly, and limited spatiotemporally. Previous studies have highlighted potentially serious measurement errors while using traditional approaches, particularly in developing and emerging economies [6]. In recent days, the increasing availability and accessibility of new cutting-edge technology and data are of great use for day-to-day decision-making processes. As a boon from the current "Information Age", a massive amount of data is now available via digital technologies and novel data sources [7]. Recent studies have demonstrated that new data sources such as social media can provide more insights in addition to the results from traditional surveys [8]. Paid and free data from online social media sources and remote sensing cater near-real-time data. Such data have been increasingly used for a huge range of applications including human settlement mapping [9,10], land use and land cover mapping [11], understanding socio-economic conditions [12], tourism studies [13-15], etc. In recent years, many studies have proposed various ways to discover tourism destinations and activities. Geo-tagged data from online social media have been extensively used in studies locating popular touristic sites [16-20], map tourist behaviors [21,22], comparing domestic and foreign tourists $[18,21]$ and discovering obscure sightseeing locations [23]. This is possible because more and more data, i.e., digital footprint, is being generated by the unprecedented use of GPS-equipped smart devices by the users (both residents and tourists) and uploaded to the Internet as geo-tagged information. Researchers can access such geo-tagged contents from social media sites, analyze them, and discover interesting spatiotemporal patterns.

Data scarcity is a common problem in many studies. Often, it is advised to use more data than reasonably required while applying statistical methods. For machine learning problems, low quantities of data can negatively affect the performance of algorithms and their strength to generate useful outcomes. Many machine learning practitioners emphasize the need to get and use as much data as possible. For instance, Ester et al. [24], explained that data clusters and noise points can be identified by observing the high contrast in data density i.e., within cluster density is much higher than outside density. A larger amount of input data will generate denser clusters than fewer data. This implies that the use of large input data sets ensures better accuracy than smaller data sets. Much of the existing literature discusses exploring Area of Interests (AOIs) and places with abundant data availability, i.e., over tens of thousands of data points $[16,17,19,25]$. For a case in point, Yingjie et al., performed spatial clustering of geo-tagged social media data from cities such as New York [25]. A minimum point density threshold of $2 \%$ was used to distinguish clusters and noise. However, in remote areas with less social media penetration, this threshold may have to be lowered due to the data scarcity issue.

Essentially all the popular clustering methods such as Density-Based Spatial Clustering of Applications with Noise (DBSCAN) require input parameters (e.g., cluster density, inter-data distance) which influence the formation of clusters but estimating the optimal values for such parameters is a non-trivial task. Moreover, it may not be logical to use a single global value for such parameters to define the intrinsic cluster structures, as different local parameters may be required to identify clusters in different regions. For instance, while identifying social media clusters, a densely populated area with better infrastructure will possibly have relatively denser data points than other less populated areas. Hence, it is indispensable to tailor approaches to cope with such problems and estimate optimal (or multiple) parameter values as needed.

Most of the literature focuses on identifying tourism areas of interest, but it is indispensable to know if those areas cater minimum essential facilities to travelers such as food and shelter. Without such knowledge, chances may arise when visitors get stranded in uninhabited locations at odd hours. This study focuses on discovering tourism areas of interest (TAOI) while ensuring the accessibility to minimum essential facilities such as a bed and breakfast. This requirement is fulfilled by spatial modeling of different types of VGI (Volunteered Geographic Information), i.e., tweets and OpenStreetMap (OSM) data, and nighttime light (NTL) remote sensing data. First, we automate 
the process of estimating the optimal combinations of clustering parameters. Next, relevant tweet clusters related to tourism are discerned from other merely popular clusters by analyzing foreigner participation, building footprint, and NTL data. Finally, the usefulness of the building footprint data and NTL data in discovering TAOIs evaluated in urban and rural places of Nepal. Despite the studied data set being sparser, we still managed to achieve good results. Also, the proposed framework works in an unsupervised manner without relying on any knowledge of tourism spots from external data sources such as Lonely Planet (https:/ /www.lonelyplanet.com/) and TripAdvisor (https://www.tripadvisor.com).

\section{Related Works}

\subsection{Volunteered Geographic Information}

Volunteered Geographic Information is user-generated digital traces including both text and multimedia, about user's geographical information. VGI has emerged expeditiously with the advancement in information and communication technology. It contains rich spatiotemporal information generated by the human sensors and provides a way to explore and understand the socio-economic conditions of a place [26,27]. Though there have been concerns regarding information bias and lack of standard quality control, several studies have empirically revealed that VGI is of equally good quality as authoritative data $[8,28,29]$. Several researchers have used VGI resources including geo-tagged contents (e.g., tweets and photos) [16-18,30], check-in data [18,30], OSM [31] and so on. VGI has been used in a variety of applications such as tourism studies [16-18,30], urban studies [19,25,32,33], land use and land cover detection [11,34] and mobility analysis [35].

\subsection{Extracting Interesting Regions from VGI}

Visitors are very choosy in selecting the locations to visit as it is almost impossible for them to explore the whole area on an average 2-3-day visit to a place [36]. Hence, it is necessary for them to choose some attractions to visit and others to skip. This results in the formation of typical spatial patterns of tourism sites. Studies exploring such patterns reveal that visitors tend to be attracted to limited areas which have the main tourist attractions (e.g., museums, parks, historical buildings, etc.), leisure, shopping and lodging services [37]. The conventional method to elicit such information was through surveys. However, surveys are inherently limited in capturing visitor spatiotemporal behavior. Online social media data makes available a huge amount of spatial and temporal data which not only compliments but also overcomes the limits of traditional data sources. Hence, visitors' spatial footprints captured by various social media sites can be investigated to study tourism patterns.

Spatial clustering has proved to be an indispensable technique for data aggregation. Current literature has a rich set of methods ranging from the classic K-means [38] clustering algorithm to the popular DBSCAN algorithm [24]. K-means is a popular clustering algorithm which have many variants such as K-medoids [39]. K-means and K-medoids are used to solve spatial clustering problems such as Multivariate Clustering (implemented in ARCGIS (https:/ / pro.arcgis.com/en/pro-app/toolreference/spatial-statistics/how-multivariate-clustering-works.htm)). K-means clustering algorithm requires the number of clusters as input, which is an important factor in cluster quality. However, the number of clusters cannot be determined a priori in many scenarios. DBSCAN has emerged to be a better alternative as it is based on data density and not the number of clusters. It can move out sparsely distributed data and group data in arbitrary shape and arbitrary size. Furthermore, it can identify outliers as noise, unlike K-means which just dumps them into some cluster even if the data shows contrasting characteristics. The DBSCAN algorithm requires two parameters: a minimum number of points (minPts) in a cluster and the search radius (eps) that a data point can influence. Many algorithms improve and extend the DBSCAN algorithm. A new method called ST-DBSCAN was proposed for discovering clusters from spatiotemporal data [40]. It is based on three attributes of data i.e., spatial, non-spatial, and temporal attributes. The original DBSCAN algorithm uses only one 
distance parameter to cluster similar data, but ST-DBSCAN requires one additional distance parameter. One distance parameter determines the closeness of the points in spatial scale while the other indicates the similarity of non-spatial attributes. Also, a concept of density factor is used to deal with the fixed density problem in the original DBSCAN algorithm. P-DBSCAN is an extension of DBSCAN algorithm using geo-tagged photos for detecting attractive areas based on the photo owner's density in the region [41]. This method is adaptive and flexible but focuses only on finding interesting points of interest (POIs). HDBSCAN extends DBSCAN by modifying it into a hierarchical clustering algorithm and then devising a way to obtain flat clusters [42]. It is more data-driven and hence automates the distance parameter. It requires only one parameter i.e., (minPts) and works well with clusters of different densities but compromises performance. Moreover, spatial point pattern methods, such as the Local Moran [43] (used for Cluster and Outlier Analysis in ArcGIS (https:/ / pro.arcgis.com/en/proapp/tool-reference/spatial-statistics/cluster-and-outlier-analysis-anselin-local-moran-s.htm)) and Getis-Ord Gi [44] (used for Hot Spot Analysis in ArcGIS (https:/ / pro.arcgis.com/en/pro-app/toolreference/spatial-statistics/hot-spot-analysis.htm)) has also been used for detecting spatial clusters. However, generic clustering algorithms such as DBSCAN perform better in delineating aggregated data and shaping generated clusters [45]. Also, Dehuri et al. showed that if the clusters are of arbitrary shape, DBSCAN algorithm performs better than self-organizing map [46].

With the global availability of geo-information from different VGI sources, numerous studies have been done to discover and understand hotspots and areas of interest. Yang et al., recommended a self-tuning clustering method which can automatically determine the number of clusters without any subjective parameters such as the number of POIs, the shape of POIs and size of POIs [47]. Important tourist locations were assumed to be highly photographed. Hence, such interesting POIs were identified from the collection of geo-tagged photos by applying spectral clustering by self-tuning the necessary parameters. Laptev et al. suggested a parameter-free method for recommending AOIs by analyzing the density of geo-tagged photos in the selected region [48]. The algorithm required a single input parameter i.e., available time for travel. The quality of the result (i.e., recommended AOIs) was judged based on the number of POIs that can be observed within a given time. Hu. et al., proposed a coherent framework for discovering urban AOI based on geo-tagged Flickr photos [25]. Three well developed and three fast developing cities were examined to identify AOI using DBSCAN clustering algorithm and describe them using the textual tags and suitable photos. Korakakis et al. applied HDBSCAN for extracting AOIs [49]. They aimed to solve the "Tourist Trip Design Problem" and automatically extract popular POIs and AOIs to construct travel routes by using social knowledge embedded in the Flickr photos and its metadata. The extracted route aims to maximize user travel experience based on the user input such as start point, endpoint, and available time budget. Chen et al. extracted and visualized the dynamics of urban area of interest using the metadata from geo-tagged Flickr images in London [19]. A variant of DBSCAN clustering, i.e., HDBSCAN clustering, was applied to cluster the spatial data and explore their spatial and temporal insights. Sun et al., proposed a way to identify city centers using traveler flows obtained from check-in data [32]. Three algorithms (DBSCAN, Local Getis-Ord and Grivan-Newman) were implemented to get the best estimation of city centers. DBSCAN demonstrated better performance in delineating geometrically regular boundaries. Koutras et al. studied the top spots in an urban area and the tourists' attitude towards visiting those spots [33]. Density-based spatial clustering was applied on geo-tagged Flickr photos inside Athens. Lee et al. mapped the visitors' trajectory and hotspots in the Grand Canyon National Park using Kernel Density Estimation and Dynamic Time Warping to the geo-tagged flicker images [22]. Hasnat et al. discovered the spatial patterns of tourist destination choices by applying K-means, Mean-Shift and DBSCAN algorithm on the Twitter data collected inside Florida [50]. Encalada et al. analyzed the spatial distribution of the geo-tagged photos in Lisbon City and extracted places of tourist attractions and important tourism variables such as monuments [17]. They applied Local Moran clustering and multiple linear regression for the purpose. Kuo et al. proposed a new method, i.e., Spatial Overlap algorithm, which considers the spatiotemporal properties and other metadata of the Flickr photos to 
extract and understand POIs/ROIs by efficiently eliminating noises [51]. Naming and merging of the clusters based on the photo attributes were done for obtaining better results. Moreover, this proposed algorithm applied local maximum to extract attractive footprints to deal with the problem in dense areas. Maeda et al. extracted the location of tourist destinations in Japan using geo-tagged tweets via attractiveness and originality of each place of interest [18]. Attractiveness was computed using DBSCAN clustering and gravity modeling. Originality was estimated by applying text analysis. Also, preference comparison of the foreigner and domestic tourists was done by relating FourSquare places characteristics.

Our research differs from previous works in several aspects. Many studies have used social media data from sources such as Flickr, Panoramio, FourSquare, Gowalla, Sina Weibo, etc. in different domains. However, to date most research has concentrated on attempting to derive the important AOIs and attractions of given regions, assuming the availability of social media data in abundance. Few of the works have used geo-tagged tweets and mainly focus on exploring urban AOIs and places. Limited work has been done to explore the area of interest in remote places and places with relatively less social media reach. Also, we came across many studies that use some sort of spatial clustering algorithm that requires some hyperparameters such as search distance. Though some of them have proposed ways to estimate or automate such parameters, they rely on some specific assumptions which are not suitable for our case (to be discussed in upcoming Section 3.2.1) [40]. To our knowledge, none of them suggest an easy way to automate the estimation of such parameter values which can work across different scarce data regions. However, we proposed a way to estimate such parameters for identifying TAOIs and prime locations. Furthermore, the recommended approach not just discovers TAOIs but also examines the accessibility to minimum essential facilities. We examined the proposed approach in sparse data regions in Nepal and further refined the results by using the freely available data from OSM and NTL. Hence, the proposed method is novel, and it encourages the discovery of TAOIs by fusing freely available data from VGI and remote sensing.

\section{Methodology}

In this section, we propose a framework devised to identify and map TAOIs from the subset of geo-tagged tweets. Our methodology consists of three main parts: data acquisition, cluster detection and TAOI identification as shown in Figure 1. Nighttime light satellite images, geo-tagged tweets, OSM data, and other ancillary data sets such as shapefiles are collected and used to identify TAOIs.

\subsection{Tourism Area of Interest (TAOI)}

A TAOI is defined as a center of attraction for many people and contains interesting tourist attractions such as restaurants, hotels, landmarks, leisure zones, and so on. Many people visit such areas and share their ideas and experiences in social media. Hence, many social media contents (e.g., geo-tagged tweets) are generated in such areas. Some TAOIs cater the maximum tourism facilities and become prime attractions while others may not act as a prime attraction but still provide basic essential facilities to the visitors within accessible locations. It is important to distinguish between such prime and non-prime regions within the span of a TAOI because non-prime areas are generally less crowded and provide economical tourism facilities.

Clustering of geo-tagged tweets is employed to extract dense regions of tweet activity as candidates for TAOIs. Also, it is essential that a minimum number of tweets are published by foreigners. Upon selecting such clusters, the final set of TAOIs is identified by ensuring the presence of basic infrastructure and services in the vicinity by examining freely accessible geo-information from OSM and NTL. 


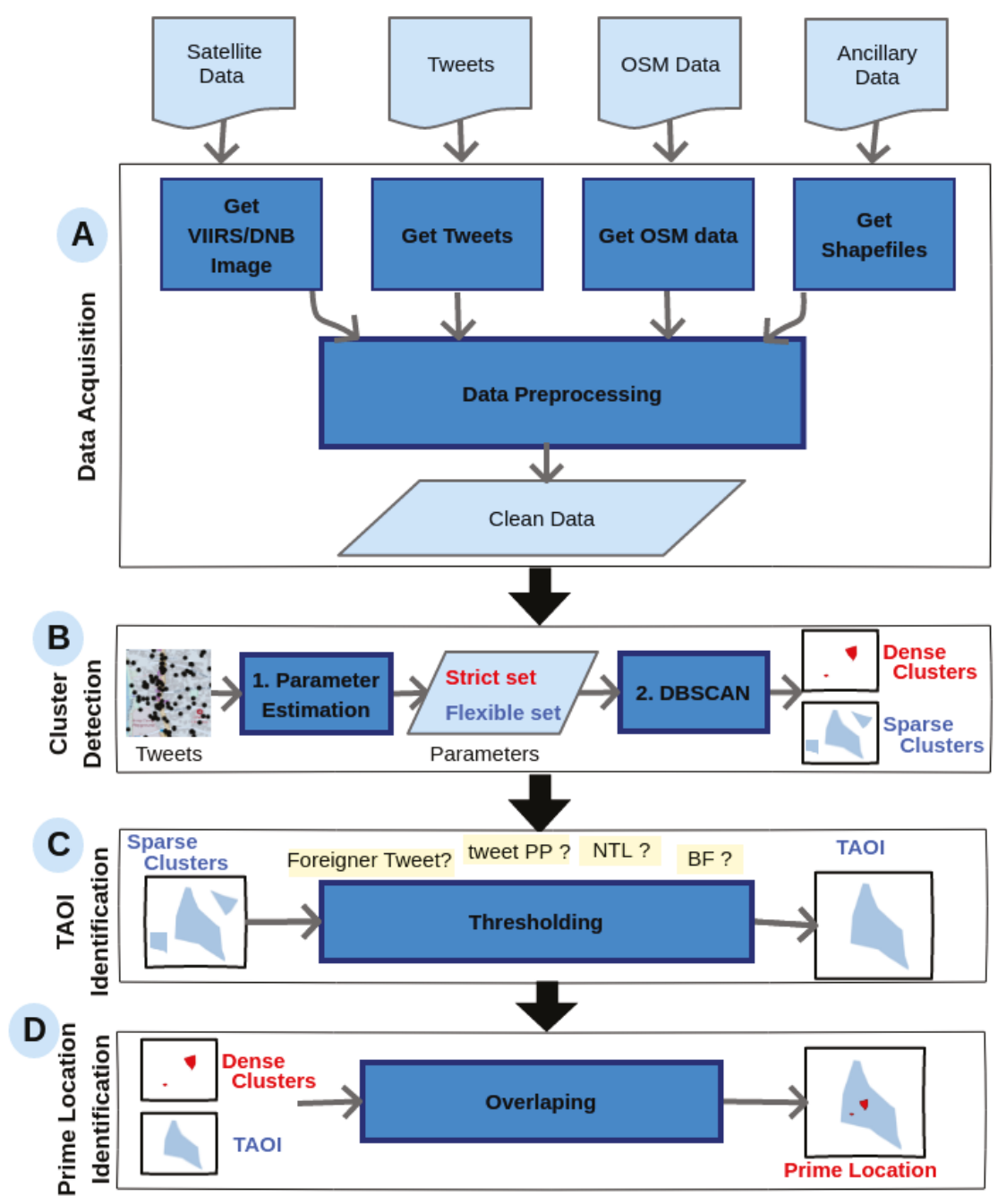

Figure 1. Overall Methodology.

\subsection{TAOI Identification Algorithm}

Geo-tagged tweets are aggregated using the clustering algorithm to identify and delineate the extent of active regions in social media. Clustering is a data mining technique of exploratory data analysis for discovering interesting patterns in data. It has been successfully used for finding dense regions such as the spatial hotspots. A plethora of clustering algorithms exist in the literature; however, we assume the following requirements for our choice:

- The algorithm should work in an unsupervised way as the exact number of clusters are not known beforehand.

- Clusters can have an arbitrary number, shape, or size (point percent) depending on the context (e.g., study area, data source, etc.)

- Method should detect high-intensity gathering of tweets all over the study area.

- The algorithm must aggregate significant data while discarding any outliers and noise. 
DBSCAN algorithm adheres to the above specifications. Also, the comparative summary of different clustering algorithms in Table 1 indicates DBSCAN algorithm as a suitable candidate. Hence, DBSCAN algorithm is selected for generating prominent tweet agglomerations. After the determination of active tweet clusters, TAOIs are selected by examining building footprints, nighttime light intensity as well as non-local user presence within the extent of the clusters.

Lately, Yan et al. proposed a model based on the probabilistic model to explore the geographic distribution of visitors by examining the underlying mechanisms behind it [52]. They suggested to couple maximum entropy modeling with geo-tagged photos to determine the location of tourists in relation to different environmental factors. They quantified the correlations between tourism presence and various environmental factors which seem very useful to envision the impact of abnormal scenarios such as a disaster and other crisis. Differing from our study, this approach modeled tourist distributions in a probabilistic way and determined touristic regions based on the probabilities. Our proposed approach outputs TAOIs in a binary form; however, it does not declare the presence or absence of tourist but tries to identify interesting places of interest (i.e., areas with tourism facilities such as hotels, viewpoints, landmarks, etc.) based on the digital footprint of the travelers. The underlying method accepts the fact that such tourism traces may be present at other places also (even if the proposed method do not highlight them as interesting TAOI), for example, the DBSCAN clustering algorithm identifies them as outliers i.e., isolated points which are not qualified as an interesting cluster. Though expressing AOIs with probabilities seem more informative, it may complicate the understanding. Moreover, Yan et al. accomplished their study in data rich region, i.e., San Diego city, by collecting over hundreds of thousands of data points because probabilistic models commonly require very large dataset to detect statistically significant relationships. Hence, the proposed method highlights the interesting tourist hotspots in scarce data regions in an easy and unambiguous way.

Table 1. Comparison of DBSCAN with other clustering methods.

\begin{tabular}{|c|c|}
\hline Algorithm & Comments \\
\hline DBSCAN [24] & $\begin{array}{l}\text { - Works in an unsupervised way as the exact number of clusters are not } \\
\text { known beforehand. } \\
\text { - Clusters can have an arbitrary number, shape, or size. } \\
\text { - Detects high-intensity gathering of points all over the study area. } \\
\text { - Aggregates significant data while discarding any outliers and noise. } \\
\text { - Require minimum domain knowledge to determine the input parameters. }\end{array}$ \\
\hline $\begin{array}{l}\text { Classic clustering } \\
\text { method such as K-means [38] } \\
\text { and K-medoids [39] }\end{array}$ & $\begin{array}{l}\text { - Require pre-knowledge of the number of clusters to be generated. } \\
\text { - Cannot identify outliers as noise. } \\
\text { - Final result is sensitive to initial starting values. } \\
\text { - Assumes the true underlying clusters are globular. }\end{array}$ \\
\hline $\begin{array}{l}\text { Spatial Point Processing } \\
\text { methods such as Local Moran[43] } \\
\text { and Getic-ord Gi[44] }\end{array}$ & $\begin{array}{l}\text { - Cannot outperform generic clustering algorithms ( e.g., DBSCAN) } \\
\text { in delineating aggregated data and shaping generated clusters. }\end{array}$ \\
\hline Self-Organizing Maps [46] & $\begin{array}{l}\text { - If the clusters are of arbitrary shape, DBSCAN algorithm performs better } \\
\text { than the self-organizing map. }\end{array}$ \\
\hline Mean-Shift Algorithm [53] & - Cannot identify outliers as noise. \\
\hline Kernel Density Estimation [54] & $\begin{array}{l}\text { - Does not generate a clear hard-lined definitions between points in different } \\
\text { clusters. }\end{array}$ \\
\hline Affinity Propagation [55] & - Assumes the true underlying clusters are globular. \\
\hline Spectral clustering[56] & - Require pre-knowledge of the number of clusters to be generated. \\
\hline
\end{tabular}

\subsubsection{Clustering Terminology and Mechanism}

As discussed in the preceding section, DBSCAN algorithm is chosen for generating tweet clusters. It is an extensively used density-based clustering algorithm. Figure 2 shows a basic representation of a 
DBSCAN cluster where each point represents a geo-tagged tweet. The fundamental terminologies are described as follows:

(i) The set of tweet points to be clustered is $D, D=\{p: p$ (latitude, longitude) $\}$ where $p$ denotes the location of any tweet.

(ii) The tweet density of a point $p$ is determined by the number of neighboring tweets within distance eps from the point $p$.

(iii) The eps-Neighborhood of $p$ is represented by $N(p)=\{q: q \in D$, dist $(p, q)<e p s\}$, where dist gives the distance between the two points $p$ and $q$.

(iv) A point $p$ is a core point if it has a minimum of minPts points within its neighborhood such that: $|N(p)| \geq$ minPts.

(v) A point $q$ is a border point if it has fewer than minPts points within its neighborhood but lies within the neighborhood of core point $p$.

(vi) Any tweet point $r$ which is neither a core nor a border point is considered to be a noise point.

(vii) A point $p$ is directly density reachable from another points $q$ if $p$ is within eps-Neighborhood of $q$ and $q$ is a core point such that:

(a) $p \in N(q)$.

(b) $|N(q)| \geq \operatorname{minPts.}$

(viii) A point $p$ is density reachable from another points $q$ if there is a series of core points leading $p$ to $q$ such that: $p_{1}=p, p_{2}, \ldots, p_{n}=q$, where $p_{i}$ denote core points.

(ix) A cluster $C$ is a non-empty subset of $D$ where each point is density reachable such that:

(a) $\forall p, q$ if $q \in C$ and $p$ is density reachable from $q$ then $p \in C$.

(b) $\forall p, q \in C, \exists r \in C$ so that both $p$ and $q$ are density reachable from $r$.

DBSCAN is simple and efficient algorithm for clustering large-scale data. The basic mechanism for identifying clusters follows the steps listed below:

(i) Start with an arbitrary point

(a) Determine the neighborhood points adhering to eps and minPts requirements.

(1) Recursively apply step (a) for all new neighboring points.

(b) Density reachable and density connected points are used to create a new cluster. Any other points are marked as noise. If the noise point satisfies eps and minPts for a different point in later iterations then it can still be a cluster point.

(c) All points within the cluster are marked visited.

(ii) Repeat step (i) with new unvisited points until all the points are marked visited or noise. 


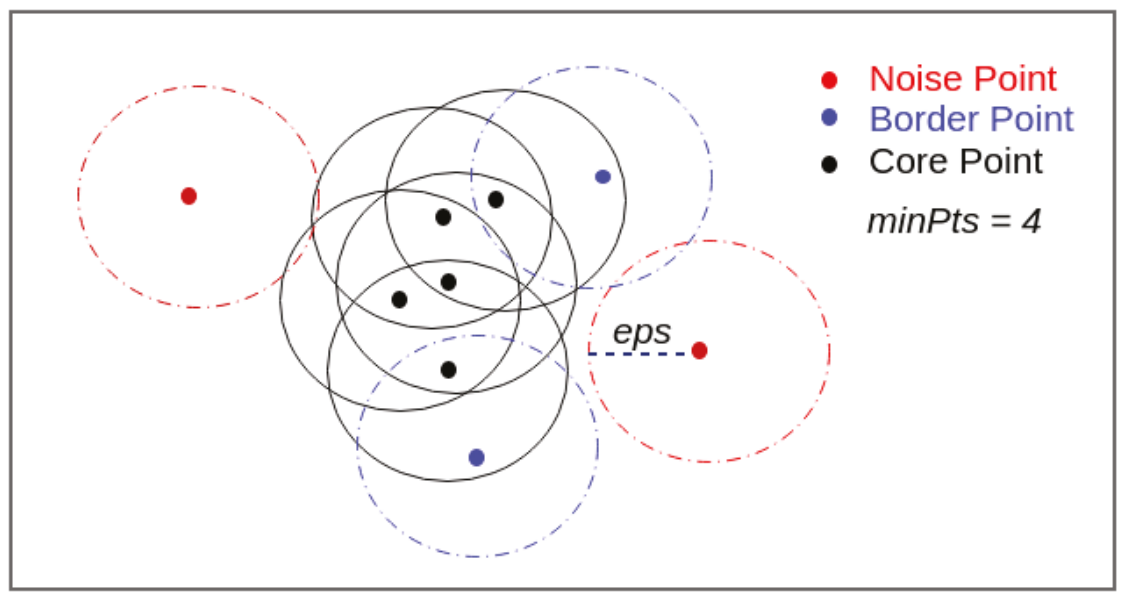

Figure 2. A single DBSCAN cluster with Core, Border, and Noise Points.

\subsubsection{Tuning of Clustering Parameter}

DBSCAN clustering outcomes are determined by two parameters: eps (the search distance or radius) and $\min P t s$ (the minimum data count within the search radius). These parameters define a minimum density threshold, such that clusters are discovered at locations where the data density exceeds the minimum threshold. To use DBSCAN proper values of eps and minPts must be chosen. The eps parameter is associated with the geographic scale of the study area. A larger value for eps results in broader clusters, while a smaller value establishes narrower clusters. The minPts parameter specifies the minimum number of points (i.e., cluster members) required to produce a new cluster. A larger value of minPts assures a more robust cluster but may exclude some potentially smaller areas as it attempts to merge them in a larger one. On the other hand, a smaller value extracts many clusters, but the resultant clusters may include noise as well.

Generally, a minimum minPts can be obtained from the number of dimensions in the data set, as minPts $\geq$ dimensions +1 . The extremely low values of minPts $\leq 2$ do not provide a significant gain of using DBSCAN clustering and hence minPts must be set to be at least 3. Larger values are considered more robust to noise and yield more significant clusters. In general, minPts $=2 *$ dimensions is suggested while larger values are encouraged for larger data sets containing noise and duplicates [57]. A single absolute value for minPts may not be appropriate (e.g., comparing different places with varying numbers of tweets) therefore a percentage of the tweets is taken as a general approach [25]. The distance parameter eps for DBSCAN is comparatively more difficult to set. DBSCAN algorithm inherently encourages the smallest possible value for eps. It is important to consider the application domain knowledge and the target geography while setting this parameter. Also, researchers advocated that if eps is based on domain knowledge then minPts may vary, to get different insights into the data. Many studies in the literature follow a trial-and-error approach with various values and compare the clustering results with the background knowledge of the study area in order to select some absolute values as the parameters (e.g., eps $=100$ and minPts $=10$ ) $[19,25,49]$. Others such as Briant et al. use some heuristics (i.e., $\operatorname{minPts}=\ln (n))$ to determine such clustering parameters [40]. Such tricks may not be suitable because this study requires the formation of maximum numbers of clusters as possible. Apart from being sparse data region, the number of tweets and geographic characteristics of the selected study areas differ significantly. Hence, it is necessary to test the different possible combinations of parameters to estimate reasonable values. To address this problem of parameter estimation, we proposed two objective functions: 
(i) StrictParameters (i.e., lowest eps and highest minPts): The strict version of parameters identifies groups with the smallest possible extent and utmost tweet density (i.e., Dense Clusters). The Dense Clusters are supposed to have the highest user attention and are the potential regions for prime tourism locations.

(ii) MaxParameters (i.e., parameter combination yielding maximum clusters): A maximum number of clusters may be obtained with relaxed DBSCAN parameters and such accumulations (i.e., Sparse Clusters) are the candidate TAOI locations.

\subsubsection{Cluster Detection}

The input data points along with the parameters estimated by MaxParameters and StrictParameters are provided to DBSCAN clustering algorithm which examines the input and generates relevant clusters. The best part of DBSCAN clustering is that it can identify and avoid irrelevant noise points from appearing in the output clusters. Once DBSCAN clustering identifies the Dense and Sparse clusters, the next step is to build a perceptual polygon to enclose the extracted cluster points. This helps in distinguishing the area of interest along with the sets of important points. Various researchers have used convex hulls for the construction of boundaries that envelop all geo-tagged data within a cluster [58]. However, convex hulls may contain unnecessary empty areas unoccupied by the selected cluster points [59]. Hence, to delineate the cluster shape more accurately, we adopted a chi-shape concave hull algorithm by Duckham et al. [60] as described in previous study [25].

\subsubsection{Identifying TAOIs by Cluster Pruning}

While active cluster regions have been extracted based on tweet density, the TAOIs of each region remains vague. This section aims to explore the extracted clusters and characterize them as TAOIs. Related studies have used geo-tagged social media data for mining tourist locations without separating the data from local and non-local users [20,49]. Also, previous studies in the proposed area (i.e., Nepal) illustrated that approximately one third portion of the tweets was contributed by non-local users [61]. Hence, to avail more input for the underlying clustering algorithm, the proposed approach makes use of the tweets posted by both local and non-local users in the beginning. Later, a strict requirement is imposed so that foreign user tweet must be present in the selected TAOIs. Twitter user profile information is examined to confirm if the tweets are published by local or foreign users. False user profile information can degrade the location inference; however, several past studies have used such data as a source for ground truth in their studies $[62,63]$. Jurgens et al., experimentally illustrated that there are not so many false location information [64]. Furthermore, recent studies have indicated that NTL data is highly correlated with urban infrastructures and socio-economic activities such as tourism $[65,66]$. Therefore, regions with either enough BF (or enough NTL intensity) are assumed to ensure minimum infrastructure necessary for tourism activities. Also, it has been observed that higher tweet percentage is associated with active tourism areas. Hence, the following criteria are defined to distinguish TAOIs from ordinary tweet clusters:

1. MaxParameters must be used to generate Sparse Clusters from geo-tagged tweets.

2. Sparse Clusters must satisfy sufficient amount of foreigner tweets (FT) and tweet point percentage (PP).

3. Lastly, BF and NTL thresholds must be fulfilled. Based on the contribution of BF and NTL, three different methods are defined to identify TAOIs as follows:

(a) tClust_B : Clusters must adhere to minimum BF threshold.

(b) tClust_N : Clusters must maintain minimum NTL threshold.

(c) tClust_NB:Clusters must adhere to minimum NTL threshold as well as BF threshold.

Any clusters which does not meet these requirements are pruned out from the list of TAOIs. Once TAOIs are discovered, prime tourism locations are identified as follows: 
1. StrictParameters must be used to generate Dense Clusters from geo-tagged tweets.

2. Dense Clusters must satisfy sufficient amounts of NTL, foreigner tweets, tweet point percentage, and building footprint requirements. Any cluster which does not meet these requirements are pruned away.

3. The overlap of the selected Dense Clusters with TAOIs, if present, identifies the location of the prime tourism spots.

\section{Experiment Setup}

This section describes the experimental setup of the research which includes the details about the study area, data sources and implementation tools used.

\subsection{Study Area}

The spatial area of interest for this study is Nepal. Nepal is the land of ten UNESCO World Heritage sites (https:/ / whc.unesco.org/en/statesparties/np). Seven are in Kathmandu Valley and one each in Lumbini (Birthplace of Lord Buddha), Chitwan National Park, and Sagarmatha National Park. Tourism is regarded as one of the most promising sectors for sustainable development, yet its total contribution to the economy is very low. The total contribution of tourism to the economy in 2017 was $7.8 \%$ and total employment including jobs indirectly supported by the industry was $6.6 \%$ [2] of the workforce.

This research is carried out by using geo-tagged tweets over two well-known tourism destinations in Nepal, i.e., Kaski district and Solukhumbu district. Nepal Government has categorized administrative sectors into urban municipalities and rural municipalities based on criteria such as infrastructure, population, and revenue. Kaski contains one urban municipality (i.e., Pokhara city) and four rural municipalities and Solukhumbu district consist of a total of eight rural municipalities (http://mofaga.gov.np/). Both Kaski and Solukhumbu district are popular tourist destinations in Nepal. Table 2 summarizes the population, area, location, and tourism importance and Figure 3 presents their location on the map of Nepal. Pokhara city of Kaski district is a popular destination for national and international tourists. Pokhara is the second largest city in the country and the capital of Province 4. Solukhumbu District of Province 1 lies in the eastern part of Nepal and is very rural compared to Kaski. Mount Everest, the highest peak on earth, lies in the northern part of this district, within the region of Sagarmatha National Park. Everest region is globally popular trekking destination.

Table 2. Geo-demographic summary of the selected regions $[67,68]$.

\begin{tabular}{lll}
\hline & \multicolumn{1}{c}{ Kaski } & \multicolumn{1}{c}{ Solukhumbu } \\
\hline Area $($ sq.km.) & 2017 & 3312 \\
\hline Population & 492,098 & 105,886 \\
\hline Bounding Box & $(83.70,28.08)$, & $(86.36,27.34)$, \\
(degrees) & $(84.28,28.61)$ & $(87.01,28.11)$ \\
\hline & - Pokhara, the tourism capital of Nepal. & - Mount Everest. \\
& - Pokhara ranked 7 in “Top Experiences & - World heritage site: Sagarmatha National \\
Features & in Nepal” by Lonely Planet [69]. & Park. \\
& - A part of Mount Annapurna and & - 'Everest Base Camp Trek' ranked 2 in “Top \\
& range. & Experiences in Nepal” by Lonely Planet [69].
\end{tabular}




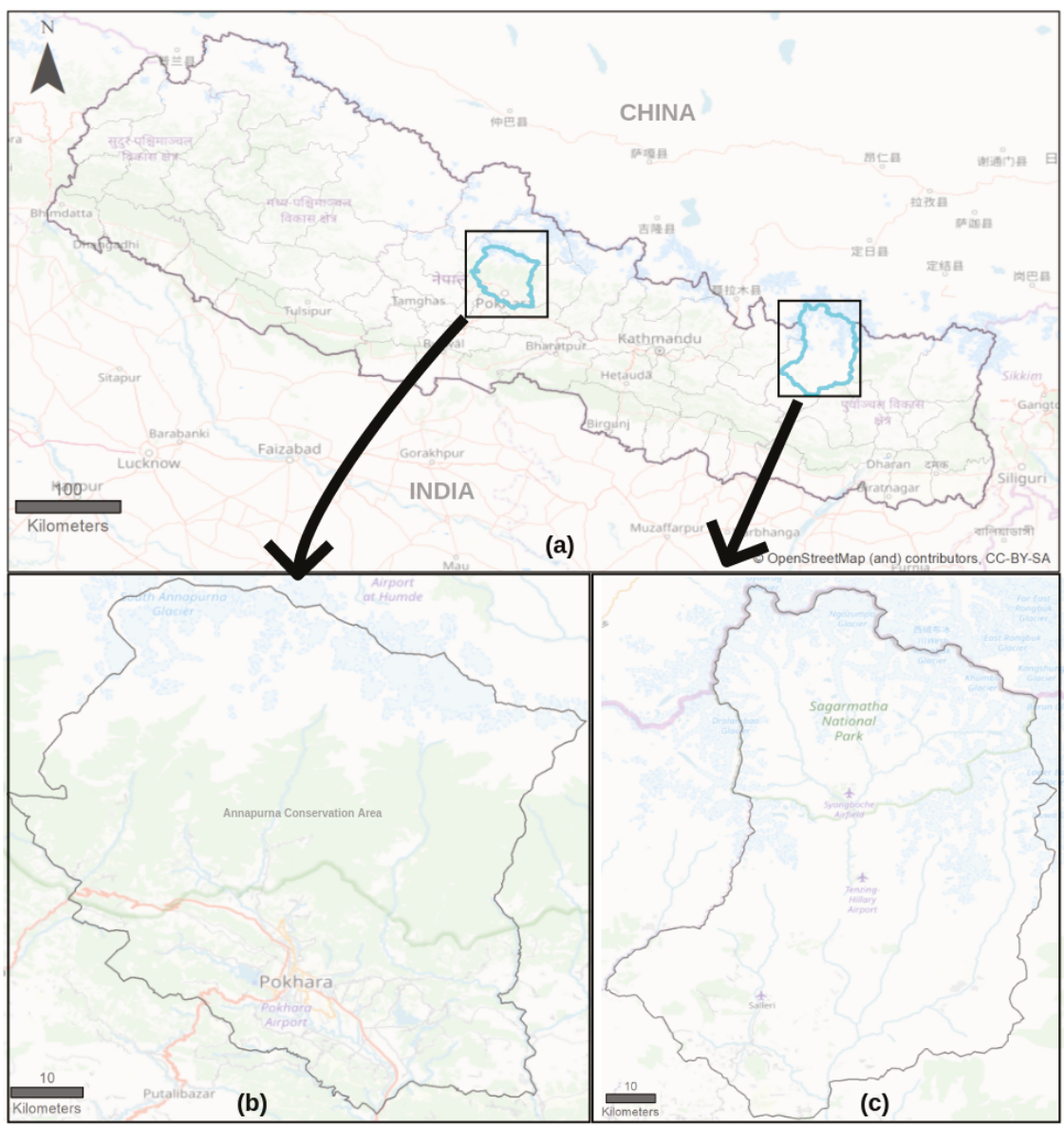

Figure 3. Map highlighting selected study areas in Nepal: (a) Nepal map, (b) Kaski district map and (c) Solukhumbu district map).

\subsection{Data Acquisition}

Freely available geospatial data from various sources such as Twitter, OpenStreetMap, and earth observation satellites were collected and made available for further processing. A comprehensive description for each of them is provided in this section.

\subsubsection{Twitter}

We chose Twitter for this study as it has a simple and well-defined public interface for extracting data. Twitter is one of the most popular social networking sites as well as a micro-blogging site. Twitter provides a platform for online users to share a text message up to 280 characters which is popularly known as a tweet. An average of 500 million tweets containing rich data such as texts, images, links, and videos are posted every day on Twitter [70]. Most of its content is public in nature, unlike other popular social media sites such as Facebook. Cesare et al., [71] investigated 60 pieces of existing research literature for social media sites to review user demographic traits. More than half, i.e., thirty-nine (i.e., 65\%) of the studies, focused on Twitter, two on Facebook, and so on. The popularity of Twitter in the research and academic community is attributed to various characteristics such as message size, metadata, availability and accessibility [72]. Furthermore, Puschmann et al., 
pointed out various reasons for considering Twitter as a reasonable source for research data [73]. It is considered a global phenomenon with a growing number of users and posts every day. Twitter is deeply rooted in our media ecology and it is used by the public as well as politicians, journalists, and marketers. An investigation on the social media usage in Nepal reported that Facebook, Twitter, and YouTube were the most popular social media sites during the first quarter of 2017 [74]. Twitter is used by government offices, celebrities, authorities, politicians, and the general public for sharing and accessing information. Nepal Police (https:/ / twitter.com/NepalPoliceHQ) started its Twitter activities on April 2015, i.e., during Nepal Earthquake 2015.

Using the free Streaming Twitter API, we collected the geo-tagged tweets within Nepal and stored only the tweets whose location data is recorded via GPS on mobile devices as they provide the best location accuracy [75]. Sometimes, the sample data made available via the free streaming service has been questioned for its representativeness. Morstatter et al., conducted various examinations and conveyed that the Streaming API provides the approximate set of the geo-tagged tweets even if the amount of geo-tagged tweets is relatively very small (i.e., around 1\%) [76,77].

In this study, the original Twitter data set contains 89,228 geo-tagged tweets within Nepal covering the study area for 685 days between March 2017 and June 2019. Data collection was interrupted for some period in the middle due to problems in the data collection server. Not all the collected tweets are usable since some of them may have been generated by spammers. Frias-Martinez et al. assumed that some mobile advertising agents may publish a huge number of tweets on a daily basis and proposed a way to filter such tweets so that any GPS location which publishes more than 20 tweets per user in one day should be removed [78]. Furthermore, Zhao et al. defined spam tweets as any geolocated tweets that are published in more than one far-away $(>500 \mathrm{~km})$ locations within a very short time interval $(<1 \mathrm{~h})$ by the same user. Such tweets are supposed to be generated either by the hackers or due to errors in the Twitter system or GPS devices [79]. Hence, from the total collection of tweets, such tweets were filtered away. Table 3 provides a summary of the total tweets collected in the whole of Nepal and the selected study areas during the study period. Figure 4 shows the plot of geo-tagged tweets over the map of Nepal indicating its distribution in different parts of the country.

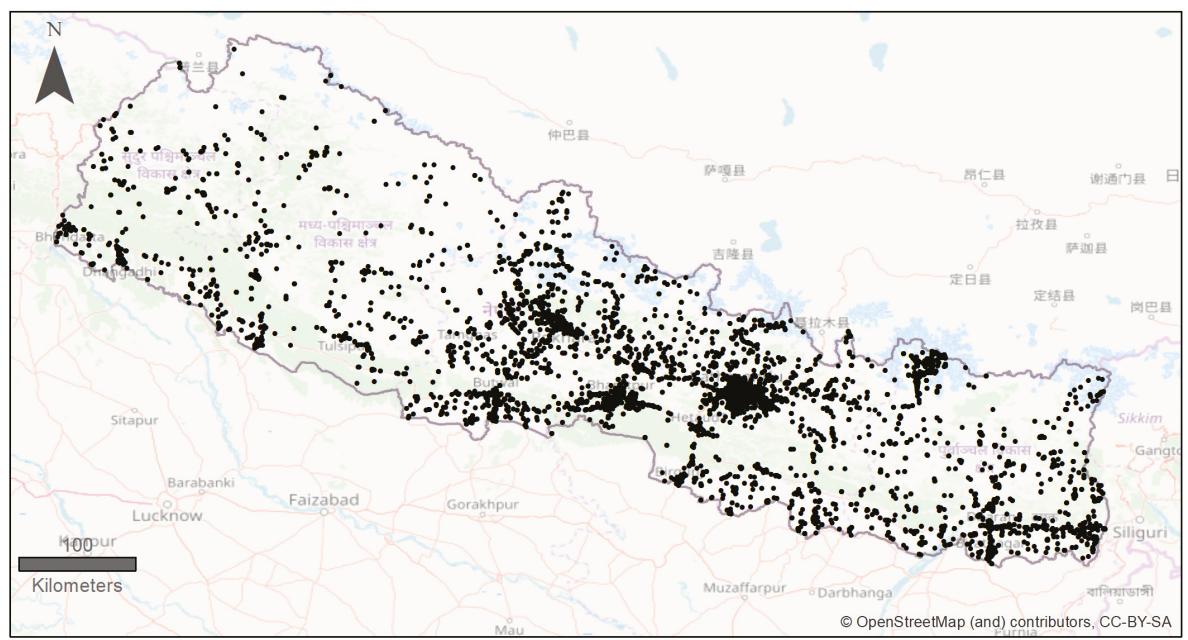

Figure 4. Map of Nepal showing the locations of geo-tagged tweets. 
Table 3. Summary statistics of collected tweets.

\begin{tabular}{cccc}
\hline Place & Total Tweets & Total Users & $\begin{array}{c}\text { Average Tweets } \\
\text { Per User }\end{array}$ \\
\hline Kaski & 8787 & 2150 & 4 \\
Solukhumbu & 5472 & 718 & 7 \\
Nepal & 89,228 & 14,216 & 6 \\
\hline
\end{tabular}

\subsubsection{Building Footprint}

OpenStreetMap project provides an easily accessible platform that enables free access to geo-information across the world. It is a crowdsourcing platform where volunteers from around the world can contribute in the generation of the geographic data. Though OSM has no strict quality control mechanism, studies have indicated that data obtained from OSM are good enough and comparable to authoritative data to some extent [28]. OpenStreetMap encodes data in different formats such as points, polylines, and polygons. Points usually indicate the point of interests such as shops, post office, and bus stops. Polylines store information such as roadways, waterways, and railways. Polygons represent features such as buildings, parks, or forests. At present, several research communities use this free global dataset for investigating and solving problems in different domains such as spatial computing, urban planning, and geographic information system, ecology, etc. This free dataset has opened boundless possibilities in commercial as well as academic research.

The proposed study uses OSM building footprint data. Building footprint data indicates an area on a site that is covered by the building structure. The perimeter of the building plan defines the extent of the built-up structure. The building footprint differs from the general built-up areas because it excludes any other regions used by other natural and man-made structures such as landscapes, parking areas, and other non-building facilities. The OSM data is freely downloadable from geofabrik website (http:/ / download.geofabrik.de/asia/nepal.html). The count of building footprint data for Solukhumbu district and Kaski district were 35,348 and 137,945 respectively as of 23 June 2019.

\subsubsection{Nighttime Light}

Nighttime Light remote sensing is a technique using satellite sensors to acquire city lights, fishing vessel lights, gas flares, and burning biomass. It is important for studying social issues such as poverty, environment, and ecology because NTL reflects real human activities. NTL remote sensing is used as an important supplementary dataset to the census in decision-making processes. It is observed that the increase in NTL intensity and extent tends to be correlated with economic growth. NTL can be used to investigate different types of development issues related to human activities, including ecological pressures, the degree of country-level economic activities, the rate of city-level urbanization, and light pollutions [80].

One of the main drivers for the growth in NTL research is the development of satellite sensors which can capture imagery data during the nighttime. The Visible Infrared Imaging Radiometer Suite (VIIRS) Day /Night Band (DNB) sensor onboard the Suomi National Polar-orbiting Partnership (NPP) satellite platform became operational in 2012. Using advanced processing schemes (e.g., excluding/correcting data impacted by stray light), NOAA-NGDC https://ngdc.noaa.gov/eog/ viirs/download_viirs_ntl.html is producing global composite products featuring average radiance values at 15 arc-sec (about $450 \mathrm{~m}$ ) spatial resolution [81]. The radiance values are measured in floating point and its unit is nanoWatts $/\left(\mathrm{cm}^{2} . \mathrm{sr}\right)$. We use the annual composite which excludes any data impacted by stray light, ephemeral lights and background (non-lights). It has been extensively used in understanding the various anthropogenic phenomenon. Also, correlating the NTL brightness with other variables such as buildings and settlement areas, bridges and communication routes, and demographic and socio-economic activities have generated good results which have proved useful for decision making $[65,66,82,83]$. 
The annual composite average radiance images are available for the years 2015 and 2016 only (as of June 2019). The radiance images are dis-aggregated to obtain the average radiance values for each district of Nepal in 2015 and 2016, respectively. The Pearson correlation coefficient measure of 0.995 is obtained for 2015 and 2016 (Table 4). This hints that the annual composite nighttime light images have a strong association. Figure 5 shows the map of the annual composite average radiance image in Nepal during 2016. It can be observed that the overall nighttime light intensity in Nepal is very low. Close observation in Kaski district reveals very faint lighting around Pokhara city. Solukhumbu district seems to be the least illuminated region. Recent studies have suggested that nighttime light data can act as a good indicator for an urban area at national or regional scale [83]. Therefore, the Kaski district is considered more urbanized than the Solukhumbu district.

Table 4. Pearson correlation of nighttime light intensity of different years; NTL 2015: Nighttime lightintensity for year 2015; NTL 2016: Nighttime light intensity for year 2016.

\begin{tabular}{ccc}
\hline & NTL 2015 & NTL 2016 \\
\hline NTL 2015 & 1 & 0.995 \\
NTL 2016 & 0.995 & 1 \\
\hline
\end{tabular}

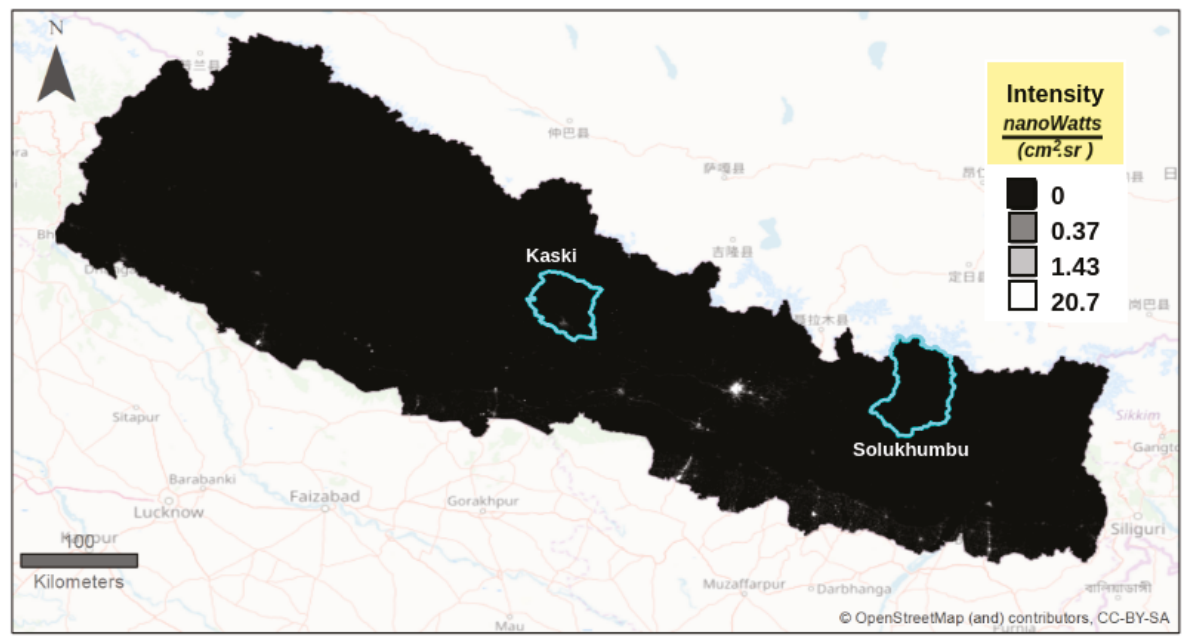

Figure 5. Average Radiance NTL Map highlighting Kaski and Solukhumbu district in Nepal (2016).

\subsection{Softwares}

The system implementation is done using free and open source resources. Programming languages and modules in Python and Java were used. Tweepy (https:/ / www.tweepy.org/) Python library was used for collecting tweets. QuickOSM (https:/ / plugins.qgis.org/plugins/QuickOSM/) Python module for QGIS was used for collecting data from OSM. PyProj (https:/ / pypi.org/project/ pyproj/) module was used for performing cartographic transformations between different projection systems. Shapely (https://pypi.org/project/Shapely/) module was used for manipulation and analysis of geometric objects. Java implementation of DBSCAN [25] was used for clustering and extent extraction. QGIS was used for visualization of spatial data and results. Additionally, other Python modules like pandas (https:/ / pandas.pydata.org/), JSON (https:/ / docs.python.org/2/ library/json.html), haversine (https://pypi.org/project/haversine/), functools (https:/ /docs.python. org/3/library/functools.html) were also used. 


\section{Results}

The proposed technique is applied to discover TAOIs using geo-tagged tweets, OSM, and NTL data. This section illustrates the experimental results of applying the proposed TAOI identification method in two different districts of Nepal.

\subsection{Selection of Clustering Parameters}

The objective functions, StrictParameters and MaxParameters, help in the selection of appropriate eps and minPts values required by the DBSCAN algorithm. The knowledge of the study area can guide in determining better values for maximum and minimum eps and minPts. We examined the geographic features of the study area as a guide to estimate a set of minimum and maximum values for the DBSCAN parameters. Minimum eps was set based on the average diameter of tourism features in the study area. Maximum eps was set based on the nearest neighbor distance among the tourism features in the study area. Maximum minPts value was taken referring to the values used by similar studies in more data dense regions. We examined 12,100 sets of eps and minPts. A total of 22 different values for eps from $25 \mathrm{~m}$ to $550 \mathrm{~m}$ with an increment of 25 meters were examined. For minPts, 550 different values from $0.01 \%$ to $5.5 \%$ with increments of $0.01 \%$ were examined. The set of StrictParameters and MaxParameters and the number of Dense Clusters and Sparse Clusters obtained are listed in Table 5. Optionally, in short of the knowledge of the target geography, the desired values for eps and minPts can be estimated without the need for any user input. The objective functions choose appropriate eps by examining a range of possible values from minimum (i.e., zero) to maximum (i.e., length of the study area) distance values. Similarly, minPts is also selected by scanning minimum data points (i.e., 3 data points as defined by DBSCAN algorithm) to maximum possible data points in the input. In this way, the objective functions, i.e., StrictParameters and MaxParameters, estimate the required values for generating Dense and Sparse clusters.

Table 5. Tweet Clusters obtained by applying StrictParameters and MaxParameters.

\begin{tabular}{ccccccc}
\hline \multirow{2}{*}{ Place } & \multicolumn{3}{c}{ StrictParameters } & \multicolumn{3}{c}{ MaxParameters } \\
\cline { 2 - 7 } & eps $(\mathbf{m})$ & minPts $(\%)$ & Clusters & eps $(\mathbf{m})$ & minPts $(\%)$ & Clusters \\
\hline Kaski & 25 & 0.11 & 1 & 175 & 0.01 & 75 \\
Solukhumbu & 25 & 0.51 & 1 & 350 & 0.01 & 44 \\
\hline
\end{tabular}

\subsection{TAOI Identification}

Table 6 illustrates the significance of different input variables in distinguishing TAOIs. The selected thresholds for foreign tweet presence, NTL, and building footprint is one or more. Similarly, the minimum requirement for tweet point percentage is $0.06 \%$ for Kaski and $0.09 \%$ for Solukhumbu district. The threshold values are estimated based on the knowledge gathered from various tourist maps and trekking guides published by recommended agencies such as the Nepal Tourism Board and Milestone Guides [84,85]. Furthermore, seven local tourism experts (i.e., tourist guides, backpackers and hotel staffs and owners) were also consulted while setting the threshold values. Local experts verified whether the extents of the identified TAOIs contain touristic spots or not. For Kaski, DBSCAN with MaxParameters discovered a total of 75 tweet clusters. Out of the 75 clusters, only 47 of them contain tweets from foreigners, 60 clusters have sufficient NTL values, 68 clusters pass BF threshold and 68 clusters confirm the minimum tweet PP requirement. Much of the tweet clusters in Solukhumbu have foreigner tweet presence while almost half of the clusters in Kaski do not have a significant contribution from foreigners. Inversely, many clusters in Kaski seem to be formed around built-up areas while the cluster presence around built-up regions in Solukhumbu is much low. Also, NTL acted as a stronger discriminator in Solukhumbu district. This is because Solukhumbu is extremely rural in 
comparison with Kaski district. Almost one third of the clusters in Kaski and less than a quarter of the clusters in Solukhumbu were below the PP threshold.

Table 6. Effect of different data sources on cluster selection; NTL: Nighttime light intensity; PP: Tweet Point Percentage; FT: Foreigner Tweet; BF: Building Footprint.

\begin{tabular}{cccccc}
\hline \multirow{2}{*}{ Place } & \multirow{2}{*}{ Total } & \multicolumn{4}{c}{ Clusters Confirming } \\
\cline { 3 - 6 } & Clusters & FT & NTL & BF & PP \\
\hline Kaski & 75 & 47 & 60 & 68 & 68 \\
Solukhumbu & 44 & 39 & 7 & 26 & 40 \\
\hline
\end{tabular}

Table 7 summarizes the result obtained by applying different TAOI identification methods. The first row shows the outcomes of the TAOI selection methods for Kaski. Out of the 75 tweet clusters, tClust_B selected 41 of them as TAOIs and eliminated 34 clusters. Similarly, tClust_N elected 33 clusters as TAOIs and disqualified 42 clusters. In addition, $t$ Clust_NB nominated 28 clusters as relevant ones and excluded 47 of them from the result. For each study area, single prime locations were identified. The TAOI determination methods $t$ Clust_ $N$ and $t C l u s t \_N B$ elected comparatively few TAOIs in the rural region (i.e., Solukhumbu district) because most of the touristic spots in the district are located in and around the Sagarmatha National Park which does not have enough NTL illumination. A closer inspection revealed some level of human settlement in few of the un-lit regions. Hence, the mandatory requirement of NTL threshold seems biased for such areas. However, the method based on the building footprints, i.e., $t$ Clust_B, behaved in a much unbiased manner while selecting TAOIs. Further exploration of the $t$ Clust_B TAOIs and OSM tourism features was made. Table 8 provides a summary of the overlapping between the TAOIs and the polygons labeled as tourism in OpenStreetMap. Out of the 41 TAOIs in Kaski, only 19 of them overlapped with tourism polygons in OSM. Accordingly, about half of the TAOIs in Solukhumbu do not find any related features in OSM. Surprisingly enough, none of the prime locations showed any overlay of OSM tourism features. Close inspection of the study areas uncovered the fact that OSM data is not complete and consistent in the regions which resulted in a low agreement between the discovered touristic spots and OSM data.

Table 7. Summary of TAOIs identified by tClust_B(FT \& PP \& BF), tClust_N(FT \& PP \& NTL) and tClust_NB(FT \& PP \& NTL \& BF).

\begin{tabular}{ccccccccc}
\hline \multirow{2}{*}{ Place } & \multirow{2}{*}{ Total } & \multicolumn{2}{c}{ tClust_B } & \multicolumn{2}{c}{ tClust_N } & \multicolumn{2}{c}{ tClust_NB } & \multirow{2}{*}{\begin{tabular}{c} 
Prime \\
\cline { 3 - 8 }
\end{tabular}} \\
\cline { 3 - 8 } & Clusters & TAOI & Pruned & TAOI & Pruned & TAOI & Pruned & Location \\
\hline Kaski & 75 & 41 & 34 & 33 & 42 & 28 & 47 & 1 \\
Solukhumbu & 44 & 24 & 20 & 7 & 37 & 5 & 39 & 1 \\
\hline
\end{tabular}

Table 8. Summary of TAOIs and OSM tourism feature overlap.

\begin{tabular}{ccccc}
\hline \multirow{2}{*}{ Place } & \multicolumn{2}{c}{ tClust_B } & \multicolumn{2}{c}{ Prime Locations } \\
\cline { 2 - 5 } & TAOIs & Overlap & Locations & Overlap \\
\hline Kaski & 41 & 19 & 1 & 0 \\
Solukhumbu & 24 & 13 & 1 & 0 \\
\hline
\end{tabular}

The spatial distribution of identified TAOIs depicted the locations of notable tourism spots where prominent natural and man-made landmarks are located. This result supports our assumption that important landmarks receive more user attention in reality, and accordingly in the social media than regular areas. To illustrate this claim, concrete evidence from our study area are discussed. Figure 6 shows Lakeside, a part of Pokhara city, which is the most touristic region in the Kaski district. Detail investigation about Lakeside revels (i) high social media activity (ii) dense OSM building footprint and 
(iii) comparatively high NTL in the region. Such a place should contain TAOIs as per the definition proposed in Section 3.1. Accordingly, the proposed TAOI mapping scheme identified Lakeside as a touristic region. Furthermore, a prime location was discovered in the core of Lakeside (i.e., a red polygon within). On a par with our assumption, this identified prime location provides better tourism services and facilities as well as active social media presence in the whole region. This is the most prominent tourist location which lies within 100 meters of 'Phewa Lake'. Phewa Lake is the main tourist attraction in Lakeside and is listed as the top attraction in Pokhara by travel website TripAdvisor (https: / / www.tripadvisor.com/Attractions-g293891-Activities-Pokhara_Gandaki_Zone_ Western_Region.html). Next, we examined some of the tourism facilities at an obscure location, i.e., very far from the prime location, but within the extent of the TAOI. For example, 'Hotel Mountain View' is located at the bottom end of TAOI i.e., most distant from the prime tourism centers. Though the hotel is not at the main road (i.e., Lakeside Road), visitors find its location good enough to stay as its cost is economical and the hotel is situated away from crowded locations (expressed in the hotel reviews in TripAdvisor (https: / / www.tripadvisor.com/Hotel_Review-g293891-d5982537-ReviewsHotel_Mountain_View-Pokhara_Gandaki_Zone_Western_Region.html).

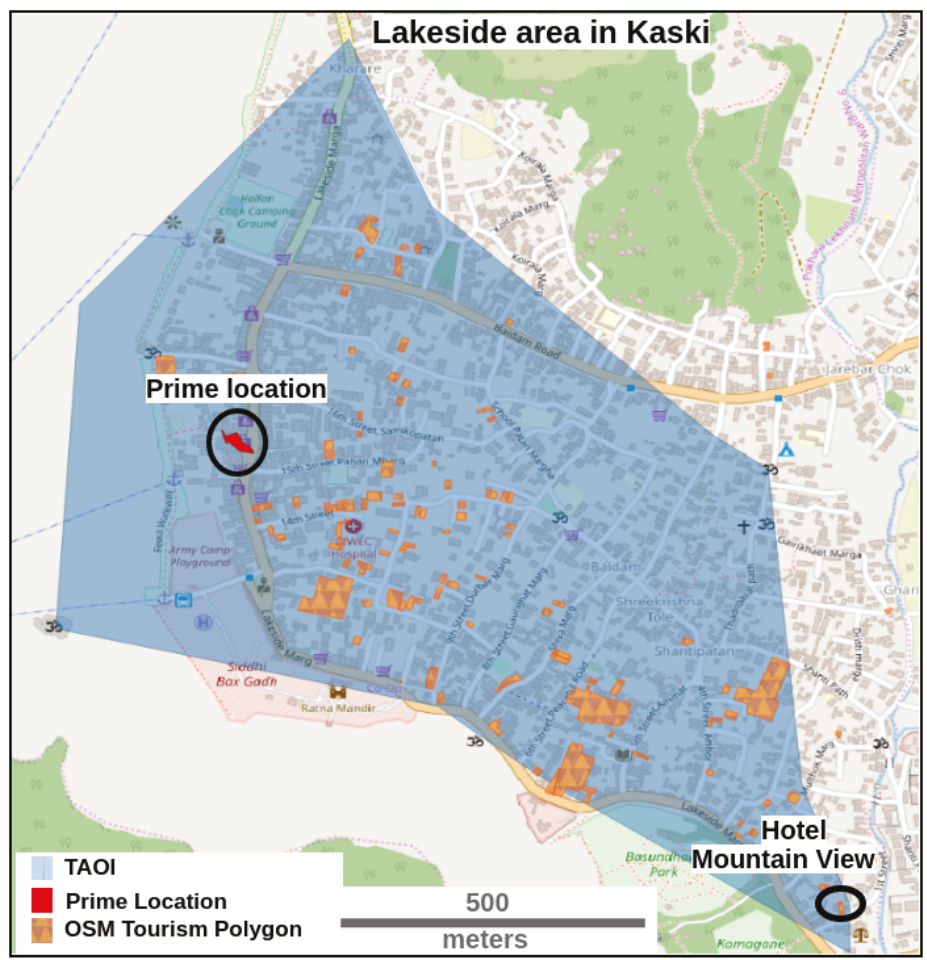

Figure 6. Lakeside region as an important TAOI in Kaski with a prime location.

Similarly, Figure 7 displays the spread of TAOI within Namche Bazaar region in the Solukhumbu district. Namche Bazaar is an important tourism hub located in the rural municipality of the district. It is situated inside the Sagarmatha National Park area, a world heritage site. The shadowed region indicates the stretch of TAOI, and the enclosed red polygon represents the prime location. An interesting pattern divulged while observing the spatial distribution of TAOIs in the Solukhumbu district. As evident in Figure 8a the distribution of TAOIs follow the trekking routes in the Everest Base Camp (EBC) region. In addition, relaxation of the thresholds resulted in the pattern becoming more 
significant (e.g., Figure 8b-d). The TAOIs which conforms to the defined threshold is relatively less in number but are in areas that provide minimum infrastructures and services. Only five TAOIs in the whole district and three TAOIs in the EBC region were found to be illuminated during the night. In fact, these illuminated TAOIs represent district headquarter Salleri (TAOI_25), neighborhood along Phaplu airport (TAOI_31) and other important locations along the Everest trekking route that act as indispensable tourism centers in the region i.e., Namche Bazzar (TAOI_12), Lukla area (TAOI_7) and Tengboche region (TAOI_6).

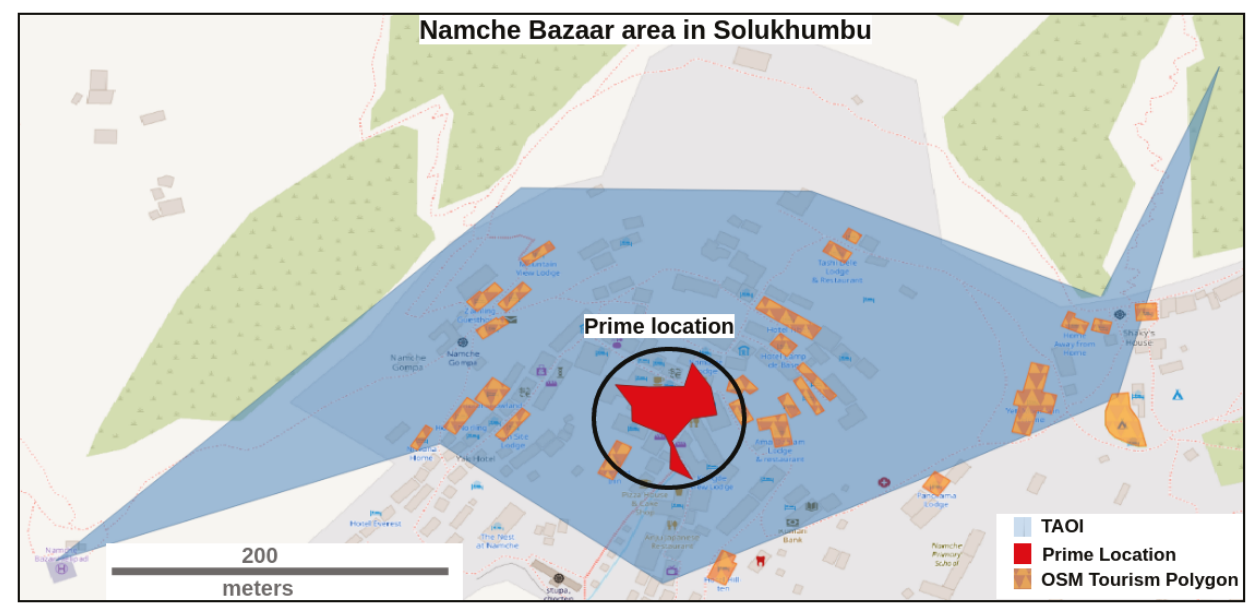

Figure 7. Namche Bazaar as an important TAOI in Soukhumbu with a prime location.

\subsection{Validation}

To evaluate and compare the performance of the proposed methods, standard measures of quality i.e., F1 score is used. The accuracy assessment is performed by selecting well-known tourism sites of Nepal. Pokhara city, one of the best urban tourism destinations in Nepal, is selected from the Kaski district. EBC region which is popular for remote area trekking is chosen from the Solukhumbu district. The choice of these areas represents well-known urban and remote tourist destinations in Nepal. The ground truth data were collected from tourist maps and trekking guides published by recommended agencies such as the Nepal Tourism Board and Milestone Guides [84,85]. Figure 9a,b respectively show the confusion matrices for the EBC region and Pokhara city by comparing ground truth data against the TAOIs recommended by the proposed $t C l u s t \_B$ method. In the EBC region, 22 TAOIs are correctly recognized and 17 TAOIs were not identified by $t C l u s t \_B$. Similarly, in Pokhara 20 clusters were recognized as TAOI correctly, 9 TAOIs were missed, and 7 clusters were incorrectly identified as TAOIs. F1 scores of 0.72 and 0.74 were calculated for EBC region and Pokhara, respectively. For EBC, the model was able to determine the tourist regions correctly as indicated by the high precision score. However, the recall value hints that $t C l u s t \_B$ sometimes make incorrect predictions by categorizing valid regions as invalid. In the case of Pokhara city, a decrease in precision and an increase in recall values were observed. However, the overall F1 score depicted the consistency of the method over different areas. Figure 10 provides a comparative overview of the three proposed methods for TAOI detection. It is determined that $t C l u s t \_B$ method offers the best performance among all. 


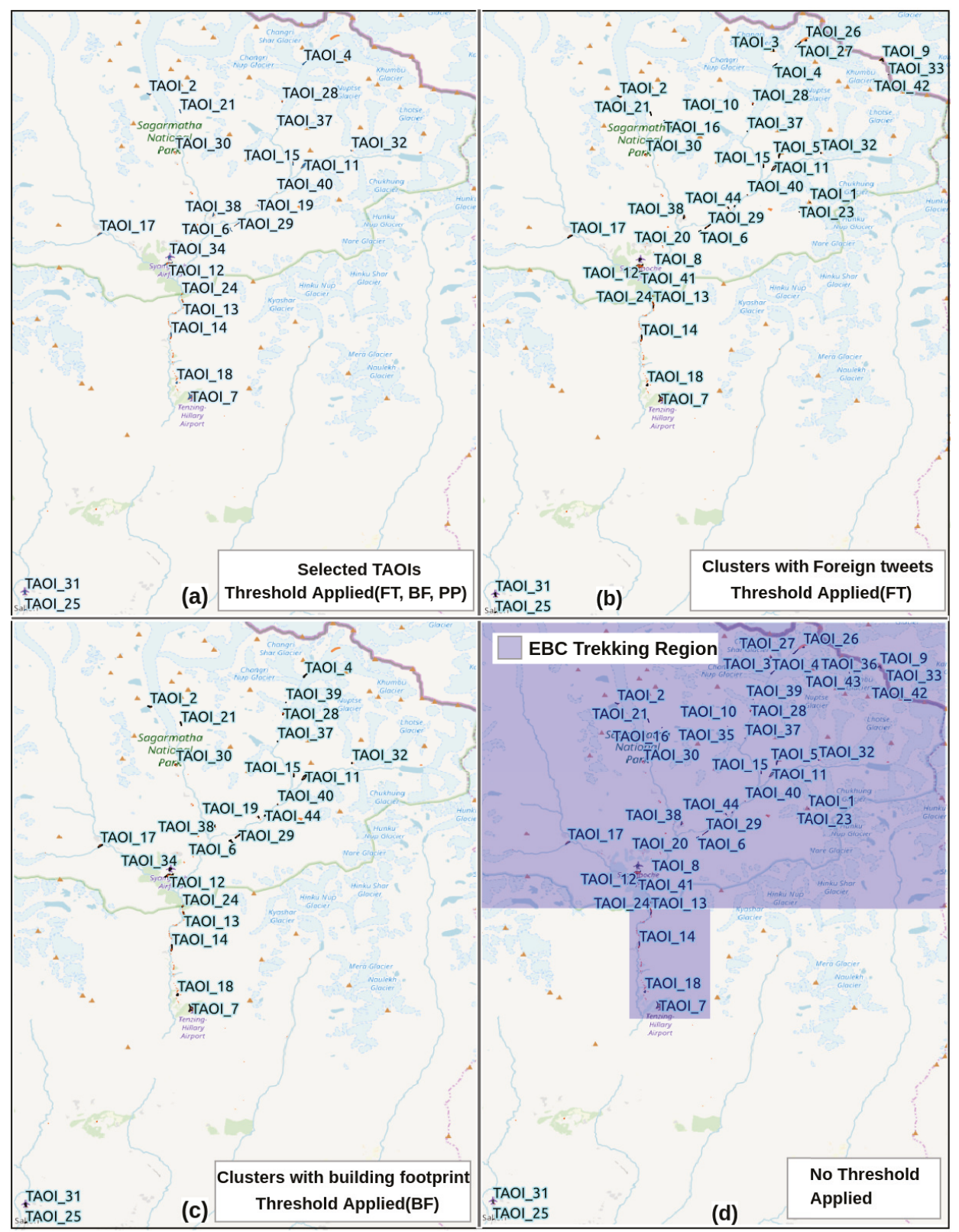

Figure 8. Distribution of TAOIs in Solukhumbu. (a) All selected TAOIs, (b) all tweet clusters with foreigner tweets, (c) all tweet clusters around building footprints, (d) all tweets clusters (no threshold). 


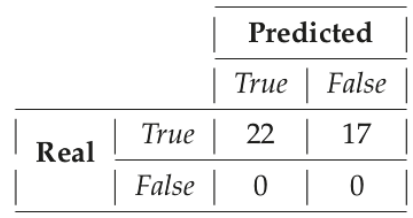

(a)

\begin{tabular}{|c|c|c|c|}
\hline & \multicolumn{2}{|c|}{ Predicted } \\
\hline & & True & False \\
\hline \multirow{2}{*}{ Real } & True & 20 & 9 \\
\hline & False & 7 & 0 \\
\hline
\end{tabular}

(b)

Figure 9. Confusion Matrices for (a) EBC Region and (b) Pokahra City.

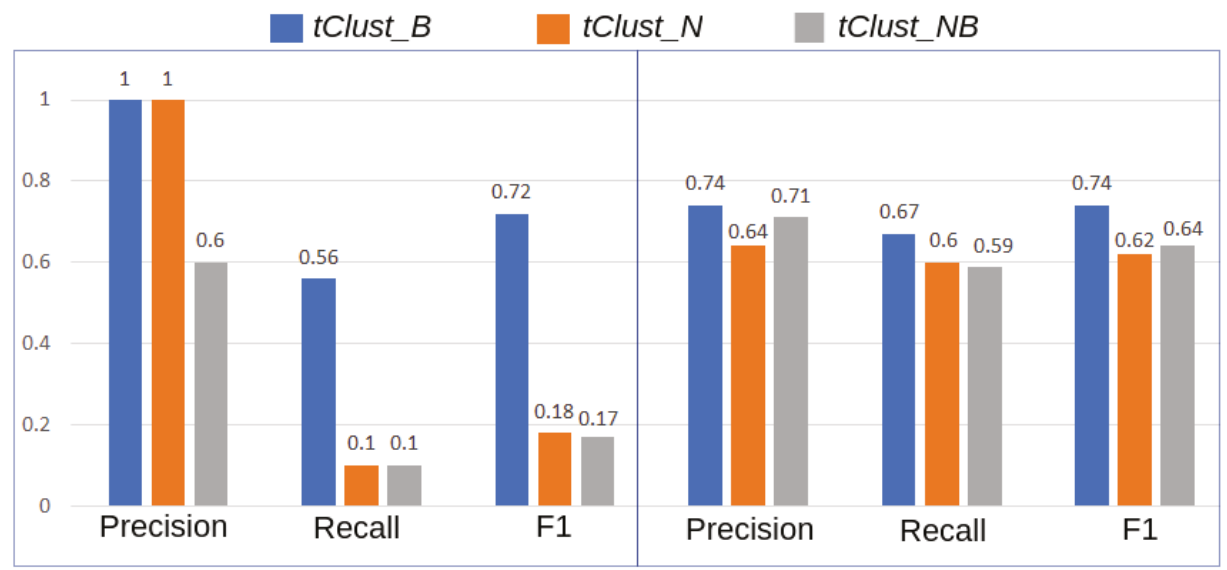

(a)

(b)

Figure 10. Performance comparison of TAOI detection using tClust_B(FT \& PP \& BF), tClust_N(FT \& PP \& NTL) and tClust_NB(FT \& PP \& NTL \& BF): (a) EBC Region (b) Pokhara.

\section{Discussion}

The proposed approach automatically searches and identifies touristic places of interest based on collective knowledge. OpenStreetMap and Nighttime light remote sensing data are used to enhance the knowledge obtained from the social media data. Results show that our approach can identify major tourist attractions in different rural and urban tourism destinations in Nepal.

\subsection{Significance of the Data Sources Used}

Previous works implemented using density-based clustering for mining important tourist locations (such as $[20,49]$ ) do not examine if the social media clusters are merely popular or actually related to tourism. Our proposed framework uses multiple data sources such as geo-tagged tweets, NTL data, and building footprint data to discard non-touristic social media clusters. For example, in the proposed study, the presence of a foreigner tweet was essential for the tweet cluster to become touristic. Additional consideration of infrastructures such as buildings in the locale of the identified clusters ensured the availability of minimum essential services for the travelers. Tables 6 and 7 clearly shows that not all spots merely popular in the social media are relevant to tourism.

Furthermore, Table 6 indicates that rural region (i.e., Solukhumbu district) have far less tweet clusters than urban region(Kaski district). It is clear that most of the rural clusters have foreigner tweet presence but those clusters are poorly lit during the night. However, most of the clusters in the urban are located around built-up areas and are illuminated during the night. Furthermore, a detail investigation of the significance of different data sources and the $t C l u s t \_B$ selected TAOIs in the rural region (i.e., EBC region) and urban region (i.e., Pokhara city) are examined. The contribution of each input data source in the final TAOI selection using $t$ Clust_B are presented in Figure 11. All the built-up 
areas with enough tweet density in the rural region are touristic. In contrast, only half of the clusters with building footprints in the urban region are touristic. This is because more local users publish tweets in urban than in rural. Moreover, almost all clusters with NTL exist around areas with buildings. Though most urban TAOIs have noticeable NTL, it is not the case in the rural TAOIs. However, NTL presence ensures the existence of better infrastructure and facilities in the neighborhood $[83,86]$. Hence, NTL availability indicates crucial information useful for identifying prime touristic locations.

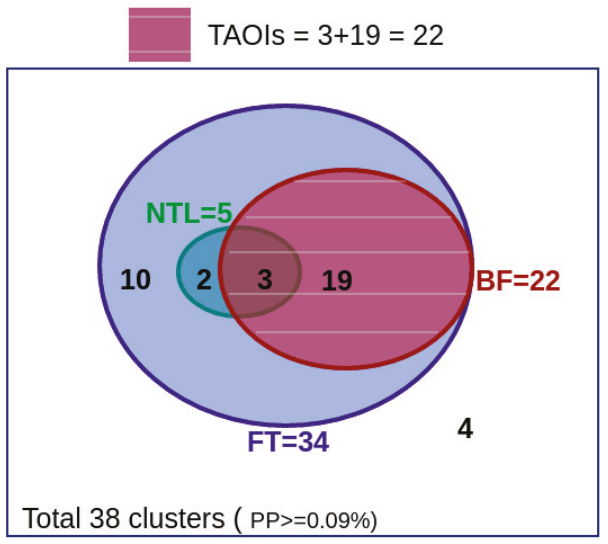

(a)

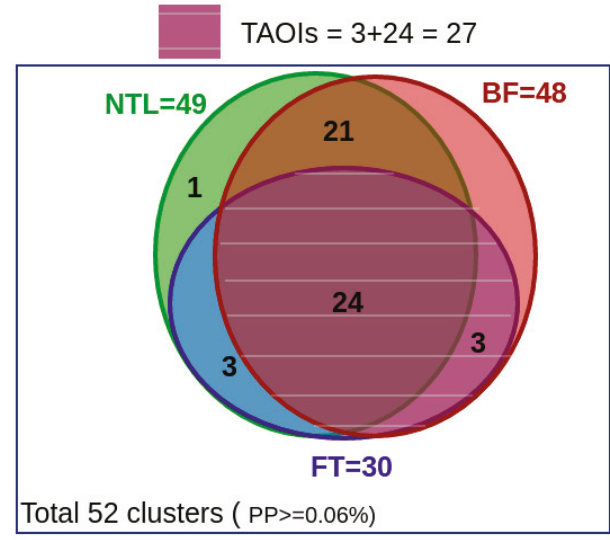

(b)

Figure 11. $t$ Clust_B TAOIs selection from clusters with sufficient tweet PP in (a) rural region (EBC, Solukhumbu) and (b) urban region (Pokhara, Kaski).

\subsection{Significance of Cluster Pruning}

It is important to recognize the desired tourism areas and eliminate any non-tourism clusters from the result. However, it is equally important that the selected TAOIs provide minimum essential services (e.g., overnight accommodation and breakfast) to the travelers. Korakakis et al. identified tourist locations without guaranteeing such facilities [49]. Although Majid et al., examined the context of travelers such as time and whether but no consideration was made for such essential requirements [20]. An example in our study clarifies the need to ensure the availability of minimum shelter requirements in the tourism spots. For instance, 'Kala Patthar' (i.e., TAOI_3 in Figure 8b), located in Solukhumbu district, is a very popular spot among travelers and caters with an amazing view of the snow-capped mountains including the Mount Everest. Though this place has extreme geography and harsh climatic conditions, a lot of geo-tagged tweets, from foreigners, were found forming a significant cluster; however, it was not included in the final list of TAOIs because this place does not have any infrastructure support for serving the travelers. The BF check helped to prune this spot from the final result.

Similarly, one of the active tweet clusters in Kaski district was identified around a collage area which was not selected as a final TAOI because the area does not fulfill the FT requirement. Field examination revealed that this particular spot does not contain any tourism facilities. Similarly, many other clusters were pruned out of the final result due to the absence of sufficient tweet points or foreigner tweets or building footprint data. Table 7 provides the number of clusters filtered out as non-tourism clusters while identifying interesting TAOIs in the different regions. Hence, the thresholding step is crucial in filtering out irrelevant tweet clusters from the final selection.

Krikigianni et al. suggested the high NTL areas are relatively more urbanized and maintain more tourism infrastructure and services than the low-lit areas [65]. Also, a relatively small number of TAOIs were identified in the rural district than in urban districts. In our investigation, the NTL threshold acted as a stronger discriminator in the rural regions than in the urban districts. It was evident that 
NTL (i.e., $t$ Clust_N and $t C l u s t \_N B$ ) could not act as a good proxy for monitoring TAOIs in rural regions where there is very low or even no nighttime light illumination. Hence, the model suggested by Krikigianni et al. may not provide good insights in such places. However, our proposed model (i.e., $t$ Clust_ $B$ which uses the building footprint information served as a better alternative to NTL by providing a fair decision while discovering TAOIs in both rural and urban region. Furthermore, thresholding may be improved by including other socio-economic and geographical features such as accessibility, population, etc. Exploration in this direction will be done in the future.

\subsection{TAOI Categorization}

This study focuses on identifying important touristic spots which can fulfill the basic needs of a travelers. This indicates that the vicinity of the discovered TAOIs must serve, at least, a basic bed and breakfast facility. Also, an approach for examining the existence of prime attractions within the extents of a TAOI has been explored in this research. Guidelines for identifying less crowded and economical alternatives for those prime locations have also been discussed. However, naming and categorizing the TAOIs based on their inherent features would be more informative. Analyzing the textural data shared by the users in social media can be helpful in labeling the TAOIs with categories, for example, TAOI_7 (Location Name: Lukla, Details: airport, hotels, mountain scene).

\subsection{Cost Effective and Quicker Alternative to Traditional Methods}

Buntain et al. suggested that social media data such as tweets can provide better insights in understanding social phenomena more rapidly and at lower cost than ground surveys [87]. For instance, Figure 12 shows newly emerged touristic hubs in the Kaski district. These spots which lie along the coast of Phewa lake, used to be non-touristic in the past. The proposed framework successfully discovered these newly formed tourism sites although most of the traditional data repositories are still outdated. Hence, an exhaustive investigation of the false positive results generated by our system may give more insights. For instance, the presence of foreigner tweets in non-touristic regions may signify an emerging tourist attraction.

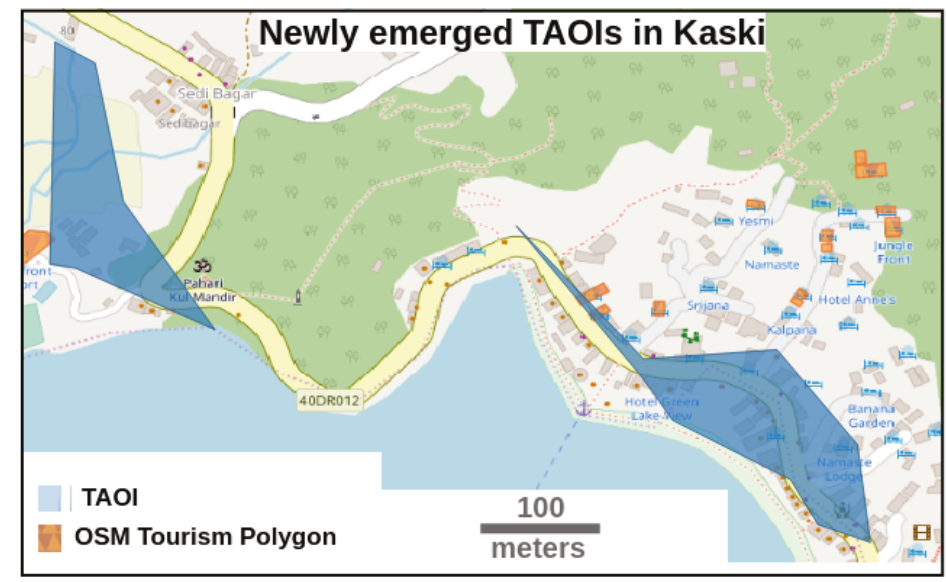

Figure 12. Newly emerged TAOI in Kaski.

\subsection{Comparison with OSM}

OSM is a popular crowdsourced project that acts as a free source for geodata. In OSM, polygons (or closed ways) are used to depict the boundaries of areas such as parks, buildings, or forests. The quality and coverage of OSM data are not consistent globally [28,88]. For instance, it is surprising that many TAOIs identified do not have any such OSM tourism features. Furthermore, the prime 
locations discovered in Kaski and Solukhumbu do not have any tourism polygon feature mappings in the OSM. However, field survey uncovered the fact that though there are several physical structures (e.g., buildings) on site, many of them do not have a proper mapping in the OSM. Many tourism facilities such as hotels, restaurants, viewpoints, etc. were observed on the ground and most of them were present in the OSM but proper labeling was not used. This means OSM lacks the representation of such features, or even if some features are represented they are not annotated correctly with the 'tourism' tag. Hence, social media data can act as a more up-to-date and closer to real-time tool for identification of active tourism centers.

\subsection{Impact of Location Accuracy of Geo-Tagged Tweets}

Our assumption of geo-tagged location is based on the capacity of smartphone which may compromise location accuracy up to 13 meters $[89,90]$. The underlying DBSCAN clustering algorithm generates social media clusters with reference to the location of geo-tagged tweets. The search distance parameter (i.e., eps) of the algorithm controls the formation of the clusters based on the horizontal distance among the geo-tweets. Therefore, the positional changes in the tweet points may affect the formation of the clusters. Many previous studies (e.g., $[17,18,50,58]$ ) which use manually selected static values for the clustering parameters may not adapt well in such cases. However, our proposed approach works well even in such scenarios because the parameters for clustering algorithm are estimated by using objective functions StrictParameters and MaxParameters. Moreover, the positional accuracy of the tweets may affect the geometry of the cluster formed and if the cluster size becomes smaller then fewer TAOI may be detected in regions with sparse building footprints.

\subsection{Limitations in Coverage}

Though we were able to get good results, it should be considered in the light of limitations of the input data and method used. The quality of OSM data has often been questioned for consistency and completeness. Though nighttime light images are used as a proxy in various research studies, it is of low resolution and it cannot capture activities unrelated to light. Furthermore, Twitter usage does not include all online activities because not all Internet users participate in this social network. However, the remotely sensed data and VGI data used are publicly accessible and consistently available across the world. Therefore, we assume nighttime light remote sensed data and VGI data can still act as a proxy for our purpose. Also, there is some room for improvements in the method. The DBSCAN clustering algorithm used for identifying TAOIs is not density adaptive. The process of NTL, BF, and tweet percentage threshold estimation is manual. Tuning of the threshold values is expected to improve results.

\section{Conclusions}

Twitter provides freely accessible geo-tagged volunteered geographic information which can be investigated across various domains of human activities. This study demonstrated that tweets hold great potential for mapping touristic areas even in remote and data deficient regions. Freely available OSM data and remotely sensed nighttime light data helped in enriching the TAOI discovery process. In this study, we collected and cleaned the geo-tagged tweets, and then applied clustering to get the spatial distribution of the social media clusters. Next, tourism related clusters were distinguished from normal clusters by exploring different Twitter metadata along with OSM and NTL data. The final list of selected TAOIs were not only popular among the travelers but also ensured the availability of essential tourism infrastructure in the vicinity. The proposed framework was examined in two different tourism districts in Nepal with satisfactory results. This framework not only identified popular TAOIs but also helped in spotting prime tourism locations in the neighborhood.

Our proposed analytic framework will enhance the state-of-the-art tourism studies in several directions. First, by discovering tourism areas of interest in data deficient remote areas by fusing free social media data and other free data sources such as OSM and NTL. Secondly, by devising a 
method to estimate clustering parameters values which can be applied across different scarce data regions. Thirdly, by ensuring the availability of minimum essential facilities in the recommended touristic sites. Moreover, useful guidelines were prescribed for identifying obscure alternatives apart from prime touristic locations. Most importantly, the approach outlined in this paper presents a novel methodology previously unused in tourism attraction studies.

The proposed approach examined the discovery of TAOIs from social media clusters which were located mainly in built-up regions (or well-lit regions). Some of the TAOIs with fewer media attention or located in non-built ( or non-lit) regions may be discarded. A simple way to include such regions in the final selection is by softening the threshold values. However, optimal threshold selection and method universalization will be explored in future iterations of our work. Further improvements are to be made in other aspects such as the elimination of bias arising from the inherent characteristics of selected data sources (Twitter, OSM, remote sensing images).

Author Contributions: B.D. and H.M. contributed to the overall study design and supervised all research. B.D. completed the data collection and development of analysis tools. B.D. and H.M. contributed to the data analysis and the manuscripts. A.W. and S.M.K. reviewed and provided critical comments for the improvement of the work.

Funding: We would like to acknowledgment the Japanese Government Scholarship. This study is supported by the Japanese Government Scholarship at the Asian Institute of Technology (grant: August 2016).

Acknowledgments: We thank Dhiraj Pahari and Niraj Pahari for assisting us during the data collection and study area surveys. Their knowledge and network on Nepalese Tourism and helpful comments has greatly contributed in the study. The authors would also like to thank Michelle Han for her help in editing the manuscript.

Conflicts of Interest: The funders had no role in the design of the study; in the collection, analyses, or interpretation of data; in the writing of the manuscript; or in the decision to publish the results.

\section{References}

1. Ashley, C.; De Brine, P.; Lehr, A.; Wilde, H. The Role of the Tourism Sector in Expanding Economic Opportunity; John F. Kennedy School of Government, Harvard University Cambridge: Cambridge, MA, USA, 2007.

2. WTTC. World Travel and Tourism Report 2018 Report for Nepal. Available online: https://www.wttc.org/-/ media/files/reports/economic-impact-research/countries-2018/nepal2018.pdf (accessed on 18 March 2019).

3. United Nations. Sustainable Tourism. Available online: https://sustainabledevelopment.un.org/topics/ sustainabletourism (accessed on 27 June 2019).

4. Morrison-Saunders, A.; Hughes, M.; Pope, J.; Douglas, A.; Wessels, J.A. Understanding visitor expectations for responsible tourism in an iconic national park: Differences between local and international visitors. J. Ecotourism 2019, 1-11. [CrossRef]

5. Martín Martín, J.M.; Guaita Martínez, J.M.; Molina Moreno, V.; Sartal Rodríguez, A. An Analysis of the Tourist Mobility in the Island of Lanzarote: Car Rental Versus More Sustainable Transportation Alternatives. Sustainability 2019, 11, 739. doi:10.3390/su11030739. [CrossRef]

6. Henderson, J.V.; Storeygard, A.; Weil, D.N. Measuring economic growth from outer space. Am. Econ. Rev. 2012, 102, 994-1028. [CrossRef] [PubMed]

7. Bennett, P.; Giles, L.; Halevy, A.; Han, J.; Hearst, M.; Leskovec, J. Channeling the deluge: Research challenges for big data and information systems. In Proceedings of the 22nd ACM International Conference on Information \& Knowledge Management, San Francisco, CA, USA, 27 October-1 November 2013; pp. 2537-2538.

8. Heikinheimo, V.; Minin, E.D.; Tenkanen, H.; Hausmann, A.; Erkkonen, J.; Toivonen, T. User-generated geographic information for visitor monitoring in a national park: A comparison of social media data and visitor survey. ISPRS Int. J. Geo-Inf. 2017, 6, 85. [CrossRef]

9. Jendryke, M.; Balz, T.; McClure, S.C.; Liao, M. Putting people in the picture: Combining big location-based social media data and remote sensing imagery for enhanced contextual urban information in Shanghai. Comput. Environ. Urban Syst. 2017, 62, 99-112. [CrossRef]

10. Miyazaki, H.; Nagai, M.; Shibasaki, R. Development of Time-Series Human Settlement Mapping System using Historical Landsat Archive. Int. Arch. Photogramm. Remote. Sens. Spat. Inf. Sci. 2016, 41, 1385. [CrossRef] 
11. Sitthi, A.; Nagai, M.; Dailey, M.; Ninsawat, S. Exploring land use and land cover of geotagged social-sensing images using naive bayes classifier. Sustainability 2016, 8, 921. [CrossRef]

12. Preoţiuc-Pietro, D.; Volkova, S.; Lampos, V.; Bachrach, Y.; Aletras, N. Studying user income through language, behaviour and affect in social media. PLoS ONE 2015, 10, e0138717. [CrossRef] [PubMed]

13. Levin, N.; Lechner, A.M.; Brown, G. An evaluation of crowdsourced information for assessing the visitation and perceived importance of protected areas. Appl. Geogr. 2017, 79, 115-126. [CrossRef]

14. Park, J.H.; Lee, C.; Yoo, C.; Nam, Y. An analysis of the utilization of Facebook by local Korean governments for tourism development and the network of smart tourism ecosystem. Int. J. Inf. Manag. 2016, 36, 1320-1327. [CrossRef]

15. Del Vecchio, P.; Mele, G.; Ndou, V.; Secundo, G. Creating value from social big data: Implications for smart tourism destinations. Inf. Process. Manag. 2018, 54, 847-860. [CrossRef]

16. García-Palomares, J.C.; Gutiérrez, J.; Mínguez, C. Identification of tourist hot spots based on social networks: A comparative analysis of European metropolises using photo-sharing services and GIS. Appl. Geogr. 2015, 63, 408-417. [CrossRef]

17. Encalada, L.; Boavida-Portugal, I.; Cardoso Ferreira, C.; Rocha, J. Identifying tourist places of interest based on digital imprints: Towards a sustainable smart city. Sustainability 2017, 9, 2317. [CrossRef]

18. Maeda, T.; Yoshida, M.; Toriumi, F.; Ohashi, H. Extraction of Tourist Destinations and Comparative Analysis of Preferences Between Foreign Tourists and Domestic Tourists on the Basis of Geotagged Social Media Data. ISPRS Int. J. Geo-Inf. 2018, 7, 99. doi:10.3390/ijgi7030099. [CrossRef]

19. Chen, M.; Arribas-Bel, D.; Singleton, A. Understanding the dynamics of urban areas of interest through volunteered geographic information. J. Geogr. Syst. 2018. doi:10.1007/s10109-018-0284-3. [CrossRef]

20. Majid, A.; Chen, L.; Mirza, H.T.; Hussain, I.; Chen, G. A system for mining interesting tourist locations and travel sequences from public geo-tagged photos. Data Knowl. Eng. 2015, 95, 66-86. [CrossRef]

21. Vu, H.Q.; Li, G.; Law, R.; Ye, B.H. Exploring the travel behaviors of inbound tourists to Hong Kong using geotagged photos. Tour. Manag. 2015, 46, 222-232. doi:10.1016/j.tourman.2014.07.003. [CrossRef]

22. Lee, J.Y.; Tsou, M.H. Mapping Spatiotemporal Tourist Behaviors and Hotspots Through Location-Based Photo-Sharing Service (Flickr) Data. In Lecture Notes in Geoinformation and Cartography, Proceedings of the Progress in Location Based Services 2018, LBS 2018, Zurich, Switzerland, 15-17 January 2018; Kiefer, P., Huang, H., Van de Weghe, N., Raubal, M., Eds.; Springer International Publishing: Cham, Switzerland, 2018; pp. 315-334.

23. Zhuang, C.; Ma, Q.; Liang, X.; Yoshikawa, M. Discovering obscure sightseeing spots by analysis of geo-tagged social images. In Proceedings of the 2015 IEEE/ACM International Conference on Advances in Social Networks Analysis and Mining 2015, Paris, France, 25-28 August 2015; pp. 590-595.

24. Ester, M.; Kriegel, H.P.; Sander, J.; Xu, X. A density-based algorithm for discovering clusters in large spatial databases with noise. In Proceedings of the Second International Conference on Knowledge Discovery and Data Mining (KDD'96), Portland, OR, USA, 2-4 August 1996; Simoudis, E., Han, J., Fayyad, U., Eds.; AAAI Press: Palo Alto, CA, USA, 1996; Volume 96, pp. 226-231.

25. Hu, Y.; Gao, S.; Janowicz, K.; Yu, B.; Li, W.; Prasad, S. Extracting and understanding urban areas of interest using geotagged photos. Comput. Environ. Urban Syst. 2015, 54, 240-254. [CrossRef]

26. Liu, Y.; Liu, X.; Gao, S.; Gong, L.; Kang, C.; Zhi, Y.; Chi, G.; Shi, L. Social sensing: A new approach to understanding our socioeconomic environments. Ann. Assoc. Am. Geogr. 2015, 105, 512-530. [CrossRef]

27. Goodchild, M.F. Citizens as sensors: The world of volunteered geography. GeoJournal 2007, 69, $211-221$. [CrossRef]

28. Haklay, M. How good is volunteered geographical information? A comparative study of OpenStreetMap and Ordnance Survey datasets. Environ. Plan. Plan. Des. 2010, 37, 682-703. [CrossRef]

29. Li, L.; Goodchild, M.F. Constructing places from spatial footprints. In Proceedings of the 1st ACM SIGSPATIAL International Workshop on Crowdsourced and Volunteered Geographic Information, Redondo Beach, CA, USA, 6 November 2012; pp. 15-21.

30. Salas-Olmedo, M.H.; Moya-Gómez, B.; García-Palomares, J.C.; Gutiérrez, J. Tourists' digital footprint in cities: Comparing Big Data sources. Tour. Manag. 2018, 66, 13-25. doi:10.1016/j.tourman.2017.11.001. [CrossRef]

31. Estima, J.; Painho, M. Exploratory analysis of OpenStreetMap for land use classification. In Proceedings of the Second ACM SIGSPATIAL International Workshop on Crowdsourced and Volunteered Geographic Information, Orlando, FL, USA, 5 November 2013; pp. 39-46. 
32. Sun, Y.; Fan, H.; Li, M.; Zipf, A. Identifying the city center using human travel flows generated from location-based social networking data. Environ. Plan. Plan. Des. 2016, 43, 480-498. [CrossRef]

33. Koutras, A.; Nikas, I.A.; Panagopoulos, A. Towards Developing Smart Cities: Evidence from GIS Analysis on Tourists' Behavior Using Social Network Data in the City of Athens. In Smart Tourism as a Driver for Culture and Sustainability; Katsoni, V.; Segarra-Oña, M., Eds.; Springer International Publishing: Cham, Switzerland, 2019; pp. 407-418.

34. Xing, H.; Meng, Y.; Hou, D.; Song, J.; Xu, H. Employing crowdsourced geographic information to classify land cover with spatial clustering and topic model. Remote. Sens. 2017, 9, 602. [CrossRef]

35. Li, Y.; Li, Q.; Shan, J. Discover patterns and mobility of Twitter users-A study of four US college cities. ISPRS Int. J. Geo-Inf. 2017, 6, 42. [CrossRef]

36. Mazanec, J. Segmenting city tourists into vacation styles. In International City Tourism: Analysis and Strategy; Pinter: London, UK, 1997; pp. 114-128.

37. Shoval, N.; Raveh, A. Categorization of tourist attractions and the modeling of tourist cities: Based on the co-plot method of multivariate analysis. Tour. Manag. 2004, 25, 741-750. [CrossRef]

38. MacQueen, J. Some methods for classification and analysis of multivariate observations. In Proceedings of the Fifth Berkeley Symposium on Mathematical Statistics and Probability, Oakland, CA, USA, 21 June-18 July 1965; University of California Press: Berkeley, CA, USA, 1967; Volume 1, pp. 281-297.

39. Rousseeuw, P.J.; Kaufman, L. Clustering by means of medoids. In Statistical Data Analysis Based on the L1 Norm and Related Methods; Dodge, Y., Ed.; North-Holland/Elsevier: Amsterdam, The Netherlands, 1987; pp. 405-416.

40. Birant, D.; Kut, A. ST-DBSCAN: An algorithm for clustering spatial-temporal data. Data Knowl. Eng. 2007, 60, 208-221. [CrossRef]

41. Kisilevich, S.; Mansmann, F.; Keim, D. P-DBSCAN: A density based clustering algorithm for exploration and analysis of attractive areas using collections of geo-tagged photos. In Proceedings of the 1st International Conference and Exhibition on Computing for Geospatial Research \& Application, Washington, DC, USA, 21-23 June 2010; p. 38.

42. Campello, R.J.; Moulavi, D.; Sander, J. Density-based clustering based on hierarchical density estimates. In Proceedings of the Pacific-Asia Conference on Knowledge Discovery and Data Mining, Gold Coast, Australia, 14-17 April 2013; Springer: Berlin/Heidelberg, Germany, 2013; pp. 160-172.

43. Anselin, L. Local indicators of spatial association-LISA. Geogr. Anal. 1995, 27, 93-115. [CrossRef]

44. Getis, A.; Ord, J. The Analysis of Spatial Association by Use of Distance Statistics, Geographycal Analysis. In Perspectives on Spatial Data Analysis; Springer: Berlin/Heidelberg, Germany, 1992.

45. Wang, T.; Ren, C.; Luo, Y.; Tian, J. NS-DBSCAN: A Density-Based Clustering Algorithm in Network Space. ISPRS Int. J. Geo-Inf. 2019, 8, 218. [CrossRef]

46. Dehuri, S.; Mohapatra, C.; Ghosh, A.; Mall, R. Comparative study of clustering algorithms. Inf. Technol. J. 2006, 5, 551-559. [CrossRef]

47. Yang, Y.; Gong, Z. Identifying points of interest by self-tuning clustering. In Proceedings of the 34th International ACM SIGIR Conference on Research and Development in Information Retrieval, Beijing, China, 24-28 July 2011; pp. 883-892.

48. Laptev, D.; Tikhonov, A.; Serdyukov, P.; Gusev, G. Parameter-free discovery and recommendation of areas-of-interest. In Proceedings of the 22nd ACM SIGSPATIAL International Conference on Advances in Geographic Information Systems, Dallas, TX, USA, 4-7 November 2014; pp. 113-122.

49. Korakakis, M.; Spyrou, E.; Mylonas, P.; Perantonis, S.J. Exploiting social media information toward a context-aware recommendation system. Soc. Netw. Anal. Min. 2017, 7, 42. [CrossRef]

50. Hasnat, M.M.; Hasan, S. Identifying tourists and analyzing spatial patterns of their destinations from location-based social media data. Transp. Res. Part Emerg. Technol. 2018, 96, 38-54. [CrossRef]

51. Kuo, C.L.; Chan, T.C.; Fan, I.; Zipf, A. Efficient Method for POI/ROI Discovery Using Flickr Geotagged Photos. ISPRS Int. J. Geo-Inf. 2018, 7, 121. [CrossRef]

52. Yan, Y.; Kuo, C.L.; Feng, C.C.; Huang, W.; Fan, H.; Zipf, A. Coupling maximum entropy modeling with geotagged social media data to determine the geographic distribution of tourists. Int. J. Geogr. Inf. Sci. 2018, 32, 1699-1736. [CrossRef]

53. Cheng, Y. Mean shift, mode seeking, and clustering. IEEE Trans. Pattern Anal. Mach. Intell. 1995, 17, 790-799. [CrossRef] 
54. Rosenblatt, M. Remarks on some nonparametric estimates of a density function. In The Annals of Mathematical Statistics; JSTOR: New York, NY, USA, 1956; pp. 832-837. doi:10.1214/aoms/1177728190.

55. Dueck, D. Affinity Propagation: Clustering Data by Passing Messages. Ph.D. Thesis, University of Toronto, Toronto, ON, Canada, 2009.

56. Ng, A.Y.; Jordan, M.I.; Weiss, Y. On spectral clustering: Analysis and an algorithm. In Advances in Neural Information Processing Systems; MIT Press: Cambridge, MA, USA, 2001; pp. 849-856.

57. Schubert, E.; Sander, J.; Ester, M.; Kriegel, H.P.; Xu, X. DBSCAN revisited, revisited: Why and how you should (still) use DBSCAN. ACM Trans. Database Syst. (TODS) 2017, 42, 19. [CrossRef]

58. Zhou, X.; Xu, C.; Kimmons, B. Detecting tourism destinations using scalable geospatial analysis based on cloud computing platform. Comput. Environ. Urban Syst. 2015, 54, 144-153. [CrossRef]

59. Akdag, F.; Eick, C.F.; Chen, G. Creating Polygon Models for Spatial Clusters. In Foundations of Intelligent Systems; Andreasen, T.; Christiansen, H.; Cubero, J.C.; Raś, Z.W., Eds.; Springer International Publishing: Cham, Switzerland, 2014; pp. 493-499.

60. Duckham, M.; Kulik, L.; Worboys, M.; Galton, A. Efficient generation of simple polygons for characterizing the shape of a set of points in the plane. Pattern Recognit. 2008, 41, 3224-3236. [CrossRef]

61. Devkota, B.; Miyazaki, H. An Exploratory Study on the Generation and Distribution of Geotagged Tweets in Nepal. In Proceedings of the 2018 IEEE 3rd International Conference on Computing, Communication and Security (ICCCS), Kathmandu, Nepal, 25-27 October 2018; pp. 70-76.

62. Yamaguchi, Y.; Amagasa, T.; Kitagawa, H. Landmark-based user location inference in social media. In Proceedings of the First ACM Conference on Online Social Networks, Boston, MA, USA, 7-8 October 2013; pp. 223-234.

63. Chong, W.H.; Lim, E.P. Fine-grained Geolocation of Tweets in Temporal Proximity. ACM Trans. Inf. Syst. (TOIS) 2019, 37, 17. [CrossRef]

64. Jurgens, D. That's what friends are for: Inferring location in online social media platforms based on social relationships. In Proceedings of the Seventh International AAAI Conference on Weblogs and Social Media, Atlanta, GA, USA, 28 June 2013.

65. Krikigianni, E.; Tsiakos, C.; Chalkias, C. Estimating the relationship between touristic activities and night light emissions. Eur. J. Remote. Sens. 2019, 52, 233-246. [CrossRef]

66. Checa, J. Urban Intensities. The Urbanization of the Iberian Mediterranean Coast in the Light of Nighttime Satellite Images of the Earth. Urban Sci. 2018, 2, 115. [CrossRef]

67. Nepal, S.K.; Kohler, T.; Banzhaf, B.R. Great Himalaya: Tourism and the Dynamics of Change in Nepal; Swiss Foundation for Alpine Research: Zurich, Switzerland, 2002.

68. Central Bureau of Statistics, Government of Nepal. National Population and Housing Census 2011. Available online: https://unstats.un.org/unsd/demographic/sources/census/wphc/Nepal/Nepal-Census-2011Vol1.pdf (accessed on 18 March 2019).

69. LonelyPlanet. Top Experiences in Nepal. Available online: https://www.lonelyplanet.com/nepal (accessed on 7 April 2019).

70. InternetLiveStats. Twitter Usage Statistics. 2013. Available online: http://www.internetlivestats.com/ twitter-statistics / (accessed on 18 March 2019)

71. Cesare, N.; Grant, C.; Nsoesie, E.O. Detection of User Demographics on Social Media: A Review of Methods and Recommendations for Best Practices. arXiv 2017, arXiv:1702.01807.

72. Burghardt, M. Tools for the Analysis and Visualization of Twitter Language Data. Available online: https:/ / epub.uni-regensburg.de/35669/ (accessed on 18 March 2019).

73. Puschmann, C.; Bruns, A.; Mahrt, M.; Weller, K.; Burgess, J. Epilogue: Why Study Twitter? In Twitter and Society; Peter Lang: New York, NY, USA, 2014; Volume 89, pp. 425-432.

74. SocialAves. Social Media Landscape Nepal. 2017. Available online: https://socialaves.com/social-medialandscape-nepal/ (accessed on 18 March 2019)

75. Li, L.; Goodchild, M.F.; Xu, B. Spatial, temporal, and socioeconomic patterns in the use of Twitter and Flickr. Cartogr. Geogr. Inf. Sci. 2013, 40, 61-77. [CrossRef]

76. Morstatter, F.; Pfeffer, J.; Liu, H.; Carley, K.M. Is the sample good enough? comparing data from twitter's streaming api with twitter's firehose. arXiv 2013, arXiv:1306.5204. 
77. Morstatter, F.; Pfeffer, J.; Liu, H. When is it biased? Assessing the representativeness of twitter's streaming API. In Proceedings of the 23rd International Conference on World Wide Web, Seoul, Korea, 7-11 April 2014; pp. 555-556.

78. Frias-Martinez, V.; Soto, V.; Hohwald, H.; Frias-Martinez, E. Characterizing urban landscapes using geolocated tweets. In Proceedings of the 2012 International Conference on Privacy, Security, Risk and Trust and 2012 International Confernece on Social Computing, Amsterdam, The Netherlands, 3-5 September 2012; pp. 239-248.

79. Zhao, N.; Cao, G.; Zhang, W.; Samson, E.L. Tweets or nighttime lights: Comparison for preeminence in estimating socioeconomic factors. ISPRS J. Photogramm. Remote. Sens. 2018, 146, 1-10. [CrossRef]

80. Elvidge, C.D.; Safran, J.; Tuttle, B.; Sutton, P.; Cinzano, P.; Pettit, D.; Arvesen, J.; Small, C. Potential for global mapping of development via a nightsat mission. GeoJournal 2007, 69, 45-53. [CrossRef]

81. Baugh, K.; Hsu, F.C.; Elvidge, C.D.; Zhizhin, M. Nighttime lights compositing using the VIIRS day-night band: Preliminary results. Proc. Asia-Pac. Adv. Netw. 2013, 35, 70-86. [CrossRef]

82. Li, X.; Elvidge, C.; Zhou, Y.; Cao, C.; Warner, T. Remote sensing of night-time light. Int. J. Remote. Sens. 2017, 38, 5855-5859. [CrossRef]

83. Mellander, C.; Lobo, J.; Stolarick, K.; Matheson, Z. Night-time light data: A good proxy measure for economic activity? PLoS ONE 2015, 10, e0139779. [CrossRef]

84. Board, N.T. Greater Pokhara Valley Lake side and City Map. In Greater Pokhara Valley and City Map; Nepal Map Publisher: Kathmandu, Nepal, 2011.

85. Banerjee, P.S. Everest Trekking Maps and Complete Guide; Milestone Himalayan Series; Milestone Books: Calcutta, India, 2017.

86. Nel, O.; López, J.; Martín, J.; Checa, J. Energy and urban form. The growth of European cities on the basis of night-time brightness. Land Use Policy 2017, 61, 103-112. [CrossRef]

87. Buntain, C.; McGrath, E.; Golbeck, J.; LaFree, G. Comparing Social Media and Traditional Surveys around the Boston Marathon Bombing. In Proceedings of the \#Microposts: 6th Workshop on Making Sense of Microposts, Montréal, QC, Canada, 11-15 April 2016; pp. 34-41.

88. Zheng, S.; Zheng, J. Assessing the completeness and positional accuracy of OpenStreetMap in China. In Thematic Cartography for the Society; Springer: Cham, Switzerland, 2014; pp. 171-189.

89. Tomaštík, J.; Saloň, Š.; Piroh, R. Horizontal accuracy and applicability of smartphone GNSS positioning in forests. For. Int. J. For. Res. 2016, 90, 187-198. [CrossRef]

90. Merry, K.; Bettinger, P. Smartphone GPS accuracy study in an urban environment. PLoS ONE 2019, 14, e0219890. [CrossRef] [PubMed]

(C) 2019 by the authors. Licensee MDPI, Basel, Switzerland. This article is an open access article distributed under the terms and conditions of the Creative Commons Attribution (CC BY) license (http:/ / creativecommons.org/licenses/by/4.0/). 



\title{
Importance of Social Media in the Image Formation of Tourist Destinations from the Stakeholders' Perspective
}

\author{
Carlos de las Heras-Pedrosa ${ }^{1}$, Elena Millan-Celis ${ }^{1}$, Patricia P. Iglesias-Sánchez ${ }^{2}$ and \\ Carmen Jambrino-Maldonado ${ }^{2, *}$ \\ 1 Department of Advertising and Public Relations, Universidad de Málaga, 29071 Málaga, Spain; \\ cheras@uma.es (C.d.1.H.-P.); elenamillan@uma.es (E.M.-C.) \\ 2 Department of Economics and Business Administration, Universidad de Málaga, 29071 Málaga, Spain; \\ patricia.iglesias@uma.es \\ * Correspondence: mcjambrino@uma.es
}

Received: 6 April 2020; Accepted: 14 May 2020; Published: 17 May 2020

\begin{abstract}
This research aimed to provide a more informed and systematic basis on which to develop the positioning strategy in social media due to the interactive capacity and influence that social media has in the success of tourist destinations. In particular, we investigated the role of stakeholders. We carried out an exploratory study using a mixed method which included interviews and an analysis of the activity conducted on the official social media accounts (Facebook, Twitter, and Instagram) of the Spanish regions of Andalusia, Catalonia, and Valencia. The findings provide insight into how tourist destinations promote their image through the use of social media. Social media was found to be a strategic platform for enhancing brand image and achieving tourist engagement. Additionally, the role of stakeholders in supporting and facilitating the image destination strategy is worth highlighting. This study shows that the results achieved by social media can be improved by identifying all stakeholders and defining a content generation strategy by integrating and adding value.
\end{abstract}

Keywords: tourist destinations; social media; destination image; stakeholders

\section{Introduction}

Tourism is an economic activity that represents an important revenue source globally and is the third most important activity in the exportation category. According to the Spanish Instituto Nacional de Estadística (INE) [1], Spain banked 87,856 million EUR in tourist revenue, an increase of 3.3\% compared with 2017.

The economic significance of tourism, along with the spread of globalization, given the constant demand for information in this sector, makes the promotion of tourist destinations fundamental. Yet, the speed of accessing information and the fast updating of technologies increasingly alter the behaviours of users and directly affect the business models of tourist destinations.

According to INE [1] data, currently, over $80 \%$ of users search for information on products and services prior to making a purchase [1]. This is the case when it comes to travel. Thus, the process of planning the trip is increasingly being more dominated by online tools and travellers' opinions.

The tourist industry is one of the sectors where the use of new communication technologies has had the greatest impact [2,3]. Therefore, global tourist destinations are competing to attract tourists. The main aspects involved in attracting travellers and enhancing the visibility of cities are having an online presence and successfully managing social media. However, it is not enough just to understand how tourist destinations use social media to promote themselves. It is necessary to know how stakeholders contribute to the promotion of tourist destinations and brand image. 
According to the Annual Social Media Report [4], eight out of every 10 users consider social media to be a good setting to interact with brands. Due to the economic crisis and the rise of social media, destinations have increased their online communication investment to the detriment of traditional methods. This is the reason why this study solely focuses on the online dimension of promoting tourist destinations.

It is well known that the literature about destination image [5-14] and social media in tourism [15-17] is very extensive. Especially, there has been an increase in studies in recent years, as both concepts have been adopted together [18]. However, to date, there has been little research focused on the tourist destination brand image development concept from the stakeholder point of view. Many studies have been based on how destinations use social media to promote themselves and boost their image [19], the image perceived by tourists as a consequence of social media strategies carried out [20], or how tourists become co-creators of the image of a destination though social media platforms [21-23]. However, there are other stakeholders that have been unnoticed or simply not studied through a holistic and integrated manner. This is the reason why our work uses the triple helix model to address this issue as a central axis.

The main objective of this research work is to provide insight into how tourist destinations promote their image through the use of social media and how they communicate with stakeholders. We also consider what type of content is shared to the public.

This study is divided into six sections. After this introduction, a review of the previous literature focused on social media used by tourism destinations is made and some research questions are asked. Secondly, an approach based on the stakeholder perspective using a mixed method is introduced in the methodology section. Specifically, qualitative content analysis and interviews are combined with quantitative data obtained using online tools. The fourth section deals with the empirical work and shows that social media can be used as strategic platforms to enhance brand image and generate value. The fifth part contains the main contribution. It should be pointed out that the role of stakeholders supports destinations in their image strategies. In addition, all content generated by users (UGC) involves tourists who help to form the image of a destination through social media. Finally, some limitations of the research and tips about the use of social media by tourism destinations are proposed.

\section{Literature Review}

\subsection{Social Media in the Development of Destination Image}

The World Tourism Organization [24] defines a tourist destination, from a merely geographical perspective, as follows: "a place where demand travel to consume the tourist product" (p. 20). Although tourist destinations have been widely explored in different studies, it is recognized that, as a multiactivity grouping, they compete based on a spatial cooperation strategy to offer integrated tourist products that are assessed and judged by potential tourists; this is significantly broader than the previous definition [25]. Others, such as Murphy, et al. [26], consider tourist destination to be products but offer an unparalleled experience to tourists. Gunn and Var [27] uses the term "experience" when attempting to understand what a tourist destination is. This is the result of a process where tourists use multiple tourist services during their visit.

Therefore, a tourist destination should not be based simply on a geographical definition $[14,24]$ or on an agglomeration of services or products [26]. Tourist destinations contain the six As: attractions, accessibility, amenities, available packages, activities, and ancillary services [28].

From a tourism stakeholder approach, a destination is defined as an open system of multiple interdependent stakeholders, among whom different interactions occur and whose decisions and actions have different levels of influence and impact on others [29].

Destination image has acquired a special key role in diverse research fields such as anthropology, sociology, geography, and marketing due to the impact that it has on human behavior [30]. 
Destination image is a concept that includes the sum of all the beliefs, ideas, and impressions that a tourist has about a destination [14,31]. Previous studies argued that it plays a crucial role in the configuration of tourist preferences and opinions regarding visiting tourist destinations [32]. Destination image has been used as a strategy by destination marketers to differentiate their brand from other competitors $[33,34]$. Considering the intangible nature of destination experience, Pike [35] stated that an image is one way to compete with other destinations.

At this point, it should be highlighted that destination image is a rather multidimensional and complex concept that involves both tourist supply and demand forces $[13,36,37]$. The characteristics of the cultural and natural heritage of a destination are the fundamental attributes that define its identity and, therefore, contribute to the formation of its image as a tourist destination [38].

Iconic elements and attributes can be considered tools for the economic and sustainable development of a tourism destination [39]. These elements of authenticity and mental insight build the image of iconic attractions [40-42] and serve as universally recognized symbols or represent their locations, culture, and natural heritage, promoting a positive image between visitors and local residents.

Destinations that include iconic elements in their promotions such as culture, heritage, gastronomy, or buildings and attractions provide a memorable image that potential visitors will associate with them [43]. The use of tourist icons is common in the positioning of the destination and are the result of a long-term communication and marketing process that involves stakeholders [44].

The classification of a place as a tourism destination has been widely identified as being a result of characteristics and emotional evaluations associated with it. Gallarza et al [30] stated that destination image is complex, multiple, relativistic, and dynamic. Boo, Busser, and Baloglu [45] demonstrated the brand of a destination with a model oriented toward the promotion and communication of all positive values that a destination can offer to tourists. They found that the more attractive the destination image is, the more likely a destination will be chosen [46]. These authors stated that an effective communication strategy will project a strong image online as well as facilitate the differentiation of a destination from its competitors.

One of the research subjects in the area of tourist destinations is the capacity of Destination Marketing Organisations (DMO) to develop or coordinate the destination brand [47,48].

In her critical dissertation, L'Etang [49] summarized the relationship between tourism and DMO in three points: First, the impact of tourism in terms of the social and economic development of the destination and how DMO must contribute to the sustainable development of a destination; second, the vulnerability of tourism in the face of crises because of its intangibility. The tourism sector is very sensitive to actions, crisis or events. A clear example is the catastrophic effect of terrorist attacks carried out in tourism destinations [50]. Third, L'Etang [49] placed great emphasis on the promotion of a destination by means of a mega-event or iconic attributes.

The perceived destination image has been said to be composed of three components over the last few decades: affective, cognitive, and behavioral [14,36,51]. Once an overall tourist destination brand image is formed, a tourist is able to make a destination vacation choice; the brand image directly affects tourists' intentions to visit a destination [52,53].

Value generation can be provided by social media interactivity, therefore enhancing the destination image in tourists' minds [17]. However, DMOs should be consistent in their use of social media to create a positive image among all stakeholders.

The Internet has become an essential tool for users in the decision-making process, allowing them to search for information on products and services, to compare and assess the alternatives, and finally, to make bookings on the spot [54]. Likewise, "Web 2.0" adds a wide range of electronic applications (social media sites, online recommendation sites, blogs, and video and photo sharing platforms) that enable interactions between users and between users and companies [55].

Therefore, something that is quite clear is that using social media means having an element that enables interactivity. As Roca [56] explained, "It is not enough to be present and argue, 
starting conversations and listening is also needed, not merely [telling the users] things because what they want is to be allowed to be involved in opinion forming" (p. 70). Based on the above, the following research questions were formulated:

RQ1. What kind of content is shared by the official profiles of destinations selected in social media?

RQ2. How is social media used to promote tourist destinations?

RQ3. How does social media management affect the official profiles or accounts analyzed in terms of engagement?

The appearance of Web 2.0 has allowed tourists to have access to and control powerful mouth-to-mouth electronic tools and has given them the opportunity to express personal opinions to an audience of millions of possible other tourists [15,57].

DMOs can use social media as a place where they can obtain information about their needs as consumers and establish the relationship between the brand and consumers at an insignificant cost $[58,59]$. It can even be used to promote their brand image or create the idea that their destination is the next destination that a tourist needs to visit [7,60] In line with Ren, Pritchard and Morgan [61], tourism destination brand image development helps to improve marketing effectiveness and attract visitors [61-63]

Social media is also recognised as a communication and public relations tool [64] and, as such, it allows effective communication of destination brands and aids in the development of a relationship with users [65]. Furthermore, many studies reinforce the idea that conversations between users and tourist accounts in social media help to create territories and allow the latter to identify with the users [66-68]. This shows that social media has positive effects on the relationship between a user and a brand, which leads to greater credibility and loyalty [68]. In recent studies [16,22], the impact of destination branding has been highlighted. Destination image is a factor that influences tourist destinations in a positive way [8-10]. As a consequence, social media should be considered as an active component in the development of destination image $[69,70]$.

Social media is considered a useful tool for DMO even though its use is often limited or not fully exploited [18,71]. A greater and better effort may be needed to bring people to the brand community based on social media and to retain them as loyal consumers [72]. This leads us to propose the following hypotheses:

H1. Tourist destination promotes its image on social media through its icons and main attributes.

H2. Social media supports the management of destination image, generating useful content for tourists.

The communication department takes this concept in account. The Andalusian Tourism Promotion Plan of Junta de Andalucía [73] acknowledges that "social media now plays a key role in the everyday life of a great part of the population, both in Spain and abroad. All economic sectors consider social media to be a fundamental factor to promote their business and that is no less true for tourist activity" (p. 75).

\subsection{User-Generated Content}

The impact of the content generated by users is growing rapidly and its role has become increasingly more fundamental for the success of a destination [12,74]. This was partly explained by De Bruyn and Lilien [75] who reinforced the idea that this type of content is under high demand and creates expectations about a tourist destination [75,76]. User-Generated Content (UGC), especially regarding tourist experiences, is transmitted through social media [6], allowing tourists, particularly younger people, to be involved in forming the image of any travel destination [16,71,77].

UGC in social media can be highly useful for understanding the needs and wants of tourists and specific suggestions for the products/services they consume [78]. Assessing individuals' motivations allows their choices, needs, and expectations to be understood, and this has been widely explored in tourism $[79,80]$. 
Blogs, social media, and videos are some of the elements that allow the users themselves to become influencers [81]. According to Standing et al. [82], travel 2.0 has been revolutionized because there is a large amount of UGC in the form of travel blogs and online travel reviews (OTR) that allows other users to experience the events that travellers share first-hand through social media platforms during their stay at a destination.

Theoretical frameworks allow us to accept that all content created by users takes part in the processes of building and promoting destination image [12,23,55,77,83,84].

According to Marine-Roig and Clavé [85], tourism data are considered to be a good source of information for national tourism organizations (NTOs) and other policymakers, destination marketing organizations (DMOs), and stakeholders, because they consist of opinions that are freely expressed by tourists who have visited the destination (p. 203).

At this point, another research question emerges:

RQ4. User-generated content is the key to

RQ4.1 Gaining more interactions

RQ4.2 Understanding stakeholders' interests and expectations

\subsection{Role of Tourism Stakeholders}

Definitions of the term range from those that only understand stakeholders as actors of organizations [86,87] to other authors who include any actor [88].

One of the first authors to offer a broad stakeholder vision was Freeman [89], who stated that a stakeholder is "any group or individual who can affect, or is affected by, the achievement of a corporation's purpose" (p. vi). Furthermore, there are those who differentiate stakeholders into strategic and moral stakeholders [90]. A reviewing about classifications of other authors has been carried out (Table 1).

Table 1. Types of stakeholders identified by authors. Source: Own elaboration.

\begin{tabular}{|c|c|}
\hline Authors & Stakeholders Identified \\
\hline Timur and Getz [91]; Sun [92] & $\begin{array}{l}\text { Government bodies, tourist and hospitality firms, tourists (the } \\
\text { key pieces to the tourism industry) and residents }\end{array}$ \\
\hline García, Gómez and Molina [93] & $\begin{array}{l}\text { Local residents of the destination, employees, investors, tourists } \\
\text { and travel intermediaries like airlines, travel agencies, and so on }\end{array}$ \\
\hline Sheehan, Ritchie and Hudson [94] & $\begin{array}{c}\text { Three pertinent destination stakeholders-the city or } \\
\text { government, hotels, and the destination marketing } \\
\text { organisations (DMOs) }\end{array}$ \\
\hline Bitsani and Kavoura [95] & $\begin{array}{c}\text { Tourism hosts a community including locals who are also } \\
\text { tourism entrepreneurs }\end{array}$ \\
\hline Simpson [96] & $\begin{array}{l}\text { Government, private sector, national government organisations } \\
\text { (NGOs) and communities }\end{array}$ \\
\hline Clarkson [97]; Dodds and Ko, [98] & Multiple primary and secondary tourism stakeholders \\
\hline
\end{tabular}

Six large groups of stakeholders common to all were identified (Table 2): the public sector, private sector (tourism companies and other local businesses), tourists (international and domestic), civil society [29,99], and opinion leaders and universities [100]. 
Table 2. Examples of stakeholders from tourism destinations observed in the study. Source: Own elaboration.

\begin{tabular}{|c|c|}
\hline Primary Stakeholders & Stakeholders on Tourism Destinations \\
\hline Public sector (DMOs) & $\begin{array}{l}\text { Public institutions and government agencies that } \\
\text { carry out strategies to promote tourist regions }\end{array}$ \\
\hline Opinion leaders & $\begin{array}{c}\text { Influencers, politicians, businessmen, investors, } \\
\text { directors of media }\end{array}$ \\
\hline Civil society & Citizens from destinations \\
\hline University & $\begin{array}{l}\text { Academics and experts in promoting tourism } \\
\text { destinations in social media }\end{array}$ \\
\hline Tourists & $\begin{array}{l}\text { National and foreign tourists with social } \\
\text { media accounts }\end{array}$ \\
\hline Private sector & Companies and entrepreneurs in the tourism sector \\
\hline
\end{tabular}

This is, in part, essential, since, as stated in the Stakeholder Theory (1984) [89], the knowledge of the different agents that interact in the participatory procedures of tourist destinations is necessary for the understanding of their interests and expectations, as well as the interdependencies and interactions that occur between them.

In tourism, considering the role of stakeholders in communication management is important to achieve a positive image of the destination $[49,101,102]$. Thus, the following research question is proposed:

\section{RQ5. How do stakeholders participate in the promotion of a tourist destination?}

Sautter and Leisen [103] claimed that, in destination management, all stakeholders should be considered and this will lead to significant returns of tourism in the long term $[104,105]$. Stakeholder management of investor, tourist, and resident attractions first requires the identification of expectations and their interests [106].

As Saxton and Waters [107] noted in their recent work, the increasingly wide adoption of social media offers the opportunity to observe stakeholders' online engagement corresponding to organizational communication. Capriotti [108] stated that the use of indicators is key when analyzing and auditing the corporate image perceived by the different stakeholders as well as the quality of the interactions and the real effects of the corporate identity.

Gartner [34] made important contributions by proposing destination image as a cyclical process in which stakeholders play a key role [109]. Chon [110] suggested that it depends particularly on tourists' cognitive perception, while other authors [14] considered that both operate together to produce a composite destination image. The current trend in the tourist market is that tourists have become creators of original content for tourist destinations and, therefore, have become very powerful and influential stakeholders [14,111,112].

Marketing efforts should be tailored to the needs of each stakeholder (tourists, residents, tourism sector and government); thus, diluting the image of each of the various stakeholders is essential for the success of destination promotion $[11,113]$.

\section{Methods}

All information was gathered using a qualitative approach through interviews combined with a quantitative approach with the data obtained through online tools and content analysis. The goal of these mixed methods was to provide useful practical recommendations for all Spanish tourist destinations on the use of social media. Mixed methods are a research design (or methodology) in which a researcher collects, analyses, and mixes (integrates or connects) quantitative and qualitative data in a single study or a multiphase program of inquiry [114]. 
Bryman [115] also suggested several practical reasons to consider mixed methods such as attractivity to stakeholders, improving the chance of getting funding, etc. In addition, Cai [116] highlighted the fact that the use of mixed methods could be a way to make both researchers and research distinctive in this research field.

In spite of the fact that a mixed methods approach was used, the qualitative part played the main role in this research. Its application is recommended for the studied phenomenon $[9,21]$. Quantitative analysis was chosen to classify and measure the main social media metric, engagement [60], while the qualitative method was used to gain depth in issues that could be lost during statistical processing [18].

This study began with a content analysis, an ideal tool for quantitative research, in order to use certain types of data to formulate reproducible interferences in other contexts [117]. Its usefulness for compiling, processing, and assessing large amounts of information [118] and its frequent use when describing the components of the media messages [119], made this the most appropriate investigation method for our work. We also took the model of de las Heras-Pedrosa et al. [120] into account, as this is a useful way to identify stakeholders in tourist destinations and to determine the research techniques to be applied in each case. The analysis process was partly based on the interactive model of Miles and Huberman [121], using the phases of data collection, data reduction, data display, and conclusions. These phases are shown in Figure 1.

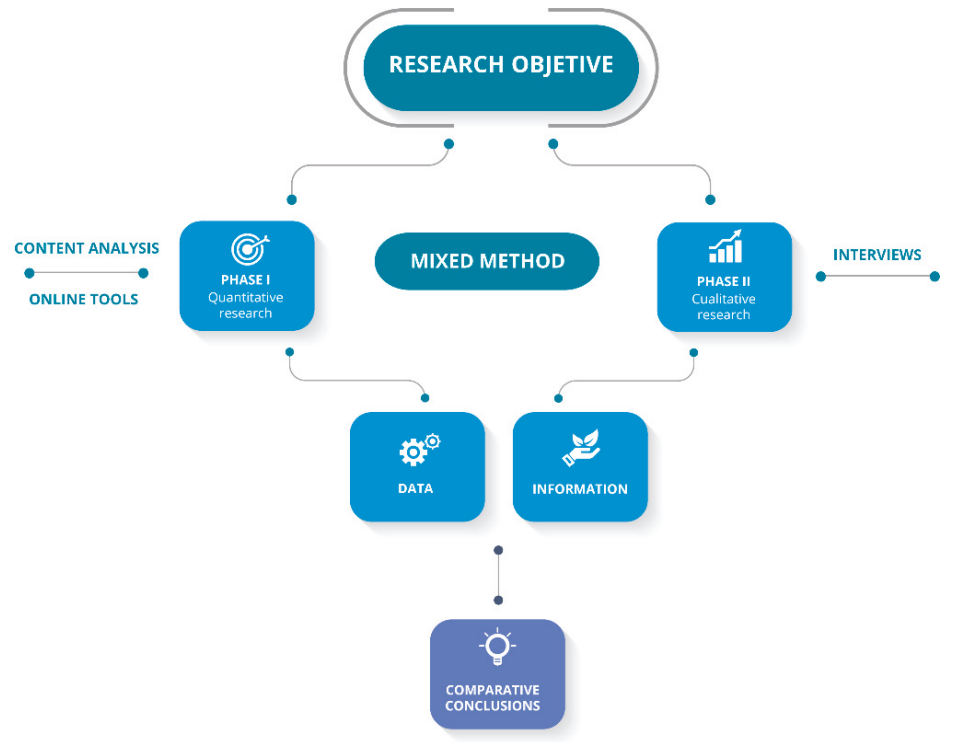

Figure 1. Research phases followed. Source: Own elaboration.

Social media from the official tourism websites of Andalusia, Catalonia, and Valencia were analysed. These destinations were selected as they are the main Spanish mainland tourist destinations according to data from INE [1], as shown in Figure 2. The cases selected may be representative of national tourist, as they include data taken from a large-scale study. The empirical evidence showed that there are extensive relationships and logic among the constructs [122]. Analysing this content means the public sector is taking government-determined actions further with tourism policies that represent government initiatives to develop tourism [123]. 


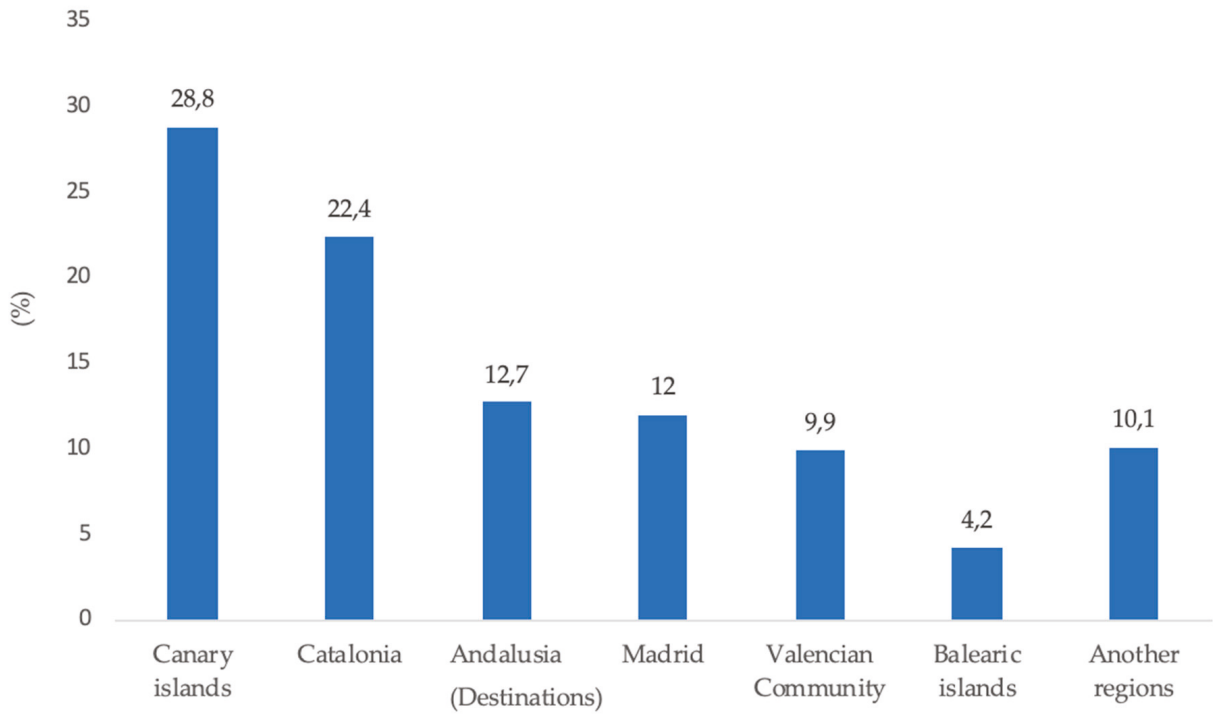

Figure 2. Percentage of tourists who have visited Spanish destinations. Source: Own elaboration based on INE [1].

The social media selected for the analysis were the Facebook, Twitter, and Instagram accounts of the aforementioned destinations, because they are the most frequently used according to the Annual Social Media Report for Spain [4].

Destinations were specifically chosen as comparable destinations due to their similarities. Moreover, all DMOs manage their official social media accounts in Spanish. On the other hand, the Summer Vacation Value Report 2018 of TripAdvisor [124] revealed the 10 main international and national destinations for Spaniards and the three main sun and beach destinations used in this study stood out.

\subsection{Content Analysis}

Content analysis is one of the most frequently used techniques to research the promotion of social media tourist destinations. It is the set of procedures that is used to interpret communication products (messages, texts, or speech) that come from previously recorded communication processes that seek to prepare and process relevant data on the very conditions in which those texts have been produced [125].

The qualitative method mainly used in this work focused on observing, directly and inversely, the posts on the official profiles of the selected tourist destinations on Facebook, Twitter, and Instagram, along with the corporate blog of each one. A total of 1721 posts were quantified from the three communities over a 60-day period, from March 1 to April 31 (2018), a period of great tourist influx due to the Fallas and Holy Week - events and festivities that attract large numbers of tourists. The development of these festivities is considered to be the peak season, and it can represent what happens in destinations at other times of the year. In total, exactly 200 publications, taken randomly within the sample, were analysed. They were typed and recorded, and aspects such as general data, tourist destinations, social media, dates, and posts and their content were analysed. The content included the resources used (photos or videos) and what was transmitted, for example, information and suggestions.

The analysis of the data was based mainly on interview transcripts with the support of Atlas.ti software for qualitative analysis. This IT tool is useful for understanding the behavior and strategies of tourism destinations in social media. The analysis of interviews was based on the grounded theory 
approach [126-128]. First, all publications were quantified, and the frequency and types of content shared were the focuses. Finally, the level of engagement was stored and coded. Codification was conducted using the following procedure: these topics were linked with tags (word or sentences) that described the essence of the specific question. This meant that the most relevant topics from previous literature were used to support this proposal. The grounded theory approach, together with the proposed data structure, showed the dynamic relationships between concepts, themes, and dimensions. In the conclusion and discussion sections, the resulting model is compared with the existing empirical framework to highlight the contribution of this research work to the phenomenon of social media management in order to promote destinations.

Furthermore, the aim of this study was to delve further into the most promoted areas in social media and, therefore, six specific categories to be observed in the posts or tweets were defined (Figure 3): nature, tangible heritage, urban landscape, intangible heritage, leisure, sun and beach, businesses, sports, technology, service, tourism agenda, and non-tourist information. Additionally, words such as happiness, thrilling, daring, energetic, imaginative, and charming were used to analyze the alleged emotions transmitted in each of the posts.

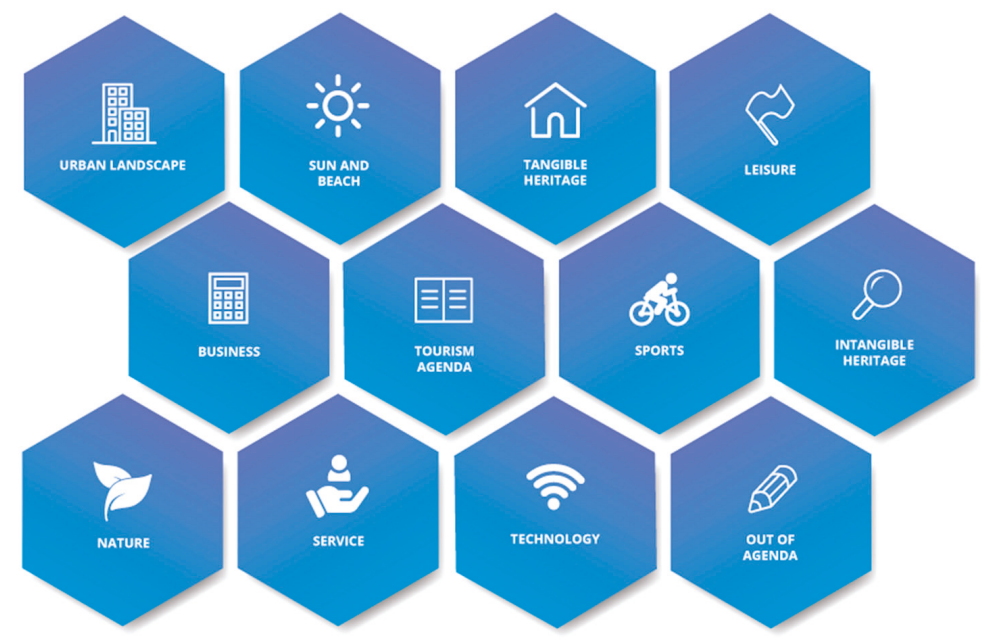

Figure 3. Content categories of posts. Source: Own elaboration based on Paniagua et al. (2019) [129].

The categories and datasheet used for the content analysis were based on the proposal by Paniagua et al. [129]. They were both applied specifically when identifying the theme of each social media post and the types of tourism promoted. In addition, the specific codes selected to determine the above categories were deduced from this study. To ensure inter-coder reliability in this analysis, in accordance with Olson et al [130], two researchers independently coded the content of the official SM accounts studied. After the whole process, a selected number of content units were compared to test the reliability.

\subsection{Interviewing Experts in Promoting Online Destinations}

Interviewing experts is one of the most common qualitative techniques used. In this case, we opted to use interviews to allow in-depth contact to be collected from the architects of the implementation of social media strategies (Table 3). All interviews were carried out by telephone. 
Table 3. Questionnaire and objectives in the interviews. Source: Own elaboration.

\begin{tabular}{|c|c|}
\hline Questions & Objectives \\
\hline $\begin{array}{l}\text { The first questions were on the personal details of the person } \\
\text { being interviewed: name and surname(s), professional profile, } \\
\text { and institution to which they belong. }\end{array}$ & These initial questions identified the experts. \\
\hline $\begin{array}{l}\text { Block 1: How important do you believe it is to implement a } \\
\text { social media strategy for tourist destinations? }\end{array}$ & $\begin{array}{l}\text { The aim of this question was to get an overall picture of how } \\
\text { the interviewees perceived the management of the social } \\
\text { media communication. }\end{array}$ \\
\hline $\begin{array}{l}\text { Block 2: Score the social media strategy established for each of } \\
\text { the selected destinations from } 1 \text { to } 10 \text {. }\end{array}$ & $\begin{array}{c}\text { This question sought to establish the experts' and professionals' } \\
\text { Assessment of social media management }\end{array}$ \\
\hline $\begin{array}{l}\text { Block 3: What are the reasons for using social media to a great } \\
\text { extent when promoting a destination (most effective content } \\
\text { for each type of social media, tone etc.)? }\end{array}$ & $\begin{array}{l}\text { The aim of this section was to analyse the interviewees' } \\
\text { perceptions of how a tourist destination should use } \\
\text { social media. }\end{array}$ \\
\hline $\begin{array}{l}\text { Block 4: How would you identify that stakeholders are taken } \\
\text { into account when plotting destination strategies? }\end{array}$ & $\begin{array}{c}\text { This aim of this question was to identify the way in which } \\
\text { stakeholders are considered when promoting a } \\
\text { tourist destination. }\end{array}$ \\
\hline $\begin{array}{l}\text { Block 5: If you had to make a recommendation or give general } \\
\text { advice to improve the promotion of a destination, what would } \\
\text { it be? }\end{array}$ & $\begin{array}{l}\text { This final question sought to pinpoint the most important } \\
\text { thing about social media management by a tourist destination }\end{array}$ \\
\hline
\end{tabular}

Interviews were based on the triple helix model [131,132], a collaborative model between the private and public sectors and the university. This model was used to analyse new aspects of knowledge based on collaborative relationships between the three entities.

In this case, the triple helix method was fundamental when choosing whom to interview (Figure 4) to ensure that the interviewees represented all stakeholders. In total, eight interviews from experts belonging to the public sector, the private sector, and university were conducted.

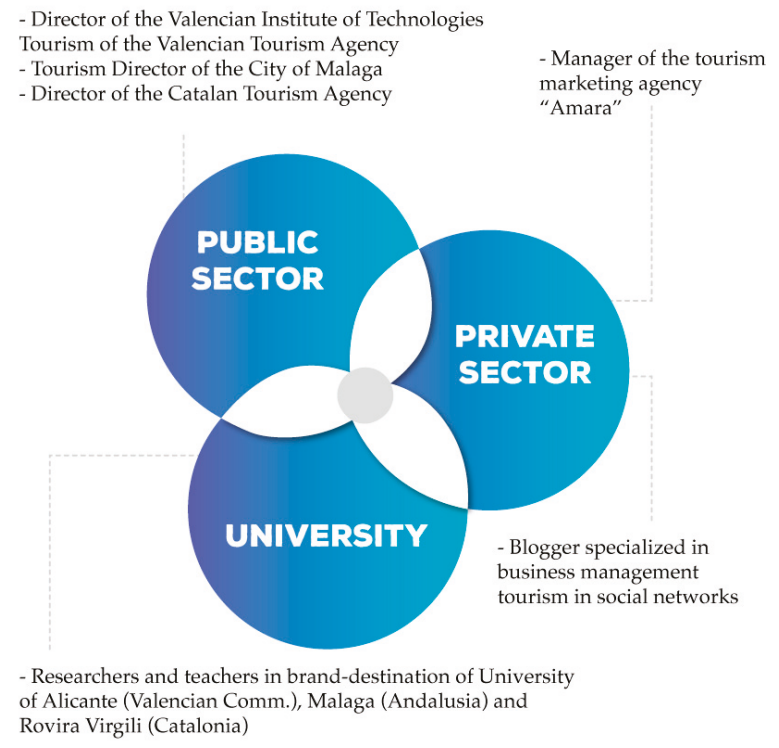

Figure 4. Triple Helix Model and managers interviewed. Source: Own elaboration.

All interviews focused on the strategies used to promote destinations in those spaces, the communications posted, and the interactions obtained. 


\subsection{Analysis Based on Data from Online Tools}

The quantitative part of the analysis was based on different tools that can provide data to be interpreted in order to take stock and put forward proposals. Those tools, or rather the data in social media profiles obtained with them, were used to establish the activity of the users with those profiles and that of their administrators.

It would not be the first time that online tools have been used to analyse the social media activity of tourist destinations, as they have been used to measure data quantitatively and assess the effectiveness of social media engagement [133].

On this occasion, Keyhole was used to analyse Instagram and Fanpage Karma to study the interactivity and impact of the content shared in Facebook. Finally, Twitonomy was used to analyse Twitter content, which provided very interesting data on the use of links or profile visibility, among others.

\section{Results}

\subsection{Type of Content Shared on Social Media}

A content analysis of all posts was warranted to begin to establish the real activity of the official tourism social media accounts of the three destinations and the communication strategies implemented. Therefore, the content was first analysed according to the attitude deduced from the post, and it was noted that nearly half of the posts sought to provide information to the followers, questions were asked in $19 \%$, and, to a lesser extent, queries were answered and thanks given.

Photographs were the most frequent type of content shared, and they were often accompanied by links to official tourism websites (Figure 5). Computer graphics, which were a mixture of image and text, came in third place and were particularly used for events and other types of calendar activity. However, contrary to what might have been expected, videos were not placed in a top spot.

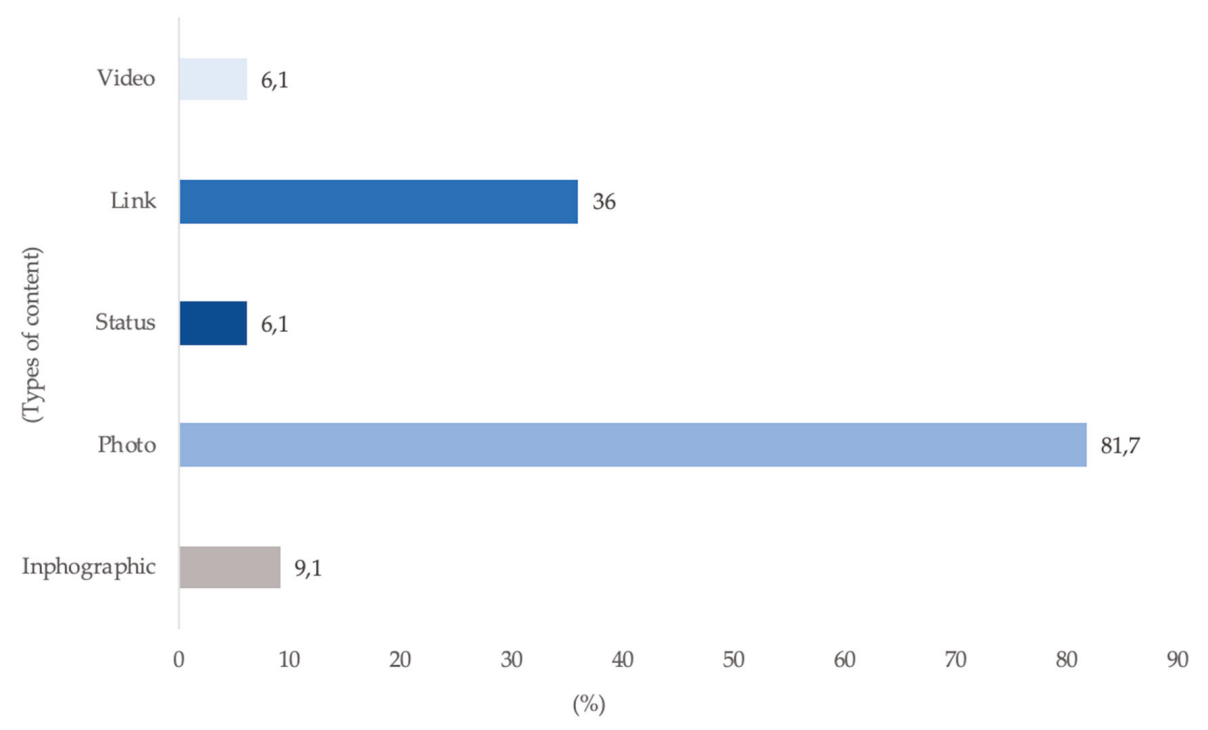

Figure 5. Type of content shared in social media. Source: Own elaboration.

Each theme covered by the posts then had to be considered and analysed in greater depth. Many of the posts (37\%) focused on places that could be visited within each of the destinations in question. Similarly, it should be mentioned that many posts focused on nature $(27.2 \%)$, and $11 \%$ focused on 
architecture. Monuments (10.2\%) were also a central topic of the posts, along with history (4.1\%) and religion (8.8\%). Museums featured less $(2.7 \%)$, despite being a major tourist attraction in many cities.

The data showed that over half of the posts referred to popular culture and traditions, an example of which would be the Fallas in Valencia or Holy Week in Andalusia, which were centre stage for longer periods of time in each of the social media analysed. However, gastronomy was also prioritized, and, as a main tourism attraction, in a great number of Spanish cities, it was covered by nearly $35 \%$ of the publications.

Regarding the types of tourism, most posts (58\%) were on cultural and calendar aspects (Figure 6). Likewise, coastal images, followed by beaches, the sea, and the sun were identified as the most commonly promoted aspects $(13 \%)$. Just over $10 \%$ of the total posts analysed were about mountains or inland tourism. There were fewer posts with religious, sports, and culinary themes. The tourism industry should continue to promote tourist destinations using iconic and unique experiences, and this should incorporate native flora and fauna as well as distinctive built and natural attractions [3].

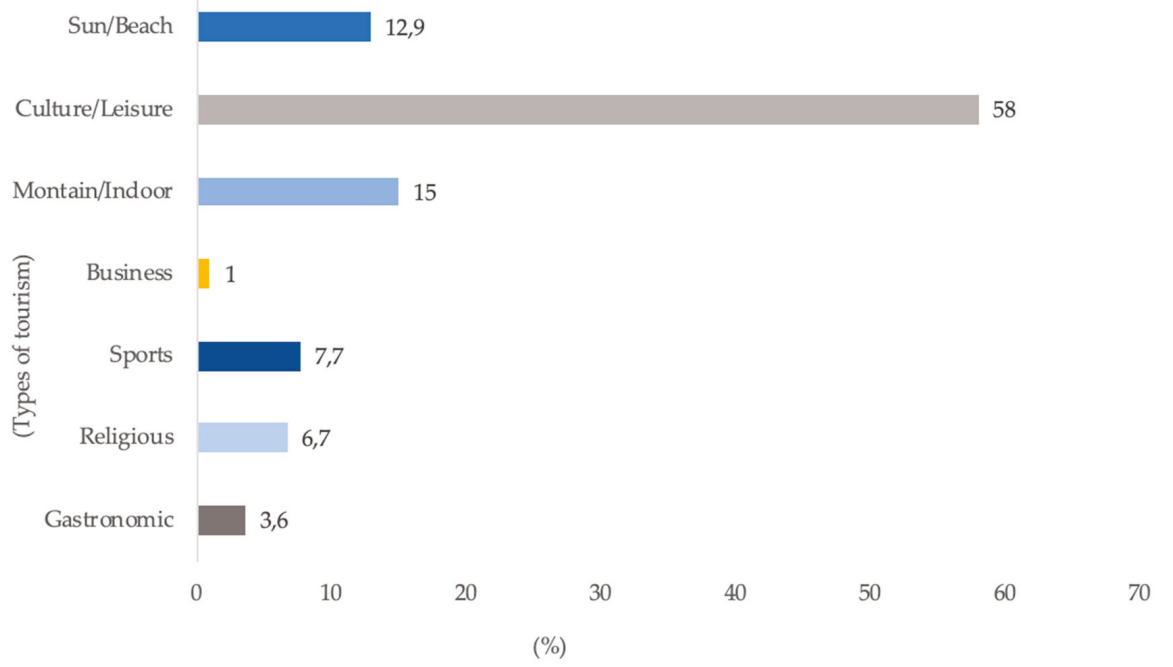

Figure 6. Types of tourism promoted in posts. Source: Own elaboration.

The study also analysed the number of times that new technologies were mentioned, and over $60 \%$ of posts included hashtags or referred to technologies such as "timelapse" in some way.

Hashtags were used to organise events and activities or to organise the information and curious facts about different events and traditions. Posts also recommended and retweeted some television programmes or other third-party content, particularly in Valencia.

A very important aspect to analyse was whether the posts were to do with events on the calendar, contained basic tourist information, or whether they were institutional or had nothing to do with tourism. Over half $(60 \%)$ of the content studied was information of tourist interest. However, $20 \%$ referred to calendar activity. It is also significant that the same percentage was non-tourist information; in other words, the posts were, for example, "Good morning" updates.

In general, the activity of the three official accounts focused on proposing plans or providing information on scheduled events. They also directly recommended visiting a specific place in the selected regions. This means that the studied DMOs prioritise showcasing the tourist attractions over promoting their own brands as a destination and triggering emotional impacts to establish an in-depth relationship with the public. 
Furthermore, the posts mention tourist entities and other local or provincial tourism accounts. This may be because their mutual promotion of each other provides greater visibility.

\subsection{Expert Assessment of the Promotion of Tourist Destinations}

It was considered relevant to compare the data collected in the content analysis period with the assessments of experts whose day-to-day work means they are familiar with promoting tourist destinations through social media.

The experts began by assessing the importance of implementing a strategy to promote tourist destination using a particular type of media. They were asked why they considered the management of the social media profiles of the destinations to be essential. There was a great variety of answers, which can be seen in Figure 7.

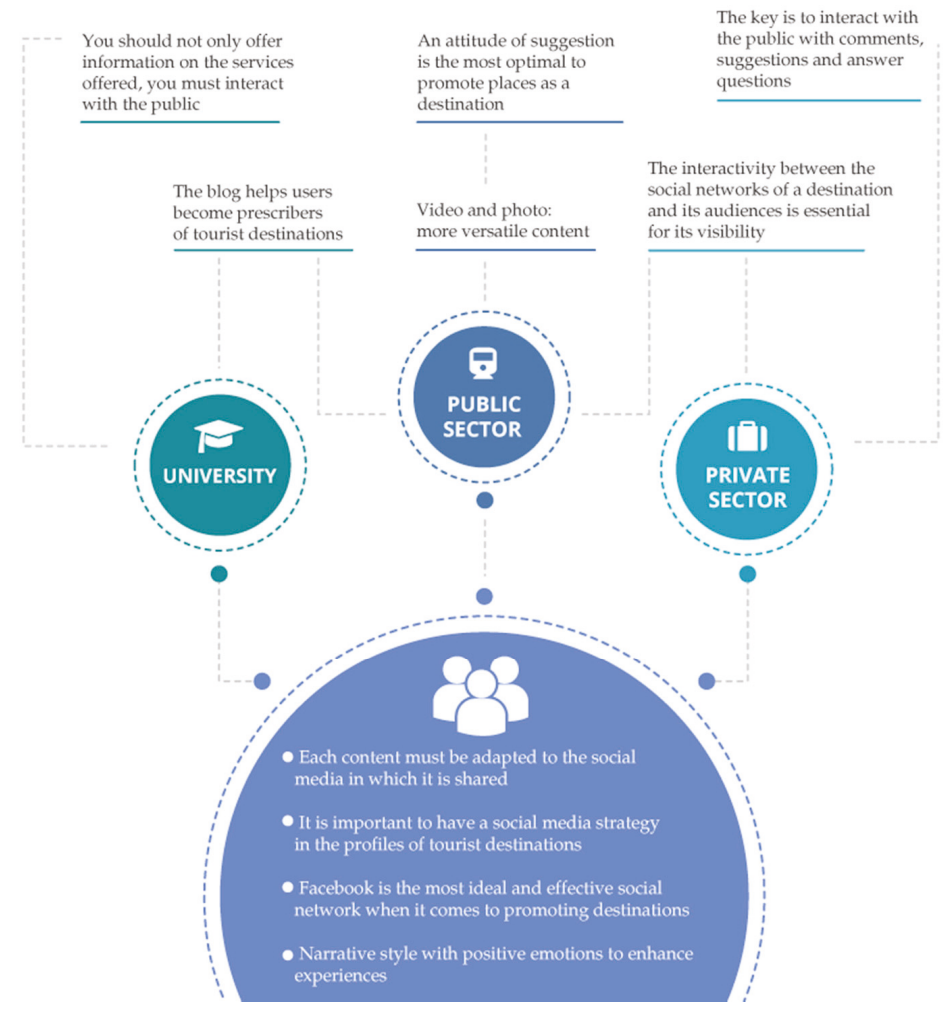

Figure 7. Experts' opinions according to sector. Source: Own elaboration.

The following questions focused on the central area of the research, aiming to analyse the content. First, the majority of the experts agreed that video is the most effective type of content to share on the official profiles of destinations, which coincides with the forecast of the 12th Cisco Visual Networking Index [134], which stated that video would lead the way and would represent $70 \%$ of all Internet traffic in Spain by 2021, compared with $60 \%$ in 2016 . However, they stressed that each type of content has to adapt to each social media platform.

Facebook was the social platform that the experts identified as being the most appropriate and effective when promoting tourist destinations. The academics also considered that in addition to 
information being offered on the available services, interactivity with the general public should be also facilitated and enhanced.

The professionals of the public sector also expressed their opinions on the most effective types of content to use according to the attitude of the message. It was highlighted that a suggestive attitude is the most optimal way to promote a place as a destination. Meanwhile, the comments of the private sector were more decisive. Even though they differed in that regard, they all expressed the same opinion: that experiences need to be enhanced by using a narrative style including feelings and experiences, for example, words such as happiness, thrilling, and daring.

The experts were encouraged to comment on the importance of sharing information from an official and specialist tourism blog through the official profiles of the destinations. Above all, both the academics and the Public Sector professionals considered that tourists are demanding more information on destinations to aid them in preparing their own travel plans, and blogs greatly help to create loyalty if useful information can be found there. Thus, blogs help to enrich the narrative and convert users into influencers based on their experiences.

An aspect in which interviewees from the private and public sectors coincided was that interactivity between the social media profiles of the destinations and their audiences is fundamental for their visibility. Thus, regarding the importance of the customer service channel, they considered it to be important but not vital.

Lastly, all experts interviewed agreed that tourist destinations use social media, above all, as an information channel to disseminate tourist content rather than a way of interacting with tourists to promote specific services of the tourist industry or deal with queries.

\subsection{Data Obtained Using Online Tools}

Within the period analysed, the images, links, and videos were the types of content with the most comments, reactions, and shares on Facebook (Table 4).

Table 4. Comparison of data obtained using online tools on Facebook. Source: Own elaboration.

\begin{tabular}{cccc}
\hline Regions & No. of Fans & Commitment & Interaction with the Posts \\
\hline Andalusia & $71 \mathrm{~K}$ & $0.41 \%$ & $0.22 \%$ \\
\hline Valencia & $194 \mathrm{~K}$ & $0.22 \%$ & $0.11 \%$ \\
\hline Catalonia & $508 \mathrm{~K}$ & $0.87 \%$ & $0.39 \%$ \\
\hline
\end{tabular}

Catalonia had the greatest number of followers with over two posts per day and a very strong weekly evolution (23\%). The interaction was striking, with $0.87 \%$, compared to the Facebook analysis of other communities. Catalonia nearly always posted images on Facebook, as was the case with the other regions analysed.

On Twitter (Table 5), Valencia was in second place with a large number of fans; however, it was the one that posted the most tweets, on average. The past performance of the account of Catalonia was very different; it obtained more "likes" per post even though it had fewer fans.

Table 5. Comparison of data obtained using online tools on Twitter. Source: Own elaboration.

\begin{tabular}{ccccc}
\hline Regions & No. of Fans & Average Number of Tweets & Average Number of Likes & Average Number of Retweets \\
\hline Andalusia & $94 \mathrm{~K}$ & $51.9 \mathrm{~K}$ & 21.2 & 560 \\
\hline Valencia & $96.7 \mathrm{~K}$ & $23.1 \mathrm{~K}$ & 20.1 & 6.2 \\
\hline Catalonia & $66 \mathrm{~K}$ & $17.8 \mathrm{~K}$ & 27.6 & 24.1 \\
\hline
\end{tabular}

Valencia was the winner in terms of the number of fans, but the fact that it did not obtain many retweets was remarkable. Retweeting from the official Twitter accounts of the three destinations was to 
local or regional tourism accounts, which could be to promote each other. The main type of activity conducted by all of them was, above all, showcasing plans through Twitter.

On Instagram (Table 6), significant engagement was achieved. In the same way as with the results obtained from the content and expert analysis, images were the content that best functioned on Instagram and, specifically, carrousel photos, which are several images put together in a single post, were used.

Table 6. Comparison of data obtained using online tools on Instagram. Source: Own elaboration.

\begin{tabular}{cccccc}
\hline Regions & No. of Fans & $\begin{array}{c}\text { Average Number } \\
\text { of Likes }\end{array}$ & $\begin{array}{c}\text { Average Number } \\
\text { of Comments }\end{array}$ & $\begin{array}{c}\text { Average } \\
\text { Engagement }\end{array}$ & Total Posts \\
\hline Valencia & 28,839 & 28,558 & 3.4 & 750 & 993 \\
\hline Andalusia & 28,646 & 560 & 5.8 & 400 & 1071 \\
\hline Catalonia & 248,712 & 5035 & 24.1 & 8000 & 2580 \\
\hline
\end{tabular}

Catalonia was the region that achieved the greatest engagement on Instagram with an average of 5035 likes and 24.1 comments per post.

Quantitative analysis allowed us to determine the content generation focus of these official tourism accounts and how they use their own icons or attributes to promote destinations. @ComunitatValenciana suggested the use of the hashtag \#navidadenvivo or \#semanasanta for their followers, while @CatalunyaExperience chose \#Terresdelebre. The most popular hashtags are shown in the following Figures 8-10. All regions used hashtags chosen according their popularity and their coincidence with the names of specific cities, for example, \#tarragona in Catalonia or \#sevilla in Andalusia.

\section{\# Hashtags most used}

$\begin{array}{lll}\text { \#mediterráneoenvivo } & 180 & \text { \#gastro } \\ \text { \#planes } & 64 & \text { \#cvquiz } \\ \text { \#quizcv } & 50 & \text { \#conniños } \\ \text { \#semanasanta } & 11 & \text { \#navidadenvivo } \\ \text { \#btt } & 9 & \text { \#receta }\end{array}$

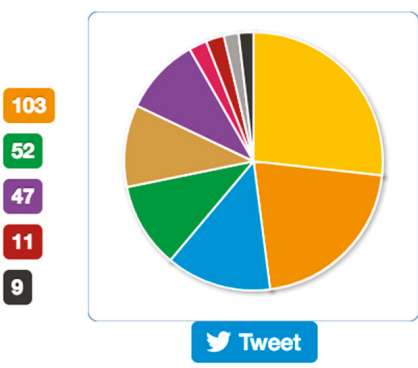

Figure 8. The most frequently used hashtags by the DMO in Valencia. Source: In-house elaboration with support of Twitonomy.

\section{\# Hashtags most used}

$\begin{array}{lllr}\text { \#incostabrava } & 91 & \text { \#barcelona } & \mathbf{6 1} \\ \text { \#catalunya } & \mathbf{5 5} & \text { \#catalunyaexperience } & \mathbf{5 1} \\ \text { \#bondia } & 50 & \text { \#terresdelebre } & \mathbf{5 0} \\ \text { \#girona } & \mathbf{8 4} & \text { \#tarragona } & \mathbf{3 0} \\ \text { \#deltadelebre } & 80 & \text { \#ebre } & \mathbf{2 9}\end{array}$

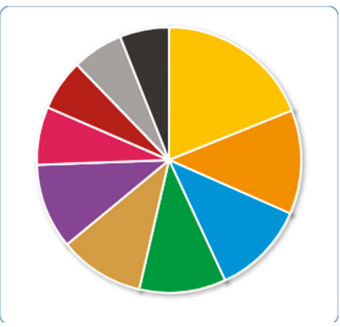

Figure 9. The most frequently used hashtags by the DMO in Catalonia. Source: In-house elaboration with support of Twitonomy. 


\section{\# Hashtags most used}

$\begin{array}{lcl}\text { \#viveandalucía } & 861 & \text { \#andalucía } \\ \text { \#intensamente } & 288 & \text { \#sevilla } \\ \text { \#málaga } & 168 & \text { \#cádiz } \\ \text { \#córdoba } & 158 & \text { \#instagram } \\ \text { \#granada } & 186 & \text { \#huelva }\end{array}$

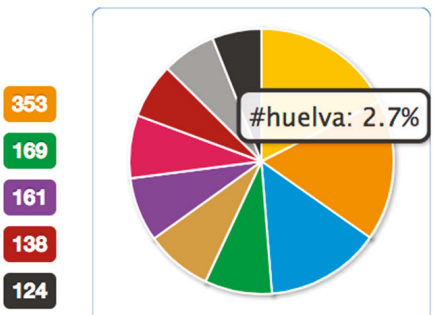

Figure 10. The most frequently used hashtags by the DMO in Andalusia. Source: In-house elaboration with support of Twitonomy.

The information provided by the following word clouds (Figures 11-13) generated by the online analysis tool also shows that hashtags, such as \#Jornadas, \#Sant, \#festes, \#festival, \#fiesta, and \#SemanaSantaAndalucía, were used to promote festivities and events in the period analysed.

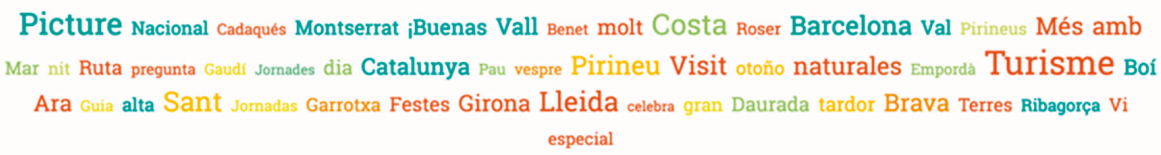

Figure 11. Cloud tag of the words used most frequently on Facebook by Catalonia. Source: In-house elaboration with support of FanPage Karma.

\section{hoy Gastronómico Alicante Village mundo Festival celebran vela Parque otoño Valenciana Dénia parcs Visit septiembre GVA Race Gastronòmic nueva volver octubre Comunitat espera Turisme Dia Salida Valencia DNA disfrutar city BTT Castellón Benassal Vueta D’na Spain Benidorm Peñiscola centres Blanca semana ocean València Puerto Naturals puertoDeEncuentro Costa MediterranéoEnVivo fiesta mesa}

Figure 12. Cloud tag of the words used most frequently on Facebook by Valencia. Source: In-house elaboration with support of FanPage Karma.

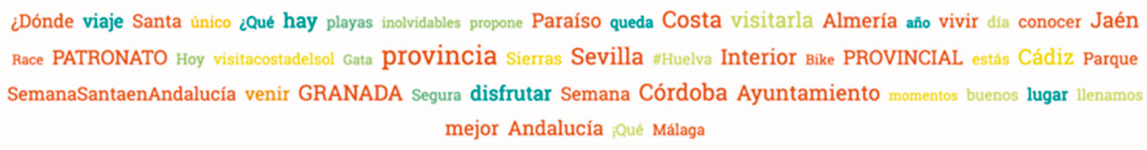

Figure 13. Cloud tag of the words used most frequently on Facebook by Andalusia. Source: In-house elaboration with support of FanPage Karma.

The largest words are the most used by destinations and the smallest are less used. The words in the same colour usually go together in a post.

It was found that some concepts are common among all social media strategies to promote image, mainly cities, moments, or events of special tourist interest and some motivations for tourists when sharing posts about nature, interior aspects, bikes, sailing, or gastronomy. They also tried to link content with positive feelings related to travel: enjoying, relaxing, good times, etc.

The hashtags and their engagement also drew on resources related to knowing the types of social media content from official account of tourist destinations valued most by tourists. Top hashtag engagement was associated with a useful plan such as \#relax or \#spanatural, unique places to visit 
such as \#granada, \#costablanca, and \#acantilados, and festivities or events, such as \#montserrat or \#santatecla (Figures 14-16).

\section{Top Hashtags by Engagements}

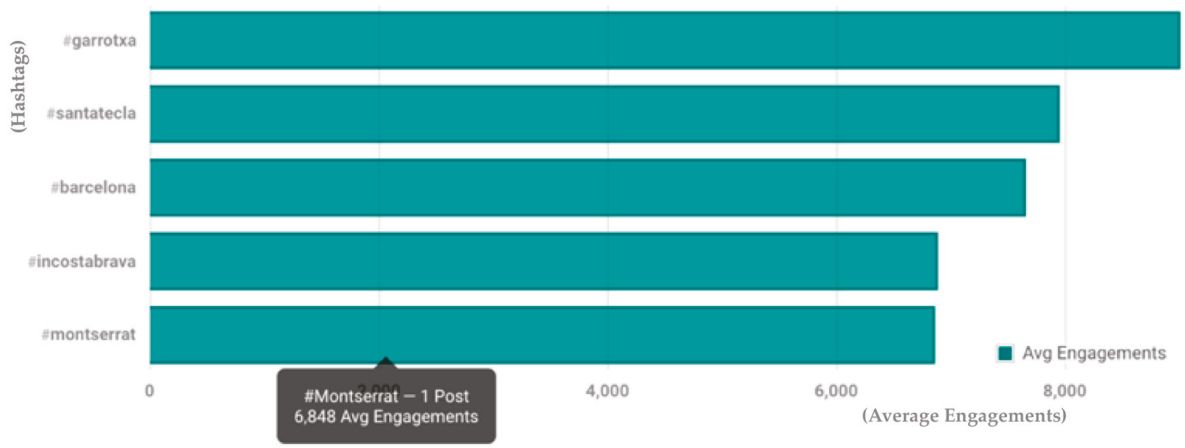

Figure 14. Engagement with hashtags from Catalonia. Source: In-house elaboration with support of Keyhole.

\section{Top Hashtags by Engagements}

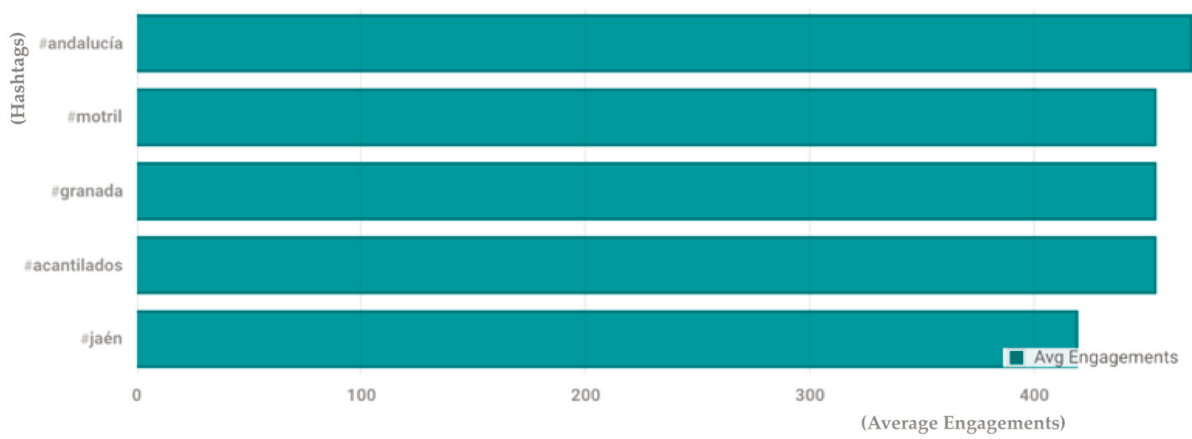

Figure 15. Engagement with hashtags from Andalusia. Source: In-house elaboration with support of Keyhole.

Thus, the great capacity of interactions to drive a destination brand is not being optimized. An association between interactions (likes and comments) and followers was predicted using the Chi-Square Test of Independence $(\alpha=0.05)$. Since the $\mathrm{p}$-value was less than our chosen significance level $\alpha=0.05$, we can reject the null hypothesis and conclude that there is an association between the number of followers and the total number of interactions (likes and comments). 


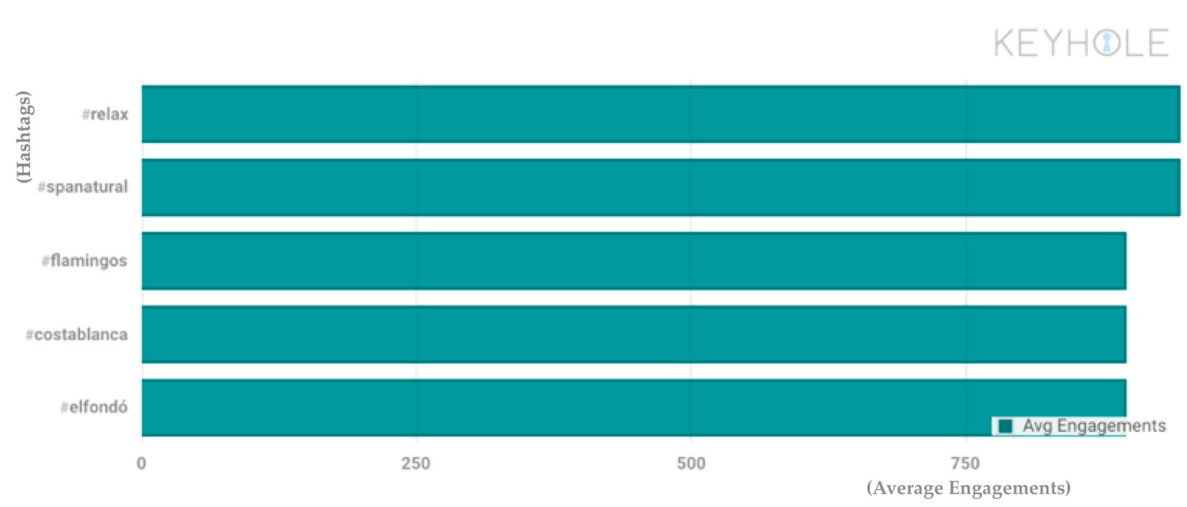

Figure 16. Engagement with hashtags from Valencia. Source: In-house elaboration with support of Keyhole.

\section{Discussion and Conclusions}

This research work provides a number of new insights into the field of destination management through social media, paying particular attention to image creation and promotion as well as stakeholder contribution. First, we revealed that social media effort is concentrated on showing, persuading, and attracting tourists to the icons and attributes of a destination. In some way, destination image is based on this and the social media content reproduces and enhances these features. However, tourist sites and monuments share centre stage with characteristic events and traditions. This finding is in line with studies by Cantos et al. [38] and Bregoli [47] that highlighted the cultural and natural heritage of a region as being fundamental attributes that define its identity and contribute to the formation of its image as a tourist destination. Moreover, special emphasis is placed on experiences, how to enjoy the destination, and the ways in which to do this, depending on who the tourist is (familial, focused on nature, etc.). Consequently, the first hypothesis was validated, giving further support to previous literature results. Baloglu and McCleary [14] highlighted these components as being key factors in the destination image, and more recent research works showed that these factors make up the core of the content on social media $[17,18,22,30]$.The value of events was also suggested by Ghazali and Cai [6], and Pike [35] drew the same conclusion about experiences as this study.

Second, the content analysis and statistical correlations support hypothesis 2 . Social media platforms are interactive and dynamic, and they allow destinations to manage their image $[15,68]$. The content producers are aware of the strategic contribution of social media for their image, and they try to generate useful content for tourists. The empirical analysis provided evidence that more than $20 \%$ of the content shared is focused on proposing plans or providing information about scheduled events. We found that the number of followers, as a direct consequence of attractive content, rather than the number of posts, is not a major factor in whether a positive relation with interactions and interest in the destination on their social media is attained. This issue agrees with the findings of Sanders and Stappers [68] and Luo and Zhong [72] regarding the types of social media content that ensure customer interest and positive effects on relations between users and brands, thereby leading to greater levels of credibility and loyalty, but it also highlights that DMOs should be consistent in creating value in social media to create a positive image for all stakeholders [17,66-68].

All of the abovementioned information also answers our first two research questions. This study provides a better understanding of how social media is used by destinations by identifying the main common topics and strategies of engagement with tourists. 
It should be stressed that there were very similar behaviour patterns in the management of social media of the three accounts analysed. The accounts focused on places of interest in the image strategies; moments or events of special tourist interest; lifestyle attributes or motivations for tourists, such as biking, sailing, or gastronomy, and the use of words to link positive feelings related to travel. As a result of the content analysis, the interactions of the posts in social media were found to differ from what they could be; this has also been a common finding in other studies [18,71]. Referring to content shared by each official account, large numbers of photographs, banners, and images were found with a lot of reactions, comments, and shares. Despite this, videos were the type of content that stood out with regard to interactions, albeit to a lesser extent because much fewer were posted. Videos were the most frequently used type of content. According to the experts interviewed, they are the most effective type of content to share on the official profiles of the destinations. Furthermore, videos are the most reliable way to provide information and are relevant for users who browse the web [135]. It is important for the tourist destinations to continue working on good social media management practices due to their interactive capacity and the influence they have on tourist decision-making [71,77]. This finding is in line with Huertas and Marine-Roig [17], and it also highlights that DMOs should be consistent in generating valuable content in social media to create a positive image among all stakeholders.

Furthermore, the promotion of the whole destination overall should be further encouraged, rather than focusing on the cities separately, while the image continues to be decisive in the differentiation of these and is crucial for value creation. Regarding engagement, it was shown that tourists are motivated to participate when a destination offers a broader and more varied range of attractions and activities, the community interaction is higher, and the content is relevant for their interests and profile (style of travelling). Likewise, gaining tourist involvement through content related to destinations is suggested as an effective resource for building and promoting a destination's image. As result of this, attention should be paid to content generated by tourists on destinations' official accounts as well as outside of these [22].

These conclusions coincide with and complement those of Stepchenkova and Li, [7], because they reinforce the identification of key issues for tourists related to destination choice. Moreover, in line with Huertas and Marine-Roig [17] and Leung et al. [15], it should be emphasised that being present on social media is a great way to promote destinations at a very low price. In any event, this study, as also stated by Usakli et al [19], shows that destinations are still facing challenges in using social media management to showcase their potential and achieve greater success with their strategies. The analysis allows us to conclude that further analysis is needed due the exploratory focus of this study.

The final finding is related to UGC (RQ4), and it is one of the main contributions of this study. Previous literature focused on how UGC is a key factor for managing destination image and general strategies $[5,83-85,136]$. UGC is a fundamental way to involve tourists in forming image destination through social media $[12,71,74,77,137]$. It allows their needs and expectations [78-80] regarding their experience in each destination to be understood and indicates the types of content they value in social media from official accounts and DMOs in charge of promoting destinations [21].

However, the extent analysis was carried out on collaborative and external platforms, which means sources outside of destinations' official accounts. This is the main difference and the source of the novelty in the analysis of the UGC issue in this study. Tourists generate content for destinations' social media as result of posts proposed by DMOs. Currently, destinations are aware of content created by users $[13,85]$, but they are not working hard enough on motivating tourists to contribute to the formation and improvement of a destination's image. This factor cannot be analysed separately, and an integrative and coordinated effort should be used as a brand strategy. As Hidalgo Alcazar et al. [136] discerned, "tourism destinations need to disseminate favorable experiences to tourists, and these experiences include advice and comments from other travellers" (p.162). However, as claimed in this study, the benefits from including evaluations and experiences of tourists on destinations channels can reinforce and improve the engagement with and attractiveness of destinations. Thus, this content becomes a source of information and recommendations since tourists use it to share 
their experiences [138]. Moreover, the content generated by users is actually the key to gaining more interactions [22], and this content is more reliable than a destination's own content [81]. To sum up, one of the keys to the promotion of a tourist destination is the content generated by users. Consequently, it is proposed that DMOs should strengthen and prioritise two-way communication with tourists to better promote their image. It possible to take a similar approach to that described by Roca [56], who recommended the use of a concerted effort to create a structured model for image promotion in order to obtain maximal results from social media.

Last, but not least, some reflections about stakeholders are presented. Stakeholders are a main issue of the initial focus of this study. Although tourism literature has widely discussed the roles of stakeholders and their capacity to construct a positive destination image [49,91,100-102], this study introduced stakeholders' roles in the interactivity of social media. Moreover, the application of the triple helix model for the analysis of stakeholders provides a clear distinction from previous literature. The interviews carried out allowed us to answer RQ5. A better understanding of the crucial role of stakeholders in content creation was also obtained. Most research works have focused on the role of tourists as central stakeholders, but other groups involved in destination activity should be taken into account, especially if the destination image is being analysed $[29,98]$. Based on the results, different stakeholders are valuable sources of trust, and content generated by them could attract potential tourists and contribute to the promotion of a destination. An interesting insight drawn from the study is that all stakeholders seem to be related and, consequently, they do not have to be approached separately. For example, it appears that universities can be a key partner for the creation and promotion of a destination's image in collaboration with DMOs and can be used as a source of knowledge for tourists.

In short, this paper demonstrates that social media platforms are strategic tools for destination branding and image formation and promotion. However, the novel factor of this study compared with previous literature is the acknowledgement that stakeholders play a main role. Tourists are not the only group to take into account. DMOs should have a wide and holistic vision of stakeholders. Therefore, their identification should be aligned with their involvement in social media strategies to achieve the goal of differentiation through a destination's image. If social media has an impact through its content, user contributions could increase and destinations will be able to exploit their attractions (icons, events, experiences) further and improve their positioning in the minds of tourists. Further, the insights provided in this exploratory study demonstrate the challenges that DMOs face in social media management.

\section{Practical Implications}

This research work provides the main practical implications for destination image management. DMOs can better manage and differentiate their social media content strategy through useful content, and the reinforcement of icons from the destination contributes highly to the formation of an image. Likewise, based on experiences and connections between a style of travelling and the type of content can improve performance in terms of a destination's competitive advantage. User-generated content is essential for promotional and marketing activities, as it is an effective way to create trust in tourists' minds. However, the involvement of all stakeholders should be appreciated and taken into account to achieve a successful result for image promotion. The analysis of destination image and the understanding and management of stakeholders' contributions indicated that operational strategies should be established to improve a destination's image promotion and to establish a deep relationship with the public.

\section{Limitation and Future Research Directions}

The current study contributes to the importance of social media in destination marketing and image formation from the perspective of stakeholders in several ways. To delve into a better understanding of the phenomenon it could be suggested to include the visitor point of view. 
It is likely that not all factors that influence the creation of the image of a tourism destination by visitors using social media management were included in the conceptual model of this research.

Third, the active role of social media and the benefits associated with its incorporation into marketing and communication strategies needs to be further examined, since the literature shows that companies have not achieved the full potential of social media, not to mention the limited research that has been done in the tourism sector [139-141].

In addition, despite the results of this exploratory approach with a mixed method, further studies are required.

Some additional areas of study are proposed. Future research could explore how visitors perceive tourist destinations through social media using surveys. This could provide the opportunity to establish a comparison between visitors and the public and private sectors. Secondly, future scholars could analyse the importance of the dimensions of process quality and the quality of results. For example, longitudinal research should emphasize the interactions that occur between the public and the social media of tourist destinations, as this could ultimately give more information about image creation. Social media allows effective communication of destination brands and the development of a relationship between users and these brands [34].

Finally, future studies might consider the association between image representation and other factors that could lead customers to decide to visit one tourist destination over another.

Author Contributions: C.d.l.H.-P., E.M.-C., P.P.I.-S. and C.J.-M. contributed equally to this paper. All authors have read and agreed to the published version of the manuscript.

Funding: This research has received funds from Plan Propio Universidad de Málaga.

Conflicts of Interest: The authors declare no conflict of interest.

\section{References}

1. Instituto Nacional de Estadística. Estadística de movimientos Turísticos en Fronteras (FRONTUR); Instituto Nacional de Estadística: Madrid, Spain, 2019.

2. Buhalis, D.; Law, R.; Law, R. Progress in information technology and tourism management: 20 years on and 10 years after the Internet-The state of eTourism research. Tour. Manag. 2008, 29, 609-623. [CrossRef]

3. Hughes, M.; Martin, S.L.; Morgan, R.E.; Robson, M.J. Realizing Product-Market Advantage in High-Technology International New Ventures: The Mediating Role of Ambidextrous Innovation. J. Int. Mark. 2010, 18, 1-21. [CrossRef]

4. IAB Spain. Annual Social Media Report. 2018. Available online: https://iabspain.es/estudio/estudio-anualde-redes-sociales-2018/ (accessed on 1 September 2019).

5. Marine-Roig, E.; Clavé, S.A. A detailed method for destination image analysis using user-generated content. Inf. Technol. Tour. 2016, 15, 341-364. [CrossRef]

6. Ghazali, R.; Cai, L. Social Media Sites in Destination Image Formation. In Tour. Social Media: Transformations in Identity, Community and Culture (Tourism Social Science Series, Vol. 18); Emerald Group Publishing Limited: Bingley, UK, 2014; pp. 73-86. [CrossRef]

7. Stepchenkova, S.; Li, X. (Robert) Destination image: Do top-of-mind associations say it all? Ann. Tour. Res. 2014, 45, 46-62. [CrossRef]

8. Chen, C.-C.; Lai, Y.H.; Petrick, J.F.; Lin, Y.-H. Tourism between divided nations: An examination of stereotyping on destination image. Tour. Manag. 2016, 55, 25-36. [CrossRef]

9. Lee, B.; Lee, C.-K.; Lee, J. Dynamic Nature of Destination Image and Influence of Tourist Overall Satisfaction on Image Modification. J. Travel Res. 2013, 53, 239-251. [CrossRef]

10. Huete-Alcocer, N.; Ruiz, V.R.L. The role of destination image in tourist satisfaction: The case of a heritage site. Econ. Res. Ekon. Istraživanja 2019, 1-18. [CrossRef]

11. Shani, A.; Wang, Y.; Hudson, S.; Moreno-Gil, S. Impacts of a historical film on the destination image of South America. J. Vacat. Mark. 2009, 15, 229-242. [CrossRef]

12. Camprubí, R.; Guia, J.; Comas, J. The new role of tourists in destination image formation. Curr. Issues Tour. 2013, 16, 203-209. [CrossRef] 
13. Llodrà-Riera, I.; Martínez-Ruiz, M.P.; Jiménez-Zarco, A.I.; Izquierdo-Yusta, A. A multidimensional analysis of the information sources construct and its relevance for destination image formation. Tour. Manag. 2015, 48, 319-328. [CrossRef]

14. Baloğlu, Ş.; McCleary, K.W. A model of destination image formation. Ann. Tour. Res. 1999, 26, 868-897. [CrossRef]

15. Leung, D.; Law, R.; Van Hoof, H.; Buhalis, D. Social Media in Tourism and Hospitality: A Literature Review. J. Travel Tour. Mark. 2013, 30, 3-22. [CrossRef]

16. Özdemir, G.; Çelebi, D. Reflections of Destinations on Social Media. In Regional Economic Development in Russia; Springer Science and Business Media LLC: Berlin, Germany, 2015; pp. 243-249.

17. Huertas, A.; Marine-Roig, E. Destination Brand Communication Through the Social Media: What Contents Trigger Most Reactions of Users? In Proceedings of the Information and Communication Technologies in Tourism, Lugano, Switzerland, 3-6 February 2015; pp. 295-308.

18. Hays, S.; Page, S.J.; Buhalis, D. Social media as a destination marketing tool: Its use by national tourism organisations. Curr. Issues Tour. 2013, 16, 211-239. [CrossRef]

19. Usakli, A.; Koç, B.; Sönmez, S. How 'social' are destinations? Examining European DMO social media usage. J. Destin. Mark. Manag. 2017, 6, 136-149. [CrossRef]

20. I Agustí, D.P. Characterizing the location of tourist images in cities. Differences in user-generated images (Instagram), official tourist brochures and travel guides. Ann. Tour. Res. 2018, 73, 103-115. [CrossRef]

21. Munar, A.M. Social Media Strategies and Destination Management. Scand. J. Hosp. Tour. 2012, 12, 101-120. [CrossRef]

22. Iglesias-Sánchez, P.P.; Correia, M.B.; Jambrino-Maldonado, C.; Heras-Pedrosa, C.D.L. Instagram as a Co-Creation Space for Tourist Destination Image-Building: Algarve and Costa del Sol Case Studies. Sustainability 2020, 12, 2793. [CrossRef]

23. Iglesias-Sánchez, P.P.; López-Delgado, P.; Correia, M.B.; Jambrino-Maldonado, C. How do external openness and R\&D activity influence open innovation management and the potential contribution of social media in the tourism and hospitality industry? Inf. Technol. Tour. 2020, 1-27. [CrossRef]

24. World Tourism Organization. Tourism Market Trends: Europe. In WTO Commission for Europe; World Tourism Organization: Madrid, Spain, 1998; pp. 15-32.

25. Ruiz, A.V.; Olarte, R.; Iglesias, V. Evaluación de los destinos turísticos en función de su valor de marca. In Actas del XI Encuentro de Profesores Universitarios de Marketing; Universidad de Valladolid: Valladolid: Valladolid, Spain, 1999; pp. 427-450.

26. Murphy, P.; Pritchard, M.P.; Smith, B. The destination product and its impact on traveller perceptions. Tour. Manag. 2000, 21, 43-52. [CrossRef]

27. Gunn, C.; Var, T. Tourism Planning: Basic, Concepts, Case, 4th ed.; Gunn, C., Var, T., Eds.; Routledge. Taylor and Francis: London, UK, 2002.

28. Buhalis, D. Marketing the competitive destination of the future. Tour. Manag. 2000, 21, 97-116. [CrossRef]

29. Jamal, T.; Getz, D. Collaboration theory and community tourism planning. Ann. Tour. Res. 1995, 22, $186-204$. [CrossRef]

30. Gallarza, M.G.; Gil Saura, I.; García, H.C. Destination image. Ann. Tour. Res. 2002, 29, 56-78. [CrossRef]

31. Crompton, J.L. An Assessment of the Image of Mexico as a Vacation Destination and the Influence of Geographical Location Upon That Image. J. Travel Res. 1979, 17, 18-23. [CrossRef]

32. Lin, C.-H.; Morais, D.B.; Kerstetter, D.L.; Hou, J.-S. Examining the Role of Cognitive and Affective Image in Predicting Choice Across Natural, Developed, and Theme-Park Destinations. J. Travel Res. 2007, 46, 183-194. [CrossRef]

33. Tsaur, S.-H.; Yen, C.-H.; Yan, Y.-T. Destination brand identity: Scale development and validation. Asia Pac. J. Tour. Res. 2016, 21, 1-14. [CrossRef]

34. Gartner, W.C. Image Formation Process. J. Travel Tour. Mark. 1994, 2, 191-216. [CrossRef]

35. Pike, S. Destination Image Literature: 2001-2007. Acta Tur. 2007, 19, 107-125. Available online: https: //www.researchgate.net/publication/27471957_Destination_image_literature_2001_-_2007 (accessed on 30 November 2019).

36. Beerli, A.; Martín, J.D. Factors influencing destination image. Ann. Tour. Res. 2004, 31, 657-681. [CrossRef]

37. Tan, W.-K.; Wu, C.-E. An investigation of the relationships among destination familiarity, destination image and future visit intention. J. Destin. Mark. Manag. 2016, 5, 214-226. [CrossRef] 
38. Cantos, E.; Pons, R.; Tanda, J. Creación De Valor En Ciudades-Destinos Turísticos a Través De Su Imagen: Rol De La Gestión Local. Retos Turísticos 2016, 15, 153-166. Available online: http://retos.mes.edu.cu/index. php/retojs/article/view/233/203. (accessed on 23 November 2019).

39. Weidenfeld, A. Iconicity and 'flagshipness' of tourist attractions. Ann. Tour. Res. 2010, 37, 851-854. [CrossRef]

40. Grayson, K.; Martinec, R. Consumer Perceptions of Iconicity and Indexicality and Their Influence on Assessments of Authentic Market Offerings. J. Consum. Res. 2004, 31, 296-312. [CrossRef]

41. Woodside, A.G.; Cruickshank, B.F.; Dehuang, N. Stories visitors tell about Italian cities as destination icons. Tour. Manag. 2007, 28, 162-174. [CrossRef]

42. Tang, L.; Morrison, A.M.; Lehto, X.; Kline, S.; Pearce, P.L. Effectiveness criteria for icons as tourist attractions: A comparative study between the United States and China. J. Travel Tour. Mark. 2009, 26, 284-302. [CrossRef]

43. Maitland, R.; Newman, P. Developing metropolitan tourism on the fringe of central London. Int. J. Tour. Res. 2004, 6, 339-348. [CrossRef]

44. Becken, S. The role of tourist icons for sustainable tourism. J. Vacat. Mark. 2005, 11, 21-30. [CrossRef]

45. Boo, S.; Busser, J.; Baloğlu, Ş. A model of customer-based brand equity and its application to multiple destinations. Tour. Manag. 2009, 30, 219-231. [CrossRef]

46. Echtner, C.M.; Ritchie, J.B. The Measurement of Destination Image: An Empirical Assessment. J. Travel Res. 1993, 31, 3-13. [CrossRef]

47. Bregoli, I. Effects of DMO Coordination on Destination Brand Identity. J. Travel Res. 2012, 52, $212-224$. [CrossRef]

48. Fernández-Cavia, J.; Rovira, C.; Díaz-Luque, P.; Cavaller, V. Web Quality Index (WQI) for official tourist destination websites. Proposal for an assessment system. Tour. Manag. Perspect. 2014, 9, 5-13. [CrossRef]

49. L'Etang, J.; Falkheimer, J.; Lugo, J. Public relations and tourism: Critical reflections and a research agenda. Public Relat. Rev. 2007, 33, 68-76. [CrossRef]

50. Kozak, M.; Crotts, J.C.; Law, R. The impact of the perception of risk on international travellers. Int. J. Tour. Res. 2007, 9, 233-242. [CrossRef]

51. Agapito, D.; Valle, P.O.D.; Mendes, J. The Cognitive-Affective-Conative Model of Destination Image: A Confirmatory Analysis. J. Travel Tour. Mark. 2013, 30, 471-481. [CrossRef]

52. Степченкова, C.; Mills, J.E. Destination Image: A Meta-Analysis of 2000-2007 Research. J. Hosp. Mark. Manag. 2010, 19, 575-609. [CrossRef]

53. Tasci, A.D.A.; Gartner, W.C. Destination Image and Its Functional Relationships. J. Travel Res. 2007, 45, 413-425. [CrossRef]

54. Casaló, C.; Flavián, M. Observación y participación activa en comunidades virtuales comerciales: El caso del sector turismo. Rev. Española Investig. Mark. ESIC 2011, 15, 113-134.

55. Crespo, Á.H.; Gutiérrez, H.S.M.; Mogollón, J.H. Perceived influence on behavior of user-generated content on social network sites: An empirical application in the hotel sector. Rev. Española Investig. Mark. ESIC 2015, 19, 12-23. [CrossRef]

56. Roca, M. La transformación de la industria publicitaria en la era digital. Percepciones de los profesionales en Estados Unidos: Retos y oportunidades. Telos Cuad. Comun. Innovación 2014, 99, 64-74.

57. King, R.A.; Racherla, P.; Bush, V.D. What We Know and Don't Know About Online Word-of-Mouth: A Review and Synthesis of the Literature. J. Interact. Mark. 2014, 28, 167-183. [CrossRef]

58. Kim, E.; Sung, Y.; Kang, H. Brand followers' retweeting behavior on Twitter: How brand relationships influence brand electronic word-of-mouth. Comput. Hum. Behav. 2014, 37, 18-25. [CrossRef]

59. Webb, J.W.; Ireland, R.D.; Hitt, M.A.; Kistruck, G.M.; Tihanyi, L. Where is the opportunity without the customer? An integration of marketing activities, the entrepreneurship process, and institutional theory. J. Acad. Mark. Sci. 2010, 39, 537-554. [CrossRef]

60. Peters, K.; Chen, Y.; Kaplan, A.M.; Ognibeni, B.; Pauwels, K. Social Media Metrics-A Framework and Guidelines for Managing Social Media. J. Interact. Mark. 2013, 27, 281-298. [CrossRef]

61. Ren, C.; Pritchard, A.; Morgan, N. Constructing tourism research. Ann. Tour. Res. 2010, 37, 885-904. [CrossRef]

62. D'Angella, F.; Go, F.M. Tale of two cities' collaborative tourism marketing: Towards a theory of destination stakeholder assessment. Tour. Manag. 2009, 30, 429-440. [CrossRef]

63. Hankinson, G. Relational network brands: Towards a conceptual model of place brands. J. Vacat. Mark. 2004, 10, 109-121. [CrossRef] 
64. Xifra, J.; Huertas, A. Blogging PR: An exploratory analysis of public relations weblogs. Public Relat. Rev. 2008, 34, 269-275. [CrossRef]

65. Laroche, M.; Habibi, M.R.; Richard, M.-O. To be or not to be in social media: How brand loyalty is affected by social media? Int. J. Inf. Manag. 2013, 33, 76-82. [CrossRef]

66. Marine-Roig, E.; Ferrer-Rosell, B. Measuring the gap between projected and perceived destination images of Catalonia using compositional analysis. Tour. Manag. 2018, 68, 236-249. [CrossRef]

67. Zwass, V. Co-Creation: Toward a Taxonomy and an Integrated Research Perspective. Int. J. Electron. Commer. 2010, 15, 11-48. [CrossRef]

68. Sanders, E.B.-N.; Stappers, P.J. Co-creation and the new landscapes of design. CoDesign 2008, 4, 5-18. [CrossRef]

69. Fatanti, M.N.; Suyadnya, I.W. Beyond User Gaze: How Instagram Creates Tourism Destination Brand? Procedia Soc. Behav. Sci. 2015, 211, 1089-1095. [CrossRef]

70. Shuqair, S.; Cragg, P. The immediate impact of Instagram posts on changing the viewers' perceptions towards travel destinations. Asia Pac. J. Adv. Bus. Soc. Stud. 2017, 3, 1-12. [CrossRef]

71. Mariani, M.M.; Felice, M.; Mura, M. Facebook as a destination marketing tool: Evidence from Italian regional Destination Management Organizations. Tour. Manag. 2016, 54, 321-343. [CrossRef]

72. Luo, Q.; Zhong, D. Using social network analysis to explain communication characteristics of travel-related electronic word-of-mouth on social networking sites. Tour. Manag. 2015, 46, 274-282. [CrossRef]

73. Junta De Andalucía. Plan De Promoción Turística De Andalucía. 2012. Available online: http://www. juntadeandalucia.es/turismoydeporte/publicaciones/143463551.pdf (accessed on 5 September 2019).

74. Narangajavana, Y.; Fiol, L.J.C.; Tena, M.; Ángel, M.; Artola, R.M.R.; García, J.S. The influence of social media in creating expectations. An empirical study for a tourist destination. Ann. Tour. Res. 2017, 65, 60-70. [CrossRef]

75. De Bruyn, A.; Lilien, G.L. A multi-stage model of word-of-mouth influence through viral marketing. Int. J. Res. Mark. 2008, 25, 151-163. [CrossRef]

76. White, L. Facebook, friends and photos: A snapshot into social networking for generating travel ideas. In Tourism Informatics: Visual Travel Recommender Systems, Social Communities and User Interface Design; Sharda, N., Ed.; IGI Global: Hershey, PA, USA, 2010; pp. 115-129.

77. Akehurst, G. User generated content: The use of blogs for tourism organisations and tourism consumers. Serv. Bus. 2008, 3, 51-61. [CrossRef]

78. Ndou, V.; Del Vecchio, P. Empowering tourists to co-create services. In Projects and Forms of The European Digital Citizenship; Mancarella, M., Ed.; Tangram Edizioni Scientifiche: Trento, Italy, 2014; pp. 124-143.

79. Chan, J.K.L.; Quah, W.B. Start-up Factors for Small and Medium-sized Accommodation Businesses in Sabah, Malaysia: Push and Pull Factors. Asia Pac. J. Tour. Res. 2012, 17, 49-62. [CrossRef]

80. Song, H.; Bae, S.Y. Understanding the travel motivation and patterns of international students in Korea: Using the theory of travel career pattern. Asia Pac. J. Tour. Res. 2017, 23, 133-145. [CrossRef]

81. Castelló, A. La orientación empresarial hacia el cliente en la Web 2.0. Miguel Hernández Commun. J. 2010, 1, 203-239.

82. Standing, C.; Tang-Taye, J.-P.; Boyer, M. The Impact of the Internet in Travel and Tourism: A Research Review 2001-2010. J. Travel Tour. Mark. 2014, 31, 82-113. [CrossRef]

83. Agrawal, A.K.; Kaushik, A.; Rahman, Z. Co-creation of Social Value through Integration of Stakeholders. Procedia Soc. Behav. Sci. 2015, 189, 442-448. [CrossRef]

84. Gretzel, U.; Kang, M.; Lee, W. Differences in Consumer-Generated Media Adoption and Use: A Cross-National Perspective. J. Hosp. Leis. Mark. 2008, 17, 99-120. [CrossRef]

85. Marine-Roig, E.; Clavé, S.A. Perceived image specialisation in multiscalar tourism destinations. J. Destin. Mark. Manag. 2016, 5, 202-213. [CrossRef]

86. Brenner, S.N.; Cochran, P. The Stakeholder Theory of the Firm. Proc. Int. Assoc. Bus. Soc. 1991, 2, 897-933. [CrossRef]

87. Pfeffer, J.; Salancik, G.R. The External Control of Organisations; Harper and Row: New York, NY, USA, 1978.

88. Shankman, N.A. Reframing the Debate Between Agency and Stakeholder Theories of the Firm. J. Bus. Ethics 1999, 19, 319-334. [CrossRef]

89. Freeman, E. Strategic Management: A Stakeholder Approach; Cambridge University Press: Cambridge, UK, 2010. 
90. Goodpaster, K.E. Ethical Imperatives and Corporate Leadership. Ruffin 1991, 6, 89-110.

91. Timur, S.; Getz, D. A network perspective on managing stakeholders for sustainable urban tourism. Int. J. Contemp. Hosp. Manag. 2008, 20, 445-461. [CrossRef]

92. Sun, H. Destination and Event Marketing: A Case Study in The 2007 c\&d Xiamen International Marathon, Xiamen, China. Master's Thesis, University of Waterloo, Waterloo, ON, USA, 2007.

93. García, J.A.; Gómez, M.; Molina, A.; Collado, A.M. A destination-branding model: An empirical analysis based on stakeholders. Tour. Manag. 2012, 33, 646-661. [CrossRef]

94. Sheehan, L.; Ritchie, J.R.B.; Hudson, S. The Destination Promotion Triad: Understanding Asymmetric Stakeholder Interdependencies Among the City, Hotels, and DMO. J. Travel Res. 2007, 46, 64-74. [CrossRef]

95. Bitsani, E.; Kavoura, A. Connecting Oenological and gastronomical tourisms at the Wine Roads, Veneto, Italy, for the promotion and development of agrotourism. J. Vacat. Mark. 2012, 18, 301-312. [CrossRef]

96. Simpson, M.C. Community Benefit Tourism Initiatives-A conceptual oxymoron? Tour. Manag. 2008, 29, 1-18. [CrossRef]

97. Clarkson, M.B.E. A Stakeholder Framework for Analyzing and Evaluating Corporate Social Performance. Acad. Manag. Rev. 1995, 20, 92. [CrossRef]

98. Dodds, R.; Ko, S. Assessing Stakeholders' Views of Tourism Policy in Prince Edward County. Environ. Manag. Sustain. Dev. 2012, 1, 1. [CrossRef]

99. Liu, Z. Sustainable Tourism Development: A Critique. J. Sustain. Tour. 2003, 11, 459-475. [CrossRef]

100. Jamal, T.; Stronza, A.L. Collaboration theory and tourism practice in protected areas: stakeholders. Structuring and sustainability. 2009, 1, 459-475.

101. Huertas, A. Aplicación de la Web 2.0 a Los Destinos TuríSticos. Implantación y Diferencias; TURITEC: Málaga, Spain, 2008.

102. Del Vasto-Terrientes, L.; Valls, A.; Slowinski, R.; Zielniewicz, P. ELECTRE-III-H: An outranking-based decision aiding method for hierarchically structured criteria. Expert Syst. Appl. 2015, 42, 4910-4926. [CrossRef]

103. Sautter, E.T.; Leisen, B. Managing stakeholders a Tourism Planning Model. Ann. Tour. Res. 1999, 26, 312-328. [CrossRef]

104. Formica, S.; Kothari, T.H. Strategic Destination Planning: Analyzing the Future of Tourism. J. Travel Res. 2008, 46, 355-367. [CrossRef]

105. Wang, Y.; Xiang, Z. Toward a Theoretical Framework of Collaborative Destination Marketing. J. Travel Res. 2007, 46, 75-85. [CrossRef]

106. Jambrino-Maldonado, C.; De Las Heras-Pedrosa, C. Building of Corporate reputation observatory for a tourism destination. Tour. Manag. Stud. Int. Conf. Algarve 2012, 1, 66-76.

107. Saxton, G.D.; Waters, R.D. What do Stakeholders Like on Facebook? Examining Public Reactions to Nonprofit Organizations' Informational, Promotional, and Community-Building Messages. J. Public Relations Res. 2014, 26, 280-299. [CrossRef]

108. Capriotti, P. Planificación Estratégica De La Imagen Corporativa, 4th ed.; Instituto de Investigación en Relaciones Públicas: Málaga, Spain, 2013.

109. Li, M.; Cai, L.A.; Lehto, X.; Huang, J.Z. A Missing Link in Understanding Revisit Intention-The Role of Motivation and Image. J. Travel Tour. Mark. 2010, 27, 335-348. [CrossRef]

110. Chon, K.-S. The role of destination image in tourism: A review and discussion. Tour. Rev. 1990, 45, 2-9. [CrossRef]

111. Glińska, E. Sposoby Badania Wizerunku Miejsca. Samorz. Teryt. 2011, 11, 33-44.

112. Środa-Murawska, S.; Szymańska, D. Cdfmr The concentration of the creative sector firms as a potential basis for the formation of creative clusters in Poland. Bull. Geogr. Socio Econ. Ser. 2013, 20, 85-93. [CrossRef]

113. Dwivedi, A.; Merrilees, B.; Miller, D.; Herington, C. Brand, value and relationship equities and loyalty-intentions in the Australian supermarket industry. J. Retail. Consum. Serv. 2012, 19, 526-536. [CrossRef]

114. Creswell, J. Educational Research: Planning, Conducting, and Evaluating Quantitative and Qualitative Research; Pearson Education: Upper Saddle River, NJ, USA, 2007.

115. Bryman, A. Barriers to Integrating Quantitative and Qualitative Research. J. Mix. Methods Res. 2007, 1, 8-22. [CrossRef]

116. Sabharwal, N.S.; Malish, C.M. Mixed Methods Approaches and Qualitative Methodology for Higher Education Policy Research; Oxford University Press (OUP): Oxford, UK, 2018; pp. 29-50. 
117. Krippendorff, K. Content Analysis: An Introduction to its Methodology, 2nd ed.; SAGE Publications: London, UK, 2004.

118. Sánchez, J.J. Análisis De Contenido Cuantitativo De Medios; Berganza, M.R., Ruiz, J.A., Eds.; Investigar en Comunicación, McGraw Hill: Madrid, Spain, 2005.

119. Igartua, J.J. Métodos Cuantitativos De Investigación En Comunicación; Bosch: Barcelona, Spain, 2006.

120. De las Heras-Pedrosa, C.; Jambrino-Maldonado, C.; Iglesias-Sánchez, P.; Lugo-Ocando, J. Importance of Public Relations for The Reputation in a Smart Tourist Destination. A Proposal of a Sustainable Model. Revista Internacional de Relaciones Públicas 2019, 9, 117-128. [CrossRef]

121. Lotto, L.S.; Miles, M.B.; Huberman, A.M. Qualitative Data Analysis: A Sourcebook of New Methods. Educ. Eval. Policy Anal. 1986, 8, 329. [CrossRef]

122. Eisenhardt, K.M.; Graebner, M.E. Theory Building from Cases: Opportunities And Challenges. Acad. Manag. J. 2007, 50, 25-32. [CrossRef]

123. Edquist, C.; Hommen, L.; McKelvey, M. Innovation and Employment; Edward Elgar Publishing: Chalone, UK, $2001 ;$ p. 214.

124. TripAdvisor. Claves Del Verano 2018: Destinos De Playa, Mayor Presupuesto y Reservas Anticipadas. 2018. Available online: https://tripadvisor.mediaroom.com/press-releases?item=125414 (accessed on 1 October 2019).

125. Piñuel, J.L. Epistemología, metodología y técnicas del análisis de contenido. Estud. Sociolingüística 2002, 3, $1-42$.

126. Corley, K.G.; Gioia, D.A. Identity ambiguity and change in the wake of a corporate spin-off. Admin. Sci. Quart. 2013, 49, 173-208.

127. Corbin, J.; Strauss, A. Basics of Qualitative Researc: Techniques and Procedures for Developing Grounded Theory, 4th ed.; SAGE Publications: London, UK, 2015.

128. Strauss, A.; Corbin, J. Basics of Qualitative Research: Grounded Theory Procedures and Techniques, 2nd ed.; SAGE Publications: Newbury Park, CA, USA, 1990.

129. Paniagua, F.J.; Rabassa, N.; Huertas, A.; Setó, D.; Lozano, N.; Marine, E.; Míguez, M.; Ariste, S. La Comunicación de Los Destinos Turísticos y Sus Marcas a Través de Los Medios Sociales; Universitat Rovira i Virgili: Tarragona, Spain, 2016.

130. Olson, J.D.; McAllister, C.; Grinnell, L.D.; Gehrke Walters, K.; Appunn, F. Applying Constant Comparative Method with Multiple Investigators and Inter-Coder Reliability. Qual. Rep. 2016, 21, 26-42.

131. Sarpong, D.; Razak, A.A.; Alexander, E.; Meissner, D. Organizing practices of university, industry and government that facilitate (or impede) the transition to a hybrid triple helix model of innovation. Technol. Forecast. Soc. Chang. 2017, 123, 142-152. [CrossRef]

132. Leydesdorff, L.; Etzkowitz, H. Emergence of a Triple Helix of university-industry-government relations. Sci. Public Policy 1996, 23, 2793-2896. [CrossRef]

133. Huertas, A.; Setó-Pamies, D.; Míguez-González, M.-I. Comunicación de destinos turísticos a través de los medios sociales. Prof. Inf. 2015, 24, 15-21. [CrossRef]

134. Cisco Visual Networking Index. Global Mobile Data Traffic Forecast Update, 2017-2022. 2017. Available online: https:/www.cisco.com/c/en/us/solutions/collateral/service-provider/visual-networkingindex-vni/white-paper-c11-738429.pdf (accessed on 10 September 2019).

135. Teixeira, J.E. The Role of Promotional Touristic Videos in the Creation of Visit Intent to Barcelona. Int. J. Sci. Manag. Tour. 2017, 3, 463-490.

136. Hidalgo Alcazar, M.C.; Sicilia Piñero, M.; Ruiz de Maya, S. The effect of user-generated content on tourist behavior: The mediating role of destination image. Tour. Manag. Stud. 2014, 10, 158-164. Available online: https://tmstudies.net/index.php/ectms/article/view/616/1131 (accessed on 30 November 2019).

137. Lo, I.S.; McKercher, B.; Lo, A.; Cheung, C.; Law, R. Tourism and online photography. Tour. Manag. 2011, 32, 725-731. [CrossRef]

138. Lalicic, L.; Huertas, A.; Moreno, A.; Gindl, S.; Jabreel, M. Do DMOs Communicate Their Emotional Brand Values? A Comparison Between Twitter and Facebook. Inf. Commun. Technol. Tour. 2017, 159-171. [CrossRef]

139. Danias, K.; Kavoura, A. The role of social media as a tool of a company's innovative communication activities. Małopolska Sch. Econ. Tarnów Res. Pap. Collect. 2013, 23, 75-83. 
140. Hay, B. Twitter Twitter-But Who is Listening? A Review of the Current and Potential Use of Twittering as a Tourism Marketing Tool; Unpublished Report; Queen Margaret University: Edinburgh, Scotland, 2011.

141. Katsoni, V.; Laloumis, D. The influence of online reviews on customers and travel agencies. Małopolska Sch. Econ. Tarnów Res. Pap. Collect. 2013, 23, 123-130.

(C) (

(C) 2020 by the authors. Licensee MDPI, Basel, Switzerland. This article is an open access article distributed under the terms and conditions of the Creative Commons Attribution (CC BY) license (http://creativecommons.org/licenses/by/4.0/). 

Article

\title{
The Moderating Effect of Social Media Use on Sustainable Rural Tourism: A Theory of Planned Behavior Model
}

\author{
Yeajin Joo, Hwayoon Seok and Yoonjae Nam * \\ Department of Culture, Tourism \& Content, Kyung Hee University, Seoul 130-701, Korea; \\ jyj6241@khu.ac.kr (Y.J.); vipshy5734@khu.ac.kr (H.S.) \\ * Correspondence: ynam@khu.ac.kr
}

Received: 22 April 2020; Accepted: 14 May 2020; Published: 17 May 2020

\begin{abstract}
Using the theory of planned behavior (TPB) model, this research aimed to investigate which important factors affect the potential behavior of rural tourism tourists. It also sought to identify the effects of social networking service (SNS) use on the rural tourism using TPB model. For analysis, a partial least squares-structural equation model (PLS-SEM) was used. The results revealed that tourists were influenced by subjective norm and perceived behavior control, of which the effect of subjective norm was greater. Consumers' use of SNS played a notable role in contributing to the strength of the relationship between intention to visit and subjective norm. Promoters and marketers of sustainable tourism can use the results to make more effective decisions in their businesses.
\end{abstract}

Keywords: sustainable tourism; rural tourism; social networking service; theory of planned behavior; social media use

\section{Introduction}

Since the 1990s, as industries have become more service-oriented, companies have made it a priority to understand and relate to their consumers more than ever before [1,2]. The tourism industry is no exception $[3,4]$.

Some research $[5,6]$ has revealed the decision-making process for sustainable tourism. However, papers about the decision-making process of rural tourism tourists are rare. To fill this gap, this study uses the theory of planned behavior (TPB) model to understand the decision-making process with rural tourism data. The TPB model has traditionally been used to analyze the decision-making process in many different fields, including tourism [7].

There has been another big paradigm shift, evoked by the information and communication technology (ICT) development. Social media has changed the entire tourism business [8,9]. Using social networking services (SNS) is not just personal: through social networking services (SNS), people can collect information, share tour experiences, and influence others to make decisions. Tourism is an informative-intensive industry, and information distribution and advertising on social media play a key role [10].

Many studies (e.g., [8,11-13]) have stated that SNS has a large effect on tourist behavior. but there are not enough papers on the effect of SNS on sustainable tourism. Hence, this study seeks to investigate how tourists' use of social media can affect their behavior, especially in sustainable tourism.

Even though there are some studies (e.g., $[6,14]$ ) that used the TPB model to examine the decision-making process of sustainable tourism tourists, those studies did so without focusing on the role of social media in rural tourism. In conclusion, the objective of this paper is to examine the role of social media use on rural tourism using the TPB model. First, the study analyzes the TPB model of rural tourism in order to understand what the important factors are for tourists. The study then 
analyzes the effect of social media use on the rural tourism TPB model. Thus, the results will provide useful insights for sustainable tourism destination marketers as well as for the industry at large.

\section{Literature Review}

\subsection{Sustainable Tourism and Rural Tourism}

Sustainability has been an increasing concern for the tourism industry since the late 1980s [15]. The main issues of sustainable tourism are ecological problems and the capacity of the destination [16]. In the 1990s, sustainable tourism meant the preservation of a site by preventing overdevelopment of the industry at the risk of harming its ecology [17]. To make this possible, the volume of visitors and the tour-related businesses that directly contributed to a site were regulated [18]. Muller [19] mentioned that, for qualitative growth to take place, sustainable tourism should be approached from five different perspectives: economic health; subjective wellbeing of the locals; unspoiled nature/protection of resources; healthy culture; and optimization of guest satisfaction.

From the late 1990s, the definition has been expanded to accommodate concerns beyond solely protecting the environment, such as those covered under the sustainability triple bottom line [20]. Therefore, sustainable tourism has three sustainability aspects based on the triple bottom line. First is the environmental aspect, which is related to essential ecological processes, conservations of natural heritage and biodiversity [21]. Second is the social-cultural aspects, which are related to crime, local culture, and crowding. The third is the economic aspect, which is related to foreign investors and the local labor market [22]. According to the World Tourism Organization (UNWTO) [23], the definition of sustainable tourism is: "Tourism that takes full account of its current and future economic, social and environmental impacts, addressing the needs of visitors, the industry, the environment and host communities."

A typical example of sustainable tourism is rural tourism [11]. Lane [24] defined rural tourism as having five conditions. Understandably, the destination should be located in a rural area. Second, the community of the destination should have traditional societies and practices. Third, the area should be rural in scale (that is to say, a small-scale based society). Fourth, rural tourism development should make progress slowly and organically and should be controlled locally. Finally, rural tourism should contain a variety of aspects, including rural environment, economy, history, and location.

Researchers have been interested in studying the development of rural tourism for some time. Embacher [25] studied the marketing of agritourism. As tourism grew into a larger source of profit for the average farm, the term "farm holiday" was coined. Nationwide efforts started to be made to brand this emerging market. Clarke [26] sought to research the marketing structure of the Farm Holiday Bureau. There are three levels to the marketing structure: national office, local area group, and the individual provider. The head office engages in a more formal style of marketing while, on a smaller scale, individual providers utilize instinctive styles to promote themselves. Rural tourism contains many layers for which marketing purposes may vary, but these roles, when brought together as a system, should be fully symbiotic and sustainable $[25,26]$.

Many approaches have been used in an effort to develop rural tourism. Byrd, Bosley, and Dronberger [27] identified the stakeholder categories as residents, entrepreneurs, tourists, and government officials. Their study tried to distinguish differences in stakeholders' attitudes. Iorio and Corsale [28] thought of ways in which a rural community could make rural tourism a stable income. Fons, Fierro, and Patino [11] made projections of possible paths that the industry could take using SWOT strategy analysis. Boley, McGehee, and Hammett [29] used the importance-performance analysis(IPA) to examine sustainable tourism through the eyes of a resident. After, researchers proposed initiatives for sustainable tourism development. Puska, Sadic, and Stojanovic [30] suggested a model that can evaluate rural touristic destinations in order to improve attractiveness. The evaluation was conducted in three parts (economic and social factors, cultural and historical factors, and the natural-ecological environment). 
There are also studies that have focused on tourists' behavior and the attributes of rural tourism. Pina and Delfa [31] made distinctions between accommodation in specific rural tourist destinations. Their research then made a cluster analysis based on tourists' features. Maestro, Gallego, and Requejo [32] conducted a study to see what kind of effect the attitude towards rural tourism had on the perceived quality and satisfaction of rural tourism accommodation. They found that the perceived quality of rural tourism accommodation was multidimensional. Attitude toward rural tourism affected tourists' impressions of the accommodation's quality, and the experience affected the relationship between the attitude and perception of quality. Loureiro [33] studied tourists of rural lodging sites to see how the rural tourism experience economy was affecting place attachment and behavior intentions. The behavior intentions variable consisted of the sharing of tourism experiences, passive recommendation of tourism, active recommendation of tourism (an unsolicited recommendation of tourism), and thoughts regarding revisitation. It was found that the rural experience economy affected pleasant arousal and memory factors. The two factors influenced place attachment and behavior intention. Although there have been steady efforts to research rural tourism in terms of its development and tourists' behavior so far, still few studies regarding the effect of social media on the rural tourism have been conducted. Thus, this study attempts to identify the effects of tourists' SNS use on the rural tourism for the sustainability.

\subsection{Theory of Planned Behavior}

Ajzen [34] developed the model known as the theory of planned behavior (TPB) not to predict, but to understand human behavior. People make decisions through a very complicated process. However, when there is intent to engage in behavior, there are three major variables at play that can be identified. The first is the attitude toward engaging in a behavior. The second is how others are likely to view the behavior, known as the subjective norm. The third is how much control a subject believes they have in engaging in a behavior, otherwise known as perceived behavior control.

Ajzen's TPB model has since been validated through much research (e.g., [14,35]) as well as through extensive application within the tourism area. Quintal, Lee, and Soutar [35] conducted a study regarding Asian tourism (Korea, China, and Japan) to Australia using the TPB model with "intent of visit" as the dependent variable. They also tested to see the effects of perceived risk and perceived uncertainty on the three variables (attitude, subjective norm, and perceived behavior control) described in the TPB model. For Japanese tourists, all three were affected, while for Korean and Chinese tourists, attitude was the only unaffected variable. Ajzen [34] also suggested that the travel decision-making process can be known through the use of the TPB model. Since then, tourism researchers have applied the TPB model behavior. Sparks [36] studied wine tourism. The TPB model was used to see if visitors would consider another wine trip within a year of their last visit. The researchers divided attitude into emotional attitude and attitude toward past wine holidays. The results showed that, with the exception of emotional attitude, other TPB factors had a direct effect. Jalivand and Samiei [14] examined the impact of electronic word of mouth (eWOM) on a tourism destination choice using the TPB model. According to the researchers, as WOM became more digitized, its effects were amplified, making it much more significant. To prove this point, a study was conducted on tourists visiting the Iranian city of Isfahan. The results showed that all TPB model paths were significant. Electronic WOM had an effect on attitude, subjective norm, and perceived behavior control.

As the TPB model was increasingly used for general tourism, there was also an increase in researchers who adopted the model to study sustainable tourism. Kuo and Dai [5] investigated low-carbon tourism behavior through the use of the TPB model. In the model, behavior intention meant intention to visit low-carbon tourism sites in the future. Then, the final dependent variable was decided as the preference for low-carbon tourism and transportation. Instead of working with tourists, Hamid and Isa [7] worked with entrepreneurs as their main subject. Using the TPB model, the researchers asked tourism-related stakeholders about whether or not they had thoughts about participating in sustainable tourism, and showed what affected stakeholders' thoughts. 
Meng and Choi [6] used the TPB model to study slow tourism. They created an extended TPB model by adding two more factors to the original: authentic perception and environmental concerns. It was found that the three factors of TPB (attitude, subjective norm, and perceived behavior control) had significant influences on behavior intention. Of the two additional factors, authentic perception was also found to have a notable impact. Lee and Jan [37] created a theoretical framework to investigate ecotourism behavior. The framework was composed of four theories, with TPB as one of them. As a result, all hypotheses related to the TPB model were supported. Eom and Han [38] applied the TPB to community-based tourism, which had social-cultural sustainability. The study aimed to examine the formation of tourists' participating intentions of a tour program and the moderating effects of gender and age. Ashraf, Hou, Kim, and Ashraf [39] developed a TPB model with resultant self-transcendence and conservation variables. The final dependent variable of the model was visiting intention toward ecofriendly tour destinations. From the literature review, this study suggests that the TPB model has been employed as a useful tool for investigating tourists' behavior. Thus, this study attempts to analyze the how attitude, perceived behavior control and subjective norm from TPB model have influenced on tourists' behavior for rural tourism.

\subsection{Social Media and Tourism}

People who promote tour destinations have determined the importance of social media. Most rural tourism businesses are too small to afford massive marketing [40]. However, even small tour destinations can use social media for promotion. So, social media promotion is especially important for rural tourism. It is easy to find promotions done via social media. Tourists get information from social media and upload information about trips.

Zeng and Gerritsen [41] conducted a bibliometric analysis of social media in tourism. They found that the amount of social media-related research in tourism quickly increased after 2010. They categorized research using six themes, including social media and tourists' behavior. Leung, Law, Van, and Buhalis [12] analyzed social media research in the tourism field conducted from 2007 to 2011. Their results showed that social media research was conducted regarding both supply and demand. On the demand side, lots of research investigated how social media affected people looking to make travel plans. On the supply side, there were many studies about social media being used as a strategy. Though, after 2010s, the social media studies for tourism have mainly focused on customer satisfaction and behavior, recently some new topics, such as eWOM, big data, online review, and digital marketing, have been adopted for the tourism research [42,43].

The following research was conducted to see the effect of social media on tourism. Xiang and Gretzel [13] found that social media plays a large role in the online tourism domain when people are making travel plans. Moreover, this research looked into the relationship between social media and key search queries used by tourists. Milano, Baggio, and Piattelli [8] measured to see the effect of Facebook and Twitter on the popularity of tourist websites. Their results showed that Facebook had a significant role in increasing the number of visitors to tourist websites. Fotis, Buhalis, and Rossides [44] tried to see the effect of social media on the process of planning a holiday trip. It was seen that the use of social media had an effect on tourists revising their initial plans. People used social media the most once their holidays were over, when they would share their travel experiences with others. Social media users believed travel information found on social media and created by other users to be more trustworthy than information from other sources. Narangajavana, Fiol, Tena, Artola, and Garcia [45] researched the relationship between social media use and tourist expectation. Tourists with a higher intensity of social media use viewed user-generated content as more frequently. Also, motivation for receiving user-generated content influenced the general reputation of user-generated content, and the heightened reputation in turn influenced tourists' expectations.

At times, researchers have studied social media marketing to provide useful insights for tourism marketers. Pesonen [9] compared small companies and big companies in their use of social media for marketing. The results showed that smaller companies generally either had a large amount of useless 
content or were far less developed. After examining the social marketing strategies of 10 of the most popular countries for international visits, Hays, Page, and Buhalis [10] spoke about what the right way to do social marketing. Kavoura and Stavrianea [46] saw social media's role as creating an online tourist community within the tourism industry, and spoke of the importance of marketing from this point of view.

Generally, people use social media for socializing and creating relationships, updating news, and searching for information [47]. The most common use of social media by tourists is posting tour pictures and experiences [44]. As the importance of social media use in tourism grows, researchers want to understand the motivation behind social media use [48,49]. Munar and Jacobsen [48] suggested that motivations for sharing online content included individual action and personal cognition, self-centered motivation, and community-related motivation. Kim and Fesenmaier [50] focused on the sharing of tourists' experiences. Through the social media of tourists, researchers were able to examine the relationship between the sharing of experiences and the rating of the experiences. Hausmann, Toivonen, Slotow, Tenkanen, Moilanen, Heikinheimo, and Di [51] used social media data to see if it was possible to check the preference of environment-friendly tourist sites. The social media data showed specific features. It was concluded that tourism-related content shared on social media is significant enough to be used in place of traditional surveys to provide research data.

\section{Research Model and Hypothesis}

The validity of the TPB model was affirmed through the literature review. Social media use influences travelers' decision-making processes [12]. Also, people who wanted to improve their image in the eyes of their neighbors had a high possibility of visiting a sustainable tourism destination [52]. Hence, this study proposed hypotheses to understand the rural tourism tourist with the TPB model and the role of social media use. Drawn from the literature review above, a research framework was formulated, as shown in Figure 1.

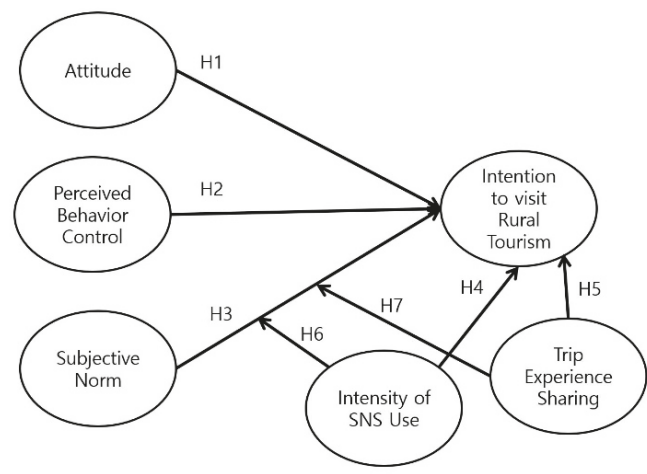

Figure 1. Theoretical model.

The following shows the research questions and hypotheses of this study.

Research Question 1. Can the TPB model be applied to rural tourism?

Hypothesis 1. Attitude toward rural tourism will have a direct effect on tourists' intention to visit a rural tourism site.

Hypothesis 2. Perceived behavior will have a direct effect on tourists' intention to visit a rural tourism site.

Hypothesis 3. Subjective norm will have a direct effect on tourists' intention to visit a rural tourism site. 
Research Question 2. How does social media use affect rural tourism in relation to the TPB model?

Hypothesis 4. Intensity of social media use will have a direct effect on the intention of tourists to visit a rural tourism site.

Hypothesis 5. Intensity of tourist trip experience sharing will have a direct effect on the intention of tourists to visit a rural tourism site.

Hypothesis 6. Intensity of social media use will have moderate effects on the subjective norm and intention relationship.

Hypothesis 7. Intensity of tourist trip experience sharing will have moderate effects on the subjective norm and intention relationship.

\section{Method}

\subsection{Sampling and Procedure}

A partial least squares-structural equation model (PLS-SEM) was employed to test the theoretical model because the study model contained moderating path hypotheses. A PLS-SEM was employed for the following reasons. First, although the TPB model is a powerful model, the theoretical model includes new paths: H4, H5, H6, and H7. PLS is an appropriate method of analysis for exploratory and theory development [53]. Second, a PLS-SEM is suited for analyzing moderating effects. Chin, Marcolin, and Newsted [54] employed a PLS-SEM to test the moderating effect based on the product indicator method developed by Kenny and Judd [55]. Estimating the measurement model, structural model, and the moderating effect all at once is possible through the use of a PLS-SEM [56].

A sample of people living in South Korea participated in order to test the proposed hypotheses. The sample data were collected using an online survey. The study collected data through a top-ranking Korean internet survey firm which has a nationwide panel of over one million online. This study collected 323 responses from the participants over a one-week period in Nov 2019. Insincere respondents who completed the questionnaire too quickly and only checked answers in the same pattern for the purpose of reward were removed. Table 2 summarizes the characteristics of respondents. The data were analyzed with SPSS (Version 23.0, IBM Corp., Armonk, NY) and SmartPLS (Version 3, SmartPLS $\mathrm{GmbH}$, Boenningstedt, Germany). As the model has only reflective factors, a consistent PLS analysis was conducted [57].

\subsection{Measurement}

Table 1 shows the study organized into number of questions used to measure the variables, as well as the papers that were referenced in the process of creating these questions. All questions were made up of a seven-point Likert scale.

Table 1. The measurement references.

\begin{tabular}{ccc}
\hline Variable & Number & Reference \\
\hline Attitude & 5 & {$[5,6,59,60]$} \\
\hline Subjective norm & 4 & {$[5,6,59,60]$} \\
\hline Perceived behavior control & 4 & {$[5,6]$} \\
\hline Intention & 4 & {$[6,35]$} \\
\hline Intensity of social media use & 2 & {$[45]$} \\
\hline Trip experience sharing & 2 & {$[58]$} \\
\hline
\end{tabular}


Narangajavana, Fiol, Tena, Artola, and Garcia [45] set the intensity of social media use as an independent variable, which was comprised of how often people connect to social media and how many hours they spend on it. Boley, Magnini, and Tuten [58] explained that there is a relationship between trip picture posting behavior and buying souvenirs. To measure the trip picture posting variable, participants were asked whether or not they had used social networking sites to post and share travel photographs online.

Measurement of the TPB factors was devised with reference to the results of preexisting studies. In Quintal, Lee, and Soutar [35], tourists were asked if they had any intention of visiting a given tourism location within two years.

\section{Results}

\subsection{Sample Profile}

Table 2 summarizes the sample characteristics. The mean age of the sample was 39.3 years, and $48.9 \%$ of the sample were men, whereas $51.1 \%$ were women. More than half of the sample was not married (55.7\%). Of the respondents, $24.8 \%$ had two children. In addition, $57.9 \%$ lived in the capital city (Seoul) or big cities. Income was low to medium, as $29.4 \%$ of the sample earned a monthly income of more than $\$ 3400$. Most of the respondents had graduated from university and above (74.9\%).

Table 2. Demographics of respondents $(n=323)$.

\begin{tabular}{ccc}
\hline Demographics & Frequency & Percentage (\%) \\
\hline Sex & 158 & 48.9 \\
Male & 165 & 51.1 \\
Female & & \\
Age & 78 & 24.1 \\
20-29 & 80 & 24.8 \\
$30-39$ & 84 & 26 \\
40-49 & 81 & 25.1 \\
50 and above & & \\
Marriage & 143 & 44.3 \\
Married & 180 & 55.7 \\
Single & & \\
Child & 165 & 51.1 \\
0 & 66 & 20.4 \\
1 & 80 & 24.8 \\
2 & 10 & 3.1 \\
3 & 2 & 0.6 \\
4 & &
\end{tabular}


Table 2. Cont.

\begin{tabular}{ccc}
\hline Demographics & Frequency & Percentage (\%) \\
\hline Education & & \\
High school & 34 & 10.5 \\
College & 47 & 14.6 \\
University & 199 & 61.6 \\
Graduate school and above & 43 & 13.3 \\
\hline Residential district & & \\
Capital city (Seoul) & 110 & 34.1 \\
Political capital city (Sejong) & 2 & 0.6 \\
City & 75 & 23.2 \\
Rural & 136 & 42.1 \\
\hline Monthly income & & \\
$\$ 849$ and below & 36 & 11.1 \\
$\$ 850-\$ 1699$ & 42 & 13.0 \\
$\$ 1700-\$ 2549$ & 92 & 28.5 \\
$\$ 2550-\$ 3399$ & 58 & 18.0 \\
$\$ 3400-\$ 4249$ & 39 & 12.1 \\
$\$ 4250-\$ 5099$ & 25 & 7.7 \\
$\$ 5100$ and above & 31 & 9.6 \\
Total & 323 & 100.0 \\
\hline
\end{tabular}

\subsection{Measurement Model}

This study ran confirmatory factor analysis using SmartPLS (Version 3, SmartPLS GmbH, Boenningstedt, Germany). Convergent validity and discriminant validity were used to evaluate the measurement model. Table 3 shows the results of the measurement model convergent validity statistics. For secure convergent validity, the statistics should meet the three permissible levels. First, all factor loadings should be bigger than 0.7. Second, composite reliability (CR) should be bigger than 0.7 . Average variance extracted (AVE) of each latent variable should be bigger than 0.5 [61]. Both the factor loadings and composite reliability of all factors exceeded 0.7. The lowest value of AVE was 0.668. Cronbach's alpha should be larger than 0.7 [62]. Also, rho_A needs to be larger than 0.7. The results of the measurement model statistics denote that the model can be analyzed.

Table 3. The measurement model statistics.

\begin{tabular}{|c|c|c|c|c|c|}
\hline Items & $\begin{array}{c}\text { Cronbach's } \\
\text { Alpha }\end{array}$ & Rho_A & $\begin{array}{l}\text { Composite } \\
\text { Reliability }\end{array}$ & AVE & Factor Loading \\
\hline \multirow[t]{5}{*}{ Attitude } & 0.940 & 0.943 & 0.940 & 0.798 & \\
\hline & \multirow{4}{*}{\multicolumn{4}{|c|}{$\begin{array}{l}\text { Going to rural tourism sites is good } \\
\text { Going to rural tourism sites is valuable } \\
\text { Going to rural tourism sites is beneficial } \\
\text { Going to rural tourism sites is enjoyable }\end{array}$}} & 0.869 \\
\hline & & & & & 0.830 \\
\hline & & & & & 0.919 \\
\hline & & & & & 0.950 \\
\hline Subjective norm & 0.935 & 0.935 & 0.934 & 0.781 & \\
\hline \multicolumn{5}{|c|}{ Most people who are important to me think I should go to rural tourism sites } & 0.898 \\
\hline \multicolumn{5}{|c|}{ Most people who are important to me approve of me going to rural tourism sites } & 0.862 \\
\hline \multicolumn{5}{|c|}{ Most people who are important to me support that I go to rural tourism sites } & 0.876 \\
\hline \multicolumn{5}{|c|}{ Most people who are important to me agree with me about going to rural tourism sites } & 0.898 \\
\hline Perceived behavior control & 0.854 & 0.860 & 0.857 & 0.668 & \\
\hline \multicolumn{5}{|c|}{ I am capable of going to rural tourism sites } & 0.823 \\
\hline \multicolumn{5}{|c|}{ I am confident that, if I want to, I can go to rural tourism sites } & 0.859 \\
\hline \multicolumn{5}{|c|}{ I have enough resources, time, and opportunities to go to rural tourism sites } & 0.766 \\
\hline
\end{tabular}


Table 3. Cont.

\begin{tabular}{|c|c|c|c|c|c|}
\hline Items & $\begin{array}{l}\text { Cronbach's } \\
\text { Alpha }\end{array}$ & Rho_A & $\begin{array}{l}\text { Composite } \\
\text { Reliability }\end{array}$ & AVE & Factor Loading \\
\hline Intention & 0.976 & 0.977 & 0.976 & 0.912 & \\
\hline \multicolumn{5}{|c|}{ I intend to travel to rural tourism sites in the next two years } & 0.982 \\
\hline \multicolumn{5}{|c|}{ I am planning to travel to rural tourism sites in the next two years } & 0.911 \\
\hline \multirow{2}{*}{\multicolumn{5}{|c|}{$\begin{array}{l}\text { I will make an effort to travel to rural tourism sites in the next two years } \\
\text { I will certainly invest time and money to travel to rural tourism sites in the next two years }\end{array}$}} & 0.955 \\
\hline & & & & & 0.970 \\
\hline Intensity of SNS use & 1 & 1 & 1 & 1 & \\
\hline \multicolumn{5}{|c|}{ How many hours do you spend per week on social media? } & 1 \\
\hline Trip experience sharing & 0.914 & 0.930 & 0.919 & 0.851 & \\
\hline \multicolumn{5}{|c|}{ How often do you upload photos of your trip on SNS? } & 0.849 \\
\hline \multicolumn{5}{|c|}{ Do you write about your trip experience on SNS? } & 0.991 \\
\hline $\begin{array}{l}\text { Subjective norm * Intensity } \\
\text { of SNS use }\end{array}$ & 1 & 0.869 & 1 & 1 & \\
\hline $\begin{array}{l}\text { Subjective norm }{ }^{*} \text { Trip } \\
\text { experience sharing }\end{array}$ & 1 & 0.935 & 1 & 1 & \\
\hline
\end{tabular}

Table 4 shows the result of the discriminant validity test. To get a discriminant validity, the square root of the AVE for each variable should exceed the inter-construct correlations [53,61]. It is easy to see that all factors have discriminant validity because all correlations are lower than the square roof of the AVE, the values for which are shown in bold. Also, the results of the model heterotrait-monotrait ratio (HTMT) indicate that all variables did not contain 1 between a $2.5-97.5 \%$ confidence interval. In conclusion, the study model gained acceptable discriminant validity for analysis.

Table 4. Discriminant validity.

\begin{tabular}{|c|c|c|c|c|c|c|c|c|}
\hline Variables & $\begin{array}{c}1 . \\
\text { Attitude }\end{array}$ & $\begin{array}{c}2 . \\
\text { Intention }\end{array}$ & $\begin{array}{c}3 . \\
\text { Perceived } \\
\text { Behavior } \\
\text { Control }\end{array}$ & $\begin{array}{c}4 . \\
\text { Subjective } \\
\text { Norm }\end{array}$ & $\begin{array}{c}5 . \\
\text { Trip } \\
\text { Experience } \\
\text { Sharing }\end{array}$ & $\begin{array}{c}6 . \\
\text { Subjective } \\
\text { Norm * Trip } \\
\text { Experience } \\
\text { Sharing }\end{array}$ & $\begin{array}{c}7 . \\
\text { Subjective } \\
\text { Norm * } \\
\text { Intensity of } \\
\text { SNS Use }\end{array}$ & $\begin{array}{l}\text { 8. Intensity } \\
\text { of SNS Use }\end{array}$ \\
\hline 1 & 0.893 & & & & & & & \\
\hline 2 & 0.663 & 0.955 & & & & & & \\
\hline 3 & 0.670 & 0.640 & 0.817 & & & & & \\
\hline 4 & 0.794 & 0.744 & 0.677 & 0.884 & & & & \\
\hline 5 & 0.312 & 0.337 & 0.333 & 0.383 & 0.923 & & & \\
\hline 6 & -0.049 & 0.040 & -0.031 & -0.115 & 0.013 & 1.000 & & \\
\hline 7 & -0.048 & -0.082 & -0.133 & -0.125 & -0.069 & 0.401 & 1.000 & \\
\hline 8 & 0.123 & 0.127 & 0.170 & 0.138 & 0.325 & -0.067 & -0.099 & 1.000 \\
\hline
\end{tabular}

Note: Values on the diagonal (in bold) represent the square root of AVE; lower diagonal values indicate factor correlations.

Generally, a PLS-SEM does not use model fit indices, contrary to CB-SEM. To clarify, the model of goodness-of-fit (GOF) is an assessment tool that measures how well a model reproduces the covariance matrix among the indicators $[63,64]$. GOF values of $0.1,0.25$, and 0.36 indicate small, moderate, and large model fits, respectively $[65,66]$. The GOF of this study model came out to be 0.729 , which suggests that it is highly applicable.

\subsection{Structural Model}

Multicollinearity between each of the endogenous variables should be confirmed absent prior to SEM analysis [67]. This can be shown by checking VIF(variance inflation factors) statistics. All VIF values of the model should be lower than 5 [68]. Inner VIF of the model was as follows: attitude 3.004; subjective norm 3.209; perceived behavior control 2.09; intensity of SNS-use 1.134; trip experience sharing 1.303; moderating variable of intensity of SNS-use 1.224; and moderating variable of trip experience sharing 1.240. All VIF values were not greater than 5 .

Before looking in detail at the structural model result, we can check the predictive power of the study model. In this study model there was one endogenous variable: intention to visit a rural tourism 
site. The $\mathrm{R}$ squared of the intention variable was 0.606 ( $\mathrm{R}$ squared adjusted: 0.598 ). An $\mathrm{R}$ squared of 0.75 means relevant, 0.5 means moderate, and 0.25 means weak [69]. So, the $\mathrm{R}$ squared of this study model was moderate. The cross-validated redundancy can be confirmed by test $\mathrm{Q} 2$, where $>0$ shows predictive relevance [33]. The test Q2 result of the study was 0.0501 .

The results from Table 5 suggest that $\mathrm{H} 2, \mathrm{H} 3$, and $\mathrm{H} 7$ were supported. The findings demonstrate that the perceived behavior control and subjective norm variables have positive and significant effects on intention to visit rural tourism sites. The $\beta$ values of perceived behavior control and subjective norm were 0.206 and 0.515 . The $\mathrm{T}$ values were 2.454 and 5.526 . The subjective norm variable was than twice the $\beta$ value of perceived behavior control. The moderating effect of intensity of SNS-use was non-significant for subjective norm on visit intention, but the moderating effect of trip experience sharing was significant $(\beta=0.118, \mathrm{t}=2.886)$.

Table 5. Result of the structural model.

\begin{tabular}{|c|c|c|c|c|c|}
\hline $\mathbf{H}$ & Path Estimate & Path Coeff $(\beta)$ & T Statistics & $p$-Values & F Square \\
\hline H1 & Attitude $>$ Intention & 0.110 & 1.064 & 0.288 & 0.010 \\
\hline $\mathrm{H} 2$ & $\begin{array}{c}\text { Perceived behavior control > } \\
\text { Intention }\end{array}$ & 0.206 & 2.454 * & 0.014 & 0.052 \\
\hline H3 & Subjective norm $>$ Intention & 0.515 & $5.526^{* * *}$ & 0.000 & 0.210 \\
\hline $\mathrm{H} 4$ & $\begin{array}{c}\text { Intensity of SNS use > } \\
\text { Intention }\end{array}$ & 0.003 & 0.058 & 0.954 & 0.000 \\
\hline H5 & $\begin{array}{c}\text { Trip experience sharing }> \\
\text { Intention }\end{array}$ & 0.032 & 0.678 & 0.498 & 0.002 \\
\hline H6 & $\begin{array}{l}\text { Subjective norm * Intensity of } \\
\text { SNS use > Intention }\end{array}$ & -0.031 & 0.656 & 0.512 & 0.002 \\
\hline H7 & $\begin{array}{c}\text { Subjective norm * Trip } \\
\text { experience sharing }>\text { Intention }\end{array}$ & 0.118 & $2.886^{* *}$ & 0.004 & 0.032 \\
\hline
\end{tabular}

The F square indices of $0.02,0.15$, and 0.35 indicate a small, medium, and large effect size, respectively [70]. It was observed that the values obtained for the perceived behavior control variables, and the moderating variable of trip experience sharing had a small effect size. The subjective norm variable had a medium effect on intention.

\section{Discussion}

Through the TPB model analysis of rural tourism, the role of social media use in the rural tourism decision-making process has been found. The TPB model has been used in numerous other studies regarding tourism, but this study is significant because of its application in studying sustainable tourism-more specifically, rural tourism. According to the TPB model results, Hypothesis 2 and 3 were supported, but not Hypothesis 1.

Attitude did not have any influence on tourists' intentions regarding rural tourism. Although, from their education, people have a positive image of rural tourism as a type of sustainable tourism, this image does not become a major motivating factor that leads to an actual tour. It might be that there is a considerable gap between tourists' certainty of the importance of sustainable tourism and their willingness to make personal sacrifices for the cause. Moreover, the image of overall tourism destinations has a significant effect on tourists' behavior intentions [71]. Unlike travel to popular locations represented by breathtaking monuments and sights to behold, rural tourism is a relatively modest experience without special images. Although it may have a positive social image, the attitude towards it may not be enough to sway tourists' intent.

The results showed that perceived behavior control has an influence on the intent of the consumer. Tourism is something that people can live without. Also, taking a trip requires time and money to spare. Sustainable tourism especially may be not a first choice for people who have not had a trip before. Therefore, if sustainable tourism is marketed, targeting the upper-middle class would be effective. 
Intention to travel to rural sites was much more heavily affected by subjective norms. The same result was shown in research papers applying the TPB model to other sustainable tourism locations $[6,14,39]$. This indicates that creating a good public image of going to sustainable tourism sites is important in order to increase popularity.

This study investigated Hypotheses 4 to 7 , because the study wanted to know how social media use affects rural tourism using the TPB model. Hypotheses 4 and 5 were not supported, which related to the social media use variables' direct effect on visit intention. The moderating effects of social media use were partly supported. Hypothesis 6 did not have a significant result. Hypothesis 7 was verified. As mentioned above, the factor that had the most influence within the TPB model was subjective norm. Therefore, it is important to understand the relationship between subjective norm and tourists' intent, as well as the moderating effect of social media. According to the results, frequency of use did not have a significant moderating effect between subjective norm and tourists' intent, but it was found that the sharing of trip experience did. What matters is how social media is used, not how often it is used. Long duration of social media use does not necessarily indicate the users' level of caring about other people, since the SNS platform can be used in such a large variety of ways.

\section{Conclusions}

This study revealed the decision-making process of rural tourism and role of social media in the process. When potential tourists consider rural tourism, what actually helps push visit intention is society atmosphere and circumstances of individuals. Thus, it can be said that, in order to assist the development of sustainable tourism, marketers should emphasize content related to subjective norms. Marketing strategies should cultivate a social mood and showcase functional benefits of rural/sustainable tourism itself instead of merely describing the appeals of specific tourist locations.

If marketers were to focus more on encouraging the sharing of tourism experiences, this could aid sustainable tourism growth. Developments could be made to help facilitate and encourage the act of sharing via social media. More physical evidence, such as enticing photo zones and monuments, are some examples. Also, marketers can host some events, including social media sharing activities, such as giving prizes when people upload posts. In particular, events that can lead to sustainable public benefits beyond personal experience may encourage more powerful subjective norm effects.

\section{Limitations and Future Studies}

This research used the TPB model to examine the influence of social media on rural tourism, but it had a couple limitations. First is a sample problem. Despite the using a large company which conducted the survey with nationwide sample in Korea, the survey method used in the study relied on self-reports. Since rural tourism in Korea has not yet been popular enough to be known to many tourists, we were not able to assess tourists who had experienced the rural tourism enough. Thus, that is, only relatively highly interested people who were willing to experience rural tourism participated in the survey, which makes it difficult to say that the subjects of this study represent a truly random sample and can be generalized. Every participant was a potential tourist mentally simulating the scenario of engaging in rural tourism, and not an actual tourist. Second, for the moderating effect, only two variables were considered: the duration of social media use and the sharing of experience. These are only two pieces of the bigger picture. Perhaps, in a future study, a more extensive study model could be developed consisting of a larger variety of representative variables that can be examined.

Author Contributions: Data curation, H.S.; Project administration, Y.N.; Supervision, Y.N.; Writing-original draft, Y.J.; Writing-review and editing, Y.N. All authors have read and agreed to the published version of the manuscript.

Funding: This research received no external funding.

Conflicts of Interest: The authors declare no conflict of interest. 


\section{References}

1. Liu, W.; Bai, E.; Liu, L.; Wei, W. A framework of sustainable service supply chain management: A literature review and research agenda. Sustainability 2017, 9, 421. [CrossRef]

2. Vargo, S.L.; Lusch, R.F. Evolving to a new dominant logic for marketing. In The Service-Dominant Logic of Marketing; Routledge: Abingdon, UK, 2014; pp. 21-46.

3. Pantano, E.; Priporas, C.V.; Stylos, N.; Dennis, C. Facilitating tourists' decision making through open data analyses: A novel recommender system. Tour. Manag. Perspect. 2019, 31, 323-331. [CrossRef]

4. Smallman, C.; Moore, K. Process studies of tourist's decision-making. Ann. Tour. Res. 2010, 37, $397-422$. [CrossRef]

5. Kuo, N.-W.; Dai, Y.-Y. Applying the Theory of Planned Behavior to Predict Low-Carbon Tourism Behavior. Int. J. Technol. Hum. Interact. 2012, 8, 45-62. [CrossRef]

6. Meng, B.; Choi, K. Extending the theory of planned behaviour: Testing the effects of authentic perception and environmental concerns on the slow-tourist decision-making process. Curr. Issues Tour. 2016, 19, 528-544. [CrossRef]

7. Hamid, M.A.; Isa, S.M. The Theory of Planned Behaviour on Sustainable Tourism. Appl. Environ. Biol. Sci. 2015, 5, 84-88.

8. Milano, R.; Baggio, R.; Piattelli, R. The effects of online social media on tourism websites. In Proceedings of the ENTER, Innsbruck, Austria, 26-28 January 2011; pp. 471-483.

9. Pesonen, J. Tourism marketing in facebook: Comparing rural tourism SME's and larger tourism companies in Finland. In Proceedings of the ENTER, Innsbruck, Austria, 26-28 January 2011; pp. 537-546.

10. Hays, S.; Page, S.J.; Buhalis, D. Social media as a destination marketing tool: Its use by national tourism organisations. Curr. Issues Tour. 2013, 16, 211-239. [CrossRef]

11. Fons, M.V.S.; Fierro, J.A.M.; y Patiño, M.G. Rural tourism: A sustainable alternative. Appl. Energy 2011, 88, 551-557. [CrossRef]

12. Leung, D.; Law, R.; van Hoof, H.; Buhalis, D. Social Media in Tourism and Hospitality: A Literature Review. J. Travel Tour. Mark. 2013, 30, 3-22. [CrossRef]

13. Xiang, Z.; Gretzel, U. Role of social media in online travel information search. Tour. Manag. 2010, 31, 179-188. [CrossRef]

14. Jalilvand, M.R.; Samiei, N. The impact of electronic word of mouth on a tourism destination choice. Internet Res. 2012. [CrossRef]

15. WCED. Our common future; Oxford University Press: New York, NY, USA, 1987.

16. Manning, E.W.; Dougherty, T.D. Sustainable tourism: Preserving the golden goose. Cornell Hotel Restaur. Adm. Q. 1995, 36, 4-42. [CrossRef]

17. Butler, R.W. Sustainable tourism: A state-of-the-art review. Tour. Geogr. 1999, 1, 7-25. [CrossRef]

18. Hobson, K.; Essex, S. Sustainable Tourism: A View from Accommodation Businesses. Serv. Ind. J. 2001, 21, 133-146. [CrossRef]

19. Müller, H. The thorny path to sustainable tourism development. J. Sustain. Tour. 1994, 2, 131-136. [CrossRef]

20. Elkington, J. Enter the triple bottom line. In The Triple Bottom Line; Routledge: Abingdon, UK, 2013; pp. $23-38$.

21. UNEP UNWTO. Making Tourism More Sustainable — A Guide for Policy Makers; UNEP UNWTO: New York, NY, USA, 2005.

22. Cottrell, S.; van der Duim, R.; Ankersmid, P.; Kelder, L. Measuring the Sustainability of Tourism in Manuel Antonio and Texel: A Tourist Perspective. J. Sustain. Tour. 2004, 12, 409-431. [CrossRef]

23. UNWTO Home Page. Available online: https://www.unwto.org/sustainable-development (accessed on 14 April 2020).

24. Lane, B. What is rural tourism? J. Sustain. Tour. 1994, 2, 7-21. [CrossRef]

25. Embacher, H. Marketing for Agri-tourism in Austria: Strategy and realisation in a highly developed tourist destination. J. Sustain. Tour. 1994, 2, 61-76. [CrossRef]

26. Clarke, J. Marketing Structures for Farm Tourism: Beyond the Individual Provider of Rural Tourism. J. Sustain. Tour. 1999, 7, 26-47. [CrossRef]

27. Byrd, E.T.; Bosley, H.E.; Dronberger, M.G. Comparisons of stakeholder perceptions of tourism impacts in rural eastern North Carolina. Tour. Manag. 2009, 30, 693-703. [CrossRef] 
28. Iorio, M.; Corsale, A. Rural tourism and livelihood strategies in Romania. J. Rural Stud. 2010, 26, 152-162. [CrossRef]

29. Boley, B.B.; McGehee, N.G.; Tom Hammett, A.L. Importance-performance analysis (IPA) of sustainable tourism initiatives: The resident perspective. Tour. Manag. 2017, 58, 66-77. [CrossRef]

30. Puska, A.; Sadic, S.; Maksimovic, A.; Stojanovic, I. Decision support model in the determination of rural touristic destination attractiveness in the Brcko District of Bosnia and Herzegovina. Tour. Hosp. Res. 2020, 19. [CrossRef]

31. Pina, I.P.A.; Delfa, M.T.D. Rural tourism demand by type of accommodation. Tour. Manag. 2005, 26, 951-959. [CrossRef]

32. Maestro, R.M.H.; Gallego, P.A.M.; Requejo, L.S. The moderating role of familiarity in rural tourism in Spain. Tour. Manag. 2007, 28, 951-964. [CrossRef]

33. Loureiro, S.M.C. The role of the rural tourism experience economy in place attachment and behavioral intentions. Int. J. Hosp. Manag. 2014, 40, 1-9. [CrossRef]

34. Ajzen, I. The theory of planned behavior. Organ. Behav. Hum. Decis. Process. 1991, 50, 179-211. [CrossRef]

35. Quintal, V.A.; Lee, J.A.; Soutar, G.N. Risk, uncertainty and the theory of planned behavior: A tourism example. Tour. Manag. 2010, 31, 797-805. [CrossRef]

36. Sparks, B. Planning a wine tourism vacation? Factors that help to predict tourist behavioural intentions. Tour. Manag. 2007, 28, 1180-1192. [CrossRef]

37. Lee, T.H.; Jan, F.H. Ecotourism Behavior of Nature-Based Tourists: An Integrative Framework. J. Travel Res. 2018, 57, 792-810. [CrossRef]

38. Eom, T.; Han, H. Community-based tourism (TourDure) experience program: A theoretical approach. J. Travel Tour. Mark. 2019, 36, 956-968. [CrossRef]

39. Ashraf, M.S.; Hou, F.J.; Kim, W.G.; Ahmad, W.; Ashraf, R.U. Modeling tourists' visiting intentions toward ecofriendly destinations: Implications for sustainable tourism operators. Bus. Strategy Environ. 2020, 29, 54-71. [CrossRef]

40. Lane, B. Sustainable rural tourism strategies: A tool for development and conservation. J. Sustain. Tour. 1994, 2, 102-111. [CrossRef]

41. Zeng, B.; Gerritsen, R. What do we know about social media in tourism? A review. Tour. Manag. Perspect. 2014, 10, 27-36. [CrossRef]

42. Nusair, K. Developing a comprehensive life cycle framework for social media research in hospitality and tourism. Int. J. Contemp. Hosp. Manag. 2020, 32, 1041-1066. [CrossRef]

43. Nusair, K.; Butt, I.; Nikhashemi, S.R. A bibliometric analysis of social media in hospitality and tourism research. Int. J. Contemp. Hosp. Manag. 2019, 31, 2691-2719. [CrossRef]

44. Fotis, J.N.; Buhalis, D.; Rossides, N. Social Media Use and Impact during the Holiday Travel Planning Process; Springer-Verlag: Berlin/Heidelberg, Germany, 2012.

45. Narangajavana, Y.; Callarisa Fiol, L.J.; Moliner Tena, M.Á.; Rodríguez Artola, R.M.; Sánchez García, J. The influence of social media in creating expectations. An empirical study for a tourist destination. Ann. Tour. Res. 2017, 65, 60-70. [CrossRef]

46. Kavoura, A.; Stavrianea, A. Economic and Social Aspects from Social Media's Implementation as a Strategic Innovative Marketing Tool in the Tourism Industry. Procedia Econ. Financ. 2014, 14, 303-312. [CrossRef]

47. Cho, I.; Kim, J.K.; Park, H.; Lee, S.M. Motivations of Facebook Places and store atmosphere as moderator. Ind. Manag. Data Syst. 2014. [CrossRef]

48. Munar, A.M.; Jacobsen, J.K.S. Motivations for sharing tourism experiences through social media. Tour. Manag. 2014, 43, 46-54. [CrossRef]

49. Seidman, G. Self-presentation and belonging on Facebook: How personality influences social media use and motivations. Personal. Individ. Differ. 2013, 54, 402-407. [CrossRef]

50. Kim, J.; Fesenmaier, D.R. Sharing tourism experiences: The posttrip experience. J. Travel Res. 2017, 56, $28-40$. [CrossRef]

51. Hausmann, A.; Toivonen, T.; Slotow, R.; Tenkanen, H.; Moilanen, A.; Heikinheimo, V.; Di Minin, E. Social Media Data Can Be Used to Understand Tourists' Preferences for Nature-Based Experiences in Protected Areas. Conserv. Lett. 2018, 11. [CrossRef]

52. Swarbrooke, J.; Horner, S. Consumer Behaviour in Tourism; Routledge: Abingdon, UK, 2007. 
53. Chin, W.W. The partial least squares approach to structural equation modeling. Mod. Methods Bus. Res. 1998, 295, 295-336.

54. Chin, W.W.; Marcolin, B.L.; Newsted, P.R. A partial least squares latent variable modeling approach for measuring interaction effects: Results from a Monte Carlo simulation study and an electronic-mail emotion/adoption study. Inf. Syst. Res. 2003, 14, 189-217. [CrossRef]

55. Kenny, D.A.; Judd, C.M. Estimating the nonlinear and interactive effects of latent variables. Psychol. Bull. 1984, 96, 201. [CrossRef]

56. Xiao, L.; Mou, J. Social media fatigue -Technological antecedents and the moderating roles of personality traits: The case of WeChat. Comput. Hum. Behav. 2019, 101, 297-310. [CrossRef]

57. Dijkstra, T.K.; Henseler, J. Consistent partial least squares path modeling. Mis Q. 2015, 39, 297-316. [CrossRef]

58. Boley, B.B.; Magnini, V.P.; Tuten, T.L. Social media picture posting and souvenir purchasing behavior: Some initial findings. Tour. Manag. 2013, 37, 27-30. [CrossRef]

59. Bagozzi, R.P.; Dholakia, U.M.; Basuroy, S. How effortful decisions get enacted: The motivating role of decision processes, desires, and anticipated emotions. J. Behav. Decis. Mak. 2003, 16, 273-295. [CrossRef]

60. Kassem, N.O.; Lee, J.W.; Modeste, N.N.; Johnston, P.K. Understanding soft drink consumption among female adolescents using the Theory of Planned Behavior. Health Educ. Res. 2003, 18, 278-291. [CrossRef] [PubMed]

61. Fornell, C.; Larcker, D.F. Evaluating structural equation models with unobservable variables and measurement error. J. Mark. Res. 1981, 18, 39-50. [CrossRef]

62. Nunnally, J.C. Psychometric Theory, 2nd ed.; McGraw-Hill: New York, NY, USA, 1978.

63. Lee, H.; Yang, S.-B.; Koo, C. Exploring the effect of Airbnb hosts' attachment and psychological ownership in the sharing economy. Tour. Manag. 2019, 70, 284-294. [CrossRef]

64. Tenenhaus, M.; Amato, S.; Esposito Vinzi, V. A global goodness-of-fit index for PLS structural equation modelling. In Proceedings of the XLII SIS Scientific Meeting, Padova, Italy, June 2004; pp. 739-742.

65. Tenenhaus, M.; Vinzi, V.E.; Chatelin, Y.-M.; Lauro, C. PLS path modeling. Comput. Stat. Data Anal. 2005, 48, 159-205. [CrossRef]

66. Wetzels, M.; Odekerken-Schröder, G.; Van Oppen, C. Using PLS path modeling for assessing hierarchical construct models: Guidelines and empirical illustration. Mis Q. 2009, 33, 177-195. [CrossRef]

67. Cassel, C.; Hackl, P.; Westlund, A.H. Robustness of partial least-squares method for estimating latent variable quality structures. J. Appl. Stat. 1999, 26, 435-446. [CrossRef]

68. Martínez-Navalón, J.G.; Gelashvili, V.; Debasa, F. The impact of restaurant social media on environmental sustainability: An empirical study. Sustainability 2019, 11, 6105. [CrossRef]

69. Henseler, J.; Ringle, C.M.; Sinkovics, R.R. The use of partial least squares path modeling in international marketing. In New Challenges to International Marketing; Emerald Group Publishing Limited: Bingley, UK, 2009.

70. Cohen, J. Statistical Power Analysis for the Behavioral Sciences, (L. Erlbaum Associates, Hillsdale, NJ); Erlbaum Associates: Hillsdale, NJ, USA, 1988.

71. Wang, C.-Y.; Hsu, M.K. The relationships of destination image, satisfaction, and behavioral intentions: An integrated model. J. Travel Tour. Mark. 2010, 27, 829-843. [CrossRef]

(C) 2020 by the authors. Licensee MDPI, Basel, Switzerland. This article is an open access article distributed under the terms and conditions of the Creative Commons Attribution (CC BY) license (http://creativecommons.org/licenses/by/4.0/). 
Article

\title{
The Effect of Social Media Usage Characteristics on e-WOM, Trust, and Brand Equity: Focusing on Users of Airline Social Media
}

\author{
Eun Ju Seo ${ }^{1}$, Jin-Woo Park ${ }^{2, *}$ and Yu Jin Choi ${ }^{3}$ \\ 1 International Service Office Incheon, Asiana Airlines, Incheon 22382, Korea; seoej@flyasiana.com \\ 2 School of Business, Korea Aerospace University, Goyang-si 10540, Korea \\ 3 Airport Industry Technology Research Institute, Incheon International Airport Corporation, Incheon 22384, \\ Korea; cyj@airport.kr \\ * Correspondence: jwpark@kau.ac.kr
}

Received: 6 January 2020; Accepted: 21 February 2020; Published: 24 February 2020

\begin{abstract}
Brand equity is a valuable intangible asset for companies, yet is increasingly difficult in managing in an era with hard to control social media. The purpose of this study was to investigate the effect of social media usage characteristics on electronic word-of-mouth (e-WOM), trust, and brand equity by dividing the characteristics to personality, social, and information. A survey was administered to 430 respondents who had experience of using airline social media and the collected data was analyzed using structural equation modeling. The results showed that the personality and informational characteristics from social media usage had statistically significant effects on e-WOM. It was found that the e-WOM had significant effects on trust and also on brand awareness. The trust was shown to have a statistically significant effect on brand awareness and brand image. Therefore, this study categorizes social media usage characteristics into three characteristics: personality characteristics, social characteristics, and information characteristics, and each of these usage characteristics present a strategy to improve actual brand equity of airline through e-WOM and trust in empirical methods. The findings of this study are expected to provide fundamental data for the development of strategies related to airline social media. In addition, this study has implications for suggesting to improve brand equity through e-WOM and trust.
\end{abstract}

Keywords: social media usage characteristics; Big Five personality traits; personality characteristics; social characteristics; information characteristics; e-WOM; trust; brand equity; brand awareness; brand image

\section{Introduction}

Social media is a space that allows consumers to discuss freely exchanged opinions. These interactions between consumers in social media are fundamentally changing the communication between brands and customers [1,2]. Social media has dramatically changed the process for how brand contents is created distributed and consumed, and has made it possible for the brand images created by marketers to be delivered online via consumers [2-4]. Social media is also fundamentally changing the way consumers acquire the information they want, how they buy products, and how they discuss social issues and pursue social change.

In the past, the public mostly relied on the service of travel products offered by travel agencies for overseas trips, but nowadays, the range of choices for travel destinations, hotel reservations, and flight ticket purchases have become diversified and subdivided through social media and the Internet. There has been a rapid increase in the number of cases where travel plans are set up, flight tickets are purchased, and hotel rooms are reserved through various travel information delivered through social 
media. In addition, information can be viewed and shared at any time or place so people are making faster decisions while preparing for overseas trips.

According to Socialbakers' statistics on usage levels of social media in the aviation industry based on Facebook fans as of June 2019, Qatar Airways has the world's largest number of fans, at 14 million followed by KLM with about 13 million. Compared to foreign cases, the usage of social media in marketing and the awareness of its importance in Korean airlines seem to be very low, considering the small size of fans (Asiana Airlines 570,000, Jeju Air 500,000, and Eastar Airlines 470,000). Thus, the use of social media by airlines in Korea is limited compared to that of overseas airlines. Major Korean airlines have official social media accounts, but their activities and marketing are less active than overseas. The use of airline social media by passengers is likely to increase continuously and so will the importance of social media in airline marketing.

Unlike other industries, the products of the airline industry consist of seats and service, which are material and intangible as comprehensive service ones, so there are no leftover stocks and the produced amount cannot be controlled. Even if products are not made or sold, it carries a unique characteristic where a definite amount of expense is spent, so it is important to attract potential customers through different marketing activities and, in the long term, enhance brand values. Seo and Park [5] examined the influence of corporate social media activities on customer response through brand equity in the social media marketing aspect of the airline industry. Studies on social media have been being actively conducted, however few studies have been conducted that systematically characterize the use of social media [6-10]. In particular, there is still a lack of research on how the use of social media can have an effect on corporate performance. This study shows that the characteristics of personality, social, and information characteristics in terms of users of social media examined the impact on companies through electronic word-of-mouth (e-WOM). The studies to identify the factors in characteristics of airline social media usage and how those characteristics affect airlines are likely to provide very important implications for developing strategies related to airline social media. The purpose of this study was, therefore, to analyze empirically the effects of the characteristics of airline social media usage on electronic word-of-mouth (e-WOM), trust, and brand equity.

\section{Theoretical Background}

"Media" is a collective term for tools that mediate human communication [11]. Social media is a concept that encompasses social network services (SNSs) such as Facebook, Twitter, and LinkedIn that share the connections formed through registered personal profiles [12] and is one of the interactive media that has enhanced the function of producing and sharing contents, in addition to the network function of existing SNS [13]. Richter and Koch [14] defined social media as "an online application, platform, and media that facilitate the interaction, collaborative work, and contents sharing". The marketing activities using social media provide companies with the opportunities to build individual relationships with consumers and to reach closer to them [15]. Social media studies in the tourism industry may be divided, by their perspectives, into consumer and supplier ones, or, by the variables, into ones to identify the prerequisites for using social media and the impact of social media $[16,17]$. The $\mathrm{e}-\mathrm{WOM}$ in social media varies depending on the personality characteristics of individual users, social networks, and information characteristics provided. This study, following the findings of previous studies, defines social media usage characteristics as personality, social, and information ones.

The study of personality characteristics centers on predictions of consumer reactions and behaviors in specific consumption situations and has been actively performed in the field of consumer behaviors and consumer psychology [18]. In the field of personality psychology, researchers have studied to discover universal and important characteristics that can distinguish individual differences in human personality, and there are various theories of physiological and developmental aspects of personality characteristics [19,20]. Although the theoretical and methodological underpinnings of the Big Five model are not completely without dispute [21], it is regarded as acknowledging at least some of the essential aspects of personality [22]. 
Neuroticism is defined as a "measurement of controlling emotion and feeling" [22]. A low level of neuroticism indicates easy control of feelings, while a high level includes acute sensitivity, nervousness, and concern [23]. Extroversion is the positive emotions and feelings that occur in social situations. Those who have low Extroversion (Introversion) tend to be shy and respond in an introversive fashion when meeting or socializing with people [18]. Openness to Experience is an attitude to novel experiences. Those who have a high level of openness are curious, creative and innovative, while those who have a low level show a clear preference for being customary and consistent, and practical and traditional [24,25]. Agreeableness is a concept meaning trust, concern, and generous sentiment toward others or the degree of maintaining a comfortable and harmonious relationship with others. This trait is expressed as trust, empathy, and is contrasted with cynicism, disrespect, and hostility [26]. Conscientiousness refers to the individual tendency to keep rules, norms, principles, and others of the organization. Higher Conscientiousness is associated with stronger self-restraint, ambition, and hard-work [27], and a stronger willingness to complete a task [28].

All members of society may build trust within relationships with their families, work colleagues, neighborhoods, and community groups, and through these social networks, increase their quality of life and reach social achievement. Social capital is formed through these interpersonal or intergroup relationships, a concept that has been studied in many disciplines related to social networks, trust, mutual cognition, and emotional support [29-32]. Bridging social capital refers to a human network created through voluntary engagement in relationships between individuals from different backgrounds and enables connections between employers regardless of race, gender, occupation, income, and religion [31]. Structural holes are created between members who are not tangled with each other based on interest. Such members do not have a direct relationship and provide a variety of non-overlapping information to each other, and are thus likely to acquire useful new information and knowledge [33]. In contrast, a social network composed of people with similar backgrounds and characteristics, such as family and friends, is referred to as bonding social capital. This relationship is closely linked by sticky emotion, providing emotional support and practical assistance to each other [34]. In this kind of relationship, members are linked by strong internal ties, deep trust, mutual dependence, and active emotion [35]. Bridging social capital is helpful for smooth social activities, while bonding social capital strengthens one's identity and is essential for complementary relationships [36].

Information can be defined as data where analyzed or contextualized messages are conveyed and differences follow depending on the recognition of the recipients [37], and due to the recent development of social media, the public can approach information more freely so it results in the production and delivery of information that is incomparably massive in quantity compared to the past [38]. The information quantity signifies the actual amount of information $[39,40]$, and it has mostly been researched in two aspects, including the number of choices or the amount of information that is provided for each decision [41]. The information credibility is the most fundamental element of the personal persuasion process [42] and can be defined as the extent to which the recipients recognize that the origin of information has knowledge, skills, and experience related to the product or service and will provide unbiased opinions and objective information [43]. The information quality is the extent to which the corresponding information helps a decision maker in evaluating a product or service and defined as the usefulness of the information in decision-making [44].

e-WOM has long been regarded as an influential marketing tool [45] and social media is recognized as the best platform for e-WOM [46,47]. E-WOM is similar to the traditional offline WOM in that it is an interactive communication process for exchanging experiences and information about products or services, while differing from offline WOM in that it is based on the Internet [48]. e-WOM is generally defined as the act of meeting and sharing opinions with each other on the Internet and exchanging the ratings for services [49-51], which provide usability, accessibility, and persistence for information that were unavailable in traditional offline WOM [52]. Consumers search for information posted by people who have used a product or service they are planning to purchase in attempts to reduce fear or anxiety about failures by verifying relevant information [53]. The information disseminated through these 
WOMs tends to be accepted as fair and unexaggerated one [54]. WOM is very important because it shapes consumers' attitudes toward brands [55]. e-WOM considers positive WOM as expressed in customers' willingness to recommend the product to others in this study.

Trust is a concept that plays an important role in social and economic interactions in which uncertainty and dependence exist and have been widely studied in various social sciences [56]. Robinson [57] argued that trust is the expectation and belief future behaviors of others will be favorable or, at least, non-hostile. In contrast with these definitions of trust in terms of emotion or psychological state, there are other definitions from the behavioral perspective. Mishra [58] defined trust as a party's willingness to be vulnerable to another party based on the belief that the other party is competent, open, concerned, and reliable. Trust is basically defined as taking risks, and in some situations, choosing one option in spite of the possibility of loss by others [59]. Consequently, trust has two components as the confident expectations of the brand's reliability and good intentions to the brand even in unexpected problems [60]. Trust in service providers is an essential factor in determining the quality of successful relationships [61] and is an important variable in forming relationships with customers [62].

The groundwork for customer-based brand equity (CBBE) was set in the early 1990s by Aaker [63] and Keller [64,65] and has received ample attention in many different fields. Keller [64] insisted that customer-based brand equity occurs when the customer is familiar with the brand, and this gives the customers a favorable, powerful, and/or unique memory about the brand image. Keller [64], accordingly, defined brand equity as the customer's response to brand knowledge and presented the components of brand equity as brand awareness including brand recall, brand recognition, and brand image including the type, preference, strength, and uniqueness associated with the brand. Brand equity may be, therefore, defined as a measure of the favorable outcome in a market that would not have occurred if the product or service had no such brand. Brand equity is formed when customers are aware of the brand and have a unique and positive image associated with the brand [63]. Therefore, brand equity is an important intangible asset of a company and is a higher level of concept than other concepts related to the brand, such as brand love or brand loyalty. In previous studies [66,67], the influence of brand love and brand loyalty on brand equity as parameters were conducted.

Brand awareness is often regarded as a fundamental concept of brand equity because it is an important starting point and the basis of forming brand loyalty by familiarity with a brand generated by brand awareness [68]. Aaker [69] defines brand awareness as a concept that represents the degree to which consumers perceive a brand differently from other brands, and Keller et al. [70] explained that brand awareness is the ability of potential customers to recognize and recall a particular brand in a product category. Brand awareness refers to the consumer's ability to identify a brand under different environmental conditions, that is, the ability to remember a brand [71], which forms the basis for building an image, attitude, and trust in the brand and is a very important concept in that it is the first step in the purchase where a product or service is included in the consumer's consideration sets. Brand awareness, therefore, plays a role in providing consumers with confidence in the quality of products with the brand [72].

Brand image is a positive or negative feeling that a consumer has about a brand, confidence in the brand, or a psychological structure of the consumer, and is defined as a set of beliefs that the consumer has about the brand [73]. Low and Lamb [74] insisted that brand image is a belief about the function and symbol of a brand' and is composed of a symbolic, social, and psychological image. Dobni and Zinkhan [75] defined brand image as logical or emotional perception reflecting the brand association of the consumer. The positive brand image is made possible by a marketing program that connects strong, favorable, and unique associations with the brand in the memory of the consumer and may be generated by, in addition to the information controlled by the marketing manager, various channels such as the direct experiences of consumers, opinions, and WOM of other consumers [65]. Brand image is composed of a combination of physical, emotional, and psychological elements of the consumer, and is created by combining associations with the brand [64]. 
Consumers with different personalities may respond differently to an identical product or service [76]. Noh et al. [77] examined the influence of WOM by inputting personality traits of users using online in their 20-30s as situation variables. Ju and Suh [78] examined consumer commitment to an online shopping-mall and its effects on WOM intention and reported that the Big Five Personality traits (excluding Openness) had a significant effect on WOM intention through commitment. Bachrach et al. [79], in their study on Facebook and personality factors, investigated the personality, Facebook profile, network size, photos, and number of events and reported that Extroversion was the strongest predictor of WOM intention and followed by Neuroticism, Openness, Conscientiousness, and Agreeableness. This study, based on these previous studies, developed the following hypothesis about the effect of personality characteristics of social media usage of consumers.

Hypothesis 1 (H1). The personality characteristics of social media usage have a significant positive effect on $e-W O M$.

The management of e-WOM through social media has a complicated structure inevitably because the users as consumers simultaneously produce the value and meaning of marketing information [80]. It was also suggested that this variable should be measured and expanded at the individual and social levels because the consumers, in e-WOM processes, jointly produce and share social capital [81]. Ellison et al. [12] investigated whether offline social capital is generated also through online tools and demonstrated that SNS plays a role in shaping and maintaining social capital accumulated through relationships between people. Lee [82] studied, focusing on the comparison between Facebook and Twitter, the e-WOM behaviors and demonstrated that the bridging and bonding social capitals had a significant effect on e-WOM where the former had a stronger effect. This study, based on these previous studies on social characteristics, developed the following hypotheses about the effect of social characteristics of social media usage of consumers.

Hypothesis 2 (H2). The social characteristics of social media usage have a significant positive effect on e-WOM.

Information and consumers have significant effects on e-WOM [83]. As has already been demonstrated in several studies, greater amounts of online reviews lead to more information that consumers acquire about the product or service, resulting in more pervasive e-WOM and higher sales [83-86]. Wen [87] set online information characteristics as accuracy, vividness, and neutrality in the study on the effect of e-WOM information characteristics on WOM acceptance, WOM spread, and purchase intention by product type, and reported that the accuracy of the information had effects on WOM acceptance, and consequently on WOM spread and purchase intention. In a study measuring the effect of tourism e-WOM information quality and reliability and community interaction on the usefulness of WOM and the WOM effect, it was found that the information quality and information reliability had a positive effect on WOM [88]. This study, based on these previous studies, developed the following hypothesis about the effect of the information characteristics of social media usage of consumers.

Hypothesis 3 (H3). The information characteristics of social media usage have a significant positive effect on e-WOM.

WOM forms the attitude of consumers toward a brand [55] and the information disseminated through WOMs tends to be accepted as fair and unexaggerated [54]. e-WOM may have a stronger effect compared to offline WOM in some situations. e-WOM is regarded as a reliable and fair source of information, shaping consumer expectations, affecting attitudes, purchasing decisions, and post-evaluation [89]. Ladhari and Michaud [90] studied the influence of e-WOM on hotel booking intentions, attitudes, trust, and website rating, and demonstrated that more positive WOM for hotels has an association with higher trust in hotels. Cha [91] studied the development of a communication 
paradigm model for the e-WOM of restaurant information and argued that positive e-WOM builds confidence in the sender. Bickart and Schindler [45] investigated Internet forums and reported that Power Blogs act as leaders in online oral communication and that consumers trust information gained from Power Blogs more compared to those from typical company websites, resulting in some effect on brand evaluation.

The most important feature of social media is that users, without constraint on time and place, directly produce and consume desired content. This means that more active and vigorous participation of consumers than company-led one-way communication is possible, and such participation is likely to have strong effects on consumers' awareness and attitudes toward the brand. The recommendation to other consumers about their experiences after online purchasing or using products or services is a typical process of e-WOM in this digital age and has been continuously expanded [92].

The studies on the relationship between e-WOM and brand equity factors have been performed continued in the service field. Yim [93] demonstrated that e-WOM had a significant effect on brand awareness in a study of family restaurant brands. Kim [94] confirmed that positive WOM activities of marketing communication in the relationship between marketing communication and service brand equity factors had a positive effect on brand awareness and brand image. Godey et al. [6] investigated luxury brands' social media marketing efforts and demonstrated that e-WOM influenced brand awareness and brand image by classifying the e-WOM as a social media marketing factor. In the aviation industry field, Lee [95] conducted a study on the influence of WOM activities on brand image and brand awareness. This study, based on these previous studies, developed the following hypothesis about the effect of e-WOM.

Hypothesis 4 (H4). The e-WOM has a significant positive effect on trust.

Hypothesis $\mathbf{5}$ (H5). The e-WOM has a significant positive effect on brand awareness.

Hypothesis 6 (H6). The e-WOM has a significant positive effect on brand image.

According to Forunier et al. [96], as with people engaging with each other in everyday life, consumers engage in personal relationships with the brands they purchase. The trust is part of the brand and consumer relationship and is one of the most influential factors in actual online consumption. Trust is the willingness to trust in, rely on, and exchange with others and, in the situation of dangerous and uncertain services, is a valuable factor for successful long-term relationships [97]. Consumers have a rigid characteristic of not participating in behaviors that they consider to be worthless [98]. Trust is formed through the entire process of recognizing, purchasing, using, and experiencing the brand and is an interaction between consumer and brand at equal positions. Kim et al. [99], in their study on hospital service, argued that brand equity could be formed through customer satisfaction, trust, and commitment, and had a positive effect on improving the overall image of the hospital. Han et al. [100] studied brand equity, brand reputation, and brand trust, and reported that brand awareness had a positive effect on brand trust through brand reputation. Fatma et al. [101], in a study of company reputation and brand equity through company social responsibility activities, found a significant effect of trust on brand equity. Loureiro [102] investigated the brand equity and demonstrated that trust has a positive effect on brand awareness. This study, based on these previous studies, developed the following hypothesis about the effect of trust.

Hypothesis 7 (H7). The trust has a significant positive effect on brand awareness.

Hypothesis 8 (H8). The trust has a significant positive effect on brand image. 


\section{Methodology}

The purpose of this study was to identify the influence of personality, social, and information characteristics of social media usage on brand equity through e-WOM and trust. For the purpose, the empirical analysis method using structural equations was performed and, as shown in Figure 1, the research model is presented.

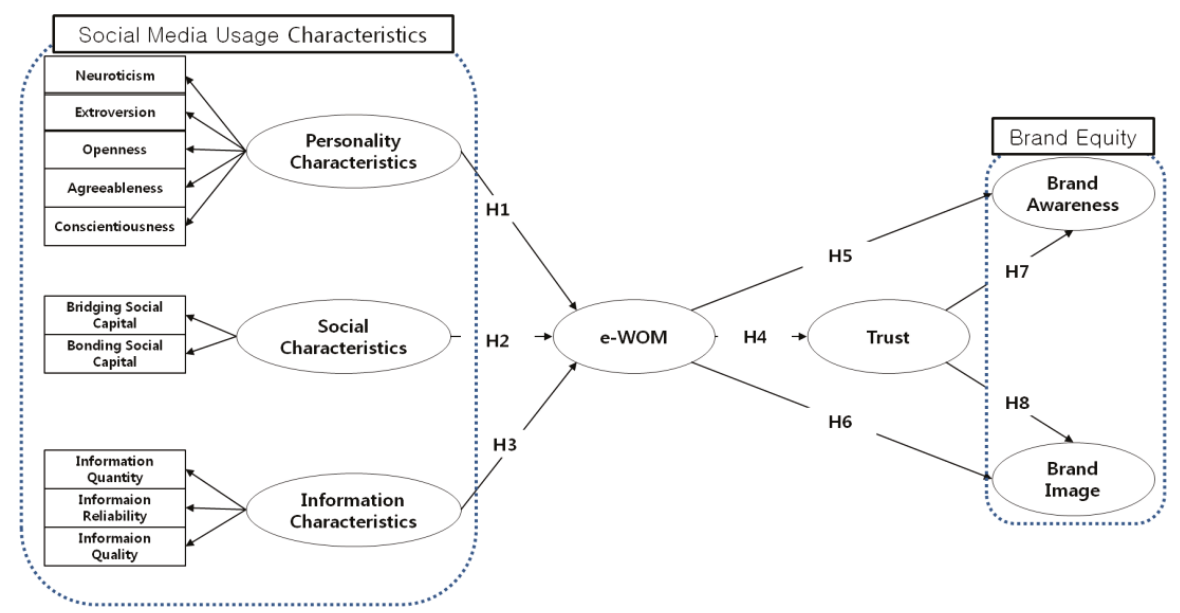

Figure 1. Research model.

The questionnaire used in this study was prepared by conducting a preliminary survey based on existing literature studies, supplementing, and revising. The items of the questionnaire were classified into personality characteristics (15), social characteristics (8), information characteristics (9), e-WOM (3), trust (3), brand awareness (3), and brand image (3). A total of 44 items were asked. Royse [103] compared the pros and cons of quantitative research and qualitative research, and while quantitative research secures objectivity through theory-based research results, qualitative research overly depends on subjective judgment and the research results show a lack of objectivity. Therefore, this study started research about social media characteristics and the theoretical background of e-WOM and gathered structured questionnaires as the data in order to identify a universal law. In addition, the demographic characteristics and airline usage were also asked. The survey scale was the Likert five-point scale, with five being very true and one being not at all. The survey was conducted on Koreans who had experience of using airlines using the convenience sampling method, from Aug. 11 to Sep. 15, 2017. In this study, non-probabilistic sampling was used. This is a common technique in social science and statistical research. Based on the probability sampling method, there are advantages including how it is relatively easy to carry out a very difficult investigation and useful to quickly comprehend certain trends [104]. Sampling was difficult to investigate because we chose a specific target. The investigation took about a month. The statistical analysis method of this study is considered very appropriate. Non-probability sampling methods have been used in various fields for longer than probability sampling, and the use of non-probability sampling methods is increasing. Non-probability sampling methods are widely used in research and research fields [105].

The random sampling method was applied in this study to ensure internal consistency and solve sample selection bias-related issues. In order to obtain a genuine response, the researcher collected a sample with data by conducting a face-to-face survey. Through this process, a total of 450 questionnaires were distributed and 442 of them were collected. After excluding twelve ones with missed or inappropriate data, 430 questionnaires were used in the final analysis. This is because when 
the data is systematically lost due to omission, the results may not characterize the population and also be affected by validity and reliability [106]. The items of the questionnaire are shown in Table 1 .

Table 1. Questionnaire items.

\begin{tabular}{|c|c|c|}
\hline Variable & Item & Related Studies \\
\hline \multirow{5}{*}{$\begin{array}{l}\text { Personality } \\
\text { Characteristics } \\
\text { of Social } \\
\text { Media Usage }\end{array}$} & $\begin{array}{c}\text { Neuroticism } \\
\text { I have calm characteristics. } \\
\text { I am emotionally stable. } \\
\text { I do not become angry easily. }\end{array}$ & \multirow{5}{*}{$\begin{array}{c}\text { Alan, A. K., and Kabadayı, E. } \\
\text { T. (2016) [7] }\end{array}$} \\
\hline & $\begin{array}{l}\text { Extroversion } \\
\text { I am extroversive. } \\
\text { I am enthusiastic. } \\
\text { I am active. }\end{array}$ & \\
\hline & $\begin{array}{l}\text { Openness } \\
\text { I like new kinds of experience. } \\
\text { I like change. } \\
\text { I am creative. }\end{array}$ & \\
\hline & $\begin{array}{c}\text { Agreeableness } \\
\text { I am kind. } \\
\text { I am generous. } \\
\text { I like emotional interaction with others. }\end{array}$ & \\
\hline & $\begin{array}{c}\text { Conscientiousness } \\
\text { I am sincere. } \\
\text { I am trustworthy. } \\
\text { I have habits of readiness and strict. }\end{array}$ & \\
\hline \multirow{2}{*}{$\begin{array}{l}\text { Social } \\
\text { Characteristics } \\
\text { of Social } \\
\text { Media Usage }\end{array}$} & $\begin{array}{c}\text { Bridging Social Capital } \\
\text { Airline social media makes me to be } \\
\text { interested in news, } \\
\text { Airline social media makes me to be curious } \\
\text { about another world. } \\
\text { Airline social media makes me to want to } \\
\text { try something new. } \\
\text { Airline social media makes me to be } \\
\text { attention to people who think differently } \\
\text { from me. }\end{array}$ & \multirow{2}{*}{$\begin{array}{c}\text { Su, C. C., and Chan, N. K. } \\
\text { (2017). [8]; } \\
\text { Kim, B., and Kim, Y. (2017). } \\
\text { [9] }\end{array}$} \\
\hline & $\begin{array}{l}\text { Bonding Social Capital } \\
\text { Airline social media is a good candidate to } \\
\text { be recommended for airline usage and } \\
\text { travel information. } \\
\text { Airline social media advises me in making } \\
\text { important decisions about airline usage and } \\
\text { travel information } \\
\text { Airline social media is helpful in solving } \\
\text { problems related to airline usage and travel } \\
\text { information. } \\
\text { Airline social media is helpful when airline } \\
\text { usage and travel information are sought in } \\
\text { a hurry. }\end{array}$ & \\
\hline
\end{tabular}


Table 1. Cont.

\begin{tabular}{|c|c|c|}
\hline Variable & Item & Related Studies \\
\hline \multirow{3}{*}{$\begin{array}{l}\text { Information } \\
\text { Characteristics } \\
\text { of Social } \\
\text { Media Usage }\end{array}$} & $\begin{array}{c}\text { Information Quantity } \\
\text { media.Airline social media has a high } \\
\text { amount of information. } \\
\text { Social media has high amount of } \\
\text { information about airlines. } \\
\text { Many people post much information about } \\
\text { airline on social }\end{array}$ & \multirow{3}{*}{$\begin{array}{c}\text { Erkan, I., and Evans, C. } \\
\text { (2016). [10] }\end{array}$} \\
\hline & $\begin{array}{l}\text { Information Reliability } \\
\text { I think that the information on airline social } \\
\text { media is credible. } \\
\text { I think that the information on airline social } \\
\text { media is accurate. } \\
\text { I think that the information on airline social } \\
\text { media is influential. }\end{array}$ & \\
\hline & $\begin{array}{l}\text { Information Quality } \\
\text { I think that the information on airline social } \\
\text { media is easy to understand. } \\
\text { I think that the information on airline social } \\
\text { media is certain. } \\
\text { I think that the quality of information on } \\
\text { airline social media is excellent in general. }\end{array}$ & \\
\hline e-WOM & $\begin{array}{l}\text { I will post positive comments about this } \\
\text { airline on my social media. } \\
\text { I will recommend to use this airline through } \\
\text { my social media. } \\
\text { I will recommend to use this airline to my } \\
\text { social media acquaintances. }\end{array}$ & $\begin{array}{l}\text { Goyette, I., Ricard, L., } \\
\text { Bergeron, J., and Marticotte, } \\
\text { F. (2010). [107] }\end{array}$ \\
\hline Trust & $\begin{array}{l}\text { This airline is reliable. } \\
\text { I think that this airline does not hide the } \\
\text { important information I needs to know. } \\
\text { I believe this airline respects its promises. }\end{array}$ & $\begin{array}{l}\text { Delgado-Ballester, E., and } \\
\text { Munuera-Alemán, J. L. } \\
\text { (2005). [60] }\end{array}$ \\
\hline \multirow[b]{2}{*}{ Brand Equity } & $\begin{array}{l}\text { Brand Awareness } \\
\text { I can always recognize this airline brand. } \\
\text { I am familiar with this airline characteristic. } \\
\text { I remember this airline logo certainly. }\end{array}$ & \multirow{2}{*}{$\begin{array}{l}\text { Godey, B., Manthiou, A., } \\
\text { Pederzoli, D., Rokka, J., } \\
\text { Aiello, G., Donvito, R., and } \\
\quad \text { Singh, R. (2016). [6] }\end{array}$} \\
\hline & $\begin{array}{l}\text { Brand Image } \\
\text { This airline is a leading one in their industry. } \\
\text { I have an impressive experience of this } \\
\text { airline. } \\
\text { The airline is a customer-centered company. }\end{array}$ & \\
\hline
\end{tabular}

This study, to identify characteristics of passengers who have experience using airline social media, analyzed data using the SPSS 21.0 program and AMOS 20.0 program, which have been widely used as statistical packages of social sciences. The frequency analysis was conducted to identify the characteristics of the sample, and confirmatory factor analysis was performed to further analyze the validity of the measurement model. The secondary forms of concepts that are composed of sub-factors were each analyzed by confirming factor analysis to verify the internal validity and then converted to the primary form. In addition, Using Cronbach's $\alpha$, by scales, the refinement of the scale was carried out. The concentration and discriminant validity were verified, and the hypothesis of this study model was verified through structural equations. 


\section{Empirical Analysis}

The valid samples of this study are 430 subjects, and Table 2 shows the demographic characteristics of the subjects. The females were somewhat higher, with $n=235(54.7 \%)$ and males were $n=195$ (45.3\%). The most frequent age group was $\mathrm{n}=159(37.0 \%)$ for $31-40$ years old and followed by $\mathrm{n}=149$ (34.6\%) for $21-30, n=75(17.4 \%)$ for $41-50$ years old, $n=30(7.0 \%)$ for 51 years old or older, and $n=17$ $(4.0 \%)$ for younger than 20 years old. This confirmed that those in their $20 \mathrm{~s}$ and $30 \mathrm{~s}$ who are active in social and economic activities are the main generations of using social media. The distribution of airlines that subjects have experience of using social media showed $n=188(43.7 \%)$ for Korean Air, $n=$ $154(35.8 \%)$ for Asiana Airlines, $\mathrm{n}=72$ for low-cost domestic airlines $(16.7 \%), \mathrm{n}=11$ for foreign airlines $(2.6 \%)$, and $n=5(1.2 \%)$ for foreign low-cost airlines $(1.2 \%)$, indicating that major airlines are focusing on SNS as part of their marketing strategy.

Table 2. Demographic characteristics of subjects.

\begin{tabular}{|c|c|c|c|}
\hline \multicolumn{2}{|c|}{ Variable } & Frequency (n) & Percentage (\%) \\
\hline \multirow{2}{*}{ Gender } & Male & 195 & 45.3 \\
\hline & Female & 235 & 54.7 \\
\hline \multirow{5}{*}{ Age } & Under 21 & 17 & 4.0 \\
\hline & $21-30$ & 149 & 34.6 \\
\hline & $31-40$ & 159 & 37.0 \\
\hline & $41-50$ & 75 & 17.4 \\
\hline & 51 and Over & 30 & 7.0 \\
\hline \multirow{5}{*}{ Purpose of Using Airline } & Work or Business & 34 & 7.9 \\
\hline & Tour and Vacation & 367 & 85.3 \\
\hline & Education and Meeting & 9 & 2.1 \\
\hline & Visiting Friend and Relatives & 15 & 3.5 \\
\hline & Others & 5 & 1.2 \\
\hline \multirow{5}{*}{ Social Media Usage } & Asiana Airlines & 154 & 35.8 \\
\hline & Korean Air & 188 & 43.7 \\
\hline & Foreign Airlines & 11 & 2.6 \\
\hline & Domestic Low-Cost Airlines & 72 & 16.7 \\
\hline & Foreign Low-Cost Airlines & 5 & 1.2 \\
\hline \multirow{6}{*}{$\begin{array}{l}\text { Frequency of Using } \\
\text { Airlines per Year }\end{array}$} & 1 & 149 & 34.6 \\
\hline & $2-3$ & 163 & 37.9 \\
\hline & $4-5$ & 77 & 17.9 \\
\hline & $6-7$ & 14 & 3.3 \\
\hline & $8-9$ & 7 & 1.6 \\
\hline & 10 and Over & 20 & 4.7 \\
\hline \multicolumn{2}{|c|}{ Total Respondents } & 430 & $100 \%$ \\
\hline
\end{tabular}

As the results of confirmatory factor analysis to verify the single-dimensionality, conceptual validity and reliability of the measurement items are shown in Table 3. The Squared Multiple Correlations (SMC) value of all the measured items except Image 2 appeared to be 0.5 or more, and the standardized factor loadings of the measured items were 0.7 or more, ensuring the concentration validity [108]. In addition, all Cronbach' $\alpha$ values were more than 0.7 , ensuring content validity. For the result of confirmatory factor analysis for each item, $\chi^{2}=552.814, \mathrm{df}=168, \mathrm{CMIN} / \mathrm{DF}=3.291, \mathrm{p}=$ 
$0.000, \mathrm{GFI}=0.889, \mathrm{NFI}=0.890, \mathrm{IFI}=0.920, \mathrm{CFI}=0.920, \mathrm{RMR}=0.04$, and $\mathrm{RMSEA}=0.073$, indicating that the research model of this study is fit.

Table 3. Results of confirmatory factor analysis.

\begin{tabular}{|c|c|c|c|c|c|c|}
\hline \multicolumn{2}{|c|}{ Constructs } & \multirow{2}{*}{$\begin{array}{c}\text { Variable } \\
\text { Neu. }\end{array}$} & \multirow{2}{*}{\multicolumn{2}{|c|}{$\begin{array}{c}\begin{array}{c}\text { Regression } \\
\text { Coefficient } \\
\text { (C.R.) }\end{array} \\
1.357(6.287)\end{array}$}} & \multirow{2}{*}{$\begin{array}{l}\text { S.C.R } \\
0.553\end{array}$} & \multirow{4}{*}{$\begin{array}{r}\alpha \\
0.734\end{array}$} \\
\hline \multirow{10}{*}{$\begin{array}{c}\text { Characteristics } \\
\text { of } \\
\text { social Media }\end{array}$} & \multirow{5}{*}{ Personality } & & & & & \\
\hline & & Ext. & 0.535 & $1.489(5.971)$ & 0.657 & \\
\hline & & Ope. & 0.420 & 1.000(Fix) & 0.561 & \\
\hline & & Agr. & 0.711 & $1.997(6.955)$ & 0.843 & \\
\hline & & Con. & 0.601 & $1.765(6.897)$ & 0.775 & \\
\hline & \multirow{2}{*}{ Social } & Bridging & 0.624 & $0.939(16.688)$ & 0.79 & \multirow{2}{*}{0.830} \\
\hline & & Bonding & 0.809 & 1.000(Fix) & 0.899 & \\
\hline & \multirow{3}{*}{ Information } & Quantity & 0.446 & $0.799(15.351)$ & 0.668 & \multirow{3}{*}{0.828} \\
\hline & & Reliability & 0.787 & 1.000(Fix) & 0.887 & \\
\hline & & Quality & 0.72 & $0.941(21.416)$ & 0.848 & \\
\hline \multirow{3}{*}{\multicolumn{2}{|c|}{ Trust }} & Trust 1 & 0.519 & 1.000(Fix) & 0.72 & \multirow{3}{*}{0.834} \\
\hline & & Trust 2 & 0.674 & $1.294(15.689)$ & 0.821 & \\
\hline & & Trust 3 & 0.735 & $1.25(16.178)$ & 0.857 & \\
\hline \multirow{3}{*}{\multicolumn{2}{|c|}{ e-WOM }} & e-WOM1 & 0.681 & 1.000(Fix) & 0.825 & \multirow{3}{*}{0.908} \\
\hline & & e-WOM2 & 0.875 & $1.163(23.663)$ & 0.935 & \\
\hline & & e-WOM3 & 0.75 & $1.051(21.767)$ & 0.866 & \\
\hline \multirow{5}{*}{ Brand Equity } & \multirow{3}{*}{ Awareness } & Awe. 1 & 0.617 & 1.000(Fix) & 0.786 & \multirow{3}{*}{0.820} \\
\hline & & Awe. 2 & 0.553 & $1.096(14.985)$ & 0.743 & \\
\hline & & Awe. 3 & 0.654 & $1.204(16.135)$ & 0.809 & \\
\hline & \multirow{2}{*}{ Image } & Image 1 & 0.461 & $0.929(12.91)$ & 0.729 & \multirow{2}{*}{0.663} \\
\hline & & Image 3 & 0.532 & 1.000(Fix) & 0.679 & \\
\hline
\end{tabular}

Note: $\alpha=$ Cronbach' $^{\prime} \alpha$ S.C.R $=$ Standardized C.R.

To test the hypotheses of this study, the structural equation model analysis was performed. The estimated fit and path coefficient of the structural model were $\chi 2=688.229, \mathrm{df}=178, \mathrm{CMIN} / \mathrm{DF}=$ 3.866, $\mathrm{p}<0.001, \mathrm{GFI}=0.863, \mathrm{AGFI}=0.822, \mathrm{RMR}=0.062, \mathrm{CFI}=0.894, \mathrm{TLI}=0.875$, and $\mathrm{RMSEA}=0.082$, indicating a satisfactory level of fitness when compared with the general criteria. The structural model presented in this study is considered as appropriate, and the hypothesis testing results are presented in Figure 2.

Among the characteristics of social media usage, only personality and information characteristics are showed significant influence on e-WOM. In particular, it was confirmed that the information characteristics showed more influence than the personality characteristics because the information characteristics were $\beta=0.472$ and C.R. $=5.598$ ( $p<0.001)$. In other words, if the information quantity, reliability, and quality of airline social media are good, it can be seen that they leave a positive review online. However, social characteristics were found to have no significant effect on e-WOM. The relationship between e-WOM and brand equity (brand awareness, brand image) yielded different results. The e-WOM had a significant effect on brand awareness, but not on the brand image. The hypothesis that e-WOM has a significant effect on trust was supported with $\beta=0.562$ and C.R. $=10.036$ $(p<0.001)$. The relationship between trust and brand equity was found to be statistically significant. 
The influence of trust on brand awareness amongst brand equity was $\beta=0.492, C R=7.241(p<0.001)$, and it was displayed as $\beta=0.91$ and $C R=12.263(\mathrm{p}<0.001)$ for brand image, supporting both H7 and H8. Accordingly, this shows that brand image is built through trust. The explanatory power was $38.8 \%$ and $77.6 \%$, respectively.

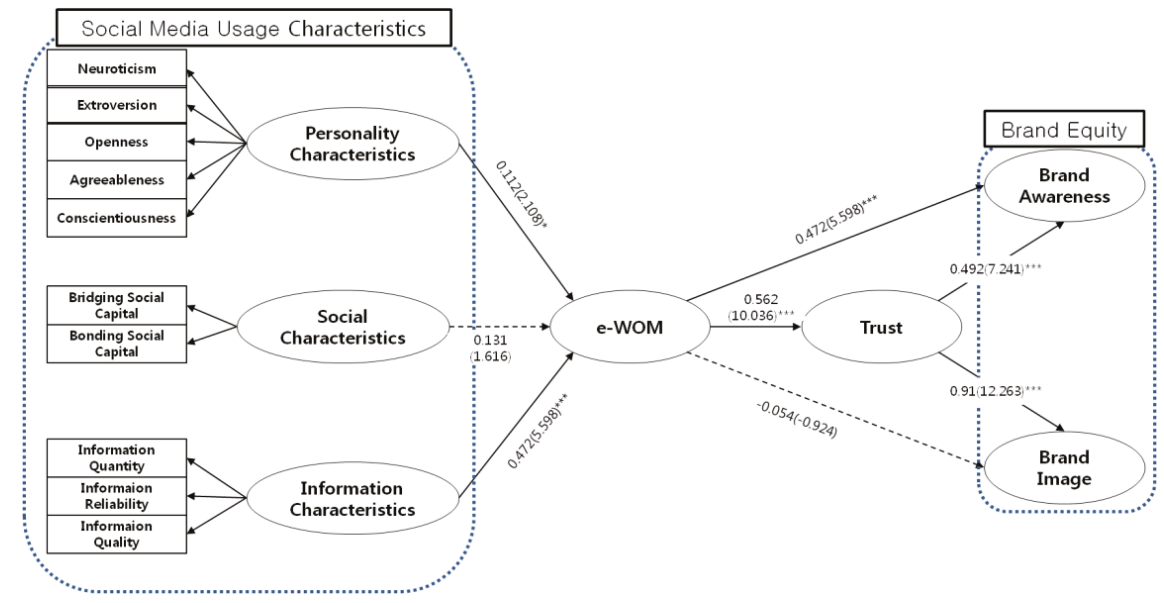

Figure 2. Results of testing hypotheses. ${ }^{*} p<0.05,{ }^{* * *} p<0.01$.

\section{Conclusions}

The purpose of this study was to investigate the influence of social media usage characteristics on brand awareness and brand image of brand equity through e-WOM and trust. Through previous studies, social media usage characteristics were derived into personality characteristics (Openness, Conscientiousness, Extroversion, Agreeableness, and Neuroticism), social characteristics (bridging social capital and bonding social capital), and information characteristics (information quantity, Reliability, and quality). The results of this study are consistent with the previous studies in a large context $[90,101,109]$, but the differences are shown according to the characteristics of the samples and the market situation $[7,82,88]$.

In order to present the empirical results, a survey of 430 passengers with experience using airline social media was analyzed. First, personality characteristics among social media usage characteristics were found to have a significant effect on e-WOM. Airlines should apply customers' social media activities and online conversations to understand customer preferences and also manage customer data. Protection of personal information is an important issue for this, but the future lies in ensuring that airlines can use social media data and convert them into actionable insights that will yield closer relationships with customers through greater personalization. For this to be achieved, airlines are required to make a significant investment in resources and professional training, especially with regards to the application of social media data. Second, the social characteristics of social media did not have a significant effect on e-WOM. This means that the personality or disposition of the individual affects the e-WOM, but the social connection or cohesion does not affect the e-WOM in relation to the characteristics of the airline's use of social media. It may be interpreted that any distortions or intentions due to social relationships have no effects on the e-WOM. Third, the information characteristics of social media have a significant effect on e-WOM. Contrary to social characteristics, and information characteristics have a close effect on e-WOM. This indicates that the higher quality, reliability, and amount of information are associated with a more active role of e-WOM. Accordingly, the airlines should reinforce the functions of the links for the information in the technical aspect and also deliver news through various social media channels and constantly provide events in order to attract interest from potential customers. 
Plenty of high quality and reliable information should be provided to customers, both quickly and actively, to strengthen the sales strategies of direct sales without distributors. Fourth, e-WOM has a significant effect on trust, suggesting that the more positive WOM about the airline in social media is associated with higher trust in that airline. Many studies have already demonstrated how important trust is to brands and consumer relationships, which gives directions on how airlines should use social media marketing. Fifth, e-WOM had a significant effect on brand awareness, but not on brand image, indicating that the e-WOM does not directly change the image of airline brands, but has a direct effect on brand awareness. It was also found that the building of trust using e-WOM may have an effect on brand image. Lastly, trust has a significant effect on both brand awareness and brand image of brand equity. Trust has already been shown to be a decisive factor in boosting brand equity and that brand equity has a direct bearing on consumer purchase intentions. This shows that e-WOM generated through the use of social media has a significant impact on airline brand equity through trust.

The airline industry is a part of the service division that plays an important role in the tourism and air transport industry and shows consistent growth through technological and economic development. However, the emergence of a mega carrier due to the merger between former airlines and the market share of low-cost carriers is causing fierce competition in the industry. Airlines need new ways to create competitive advantage and various strategies are required in order for the airlines to attract and maintain more customers. Based on the circumstances, the following is the significance of this study. This study focused on investigating the application of social media in the airline industry in regard to how this particular field turned to the researches done by other industries due to the lack of research about social media targeted on the airline industry. This study analyzed the influence of the users' personality characteristics, social characteristics, and information characteristics on e-WOM, trust, and brand equity in respect of social media application. The study provides an academic base on social media in the airline industry henceforward.

The following is a suggestion for the specific practice. First, the importance of social media marketing should be recognized and dedicated personnel and division should be designated to focus on social media marketing. Second, social media marketing that customers can join should be implemented and attention should be provided to the data collection and management of the users. Third, various reliable information should be provided and marketing activities should be executed to activate e-WOM and create differentiation that is worthy of gaining trust as well as construct brand equity that can make way for cognitive and emotional experiences to the consumers.

As a result, this study suggests that companies should consider customers' personality characteristics for social media marketing activities and also apply the information quantity, reliability, and quality to make e-WOM active and gain trust. The marketing manager and decision maker should make investments to consistently collect and analyze data in order to learn customers' personal tastes and preferences, and if sufficient amount of information and credible data are provided to the customers through social media, then significant synergy effect can be expected to construct advanced brand equity. Therefore, the result of this study provides support to establish operational strategies as to how airline companies should give attention to social media marketing activities and conduct research about the strategies of the application measures along with the methods that should be applied to build positive word-of-mouth online and reliability about the company.

The limitations of this study and future research subjects are as follows. First, the survey was conducted only on Korean passengers of domestic airlines and lacked representativeness of the sample. That $7.9 \%$ of the surveys were included using foreign airline social media is insignificant. In the future, comprehensive studies should be conducted on foreign cases and airlines. Second, the definition of $\mathrm{e}-\mathrm{WOM}$ was studied as a positive meaning, so it could display preponderance in the research. It is anticipated for future research to consider the directivity of e-WOM and the differences that follow the varied characteristics. Third, although empirical studies demonstrated that social media marketing activities have a significant effect on e-WOM or trust, there is a lack of studies on how these results affect to make the actual profit or management performance of companies. The overcome of these 
limitations in future studies is expected to be greatly helpful not only to aviation companies but also to marketers in all areas concerned with social media marketing strategies and decision-making. The results of this study confirm how social media usage characteristics affect brand equity through e-WOM and trust, and demonstrate the importance of using social media in corporate activities of airlines. Despite the limitations of this study, it theoretically and statistically was confirmed that results will have great significance in future social media research directions, and proposals for airline operation strategies will be of practical help to management.

Author Contributions: Conceptualization, E.J.S. and J.-W.P.; methodology, E.J.S., J.-W.P. and Y.J.C.; data collection and analysis, E.J.S. and Y.J.C.; supervision of the research, J.-W.P.; writing-original draft, E.J.S.; writing-review \& editing E.J.S., J.-W.P. and Y.J.C. All authors have read and agreed to the published version of the manuscript.

Funding: This research received no external funding.

Conflicts of Interest: The authors declare no conflicts of interest.

\section{References}

1. Gallauter, J.; Ransbotham, S. Social Media and Customer Dialog Management at Starbucks. MIS Q. Exec. 2010, 9, 197-212.

2. Kaplan, A.M.; Haenlein, M. Users of the World, Unite! The Challenges and Opportunities of Social Media. Bus. Horizons 2010, 53, 59-68. [CrossRef]

3. Kietzmann, J.; Hermkens, K.; McCarthy, I.; Silvestre, B.S. Social Media? Get Serious! Understanding the Functional Building Blocks of Social Media. Bus. Horizons 2011, 54, 241-251. [CrossRef]

4. Hanna, R.; Rohm, A.; Crittenden, V.L. We're All Connected: The Power of the Social Media Ecosystem. Bus. Horizons 2011, 54, 265-273. [CrossRef]

5. Seo, E.-J.; Park, J.-W. A Study on the Effects of Social Media Marketing Activities on Brand Equity and Customer Response in the Airline Industry. J. Air Transp. Manag. 2018, 66, 36-41. [CrossRef]

6. Godey, B.; Manthiou, A.; Pederzoli, D.; Rokka, J.; Aiello, G.; Donvito, R.; Singh, R. Social Media Marketing Efforts of Luxury Brands: Influence on Brand Equity and Consumer Behavior. J. Bus. Res. 2016, 69, 5833-5841. [CrossRef]

7. Alan, A.K.; Kabadayı, E.T. The Effect of Personal Factors on Social Media Usage of Young Consumers. Procedia Soc. Behav. Sci. 2016, 235, 595-602. [CrossRef]

8. Su, C.C.; Chan, N.K.; Chao, C.S.; Keung, C.N. Predicting Social Capital on Facebook: The Implications of Use Intensity, Perceived Content Desirability, and Facebook-Enabled Communication Practices. Comput. Hum. Behav. 2017, 72, 259-268. [CrossRef]

9. Kim, B.; Kim, Y. College students' Social Media Use and Communication Network Heterogeneity: Implications for Social Capital and Subjective Well-Being. Comput. Hum. Behav. 2017, 73, 620-628. [CrossRef]

10. Erkan, I.; Evans, C. The Influence of EWOM in Social Media on consumers' Purchase Intentions: An Extended Approach to Information Adoption. Comput. Hum. Behav. 2016, 61, 47-55. [CrossRef]

11. Kim, J.T. Media and Man. Communication Books; SEL: Seoul, Korea, 1998.

12. Boyd, D.M.; Ellison, N. Social Network Sites: Definition, History, and Scholarship. J. Comput. Commun. 2007, 13, 210-230. [CrossRef]

13. Safko, L.; Brake, D. The Social Media Bible: Tactics, Tools and Strategies for Business Success; Wiley: New York, NY, USA, 2009.

14. Richter, A.; Koch, M. Social Software-Status Quo and Zukunft, Fak. Fur Informatik; University der Bundeswehr München: Munich, Germany, 2007.

15. Kelly, L.; Kerr, G.; Drennan, J. Avoidance of Advertising in Social Networking Sites. J. Interact. Advert. 2010, 10, 16-27. [CrossRef]

16. Leung, D.; Law, R.; Van Hoof, H.; Buhalis, D. Social Media in Tourism and Hospitality: A Literature Review. J. Travel Tour. Mark. 2013, 30, 3-22. [CrossRef]

17. Zeng, B.; Gerritsen, R. What Do We Know about Social Media in Tourism? A Review. Tour. Manag. Perspect. 2014, 10, 27-36. [CrossRef]

18. Costa, P.T.; McCrae, R.R. The Revised NEO Personality Inventory (NEO-PI-R); SAGE Publications: Southend Oaks, CA, USA, 2012; pp. 179-198. [CrossRef] 
19. Min, K. Personality Psychology; Bobmunsa: Paju, Korea, 2002.

20. Liu, D.; Campbell, W.K. The Big Five Personality Traits, Big Two Metatraits and Social Media: A Meta-Analysis. J. Res. Pers. 2017, 70, 229-240. [CrossRef]

21. Block, J. The Five-Factor Framing of Personality and Beyond: Some Ruminations. Psychol. Inq. 2010, 21, 2-25. [CrossRef]

22. Hughes, D.J.; Rowe, M.; Batey, M.; Lee, A. A Tale of Two Sites: Twitter Vs. Facebook and the Personality Predictors of Social Media Usage. Comput. Hum. Behav. 2012, 28, 561-569. [CrossRef]

23. McCrae, R.R.; Costa, P.T., Jr. The Five-Factor Theory of Personality; Guilford Press: New York, NY, USA, 2008.

24. John, O.P.; Srivastava, S. The Big Five Trait Taxonomy: History, Measurement, and Theoretical Perspectives. In Handbook of Personality: Theory and Research; Pervin, L.A., John, O.P., Eds.; Guilford Press: New York, NY, USA, 1999; pp. 102-138.

25. Ryan, T.; Xenos, S. Who Uses Facebook? An Investigation into the Relationship Between the Big Five, Shyness, Narcissism, Loneliness, and Facebook Usage. Comput. Hum. Behav. 2011, 27, 1658-1664. [CrossRef]

26. Costa, P.T.; McCrae, R.R. The Five-Factor Model and the NEO Inventories. Oxf. Handb. Personal. Assess. 2009, 299-322. [CrossRef]

27. McCrae, R.R.; Costa, P.T., Jr. Personality in Adulthood; Informa UK Limited: Colchester, UK, 2003. [CrossRef]

28. Chan, D.; Schmitt, N. Situational Judgment and Job Performance. Hum. Perform. 2002, 15, 233-254. [CrossRef]

29. Adler, P.S.; Kwon, S.-W. Social Capital: Prospects for a New Concept. Acad. Manag. Rev. 2002, 27, 17-40. [CrossRef]

30. Lin, N. Social Capital: A Theory of Social Structure and Action; Cambridge University Press: New York, NY, USA, 2002.

31. Etzioni, A.; Putnam, R.D. Is Bowling Together Sociologically Lite? Bowling Alone: The Collapse and Revival of American Community. Contemp. Sociol. A J. Rev. 2001, 30, 223. [CrossRef]

32. Williams, D. On and Off the 'Net: Scales for Social Capital in an Online Era. J. Comput. Commun. 2006, 11, 593-628. [CrossRef]

33. Burt, R.S. The Contingent Value of Social Capital. Adm. Sci. Q. 1997, 42, 339. [CrossRef]

34. Steinfield, C.; Ellison, N.; Lampe, C. Social Capital, Self-Esteem, and Use of Online Social Network Sites: A Longitudinal Analysis. J. Appl. Dev. Psychol. 2008, 29, 434-445. [CrossRef]

35. Briggs, X.D.S. Brown Kids in White Suburbs: Housing Mobility and the Many Faces of Social Capital. Hous. Policy Debate 1998, 9, 177-221. [CrossRef]

36. Wilken, R. "Bonds and Bridges: Mobile Phone Use and Social Capital debates." Mobile Communication: Bringing Us Together and Tearing Us Apart; Transaction Publishers: New Brunswick, NJ, USA, 2011.

37. Ahituv, N.; Riley, H.N.; Neumann, S. Principles of Information Systems for Management; McGraw-Hill Professional: Columbus, OH, USA, 1993.

38. A Thelwall, M.; Buckley, K.; Paltoglou, G. Sentiment Strength Detection for the Social Web. J. Am. Soc. Inf. Sci. Technol. 2011, 63, 163-173. [CrossRef]

39. Chewning, E.G.; Harrell, A.M. The Effect of Information Load on Decision makers' Cue Utilization Levels and Decision Quality in a Financial Distress Decision Task. Account. Organ. Soc. 1990, 15, 527-542. [CrossRef]

40. Eppler, M.J.; Mengis, J. The Concept of Information Overload: A Review of Literature from Organization Science, Accounting, Marketing, MIS, and Related Disciplines. Inf. Soc. 2004, 20, 325-344. [CrossRef]

41. Chen, Y.-C.; Shang, R.-A.; Kao, C.-Y. The Effects of Information Overload on consumers' Subjective State towards Buying Decision in the Internet Shopping Environment. Electron. Commer. Res. Appl. 2009, 8, 48-58. [CrossRef]

42. Wathen, C.N.; Burkell, J. Believe It or Not: Factors Influencing Credibility on the Web. J. Am. Soc. Inf. Sci. Technol. 2002, 53, 134-144. [CrossRef]

43. Pan, X.; Ratchford, B.T.; Shankar, V. Can Price Dispersion in Online Markets Be Explained by Differences in E-Tailer Service Quality? J. Acad. Mark. Sci. 2002, 30, 433-445. [CrossRef]

44. Chen, C.C.; Tseng, Y.-D. Quality Evaluation of Product Reviews Using an Information Quality Framework. Decis. Support Syst. 2011, 50, 755-768. [CrossRef]

45. Bickart, B.; Schindler, R.M. Internet Forums As Influential Sources of Consumer Information. J. Interact. Mark. 2001, 15, 31-40. [CrossRef]

46. Canhoto, A.; Clark, M. Customer Service 140 Characters at a Time: The users' Perspective. J. Mark. Manag. 2013, 29, 522-544. [CrossRef] 
47. Erkan, I. The Influence of Electronic Word of Mouth in Social Media on Consumers' Purchase Intentions. Ph.D. Thesis, Brunel University, London, UK, June 2016.

48. Katz, E.; Lazarsfeld, P.F.; Roper, E. Personal Influence: The Part Played by People in the Flow of Mass Communications; Routledge: London, UK, 2017.

49. King, R.A.; Racherla, P.; Bush, V.D. What We Know and Don't Know About Online Word-of-Mouth: A Review and Synthesis of the Literature. J. Interact. Mark. 2014, 28, 167-183. [CrossRef]

50. Yoon, S.J. A Social Network Approach to the Influences of Shopping Experiences on e-WOM. J. Elec. Comm. Res. 2012, 13, 213-223.

51. Barreto, A.M. The Word-of-Mouth Phenomenon in the Social Media Era. Int. J. Mark. Res. 2014, 56, 631-654. [CrossRef]

52. Cheung, C.M.; Lee, M.K. What Drives Consumers to Spread Electronic Word of Mouth in Online Consumer-Opinion Platforms. Decis. Support Syst. 2012, 53, 218-225. [CrossRef]

53. Pitta, D.A.; Fowler, D. Online Consumer Communities and Their Value to New Product Developers. J. Prod. Brand Manag. 2005, 14, 283-291. [CrossRef]

54. Mourali, M.; Laroche, M.; Pons, F. Antecedents of Consumer Relative Preference for Interpersonal Information Sources in Pre-Purchase Search. J. Consum. Behav. 2005, 4, 307-318. [CrossRef]

55. O'Cass, A.; Grace, D. Service Brands and Communication Effects. J. Mark. Commun. 2004, 10, $241-254$. [CrossRef]

56. Gefen, D.; Drexel University. Customer Loyalty in E-Commerce. J. Assoc. Inf. Syst. 2002, 3, 27-53. [CrossRef]

57. Robinson, S.L. Trust and Breach of the Psychological Contract. Adm. Sci. Q. 1996, 41, 574. [CrossRef]

58. Mishra, A.K. Organizational Responses to Crisis; Trust in Organizations: Sage, CA, USA, 1996.

59. Luhmann, N. Familiarity, Confidence, Trust: Problems and Alternatives. In Trust: Making and Breaking Cooperative Relations; Basil Blackwell: Oxford, UK, 2000; pp. 94-107.

60. Ballester, E.D.; Munuera-Alemán, J.L. Does Brand Trust Matter to Brand Equity? J. Prod. Brand Manag. 2005, 14, 187-196. [CrossRef]

61. E Swan, J.; Bowers, M.R.; Richardson, L.D. Customer Trust in the Salesperson. J. Bus. Res. 1999, 44, 93-107. [CrossRef]

62. Jang, H. A Comparative Study on the Structutal Interactionns Among Customer Satisfaction, Trust, Loyalty Based on Types of Internet Shopping Mall. J. Global Acad. Mark. Sci. 2007, 17, 23-49.

63. Aaker, D.A. Measuring Brand Equity Across Products and Markets. Calif. Manag. Rev. 1996, 38, 102-120. [CrossRef]

64. Keller, K.L. Conceptualizing, Measuring, and Managing Customer-Based Brand Equity. J. Mark. 1993, 57, 1-22. [CrossRef]

65. Keller, K.L. Brand Synthesis: The Multidimensionality of Brand Knowledge. J. Consum. Res. 2003, 29, 595-600. [CrossRef]

66. Sallam, M.A.; Wahid, N.A. The Effects of Satisfaction and Brand Identification on Brand Love and Brand Equity Outcome: The Role of Brand Loyalty. Eur. J. Bus. Soc. Sci. 2015, 4, 42-55.

67. Machado, J.C.; De Carvalho, L.V.; Azar, S.L.; André, A.R.; Dos Santos, B.P. Brand Gender and Consumer-Based Brand Equity on Facebook: The Mediating Role of Consumer-Brand Engagement and Brand Love. J. Bus. Res. 2019, 96, 376-385. [CrossRef]

68. Sen, S. The Effects of Brand Name Suggestiveness and Decision Goal on the Development of Brand Knowledge. J. Consum. Psychol. 1999, 8, 431-455. [CrossRef]

69. Shocker, A.D.; Aaker, D.A. Managing Brand Equity. J. Mark. Res. 1993, 30, 256. [CrossRef]

70. Keller, K.L.; Parameswaran, M.G.; Jacob, I. Parameswaran, and Isaac Jacob. Strategic Brand Management: Building, Measuring, and Managing Brand Equity; Pearson Education: London, UK, 2011.

71. Percy, L.; Rossiter, J.R. A Model of Brand Awareness and Brand Attitude Advertising Strategies. Psychol. Mark. 1992, 9, 263-274. [CrossRef]

72. Heo, J.H. The Effects of Brand Awareness, Perceived Service Quality and Physical Environment on Congruity and Purchase Intention: Focusing on Coffee Shop. Master's Thesis, Sejong University, Seoul, Korea, 2012.

73. Kotler, P.; Armstrong, G. Principles of Marketing; Pearson Education: London, UK, 2010.

74. Low, G.S.; Lamb, C.W. The Measurement and Dimensionality of Brand Associations. J. Prod. Brand Manag. 2000, 9, 350-370. [CrossRef] 
75. Dobni, D.; Zinkhan, G.M. In Search of Brand Image: A Foundation Analysis. Adv. Consum. Res. 1990, 17, 110-118.

76. Ajzen, I. The Theory of Planned Behavior. Organ. Behav. Hum. Decis. Process. 1991, 50, 179-211. [CrossRef]

77. Noh, J.-E.; Choi, Y.-S.; Choi, S.-D. The Impact of Tourism Information Characteristics and Information Seeker Personality Variables on Word-of-Mouth Effects Throughout the Online Environment: Focusing on Internet Users in the Age Groups of the 20s and 30s. J. Tour. Sci. 2016, 40, 43-62. [CrossRef]

78. Ju, H.; Suh, H. Relationships Among Personality, Commitment and Word-of-Mouth Insertion in Online Shopping. Glob. E-Bus. Assoc. 2015, 16, 29-50.

79. Bachrach, Y.; Kosinski, M.; Graepel, T.; Kohli, P.; Stillwell, D. Personality and Patterns of Facebook Usage. In the 3rd Annual ACM Web Science Conference; Association for Computing Machinery (ACM): New York, NY, USA, 2012; pp. 24-32. [CrossRef]

80. Wang, T.; Yeh, R.K.-J.; Chen, C.; Tsydypov, Z. What Drives Electronic Word-of-Mouth on Social Networking Sites? Perspectives of Social Capital and Self-Determination. Telemat. Inform. 2016, 33, 1034-1047. [CrossRef]

81. Chung, N.; Koo, C. The Use of Social Media in Travel Information Search. Telemat. Inform. 2015, 32, $215-229$. [CrossRef]

82. Lee, S.N. The Study on the Effect of Personal Characteristics, Interpersonal Influence and Social Capital on Online Word of Mouth on SNS: Mainly about Comparison between Facebook and Twitter. Master's Thesis, Hanyang University, Seoul, Korea, June 2013.

83. Knoll, J. Advertising in Social Media: A Review of Empirical Evidence. Int. J. Advert. 2015, 35, $266-300$. [CrossRef]

84. Bowman, D.; Narayandas, D. Managing Customer-Initiated Contacts with Manufacturers: The Impact on Share of Category Requirements and Word-of-Mouth Behavior. J. Mark. Res. 2001, 38, 281-297. [CrossRef]

85. Duan, W.; Gu, B.; Whinston, A.B. Do Online Reviews Matter? - An Empirical Investigation of Panel Data. Decis. Support Syst. 2008, 45, 1007-1016. [CrossRef]

86. Liu, Y. Word of Mouth for Movies: Its Dynamics and Impact on Box Office Revenue. J. Mark. 2006, 70, 74-89. [CrossRef]

87. Wen, X. The Effect of the Characteristic of Online Word-of -Mouth on Word of Mouth Acceptance, Word of Mouth Spread and Purchase Intention. Master's Thesis, Kyung Hee University, Seoul, Korea, Suwon, Korea, 2015.

88. Kim, N.; Kim, M.H. The Impact of Tourism e-WOM Information Characteristics and Community Interactivity on E-WOM Information Usefulness and e-WOM Effects. Korea Serv. Manag. Soc. 2010, 11, 17-44.

89. Litvin, S.W.; Goldsmith, R.E.; Pan, B. Electronic Word-of-Mouth in Hospitality and Tourism Management. Tour. Manag. 2008, 29, 458-468. [CrossRef]

90. Ladhari, R.; Michaud, M. EWOM Effects on Hotel Booking Intentions, Attitudes, Trust, and Website Perceptions. Int. J. Hosp. Manag. 2015, 46, 36-45. [CrossRef]

91. Cha, S.M. Developing Word-of-Mouth(WOM) Communication Paradigm Model of Restaurant Information. Master's Thesis, Yonsei University, Seoul, Korea, 2006.

92. Sénécal, S.; Nantel, J. The Influence of Online Product Recommendations on consumers' Online Choices. J. Retail. 2004, 80, 159-169. [CrossRef]

93. Yim, S.H. Research on Influence to Brand Equity and Revisit Intention by On/Off-Line Word of Mouth: Based on Family Restaurant Brands. Master's Thesis, Tourism Kyung Hee University, Seoul, Korea, Suwon, Korea, 2009.

94. Kim, J.-W. The Relation Between of Marketing Communication and Service Brand Equity. J. Korea Contents Assoc. 2008, 8, 150-163. [CrossRef]

95. Lee, S.H. The Effects of Airline Marketing Communications on Brand Knowledge and Core Customer-Based Brand Equity. Ph.D. Thesis, Sejong University, Seoul, Korea, 2012.

96. Fournier, S.; Dobscha, S.; Mick, D.G. Preventing the Premature Death of Relationship Marketing. Harv. Bus. Rev. 1997, 76, 42-51.

97. Moorman, C.; Deshpandé, R.; Zaltman, G. Factors Affecting Trust in Market Research Relationships. J. Mark. 1993, 57, 81-101. [CrossRef]

98. Moorman, C.; Zaltman, G.; Deshpande, R. Relationships Between Providers and Users of Market Research: The Dynamics of Trust Within and Between Organizations. J. Mark. Res. 1992, 29, 314-328. [CrossRef] 
99. Kim, K.H.; Kim, K.S.; Kim, D.Y.; Kim, J.H.; Kang, S.H. Brand Equity in Hospital Marketing. J. Bus. Res. 2008, 61, 75-82. [CrossRef]

100. Han, S.H.; Nguyen, B.; Lee, T. Consumer-Based Chain Restaurant Brand Equity, Brand Reputation, and Brand Trust. Int. J. Hosp. Manag. 2015, 50, 84-93. [CrossRef]

101. Fatma, M.; Rahman, Z.; Khan, I. Building Company Reputation and Brand Equity through CSR: The Mediating Role of Trust. Int. J. Bank Mark. 2015, 33, 840-856. [CrossRef]

102. Loureiro, S.M.C. The Effect of Perceived Benefits, Trust, Quality, Brand Awareness/Associations and Brand Loyalty on Internet Banking Brand Equity. Int. J. Electron. Commer. Stud. 2013, 4, 139-158. [CrossRef]

103. Royse, D. Research Methods in Social Work. Cengage Learning, 5th ed.; Thomson Learning: Belmont, CA, USA, 2008.

104. Given, L. The SAGE Encyclopedia of Qualitative Research Methods. SAGE Encycl. Qual. Res. Methods 2008. [CrossRef]

105. Baker, R.; Brick, J.M.; Bates, N.A.; Battaglia, M.; Couper, M.P.; Dever, J.A.; Gile, K.J.; Tourangeau, R. Summary Report of the AAPOR Task Force on Non-Probability Sampling. J. Surv. Stat. Methodol. 2013, 1, 90-143. [CrossRef]

106. McKnight, P.E.; McKnight, K.M.; Sidani, S.; Figueredo, A.J. Missing Data: A Gentle Introduction; Guilford Press: New York, NY, USA, 2007.

107. Goyette, I.; Ricard, L.; Bergeron, J.; Marticotte, F. e-WOM Scale: Word-of-mouth Measurement Scale for e-services Context. Can. J. of Adm. Sci./Rev. Can. Sci. De l'Adm. 2010, 27, 5-23. [CrossRef]

108. Song, J.J. SPSS/AMOS Statistical Analysis Method; 21C: Paju, Korea, 2015; ISBN 978-898-468-618-2.

109. Augusto, M.; Torres, P. Effects of Brand Attitude and EWOM on consumers' Willingness to Pay in the Banking Industry: Mediating Role of Consumer-Brand Identification and Brand Equity. J. Retail. Consum. Serv. 2018, 42, 1-10. [CrossRef]

(C) 2020 by the authors. Licensee MDPI, Basel, Switzerland. This article is an open access article distributed under the terms and conditions of the Creative Commons Attribution (CC BY) license (http://creativecommons.org/licenses/by/4.0/). 
Article

\title{
A Sustainable Solution to Overtourism in the Social Media Era: An Exploratory Analysis on the Roles and Functions of Place-Visitor Relationship (PVR)
}

\author{
Hyung Min Lee ${ }^{1}$, Jinwoo Park ${ }^{1}$ and Yoonjae Nam ${ }^{2, *}$ \\ 1 Department of Media Communication, College of Social Science, Sungshin Women's University, \\ Seoul 02844, Korea; hmlee@sungshin.ac.kr (H.M.L.); creativejw1019@gmail.com (J.P.) \\ 2 Department of Culture, Tourism and Content, College of Hotel and Tourism management, \\ Kyung Hee University, Seoul 02453, Korea \\ * Correspondence: ynam@khu.ac.kr; Tel.: +82-2-961-9187
}

Received: 19 March 2020; Accepted: 8 April 2020; Published: 10 April 2020

\begin{abstract}
As overtourism has become a serious threat to the tourism industry in recent years, this study attempts to extend the theoretical framework of organization-public relationship (OPR) developed in the public relations scholarship to the context of overtourism. To that end, the concept of place-visitor relationship (PVR) is theoretically suggested and empirically tested in a structural equation model. Also, statistical reliability and validity of PVR are put under investigation. The findings helped confirm the roles and functions of PVR as a potential solution to overtourism in the social media era. As an antecedent, visitors' affective tour experiences stemming from exposure to social media information significantly influenced PVR. PVR, on the other hand, significantly affected attitudes toward a place and, further, behavioral intentions toward measures against overtourism. In addition, the findings revealed that PVR consists of two sub-factors: Loyalty and relational attachment. Relationship strategies as a solution to the issue of overtourism are discussed in light of PVR.
\end{abstract}

Keywords: overtourism; social media; sustainable tourism; organization-public relationship; place-visitor relationship

\section{Introduction}

Tourism as a pollution-free and never-ending industry is considered simply a myth now. Tourism, more precisely, mass-tourism, has been accused of severe damage to the nature as well as human societies [1]. With the increasing awareness of and concern about tourism's negative impact to places and communities, several measures have been discussed and implemented worldwide. For instance, the government of Philippines banned tourists' visits to Boracay for six months due to deteriorating environmental pollution. Venice of Italy, Bali of Indonesia, Edinburgh of Scotland, Dubrovnik of Croatia, along with other famous tour sites are already imposing or planning to impose a visiting tax on tourists [2]. Places such as Salzburg of Austria and Komodo Island of Indonesia are limiting the number of visitors per day [2]. All of the cases point to the side effects of overtourism, which refers to negative impact of tourism occurring from excessive inflow of tourists into a certain place [3].

In such a context, the concept of sustainable tourism has drawn growing interest and attention both from the academia and the industry in recent years. Sustainable tourism, which is derived and evolved from the notion of sustainable development, can be defined as a tourism marketing process in which a balance between visitors' high-quality tour experiences and the host community's high quality of life is sought and valued [1,4]. Thus, sustainable tourism "would involve a coordinated attempt to manage the tourism in such a way that the long-term integrity of a region's natural and human resources will be preserved" [5]. In that sense, sustainable tourism products should be not only 
environment-friendly and community-respectful, but also economically viable and profitable [6]. To be successful, sustainable tourism requires active engagement and participation of stakeholders [7]. That is, sustainable tourism business models are feasible only when marketers, tourists, and the surrounding community strategically collaborate and build mutually beneficial relationships with one another [8]. In particular, promoting tourists' eco-friendly, culturally sensitive, and socially desirable behaviors in tour sites is one of the key factors determining success or failure of sustainable tourism $[8,9]$.

As a theoretical framework to understand how to promote tourists' good behaviors for sustainable tourism, this study looks into the concept of organization-public relationship (OPR) from the public relations scholarship and extends the theoretical concept to the context where visitors and places build and maintain emotional bonding and mutually beneficial relationships. To that end, this study aims to empirically demonstrate the roles and functions of the place-visitor relationship (PVR) in explaining and predicting visitors' behavioral intentions, such as willingness to pay a visiting tax or voluntary tourist behaviors for environmental protection, to prevent numerous side effects stemming from overtourism. Along this analytic process, construct reliability and validity of place-visitor relationship (PVR), which is assumed to consist of visitors' relational perceptions about a specific tourism place, is statistically examined as well.

The main purpose of this study, therefore, is to corroborate the theoretical as well as practical utility of PVR in a structural equation model. As potential antecedents of PVR, this study focuses on exposure to place information via social network sites (SNS) and affective tour experiences. Certainly, tourists must visit a specific place in order to develop emotional bonding and PVR. Further, one of the driving forces for tourists to have curiosity and desire to visit a certain place in recent years is tourism information shared, diffused, and consumed through social media platforms [10]. As a sequential process, this study hypothesizes that social media exposure to place information significantly leads to affective tour experiences, and affective tour experiences, in turn, are associated with PVR. It is asserted in this study that PVR as a relational outcome between visitors and a tourism place should result from affective tour experiences facilitated by social media use for tourism information. This study also suggests that PVR as a determinant should influence tourists' attitudes toward a place and their behavioral intentions for countermeasures against overtourism. By demonstrating such relationships between PVR, attitudes, and behavioral intentions, this study aims to highlight the roles and functions of PVR that can help enhance tourists' voluntarily eco-friendly behaviors in line with the core philosophy of sustainable tourism. In addition, with a series of factor analyses, the construct validity of PVR and its structural components are identified.

\section{Literature Review}

\subsection{Conceptualizing Place-Visitor Relationship (PVR)}

With the growing volume and increasingly intense competition in the tourism industry, building and maintaining mutually beneficial relationships between places and visitors in the long-term have garnered much attention in recent years [11]. When it comes to tourism marketing, the first purchase by consumers (visitors) often occur without a clear understanding of the products (places), while visitors always have many travel destinations to choose from. Places, on the other hand, should compete against other places to attract as many visitors as possible, while too many visitors or visitors with malicious behaviors have increasingly been recognized as uninvited and unwelcoming guests, as in the cases of overtourism [6,12]. For such reasons, relationships between places and visitors have considered a key factor for not only economic viability, but also ecological sustainability [13].

The concept of organization-public relationship (OPR) stemming from the public relations scholarship offers a theoretical framework for place-visitor relationship (PVR). OPR can be defined as a state that exists between an organization and its relevant publics when one's actions affect the other's economic, social, and cultural wellbeing [14]. Based the conceptual definition, numerous studies have explored sub-dimensions that constitute OPR. Sub-dimensions of OPR identified so far 
include trust, satisfaction, control mutuality, and commitment among others [15,16]. Moreover, many scholars have attempted to develop measures to evaluate relationships between an organization and its publics $[17,18]$.

This study attempts to apply the principles of OPR to the context where places and visitors build and maintain mutually beneficial relationships for sustainable tourism. As mentioned earlier, managing relationships between places and visitors has been of important scholarly as well as practical issue, but little discussion has been made to explicate OPR in the context of sustainable tourism. Furthermore, previous literature has paid scant attention to the fact that PVR can be a critical indicator in relation to the issue of overtourism. Taking into consideration that PVR conceptually shows the extent to which visitors care for a certain place, PVR appears to provide a noteworthy starting point for strategic actions against overtourism and sustainable tourism. Based on the previously explored OPR dimensions and measures, this study aims to statistically test reliability and validity of PVR. Thus, the following research questions are to be addressed.

Research Question 1 (RQ1). Is PVR statistically reliable and valid as a construct meaningful in the context of sustainable tourism?

Research Question 2 (RQ2). If so, which factors are consisting of PVR to help explain and predict positive consequences in relation to sustainable tourism?

\subsection{The Impact of Social Media on Overtourism}

The scale of tourism industry has dramatically increased in recent years, thanks to a number of factors [19]. Among such factors, the emergence of social media and consequent overflow of tourism information created, shared, and disseminated online require both scholarly and practical attention, due to its huge impact on the landscape of tourism industry $[10,20]$. More people gravitate toward, as well as take advantage of, social media, searching for relevant information and expressing their opinions. For example, Facebook, one of the leading social media, had over 1.9 billion users worldwide as of 2017 [21].

Tourism information circulated and sought in such social media has exerted significant influence on tourists' decision-making and consumer behaviors [20]. Social media have allowed tourists not only to search for information about travel destinations, but also to evaluate online content (i.e., reviews, photos, and videos) related to such places [22,23]. Social media also provides a public sphere where tourists can interact with each other and share their thoughts and feelings. From the perspective of tourism, social media offers a channel as well as platform of interaction and communication where tourists' opinions and ideas about certain places are actively generated and exchanged. Such an interactive communication process has a significant influence on tourists' consumer behaviors, specifically in choosing which destination they will visit and spend their leisure time [24,25].

The dark side of social media regarding tourism, however, is that tourists are likely to be exposed to a very limited amount of popular information-due to the power law distribution tendency of social media-so only a handful of places are even more highlighted in such a channel [26]. Such an asymmetry of tourism information exposure, in turn, may help instigate the 'the rich get richer and the poor get poorer' phenomenon among places, which is likely to worsen overtourism with more tourists coming into a few limited places. Moreover, social media sometimes expose relatively underexplored and unknown places to a number of users, which may result in business exploitation on such environmentally sensitive places [27]. On one hand, social media may aggravate overtourism by asymmetrical information circulation and exposure, which is likely to attract more visitors to only a handful of already famous and popular destinations [28]. Social media, on the other hand, could be a serious threat to sustainable tourism in general with its widespread and powerful influence [27].

Based on the abovementioned theoretical premise, this study empirically examines the relationship between social media exposure to a certain place and visitors' travel experiences in the place, particularly focusing on emotional outcomes. Previous studies showed that tourists seek relevant information 
and upload their postings in social media for emotional purposes [29,30]. A study by Hudson and colleagues [31] demonstrated that tourists' interaction through social media has a positive effect on their emotional attachment to a tour experience as well as a positive effect on word-of-mouth (WOM) intent. In view of social identity theory, it is also likely that visitors sharing the same information about a certain place may have emotional bonding not only with each other, but also with the place for which they are searching information [32,33]. Thus, this study looks into the effect of social media exposure on affective tour experiences, defined in this study as tourists' emotional outcomes and evaluation based on actual tour experiences. For such an analytic purpose, the following research hypothesis is suggested.

Hypothesis $\mathbf{1}$ (H1). The degree of visitors' social media information exposure to a place is positively related to their affective tour experiences in the place.

\subsection{The Relationship Between Affective Tour Experiences and PVR}

It is important in this study to verify whether PVR is a useful variable by establishing its relationships with potential antecedents and descendants in a theoretical model. By doing so, it can be more strongly argued that PVR is a theoretically meaningful concept in the context of sustainable tourism.

According to the theoretical framework of OPR from which PVR is both conceptually and operationally derived, PVR should be made of visitors' perceived relationship quality and satisfaction with a certain place $[16,34]$. In view of such a characteristic of PVR, visitors' affective tour experiences in a certain place should account for their perceived relationship with the place. Prior research adds theoretical support to such an argument. Actual experiences should determine one's perceptions and feelings about the relationship counterpart, and such psychologies influence one's perceived relationship quality as well as satisfaction [35].

Several studies have empirically demonstrated that affective tour experiences are substantial factors for tourists' psychologies. Pine and Gillmore [36] highlighted the role of tourists' affective experiences in forming attitudes toward places. Lasalle and Britton [37] argued that positive emotions resulting from tour experiences can elicit tourists' attachment and loyalty to the places they were traveling. Along the same vein of such findings, many scholars have argued that affective tour experiences can enhance favorable memories of as well as positive attitudes and behaviors toward the place [38-40]. Taking into account such literature, it is expected that affective tour experiences are likely to influence the ways in which visitors are forming their perceived relationships with a place they are traveling. This study presumes the following hypothesis accordingly.

Hypothesis 2 (H2). Affective tour experiences are positively related to PVR.

\subsection{The Consequences of PVR}

As OPR has been tested and proved as a reliable and valid concept to evaluate and understand publics' perceived relationship status and quality with an organization, many scholars have tried to extend the scope of research to exploring the consequences of OPR [41]. Studies along such a research stream have revealed that OPR significantly affects publics' perceptions, attitudes, and behavioral intentions across a variety of different levels and circumstances $[14,42,43]$.

Considering the main purpose of this study, which aims to demonstrate the utility of PVR as a concept and variable, especially in the context of sustainable tourism, this study puts its analytic focus on visitors' attitudes toward a place and their behavioral intentions toward protective measures against overtourism as potential consequences of PVR. As mentioned above, visitors' sustainable and responsible behaviors are one of the key factors essential for the effective implementation of measures against overtourism and, consequently, the success of sustainable tourism. It has been reported that visitors' environmentally irresponsible and culturally insensitive attitudes and behaviors are the most 
serious factors aggravating side effects of overtourism [44]. Such an issue was also addressed in recent research by Shen and colleagues [45] looking at the strategic use of SNS to promote visitors' sustainable and responsible behaviors in a place as a potential means to decrease the problems of overtourism.

In the public relations scholarship, Ki and Hon [43] empirically investigated the impact of OPR on attitudes and behavioral intentions. What is noteworthy in this study is that they designed a research model as a sequential one where OPR affects publics' attitudes toward an organization, and attitudes, in turn, affect their behavioral intentions toward the organization. In other words, attitude was regarded as a mediator between OPR and behavioral intentions in the study, based on the classic theoretical framework of hierarchy of effects model [46]. Such a hierarchy with attitude as an antecedent of behavioral intentions was advocated in the theory of planned behavior as well [47]. Empirical studies based on such a theoretical assumption have demonstrated that OPR's influence on publics' behavioral intentions was mediated by attitudes $[48,49]$.

Following the previous literature, this study attempts to empirically confirm the explanatory as well as predictive power of PVR for the variance in visitors' attitudes toward a place and behavioral intentions toward protective measures against overtourism. Thus, it is hypothesized that PVR should account for visitors' attitudes toward a place, and visitors' attitudes toward a place, in turn, should influence their behavioral intentions toward protective measures against overtourism, more specifically, intentions toward paying a visiting tax and behaving in an environmentally friendly manner while traveling. Figure 1 illustrates the hypothesized relationships between the variables.

Hypothesis 3 (H3). Sub-dimensions of PVR are positively related to visitors' attitudes toward a place.

Hypothesis 4 (H4). Visitors' attitudes toward a place are positively related to their behavioral intentions toward protective measures against overtourism.

Hypothesis 4-1 (H4-1). Visitors' attitudes toward a place positively influence their willingness to pay a visiting tax when entering the place.

Hypothesis 4-2 (H4-2). Visitors' attitudes toward a place positively influence their willingness to behave in an environmentally friendly manner when traveling in the place.

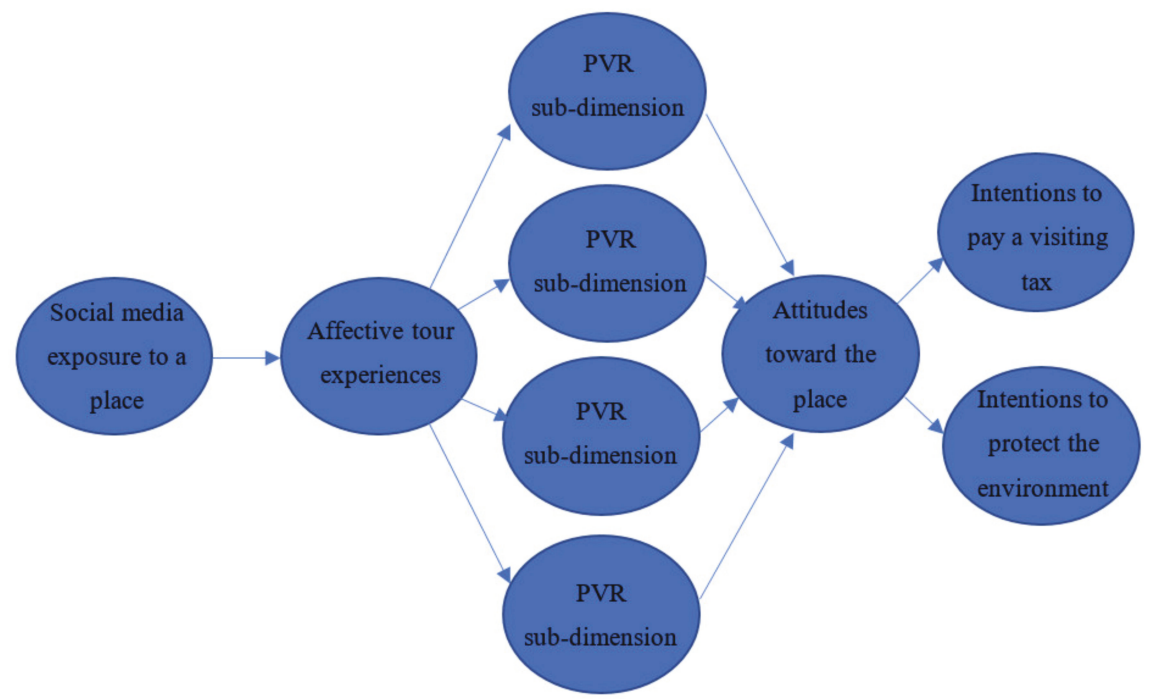

Figure 1. Hypothesized research model. PVR: Place-Visitor Relationship. 


\section{Research Design and Methods}

\subsection{Survey Overview}

A national survey was designed and conducted in South Korea to empirically collect data and statistically verify the hypothesized relationships among the variables. The survey was executed online through a research company with a country-wide panel. Survey respondents were recruited based on a random quota sampling. During a one-week period of October 2019, a total of 389 respondents completed and submitted reliable and analyzable questionnaires. Table 1 shows the demographics of the research sample.

Table 1. Sample demographics.

\begin{tabular}{cccc}
\hline \multicolumn{2}{c}{ Demographics } & $\boldsymbol{n}$ & Percentage \\
\hline \multirow{2}{*}{ Gender } & Male & 188 & 48.3 \\
& Female & 201 & 51.7 \\
\hline \multirow{4}{*}{ Age } & $20 \mathrm{~s}$ & 65 & 16.8 \\
& $30 \mathrm{~s}$ & 70 & 18.0 \\
& $40 \mathrm{~s}$ & 92 & 23.7 \\
& $50 \mathrm{~s}$ & 89 & 22.9 \\
& $60 \mathrm{~s}$ & 73 & 18.8 \\
\hline \multirow{4}{*}{ Education } & High school & 77 & 19.8 \\
& Junior college & 46 & 11.8 \\
& College & 193 & 49.6 \\
& Graduate school & 73 & 18.8 \\
\hline
\end{tabular}

Jeju Island of South Korea was selected as the tourism place for analysis. Jeju Island was chosen for several reasons. First, Jeju Island has been one of the most popular destinations for South Korean tourists for a long time [50]. Hence, Jeju Island has been familiar to most of South Korean tourists as a tourism place. Further, Jeju Island has suffered from overtourism in recent years due to overflow of both domestic and foreign travelers [51]. Through a screening question, it was confirmed that all the survey respondents had visited Jeju Island in person at least once.

\subsection{Measurements and Instruments}

Referring to previous studies [24,29,52], the degree of respondents' social media information exposure to Jeju Island was measured by three items using 7-point Likert scales. The items included 'I get a lot of Jeju Island's tourist information through social media,' 'I frequently get tourist information about Jeju Island through social media,' and 'I actively use social media to obtain tourist information about Jeju Island.'

Items to measure affective tour experiences were developed with reference to prior research as well [53-55]. Nine items measuring respondents' affective tour experiences in Jeju Island were 'I was able to escape from my ordinary life while traveling in Jeju Island,' 'I was able to have a good time with my family and friends while traveling in Jeju Isalnd,' 'I was able to relieve stress while traveling in Jeju Island,' 'While traveling in Jeju Island, I could enjoy various natural landscapes,' 'I was able to recharge my life while traveling in Jeju Island,' 'I was able to relax my mind and body while traveling in Jeju Island,' 'While traveling in Jeju Island, I could enjoy outstanding natural scenery,' 'I was able to realize the joy of sightseeing while traveling in Jeju Island,' and 'While traveling in Jeju Island, I was able to get closer with my family and friends.'

Meanwhile, items to measure PVR were adopted from previous OPR measures and tourism studies [16,55-57]. Items were slightly adjusted in consideration of the context where visitors build and maintain relationships with places. A total of 24 items were used to measure PVR in this study, 
and the items asked visitors' perceived loyalty, commitment, attachment, trust, and satisfaction for Jeju Island. Statistical reliability and validity of the items are to be assessed.

For the consequences of PVR, attitudes toward Jeju Island and behavioral intentions toward protective measures against overtourism were evaluated by three seven-point semantic differential scales, respectively. As for attitudes toward Jeju Island, three semantic differential scales (unfavorable-favorable; bad-good; negative-positive) were asked to be marked to measure respondents' attitudinal responses to Jeju Island [58,59]. In addition, behavioral intentions to pay a visiting tax were measured by three semantic differential scales (unlikely-likely; improbable-probable; impossible-possible), following the statement: 'To make a better environment, I am willing to pay a visiting tax when traveling Jeju Island.' Likewise, behavioral intentions to behave in an environmentally friendly manner were measured by three semantic differential scales (unlikely-likely; improbable-probable; impossible-possible), following the statement: 'I am willing to behave in an environmentally friendly manner when traveling Jeju Island' [58,59].

\section{Research findings}

\subsection{Descriptive Statistics and Reliability Check}

Descriptive statistics indicated that the mean score of social media information exposure regarding Jeju Island was $4.62(S D=1.33)$. The mean score of affective tour experiences was $5.64(S D=0.84)$. On the other hand, the mean score of attitudes toward Jeju Isalnd was $5.47(S D=1.08)$. The mean score of behavioral intentions to pay a visiting tax was $3.82(S D=1.52)$, whereas that of behavioral intentions to behave in an environmentally friendly manner was $5.21(S D=1.29)$.

In addition, three criteria were employed to check statistical reliability of the measurements. Internal consistency reliability, which can be evaluated by Cronbach's $\alpha$, was checked for each measurement. Construct reliability (CR) was also verified for each measurement. Further, average variance extracted (AVE) analyses were performed to confirm the degree of variance each measurement can explain. Table 2 shows the results of such reliability analyses. All the coefficients were above the statistical baselines, pointing to each measurement's satisfactory reliability $[60,61]$.

Table 2. Reliability test results.

\begin{tabular}{cccc}
\hline Measurement & Cronbach's $\alpha$ & CR & AVE \\
\hline Social media exposure & 0.93 & 0.93 & 0.81 \\
Affective tour experiences & 0.95 & 0.95 & 0.67 \\
Attitudes toward Jeju Island & 0.95 & 0.95 & 0.86 \\
Paying a visiting tax & 0.96 & 0.96 & 0.90 \\
Behaving environmentally friendly & 0.96 & 0.96 & 0.90 \\
\hline
\end{tabular}

CR: Construct reliability, AVE: average variance extracted.

\subsection{Exploratory and Confirmatory Factor Analyses: PVR}

A series of factor analyses were executed to demonstrate reliability and validity of PVR as a construct as well as to identify structural components of PVR. First, an exploratory factor analysis was conducted to verify factor loadings of each measurement item, and to identify sub-factors that may constitute the PVR construct. Second, a confirmatory factor analysis was performed to reaffirm statistical validity of each sub-factor and measuring items.

For the exploratory factor analysis, a maximum likelihood method was chosen for factor extraction [62]. As to rotation method, varimax rotation was employed as one of the most commonly available orthogonal methods [63]. The analysis showed that two sub-factors statistically stood out among the 24 items used to measure the PVR construct. The first factor of PVR consisted of 13 items. The first factor $(M=4.56, S D=0.96)$ was named 'loyalty' in this study, since most of the 13 items were used to measure loyalty to a tourism destination in previous studies. The second factor $(M=5.28$, 
$S D=1.00)$ consisted of seven items. As items used to measure trust, satisfaction, and commitment were mingled in this factor, this study named the factor 'relational attachment.' Internal consistency reliability of each factor, measured by Cronbach's $\alpha$, was statistically satisfactory as well. Among 24 items employed to measure PVR in the first place, 4 items were finally excluded, due to their low factor loadings and lack of communality. Table 3 summarizes the results of the factor analysis.

Table 3. Exploratory factor analysis.

\begin{tabular}{ccc}
\hline Items & $\begin{array}{c}\text { Loyalty } \\
\text { Factor Loadings }\end{array}$ & $\begin{array}{c}\text { Relational } \\
\text { Attachment }\end{array}$ \\
\hline Even though I know other places, I always support Jeju Island. & 0.82 \\
I believe that supporting Jeju Island is preferable to other places. & 0.78 \\
I feel strong loyalty to Jeju Island. & 0.77 \\
I will continue to support Jeju Island in the long-term. & 0.74 \\
Jeju Island keeps its promises as tourism places. & 0.72 \\
I feel comfortable with Jeju Island over time. & 0.71 \\
I choose to visit Jeju Island because I am sure it is a good place. & 0.71 & \\
I believe that Jeju Island provides the best value to its visitors. & 0.68 & \\
Visitors' loyalty to Jeju Island is pure. & 0.67 & 0.66 \\
I would like to say good things about Jeju Island to my friends. & 0.65 & \\
I feel emotional connection with Jeju Island. & 0.62 & 0.59 \\
Jeju Island does not hurt my feeling. & & 0.82 \\
Visiting Jeju Island is an exciting challenge for me. & & 0.77 \\
\hline I wish I had never entered into a relationship with Jeju Island (R). & 0.75 \\
I do not wish to continue any relationship with Jeju Island (R). & 0.75 \\
I want to keep a long-lasting relationship with Jeju Island. & & 0.73 \\
Generally speaking, I do not trust Jeju Island as a tourism place (R). & & 0.68 \\
\hline I believe that it is worthwhile to maintain a relationship with Jeju Island. & & 6.36 \\
Overall, I am satisfied with my tour in Jeju Island. & & 31.77 \\
Traveling in Jeju Island is worthwhile. & 36.87 \\
\hline Eigenvalue & 36.87 & 0.95 \\
\hline Pccumulate percentage of explained variance & \\
\hline Cronbach's $\alpha$ & & 0.93 \\
\hline
\end{tabular}

Convergent and discriminant validity of the two sub-factors of PVR were examined. The average variance extracted (AVE) value of 'loyalty' was 0.61 , which was over 0.5 , and its construct reliability (CR) value was 0.95 , which was over 0.7 , all of which met statistical standards to assure convergent validity [64]. Likewise, convergent validity of 'relational attachment' was confirmed (AVE = 0.64; $\mathrm{CR}=0.92$ ). Discriminant validity was also checked by demonstrating whether the AVEs of the sub-factors are greater than the square of correlation coefficient between the two (0.60). With such a criterion, discriminant validity of the two sub-factors was confirmed as well [65].

In addition, statistical validity of the two sub-factors of PVR was demonstrated by a confirmatory factor analysis. The first factor (loyalty) model with 13 items and the second factor (relational attachment) model with 7 items were under scrutiny. The results of goodness-of-fit analyses for each model are described in Table 4.

Table 4. Confirmatory factor analysis.

\begin{tabular}{ccccccc}
\hline Model & Chi-Square & df & RMSEA & GFI & NFI & TLI \\
\hline Loyalty & 134.177 & 60 & 0.056 & 0.948 & 0.967 & 0.976 \\
Relational Attachment & 5.746 & 3 & 0.049 & 0.996 & 0.997 & 0.991 \\
\hline
\end{tabular}

The abbreviations are defined in the text below Table 4 . 
As described in Table 4, goodness-of-fit for each model was statistically satisfactory. For each model, the values of root mean square error of approximation (RMSEA) were below 0.06, and other indices, including goodness-of-fit index (GFI), normed fit index (NFI), and Turker-Lewis index (TLI) were over 0.9 , which signaled statistically significant construct validity of PVR [66,67]. Overall, PVR was reliable and valid as a theoretical construct, according to a series of statistical tests, and it appeared to consist of two sub-factors, which were loyalty and relational attachment.

\subsection{Structural Equation Modeling and Path Analyses}

Apart from statistical reliability and validity of the PVR construct, scholarly as well as practical utility of PVR was inspected by establishing its relationships with other theoretically relatable variables in a structural equation model. More specifically, the hypothesized model attempts to demonstrate whether loyalty and relational attachment, which were identified as two sub-factors composing PVR, are influenced by visitors' affective tour experiences stemming from exposure to social media information about Jeju Island. In addition, loyalty and relational attachment are expected to positively influence attitudes toward Jeju Island, and attitudes toward Jeju Island, in turn, are hypothesized to facilitate counter-overtourism behavioral intentions.

As the first step to demonstrate the utility of PVR, statistical appropriateness of the structural equation model was estimated. Goodness-of-fit was estimated with several statistical coefficients. $\chi^{2}$ was $1452.81(p<0.01)$, and degree of freedom was $743\left(\chi^{2} / d f=1.96\right)$. RMSEA $(0.050)$, which was less than 0.06, signified statistical appropriateness of the model [66]. Furthermore, NFI (0.914), IFI (0.956), TLI (0.951), and CFI (0.956) were all over 0.90, which also confirmed statistical appropriateness of the model [68]. Overall, statistical appropriateness of the model was verified.

In addition, path analyses were performed to test statistical significance of the relationships between the variables. As shown in Figure 2, all the regression weights for the paths were statistically significant at the 0.01 level, which supported all the hypotheses suggested by the study. As theoretically assumed, the degree of exposure to social media information about Jeju Island had significantly positive influence on visitors' affective tour experiences. Affective tour experiences, on the other hand, were significantly as well as positively associated with the two sub-factors of PVR, loyalty and relational attachment to Jeju Island. In other words, the more visitors were affectively satisfied with their tour experiences in Jeju Island, the stronger they felt loyalty and relational attachment to Jeju Island. Meanwhile, PVR elicited more positive attitudes toward Jeju Island, and positive attitudes toward Jeju Island, in turn, led to visitors' favorable behavioral intentions to curb side effects of overtourism, such as willingness to pay a visiting tax and to behave in an environmentally friendly manner.

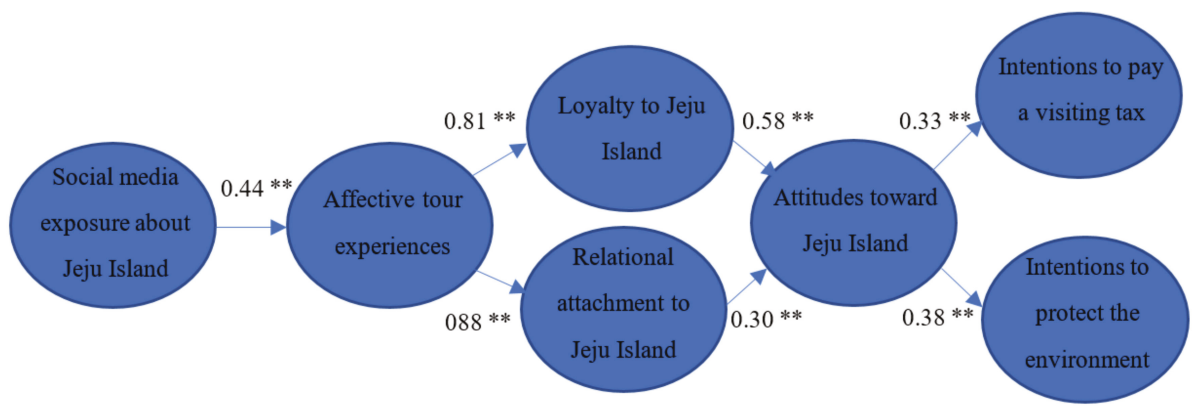

Figure 2. Structural equation modeling. ${ }^{* *}$ Path significance: $p<0.01$.

Table 5 shows the direct and indirect effects of independent variables on dependent variables in the model. As demonstrated from the path analyses, both social media exposure and affective tour experiences directly as well as indirectly affected the two sub-factors of PVR, loyalty and relational attachment to Jeju Island. Also, attitudes toward Jeju Isalnd were directly as well as indirectly influenced 
by social media exposure, affective tour experiences, and PVR. By the same token, willingness to pay a visiting tax and to behave in an environmentally friendly manner were influenced by the preceding variables both directly and indirectly.

Table 5. Direct and indirect effects.

\begin{tabular}{ccccc}
\hline Independent Variable & Dependent Variable & Direct Effect & Indirect Effect & Total Effect \\
\hline $\begin{array}{c}\text { Exposure to social network } \\
\text { services (SNS) }\end{array}$ & Tour experiences & 0.44 & 0.00 & 0.44 \\
\hline Exposure to SNS & Loyalty & 0.00 & 0.36 & 0.36 \\
Tour experiences & Relational attachment & 0.81 & 0.00 & 0.81 \\
\hline Exposure to SNS & & 0.80 & 0.39 & 0.39 \\
Tour experiences & Attitudes & 0.00 & 0.00 & 0.88 \\
\hline Exposure to SNS & & 0.00 & 0.33 & 0.33 \\
Tour experiences & & 0.58 & 0.74 & 0.74 \\
Loyalty & & 0.30 & 0.00 & 0.58 \\
Relational attachment & Willingness to pay a visiting & 0.00 & 0.30 \\
\hline Exposure to SNS & tax & 0.00 & 0.11 & 0.11 \\
Tour experiences & & 0.00 & 0.24 & 0.24 \\
Loyalty & & 0.33 & 0.19 & 0.19 \\
Relational attachment & Willingness to behave in an & 0.00 & 0.10 \\
Attitudes & environmentally friendly & 0.00 & 0.00 & 0.33 \\
\hline Exposure to SNS & manner & 0.00 & 0.12 & 0.12 \\
Tour experiences & & 0.38 & 0.12 & 0.28 \\
Loyalty & & 0.00 & 0.12 \\
Relational attachment & & & 0.38 \\
Attitudes & &
\end{tabular}

\section{Discussion}

A series of factor analyses helped confirm statistical reliability and validity of the construct of PVR as a theoretical extension of OPR to the context where a place and visitors build and maintain mutually beneficial relationships. Further, the analyses helped shed light on the structural components of PVR, which are loyalty and relational attachment to a place. According to the findings, visitors perceive their relationships with a place particularly in association with their loyalty and relational attachment to a place. To the extent the concept of OPR has contributed to the public relations scholarship, it appears that PVR could help advance the body of knowledge for the tourism scholarship by helping explain and evaluate the dynamics of relationships built and retained between a place and visitors.

Also, scholarly and practical utility of PVR was demonstrated by establishing its connections to theoretically meaningful antecedents and descendants. Specifically, PVR in this study was considered one of the sustainable solutions to overtourism, for PVR is conceptualized as a state in which visitors value their relationships with a place and care about the place's wellbeing $[14,16]$. In other words, PVR is considered an influential motive for visitors to behave in accordance with the principles of sustainable development in a certain place. Moreover, the roles and functions of PVR were theorized and articulated in consideration of the impact of social media on the tourism industry in recent years.

As demonstrated by numerous previous studies $[28,69,70]$, it turned out in this study that social media has a substantial effect on visitors' perceived tour experiences. More specifically, this study empirically found that the degree of exposure to social media information about a certain place could positively influence visitors' emotional outcomes associated with their actual tour experiences. A statistically significant influence of social media exposure on affective tour experiences indicated that information posted, shared, and spread in social media about a place can not only attract more visitors to the place, but also enhance visitors' affectively satisfying tour experiences and emotional outcomes. In fact, exposure to social media information about a certain tourism place can offer diverse ways of social or parasocial interaction where emotions and experiences are exchanged and amplified 
between the place and visitors even before arrival [28]. Such social interaction between a place and visitors can lead to more satisfactory perceptions and responses with regard to tour experiences [71].

Visitors' affective tour experiences, on the other hand, were positively associated with the two sub-dimensions of PVR, loyalty and relational attachment. The more satisfactory were visitors' affective tour experiences in Jeju Island, the stronger they perceived loyalty and relational attachment toward the place. Not only do such findings intuitively make sense, but they are in parallel with prior literature highlighting the role of actual interaction and experience between two parties in the making of a relationship [72,73]. Certainly, a relationship requires actual interaction between two parties. Perceptions of such a relationship are subject to actual experiences of each other's interactive behaviors. Also, it is noteworthy that such firsthand tour experiences are more powerful and influential than simple information exposure in social media in explaining the variances in PVR. The findings shed light on the possibility that visitors are likely to form and develop PVR, such as loyalty and relational attachment to Jeju Island, as they have quality tour experiences at an emotional level.

Loyalty and relational attachment to Jeju Island, in turn, had a significantly positive influence on visitors' attitudes toward Jeju Island. Referring to previous studies [17,41,43], this study theorized attitudes toward a place as a dependent variable of PVR, and, as hypothesized, visitors' attitudes toward Jeju Island, which conceptually reflect visitors' emotional judgment for the place, were significantly affected by the level of loyalty and relational attachment. Visitors' attitudes toward Jeju Island tend to get better as a result of their stronger loyalty and relational attachment to the place. This study empirically demonstrated the role and function of PVR in explaining and predicting visitors' attitudes toward a place.

In addition, visitors' attitudes toward Jeju Island were significantly related to visitors' behavioral intentions to alleviate side effects of overtourism. Based on the hierarchy of effects model and theory of planned behavior, this study hypothesized that attitudes toward Jeju Island will lead to visitors' willingness to support countermovement against overtourism. The findings showed that visitors are more likely to pay a visiting tax as well as to voluntarily protect the environment of Jeju Island if they maintain more positive and favorable attitudes toward the place. Moreover, according to the structural equation model, PVR indirectly affected such behavioral intentions through attitudes. Thus, the relationships between the two sub-dimensions of PVR and behavioral intentions were mediated by attitudes toward Jeju Island. In sum, PVR can elevate attitudes toward a place, which, in turn, positively encourage visitors to behave against overtourism.

Such a result demonstrated the roles and functions of PVR as a sustainable solution to overtourism. It has been well-documented that quality OPR helps establish a sustainable and mutually beneficial environment both for an organization and its publics $[14,16,34]$. Likewise, this study showed that PVR can be an effective indicator for such a sustainable relationship built and maintained between a place and its visitors. Scholars and practitioners alike have discussed numerous approaches and strategies to deal with problems arising from overtourism. No such measures can be successful, however, unless visitors take the problem of overtourism seriously and act responsibly and voluntarily to solve such problems from the perspective of a relationship partner. PVR can be understood as an indicator to gauge visitors' willingness to protect the environment of a certain place and, further, to safeguard the longevity of mutually beneficial relationships with the place. The more so visitors value and cherish their relationships with a place, the more likely they act in favor of protecting and supporting the place and its community.

From a strategic and practical viewpoint, therefore, places need to enhance and strengthen visitors' perceived relationships. Public relations strategies and tactics could be useful not only for measures against overtourism, but also for place marketing and tourism in general. When a place is recognized as unique, special, and irreplaceable, visitors are likely to cherish their relationships with such a place. An emotional appeal is important for PVR as demonstrated in this study. Personification of a place could be an effective approach to promote visitors' perceived relationships as well. According to the 
findings, sustainable tourism with economic viability as well as environmental preservation calls for visitors' loyalty and relational attachment to a place.

\section{Conclusions}

The problems and conflicts caused by overtourism have become a serious threat to the tourism industry. Such problems and conflicts are of particular significance, because they can possibly destroy mutually beneficial relationships between visitors and residents living in the local community [74]. As seen from various cases, local residents do not welcome too many visitors into their territory and do not appreciate any potential depletion or pollution caused by excessive tourist traffic. Some extremists have protested against tourism and have even manifested acts of vandalism [75]. Visitors, on the other hand, are likely to feel intimidated and insulted from such reactions from the locals. Certainly, such experiences may prevent visitors from considering revisit or posting positive word-of-mouth on social media. Within such a process, overtourism hampers sustainable tourism.

What is ideally critical to solve such problems and conflicts, therefore, is mutual understanding and respect between visitors and a local community. PVR suggested in this study came from such a recognition. Based on the theoretical framework of OPR, PVR helps indicate and evaluate visitors' perceived relationship quality with a certain place. It also helps understand visitors' psychological structures regarding their perceived relationships with a place. According to the empirical findings of this study, visitors of Jeju Island perceive their relationships with the place in terms of loyalty and relational attachment. As long as visitors possess loyalty and relational attachment to Jeju Island, it is not likely that they cause too much damage to the local community. The local community, on the other hand, is likely to welcome and appreciate visitors' goodwill and responsible behaviors while traveling. Theoretically speaking, such mutually beneficial relationships built and maintained between visitors and a place should be an effective solution to problems and conflicts caused by overtourism.

Although this study presents a relatively new and fresh perspective looking at the issue of overtourism, it should be mentioned that this study has several methodological limitations as well. Despite the nation-wide survey based on random quota sampling, generalizability is still an issue, since survey respondents were recruited from a pool constructed and managed by a research company. External validity, therefore, should be tested again elsewhere with a more representative sample. An analysis in a different cultural context, with different units of observation could strengthen external validity of the findings. Additionally, the utility of PVR can be tested and demonstrated within a different theoretical model, taking into account PVR's relationships with other meaningful variables, such as word-of-mouth, donation to a community, or other eco-friendly behaviors. Future studies could be designed and implemented along such a line of research.

Author Contributions: H.M.L. held the leading position in writing, while Y.N. and J.P. supported writing, reviewing, and editing. All authors read and approved the final manuscript. Conceptualization, H.M.L. and Y.N.; methodology, H.M.L., Y.N., and J.P.; data curation, H.M.L. and J.P.; formal analysis, Y.N. and J.P.; investigation, H.M.L.; writing — original draft preparation, H.M.L.; writing—review and editing, Y.N. and J.P. funding acquisition, H.M.L. All authors have read and agreed to the published version of the manuscript.

Funding: This research was supported by the Sungshin Women's University Research Grant of 2019.

Conflicts of Interest: The authors declare no conflict of interest.

\section{References}

1. Choi, H.C.; Sirakaya, E. Measuring residents' attitude toward sustainable tourism: Development of sustainable tourism attitude scale. J. Travel Res. 2005, 43, 380-394. [CrossRef]

2. Koens, K.; Postma, A.; Papp, B. Is overtourism overused? Understanding the impact of tourism in a city context. Sustainability 2018, 10, 4384. [CrossRef]

3. UNWTO. Overtourism? Understanding and Managing Urban Tourism Growth beyond Perceptions; UNWTO: Madrid, Spain, 2018. 
4. McIntyre, G. Sustainable Tourism Development: Guide for Local Planners; World Tourism Organization: Madrid, Spain, 1993.

5. Dinan, C.; Sargeant, A. Social marketing and sustainable tourism: Is there a match? Int. J. Tour. Res. 2000, 2,1-14.

6. Ayuso, S. Adoption of voluntary environmental tools for sustainable tourism: Analyzing the experience of Spanish hotels. Corp. Soc. Responsib. Environ. Manag. 2006, 13, 207-220. [CrossRef]

7. Waligo, V.M.; Clarke, J.; Hawkins, R. Implementing sustainable tourism: A multi-stakeholder involvement management framework. Tour. Manag. 2013, 36, 342-353. [CrossRef]

8. Clarke, J. A framework of approaches to sustainable tourism. J. Sustain. Tour. 1997, 5, 224-233. [CrossRef]

9. Neto, F. A new approach to sustainable tourism development: Moving beyond environmental protection. Nat. Resour. Forum. 2003, 27, 212-222. [CrossRef]

10. Wang, D.; Xiang, Z.; Fesenmaier, D.R. Adapting to the mobile world: A model of smartphone use. Anna. Tour. Res. 2014, 48, 11-26. [CrossRef]

11. Conze, O.; Bieger, T.; Laesser, C.; Riklin, T. Relationship intention as a mediator between relational benefits and customer loyalty in the tour operator industry. J. Travel Tour. Mark. 2010, 27, 51-62. [CrossRef]

12. Hassan, S.S. Determinants of market competitiveness in an environmentally sustainable tourism industry. J. Travel Res. 2000, 38, 239-245. [CrossRef]

13. Vogt, C.A. Customer relationship management in tourism: Management needs and research applications. J. Travel Res. 2011, 50, 356-364. [CrossRef]

14. Bruning, S.D.; Ledingham, J.A. Relationships between organizations and publics: Development of a multidimensional organization-public relationship scale. Public Relat. Rev. 1999, 25, 157-170. [CrossRef]

15. Hon, L.C.; Brunner, B. Measuring public relationships among students and administrators at the University of Florida. J. Commun. Manag. 2002, 6, 227-238. [CrossRef]

16. Huang, Y.H. OPRA: A cross-cultural, multiple-item scale for measuring organization-public relationships. J. Public Relat. Res. 2001, 13, 61-90. [CrossRef]

17. Ki, E.J.; Hon, L.C. Reliability and validity of organization-public relationship measurement and linkages among relationship indicators in a membership organization. J. Mass. Commun. Q. 2007, 84, 419-438. [CrossRef]

18. Bruning, S.D.; Galloway, T. Expanding the organization-public relationship scale: Exploring the role that structural and personal commitment play in organization-public relationship. Public Relat. Rev. 2003, 29, 309-319. [CrossRef]

19. Butler, R.W. Sustainable tourism: A state-of-the-art review. Tour. Geogr. 1999, 1, 7-25. [CrossRef]

20. Hudson, S.; Thal, K. The impact of social media on the consumer decision process: Implications for tourism marketing. J. Travel Tour. Mark. 2013, 30, 156-160. [CrossRef]

21. Kallas, P. Top 15 Most Popular Social Networking Sites (and 10 apps!). Available online: https://www. dreamgrow.com/top-15-most-popular-social-networking-sites/ (accessed on 23 November 2019).

22. Leung, D.; Law, R.; Van Hoof, H.; Buhalis, D. Social media in tourism and hospitality: A literature review. J. Travel Tour. Mark. 2013, 30, 3-22. [CrossRef]

23. Sigala, M.; Christou, E.; Gretzel, U. Social Media in Travel, Tourism and Hospitality: Theory, Practice and Cases; Ashgate Publishing Limited: Surrey, UK, 2012.

24. Chung, N.; Koo, C. The use of social media in travel information search. Telemat. Inform. 2015, 32, $215-229$. [CrossRef]

25. No, E.; Kim, J.K. Comparing the attributes of online tourism information sources. Comput. Hum. Behav. 2015, 50, 564-575. [CrossRef]

26. Ford, R.C.; Wang, Y.; Vestal, A. Power asymmetries in tourism distribution networks. Anna. Tour. Res. 2012, 39, 755-779. [CrossRef]

27. Mkono, M. Sustainability and indigenous tourism insights from social media: Worldview differences, cultural friction and negotiation. J. Sustain. Tour. 2016, 24, 1315-1330. [CrossRef]

28. Alonso-Almeida, M.; Borrajo-Millan, F.; Yi, L. Are social media data pushing overtourism? The case of Barcelona and Chinese tourists. Sustainability 2019, 11, 3356. [CrossRef]

29. Parra-Lopez, E.; Bulchand-Gidumal, J.; Gutierrez-Tano, D.; Diaz-Armas, R. Intentions to use social media in organizing and taking vacation trips. Comput. Hum. Behav. 2011, 27, 640-654. [CrossRef] 
30. Xiang, Z.; Gretzel, U. Role of social media in online travel information search. Tour. Manag. 2010, 31, 179-188. [CrossRef]

31. Hudson, S.; Roth, M.S.; Madden, T.J.; Hudson, R. The effects of social media on emotions, brand relationship quality, and word of mouth: An empirical study of music festival attendees. Tour. Manag. 2015, 47, 68-76. [CrossRef]

32. Stets, J.E.; Burke, P.J. Identity theory and social identity theory. Soc. Psychol. Q. 2000, 63, 224-237. [CrossRef]

33. Sierra, J.J.; McQuitty, S. Attitudes and emotions as determinants of nostalgia purchases: An application of social identity theory. J. Mark. Theory Pract. 2007, 15, 99-112. [CrossRef]

34. Broom, G.M.; Casey, S.; Ritchey, J. Toward a concept and theory of organization-public relationships. J. Public Relat. Res. 1997, 9, 83-98. [CrossRef]

35. Vorvoreanu, M. Online organization-public relationship: An experience-centered approach. Public Relat. Rev. 2006, 32, 395-401. [CrossRef]

36. Pine, J.; Gillmore, J.H. Welcome to the experience economy. Harv. Bus. Rev. 1998, 76, 97-105. [PubMed]

37. Lasalle, D.; Britton, T.A. Priceless: Turning Ordinary Products into Extraordinary Experiences; Harvard Business School Press: Boston, MA, USA, 2003.

38. Hennig-Thurau, T.; Gwinner, K.P.; Gremler, D.D. Understanding relationship marketing outcomes: An integration of relational benefits and relationship quality. J. Serv. Res. 2002, 4, 230-247. [CrossRef]

39. Dunn, G.; Baloglu, S.; Brewer, P.; Qu, H. Consumer e-loyalty to online travel intermediaries. J. Qual. Assur. Hosp. Tour. 2009, 10, 1-22. [CrossRef]

40. Hosany, S.; Witham, M. Dimensions of cruisers' experience, satisfaction, and intention to recommend. J. Travel Res. 2010, 49, 351-364. [CrossRef]

41. Bruning, S.D.; Lambe, K. Relationship building and behavioral outcomes: Exploring the connection between relationship attitudes and key constituent behavior. Commun. Res. Rep. 2002, 19, 327-337. [CrossRef]

42. Bruning, S.D.; Ledingham, J.A. Organizatioanl-public relationships and consumer satisfaction: The role of relationships in the satisfaction mix. Commun. Res. Rep. 1998, 15, 199-209. [CrossRef]

43. Ki, E.; Hon, L.C. Causal linkages among relationship quality perception, attitude, and behavior intention in a membership organization. Corp. Commun. 2012,17, 187-208. [CrossRef]

44. Juvan, E.; Dolnicar, S. The attitude-behavior gap in sustainable tourism. Ann. Tour. Res. 2014, 48, 76-95. [CrossRef]

45. Shen, S.; Sotiriadis, M.; Zhou, Q. Could smart tourists be sustainable and responsible as well? The contribution of social networking sites to improving their sustainable and responsible behavior. Sustainability 2020, 12, 1470. [CrossRef]

46. Lavidge, R.; Steiner, G.A. A model for predictive measurement of advertising effectiveness. J. Mark. 1961, 25, 59-62. [CrossRef]

47. Ajzen, I. The theory of planned behavior. Organ. Behav. Hum. Decis. Process. 1991, 50, 179-211. [CrossRef]

48. Bruning, S.D.; Ralston, M. The role of relationships in public relations: Examining the influence of key public member relational attitudes on behavioral intent. Commun. Res. Rep. 2000, 17, 426-435. [CrossRef]

49. Ki, E.; Hon, L.C. Testing the linkages among the organization-public relationship and attitude and behavioral intentions. J. Public Relat. Res. 2007, 19, 1-23.

50. Kim, M.; Thapa, B.; Kim, H. International tourists' perceived sustainability of Jeju Island, South Korea. Sustainability 2018, 10, 73. [CrossRef]

51. Choi, K.; Kim, M.; Chang, M.; Koo, B. Evaluation on overtourism in Jeju Island. J. Tour. Ind. Res. 2019, 39, 29-36. [CrossRef]

52. Dijkmans, C.; Kerkkhof, P.; Beukeboom, C.J. A stage to engage: Social media and corporate reputation. Tour. Manag. 2015, 47, 58-67. [CrossRef]

53. Jang, S.; Cia, L.A. Travel motivations and destination choice: A study of British outbound market. J. Travel Tour. Mark. 2002, 13, 111-133. [CrossRef]

54. Kim, S.S.; Lee, C.; Klenosky, D.B. The influence of push and pull factors at Korean national parks. Tour. Manag. 2003, 24, 169-180. [CrossRef]

55. Yoon, Y.; Uysal, M. An examination of the effects of motivation and satisfaction on destination loyalty: A structural model. Tour. Manag. 2005, 26, 45-56. [CrossRef]

56. Anwar, S.A.; Sohail, M.S. Festival tourism in the United Arab Emirates: First-time versus repeat visitor perceptions. J. Vacat. Mark. 2004, 10, 161-170. [CrossRef] 
57. Pressgrove, G.N.; McKeever, B.W. Nonprofit relationship management: Extending the organization-public relationship to loyalty and behaviors. J. Public Relat. Res. 2016, 28, 193-211. [CrossRef]

58. Osgood, C.E.; Suci, G.J.; Tannenbaum, P.H. The Measurement of Meaning; University of Illinois Press: Urbana, IL, USA, 1957.

59. Asplund, K.; Norberg, A. Caregivers' reactions to the physical appearance of a person in the final stage of dementia as measured by semantic differentials. Int. J. Aging Hum. Dev. 1993, 37, 205-215. [CrossRef]

60. Hair, J.F.; Black, W.C.; Babin, B.J.; Anderson, R.E.; Tatham, R.E. Multivariate Data Analysis; Prentice Hall: Upper Saddle River, NJ, USA, 2006.

61. Fornell, C.; Larcker, D.F. Evaluating structural equation models with unobservable variables and measurement error. J. Mark. Res. 1981, 18, 39-50. [CrossRef]

62. Fabrigar, L.R.; Wegener, D.T.; MacCallum, R.C.; Strahan, E.J. Evaluating the use of exploratory factor analysis in psychological research. Psychol. Method. 1999, 4, 272-299. [CrossRef]

63. Costello, A.B.; Osborne, J.W. Best practices in exploratory factor analysis: Four recommendations for getting the most from your analysis. Pract. Assess. Res. Eval. 2005, 10,1-9.

64. Reidy, J.; Keogh, E. Testing the discriminant and convergent validity of the mood and anxiety symptoms questionnaire using a British sample. Person. Individ. Diff. 1997, 23, 337-344. [CrossRef]

65. Zait, A.; Bertea, P.E. Methods for testing discriminant validity. Manag. Mark. 2011, 9, 217-224.

66. Hu, L.Z.; Bentler, P.M. Cut off criteria for fit indexes in covariance structure analysis: Conventional criteria versus new alternatives. Struct. Equ. Model. 1999, 6, 1-55. [CrossRef]

67. Bentler, P.M. Comparative fit indices in structural models. Psychol. Bull. 1990, 107, 238-246. [CrossRef] [PubMed]

68. Schumacker, R.E.; Lomax, R.G. A Beginners' Guide to Structural Equation Modeling; Lawrence Erlbaum Associates: Mahwah, NJ, USA, 1996.

69. Tham, A.; Croy, G.; Mair, J. Social media in destination choice: Distinctive electronic word-of-mouth dimensions. Soc. Med. Tour. Mark. 2013, 30, 144-155. [CrossRef]

70. Ye, Q.; Law, R.; Gu, B.; Chen, W. The influence of user-generated content on traveler behavior: An empirical investigation on the effects of e-word-of-mouth to hotel online bookings. Comput. Hum. Behav. 2011, 27, 634-639. [CrossRef]

71. Lim, Y.; Chung, Y.; Weaver, P.A. The impact of social media on destination branding: Consumer-generated videos versus destination marketer-generated videos. J. Vacat. Mark. 2012, 18, 197-206. [CrossRef]

72. Harrison, V.S. Understanding the donor experience: Applying stewardship theory to higher education donors. Public Relat. Rev. 2018, 44, 533-548. [CrossRef]

73. Yang, S.U. An integrated model for organization: Public relational outcomes, organizational reputation, and their antecedents. J. Public Relat. Res. 2007, 19, 91-121. [CrossRef]

74. Zmyslony, P.; Kowalczyk-Aniol, J.; Dembinska, M. Deconstructing the overtourism-related social conflicts. Sustainability 2020, 12, 1695. [CrossRef]

75. Martin, J.M.M.; Martinez, J.M.G.; Fernandez, J.A.S. An analysis of the factors behind the citizen's attitude of rejection towards tourism in a context of overtourism and economic dependence on this activity. Sustainability 2018, 10, 2851. [CrossRef]

(C) 2020 by the authors. Licensee MDPI, Basel, Switzerland. This article is an open access article distributed under the terms and conditions of the Creative Commons Attribution (CC BY) license (http://creativecommons.org/licenses/by/4.0/). 

Article

\title{
Influences of SNS (Social Network Service) Uses and Musical Consumption on City Branding: A Focus on Broadway, New York and the West End, London
}

\author{
Jongwon Won ${ }^{1}$, Jong Yoon Lee ${ }^{2}$ and Jong Woo Jun ${ }^{3, *}$ \\ 1 Department of Media Communication, Soonchunhyang University, Chungnam 31538, Korea; jwon@sch.ac.kr \\ 2 Department of Photography, Visual \& Contents, Sangmyung University, Seoul 03016, Korea; \\ jongyoonlee1@smu.ac.kr \\ 3 School of Communication, Dankook University, Gyeonggi-do 16890, Korea \\ * Correspondence: jwjun@dankook.ac.kr
}

Received: 28 March 2020; Accepted: 26 April 2020; Published: 9 May 2020

\begin{abstract}
This study explored the role the musical industry plays in creating city brand images. The results showed that younger consumers were found to have more favorable visit intentions in New York and London due to their image as musical cities. Instagram users wanted to visit New York, but Twitter users had negative visit intentions in New York. Sensation-seeking orientation toward musicals influenced visit intention in New York. Broadway familiarity was linked to visit intention. In London, only sensation-seeking orientation influenced visit intention. Uses of SNS did not influence London visit intention and West End familiarity was not related to London visit intention. These results could provide academic and managerial implications for city branding.
\end{abstract}

Keywords: musicals; city branding; SNSs; orientation; sustainable tourism

\section{Introduction}

Countries, cities and communities are competing with each other in global environments. They develop marketing strategies and aim to attract residents, tourists, international students and even immigrants [1]. The term of place branding is a macro concept and country branding, city branding, and community brand are based on micro perspectives for individual places. Cities can be one of the basic units for place branding because nobody can visit a country without visiting a specific city or local places. Community brands are too small to be independent segments of place branding. Some cities are famous domestically or internationally. In this case, a certain city has its own unique identity. Identity is a critical element to be a recognizable city among other competing cities. This problem is why cities need to strategically brand their images to draw the attention of tourists or residents. City branding includes various tools along with media advertising. However, paid communication has limitations for city branding because cities have more complex and diverse identities and propositions [2].

Brand assets include various elements such as heritage, people, companies and culture. Some cities are famous for cultural attractions. European cities are famous for their cultural images stemming from traditional equity. On the other side, artificial things can be attractive sources of city branding. For example, Florida is a famous place because of Disney World, LA because of Disney Land, New York for Broadway and European cities for classical music. Broadway and the West End are well known due to their musical industries. International tourists visit Broadway and the West End to watch original performances of musical masterpieces. However, the research investigating roles of entertainment industries on city branding is limited and so it is required to fill this academic gap. 
This study tried to explore the effects of cultural industries on city branding and proposes the research model that proposes musical and cultural content could be the main reason tourists visit these cities. Using Broadway, New York and the West End, London as research targets, these relationships are investigated. The results could provide academic implications and managerial insights for city marketers and even musical producers. Artificial industries can be used as sustainable tourism attraction because they do not destroy natural resources such as mountain, beach and natural scenery, etc. Musical industries contribute an ecofriendly, sustainable tourism.

\section{Literature Review}

\subsection{City Branding}

The concept of brands has been used when companies deal with their products or services. However, the application of brands extends to various fields, and the public sector also uses brand management as a strategy. Representative examples are place brands and country brands. The term of place branding is used when dealing with particular regions. Country brands are the ultimate goals that individual countries try to build. Cities also utilize branding strategies to build more competitive brand equity. Destination branding needs to be differentiated from general place branding because destination branding is a tourism-oriented concept [3]. A destination is an object to visit for travelers, but a place can be an object to consume for various purposes.

Images are important concepts for city branding. Lynch [4] first used the concept of city images, and classified city images into paths, edges, districts, nodes, and landmarks based on the physical characteristics of cities. Appleyard retested the results of Lynch using the city of Ciudad Guyana in Venezuela, and Nasar analyzed Knoxville and Chattanooga in Tennessee to improve the old images of the two cities [4-6]. After that, Banai [7] analyzed comparative importance among adjectives, but this study had problems with generalization because the research cities were limited to college towns. Raporport stressed the complex characteristics of cities and added social and temporal characteristics along with the physical characteristics that Lynch suggested [4].

City brand equity includes brand awareness, perceived quality, brand attitudes, and brand loyalty [8]. City image studies are based on awareness of cities, and subsequent research also used city awareness using logical backgrounds and methods [9], applications to other cities [10], and the relationship between images and behaviors [11]. The goals of city branding include attracting tourists, shoppers, and global business people [1]. The ultimate goal of city branding is to build a unique city identity [12], and the concept of a creative city was suggested to make unique brand identity competing with other cities [13]. More recently, it was found that brand attitude, trust, commitment and uniqueness were important elements influencing city branding [14].

A city's brand can be built by various tools. Traditional marketing communication could make cities more noticeable, but they are not efficient communication methods. Cities are more complicated entities that are different from general companies or organizations. Non-traditional communication tools could be alternative ways to deliver favorable images to the public, consumers and tourists [2]. Cultural and entertainment approaches are helpful to build city images and brands [14]. More specifically, cinema can also provide an effective medium to deliver city images to tourism consumers [15]. The roles of cultural festivals were also supported for the city of Suwon, the capital of Gyeonggi-do, South Korea [16].

\subsection{SNS Uses}

As mass media continues to shed its power, people obtain information from alternative sources of content. SNS is a platform that is networking people based on users' common interest, hobbies and even professional opinions. SNS is defined as "a networked platform in which participants have uniquely identifiable profiles; can publicly articulate connections; and can consume, produce, and/or interact with streams of user-generated content" [17]. YouTube has become a primary information 
channel, and Facebook and Instagram also play important roles in information distribution [18]. SNS could construct virtual communities, and users could support each other by translating certain intentions into specific behaviors, through sharing goals and challenges [19]. SNS explains that social penetration happens without face-to-face relationships through online interpersonal interactions along with self-disclosure practices [20].

Many social capital studies have been done regarding the roles of SNS uses [21,22]. SNS users show higher scores on social capital dimensions such as face-to-face interactions, number of acquaintances, and bridging capital than non-users, and male users are reported deeper loneliness than female users [21]. The amount of time that users spend on SNS could differentiate users' specific behaviors and perceptions on social capital [22]. Heavy users are more likely to share information and use application programs frequently.

Individual SNSs have different characteristics. WhatsApp is a multifaceted communication tool; Facebook is used to display the socially acceptable self; Instagram is useful for stylized self-presentation; Twitter is a channel for information; and Snapchat provides an environment for spontaneous and playful connections [23]. Instagram influences a shopper's life [24]. Twitter enhances users' news knowledge, and these Twitter effects are moderated by the need for orientation and type of news [25].

Social media provides an entertainment experience to users, and competence and autonomy directly influenced enjoyment [26]. SNS is known to provide various reviews for theme parks [27]. It is found that Twitter enhanced hard news knowledge when users have a higher need for orientation [25]. Social media also motivates collaborative information seeking when people are planning group trips [28]. Different social media provide different forms of information [29].

SNS can be used as marketing communication tools, and create consumer trust toward user-created SNS content [30]. Different types of SNS users influence SNSs marketing performance, and social surveillance and self-surveillance can be factors classifying SNS users [31]. The four types of SNS users are versatile users, self-expression users, pass-along users, and introvert users. The most noticeable role of SNSs is to be helpful for tourism organizations to make more effective decisions [32]. This can be applied to an individual tourist.

According to the literature, Facebook plays roles to illustrate city personality [33]. Social media is also effective to deliver information of festivals that a city holds to promote favorable city images [16]. This study operationally defined SNS uses as daily uses of user-generated content of musicals that are posted on YouTube, Facebook, Instagram, and Twitter. According to the previous literature, it can be hypothesized that people will have positive visit intentions when they use SNS for musical information. This study will explore the effects on New York and London, cities that are famous for their musical industries.

Hypothesis 1 (H1). SNS uses will influence city visit intentions positively.

Hypothesis 1a (H1a). SNS uses will influence New York visit intentions positively.

Hypothesis $\mathbf{1 b}(\mathbf{H 1 b})$. SNS uses will influence London visit intentions positively.

\section{Musical Orientation}

People watch musicals for various purposes and motivations. Some musical fans could have a particular orientation toward musical performance. Why people use is an important issue in performance marketing. Orientation used in marketing is defined as the propensity to do predictable behaviors [34]. Two important concepts to define orientation are motivation and value [35]. Orientation also is expressed as sustainable involvement [36]. Another key concept of orientation is modality $[35,37,38]$. People's orientation (motivation and value) is presented in a typical modality. When we think about sports, people could express orientation through emotional responses [39]. 
Others could deal with sports with social motivation [40]. Ultimately, orientation can be classified into sensation-, cognition-, and socialization-seeking orientation [41].

This study uses the concept of orientation to explain the roles of musicals in a city branding context. Originally, orientation was developed for sports events, but it is useful to understand the motivation of content users [41]. Using three orientation dimensions toward musicals, the relationship between musical orientation and visit intention in both cities are proposed.

Hypothesis 2 (H2). Musical Orientation will influence city visit intentions positively.

Hypothesis 2a (H2a). Musical Orientation will influence New York visit intentions positively.

Hypothesis 2b (H2b). Musical Orientation will influence London visit intentions positively.

\section{Place Familiarity}

Familiarity refers to the cognitive structure that individuals hold toward a certain product [42]. Familiarity is also said to be the complicated and refined memory structure of stimulus through repeated processes of exposure, encoding, and representation [43]. Brand familiarity means that users have professional knowledge and experiences about a certain product or brand [44]. It means that brand familiarity involves the degree of knowledge that consumers perceive [45].

The reason why familiarity is important is that familiarity could influence consumer attitudes and behavioral intention. For example, when consumers hold knowledge about products and brands, they have confidence and hold preferences and choose a certain product and brand [42]. Even when persuasion knowledge is activated, brand familiarity decreases the negative effects [46]. Consumers have more purchase intention on familiar brands than unfamiliar ones in initial purchases [47]. This relationship between brand familiarity and purchase intention is confirmed on photo service and car insurance [48]. Another case can be found in country-of-origin effects. People who are familiar with a country or a product might lead to purchasing [49]. It is also found that consumers purchase familiar products to reduce shopping time [50].

Familiarity has been dealt with in terms of the number of visits, the quantity of information, and past experiences from tourism perspectives [51]. Place familiarity is classified into experiential familiarity and informational familiarity [51,52]. This study focuses on both experiential familiarity and informational familiarity and explores the roles of familiarity on visit intentions in New York and London.

Hypothesis 3 (H3). Place familiarity will influence city visit intentions positively.

Hypothesis 3a (H3a). Broadway familiarity will influence New York visit intentions positively.

Hypothesis $3 \mathbf{b} \mathbf{( H 3 b ) . ~ P l a c e ~ f a m i l i a r i t y ~ w i l l ~ i n f l u e n c e ~ L o n d o n ~ v i s i t ~ i n t e n t i o n s ~ p o s i t i v e l y . ~}$

\section{Methodology}

The study chose New York and London as research targets because those two cities were famous for musical industries and adequate to study relationships with city branding and tourism marketing. This study used a survey research method. Using general consumers in South Korea as a research sample, the hierarchical relationships were investigated. Research samples were collected from research panels that a professional research company provides. Among respondents, people who had never watched musicals were excluded. Collected data were analyzed using hierarchical regression and the SPSS16 package was used for statistical data processing. 


\subsection{Samples}

Sampling was done in 2019, April 1 to 15. A survey questionnaire was sent to people who had the opportunity to watch musicals at least once in the past year. A total of 339 participated in the survey. The gender of respondents are equally allocated, and ages are also allocated to 20s, 30s, 40s, and 50s evenly. Among them, males made up 175 (51.6\%), and females made up 164 (48.4\%). The ages of participants ranged from 20 to 59 , and the mean age was 39.8 years (SD = 10.7). One hundred and twelve respondents (33.0\%) visited the United States, 60 (17.7\%) visited New York, 72 (21.2\%) visited the United Kingdom, and 70 (20.6\%) visited London. All participants were Korean.

\subsection{Measurements}

SNS uses are measured by the sentence 'I use musical information on $(\ldots \ldots)^{\prime}$ using Facebook $(\mathrm{M}=3.20$, S.D. $=1.66)$, Instagram $(\mathrm{M}=3.14$, S.D. $=1.76)$, Twitter $(\mathrm{M}=2.71, \mathrm{~S} . \mathrm{D} .=1.65)$, and YouTube $(\mathrm{M}=3.75$, S.D. $=1.87)$. Other variables such as orientation, familiarity, and visit intention are measured as the previous literature has suggested. Orientation was measured using three different dimensions of socialization-, sensation-, and cognition-seeking orientation [41]. Socialization seeking includes 'I am often involved in conversations about musicals,' 'I like talking about musicals with people I know,' 'Watching musicals is a good opportunity to socialize,' 'Watching musicals is a good opportunity to socialize with one's friends' $(\mathrm{M}=4.10$, S.D. $=1.33$, alpha $=0.94)$. Sensation seeking includes, 'For me, watching musicals is a real pleasure,' 'I am always excited when I am going to watch a musical,' 'I am always enthusiastic when I think about watching musicals,' 'When I watch a musical, I sometimes feel like I am part of the musical,' 'I feel really happy when I can watch a musical' ( $\mathrm{M}=4.95$, S.D. $=1.21$, alpha $=0.95)$. Cognition seeking includes 'I consider myself as a musical expert,' 'I know very much about musicals, ' 'I am really interested in any information regarding musicals' ( $\mathrm{M}=3.49$, S.D. $=1.38$, alpha $=0.92$ ). Familiarity was measured by Kent \& Allen (1994) [53] with statements such as 'I am familiar with Broadway,' 'I experienced Broadway a lot,' 'I know Broadway well' (Broadway M = 3.12, S.D. $=1.39$, alpha = 0.92; West End M = 2.64, S.D. = 1.46, alpha = 0.97). Visit intention was measured using three items of 'provable,' 'likely,' 'possible' (New York M = 5.25, S.D. = 1.22, alpha = 0.92; London $\mathrm{M}=5.22$, S.D. $=1.23$, alpha $=0.93$ ). All measurement items are summated, and used as individual variables.

\section{Results}

\section{Hypotheses Testing}

Table 1 shows the results of the hypothesis test. A four-stage hierarchal regression analysis was implemented to verify the eight hypotheses. In the first stage, we tried to control demographic variables. In the second stage, we included four factors from SNS uses: Facebook, Instagram, Twitter, and YouTube. In the third stage, we included musical orientation such as socialization seeking, sensation seeking, and cognition seeking. In the final stage, we added city familiarity to verify the effect of place familiarity on visit intention.

In the first stage, we controlled demographic variables and verified that age has a direct effect on visit intention in New York. In the second stage, the model included SNS uses, and uses of Instagram were found to be effective at a limited level $(p<0.10)$. In the third stage, we included musical orientation and sensation-seeking orientation was statistically significant $(p<0.001)$. The results of the analysis showed that the third model had a higher coefficient of determination (17.2\%) than models 1 and 2. In addition, Instagram use, which had only a limited effect in model 2, showed a meaningful effect of $p<0.05$, and Twitter use, which did not have a meaningful effect in model 2, became statistically significant in a negative way $(p<0.05)$. The final model included familiarity with Broadway. The results of the analysis showed that Broadway familiarity influenced visit intention in New York. 
Table 1. Results of hierarchal regression analysis on visit intentions of New York.

\begin{tabular}{|c|c|c|c|c|c|}
\hline DV & IV & Model 1 & Model 2 & Model 3 & Model 4 \\
\hline \multirow{10}{*}{$\begin{array}{l}\text { Visit Intention of } \\
\text { New York }\end{array}$} & GENDER & 0.012 & 0.002 & 0.054 & 0.044 \\
\hline & AGE & $-0.142 * *$ & -0.122 * & -0.110 * & -0.120 * \\
\hline & Facebook & & 0.005 & -0.033 & -0.050 \\
\hline & Instagram & & $0.186 \#$ & $0.196^{*}$ & $0.189 *$ \\
\hline & Twitter & & -0.117 & -0.143 * & -0.162 * \\
\hline & YouTube & & 0.124 & -0.001 & -0.004 \\
\hline & Social OT & & & -0.014 & -0.029 \\
\hline & Sensation OT & & & $0.390^{* * *}$ & $0.413^{* * *}$ \\
\hline & Cognitive OT & & & 0.013 & -0.081 \\
\hline & Broadway F & & & & $0.162 *$ \\
\hline \multicolumn{2}{|c|}{ Adjusted R2 = $(\Delta \mathrm{R} 2)$} & 0.014 & $0.055(0.052)$ & $0.172(0.122)$ & $0.180(0.011)$ \\
\hline \multicolumn{2}{|c|}{$\mathrm{F}$} & 3.434 * & $4.267^{* * *}$ & $8.782^{* * *}$ & $8.426^{* * *}$ \\
\hline
\end{tabular}

Another four-stage hierarchal regression analysis was implemented to verify hypotheses for London visit intentions. In the first stage, we verified that age has a direct effect on visit intention in London. In the second stage, we could not find any statistically significant variables on visit intention in London. In the third stage, we found that the sensation-seeking orientation was statistically significant $(p<0.001)$. In the fourth stage, West End familiarity did not influence visit intention in London (see Table 2).

Table 2. Results of hierarchal regression analysis on visit intentions of London.

\begin{tabular}{|c|c|c|c|c|c|}
\hline DV & IV & Model 1 & Model 2 & Model 3 & Model 4 \\
\hline \multirow{10}{*}{$\begin{array}{l}\text { Visit Intention of } \\
\text { London }\end{array}$} & GENDER & 0.037 & 0.027 & 0.073 & 0.071 \\
\hline & AGE & -0.140 * & -0.133 * & -0.112 * & $-0.115^{*}$ \\
\hline & Facebook & & 0.032 & 0.006 & 0.004 \\
\hline & Instagram & & 0.085 & 0.102 & 0.099 \\
\hline & Twitter & & -0.031 & -0.043 & -0.049 \\
\hline & YouTube & & 0.083 & -0.034 & -0.031 \\
\hline & Social OT & & & 0.044 & 0.043 \\
\hline & Sensation OT & & & $0.343^{* * *}$ & $0.347^{* * *}$ \\
\hline & Cognitive OT & & & -0.063 & -0.078 \\
\hline & West End F & & & & 0.026 \\
\hline \multicolumn{2}{|c|}{ Adjusted $R 2=(\Delta R 2)$} & 0.015 & $0.029(0.026)$ & $0.118(0.095)$ & $0.115(0.000)$ \\
\hline \multicolumn{2}{|c|}{ F } & 3.506 * & $2.688 *$ & $6.018^{* * *}$ & $5.412 * * *$ \\
\hline
\end{tabular}

\section{Discussion and Conclusions}

This study explored the role of musicals on city brand images in New York and London. The findings of this study showed that younger consumers were found to have more favorable visit intentions in New York and London. Regarding the effects of SNS, Instagram positively influenced visit intention in New York, but Twitter negatively influenced visit intention in New York (H1a). We could not find significant effects of SNS on visit intention in London (H1b). Among the three 
musical orientation dimensions, sensation-seeking orientation influenced visit intention in New York (H2a) and London (H2b). Broadway familiarity positively influenced visit intention in New York (H3a), but West End familiarity was not related to London visit intention ( $\mathrm{H} 3 \mathrm{~b})$.

This study has some academic implications. It is found that musicals could be important antecedents of city branding. This result supported the roles of entertainment content on city marketing $[15,16]$. It is accepted that traditional advertising and marketing are not sufficient to build city brand equity, and cultural products of cities can be precious elements of city brand equity [2]. This study supported the proposition that the musical industry plays an important role in place and city brands. Musicals are an essential business in New York and London, and this physical element can be regarded as a part of city brand equity.

SNSs showed influences as distribution channels of musical information, and it was similar to the results found in the previous literature illustrating the effects of SNS on city branding [16,33]. It is well known that traditional media have lost their power, and SNSs seem to replace traditional media [20]. Online communication has already become the dominant way of facilitating interpersonal and mass communication. The findings of this study also support the novel roles of SNSs as information providers. The information delivered by SNSs could cultivate perceptions of travelers and lead them to behavioral intention. The more people use musical information, the more likely that they have visit intention. However, the roles of Instagram and Twitter are different. Instagram positively influenced visit intention of New York, but Twitter showed negative influences. It is because of the different characteristics of SNS. Instagram is used for self-presentation, and Twitter is used for information seeking [23]. These different characteristics might involve the influences of SNS.

Another finding is that place familiarity is linked to city branding, and it is in the same line of thoughts of the previous literature [44]. However, it is also found that the roles of Broadway and the West End influence city branding differently. Even though the West End is the birthplace of the musical, associations of musicals and London are weak compared to New York. It stems from the gap of existent brand power between New York City and London. New York has a strong background in various cultural products or content industries. New York is the center for broadcasting and advertising, along with musicals, and also is famous for financial services. On the contrary, London has limited associations as a cultural destination for Korean tourists. It is true that London has strong brand power, but this brand power is not built on musicals.

This study provides a couple of managerial implications. Cultural products can be used as tourist attractions for cities. For example, European cities are famous for classical music. Museums also can attract tourists to historical or natural exhibits. However, we could not find the role of the musical in the city brand of London. It is recommended for brand managers of London to build more strong associations with musicals performed in the West End. Broadway is a symbol of the musical that leads to images of New York, but the West End is as well known to international consumers or visitors. Branding the West End is an urgent task to utilize cultural images of a musical city. Successful case studies could give insightful implications.

Small cities also learn the managerial implications of cultural marketing for cities that do not have enough cultural resources. It is difficult to foster a cultural industry in a short period. In this case, cultural festivals can be an alternative option. A city can develop a cultural festival related to the city's image. The Edinburgh International Festival in Scotland is the representative case. The Munich Beer Festival is another successful case of cultural events. Well-made regional festivals could build favorable city images and even attract visitors to the city.

Another implication is the effect of individual SNS services. SNSs are now major marketing communication tools for companies and public organizations, but the selection of individual platforms needs to be strategically considered for cities. Instagram positively influenced visit intention to New York, but Twitter showed negative influences. This might be because Twitter lost its influence on entertainment content users. In this regard, information channels should be managed strategically to 
communicate with musical consumers regarding trips to musical cities. Particularly, media management of Instagram could be fruitful for marketing managers.

In sum, this study showed entertainment industries could be important elements of city branding. Particularly, the concept of orientation was suggested, and this study found specifically to be a motivation of musical influencing city visits. Additionally, roles of familiarity were supported academically, and effects of SNS content should be used in terms of practical strategies.

This study has a few limitations. The homophily phenomenon of the sampling issue could be mentioned because we used only musical fans. This could be an obstacle for generalizing the result of this study. In addition, we could not find antecedents influencing the city brand of London. Even though London has unique characteristics, a weak association between musicals and London is unexpected. Future research exploring significant elements influencing the brand of London is necessary. Further, other entertainment industries other than musicals need to be investigated in terms of city or place branding. This study used only Korean consumers, and future studies dealing with more generalizable international samples will be also fruitful.

Author Contributions: Conceptualization, J.W.; Methodology, J.W.J.; Data Curation, J.Y.L.; Writing Original Draft Preparation, J.Y.L.; Writing Review \& Editing, J.W.J.; Project Administration, J.W.J.; Funding Acquisition, J.W. All authors have read and agreed to the published version of the manuscript.

Funding: This research received no external funding.

Conflicts of Interest: The authors declare no conflict of interest.

\section{References}

1. Bagaeen, S.G. Brand Dubai: The Instant City; or the Instantly Recognizable City. Int. Plan. Stud. 2007, 12, 173-197. [CrossRef]

2. Jun, J.W. Impacts of Novelty of North Korean Cheerleaders and Olympic Engagement on Olympic and PyeongChang Attitudes: A Focus on Perspectives from Korean College Students. Inf. Soc. Media 2019, 20, 175-195.

3. Walmsley, D.; Young, M. Evaluative Images and Tourism: The Use of Personal Constructs to Describe the Structure of Destination Images. J. Travel Res. 1998, 36, 65-69. [CrossRef]

4. Lynch, K. The Image of the City; MIT Press: Cambridge, MA, USA, 1960.

5. Stann, E.J.; Appleyard, D. Planning a Pluralist City: Conflicting Realities in Ciudad Guayana. Hisp. Am. Hist. Rev. 1977, 57, 774. [CrossRef]

6. Nasar, J.L. The Evaluative Image of the City. J. Am. Plan. Assoc. 1990, 56, 41-53. [CrossRef]

7. Banai, R. A methodology for The Image of the City. Environ. Plan. B Plan. Des. 1999, 26, 133-144. [CrossRef]

8. Kladou, S.; Kehagias, J. Assessing destination brand equity: An integrated approach. J. Destin. Mark. Manag. 2014, 3, 2-10. [CrossRef]

9. Howard, R.B.; Chase, S.D.; Rothman, M. An analysis of four measures of cognitive maps. Environ. Des. Res. 1973, 2, 254-264

10. Klein, H.J. Urban Core and Inner City; Brill: Leiden, The Netherlands, 1967.

11. Downs, R.M.; Stea, D. Maps in Minds: Reflections on Cognitive Mapping; Harper \& Row, Publishers: New York, NY, USA, 1977.

12. Kavaratzis, M.; Ashworth, G.J. City Branding: An Effective Assertion of Identity or A Transitory Marketing Trick? Tijdschr. Voor Econ. En Soc. Geogr. 2005, 96, 506-514. [CrossRef]

13. Landry, C. The art of city making. Aust. Plan. 2006, 43, 47. [CrossRef]

14. Kemp, E.; Childers, C.Y.; Williams, K.H. A tale of a musical city: Fostering self-brand connection among residents of Austin, Texas. Place Brand. Public Dipl. 2012, 8, 147-157. [CrossRef]

15. Chen, S.; Shih, E. City branding through cinema: The case of postcolonial Hong Kong. J. Brand Manag. 2019, 26, 505-521. [CrossRef]

16. Yoon, S.-W.; Chung, S.W. Promoting a World Heritage Site through Social Media: Suwon City's Facebook Promotion Strategy on Hwaseong Fortress (in South Korea). Sustainability 2018, 10, 2189. [CrossRef]

17. Ellison, N.; Boyd, D.M. Sociality Through Social Network Sites; Oxford University Press: Oxford, UK, 2013; pp. 1-26. 
18. Angelovska, N. Facebook Losing Users To Pinterest, Youtube And Twitter (Market Share By Region). Available online: https:/www.forbes.com/sites/ninaangelovska/2019/01/07/facebook-loosing-users-topinterest-youtube-and-twitter-market-share-by-region/\#58ffab627746 (accessed on 7 January 2019).

19. De La Pena, A.; Domínguez, C.Q. Share, like and achieve: The power of facebook to reach health related goals. Int. J. Consum. Stud. 2015, 39, 495-505. [CrossRef]

20. Pennington, N. Building and Maintaining Relationships in the Digital Age: Using Social Penetration Theory to Explore Communication through Social Networking Sites. Ph.D. Thesis, University of Kansas, Lawrence, KS, USA, 2015.

21. Brandtzaeg, P.B.; Brandtzæg, P.B. Social Networking Sites: Their Users and Social Implications-A Longitudinal Study. J. Comput. Commun. 2012, 17, 467-488. [CrossRef]

22. Chang, T.-S.; Hsiao, W.-H. Time Spent on Social Networking Sites: Understanding User Behavior and Social Capital. Syst. Res. Behav. Sci. 2013, 31, 102-114. [CrossRef]

23. Boczkowski, P.J.; Matassi, M.; Mitchelstein, E. How Young Users Deal With Multiple Platforms: The Role of Meaning-Making in Social Media Repertoires. J. Comput. Commun. 2018, 23, 245-259. [CrossRef]

24. Hund, E.; McGuigan, L. A Shoppable Life: Performance, Selfhood, and Influence in the Social Media Storefront. Commun. Cult. Crit. 2019, 12, 18-35. [CrossRef]

25. Lee, E.-J.; Oh, S.Y. Seek and You Shall Find? How Need for Orientation Moderates Knowledge Gain from Twitter Use. J. Commun. 2013, 63, 745-765. [CrossRef]

26. Reinecke, L.; Vorderer, P.; Knop, K. Entertainment 2.0? The Role of Intrinsic and Extrinsic Need Satisfaction for the Enjoyment of Facebook Use. J. Commun. 2014, 64, 417-438. [CrossRef]

27. Ren, Q.; Xu, F.; Ji, X. Use of the pathfinder network scaling to measure online customer reviews: A theme park study. Strat. Chang. 2019, 28, 333-344. [CrossRef]

28. Fardous, J.; Du, J.T. Tourists' social interactions on social media during a group trip planning. Proc. Assoc. Inf. Sci. Technol. 2019, 56, 642-644. [CrossRef]

29. Yuan, C.; Tian, W. The Influences of Dual Social Network Site Use and Social Capital Development on Sociocultural Adaptation. Ph.D. Thesis, Cornell University, Ithaca, NY, USA, 2015.

30. Chari, S.; Christodoulides, G.; Presi, C.; Wenhold, J.; Casaletto, J.P.; Gonçalves, H.M.; Rey-Martí, A.; Roig-Tierno, N.; Miles, M.P. Consumer Trust in User-Generated Brand Recommendations on Facebook. Psychol. Mark. 2016, 33, 1071-1081. [CrossRef]

31. Park, M.-S.; Shin, J.-K.; Ju, Y. A Taxonomy of Social Networking Site Users: Social Surveillance and Self-surveillance Perspective. Psychol. Mark. 2015, 32, 601-610. [CrossRef]

32. Rydén, P.; Kottika, E.; Hossain, M.; Skare, V.; Morrison, A.M. Threat or treat for tourism organizations? The Copenhagen Zoo social media storm. Int. J. Tour. Res. 2019, 22, 108-119. [CrossRef]

33. Glińska, E.; Rudolf, W. City Brand Personality Projected by Municipalities from Central and Eastern Europe Countries-A Comparison of Facebook Usage. Sustainability 2019, 11, 5440. [CrossRef]

34. Kantanen, T. Analysis of the Contents of Theatre Visiting Orientation. In Proceedings of University of Vaasa; Research Paper; Business Administration: Vaasa, Finland, 1993.

35. Parsons, T.; Shills, E.A. Toward a General Theory of Action; Harper \& Row: New York, NY, USA, 1967.

36. Laurent, G.; Kapferer, J.-N. Les profils d'implication. Rech. Appl. Mark. 1986, 1, 41-57. [CrossRef]

37. Hirschman, E.C. Experience seeking: A subjectivist perspective of consumption. J. Bus. Res. 1984, 12, 115-136. [CrossRef]

38. Laaksonen, M. Shopping Orientation: An Attitude Structure Approach. In Proceedings of University of Vaasa; Research Paper; Business Administration: Vaasa, Finland, 1987.

39. Wann, D.L. Preliminary Validation of the Sport Fan Motivation Scale. J. Sport Soc. Issues 1995, 19, 377-396. [CrossRef]

40. Swanson, S.R.; Larson, B.V.; Janda, S. Motivations of College Students Game Attendance and Word-of-Mouth Behavior: The Impact of Gender Differences. Sport Mark. Q. 2003, 12, 151-169.

41. Pons, F.; Mourali, M.; Nyeck, S. Consumer Orientation Toward Sporting Events. J. Serv. Res. 2006, 8, $276-287$. [CrossRef]

42. Zinkhan, G.M.; Muderrisoglu, A. Involvement, familiarity, cognitive differentiation, and advertising recall: A test of convergent and discriminant validity. Adv. Consum. Res. 1985, 12, 251-256.

43. Mowen, J.C. Consumer Behaviour, 4th ed.; Prentice-Hall: Englewood Cliffs, NJ, USA, 1995.

44. Alba, J.W.; Hutchinson, J.W. Dimensions of Consumer Expertise. J. Consum. Res. 1987, 13, 411. [CrossRef] 
45. Barbara, S.; Smith, T.R. A computational process of evaluation based on the cognitive structuring of episodic knowledge. Assoc. Consum. Res. 1982, 8, 136-143.

46. Wei, M.-L.; Fischer, E.; Main, K.J. An Examination of the Effects of Activating Persuasion Knowledge on Consumer Response to Brands Engaging in Covert Marketing. J. Public Policy Mark. 2008, 27, 34-44. [CrossRef]

47. Ray, M.L.; Sawyer, A.G. Repetition in Media Models: A Laboratory Technique. J. Mark. Res. 1971, 8, $20-29$. [CrossRef]

48. Arora, R.; Stoner, C. The effect of perceived service quality and name familiarity on the service selection decision. J. Serv. Mark. 1996, 10, 22-34. [CrossRef]

49. Han, C.M.; Terpstra, V. Country-of-Origin Effects for Uni-National and Bi-National Products. J. Int. Bus. Stud. 1988, 19, 235-255. [CrossRef]

50. Biswas, A. The moderating role of brand familiarity in reference price perceptions. J. Bus. Res. 1992, 25, 251-262. [CrossRef]

51. Baloglu, S. Image variations of Turkey by familiarity index: Informational and experiential dimensions. Tour. Manag. 2001, 22, 127-133. [CrossRef]

52. Prentice, R.C. The distant familiar? Young british adults' imaginings of Australia. In Riding the Wave of Tourism and Hospitality Research; Braitwaite, R., Braithwaite, R., Eds.; Southern Cross University: Lismore, NSW, Australia, 2003.

53. Kent, R.; Allen, C. Competitive interference effects in consumer memory for advertising: The role of brand familiarity. J. Mark. 1994, 58, 97-105. [CrossRef]

(C) 2020 by the authors. Licensee MDPI, Basel, Switzerland. This article is an open access article distributed under the terms and conditions of the Creative Commons Attribution (CC BY) license (http://creativecommons.org/licenses/by/4.0/). 
Article

\title{
A Study on the Effects of Crowdfunding Values on the Intention to Visit Local Festivals: Focusing on Mediating Effects of Perceived Risk and e-WOM
}

\author{
Hun Kim and Byenghee Chang * \\ Department of Journalism and Mass Communication, Sungkyunkwan University, Seoul 03063, Korea; \\ kimhun6301@naver.com \\ * Correspondence: mediaboy@skku.edu; Tel.: +82-2-760-0687
}

Received: 6 March 2020; Accepted: 15 April 2020; Published: 17 April 2020

\begin{abstract}
Information technology is recognized as an important means of expanding the sustainability of local festivals, but most research and practices only focus on existing information technologies such as websites and social network services. This study examines the potential of crowdfunding platforms to ensure the success of local festivals and assesses how emerging information technologies impact the sustainability of the tourism industry. This study proposed four values based on the value theory that is frequently applied in consumer research. We also applied inner innovativeness as a personal characteristic and examined the effects of economic, emotional, social, altruistic, and inner innovativeness regarding film festival crowdfunding on the intention to visit the film festival. We applied perceived risk and the intention to use electronic word of mouth (e-WOM) as mediating variables. As a result, emotional, social, and altruistic values were found to significantly affect the intention to visit film festivals by mediating perceived risk. In addition, the social value was found to have positive effects on the dependent variable through the intention to use e-WOM. The results show that crowdfunding platforms are considered an important tool for promoting the festival. It is also important to develop value in favor of the festival by increasing value through marketing strategies.
\end{abstract}

Keywords: crowdfunding; consumption value; inner innovativeness; perceived risk; e-WOM; the intention to visit festival

\section{Introduction}

The sustainability of local festivals is important because they provide benefits to the community. Communities can generate economic income by discovering unique local content [1], and festivals provide an opportunity to externally promote this content [2]. However, festivals held by small cities often face budgeting issues, so it is hard to be active in marketing. In this situation, advances in information technology (IT) may act as another opportunity to promote the sustainability of the local festival industry.

During the period in which the Internet became popular, local tourism websites were used as the main platform to provide travelers with up-to-date tourist information in the region [3]. Many travelers have recently been able to get or share information about local festivals through various IT platforms. Social network services (SNS), which emerged around 2010, are regarded as one of the megatrends influencing tourism systems [4]. These platforms not only allow travelers to search for information [5] but also help them make decisions about visiting tourist attractions or festivals [6]. SNS is also rated as having the ability for travelers to engage with potential customers by sharing comments, videos, photos, and more [4]. Tourists upload their local photos to SNS, and potential customers who view this content through SNS are attracted to the region. Given that potential tourists primarily rely on the 
opinions of others, such as word of mouth (WOM), for decision-making [7], SNS are the best method to encourage them to participate in local festivals. From the festival organizers' point of view, SNS help to effectively promote local festivals [8].

The emerging crowdfunding platform is also a valuable tool to promote local festivals. Businesspeople have said that they use crowdfunding to market their projects or communicate with fans or supporters [9]. They can also increase their brand awareness through crowdfunding [10]. Many arts and cultural projects, for example, make heavy use of crowdfunding because in addition to attracting funds, they can also promote funding simultaneously [11]. A notable example is Big Issue Korea, which raised funds through crowdfunding for the Nutcracker, a play in which homeless people played a major role. The press recognized the crowdfunding by Big Issue Korea, which saw great results from the promotion [12].

Crowdfunding can be linked with SNS to maximize the promotion of local festivals [13]. Most crowdfunding sites provide the SNS information of individual operators and include the function to share crowdfunding information. Potential participants can use SNS information to determine various indicators of various crowdfunding, which can have a social impact on participants' support decisions [14]. Individuals spread crowdfunding information to others, and those exposed to it can receive festival and crowdfunding information. Therefore, it is meaningful to consider the effects of electronic word of mouth (e-WOM) and crowdfunding factors on social network services.

While crowdfunding platforms are being assessed as a means of sustainability for local festivals, previous research has focused primarily on the effect of social network services [4,5]. However, these works omit the impact of associations between one or more online services and the intention to visit a tourist destination. Online marketing tools show an important diversity, and consumers are also able to receive tourism information through a range of various online channels. This indicates the importance of investigating the effects of multiple marketing tools simultaneously. Therefore, this study constitutes the framework of study considering not only (1) e-WOM, which is the main effect of social network services, but also (2) the role of a new medium-crowdfunding.

With regard to crowdfunding factors, this study examines the influence of perceived economical, emotional, social, and altruistic values about film festival crowdfunding on the intention to visit the region of film festivals. In addition, the perceived risk of film festival crowdfunding is applied as a mediating variable. Perceived risk is considered a useful variable in exploring the user's perception of uncertainty in accepting new IT services. Therefore, it is a meaningful approach to look at the effects of perceived risks on film festival crowdfunding. Regarding the role of e-WOM, it applies the intention to use electronic word of mouth (e-WOM) as mediating variables. E-WOM was proven to be an effective way to promote tourist destinations at a low cost [15]. The intention to use e-WOM is thus a meaningful variable to examine the effects of crowdfunding as a tool for disseminating information about festivals. In addition, the framework includes inner innovativeness as one of the independent variables. This approach is to consider not only the marketing platform but also the personal characteristic as an explanatory variable.

This research model draws on the frameworks of several studies that examine factors influencing the intention to visit tourist destinations $[16,17]$. These studies assume that measures of online consumer behavior, such as e-WOM [16] and blog usage [17], mediate explanatory variables relating to the intention to visit tourist destinations. This study proposes an extended framework by applying the explanatory variables and some mediating variables suggested above considering the characteristics of the subject and factors. Thus, this framework examines tourists' perceptions, intentions, and actions as a whole. Given the continuing increase in the impact of information technology services in the tourism industry, it will help to increase sustainability in regional tourism industries with unstable conditions. 


\section{Literature Review}

\subsection{Local Festival and Crowdfunding}

Local festivals provide tourists with a variety of events that utilize cultural elements within the city for a certain period of time. During the festival, the community can introduce the community's local traditions, intangible heritage, natural scenery, and ethnic backgrounds to the tourists [18]. Thus, festivals are meaningful because the community can discover local cultural elements and use them as highly sustainable tourism resources. Local festivals also contribute to increasing the city's demand for travel [19]. Tourists visit the festival area and pay a cost to use various amenities such as transportation, accommodation, and restaurants. Thus, local festivals are also recognized as a means of promoting the local tourism industry and activating the economy [20]. In addition, local festivals not only expose a city as a tourist destination but also influence the re-creation of a city's image [21]. From a city marketing point of view, festivals are an effective approach to strengthening the identity of a city and reversing the negative image of a city [22]. In summary, local festivals are important tourist resources that generate economic benefits and enhance a city's image.

In recent years, local film festivals have become popular as one of the exciting local events. Major cities around the world, such as Cannes, Venice, and Berlin, have held international film festivals for a long time. For example, Cannes, a local small town, established the world's third film festival in 1939, following the Venice International Film Festival (1932) and Moscow International Film Festival (1935). Currently, hosting the most popular international film festival worldwide is the most powerful element defining the identity of Cannes [23]. The Cannes International Film Festival attracts approximately 200,000 tourists, including audiences and professionals, each year, generating 200 million euros' worth of economic benefits [24].

The Cannes International Film Festival attracts audiences of all ages by offering movies and popular entertainment content. The general public can take an interest in international film festivals because movies from different countries can be seen in one place. Professional groups such as directors, actors, and students may visit the city to participate in special sessions or workshops at the festival [25]. Therefore, film festivals are not just entertaining events but also culturally valuable events that can be inspired by various academic topics, such as socio-cultural, aesthetic, geopolitical, and economic issues [26].

The success of film festivals is often related to proper promotion. Promotions, in particular, are an essential element in promoting the sustainability of start-up film festivals in the locale. Marketers at local festivals, including film festivals, are actively using IT as a promotional tool. Websites and SNS are representative IT platforms for festival promotion. Recently, they have also used crowdfunding platforms as a means of promoting festivals.

Crowdfunding can be classified into four types based on investment method: lending-based, equity-based, donation-based, and reward-based [27]. Equity-based is a way of investing in the idea of a startup, and lending-based is a way of investing in a project through a small loan. Donation-based means that investors will purely support the donation with no rewards. Finally, reward-based refers to offering products instead of financial rewards. Cultural and art events such as film festivals are mainly associated with reward-based crowdfunding. For instance, 154 and 32 crowdfunding projects on festivals were posted on Tumblbug [28] and Wadiz [29], respectively, which are Korea's leading reward-based crowdfunding platforms. Although the main purpose of crowdfunding is to fund projects by recruiting small investments of many people through an online platform [30], crowdfunding also generates promotional benefits for tourism projects [31]. In particular, crowdfunding is also used as a marketing tool to implement word-of-mouth strategies on social media. Crowdfunding platforms offer the chance for participants to pitch an idea to their social network [32]. This means that crowdfunding and SNS are highly compatible. 


\subsection{Consumption Value of Crowdfunding}

Consumption value is an estimate of the utility of the product that a consumer receives when compared to the price and effort paid [33,34]. This explains whether a consumer purchases a particular product or why the consumer chooses one product over other ones [35]. Consumption value is thus a key determinant of consumer shopping behavior and product choice [36]. Previous studies segmented specific factors of value based on the concept of consumption value. For example, Park, Jaworski, and Maclnnis (1986) proposed functional, symbolic, and experiential values based on the concept of consumer needs [37]. Sheth, Newman, and Gross (1991) confirmed functional, conditional, social, emotional, and epistemic values as factors that influence consumer behavior [35]. Sweeney and Soutar (2001) proposed a multiple-item scale that includes a price value to measure the cost value of a product as well as the quality, emotional, and social values suggested in previous studies [38].

However, Smith and Colgate (2007) argued that value factors fall into four broad categories: (1) functional/instrumental, (2) experiential/hedonic, (3) symbolic/expressive, and (4) cost/sacrifice values [39]. The functional/instrumental value is related to the usefulness of a product or service, or the extent to which they perform a required function, and it encompasses concepts of functional, practical, and useful value. The experiential/hedonic value refers to the level at which a product or service creates an emotional value for a consumer. This value became an emotional or sensory value in some studies. The symbolic/expressive value is related to the degree to which consumers give psychological meaning to a product and includes the meaning of intrinsic value or possession value. Finally, the cost/sacrifice value represents the level of economic costs that consumers perceive during purchasing activities and includes factors such as economic and psychological costs. The consumption value factors presented by Smith and Colgate (2007) have academic implications because they have proposed categories in consideration of many previous studies [39].

Perceived values have been applied to describe consumers' behavior about various services and products. Some studies investigated the effect of consumption value on crowdfunding [40-42]. Schulz et al. (2015) proposed a hedonic value as a predictor of crowdfunding success, and it was confirmed that crowdfunding projects that show a high level of hedonic value are more successful than others [42]. Studies by Ho, Lin, and Lu (2014) and Moysidou and Spaeth (2016) introduced various consumption values as explanatory variables in the crowdfunding arena [40,41]. Moysidou and Spaeth (2016) categorized investor types into loan-based, equity-based, and presales-based projects based on crowdfunding investment methods and examined the factors that influence their willingness to support the project [41]. Financial, functional, informational, emotional, social, aesthetic, and novelty values were considered as independent variables. Consequently, financial and informational values were identified as factors affecting the willingness to support equity-based projects. Additionally, financial, emotional, and informational values were predictors for loan-based projects, and functional, emotional, and informational values were predictors in presales-based projects. In addition, Ho, Lin, and $\mathrm{Lu}$ (2014) also found that emotional, social, and function values have a positive effect on behavioral intentions for crowdfunding of cultural goods [40].

Previous studies commonly supported that the influence of financial, emotional, social, functional, and informational values presented by traditional value theories also influence behavioral intentions on crowdfunding. These values reflect the nature of crowdfunding as an investment platform but do not consider the characteristics of donation-based crowdfunding. Individuals are likely to participate in crowdfunding to satisfy their social approval desire rather than for monetary compensation in the reward or donation-based crowdfunding, where outputs are not monetary benefits [43]. As other-oriented factors, altruistic values are also important in the area of consumption [44].

Yet, another limitation of previous studies was that they did not consider the mediating effects between value factors and user behavior. Crowdfunding is an investment of personal money, so participants are likely to perceive financial risks. Generally, perceived risk is negatively correlated with a preference for financial instruments such as crowdfunding [45]. When crowdfunding includes high levels of value and credibility, participants perceive a low level of risk, which might positively 
affect behavior intentions [46]. In light of previous studies, this study applies economic, hedonic, social, and altruistic values as independent variables and assumes that these variables negatively affect perceived risk.

Hypothesis 1-1 (H1-1). Economic value will negatively affect perceived risk.

Hypothesis 1-2 (H1-2). Emotional value will negatively affect perceived risk.

Hypothesis 1-3 (H1-3). Social values will negatively affect perceived risk.

Hypothesis 1-4 (H1-4). Altruistic values will negatively affect perceived risk.

This study assumes that four values influence crowdfunding's e-WOM. If consumers perceive that one service provides more value than another one, they will voluntarily recommend it to others. In general, the intention to use e-WOM is determined by how a consumer evaluates a service or product. Specifically, consumers will be more likely to use word of mouth when a supplier provides value satisfaction compared to other suppliers [47].

Previous studies reported that the perceived value of consumers positively affects intention to use WOM [48,49]. Anwar and Gulzar (2011) found that perceived food value affects WOM endorsements through the mediating effects of consumer satisfaction [49]. By contrast, Abdolvand and Norouzi (2012) and Hansen et al. (2008) reported that perceived values have a direct effect on WOM without mediating effects [48,50]. Furthermore, Hansen et al. (2008) argued that the WOM behavior originated from the intention to repay the benefits provided by suppliers [50]. The relationship between perceived value and WOM is also important in emerging markets such as crowdfunding services. Customers in emerging markets determine their WOM behavior by considering how much value they have gained [51]. Potential investors are unlikely to know how much value they can return in crowdfunding before the experiences. Furthermore, WOM is likely to affect potential consumers' indirect experience of crowdfunding, and they might perceive the value. Although it is difficult to find a study that applies four types of value, the relationship between perceived values and e-WOM is supported by previous studies. Therefore, this study suggests that the perceived value of crowdfunding positively affects e-WOM.

Hypothesis 2-1 (H2-1). Economic value will positively affect $e-W O M$.

Hypothesis 2-2 (H2-2). Emotional value will positively affect e-WOM.

Hypothesis 2-3 (H2-3). Social values will positively affect e-WOM.

Hypothesis 2-4 (H2-4). Altruistic values will positively affect e-WOM.

\subsection{Innovativeness}

Inner innovativeness is defined as the degree to which an individual accepts a new idea or makes a decision about a new one [52]. The concept of inner innovativeness, based on innovation diffusion theory, adequately describes how to recognize new subjects and further adopts real targets according to the level of individual innovation [53,54]. Perceptions and behaviors regarding new technologies or services, such as crowdfunding, are likely to be determined by an individual's personality [53]. Specifically, when having a high level of inner innovativeness, the individual is better able to receive new technologies [55]. These individual tendencies have been consistently observed in the areas of media technologies such as personal computers, online games, and mobile technology [54-56]. 
Prior to the 1990s, studies of new technologies and services were primarily focused on the individual's perception of the subject instead of that individual's personal characteristics. After that, several studies examined the effects of individual characteristics such as self-efficacy [57] and intrinsic involvement [58]. For instance, Chang et al. (2006) identified the impact of internal innovation among the personal characteristics supporting online game adoption and suggested that highly innovative game players are more likely to adopt game playing [54]. In addition, several studies suggested that innovativeness leads to adoption behaviors $[55,59]$. In summary, previous research has focused primarily on examining the relationship between inner innovativeness and adoption behaviors.

However, several perceptual factors are proposed as well as adoption intentions as outcomes of inner innovativeness. First, inner innovativeness is related to perceived risk factors. In general, individuals face risks due to uncertainties about outcome behavior regarding new products or services $[60,61]$. In this situation, highly innovative individuals are willing to accept the uncertainty [53]. These risk-taking behaviors are considered typical features of highly innovative individuals [62]. Previous studies proved the negative relationships between inner innovativeness and perceived risks $[60,63]$. In summary, individuals with high levels of innovation are less likely to perceive risks and, as a result, are more likely to act aggressively on new services or products. The link between inner innovativeness and perceived risk has also been found in areas of financial transactions such as crowdfunding. Users cannot be sure of the quality of their financial transactions and, as a result, experience uncertainty [60]. Highly innovative individuals perceive lower risks than those who do not, resulting in more financial transactions.

Another outcome of inner innovativeness is the intention to use e-WOM. Traditional opinion leader research suggests that the higher the individual's inclination to innovate, the more likely they are to be opinion leaders [64]. Opinion leaders are considered active persons with a strong willingness to spread information about a particular object to others [65]. Given this, inner innovativeness can also have a positive effect on an individual's e-WOM intentions. Yoo, Jin, and Sanders (2013) classified consumer innovativeness into social, functional, hedonic, and cognitive [66]. Of these, social and cognitive innovativeness were found to affect intentions to use e-WOM. Sun et al. (2006) proposes online opinion leadership and online opinion seeking as two components of the e-WOM [67]. Online opinion leadership was identified as an outcome of inner innovativeness. In particular, Sun et al. (2006) provided significant results, showing that the effects of inner innovativeness on e-WOM are also found online [67]. In the study of tourism and experience goods, direct relationships between inner innovativeness and intention to use e-WOM are hard to find. It has been suggested that personal characteristics could affect the intention to use e-WOM in tourism [68]. Because inner innovativeness is a personal characteristic whose relationship with intention to use e-WOM has been verified in marketing studies, it can be expected that inner innovativeness will affect the intention to use e-WOM in tourism.

From a marketing point of view, an e-WOM strategy via SNS is a powerful tool that can leverage a range of features, such as the hashtag, to expand the reach of potential customers [69]. Therefore, inner innovativeness seems to affect e-WOM on SNS more clearly.

Hypothesis 3 (H3). Inner innovativeness will negatively affect perceived risk.

Hypothesis 4 (H4). Inner innovativeness will positively affect intention to use e-WOM.

\subsection{Perceived Risk}

Perceived risk has been applied consistently in analyzing consumer behavior because it has a decisive effect on behavior. Perceived risk is defined as the degree of uncertainty about the outcome of a decision [70] and anxiety about the loss of expected value [71]. In particular, individuals are more likely to perceive risk in an online environment where uncertain information is relatively 
high. High risk perception means that consumers believe themselves more likely to suffer loss as a result of using a product or service. This potential is expected to diminish consumer intentions or behaviors to buy products or services, especially in the online context [72]. Perceived risk thus leads to a negative causal relationship between consumption intentions. Individuals experience risk in an online environment for a variety of reasons, including technical complexity [73], privacy exposure [74], and uncertainty in the non-face-to-face communication environment. Previous studies on online services adopted perceived risk as the main variable $[75,76]$. The online shopping study classified risk types into economic, social, functional, personal, and privacy risks [76], and the research on e-services suggested, overall, that financial, psychological, privacy, time, and performance risks are perceived risk types. [75]. In summary, it was found that the type of risk adopted depends on the type of service or product covered by each study, but most of the previous studies consistently applied at least economic risk.

Economic risk is defined as "the potential monetary outlay associated with the initial purchase price as well as the subsequent maintenance cost of the product" [77] (p. 146). Featherman and Pavlou (2003) argued that economic risk has the highest explanatory power over other risk types [75]. Economic risk is considered as an important factor in crowdfunding research [78,79]. Kim and Jeon (2017) checked how economic risk negatively affects the intention of participating in crowdfunding [78]. Zhao et al. (2017) also found that perceived economic risk has a negative correlation with backers' funding intention in crowdfunding [79]. Therefore, if crowdfunding participants perceive that the project has high economic risks, they may be able to reconsider their next actions rather than actively investing or participating.

This study also considers perceived economic risk as a mediator. Although most festival crowdfunding is for donation-based or reward-based crowdfunding, this relates strictly to monetary spending. Thus, regardless of whether the rewards provided by crowdfunding managers are psychological or material, individuals can compare monetary expenditures and rewards. If the value of the perceived reward of a donation is lower than the monetary expenditure, perceived risk is likely to appear [71]. Thus, we consider that economic risk is a major variable because such risk is triggered by individual monetary spending.

Previous studies suggested that the intention to participate in crowdfunding was an outcome of perceived economic risks [46,78], but this study assumes that perceived economic risks for festival crowdfunding services negatively affect the intention to visit festivals. The crowdfunding platform is not only an investment platform but also an information resource for the festival. Individuals are more likely to visit film festivals based on the information. Particularly, considering that many festivals' crowdfunding offers movie tickets as a reward, which must be used on-site, participants are likely to visit the area.

Hypothesis 5 (H5). Perceived risk will negatively affect the intention to visit.

\subsection{Intention to Use e-WOM}

In the traditional sense, WOM is the interpersonal communication that occurs between the sender and the receiver, and it also describes the process of changing the recipient's behavior or attitude [38]. Recently, many studies have examined the effect of e-WOM. E-WOM is defined as "all informal communications directed at consumers through Internet-based technology related to the usage or characteristics of particular goods and services" [79] (p. 461). As such, online services are considered the most effective means of interpersonal communication [80]. As IT advances and various communication platforms such as social networks increase, individuals can exchange information seamlessly with each other. The information produced on the major platforms for e-WOM influences individual planning and decision-making [81]. In fact, e-WOM was estimated to secure 30 times more customers than traditional channels [82]. 
Given some characteristics of tourism, travelers are more likely to be influenced by e-WOM. First, tourism is an experience good [83]. Individuals try to lower the uncertainty they perceive in decision-making by considering an indirect experience regarding tourism information. Second, tourism is characterized by the information-intensive industry [84]. Tourists might try to get reliable information from many of the communications generated through e-WOM. Given these characteristics, individuals use e-WOM as an easy way to find information about travel destinations [85].

Several studies examined how e-WOM influences attitudes and behaviors to visit tourist areas [15,17]. Wang (2015) found that the intention to use e-WOM positively affected tourist visits [17]. Previous studies argued that WOM behavior includes a positive attitude and loyalty to the target [17,44]. Abubakar et al. (2017) reported that e-WOM influences the intention to re-visit the medical tourism industry [86]. Previous studies have found that those who share a positive message on the elements of a tourist experience with others are more likely to be immersed in tourism information or to have a positive understanding of it. In this sharing process, positive images can be created that arouse intention to visit [16]. It can be expected that this mechanism will appear here as it has elsewhere. Additionally, it is inferred that people with a high level of e-WOM intention are more likely to show the actual behavior, like visiting a tourist destination. According to this view, the sharing of film festival crowdfunding information is based on positive royalties for the festival. We assume that intention to use e-WOM based on the loyalty toward festival crowdfunding affects the intention to visit.

Hypothesis 6 (H6). E-WOM will positively affect the intention to visit.

\section{Research Methods}

\subsection{Sampling Procedure and Sample}

This study collected survey data from February 7 to 11, 2020, by requesting data from Marcromill Embrain, an online survey company. Korean survey respondents included those who had visited an international film festival in Korea at least once. International film festivals included Busan International Film Festival, Jeonju International Film Festival, Bucheon International Fantastic Film Festival, Jecheon International Music and Film Festival, Ulju Mountain Film Festival, DMZ International Documentary Film Festival, PyeongChang International Peace Film Festival, Muju Film Festival, Animal Film Festival in Suncheonman, Jeju Film Festival, Seoul Independent Film Festival, and Seoul International Women's Film Festival. Except for 15 samples that were unreliable responses, 447 samples were considered as sample data for the research.

To help understand film festival crowdfunding, participants reviewed the crowdfunding content uploaded to Tumblbug, which is a leading crowdfunding platform in Korea. Specifically, before responding to the questionnaire, participants read information about film festival crowdfunding, fundraising, and benefits from participating in the crowdfunding.

The quota sampling was conducted by considering the proportion of age and gender in the census population. As shown in Table 1, 221 (49.4\%) of the survey respondents were male and $226(50.6 \%)$ were female. Furthermore, $108(24.2 \%)$ of the respondents were aged 20-29, followed by those aged $30-39(112,25.1 \%), 40-49(115,25.7 \%)$, and 50-59 (112, 25.1\%). Most respondents, 314 participants $(70.2 \%)$, had a university degree followed by those who had completed graduate school or higher $(59,13.2 \%)$, those who had a high school diploma or lower $(36,8.1 \%)$, and those who were university students $(37,8.3 \%)$. Regarding income, $23 \%$ of respondents had a monthly personal income between 2,000,000-2,999,999 Korean Won (approximately US \$1688-US $\$ 2532$ ), followed by 3,000,000-3,999,999 (18.1\%), 4,000,000-4,999,999 (11.4\%), and 5,000,000-5,999,999 (11.0\%). Finally, $10.3 \%$ of respondents had a personal income of less than 1,000,000 won (See Table 1). 
Table 1. Demographic information for the sample. KRW: Korean Won.

\begin{tabular}{cccc}
\hline \multirow{3}{*}{ Gender } & Characteristics & Frequency $\mathbf{( N = 4 4 7 )}$ & \% (100) \\
\hline \multirow{3}{*}{ Age } & Male & 221 & 49.4 \\
& Female & 226 & 50.6 \\
& $20-29$ & 108 & 24.2 \\
& $30-39$ & 112 & 25.1 \\
Education & $40-49$ & 115 & 25.7 \\
& $50-59$ & 112 & 25.1 \\
& Below or high school & 37 & 8.3 \\
& University student & 37 & 8.3 \\
& University & 314 & 70.2 \\
& Graduate school or higher & 59 & 13.2 \\
& Less than 1.00 million KRW & 46 & 10.3 \\
& $1.00-1.99$ million KRW & 41 & 9.2 \\
2.00-2.99 million KRW & 103 & 23.0 \\
& $3.00-3.99$ million KRW & 81 & 18.1 \\
& $4.00-4.99$ million KRW & 51 & 11.4 \\
& $5.00-5.99$ million KRW & 49 & 11.0 \\
& $6.00-6.99$ million KRW & 25 & 5.6 \\
& $7.00-7.99$ million KRW & 21 & 4.7 \\
& $8.00-8.99$ million KRW & 9 & 2.0 \\
& $9.00-9.99$ million KRW & 9 & 2.0 \\
& Over 10 million KRW & 12 & 2.7 \\
\hline
\end{tabular}

\subsection{Measurement and Analysis Method}

The research model includes the following eight variables: (1) economic value; (2) emotional value; (3) social value; (4) altruistic value; (5) inner innovativeness; (6) perceived risk; (7) e-WOM; and (8) intention to visit film festival. We set four value factors and one personal characteristic factor as independent variables that affected perceived risk (Figure 1).

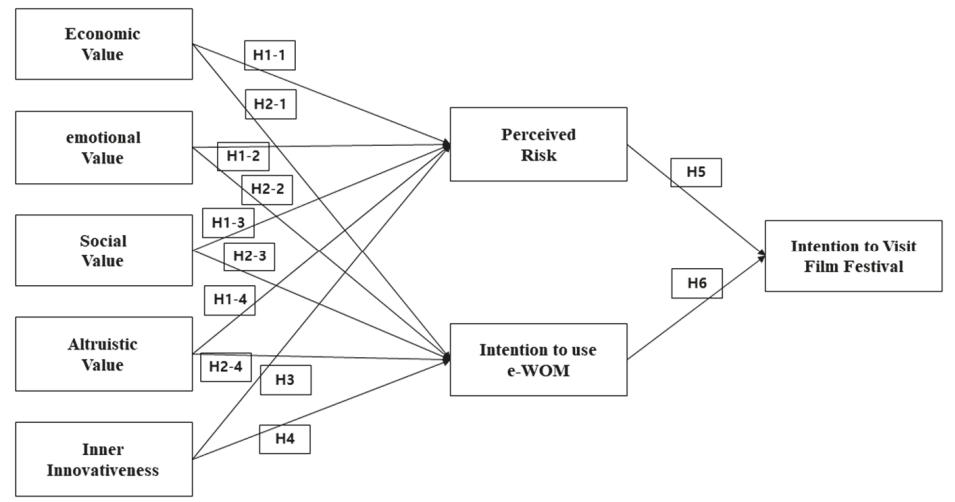

Figure 1. Research model.

The economic value describes how far it is perceived that film festival crowdfunding can provide monetary value. The emotional value describes how far it is perceived that the film festival crowdfunding can lead to a positive emotional state. The social value describes how far one expects to be recognized by social contacts for participating in the film festival crowdfunding. Economic, emotional, and social value were revised based on the Sweeney and Soutar (2001) study on shopping value [38]. The altruistic value describes how far one believes that film festival crowdfunding participation will help others. To measure the altruistic value, we considered items from Holbrook's (2006) 
study of the applicability of subjective personal introspection factors in consumption experience [87]. Inner innovativeness is "the degree to which an individual is receptive to new ideas and makes innovation decisions independently of the communicated experience of others" [52] (p. 236). Items of inner innovativeness were revised based on Chang, Lee, and Kim's (2006) study, which examined factors that influence online game adoption [54]. In an e-service adoption study, Featherman and Pavlou (2003) proposed seven types of risks, such as performance, financial, time, psychological, social, privacy, and overall risks [75]. Among them, the items of financial risk by Featherman and Pavlou (2003) were used in the study [75]. Perceived risk describes how much one expects to lose in relation to money for participating in film festival crowdfunding. Online word-of-mouth behavior is divided into opinion giving, opinion passing, and opinion seeking [88]. In this study, opinion passing was considered as observed e-WOM. E-WOM describes the willingness to share festival crowdfunding information with others on SNS. We used items based on research from Sun et al. (2006), Chu and Choi (2012), and Lee and Lee (2013) [67,89,90]. Finally, intention to visit the film festival describes the willingness to visit a local film festival in the near future. To measure this, we used the items of Perugini and Bagozzi (2001), which were based on the theory of planned behavior [91]. All questions in this study consisted of a Likert five-point scale $(1=$ strongly disagree and $5=$ strongly agree). Three items were contained for each variable (See Table 2).

Table 2. Items and scales used in questionnaire.

\begin{tabular}{|c|c|c|}
\hline Variables & Items & References \\
\hline Economic value & $\begin{array}{l}\text { I think film festival crowdfunding is reasonably priced. } \\
\text { I think participating in film festival crowdfunding will offer value for money. } \\
\text { I think participating in film festival crowdfunding is a good for the price. }\end{array}$ & \multirow{3}{*}[38]{} \\
\hline Emotional value & $\begin{array}{l}\text { Participating in festival crowdfunding will make me feel good. } \\
\text { Participating in film festival crowdfunding is something that I would enjoy. } \\
\text { Participating in festival crowdfunding would give me pleasure. }\end{array}$ & \\
\hline Social value & $\begin{array}{l}\text { My participation in festival crowdfunding would make a good impression on other people. } \\
\text { Participating in festival crowdfunding would help me to feel acceptable. } \\
\text { Participation in film festival crowdfunding would give its owner social approval. }\end{array}$ & \\
\hline Altruistic value & $\begin{array}{l}\text { Participation in film festival crowdfunding helps me make social contributions. } \\
\text { Participation in film festival crowdfunding is a kind of donation. } \\
\text { Participation in festival crowdfunding can provide pure help to the festival. }\end{array}$ & {$[44]$} \\
\hline Inner innovativeness & $\begin{array}{l}\text { I like to learn about new ideas. } \\
\text { I am interested in news stories that deal with new inventions or discoveries. } \\
\text { I am updating my computer to keep up with new technologies. }\end{array}$ & {$[54]$} \\
\hline Perceived risk & $\begin{array}{l}\text { Participation in film festival crowdfunding will be a waste of money. } \\
\text { Participation in film festival crowdfunding is relatively expensive. } \\
\text { Participation in film festival crowdfunding will be costly in the long run. }\end{array}$ & {$[75]$} \\
\hline E-WOM & $\begin{array}{l}\text { I will share the usefulness of festival crowdfunding with my SNS friends. } \\
\text { I will share the festival crowdfunding information received from my friends with other } \\
\text { SNS friends. } \\
\text { I will share a positive assessment of festival crowdfunding on social media with others. }\end{array}$ & {$[67,87,88]$} \\
\hline $\begin{array}{l}\text { Intention to visit film } \\
\text { festival }\end{array}$ & $\begin{array}{l}\text { I will visit the area where the film festival is taking place in the near future. } \\
\text { I am likely to visit a local international film festival in the near future. } \\
\text { I will visit where the international film festival is held in the area soon. }\end{array}$ & [89] \\
\hline
\end{tabular}

The general characteristics of the samples were identified using SPSS18 statistical software. In particular, frequency analysis and composite reliability were performed to verify the reliability of the measurement questions. Next, this study utilized the structural equation model (SEM). The statistical software Amos 20 was used to carry out the calculations. SEM enacts multi-equation system procedures, determining multiple indicators of concepts, continuous latent variables, errors in equations, errors of measurement, and continuous latent variables [92]. This is useful for the comprehensive examination of relationships among multiple variables, as needed in this study. First, confirmatory factor analysis (CFA) was performed to check the latent variables and ensure the absence of measurement errors by CFA. Next, the relationship between the variables was examined through the structural model. 


\section{Results}

\subsection{Measurement Model}

In this study, we applied the coefficient of Cronbach's $\alpha$ to verify the composite reliability between the measured items. In general, reliability is satisfied when Cronbach's $\alpha$ is greater than 0.7 [93]. Each variable includes three observed variables, and all the items in the model met the reliability criteria (See Table 3). Convergent validity is desirable when the average variance extracted (AVE) is greater than 0.5 [94]. As a result of the verification, all values of AVE were found to meet the criteria. To verify the indicator reliability, this study applies the criterion that every loading must be greater than 0.4 [95]. Of all the loading values, two observed variables were above 0.6 (AV_2 $=0.621$, II_3 $=0.610$ ), but most of them were greater than 0.7. In addition, all observed variables were verified to be statistically significant $(p=0.00)$. Therefore, good indicator reliability was confirmed. Regarding discriminant validity, AVE must be greater than all constructs of the square root [94]. As shown in Table 4, it was found that AVE exceeds the square of the correlation.

Table 3. Construct reliability and validity.

\begin{tabular}{|c|c|c|c|c|c|c|}
\hline \multicolumn{2}{|c|}{ Variable } & \multirow{2}{*}{$\begin{array}{c}\text { Estimate } \\
0.772\end{array}$} & \multirow{2}{*}{$\begin{array}{c}\text { Measurement Error } \\
0.273\end{array}$} & \multirow[t]{2}{*}{$\mathrm{CR}^{\mathrm{a}}$} & \multirow[t]{2}{*}{$\operatorname{AVE}^{b}$} & \multirow[t]{2}{*}{ Cronbach's o } \\
\hline & EV_1 & & & & & \\
\hline \multirow[t]{3}{*}{ Economic Value } & EV_2 & 0.712 & 0.346 & 0.847 & 0.690 & 0.818 \\
\hline & EV_3 & 0.831 & 0.186 & & & \\
\hline & EmV $\bar{V}_{-} 1$ & 0.837 & 0.214 & & & \\
\hline \multirow[t]{3}{*}{ Emotional Value } & EmV_2 & 0.847 & 0.172 & 0.919 & 0.791 & 0.881 \\
\hline & EmV_3 & 0.849 & 0.179 & & & \\
\hline & SV_1 & 0.837 & 0.253 & & & \\
\hline \multirow[t]{3}{*}{ Social Value } & SV_2 & 0.809 & 0.290 & 0.899 & 0.749 & 0.881 \\
\hline & SV_3 & 0.892 & 0.177 & & & \\
\hline & AV_1 & 0.794 & 0.216 & & & \\
\hline \multirow[t]{2}{*}{ Altruistic Value } & $\mathrm{AV} \_2$ & 0.621 & 0.443 & 0.844 & 0.647 & 0.777 \\
\hline & AV_3 & 0.784 & 0.229 & & & \\
\hline \multirow{4}{*}{$\begin{array}{c}\text { Inner } \\
\text { Innovativeness }\end{array}$} & InI_1 & 0.781 & 0.210 & & & \\
\hline & InI_2 & 0.781 & 0.188 & 0.847 & 0.652 & 0.756 \\
\hline & InI_3 & 0.610 & 0.448 & & & \\
\hline & PR_1 & 0.881 & 0.205 & & & \\
\hline \multirow[t]{3}{*}{ Perceived Risk } & PR_2 & 0.931 & 0.120 & 0.938 & 0.836 & 0.932 \\
\hline & PR_3 & 0.907 & 0.156 & & & \\
\hline & e-WOM_1 & 0.872 & 0.184 & & & \\
\hline \multirow[t]{2}{*}{ E-WOM } & e-WOM_2 & 0.890 & 0.164 & 0.922 & 0.797 & 0.903 \\
\hline & e-WOM_3 & 0.849 & 0.228 & & & \\
\hline \multirow{3}{*}{$\begin{array}{l}\text { Intention to visit } \\
\text { film festival }\end{array}$} & IVFF_1 & 0.880 & 0.13 & & & \\
\hline & IVFF_2 & 0.826 & 0.191 & 0.914 & 0.781 & 0.791 \\
\hline & IVFF_3 & 0.778 & 0.255 & & & \\
\hline
\end{tabular}

a. CR: composite reliability coefficient; b. AVE: average variance extracted; e-WOM: electronic word of mouth.

Table 4. Test of discriminant validity.

\begin{tabular}{cccccccccc}
\hline & II & EV & SV & HV & AV & PR & e-WOM & IVFF & AVE \\
\hline InI & 1 & & & & & & & & 0.652 \\
EV & 0.364 & 1 & & & & & & & 0.690 \\
SV & 0.179 & 0.424 & 1 & & & & & 0.749 \\
EmV & 0.274 & 0.799 & 0.426 & 1 & & & & 0.791 \\
AV & 0.284 & 0.520 & 0.411 & 0.521 & 1 & & & 0.647 \\
PR & 0.023 & 0.048 & 0.004 & 0.060 & 0.043 & 1 & & & 0.836 \\
e-WOM & 0.201 & 0.391 & 0.359 & 0.397 & 0.328 & 0.000 & 1 & & 0.797 \\
IVFF & 0.281 & 0.453 & 0.193 & 0.382 & 0.325 & 0.018 & 0.320 & 1 & 0.781 \\
\hline
\end{tabular}




\subsection{Hypothesis Testing}

To verify the fit of the structural model, absolute fit index, parsimonious fit index, and incremental fit index were considered. Scholars applied different criteria to assess model fit [92], but many previous studies usually applied $\chi 2 / \mathrm{df}$, RMSEA (Root Mean Square Error of Approximation), AGFI (Adjusted Goodness of Fit Index), CFI (Comparative Fit Index), TLI (Tucker Lewis Index), and NFI (Normed Fit Index) as criteria for the model fit. As a result of the model fit verification, all of the fitness indices $(\chi 2 / \mathrm{df}=2.578, \mathrm{RMSEA}=0.059, \mathrm{AGFI}=0.867, \mathrm{CFI}=0.948, \mathrm{TLI}=0.938$, NFI $=0.919)$ signaled a good model fit (See the Table 5).

Table 5. Model fit statistics.

\begin{tabular}{ccccccc}
\hline Model & $\chi^{2} / \mathbf{d f}$ & RMSEA & AGFI & CFI & TLI & NFI \\
\hline Criterion & $<3$ & $<0.08$ & $>0.80$ & $>0.90$ & $>0.90$ & $>0.90$ \\
\hline Structural model & 2.578 & 0.059 & 0.867 & 0.948 & 0.938 & 0.919 \\
\hline
\end{tabular}

The purpose of this study was to analyze the effects of value factors and inner innovativeness on the intention to visit film festivals through perceived risk and e-WOM. The results of the analysis are shown in Table 6. With regard to the value factor, all variables, except for EV (economic value), showed significant effects on perceived risk. EmV (emotional value) $(\mathrm{B}=-0.410, \rho<0.044)$ had a negatively significant effect on PR (perceived risk). It was also confirmed that AV (altruistic value) ( $\mathrm{B}=-0.306, \rho<0.025)$ negatively affected PR. However, SV (social value) $(\mathrm{B}=0.528, \rho<0.000)$, unlike the hypothesis, positively affected PR. Therefore, H1-2 and H1-4 were supported. Regarding H2-1 to H2-4, SV (B = 0.278, $\rho<0.000$ ) had statistically significant effects on e-WOM, but EV, HV, and AV had no effect on e-WOM. Thus, only H2-3 was supported and H2-1, H2-2, and H2-4 were not supported. Next, InI (inner innovativeness) did not affect both PR and e-WOM. Therefore, H3 and H4 were not supported. Regarding the effects of PR and e-WOM on the dependent variable, PR (B $=-0.099$, $\rho<0.005$ ) negatively affected IVFF (intention to visit film festival). The influence of e-WOM $(B=0.521$, $\rho<0.000$ ) was positively significant on IVFF. Thus, H5 and H6 were supported.

Table 6. Hypotheses test for structural models by the general maximum likelihood structural equation model (SEM).

\begin{tabular}{|c|c|c|c|c|c|}
\hline & Hypotheses & Estimate & $\begin{array}{l}\text { Standard } \\
\text { Error }\end{array}$ & Sig. & $\begin{array}{c}\text { Hypotheses } \\
\text { Validation }\end{array}$ \\
\hline H1-1 & $\mathrm{EV} \rightarrow \mathrm{PR}$ & -0.059 & 0.248 & 0.812 & Not supported \\
\hline H1-2 & $\mathrm{EmV} \rightarrow \mathrm{PR}$ & -0.410 * & 0.203 & 0.044 & Supported \\
\hline H1-3 & $\mathrm{SV} \rightarrow \mathrm{PR}$ & $0.528 * *$ & 0.086 & 0.000 & Not supported \\
\hline H1-4 & $\mathrm{AV} \rightarrow \mathrm{PR}$ & $-0.306^{*}$ & 0.137 & 0.025 & Supported \\
\hline $\mathrm{H} 2-1$ & $\mathrm{EV} \rightarrow \mathrm{e}-\mathrm{WOM}$ & 0.208 & 0.182 & 0.253 & Not supported \\
\hline $\mathrm{H} 2-2$ & $\mathrm{EmV} \rightarrow \mathrm{e}-\mathrm{WOM}$ & 0.206 & 0.148 & 0.164 & Not supported \\
\hline $\mathrm{H} 2-3$ & $\mathrm{SV} \rightarrow \mathrm{e}-\mathrm{WOM}$ & $0.278^{* *}$ & 0.061 & 0.000 & Supported \\
\hline $\mathrm{H} 2-4$ & $\mathrm{AV} \rightarrow \mathrm{e}-\mathrm{WOM}$ & 0.122 & 0.099 & 0.220 & Not supported \\
\hline $\mathrm{H} 3$ & $\mathrm{InI} \rightarrow \mathrm{PR}$ & -0.048 & 0.111 & 0.664 & Not supported \\
\hline $\mathrm{H} 4$ & $\mathrm{InI} \rightarrow \mathrm{e}-\mathrm{WOM}$ & 0.130 & 0.081 & 0.109 & Not supported \\
\hline H5 & $\mathrm{PR} \rightarrow \mathrm{IVFF}$ & $-0.099 *$ & 0.035 & 0.005 & Supported \\
\hline $\mathrm{H} 6$ & $\mathrm{e}-\mathrm{WOM} \rightarrow$ IVFF & $0.521 * *$ & 0.043 & 0.000 & Supported \\
\hline
\end{tabular}

\section{Discussion and Conclusions}

This study aims to empirically examine how the perceived value of crowdfunding and inner innovativeness affect the intention to visit the film festival. For this, perceived risk and e-WOM were applied as mediating variables. Structural equation modeling was applied, and data were collected 
through an online survey. As a result, 6 of 12 research hypotheses were supported. Accordingly, we confirmed through its effects that crowdfunding is an important platform for promoting sustainability at local film festivals.

First of all, emotional values (H1-2) and altruistic values (H1-4) negatively affected perceived risk. This supports the findings of previous studies, which indicate that individuals perceive low levels of risk when they have a sense of worth regarding a given service or product [46]. These variables are commonly considered to be related to emotional characteristics rather than to cognitive ones. These results indicate that consumers are more likely to obtain emotional value as a result of the crowdfunding of cultural products, such as festival crowdfunding. Another notable aspect is that the path coefficient for emotional value $(B=-0.401)$ was higher than the altruistic value $(B=-0.306)$. This implies that emotional value has greater explanatory power. Future studies should develop this framework by developing detailed factors for emotional value. Interestingly, economic value effects were not confirmed. It is expected that the effects of economic value vary in relation to the type of crowdfunding. Moysidou and Spaeth's (2016) study, which examined the impact of economic value on behavioral intention by project type, found an influence of economic value in revenue-based crowdfunding, such as equity-based and loan-based projects [41]. Individuals may not take economic value into account for donation-based or reward-based crowdfunding, including cultural goods. Economic relates to the amount of monetary benefit that an individual can receive. Individuals do not generally seek financial benefits when they participate in donation and reward-based crowdfunding. Rather, they seek to meet non-monetary needs, such as social approval or self-realization [43]. In particular, individuals' non-monetary motivations are likely to be more prominent in cultural goods, such as in relation to local film festival crowdfunding, which mainly has non-commercial purposes.

For hypotheses 2-1 to 2-4, only social values (H2-3) positively affected the intention to use e-WOM. Social values and intention to use e-WOM both rest on the concept of social relationships. This result means that perception of the value of interacting with others through participation in festival crowdfunding can affect social interactions online. Prior studies have found that perception of quality in the general product area, such as food, can affect WOM [49]. On the other hand, social value effects were identified in this study. It is unclear whether these results can be attributed to the nature of the experience or to crowdfunding type. Further research is needed.

Next, inner innovativeness was found not to affect perceived risk and e-WOM intentions. These results indicate that the consumer's inner innovativeness is not related to the perception of festival crowdfunding. Regarding perceived risks, Foxall (1988) proposed that risk perceptions vary according to the type of users [96]. This means that early adopters underestimate the risk compared to late adopters. Accordingly, future studies should identify the audience characteristics for film festival crowdfunding and examine the relationship between inner innovativeness and perceived risk for each type. Regarding e-WOM, previous studies on innovation have shown a different view of claims. For example, Summers (1970) argued that highly innovative people tend to give opinions to people [64], but Rogers (1995) argued that innovation is related to information seeking [53]. The two studies each focused on the production and acceptance of information. By contrast, Sun et al. (2006) verified that innovativeness has a significant impact on both online information leadership, which includes the concept of e-WOM, and information seeking [67]. Given the inconsistent claims of the previous studies, further research should examine the relationship between innovativeness and e-WOM.

Third, perceived risk negatively affects the intention to visit the film festival (H5). This result is consistent with the findings of previous studies, which indicated that perceived risk for crowdfunding affects actual behavior [46,78]. This also means that, if an individual underestimates the risk of festival crowdfunding, they can visit the festival. Last, the intention to use e-WOM also had a positive effect on the intention to visit the film festival (H6). This result is consistent with previous studies that have suggested that e-WOM has a positive effect on travel intention $[15,17]$. The action to share the usefulness of festival crowdfunding with friends is believed to be based on trust or interest in the film festival. This means that positive perceptions of the film festival can lead to real action, such as visiting 
a festival. However, there is also the possibility that control variables may have some bearing on the intention to use e-WOM and the intention to visit a film festival. For example, actual behavior may vary depending on perceived involvement in film festivals or crowdfunding platforms. Future research will be needed to expand the research model by exploring moderating variables.

As for $\mathrm{H} 5$ and $\mathrm{H} 6$, earlier study of tourism crowdfunding used crowdfunding behavior as a dependent variable. Our study, however, set the intention to visit a festival as an outcome variable and not a crowdfunding behavior, and found that it is affected by explanatory variables. This means that the effects on the service platform can affect consumers' behavior in relation to the topic of crowdfunding beyond the platform. If individuals have a positive perception of crowdfunding services, this may indirectly indicate that they have a positive perception of crowdfunding as well as of the service platform. Therefore, crowdfunding goes beyond investment to acting as a marketing tool, attracting individual visits to local film festivals and further enhancing the sustainability of local film festivals.

This study has theoretical implications. First, previous work has examined factors affecting the intention to participate in festival crowdfunding $[97,98]$, but overall, too little work has been done on the effects of crowdfunding in tourism. Additionally, from a marketing perspective, few analyses or in-depth discussions of festival crowdfunding have been done. This study is thus an important examination of the influence of tourism crowdfunding. Second, this study proposed a framework to examine the marketing effects of crowdfunding in tourism. It has been confirmed that the influence of certain variables varies depending on the type of crowdfunding being considered. As noted, we found that emotional factors should be taken seriously in the study of donation-based and reward-based crowdfunding. If this framework is properly modified in response to the results of this study, festival-visiting behavior can be explained in greater detail. Third, to supplement existing research trends, structural modeling is proposed in relation to perceived risk and e-WOM as mediating variables between value factors and behavioral intentions. This approach can provide a useful aid to studies dealing with online communication environments and platforms with high uncertainty, such as crowdfunding platforms.

This study provides practical guidelines to help with festival sustainability. First, this study confirmed that the crowdfunding platform can be used as a marketing tool. It was found that factors related to crowdfunding platforms, such as perceived risk and e-WOM, drive intentions to visit the festival. Therefore, even if the film festival does not need financial help, marketers should open a crowdfunding page to provide an opportunity to access the film festival crowdfunding and obtain information. Second, marketers should build their value strategy differently depending on the type of crowdfunding. Marketers encourage consumers to perceive economic value for equity-based and loan-based crowdfunding, whereas, for non-revenue types such as donation-based or reward-based, marketers should provide an opportunity to gain emotional value or the need for approval. Finally, marketers can understand consumers' e-WOM behavior and strategically use it. Crowdfunding, for example, is compatible with SNS. Moreover, users can share information about crowdfunding via SNS. Therefore, this study is expected to provide a guide for developing and implementing a marketing strategy that simultaneously utilizes SNS and crowdfunding platforms to enhance the e-WOM effect on film festivals.

Regarding limitations, in this study, film festival crowdfunding is still an emerging market, so it was difficult to conduct online surveys for actual users. Respondents to this study experienced indirect festival crowdfunding only through the experimental treatment provided by the researcher. Future researches may conduct online surveys in collaboration with film festival crowdfunding providers, who can directly contact actual users. Next, follow-up studies should take a closer look at the effects of other factors on the intention to visit a film festival. Potential consumers will recognize not only the risks directly related to the festival but also the risks of the crowdfunding investment in their decision to visit the festival. Thus, in addition to the perceived risk of participation in film festival crowdfunding, risks associated with the consumption behaviors that are seen during participation in 
the festival should also be considered. Finally, future studies should be conducted that investigate multi-dimensional inner innovativeness. Certain studies have also reviewed how well individuals accept brands or images related to innovation [54], but these have mainly involved emotional indicators, so to examine the effects of inner innovativeness, both cognitive and emotional perspectives should be considered.

Author Contributions: H.K. and B.C. conceived and designed the experiments; B.C. performed the experiments; H.K. analyzed the data; B.C. contributed reagents/materials/analysis tools; H.K. and B.C. wrote the paper. All authors have read and agreed to the published version of the manuscript.

Funding: This work was supported by the Global Research Network program through the Ministry of Education of the Republic of Korea and the National Research Foundation of Korea (NRF-2017S1A2A2041908).

Conflicts of Interest: The authors declare no conflict of interest.

\section{References}

1. Yoo, C.; Kwon, S.; Na, H.; Chang, B. Factors affecting the adoption of gamified smart tourism applications: An integrative approach. Sustainability 2017, 9, 2162. [CrossRef]

2. Johansson, M.; Kociatkiewicz, J. City festivals: Creativity and control in staged urban experiences. Eur. Urban Reg. Stud. 2011, 18, 392-405. [CrossRef]

3. Beirne, E.; Curry, P. The impact of the Internet on the information search process and tourism decision making. In Information and Communication Technologies in Tourism; Springer: Vienna, Austria, 1999; pp. 88-97.

4. Leung, D.; Law, R.; Van Hoof, H.; Buhalis, D. Social media in tourism and hospitality: A literature review. J. Travel Tour. Mark. 2013, 30, 3-22. [CrossRef]

5. Huang, Y.H.; Basu, C.; Hsu, M.K. Exploring motivations of travel knowledge sharing on social network sites: An empirical investigation of U.S. college students. J. Hosp. Mark. Manag. 2010, 19, 717-734. [CrossRef]

6. Fotis, J. Discussion of the Impacts of Social Media in Leisure Tourism: The Impact of Social Media on Consumer Behaviour: Focus on Leisure Travel. Available online: http://johnfotis.blogspot.com.au/p/projects.html (accessed on 1 March 2020).

7. Peterson, R.A.; Balasubramanian, S.; Bronnenberg, B.J. Exploring the implications of the Internet for consumer marketing. J. Acad. Mark. Sci. 1997, 25, 329. [CrossRef]

8. Hoksbergen, E.; Insch, A. Facebook as a platform for co-creating music festival experiences: The case of New Zealand's Rhythm and Vines New Year's Eve festival. Int. J. Event Festiv. Manag. 2016. [CrossRef]

9. Mollick, E.R.; Kuppuswamy, V. After the campaign: Outcomes of crowdfunding. UNC Kenan-Flagler Res. Pap. 2014, 2376997. [CrossRef]

10. Ingram, C.; Teigland, R. Crowdfunding among IT entrepreneurs in Sweden: A qualitative study of the funding ecosystem and ICT entrepreneurs' adoption of crowdfunding. Available online: http://ssrn.com/ abstract=2289134 (accessed on 1 March 2020).

11. Jeon, S.H. Crowd-funding between the movie content production through the analysis of the relationship or the successful funding case research. J. Korea Contents Assoc. 2013, 13, 81-91. [CrossRef]

12. Kim, D.H. The study on an invigoration of crowding fund - based on legal support system \& related case research. Int. Bus. Rev. 2014, 18, 105-123.

13. Park, J.Y.; Hwang, S.G. The Case and Analysis of the Viral Marketing Effect of Crowdfunding -Focused on the Design Brand Start-up. Treatise Plast. Media 2018, 21, 118-125.

14. Moisseyey, A. Effect of Social Media on Crowdfunding Project Results. Master's Thesis, University of Nebraska, Lincoln, NE, USA, May 2013.

15. Prayogo, R.R.; Kusumawardhani, A. Examining Relationships of Destination Image, Service Quality, e-WOM, and Revisit Intention to Sabang Island, Indonesia. Asia-Pac. Manag. Bus. Appl. 2017, 5, 89-102. [CrossRef]

16. Chen, Y.C.; Shang, R.A.; Li, M.J. The effects of perceived relevance of travel blogs' content on the behavioral intention to visit a tourist destination. Comput. Hum. Behav. 2014, 30, 787-799. [CrossRef]

17. Wang, P. Exploring the influence of electronic word-of-mouth on tourists' visit intention. J. Syst. Inf. Technol. 2015. [CrossRef]

18. McKercher, B.; Mei, W.S.; Tse, T.S. Are short duration cultural festivals tourist attractions. J. Sustain. Tour. 2006, 14, 55-66. [CrossRef] 
19. Smith, C.; Jenner, P. The impact of festivals and special events on tourism. Travel Tour. Anal. 1998, 4, 73-91.

20. Felsenstein, D.; Fleischer, A. Local festivals and tourism promotion: The role of public assistance and visitor expenditure. J. Travel Res. 2003, 41, 385-392. [CrossRef]

21. Kotler, P.; Haider, D.; Rein, I. Marketing Places: Attracting Investment, Industry and Tourism to Cities, States and Nations; Free Press: New York, NY, USA, 1993.

22. Liu, Y.; Chen, C. The effects of festivals and special events on city image design. Front. Archit. Civ. Eng. China 2007, 1, 255-259. [CrossRef]

23. Ooi, C.S.; Pedersen, J.S. City branding and film festivals: Re-evaluating stakeholder's relations. Place Branding Public Dipl. 2010, 6, 316-332. [CrossRef]

24. Palais Des Festivals. Available online: https://www.palaisdesfestivals.com/uploads/brochures/Press-PackCannes-Tourism-2018.pdf (accessed on 1 March 2020).

25. Báez, A.; Devesa, M. Segmenting and profiling attendees of a film festival. Int. J. Event Festiv. Manag. 2014. [CrossRef]

26. Udden, J. Film festivals. J. Chin. Cine 2016, 10, 14-17. [CrossRef]

27. Burtch, G.; Ghose, A.; Wattal, S. An Empirical Examination of the Antecedents and Consequences of Contribution Patterns in Crowd-Funded Markets. Inf. Syst. Res. 2013, 24, 499-519. [CrossRef]

28. Tumblbug. Available online: https://tumblbug.com/ (accessed on 1 March 2020).

29. Wadiz. Available online: https://www.wadiz.kr/web/wcampaign/search?keyword=festival (accessed on 1 March 2020).

30. Borst, I.; Moser, C.; Ferguson, J. From friendfunding to crowdfunding: Relevance of relationships, social media, and platform activities to crowdfunding performance. New Media Soc. 2018, 20, 1396-1414. [CrossRef] [PubMed]

31. Temelkov, Z.; Gulev, G. Role of crowdfunding platforms in rural tourism development. Sociobrains Int. Sci. Refereed Online J. Impact Factor 2019, 56, 73-79.

32. Gerber, E.M.; Hui, J. Crowdfunding: Motivations and deterrents for participation. ACM Trans. Comput. Hum. Interact. 2013, 20, 1-32. [CrossRef]

33. Bolton, R.N.; Drew, J.H. A multistage model of customers' assessments of service quality and value. J. Consum. Res. 1991, 17, 375-384. [CrossRef]

34. Johnson, M.D.; Herrmann, A.; Huber, F. The evolution of loyalty intentions. J. Mark. 2016, 70, $122-132$. [CrossRef]

35. Sheth, J.N.; Newman, B.I.; Gross, B.L. Why We Buy What We Buy: A Theory of Consumption values. J. Bus. Res. 1991, 22, 159-170. [CrossRef]

36. Zeithaml, V.A. Consumer perceptions of price, quality, and value: A means-end model and synthesis of evidence. J. Mark. 1988, 52, 2-22. [CrossRef]

37. Park, C.W.; Jaworski, B.J.; Maclnnis, D.J. Strategic brand concept-image management. J. Mark. 1986, 50, 135-145. [CrossRef]

38. Sweeney, J.C.; Soutar, G.N. Consumer perceived value: The development of a multiple item scale. J. Retail. 2001, 77, 203-220. [CrossRef]

39. Smith, J.B.; Colgate, M. Customer value creation: A practical framework. J. Mark. Theory Pract. 2007, 15, 7-23. [CrossRef]

40. Ho, H.Y.; Lin, P.C.; Lu, M.H. Effects of online crowdfunding on consumers' perceived value and purchase intention. Anthropologist 2014, 17, 837-844. [CrossRef]

41. Moysidou, K.; Spaeth, S. Cognition, emotion and perceived values in crowdfunding decision making. In Proceedings of the Open and User Innovation Conference, Boston, MA, USA, 1 August 2016.

42. Schulz, M.; Haas, P.; Schulthess, K.; Blohm, I.; Leimeister, J.M. Available online: https://www.alexandria. unisg.ch/241339/1/JML_513.pdf (accessed on 1 March 2020).

43. Kraus, S.; Richter, C.; Brem, A.; Cheng, C.F.; Chang, M.L. Strategies for reward-based crowdfunding campaigns. J. Innov. Knowl. 2016, 1, 13-23. [CrossRef]

44. East, R.; Hammond, K.; Lomax, W. Measuring the impact of positive and negative word of mouth on brand purchase probability. Int. J. Res. Mark. 2008, 25, 215-224. [CrossRef]

45. Byrne, K. How do Consumers Evaluate Risk in Financial Products? J. Financ. Serv. Mark. 2005, 10, 21-36. [CrossRef] 
46. Zhao, Q.; Chen, C.D.; Wang, J.L.; Chen, P.C. Determinants of backers' funding intention in crowdfunding: Social exchange theory and regulatory focus. Telemat. Inform. 2017, 34, 370-384. [CrossRef]

47. Dick, A.S.; Basu, K. Customer loyalty: Towards an integrated conceptual framework. J. Acad. Mark. Sci. 1994, 22, 99-113. [CrossRef]

48. Abdolvand, M.A.; Norouzi, A. The effect of customer perceived value on word of mouth and loyalty in B-2-B marketing. Res. J. Appl. Sci. Eng. Technol. 2012, 4, 4973-4978.

49. Anwar, S.; Gulzar, A. Impact of perceived value on word of mouth endorsement and customer satisfaction: Mediating role of repurchase intentions. Int. J. Econ. Manag. Sci. 2011, 1, 46-54.

50. Hansen, H.; Samuelsen, B.M.; Silseth, P.R. Customer perceived value in BtB service relationships: Investigating the importance of corporate reputation. Ind. Mark. Manag. 2008, 37, 206-217. [CrossRef]

51. McKee, D.; Simmers, C.S.; Licata, J. Customer self-efficacy and response to service. J. Serv. Res. 2006, 8, 207-220. [CrossRef]

52. Midgley, D.F.; Dowling, R.G. Innovativeness-concept and its measurement. J. Consum. Res. 1978, 4, $229-242$. [CrossRef]

53. Rogers, E.M. Diffusion of Innovation; Springer: Berlin/Heidelberg, Germany, 1995.

54. Chang, B.H.; Lee, S.E.; Kim, B.S. Exploring factors affecting the adoption and continuance of online games among college students in South Korea: Integrating uses and gratification and diffusion of innovation approaches. New Media Soc. 2006, 8, 295-319. [CrossRef]

55. Lu, J.; Yao, J.E.; Yu, C.S. Personal innovativeness, social influences and adoption of wireless Internet services via mobile technology. J. Strateg. Inf. Syst. 2005, 14, 245-268. [CrossRef]

56. Lin, C.A. Exploring personal computer adoption dynamics. J. Broadcasting Electron. Media 1998, 42, 95-112. [CrossRef]

57. Compeau, D.; Higgins, C.A.; Huff, S. Social cognitive theory and individual reactions to computing technology: A longitudinal study. Mis Q. 1999, 23, 145-158. [CrossRef]

58. Jackson, C.M.; Chow, S.; Leitch, R.A. Toward an understanding of the behavioral intention to use an information system. Decis. Sci. 1997, 28, 357-389. [CrossRef]

59. Shin, D. What do people with digital multimedia broadcasting. Int. J. Mob. Commun. 2008, 6, 258-275. [CrossRef]

60. Aldás-Manzano, J.; Lassala-Navarré, C.; Ruiz-Mafé, C.; Sanz-Blas, S. The role of consumer innovativeness and perceived risk in online banking usage. Int. J. Bank Mark. 2009. [CrossRef]

61. Mitchell, V.W.; Harris, G. The importance of consumers perceived risk in retail strategy. Eur. J. Mark. 2005, 39, 821-837. [CrossRef]

62. Nakata, C.; Sivakumar, K. National culture and new product development: An integrative review. J. Mark. 1996, 60, 61-72. [CrossRef]

63. Eastlick, M.; Lotz, S. Profiling potential adopters and non-adopters of an interactive electronic shopping medium. Int. J. Retail Distrib. Manag. 1999, 27, 209-223. [CrossRef]

64. Summers, J.O. The identity of women's clothing fashion opinion leaders. J. Mark. Res. 1970, 7, 178-185. [CrossRef]

65. Saunders, J.; Davis, J.M.; Monsees, D.M. Opinion leadership in family planning. J. Health Soc. Behav. 1974, 15, 217-227. [CrossRef] [PubMed]

66. Yoo, C.W.; Jin, S.; Sanders, G.L. Exploring the role of referral efficacy in the relationship between consumer innovativeness and intention to generate word of mouth. Agribus. Inf. Manag. 2013, 5, 27-37. [CrossRef]

67. Sun, T.; Youn, S.; Wu, G.; Kuntaraporn, M. Online word-of-mouth (or mouse): An exploration of its antecedents and consequences. J. Comput. Mediat. Commun. 2006, 11, 1104-1127. [CrossRef]

68. Kim, Y.K.; Nam, J.H. Influences of Personality of Information Seekers on Instrumentality of Word of Mouth Communication: Focused on University Students. Int. J. Tour. Hosp. Res. 2012, 26, 199-215.

69. Shin, J.; Chae, H.; Ko, E. The power of e-WOM using the hashtag: Focusing on SNS advertising of SPA brands. Int. J. Advert. 2018, 37, 71-85. [CrossRef]

70. Bauer, R.A. Consumer Behavior as Risk Taking; American Marketing Association: Chicago, IL, USA, 1960; pp. 384-398. 
71. Jacoby, J.; Kaplan, L.B. The components of perceived risk. ACR Special Volumes 1972. Available online: https://www.acrwebsite.org/volumes/12016/volumes/sv02/SV02 (accessed on 1 March 2020).

72. Gemar, G.; Soler, I.P.; Melendez, L. Analysis of the intent to purchase travel on the web. Tour. Manag. Stud. 2019, 15, 23-33. [CrossRef]

73. Higgins, S.H.; Shanklin, W.L. Seeking mass market acceptance for high-technology consumer products. J. Consum. Mark. 1992. [CrossRef]

74. Jarvenpaa, S.L.; Todd, P.A. Consumer reactions to electronic shopping on the World Wide Web. Int. J. Electron. Commer. 1996, 1, 59-88. [CrossRef]

75. Featherman, M.S.; Pavlou, P.A. Predicting e-services adoption: A perceived risk facets perspective. Int. J. Hum. Comput. Stud. 2003, 59, 451-474. [CrossRef]

76. Jarvenpaa, S.L.; Trictinsky, N.; Vitale, M. Consumer Trust in Intention Store. Inf. Technol. Manag. 1999, 1, 45-47. [CrossRef]

77. Grewal, D.; Gotlieb, J.; Marmorstein, H. The moderating effects of message framing and source credibility on the price-perceived risk relationship. J. Consum. Res. 1994, 21, 145-153. [CrossRef]

78. Kim, S.D.; Jeon, I.H. Influencing Factors on the Acceptance for Crowd Funding-Focusing on Unified Theory of Acceptance and Use of Technology. J. Korean Inst. Intell. Syst. 2017, 27, 150-156. [CrossRef]

79. Litvin, S.W.; Goldsmith, R.E.; Pan, B. Electronic word-of-mouth in hospitality and tourism management. Tour. Manag. 2008, 29, 458-468. [CrossRef]

80. Baloglu, S.; McCleary, K.W. Factors Influencing Destination: A Perspective of Foreign Tourist. Ann. Tour. Res. 1999, 26, 868-897.

81. Hudson, S.; Thal, K. The Impact of Social Media on the Consumer Decision Process: Implications for Tourism Marketing. J. Travel Tour. Mark. 2013, 30, 156-160. [CrossRef]

82. Trusov, M.; Bucklin, R.E.; Pauwels, K. Effects of word-of-mouth versus traditional marketing: Findings from an internet social networking site. J. Mark. 2009, 73, 90-102. [CrossRef]

83. Tan, W.K.; Chen, T.H. The usage of online tourist information sources in tourist information search, an exploratory study. Serv. Ind. J. 2012, 32, 451-476. [CrossRef]

84. Steinbauer, A.; Werthner, H. Consumer behaviour in e-tourism. In Information and Communication Technologies in Tourism 2007; Springer: Vienna, Austria, 2007; pp. 65-76.

85. Ladhari, R.; Michaud, M. eWOM effects on hotel booking intentions, attitudes, trust, and website perceptions. Int. J. Hosp. Manag. 2015, 46, 36-45. [CrossRef]

86. Abubakar, A.M.; Ilkan, M.; Al-Tal, R.M.; Eluwole, K.K. eWOM, revisit intention, destination trust and gender. J. Hosp. Tour. Manag. 2017, 31, 220-227. [CrossRef]

87. Holbrook, M.B. Consumption experience, customer value, and subjective personal introspection: An illustrative photographic essay. J. Bus. Res. 2006, 59, 714-725. [CrossRef]

88. Hansen, S.S.; Lee, J.K. What drives consumers to pass along marketer-generated eWOM in social network games? social and game factors in play. J. Theor. Appl. Electron. Commer. Res. 2013, 8, 53-68.

89. Chu, S.C.; Kim, Y. Determinants of consumer engagement in electronic word-of-mouth (eWOM) in social networking sites. Int. J. Advert. 2011, 30, 47-75. [CrossRef]

90. Lee, S.N.; Lee, K.Y. Determinants of eWOM behavior of SNS users with emphasis on personal characteristics, SNS traits, interpersonal influence, social capital. Korean J. Advert. Public Relat. 2013, 15, 273-315.

91. Perugini, M.; Bagozzi, R.P. The role of desires and anticipated emotions in goal-directed behaviours: Broadening and deepening the theory of planned behaviour. Br. J. Soc. Psychol. 2001, 40, 79-98. [CrossRef]

92. Bollen, K.A. A new incremental fit index for general structural equation models. Sociol Methods Res. 1989, 17, 303-316. [CrossRef]

93. Hair, J.F.; Black, W.C.; Babin, B.J.; Anderson, R.E.; Tatham, R.L. Multivariate Data Analysis; Prentice Hall: Upper Saddle River, NJ, USA, 1998.

94. Fornell, C.; Larcker, D.F. Structural equation models with unobservable variables and measurement error: Algebra and statistics. J. Market. Res. 1981, 18, 382-388. [CrossRef]

95. Churchill, G.A., Jr. A paradigm for developing better measures of marketing constructs. J. Mark. Res. 1979, 16, 64-73. [CrossRef] 
96. Foxall, G.R. Marketing new technology: Markets, hierarchies, and user-initiated innovation. Manag. Decis. Econ. 1988, 9, 237-250. [CrossRef]

97. Kim, M.J.; Bonn, M.; Lee, C.K. The effects of motivation, deterrents, trust, and risk on tourism crowdfunding behavior. Asia Pac. J. Tour. Res. 2020, 25, 244-260. [CrossRef]

98. Wang, Z.; Li, H.; Law, R. Determinants of tourism crowdfunding performance: An empirical study. Tour. Anal. 2017, 22, 323-336. [CrossRef]

(C) 2020 by the authors. Licensee MDPI, Basel, Switzerland. This article is an open access article distributed under the terms and conditions of the Creative Commons Attribution (CC BY) license (http://creativecommons.org/licenses/by/4.0/). 



\title{
Relationship between Viewing Motivation, Presence, Viewing Satisfaction, and Attitude toward Tourism Destinations Based on TV Travel Reality Variety Programs
}

\author{
Bo-Kyeong Kim * and Kyoung-Ok Kim \\ Department of Tourism Management, Pukyong National University; 45 Yongso-ro, Busan 608737, Korea; \\ okson334@hanmail.net \\ * Correspondence: ynnij@hanmail.net
}

Received: 29 April 2020; Accepted: 27 May 2020; Published: 5 June 2020

\begin{abstract}
The purpose of this study was to clarify the relationship between viewing motivation for reality programs and the viewing experience (presence) during watching, viewing satisfaction after watching, and attitudes toward presented tourism destinations. While this study notes that the empirical verification of travel reality variety programs is insufficient, various discussions are presented with regard to the grip of reality variety program fever in Korea. Notably, viewers are interested in the emotional experience related to characters and tourism destinations in reality variety programs. Therefore, we asked the following questions: (1) What kind of motivation encourages viewers to watch reality variety programs? (2) How does motivation for viewing a reality variety program affect viewing satisfaction through a certain approach (presence)? (3) How does viewing satisfaction affect one's attitude toward a program's tourism destinations? To answer these questions, we conducted a survey of 358 viewers of travel reality variety programs. The results of this study are as follows: (1) viewing motivation for travel reality variety programs consists of five factors: vicarious gratification, entertainment, information-seeking, habitual time-spending, and socializing; (2) it was confirmed that the effect of these five factors on satisfaction was mediated by presence (viewing experience); and (3) viewing satisfaction through presence affected the subsequent attitude toward presented tourism destinations.
\end{abstract}

Keywords: travel reality variety program; viewing motivation; viewing satisfaction; presence; attitude toward tourism destination

\section{Introduction}

In the past, most programs about travel were mainly documentaries focused on information delivery. However, the recent trend involves reality variety programs, which combine variety, fiction, and reality; travel programs combine entertainment and seeking fun, transforming into a new genre called "travel entertainment" [1]. It is said that this change in travel programs is leading to corresponding changes in travel pursuit and tourism trends [2]. In addition, after travel reality variety programs are broadcast, credit card usage for travel and leisure increases. This suggests that consumers' or viewers' desire to travel is indirectly connected to travel programs.

Reality variety programs are continuously increasing in terms of attractive content due to accelerated competition among media, as well as the advantage of securing viewer ratings at relatively low production costs [3-6].

Due to the format of reality travel programs, tourist destinations are shown on the screen frequently and for longer periods of time compared to drama programs and movies. Therefore, viewers are more likely to be more immersed in the presented situation than they would be with dramas and movies, 
They also form images of tourist destinations in the programs and make meaningful connections to the selection of a travel destination [7].

As such, mass media plays an important role in the travel industry, and the reality variety format is particularly important for theoretical research and has managerial aspects that can be key in the travel industry.

In particular, travel programs in the reality format are increasing and the formats are diversifying, but related previous studies are limited to the exposure effects on the tourist destinations, factors influencing the choice of destinations, and airline ticket price analysis for travel programs.

Recently, there have been several studies on how viewing motivation for reality travel programs influences future viewer behavior. However, they are limited to discussing the general relationship between viewing motivation and viewing satisfaction.

Therefore, this study aimed to investigate the effects of viewing motivation, viewing satisfaction, and attitudes toward tourism destinations, and to empirically reveal the mechanism of presence (watching experience) in a sample of men and women over the age of 18 who live in Busan and Gyeongnam provinces. To achieve this purpose, based on previous research on viewing motivation for broadcast programs, it is classified into five factors related to reality travel programs, and the examination of the effect of the five factors on satisfaction is mediated by presence (viewing experience). Lastly, viewing satisfaction through presence is confirmed with regard to its influence on subsequent attitudes toward presented tourism destinations.

\section{Literature Review}

\subsection{Viewing Motivation for Travel Reality Variety Programs}

Previous studies on viewing motivation divided it into social reward and psychological motivation [8], including information, entertainment, establishing a relationship, and recreation [9]; and entertainment, time-spending, and social learning $[10,11]$. Viewing motivation is based on uses and gratification theory $[12,13]$, which posits that viewers use or watch TV programs to satisfy their desires and needs. Unlike traditional research about the effect of mass media asking questions such as, What does media do to people? this theory conversely asks, What do people do with media? Thus, this theory is used to understand viewers' media experience and to identify their motivation in selecting and using specific media in comparison with other media.

Research on uses and gratification has been extended to studies ranging from categorizing viewing motivation for TV programs to describing viewer motivation according to the genre of programs.

According to Lim (2008) [14], college students' motivations for viewing American dramas were entertainment and rest, information, and environment/companion, while those for Korean dramas also included drama characteristics (feature) and habitual free time-spending.

Ban and Park (2014) [15] studied viewing motivation for reality dating programs and categorized it into virtual avoidance, indirect experience, pleasure, social interaction, and free time-spending. For a certain type of reality travel program, such as "Daddy! Where Are You Going?", there are four main factors of viewing motivation: interest, the attractiveness of the children, alleviating loneliness, and expressing everyday emotions [16,17]. "Hyori's B \& B," which aired in 2017 and 2018, was an observational reality travel program that recently became an entertainment trend, with entertainment, empathy, interaction, aesthetics, information, and indirect experiences selected as viewing motivations [2]. It was found that only aesthetics, information, and indirect experiences had significant effects on viewer satisfaction and intention to visit.

The results of these studies show differences in viewing motivation according to program format or genre, and that generally, but not always, it affects viewer satisfaction.

Therefore, the motivation for viewing travel reality variety programs does not only involve acquiring information about tourism destinations, but there is also a psychological motivation in comparison with other travel programs [16]. Accordingly, this study proposes vicarious gratification, 
entertainment, information-seeking, habitual time-spending, and socializing as factors of viewing motivation for travel reality variety programs based on previous studies.

\subsection{Presence}

While the definition of presence varies according to the researcher [18], it generally refers to the psychological and subjective experience of a person's feeling of being directly in the mediating environment outside their surrounding physical environment [19-21]. To be specific, there are six subtypes of this dimensionality: (1) social richness that feels the media intimately, (2) realism based on realistic expression, (3) transportation that seems to be going to a virtual media environment, (4) immersion in a virtual reality, (5) a sense of reality as a social actor within the medium who interacts with the mediated character, and (6) feelings toward the medium itself as a social actor [20]. The study of this type of presence has been extended to its role as a mediator between viewing motivation and satisfaction, as well as various media environments. According to Kim and Biocca (1997) [19] and Lombard and Ditton(1997) [22], while there are differences in the types and scopes of media, presence can be applicable to all media. In addition, presence is established by the format, genre, content, and characteristics of the media, and can be sufficiently experienced even in a traditional medium where the mediated environment is relatively unrealistic. In particular, in the case of TV media, the amount and precision of sensory channels mobilized by users, i.e., the "sensory output", creates a higher reality experience that is called presence in TV but not radio, which is only auditory [23].

Therefore, it can be said that it is difficult to explain the genre of reality travel programs only by the relationship between viewing motivation and satisfaction in terms of the realistic expression (presence as reality) of TV media $[8,24]$. In particular, in the case of reality travel programs, with travel and storytelling, viewers become more immersed in the tourism destination, which is the background of the program, making them more aware of the reality of the place depending on the level of perceived similarity and wishful identification with the characters [1]. In accordance with this view, it is critical to confirm that presence plays a mediating role in the relationship between viewing motivation and satisfaction [25].

Based on the above discussion, this paper examines the mediating role of presence in accounting for the relationship between viewing motivation and viewing satisfaction for travel reality variety programs. We accordingly set out to prove the significance of presence as a parameter that affects viewing satisfaction.

\subsection{Viewing Satisfaction}

Satisfaction with viewing media and programs starts with expectations for content. Thus, since satisfaction with a program is shaped when the viewer's expectations for that program are met, or the viewer evaluates them positively through a process of assessing the cognitive and emotional dimensions of the content, their consequent satisfaction reflects the fulfillment of their desires. Therefore, satisfaction after watching a program is a very critical parameter for understanding the viewer's media experience. Satisfaction with television viewing is formed by a combination of various factors, such as motivation and viewing experience. In fact, there has been extensive research on viewing satisfaction and viewing experience regarding television and specific programs $[11,24,26]$.

According to the expectancy value model of Palmgreen and Rayburn (1984) [27], media consumption is driven by satisfaction, or the seeking of gratification, and perceived gratification obtained afterwards. Beliefs and evaluations are modified, thereby affecting the repeated seeking of gratification. In other words, if gratification sought and obtained is consistent, viewing satisfaction increases, which leads to continued viewing. This model explains the cyclical relationship between viewing motivation, viewing behavior, and satisfaction (fulfillment) through various media.

Indeed, on travel reality programs, tourism destinations are realistically described from the tourists' perspective, and viewers' expectations and desires are satisfied by being providing rich information on tourism destinations $[10,28]$. In addition, detailed information about the region/place 
is provided while famous tourists travel directly in the program, unlike in drama and movies [6,29]. In short, as it is a place that is not merely an image, such as in a film or drama, but is directly exposed to viewers, it can be expected to arouse their expectations and intentions for future behavior.

Therefore, this study intends to expand viewing satisfaction theoretically through the specific parameter that the motivation to view travel reality variety programs affects viewing satisfaction, based on previous studies.

\subsection{Attitude toward Tourism Destinations}

Attitude is an ongoing assessment of beliefs and emotions about an object [30,31]. Attitudes are also individuals' thoughts, emotions, and tendencies regarding particular subjects, and their future behavior will vary depending on how they establish attitudes toward particular subjects [32]. In addition, it is not necessary for awareness, influence, and behavior to simultaneously exist in order to form or express an attitude; rather, such can be established when one or more components are combined [33]. Fishbein and Ajzen (1975) [31] proposed the theory of reasoned action by adding subjective norms to attitudes and behavioral intentions to clarify the human decision-making process. Ajzen (1991) [34] then developed the theory of extended planned behavior by adding perceived behavior control considering the external environment. In the tourism industry, it is important to understand tourists' decision-making, so research has been performed to identify behavioral intent through attitude [28,35-37]. In particular, decision-makers include many criteria when selecting tourism destinations, determining their preferences and behaviors concerning future destinations by comparing and evaluating the attributes of tourist sites [38].

Thus, tourists can be said to have different behaviors depending on their prior preferences [39]. Tourists are exposed to diverse mass media, actively use media to gather information about tourism destinations, and make various decisions regarding tourism, such as selecting destinations, through such information. The media sector that has attracted the most attention recently in the field of tourism is entertainment, which is indirectly connected to viewers (potential tourists) through movies and TV programs, and directly connected to the tourism industry [40]. Based on previous research, it is necessary to extend the research area into the effect of viewing satisfaction on attitudes toward tourist destinations according to the motivation for viewing travel reality variety programs.

\section{Research Model and Methodology}

\subsection{Research Model and Hypotheses Development}

Viewing motivation is a significant factor in understanding how to meet viewer expectations for a program as well as influencing attitudes and future behaviors. Taking a look at previous studies, there was a difference in viewing motivation depending on the viewer or the program format or genre. In particular, the effect on viewing satisfaction or other behavioral variables can be different through the realistic expression (presence as reality) of the place shown in a travel reality program, unlike in films and dramas $[7,40,41]$. Therefore, presence is a psychological factor of the viewer and can be said to be a weighty parameter that influences satisfaction with the use of TV or media [5]. For example, viewers motivated to pursue information on travel will have a higher level of interest in tourism destinations that are in the background of the program. Particularly, while moving along with performers, they can experience presence in the places they see on the screen, and this shows high satisfaction with the program [15].

Therefore, the following hypothesis was proposed:

Hypothesis 1 (H1). Viewing motivation for a travel reality variety program positively affects presence.

Hypothesis 1-1 (H1-1). Vicarious gratification positively affects presence.

Hypothesis 1-2 (H1-2). Entertainment positively affects presence. 
Hypothesis 1-3 (H1-3). Information-seeking positively affects presence.

Hypothesis 1-4 (H1-4). Habitual time-spending positively affects presence.

Hypothesis 1-5 (H1-5). Socializing positively affects presence.

Previous studies on viewing motivation and satisfaction found a positive relationship between these factors through the mediation of parasocial interaction and presence on the program "Cookbang" [15]. This means that high levels of involvement in, attention to, and engagement with the reality program by viewers showed a positive effect on their viewing satisfaction according to their watching experience (presence). Therefore, this reality presence can increase enjoyment and engagement even when watching a travel reality variety program, which can increase viewing satisfaction. Hence, the following hypothesis was proposed:

Hypothesis 2 (H2). Presence positively affects viewing satisfaction with a travel reality variety program.

Viewing satisfaction means that a program exceeds the viewer's expectations, and viewers are more satisfied with a program if their expectations are exceeded, and dissatisfied if their expectations are not met [11]. Satisfaction with TV viewing is influenced by the viewer's attitude and behavioral intention, because the viewing motivation or expectation for the program is satisfied or the viewer's behavior is closely related to the program [42]. Therefore, the following hypothesis was proposed:

Hypothesis 3 (H3). Viewing satisfaction with a travel reality variety program positively affects the viewer's attitude toward the tourism destination.

Based on the above hypotheses, this study's research model is shown in Figure 1.

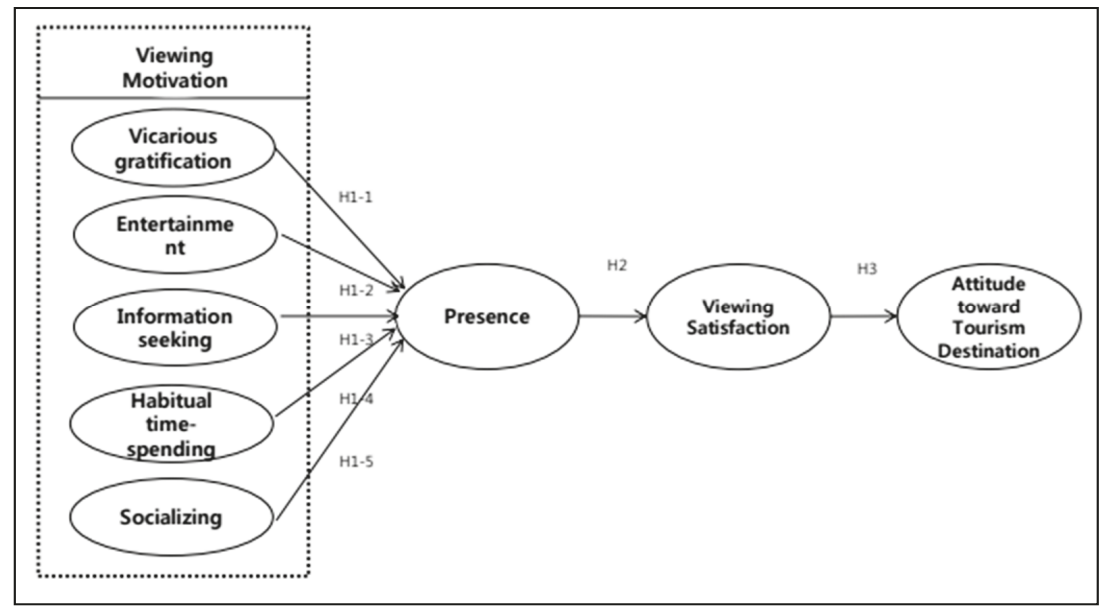

Figure 1. Research model.

\subsection{Operational Definition}

Viewing motivation was defined as "the viewer's personal viewing desire/need for the media" [8]. Next, based on the research of McQuail (2005) [36], Ebersole and Woods (2007) [43], and Jang and Kim (2016) [44], five motivational factors were derived: vicarious gratification, entertainment, information-seeking, habitual time-spending, and socializing. With slight modification, a seven-point Likert scale was used to measure these factors ( $1=$ strongly disagree to $7=$ strongly agree). Presence 
was defined as "feelings/emotions of immersing into the environment created by television programs without being able to perceive the reality of television" based on the research of Bracken (2009) [23] and Pope and Wansink (2015) [11]. With slight modification, a seven-point Likert scale was used to measure four items ( 1 = strongly disagree to $7=$ strongly agree). Viewing satisfaction was defined as follows: "the viewer uses media to satisfy his or her various needs/desire and establish satisfaction," from the uses and gratification theory of Kartz (1959) [9]. Four items were extracted and slightly modified from Kim and Rubin (1997) [5] and An and Han (2018) [16], and measured by a seven-point Likert scale $(1=$ strongly disagree to $7=$ strongly agree). Lastly, attitudes toward tourism destinations were measured by using a seven-point Likert scale ( $1=$ strongly disagree to $7=$ strongly agree) for the four items used in the survey on the impact of the series "Better Late Than Never" of the Korea Tourism Organization-International Travel Trend Report (2014).

\subsection{Data Collection and Analysis Method}

For this study, the survey was conducted via an Internet questionnaire, first confirming the experience of watching reality variety programs. Next, the authors set as samples those living in Busan and Gyeongnam provinces who had experience watching travel reality variety programs from 1-31 March 2020. The convenience sampling method was applied. Among the 382 total respondents, 358 valid questionnaires were received and used for analysis, excluding (1) nonresponse, (2) overlapping response, and (3) unfinished questionnaires. Because of the high response rate, analysis of nonrespondents was not conducted, since a $94.7 \%$ response rate was considered adequate [45]. SPSS 25.0 and AMOS 23.0 were used for the analysis.

The statistical analysis methods and procedures applied in the empirical data analysis process of this study were as follows:

First, frequency analysis was performed to examine the general demographic characteristics of the sample. Second, exploratory factor analysis was conducted to identify the factor structure, reliability, and validity of the measurement tool, and confirmatory factor analysis and discriminant validity analysis were performed to verify the fit of the factor structure. Third, Structural Equations Models (SEMs) were examined to investigate the hypotheses.

\section{Results}

\subsection{Profile of the Sample}

The demographics of the 358 participants were as follows: 188 men (52.7\%) and 170 women (47.3\%). Regarding the age groups, 103 people (29.0\%) were in their $20 \mathrm{~s}, 113$ people (31.7\%) were in their 30 s, 88 people $(24.6 \%)$ were in their 40 s, and 38 people $(10.7 \%)$ were in their 50 s. In terms of marital status, 230 (64.5\%) were married and 128 (35.5\%) were single. Regarding the distribution of occupations, 51 were homemakers (14.3\%), 84 had specialized jobs (23.7\%), 193 (54.0\%) were office workers, and $30(8.0 \%)$ had other occupations. Regarding the distribution of the amount of travel in a year, 119 people (33.5\%) traveled once, and 142 people $(39.7 \%)$ traveled two to three times a year. Regarding the number of reality variety programs watched in a week, 54 people (14.7\%) watched once per week, 177 people $(49.6 \%)$ watched two to three times, and 76 people $(21.4 \%)$ watched four to five times. Considering distribution of monthly income (in million won), 198 people (55.4\%) earned 3 to 4.99 million won and 76 people $(21.0 \%)$ earned more than 5 million won. The demographic characteristics of this study are shown in Table 1. 
Table 1. Demographic variables $(n=358)$.

\begin{tabular}{|c|c|c|c|c|c|c|c|}
\hline \multicolumn{2}{|c|}{ Classification } & \multicolumn{2}{|c|}{ FrequencyPercentage (\%) } & \multicolumn{2}{|c|}{ Classification } & \multirow{2}{*}{$\begin{array}{c}\text { Frequency } \\
119\end{array}$} & \multirow{2}{*}{$\frac{\text { Percentage }(\%)}{33.5}$} \\
\hline \multirow{2}{*}{ Gender } & Male & 188 & 52.7 & \multirow{5}{*}{$\begin{array}{l}\text { Amount of } \\
\text { travel in a year }\end{array}$} & 1 time & & \\
\hline & Female & 170 & 47.3 & & 2-3 times & 142 & 39.7 \\
\hline \multirow{5}{*}{ Age (years) } & 20 & 103 & 29.0 & & 4-5 times & 38 & 10.7 \\
\hline & 30 & 113 & 31.7 & & 6-7 times & 17 & 4.9 \\
\hline & 40 & 88 & 24.6 & & $>8$ times & 42 & 11.2 \\
\hline & 50 & 38 & 10.7 & \multirow{4}{*}{$\begin{array}{c}\text { Number of } \\
\text { reality variety } \\
\text { programs } \\
\text { watched in a } \\
\text { week }\end{array}$} & 1 time & 54 & 14.7 \\
\hline & $>60$ & 16 & 4.0 & & $2-3$ times & 177 & 49.6 \\
\hline \multirow{2}{*}{$\begin{array}{l}\text { Marital } \\
\text { status }\end{array}$} & Married & 230 & 64.5 & & $4-5$ times & 76 & 21.4 \\
\hline & Single & 128 & 35.5 & & 6-7 times & 51 & 14.3 \\
\hline \multirow{4}{*}{ Occupation } & Homemaker & 51 & 14.3 & \multirow{4}{*}{$\begin{array}{l}\text { Monthly income } \\
\text { (in million won) }\end{array}$} & $<1.49$ & 36 & 10.1 \\
\hline & Specialized job & 84 & 23.7 & & $1.5-2.99$ & 48 & 13.5 \\
\hline & Office worker & 193 & 54.0 & & $3-4.99$ & 198 & 55.4 \\
\hline & Other & 30 & 8.0 & & $>5$ & 76 & 21.0 \\
\hline Sum & & 358 & 100 & Sum & & 358 & 100 \\
\hline
\end{tabular}

\subsection{Reliability and Validity}

This study confirmed the reliability and validity of the variables before hypothesis testing. Regarding the reliability of constructs, internal consistency was found with all measuring factors with Cronbach's $\alpha$ over 0.7 [46]. The validity of the variables was verified through confirmatory factor analysis. The results are presented in Table 2. First, it was concluded that the standardized factor loading value was over 0.6 , the significance level was less than $0.1 \%$, and the AVE (average variance extracted) value was over 0.5 , indicating that there was convergent validity [47]. Comparing the AVE value with the square of the correlation coefficient (based on Table 3) showed that the AVE value of latent variables was greater than the square of correlation value. Thus, the validity of discriminant validity was assured [47].

Table 2. Results of confirmatory factor analysis of the measurement model.

\begin{tabular}{|c|c|c|c|c|c|c|}
\hline Construct & Measurement Items & $\begin{array}{l}\text { Factor } \\
\text { Loading }\end{array}$ & S.E. & C.R. & $p$-Value & Cronbach's a \\
\hline \multirow{5}{*}{$\begin{array}{l}\text { Vicarious } \\
\text { gratification }\end{array}$} & $\begin{array}{l}\text { While watching reality travel variety programs, } \\
\text { I feel assimilated with the characters. }\end{array}$ & 0.728 & & & & \multirow{5}{*}{0.902} \\
\hline & $\begin{array}{l}\text { While watching reality travel variety programs, } \\
\text { I can forget my daily life. }\end{array}$ & 0.755 & 0.074 & 13.918 & $<0.001$ & \\
\hline & $\begin{array}{l}\text { While watching reality travel variety programs, } \\
\text { I feel as though I am in the village. }\end{array}$ & 0.842 & 0.077 & 15.547 & $<0.001$ & \\
\hline & $\begin{array}{l}\text { While watching reality travel variety programs, } \\
\text { I feel like a resident of the village. }\end{array}$ & 0.854 & 0.081 & 15.765 & $<0.001$ & \\
\hline & $\begin{array}{l}\text { While watching reality travel variety programs, } \\
\text { I feel like I am traveling. }\end{array}$ & 0.840 & 0.082 & 15.513 & $<0.001$ & \\
\hline \multirow{4}{*}{ Entertainment } & $\begin{array}{l}\text { Reality travel variety programs are more } \\
\text { entertaining than other reality variety programs. }\end{array}$ & 0.701 & & & & \multirow{4}{*}{0.846} \\
\hline & $\begin{array}{l}\text { Viewing reality travel variety programs makes } \\
\text { me happy. }\end{array}$ & 0.824 & 0.066 & 14.348 & $<0.001$ & \\
\hline & $\begin{array}{c}\text { Every new episode of a reality travel variety } \\
\text { program is interesting. }\end{array}$ & 0.873 & 0.069 & 15.061 & $<0.001$ & \\
\hline & $\begin{array}{l}\text { Places that appear in reality travel variety } \\
\text { programs are more interesting than in other } \\
\text { reality variety programs. }\end{array}$ & 0.780 & 0.075 & 13.637 & $<0.001$ & \\
\hline
\end{tabular}


Table 2. Cont.

\begin{tabular}{|c|c|c|c|c|c|c|}
\hline Construct & Measurement Items & $\begin{array}{l}\text { Factor } \\
\text { Loading }\end{array}$ & S.E. & C.R. & $p$-Value & Cronbach's a \\
\hline \multirow{5}{*}{$\begin{array}{l}\text { Information- } \\
\text { seeking }\end{array}$} & $\begin{array}{l}\text { Reality travel variety programs show specific } \\
\text { information about tourism destinations. }\end{array}$ & 0.758 & & & & \multirow{5}{*}{0.863} \\
\hline & $\begin{array}{l}\text { Reality travel variety programs show cultural } \\
\text { information about tourism destinations. }\end{array}$ & 0.652 & 0.068 & 12.254 & $<0.001$ & \\
\hline & $\begin{array}{c}\text { Reality travel variety programs are educational } \\
\text { in showing the way of life in } \\
\text { tourist destinations. }\end{array}$ & 0.776 & 0.073 & 14.844 & $<0.001$ & \\
\hline & $\begin{array}{l}\text { Reality travel variety programs are beneficial } \\
\text { because they provide an overview of } \\
\text { tourism destinations. }\end{array}$ & 0.772 & 0.075 & 14.755 & $<0.001$ & \\
\hline & $\begin{array}{l}\text { Reality travel variety programs have enhanced } \\
\text { my intellectual ability to travel by making it } \\
\text { possible to know about tourism destinations. }\end{array}$ & 0.779 & 0.068 & 14.892 & $<0.001$ & \\
\hline \multirow{4}{*}{$\begin{array}{l}\text { Habitual } \\
\text { time-spending }\end{array}$} & $\begin{array}{c}\text { Reality travel variety programs are good for } \\
\text { spending time alone. }\end{array}$ & 0.820 & & & & \multirow{4}{*}{0.899} \\
\hline & $\begin{array}{l}\text { Reality travel variety programs are good to } \\
\text { watch without thinking. }\end{array}$ & 0.827 & 0.056 & 18.046 & $<0.001$ & \\
\hline & $\begin{array}{l}\text { I watch reality travel variety programs } \\
\text { habitually without a special purpose. }\end{array}$ & 0.814 & 0.059 & 17.658 & $<0.001$ & \\
\hline & $\begin{array}{l}\text { There are no other programs to watch at the } \\
\text { time this program is broadcast. }\end{array}$ & 0.863 & 0.056 & 19.176 & $<0.001$ & \\
\hline \multirow{3}{*}{ Socializing } & $\begin{array}{c}\text { I watch reality travel variety programs to } \\
\text { empathize with others. }\end{array}$ & 0.642 & & & & \multirow{3}{*}{0.746} \\
\hline & $\begin{array}{l}\text { Reality travel variety program are subjects of } \\
\text { interest among people. }\end{array}$ & 0.557 & 0.134 & 8.753 & $<0.001$ & \\
\hline & $\begin{array}{l}\text { I watch reality travel variety programs to avoid } \\
\text { being alienated from conversations with others. }\end{array}$ & 0.745 & 0.144 & 9.487 & $<0.001$ & \\
\hline \multirow{4}{*}{$\begin{array}{l}\text { Viewing } \\
\text { satisfaction }\end{array}$} & $\begin{array}{l}\text { I am satisfied with the content of reality travel } \\
\text { variety programs. }\end{array}$ & 0.650 & & & & \multirow{4}{*}{0.803} \\
\hline & $\begin{array}{l}\text { I would like to watch more reality travel } \\
\text { variety programs. }\end{array}$ & 0.783 & 0.096 & 12.844 & $<0.001$ & \\
\hline & $\begin{array}{l}\text { I am satisfied that I have gained useful } \\
\text { information about places and cultures that } \\
\text { appear in reality travel variety programs. }\end{array}$ & 0.911 & 0.101 & 14.400 & $<0.001$ & \\
\hline & $\begin{array}{l}\text { I would like my acquaintances to recommend } \\
\text { watching reality travel variety programs. }\end{array}$ & 0.907 & 0.103 & 14.349 & $<0.001$ & \\
\hline \multirow{4}{*}{ Presence } & $\begin{array}{l}\text { I feel that I am in the TV situation with } \\
\text { the characters. }\end{array}$ & 0.709 & & & & \multirow{4}{*}{0.887} \\
\hline & $\begin{array}{l}\text { I feel that the situations on TV are happening in } \\
\text { front of my eyes. }\end{array}$ & 0.815 & 0.066 & 14.020 & $<0.001$ & \\
\hline & $\begin{array}{l}\text { The characters on the TV seem to be talking } \\
\text { right in front of me. }\end{array}$ & 0.890 & 0.076 & 14.640 & $<0.001$ & \\
\hline & I feel that I am in that place on the TV. & 0.645 & 0.073 & 11.292 & $<0.001$ & \\
\hline \multirow{4}{*}{$\begin{array}{l}\text { Attitude } \\
\text { toward } \\
\text { tourism } \\
\text { destinations }\end{array}$} & $\begin{array}{c}\text { I feel that the places that appear on reality travel } \\
\text { variety programs are good. }\end{array}$ & 0.759 & & & & \multirow{4}{*}{0.882} \\
\hline & $\begin{array}{l}\text { I feel that the places that appear on reality travel } \\
\text { variety programs are good places to travel. }\end{array}$ & 0.769 & 0.068 & 14.924 & $<0.001$ & \\
\hline & $\begin{array}{c}\text { I like the places that appear on reality travel } \\
\text { variety programs. }\end{array}$ & 0.847 & 0.068 & 16.637 & $<0.001$ & \\
\hline & $\begin{array}{l}\text { I feel that the places that appear on reality travel } \\
\text { variety programs are attractive. }\end{array}$ & 0.854 & 0.067 & 16.806 & $<0.001$ & \\
\hline \multicolumn{7}{|c|}{$X^{2}=982.850, d f=467, p=0.000,(A G F I=0.909 T L I=0.924, C F I-=0.933, R M S E A=0.056)$} \\
\hline
\end{tabular}


Table 3. Correlation analysis of constructs and average variance extracted (AVE).

\begin{tabular}{|c|c|c|c|c|c|c|c|c|}
\hline & $\begin{array}{l}\text { Vicarious } \\
\text { Gratification }\end{array}$ & Entertainment & $\begin{array}{l}\text { Information- } \\
\text { Seeking }\end{array}$ & $\begin{array}{c}\text { Habitual } \\
\text { Time-Spending }\end{array}$ & Socializing & $\begin{array}{l}\text { Viewing } \\
\text { Satisfaction }\end{array}$ & Presence & $\begin{array}{c}\text { Attitude } \\
\text { toward } \\
\text { Tourism } \\
\text { Destination }\end{array}$ \\
\hline $\begin{array}{l}\text { Vicarious } \\
\text { Gratification }\end{array}$ & $(0.636)$ & & & & & & & \\
\hline Entertainment & $0.458^{* *}$ & $(0.661)$ & & & & & & \\
\hline $\begin{array}{l}\text { Information- } \\
\text { Seeking }\end{array}$ & $0.457^{* *}$ & 0.560 ** & $(0.701)$ & & & & & \\
\hline $\begin{array}{l}\text { Habitual Time- } \\
\text { Spending }\end{array}$ & $0.388^{* *}$ & $0.536^{* *}$ & $0.722 * *$ & $(0.759)$ & & & & \\
\hline Socializing & $0.452 * *$ & 0.469 ** & 0.491 ** & $0.364 * *$ & $(0.711)$ & & & \\
\hline Satisfaction & 0.464 ** & $0.528 * *$ & $0.588^{* *}$ & $0.521 * *$ & $0.593 * *$ & $(0.745)$ & & \\
\hline Presence & -0.029 & 0.003 & -0.020 & 0.020 & 0.012 & 0.049 & $(0.749)$ & \\
\hline $\begin{array}{c}\text { Attitude } \\
\text { toward } \\
\text { Tourism } \\
\text { Destination }\end{array}$ & $0.417^{* *}$ & $0.655^{* *}$ & $0.576^{* *}$ & $0.538 * *$ & $0.483 * *$ & $0.744^{* *}$ & 0.043 & $(0.758)$ \\
\hline
\end{tabular}

\subsection{Verification of Hypotheses}

Given an acceptable measurement-model fit, SEM was carried out in AMOS to investigate the overall fit of the structural model and hypotheses. The fit of the proposed model was tested using fit indices. The results $\left(\chi^{2} / \mathrm{df}=958.581 / 483, \mathrm{CMIN} / \mathrm{df}=1.984, \mathrm{RMR}=0.028, \mathrm{GFI}=0.900, \mathrm{AGFI}=0.902, \mathrm{CFI}\right.$ $=0.908, \mathrm{NFI}=0.901, \mathrm{IFI}=0.909, \mathrm{RMSEA}=0.063$ ) proved that the model was a good fit and appropriate for the sample data. Therefore, the analysis was performed without any model modification. The results of the hypotheses in this study are summarized in Table 4.

Table 4. Results of hypothesis testing.

\begin{tabular}{|c|c|c|c|c|}
\hline & Structural Paths & $\beta$ & t-Value & Hypothesis Test \\
\hline H1-1 & Vicarious Gratification $\rightarrow$ Presence & 0.453 & $4.899^{* * *}$ & Supported \\
\hline H1-2 & Entertainment $\rightarrow$ Presence & 0.286 & $4.030^{* * *}$ & Supported \\
\hline H1-3 & Information-Seeking $\rightarrow$ Presence & 0.131 & $2.182 *$ & Supported \\
\hline $\mathrm{H} 1-4$ & Habitual Time-Spending $\rightarrow$ Presence & 0.076 & 1.302 & Rejected \\
\hline H1-5 & Socializing $\rightarrow$ Presence & 0.129 & 1.937 & Rejected \\
\hline $\mathrm{H} 2$ & Presence $\rightarrow$ Viewing Satisfaction & 0.239 & $4.517^{* * *}$ & Supported \\
\hline $\mathrm{H} 3$ & $\begin{array}{l}\text { Viewing Satisfaction } \rightarrow \text { Attitude } \\
\text { toward Tourism Destination }\end{array}$ & 0.818 & $10.630^{* * *}$ & Supported \\
\hline $\begin{array}{l}\text { Model } \\
\text { fit }\end{array}$ & \multicolumn{4}{|c|}{$\begin{array}{c}\text { Chi-square } / \mathrm{df}=958.581 / 483, \mathrm{CMIN} / \mathrm{df}=1.984, \mathrm{RMR}=0.028 \\
\mathrm{GFI}=0.900, \mathrm{AGFI}=0.902, \mathrm{CFI}=0.908, \mathrm{NFI}=0.901, \mathrm{IFI}=0.909, \mathrm{RMSEA}=0.063\end{array}$} \\
\hline
\end{tabular}

Specifically, in terms of Hypothesis 1, Viewing motivation of reality travel variety programs positively affects presence, Hypothesis 1-1, which assumes that vicarious gratification positively affects presence, was positive and significant at the $5 \%$ level. Hypothesis 1-2, which assumes that entertainment positively affects presence, was positively related to presence and significant at the 5\% level. Hypothesis 1-3, which assumes that information-seeking positively affects presence, was also positive and significant at the 5\% level. Hypotheses 1-4 and 1-5, which posit that habitual time-spending and socializing positively affect presence, were not significant and not supported. Regarding Hypothesis 2, which assumes that presence positively affects viewing satisfaction of reality travel variety programs, was positive and significant at the $5 \%$ level, thus supporting this hypothesis. Hypothesis 3 , which assumes 
that viewing satisfaction of reality travel variety programs positively affects attitudes toward tourism destinations, was positive and significant at the 5\% level, supporting this hypothesis as well.

\section{Discussion and Conclusions}

\subsection{Discussion}

This study was conducted with viewers who have experience watching travel reality variety programs; the program format is travel reality show, which is a mixture of travel and reality rather than a movie or drama. This aspect seems to contribute in a way that is different from previous research. In addition, previous studies investigated only the relationship between motivation for viewing a reality program and satisfaction in various aspects, but few studies have analyzed the psychological effect on viewing motivation and satisfaction by presence, by which viewers vividly and realistically recognize the places in the programs. Thus, it can be considered that the attempt to identify presence in travel reality variety programs is a contribution to the scalability of the theoretical application utilized in this study. The results of this study are as follows.

First, among five motivational factors, vicarious gratification, entertainment, and information-seeking have a positive influence on presence. This is because of the nature of travel reality variety programs, which provide destination information and entertainment at the same time, provide accurate information on the locations, then have fun with the content itself, and the vicarious gratification provided by the indirect experience with this kind of program gives viewers a vivid sense of aliveness or presence about the place, which leads to tourists expectations toward the place. This result is meaningful, because it proves that the effect of presence is mediated in travel reality variety programs as well as reality programs $[10,48]$. Second, it was confirmed that presence had a positive effect on the satisfaction of viewing travel reality variety programs, similar to the presence of reality programs such as "Cookbang" and "Mukbang" [15,24]. It can be concluded that viewers can experience more presence in the place due to the liveliness, which is one of the features of travel variety programs, and this presence has a positive influence on viewing satisfaction. Therefore, presence can be said to be a significant parameter that can influence the relationship between viewing motivation and satisfaction. Third, a positive attitude toward the tourism destination in the program was found when the viewing satisfaction was higher in this relationship. This supports previous research on satisfaction and attitude [49]. Ultimately, in travel reality variety programs, like other reality programs, viewing satisfaction causes the viewers to have good image and expectation for the place, and finally, viewing satisfaction can lead to a positive attitude toward tourism destinations [50].

\subsection{Implications}

Above all, we note the theoretical implications of this study. First, this study divided viewing motivation into five variables based on a literature study on previous media-viewing motivation, and it is meaningful that this research considered media diversity by applying reality travel variety programs. In particular, among five motivational factors, considering the characteristics of the travel reality program, adding the variable of "vicarious gratification" as an indirect experience through the screen can also be said to be a meaningful theoretical extension from the previous research.

In addition, presence, the realistic feeling given by a reality travel variety program, was verified empirically as a mediating role between viewing motivation and viewing satisfaction. This is an attractive factor for viewers watching reality programs, so it can be applied to various genres of media in the future. This is also an empirical evidence for the claim that presence is applicable to all media, although there are differences in the type and exposure degree of media [12], and it was confirmed that the experience of presence is a highly significant variable in the travel reality program.

Lastly, it is meaningful that we reconfirmed (based on existing research) the satisfaction of viewing these TV programs and attitudes toward their tourism destinations. 
In addition, this study suggests the following practical implications. People often indirectly experience tourism destinations through mass media without actually visiting them. This indirect experience will have a great influence on future behavioral intentions [12,13]. In Korea, due to the diversification of broadcasting channels, each broadcasting company produces programs that include indirect advertisements. Indeed, since the tvN "Over Flowers Series" travel program was broadcast, the number of Korean tourists who visited the broadcast country increased by an average of $52.7 \%$ [51]. In this respect, therefore, the results of this study can provide a guideline for mapping strategies to attract tourists to certain places in terms of marketing the tourism destination itself. In particular, the exposure of places in travel reality programs is more realistic and specific than in dramas or movies. In the end, this evokes expectations of a tourism destination [16], and since it approaches the viewer with easy and comfortable images, the effect of enhancing the attractiveness of the space will be strong. Therefore, tourism destinations exposed through travel reality programs are imprinted with a positive image for viewers or potential tourists, and can also affect actual behaviors such as visiting certain locations.

\subsection{Limitations and Future Research}

Finally, we present the limitations of this study and the direction of future research. In this study, even though the questionnaire survey was conducted for respondents who had watched the programs, their memories may have been distorted over time. Therefore, subsequent research should be conducted on programs that are being broadcast to obtain more accurate results. In addition, recent travel reality variety programs may also include entertainers of specific genders or ages. In further studies, it will be necessary to consider the ages and genders of the entertainers on various programs. Lastly, the sample for the survey was limited to adults residing in Busan and Gyeongsangnam provinces. Therefore, further research should consider all regions in Korea. In future research, it is expected that more specific and diverse studies on travel reality programs will be conducted based on this study. The results of this study can then be supplemented with meaningful facts, which will enable a wider understanding of the results and generalization of previously discovered theories.

Author Contributions: Conceptualization, B.-K.K.; methodology, K.-O.K.; data curation, K.-O.K.; writingoriginal draft preparation, B.-K.K.; writing-review and editing, B.-K.K.; project administration, B.-K.K.; funding acquisition, K.-O.K. All authors have read and agreed to the published version of the manuscript.

Funding: This work was supported by the Ministry of Education of the Republic of Korea and the National Research Foundation of Korea (NRFS1A5B5A07XXXXXX).

Conflicts of Interest: The authors declare no conflict of interest.

\section{References}

1. Shah Alam, S.; Mohamed Sayuti, N. Applying the theory of planned behavior (TPB) in halal food purchasing. Int. J. Commer. Manag. 2011, 21, 8-20. [CrossRef]

2. Papacharissi, Z.; Mendelson, A.L. An exploratory study of reality appeal: Uses and gratifications of reality TV Shows. J. Broadcast. Electron. Media 2007, 51, 355-370. [CrossRef]

3. Fu, H.; Ye, B.H.; Xiang, J. Reality TV audience travel intentions, and destination image. Tour. Manag. 2016, 55, 37-48. [CrossRef]

4. Kim, J.O.; Kim, N.J. A study on the perception of elderly leisure of the reality program 'Grandpa over Flowers' older generation viewers: Focusing on the middle class. J. Tour. Sci. 2016, 40, 11-29.

5. Kim, J.; Rubin, A.M. The variable influence of audience activity on media effects. Commun. Res. 1997, 24, 107-135. [CrossRef]

6. Woodside, A.G.; Dubelaar, C. A general theory of tourism consumption systems: A conceptual framework and an empirical exploration. J. Travel Res. 2002, 41, 120-132. [CrossRef]

7. Cho, K.S.; Shin, K.H. The effects of reality programming in the selection of tour destinations for foreign travelers: Focused on the show 'grandpa rather than flowers' (Taiwan). Northeast Asia Tour. Res. 2016, $12,21-39$ 
8. Cole, T.; Leets, L. Attachment styles and intimate television viewing: Insecurely forming relationships in a parasocial way. J. Soc. Pers. Relatsh. 1999, 16, 495-511. [CrossRef]

9. Katz, E.; Blumler, J.G.; Gurevitch, M. Utilization of mass communication by the individual. In The Uses of Mass Communications: Current Perspective on Gratifications Research; Blumler, J.G., Katz, E., Eds.; Sage Kayak: Beverly Hills, CA, USA, 2019.

10. Ha, S.H.; Park, S.B. Rethinking of TV viewing Satisfaction: Relationships among TV viewing Motivation, Parasocial Interaction, and Presence. Korean J. Broadcast. Telecommun. Stud. 2007, 21, 339-379.

11. Pope, L.; Latimer, L.; Wansink, B. Viewers vs. doers. The relationship between watching food television and BMI. Appetite 2015, 90, 131-135. [CrossRef]

12. Kim, B.K.; Yhang, W.J. The Effect of Tourism Destination Placement Types in Real Variety Program on Indirect Experience and Attidute: By the Moderating Effect of Parasocial Interaction. Ph.D. Thesis, Pukyong National University, Busan, Korea, 2019.

13. Kim, D.H.; Lee, J.W. The effects of the experience of Korean Wave contents and Korean product and services on preference for the Korean Wave, Change in Korea awareness and behavior intention: Focusing on China, Japan and USA. Korean J. Bus. Adm. 2019, 32, 2107-2130. [CrossRef]

14. Lim, Y.J. A Study on the uses and gratifications on the U. S. TV dramas: Focusing on comparison to the Korean counterparts. Korean J. Commun. Inf. 2008, 41, 302-336.

15. Ban, O.S.; Park, J.Y. A Study on motivations and effects of viewing reality date programs: Focusing on $<$ The Partner > on SBS. Soc. Sci. Res. Rev. Kyunhsung Univ. 2014, 29, 47-70.

16. An, S.Y.; Han, J.S. A study on the influence of viewing motivation of a TV reality travel program on viewing satisfaction and visit intention: Moderating effect of travel involvement. Int. J. Tour. Hosp. Res. 2018, 32, 5-19.

17. Nam, J.A.; Cho, S.H. Motivations and gratifications of re-watchng terrestrial broadcasting programs. Korean Reg. Commun. Res. Assoc. 2013, 14, 275-304.

18. Lee, K.M. Presence, explicated. Commun. Theory 2004, 14, 27-50. [CrossRef]

19. Biocca, F. The cyborg's dilemma: Progressive embodiment in virtual environments. J. Comput. Mediat. Commun. 1997, 3, 3. [CrossRef]

20. March, R.; Woodside, A.G. Testing theory of planned versus realized tourism behavior. Ann. Tour. Res. 2005, 32, 905-924. [CrossRef]

21. Tessitore, T.; Pandelaere, M.; Van Kerckhove, A. The amazing race to India: Prominence in reality television affects destination image and travel intentions. Tour. Manag. 2014, 42, 3-12. [CrossRef]

22. Lombard, M.; Ditton, T. At the heart of it all: The concept of presence. J. Comput. Mediat. Commun. 1997, 3. [CrossRef]

23. Bracken, C. Presence and image quality: The case of high-definition television. Media Psychol. 2009, 7, 191-205. [CrossRef]

24. Katz, E. Mass communications research and the study of popular culture: An editorial note on a possible future for this journal. Stud. Public Commun. 1959, 2, 1-6. Available online: http://repository.upenn.edu/asc_papers (accessed on 21 March 2020).

25. Godlewski, L.R.; Perse, E.M. Audience Activity and Reality Television: Identification, Online Activity, and Satisfaction. Commun. Q. 2010, 58, 148-169. [CrossRef]

26. Kumar, V.V.; Arulchelvan, S. Motivation and impact of viewing reality Television programme: An audience study. Indian J. Sci. Technol. 2015, 8, 1-9.

27. Palmgreen, P.; Rayburn, J.D. Merging uses and gratifications and expectancy-value theory. Commun. Res. 1984, 11, 537-562. [CrossRef]

28. Han, H.; Hsu, L.T.J.; Sheu, C. Application of the theory of planned behavior to green hotel choice: Testing the effect of environmental friendly activities. Tour. Manag. 2010, 31, 325-334. [CrossRef]

29. Seo, B.J.; Kim, M.G.; Lee, C.H. The effect of TV viewers' perceived similarity, wishful identification, and para-social interaction on tourism Attitude: In the case of a travel-reality show. Tour. Res. Inst. Hanyang Univ. 2018, 1, 29-51.

30. Bettinghaus, E.P.; Cody, M.J. Persuasive Communication; Holt, Rinehart and Winston: New York, NY, USA, 1987.

31. Fishbein, M.; Ajzen, I. Belief. Attitude, Intention and Behavior: An Introduction to Theory and Research Reading; Addison-Wesley: Amherst, MA, USA, 1975; Volume 6.

32. Gerrig, R.J.; Zimbardo, P.G.; Campbell, A.J.; Cumming, S.R.; Wilkes, F.J. Psychology and Life; Pearson Higher Education AU: London, UK, 2015. 
33. Eagly, A.H.; Chaiken, S. The advantages of an inclusive definition of ttitude. Soc. Cogn. 2007, 25, 582-602. [CrossRef]

34. Ajzen, I. The theory of planned behavior. Organ. Behav. Hum. Decis. Process. 1991, 50, 179-211. [CrossRef]

35. Han, H.; Lee, S.; Lee, C.K. Extending the theory of planned behavior: Visa exemptions and the traveler decision-making process. Tour. Geogr. 2011, 13, 45-74. [CrossRef]

36. McQuail, D. Audience formation and experience. In Mass Communication Theory, 5th ed.; Sage: London, UK; Thousand Oaks, CA, USA, 2005; pp. 419-452.

37. Steuer, J. Defining virtual reality: Dimensions determining terepresence. J. Commun. 1992, 42, 73-93. [CrossRef]

38. Brown, J.R.; Israeli, A.A.; Mehrez, A. Modelling a decision maker's preferences with different assumptions about the preference structure. Tour. Econ. 2002, 8, 39-57. [CrossRef]

39. Yi, S.E.; Kang, C.H. The effect of tourist attraction exposure in a TV program on the tourist Volume: The case of tvN "Over Flowers" Series. Korean Assoc. Appl. Econ. 2018, 20, 5-35.

40. Chan, B. Film-induced tourism in Asia: A case study of Korean television drama and female viewers' motivation to visit Korea. Tour. Cult. Commun. 2007, 7, 207-224. [CrossRef]

41. Bracken, C.; Ditton, T.B. Presence and television: The role of screen size. Hum. Commun. Res. 2000, 26, 75-98. [CrossRef]

42. Lam, T.; Hsu, C.H. Theory of planned behavior: Potential travelers from China. J. Hosp. Tour. Res. 2004, 28, 463-482. [CrossRef]

43. Ebersole, S.; Woods, R. Motivations for viewing reality Television: A uses and gratifications analysis. Southwest Mass Commun. J. 2007, 23, 23-42.

44. Jang, Y.J.; Kim, M.R. Need for interaction or Pursuit of information and entertainment? The relationship among viewing motivation, presence, parasocial interaction, and satisfaction of eating and cooking broadcasts. Korean J. Broadcast. Telecommun. Stud. 2016, 30, 152-185.

45. Lindner, J.R.; Murphy, T.H. Handling nonresponse in social science research. J. Agric. Educ. 2001, 42, 43-53. [CrossRef]

46. Nunnally, J.C. Psychometric Theory, 2nd ed.; McGraw-Hill: New York, NY, USA, 1978.

47. Hair, J.F.; Black, W.C.; Babin, B.J.; Anderson, R.E.; Tatham, R.L. Multivariate Data Analysis; Pearson Prentice Hall: Upper Saddle River, NJ, USA, 2006; Volume 6.

48. Kim, D.W.; Lee, Y.J. The impact of watching motives of parenting reality TV program on user satisfaction and rewatching. J. Broadcast Eng. 2014, 19, 925-933. [CrossRef]

49. Lee, H.E.; Choi, H.S. A Study on the Effect of Entertainment Show on the Tourism. Korea Contents Soc. 2016, 215-216.

50. Quintal, V.A.; Lee, J.A.; Soutar, G.N. Risk, uncertainty and the theory of planned behavior: A tourism example. Tour. Manag. 2010, 31,797-805. [CrossRef]

51. Park, J.Y. Prospects for Korea Tourism trend 2020-2024. Korea Tour. Policy 2019, 78, 12-19.

(C) 2020 by the authors. Licensee MDPI, Basel, Switzerland. This article is an open access article distributed under the terms and conditions of the Creative Commons Attribution (CC BY) license (http://creativecommons.org/licenses/by/4.0/). 

Article

\title{
Topic Modeling of Online Accommodation Reviews via Latent Dirichlet Allocation
}

\author{
Ian Sutherland, Youngseok Sim, Seul Ki Lee, Jaemun Byun and Kiattipoom Kiatkawsin * \\ Department of Hospitality and Tourism Management, Tourism Industry Data Analytics Lab (TIDAL), \\ Sejong University, Seoul 05006; ianosutherland@gmail.com (I.S.), iamssys@gmail.com (Y.S.), \\ seulkilee@sejong.ac.kr (S.K.L.), jaemun.byun@sejong.ac.kr (J.B.) \\ * Correspondence: kiatkawsin@gmail.com
}

Received: 24 January 2020; Accepted: 27 February 2020; Published: 28 February 2020

\begin{abstract}
There is a lot of attention given to the determinants of guest satisfaction and consumer behavior in the tourism literature. While much extant literature uses a deductive approach for identifying guest satisfaction dimensions, we apply an inductive approach by utilizing large unstructured text data of 104,161 online reviews of Korean accommodation customers to frame which topics of interest guests find important. Using latent Dirichlet allocation, a generative, Bayesian, hierarchical statistical model, we extract and validate topics of interest in the dataset. The results corroborate extant literature in that dimensions, such as location and service quality, are important. However, we extend existing dimensions of importance by more precisely distinguishing aspects of location and service quality. Furthermore, by comparing the characteristics of the accommodations in terms of metropolitan versus rural and the type of accommodation, we reveal differences in topics of importance between different characteristics of the accommodations. Specifically, we find a higher importance for points of competition and points of uniqueness among the accommodation characteristics. This has implications for how managers can improve customer satisfaction and how researchers can more precisely measure customer satisfaction in the hospitality industry.
\end{abstract}

Keywords: topic modeling; latent Dirichlet allocation; tourism 4.0; online travel agency; online review; text analytics; improve customer satisfaction; inductive approach; dimensions of interest; era of big data

\section{Introduction}

Customer satisfaction is "a person's feelings of pleasure or disappointment that results from comparing a product's perceived performance or outcome with his/her expectations" [1]. Prior research in service industries has shown that customer satisfaction has a strong relationship with future repurchase intention, dubbed customer loyalty [2-4]. This link, in turn, is also suggested to have a strong impact on firm performance in service industries $[5,6]$. In the tourism industry specifically, research has shown that the quality of visitors' relationship with a destination has a positive impact on their environmentally responsible behavior as well [7]. However, the subjective nature of meeting customer expectations makes measuring customer satisfaction notoriously difficult in the service sector since many dimensions exist to shape customer expectations.

A new paradigm in the form of Industry 4.0 potentially lends itself to holding many of the answers to the barriers that such difficult problems posed in the past $[8,9]$. Industry 4.0 combines "smart objects" (such as products and machines) with internet technology, allowing for economies of scale but for small-batch-sized productions [8]. This concept has been applied to service industries as well, and in the tourism industry it has been dubbed "Tourism 4.0" among some academics and industry professionals [10-13]. Due to the recency of the Tourism 4.0 paradigm, industry and governments have adopted the concept, for example a government project by Slovenia uses the term $[10,13]$, though 
there is little academic research on the concept as of yet. Two of the key information technologies of Tourism 4.0, artificial intelligence and big data [10], are suggested by this study to be robust for identifying customers' topics of discussion by utilizing online user-generated content. As part of the greater Industry 4.0 paradigm, Tourism 4.0 is defined by an "individualization on demand" wherein a shift to a buyer's market rather than a seller's market is a defining characteristic [8] (p. 239), thereby emphasizing the customers' perspective over the sellers'.

We submit that customer satisfaction should be approached from the customer perspective. As such, we argue that online reviews offer a richer source of data than traditional survey methods for better understanding the holistic experiences of customers. Utilizing a topic modeling technique called latent Dirichlet allocation (LDA), we extract topics of discussion in online reviews because they reflect the topics that customers find important enough to discuss, but are not confined to only the service encounter itself nor confined by existing theoretical constructs of service quality. Comparing the topics between different types of accommodations and locations, a better understanding of customer satisfaction can be achieved relative to traditional, deductive approaches. Nevertheless, extant constructs of determinants of customers' experiences in hospitality literature, are critical in the validation of useful information extracted from online reviews, and therefore are presented in greater detail in Section 2.

\section{Literature Review}

\subsection{Measurement Scales of Customer Satisfaction in Hospitality}

In 1986, Parasuraman, Zeithaml, and Berry published a multi-item survey to gauge the perceptions of service quality in four service sectors and called it SERVQUAL. Subsequent research critiqued SERVQUAL on that it does not adequately cover all service settings [14], and was found not to be optimal for application in the hospitality industry [15-17]. Several industry-specific modifications of the SERVQUAL scale have been introduced to suit the specific needs of customers within the context of the hospitality industry, such as LODGQUAL [15] and HOLSERV [17]. However, these measures focus mainly on hotel customers rather than customers in other accommodation types. Some researchers argue that SERVQUAL, along with similar measures, overemphasizes the provider and fails to represent a modern view of customer satisfaction wherein not only the rational functional product/service benefits, but the irrational emotional benefits affect purchasing decisions [18-20].

As such, the Customer Experience Quality (EXQ) construct was proposed and validated to encompass a more holistic perspective of the customers' experiences [20,21]. However, implementation of EXQ has seen mixed results in the hospitality and tourism literature, such as a recent study in the tourism context that found that the structure of EXQ's four dimensions were not confirmed via confirmatory factor analysis [22]. The shortcomings of currently used scales suggest that more research into customer satisfaction dimensions is still needed.

\subsection{Dimensions of Customer Satisfaction in Hospitality}

Measurement of customer satisfaction relies on sufficiently measuring all relevant dimensions of the customer experience. As the most commonly utilized instrument, SERVQUAL measures the perception of quality of the services provided via 22 items in five subscales, which include Tangibility (physical characteristics of the service encounter), Reliability (proper performance of services), Responsiveness (employees' willingness to help), Assurance (employees' knowledge and courtesy), and Empathy (employees' ability to care) [23].

LODGQUAL identified three dimensions as important: Tangibility, Reliability, and a new dimension, Contact (representing the contact experience with employees) [15]. Similarly, HOLSERV found three dimensions: Employees (willingness to help, polite, knowledgeable, etc.), Tangibles (modern-looking equipment, fixtures and fittings, etc.), and Reliability (accurate and timely service, safe and secure stay, etc.) [17]. 
More recently, research has continued to develop using a variety of dimensions and questionnaire items for specific usage in different contexts within the hospitality industry. One research paper into Jordanian hotels used a modified SERVQUAL questionnaire called SERVPERF to evaluate hotel guests [24]. Research in Serbian 3-star hotels used SERVQUAL [25], and in Taiwan hotel guests were surveyed using another modified version of SERVQUAL [26].

\subsection{Satisfaction and Accommodation Modalities}

While a significantly large amount of research exists surrounding hotels and other traditional accommodation types, a relatively smaller amount of research has been done on specialist accommodation. While numerous studies have tried to define the concept of specialist accommodation in various ways, such as 'supplementary accommodation' [27], 'boutique accommodation' [28], and 'specialist accommodation' $[29,30]$, we adhere to the terminology of 'commercial home enterprises' referring to accommodation in which guests may stay in private homes or other facilities that often have shared living areas with a relatively high level of interaction with owners, hosts or other guests, such as B\&Bs, homestays, guesthouses, etc. [31-33]. In commercial home enterprises (CHEs), customers have different expectations since the products and services offered differ from traditional hotels [30]. Owners of commercial homes "prioritize personal values, quality of life, and personal interactions above economic gains" and it is reflected in the dimensions valued by CHE guests [34] (p. 115).

A study of bed and breakfasts in the United States found that important dimensions included personalized service, homeliness feel, bed comfort, privacy, cleanliness, and the helpfulness of advice about nearby restaurants and other attractions by the owner [35]. In South Africa, business travelers were observed as finding importance in several other dimensions for bed and breakfasts, such as professional and functional skills and abilities, amenities in general and those provided only for the room, ambiance, secure parking, and staff professionalism [36]. For bed and breakfasts in Taiwan, the facilities, service, environment, management, and emotive aspects were found to be important dimensions [37], while another Taiwanese bed and breakfast study found that the physical environment (e.g., equipment, décor, design ambiance), and personal interaction (e.g., staff expertise and problem solving skills) were important factors [38].

For guesthouses in China, a home atmosphere, facilities in the room and on the premises, cleanliness, service quality, value for money, and the location were found to be important dimensions in an analysis of online user-generated content (UGC) [39]. Similarly, another study of guesthouses in China found that sanitation, service and climate, room facilities, bedding, and shower were important dimensions [30]. It was found that for guesthouses in Ghana, atmospherics, enjoyment, entertainment, feeling of escape from daily life, efficiency, excellence, and economic value were important dimensions [40].

In Korea, an endemic, traditional type of CHE accommodation exists called a "hanok", and it is popular for both domestic and international tourists. Hanok "literally means Korean house and it refers to an abode designed according to the traditional principles of Korean architectural culture" [34]. The traditional principles are known as pungsu-jiri (i.e., Feng Shui), and they direct how the traditional hanok accommodations are constructed [41]. As such, guests have very different expectations of the product and services offered at such establishments. While research is minimal on the subject of customer satisfaction dimensions for Korean hanoks, a study showed that hanok guests value staff and facilities (e.g., staff was helpful, bathrooms were clean, etc.), emotional value (e.g., pleasant stay experience), and functional value (e.g., value for price), wherein all dimensions had either direct or indirect impacts on revisit intention and word-of-mouth [42].

\subsection{Extracting Dimensions of Interest in the Era of Big Data}

Research suggests that big data, such as online reviews, is key to solving problems of the 21st century, such as by helping to reach sustainability development goals [43] or by giving insight into consumer experiences and behaviors [44]. Online reviews offer great insight into customer experiences 
for most services and products, and recent improvements in computing along with advances in methodologies have allowed for the analysis of online reviews at scale. There is evidence showing that online product ratings do not significantly impact sales $[45,46]$. On the other hand, many studies show a correlation between sales of products and customer's online reviews [47-50]. Thus, implying that the content of online text reviews can be more important than numeric ratings. One study found that recommendations of book reviews were significant with book sales using 58,566 reviews of 610 books via multiple regression [47]. Similarly, another study determined a significant correlation exists between posted reviews and box office sales via pooled regression of 12,136 reviews of 40 movies [48]. Using the differences-indifferences method to analyze 134,904 reviews of 1636 books and 176,112 reviews of 2387 books, respectively, at different time points, it was demonstrated that improvement in book reviews can lead to an increase in relative book sales [49]. A logistic regression analysis of 31,522 reviews of 148 digital cameras indicates that reviews of products on review sites is an effective indicator of sales for high-involvement products [50]. Tobit regression on 1587 reviews of six products showed that while review depth is correlated with helpfulness, review extremity is less helpful particularly for experience goods [45]. Furthermore, no significant impact on box office revenues was found from the ratings of online reviews in a study of 95,867 reviews of 71 movies via three-stage least squares [46].

A comprehensive review of tourism literature for service quality showed that the most prominent issue in the literature from 2008 to 2019 was the impact on customer behavior from service quality [51]. In tourism and service sectors, such as the hospitality industry, studies show that the qualitative content in text of online reviews are influential to customers and offer rich content regarding customer experiences. One study used logistic regression on 5090 restaurant reviews to discover that the qualitative aspects of online reviews were the most influential factor in determining whether the reviews were useful or not [48]. Furthermore, another study used 5090 reviews of 45 restaurants in New York and London to find that the valence of the reviews has a U-shaped effect on usefulness and enjoyment, wherein negative ratings are more useful and positive ratings are associated with more enjoyment [52]. Using content analysis of 42,886 reviews of 774 Beijing hotels, customer satisfaction determinants were extracted through analyzing online reviews [53]. More recently, latent Dirichlet allocation was used to analyze 266,544 reviews from 39,287 unique reviewers of 25,670 hotels in 16 countries in order to extract the relative importance of customer experience dimensions [54]. A total of 29 dimensions were identified, of which, room experience and service quality were deemed most important [54]. In this study, we utilized the same technique to extract the dimensions of customer satisfaction within various accommodation types of South Korea in order to extract and compare the accommodation types without the confounding effect of country-specific differences.

\section{Materials and Methods}

\subsection{Data Collection and Screening}

This study applied latent Dirichlet allocation to Online Travel Agencies' (OTAs) review corpora. Using scraping techniques applied through Java, the OTA reviews of accommodations across South Korea were extracted from Booking.com, Hotels.com, and Agoda.com over the course of one year, from January 1, 2018 to December 31, 2018. Subsetting the data for only English reviews, the 120,875 observations in the English subset were then cleaned to exclude duplicates and short texts of four words or less, resulting in a final dataset for analysis of 104,161 reviews. Therefore, the final dataset utilized for analysis included text reviews from 104,161 customers and several other corresponding variables including the date of review, data source, name of accommodation, location of accommodation, review rating, and type of accommodation.

Reviews were categorized by both location and accommodation type. For location types, reviews were dubbed as urban $(n=88,692)$ for accommodations located in seven Korean metropolitan cities: Seoul, Busan, Incheon, Daegu, Daejeon, Gwangju, and Ulsan. Otherwise, reviews were classified as rural $(n=15,469)$ for accommodations located outside metropolitan cities within six provinces: 
Gyeongi, Gyeongsang, Chungcheon, Jeolla, Gangwon, and Jeju. For accommodation types, hotels and motels were dubbed hotels $(n=22,011)$, Korean hanok-style accommodations in which guests usually sleep on the floor in a traditional-style Korean house were classified as hanok $(n=966)$, and guesthouses, bed and breakfasts, and hostels were named commercial home enterprises (CHEs) $(n=8784)$.

\subsection{Analysis Tools and Techniques}

Javascript was used for the scraping of data, whereas all data handling, screening, processing, and analysis was achieved through $\mathrm{R}$ versions 3.5 and 3.6. Key $\mathrm{R}$ packages used were stringr, tidyverse, and $t m$ for handling data and preprocessing. Dictionary stemming was executed with the hunspell stemming dictionary via the hunspell package. For assessing the fit statistics of different levels of $k$, the ldatuning package was used. Training of the LDA model was accomplished through the topicmodels package. Base R coding was favored wherever applicable.

Preprocessing the text prior to modeling is a critical component of successful topic modeling. The first step in preprocessing textual data is the removing of stopwords. A list of common stopwords, such as 'the', 'a', 'and', etc. was used, as well as an extended list of stopwords specific to the dataset, such as 'lol', 'haha', etc. Tokenization of relevant word chunks and phrases was useful to avoid losing meaning in certain cases, such as 'bus stop', 'taxi stop', 'metro station', etc. Dictionary word stemming was selected over algorithmic stemming in order to minimize the occurrence of eliminating useful words, for example by avoiding short words like 'bed' being stemmed to ' $b$ ' due to the ending of 'ed'. Dictionary stemming also proved to be more successful than algorithmic stemmers by removing proper nouns, which confounded the creation of useful topics, for example it creates topics focused on location names in reviews rather than the content of the reviews.

\section{Results}

\subsection{Topic Extraction}

The number of latent topics is an important decision in topic modeling. The optimal number of topics was assessed using several metrics [55-58]. The number of topics was set at $14(k=14)$ since it is the optimal number of topics that maximizes information divergence between all pairs of topics [58]. The information divergence criterion was chosen for its sensitivity to changes in the number of latent topics which was found to yield the most practical results in exploratory tests.

After extracting 14 topics through latent Dirichlet allocation, the topics were validated in a four-step procedure. First, a panel of four professors of hospitality and tourism management and one industry practitioner individually assessed the keywords with the highest beta values for each of the 14 topics. Second, the panel was given the topics in context using the top reviews with the highest proportions of each topic. In the third step, each topic was named accordingly by each panel member individually. Fourth, the topics were discussed and voted on until the panel reached a unanimous decision on the most suitable names for each of the 14 topics.

The reviews in the dataset were given probabilities by topic via the posterior distribution of the LDA model. Each review was classified into one of the 14 topics based on the topic that comprised the highest proportion of that review. The classifications were then analyzed by region and accommodation type.

\subsection{Topic Identification}

The 14 topics that were extracted via the latent Dirichlet allocation model are summarized in Table 1. Keywords were determined by those words with the highest beta value within the topic, i.e., the words with the highest relative probability of belonging to the given topic and were organized in order from most important to least important keyword per topic. As LDA is an admixture model, the same words can belong to more than one topic. Below, we briefly introduce each topic and the meaning of each. 
Table 1. Latent topics with relevant keywords.

\begin{tabular}{|c|c|}
\hline Topic & Relevant Keywords \\
\hline Evaluation & $\begin{array}{l}\text { Stay, recommend, back, enjoy, pleasant, experience, happy, satisfy, wonderful, } \\
\text { expectation, comfort, love, plan, future, regret, exceed, mention }\end{array}$ \\
\hline Value & $\begin{array}{l}\text { Location, price, money, facility, cheap, worth, reason, quality, afford, choice, } \\
\text { budget, expensive, decent, paid, valuable, competitive, comparison }\end{array}$ \\
\hline Reception & $\begin{array}{l}\text { Check, book, day, desk, front, late, arrive, pay, quote, reception, morning, call, } \\
\text { charge, wait, receptionist, key, card, leave, request, rude, lady, guy }\end{array}$ \\
\hline Room Size & $\begin{array}{l}\text { Room, small, big, luggage, size, space, floor, large, family, double, lift, person, } \\
\text { single, upgrade, move, standard, bigger, carry, stairs, elevator }\end{array}$ \\
\hline Staff Professionalism & $\begin{array}{l}\text { Staff, friendly, helpful, service, speak, accommodate, kind, friend, polite, } \\
\text { communicate, smile, spoke, professional, language, fluent, courteous, language }\end{array}$ \\
\hline Hospitality & $\begin{array}{l}\text { Hostel, guesthouse, guest, owner, host, kind, feel, house, home, travel, people, } \\
\text { atmosphere, experience, love, warm, traditional, meet, beer, care, accommodate }\end{array}$ \\
\hline Amenities & $\begin{array}{l}\text { Breakfast, free, provide, coffee, water, towel, toiletries, include, available, kitchen, } \\
\text { basic, laundry, machine, toast, drink, tea, buffet, amenity, shampoo, fridge, egg }\end{array}$ \\
\hline Accessibility & $\begin{array}{l}\text { Hotel, airport, bus, stop, convenient, taxi, shuttle, drive, terminal, pick, limousine, } \\
\text { road, travel, transfer, international, overnight, express, layover, flight, hour }\end{array}$ \\
\hline Mobility & $\begin{array}{l}\text { Station, walk, subway, close, convenient, minute, location, distance, store, metro, } \\
\text { train, exit, convenience, market, station, nearby, street, road, connect }\end{array}$ \\
\hline Neighborhood & $\begin{array}{l}\text { Area, restaurant, shopping, close, location, food, street, nearby, quiet, local, café, } \\
\text { central, attraction, convenient, market, busy, mall, bar, sightseeing, district, safe }\end{array}$ \\
\hline Facilities & $\begin{array}{l}\text { Location, perfect, view, park, beach, beautiful, pool, facilities, relax, rooftop, gym, } \\
\text { sea, bar, spa, ocean, swimming, lounge, garden, sauna, balcony, terrace, mountain }\end{array}$ \\
\hline Ambiance & $\begin{array}{l}\text { Room, night, sleep, open, hot, air, window, light, cold, noisy, problem, dark, wall, } \\
\text { conditioner, heat, noise, warm, hear, heater, cool, floor, stuffy, fan, season, winter }\end{array}$ \\
\hline Room Condition & $\begin{array}{l}\text { Bathroom, shower, room, toilet, floor, towel, smell, bad, dirty, bath, door, pillow, } \\
\text { sheet, hair, smoke, lock, mold, housekeeping, privacy, broken, soft, hard, stain }\end{array}$ \\
\hline Decor & $\begin{array}{l}\text { Clean, nice, comfortable, spacious, bed, modern, quiet, cozy, tidy, sign, decorate, } \\
\text { interior, equipped, stylish, furnishing, chic, décor, minimalist, elegant, stiff, neat }\end{array}$ \\
\hline
\end{tabular}

General guest satisfaction topics include the overall evaluation and the value of the stay. The evaluation topic encompasses general guest evaluations in the veins of return intention (e.g., 'back', 'plan', 'future', 'regret'), meeting/exceeding expectations (e.g., 'expectations', 'satisfy', 'exceed') and recommending to others (e.g., 'recommend', 'mention'). The value topic relates to the price-quality relationship. Keywords related to the accommodation's relative quality (e.g., 'choice', 'quality', 'valuable', 'competitive', 'comparison') signify a quality comparison with competitors in relation to keywords related to price (e.g., 'price', 'money', 'cheap', 'expensive', 'afford').

Service-related topics include reception, staff professionalism, hospitality, and amenities. The topic of reception includes the tasks associated with reception such as checking in and out (e.g., 'check', 'booking', 'arrival', 'leave'), payment (e.g., 'pay', 'charge', 'quote'), and others (e.g., 'card', 'morning', 'call', 'request'), as well as includes the reception staff (e.g., 'receptionist', 'lady', 'guy', 'rude'). The staff professionalism topic encompasses staff service quality (e.g., 'friendly', 'helpful', 'service', 'polite', 'professional'), and communication ability (e.g., 'speak', 'communicate', 'fluent', 'language'). The hospitality topic emphasizes the emotional warmth of the experience (e.g., 'feel', 'experience', 'warm', 'atmosphere') and the hospitality of the owner or staff (e.g., 'owner', 'people', 'kind', 'accommodate', 'care'). The topic of amenities consists of bathroom amenities (e.g., 'towel', 'toiletries', 'shampoo'), room amenities (e.g., 'laundry', 'machine', 'fridge', 'kitchen'), free mini-bar items ('free', 'included', 'provided', 'coffee', 'tea', 'water' ), and breakfast (e.g., 'breakfast', 'toast', 'egg', 'cereal', 'fresh'). 
Location-related topics include accessibility, mobility, and the surrounding neighborhood. The topic of accessibility relates to transportation (e.g., 'airport', 'terminal', 'international', 'stop', 'transfer') to and from the accommodation from a long distance (e.g., 'overnight', 'express', 'hour', 'layover'). Specifically, modes of transportation often associated with travel to and from the airport are observed (e.g., 'shuttle', 'limousine', 'bus', 'taxi', 'flight'). The topic of mobility relates to transportation (e.g., 'station', 'street', 'distance', 'road', 'exit', 'connect') to and around the accommodation locally (e.g., 'close', 'convenient', 'minute', 'nearby'). Specifically, modes of transportation often associated with travel within a localized area or city are observed (e.g., 'subway', 'metro', 'train', 'walk'). The neighborhood topic encompasses the local attractions (e.g., 'restaurant', 'shopping', 'café', 'market', 'mall', 'bar', 'sightseeing'), centrality (e.g., 'nearby', 'local', 'centrality', 'district', 'heart', 'middle'), and local atmosphere (e.g., 'quiet', 'busy', 'crowded', 'safe') of the location.

Topics related to the physical condition of the room include room size, room condition and décor. The room size topic relates specifically to the size of the room (e.g., 'room, 'small, 'big, 'size', 'space', 'floor', 'large', 'bigger'), type of room ('family', 'double', 'single', 'person', 'upgrade', 'standard'), and changing of rooms along with moving of luggage (e.g., 'luggage', 'upgrade', 'move', 'lift', 'carry', 'stairs', 'elevator'). The room condition topic relates to the physical aspects of the room (e.g., 'bathroom', 'shower', 'toilet', 'floor', 'pillow', 'sheet', 'sink', 'wall', 'door') in relation to the condition of those physical aspects in terms of cleanliness (e.g., 'smell', 'dirty', 'change', 'hair', 'smoke', 'stain', 'wet', 'mold', 'housekeeping'), and comfort (e.g., 'hard', 'privacy', 'broken', 'soft'). The decor topic refers to the interior decoration and design of the room (e.g., 'decorate', 'interior', 'modern', 'chic', 'stylish', 'furnishing', 'minimalist', 'decor', 'elegant', 'stiff') as well as the general comfort and atmosphere (e.g., 'clean', 'comfortable', 'cozy', 'spacious', 'neat', 'environment', 'roomy').

Topics related to physical condition of the environment of the accommodation include the facilities and ambiance. The topic dubbed facilities relates to the facilities of the accommodation (e.g., 'facility', 'pool', 'rooftop', 'gym', 'bar', 'spa', 'lounge', 'sauna', 'balcony', 'garden', 'terrace'), and, to a lesser extent, the view of the surrounding area (e.g., 'view', 'beautiful', 'ocean', 'beach', 'sea', 'park', 'mountain'). The ambiance topic mainly encompasses three distinct ambient qualities of the room including sound (e.g., 'noisy', 'hear', 'sound', 'loud'), light (e.g., 'light', 'dark'), and air (e.g., 'hot', 'cold', 'heat', 'stuffy', 'ventilation'). Things that directly affect these ambient qualities of the room are also included in the topic (e.g., 'AC', 'air', 'conditioner', 'heater', 'floor', 'window', 'fan', 'thin', 'wall', 'construction', 'season', 'winter', 'summer').

\subsection{Topic Validation}

In addition to keyword analysis of words with the highest beta values in each topic, validation of the topics was achieved by analyzing the review text with the highest proportion of the given topic. For example, the review with the highest proportion of the accessibility topic starts off with, "Bad memory about Airport shuttle bus to hotel. We catched a Limousine Airport bus to [the] hotel ... but I didn't know the bus stop at [the] hotel was the opposite road, we lost our way to Myeongdong ... ", and continues discussing the author's difficulties with accessibility from the airport to the hotel. Validation of topics was achieved by analyzing the top 10 reviews for each topic by each member of the panel, in addition to the keywords, and then voting until unanimity of naming the topics was achieved by the panel. For the sake of length, the full validation of topics is not expanded upon in this paper, however the authors strongly urge the importance of validating the topics in LDA by analyzing not only the top keywords, but analyzing the words in context of the reviews by evaluating the most prominent review for each topic.

\subsection{Comparisons of Accommodation Characteristics}

The number of reviews for each topic by the characteristics of urbanization of location, and accommodation types are reported in Table 2, while Table 3 reports the proportions of reviews as a percentage of each trait. Due to the relatively small sample size of hanok reviews, a larger discrepancy 
is required for statistical significance, so differences in proportion sizes should be inferred with caution in the hanok category.

Table 2. Latent topic review frequencies by accommodation characteristic.

\begin{tabular}{llccccc}
\hline \multicolumn{1}{c}{ Topic } & Urban & Rural & Hanok & Hotel & CHE \\
\hline 1. & Evaluation & 8469 & 1439 & 99 & 2097 & 796 \\
\hline 2. & Value & 7507 & 1442 & 56 & 2085 & 662 \\
\hline 3. & Reception & 7880 & 1214 & 101 & 1833 & 814 \\
\hline 4. & Room Size & 6780 & 1083 & 76 & 1623 & 695 \\
\hline 5. & Staff Professionalism & 8159 & 1259 & 70 & 1955 & 730 \\
\hline 6. & Hospitality & 6371 & 1232 & 76 & 1641 & 667 \\
\hline 7. & Amenities & 6542 & 1143 & 70 & 1609 & 751 \\
\hline 8. & Accessibility & 5721 & 934 & 49 & 1445 & 518 \\
\hline 9. & Mobility & 5832 & 1194 & 92 & 1555 & 571 \\
\hline 10. & Neighborhood & 5504 & 1064 & 58 & 1393 & 549 \\
\hline 11. & Facilities & 4555 & 716 & 33 & 1089 & 459 \\
\hline 12. & Ambiance & 5168 & 1124 & 85 & 1246 & 595 \\
\hline 13. & Room Condition & 5538 & 895 & 55 & 1316 & 497 \\
\hline 14. & Decor & 4666 & 730 & 46 & 1124 & 480 \\
\hline
\end{tabular}

Table 3. Latent topic review proportions by accommodation characteristic.

\begin{tabular}{|c|c|c|c|c|c|}
\hline Topic & Urban $(\%)$ & Rural (\%) & Hanok (\%) & Hotel (\%) & CHE (\%) \\
\hline 1. Evaluation & $9.5^{*}$ & 9.3 & 10.2 & 9.5 & 9.1 \\
\hline 2. Value & $8.5^{* * *}$ & $9.3^{* * *}$ & $5.8^{* * *}$ & $9.5^{* * *}$ & $7.5^{* * * *}$ \\
\hline 3. Reception & $8.9^{* * *}$ & $7.8^{* * *}$ & 10.5 * & $8.3^{* * *}$ & $9.3^{* *}$ \\
\hline 4. Room Size & $7.6^{* * *}$ & $7.0^{* *}$ & 7.9 & $7.4^{* *}$ & $7.9 \bullet$ \\
\hline 5. Staff Professionalism & $9.2 * * *$ & $8.1^{* * *}$ & 7.2 & $8.9^{* * *}$ & $8.3 * *$ \\
\hline 6. Hospitality & $7.2^{* * *}$ & $8.0^{* * *}$ & 7.9 & 7.5 & 7.6 \\
\hline 7. Amenities & 7.4 & 7.4 & 7.2 & $7.3^{* * *}$ & $8.5^{* * *}$ \\
\hline 8. Accessibility & $6.5^{* * *}$ & $6.0^{*}$ & 5.1 & $6.6^{* * *}$ & $5.9 *$ \\
\hline 9. Mobility & $6.6^{* * *}$ & $7.7^{* * *}$ & $9.5^{* *}$ & 7.1 & $6.5^{*}$ \\
\hline 10. Neighborhood & $6.2^{* * *}$ & $6.9^{* * *}$ & 6.0 & 6.3 & 6.2 \\
\hline 11. Facilities & $5.1^{* * *}$ & $4.6^{* *}$ & $3.4^{*}$ & 4.9 & 5.2 \\
\hline 12. Ambiance & $5.8^{* * *}$ & $7.3^{* * *}$ & $8.8^{* * *}$ & $5.7^{* * *}$ & $6.8^{* * *}$ \\
\hline 13. Room Condition & $6.2^{* * *}$ & $5.8^{*}$ & 5.7 & $6.0 *$ & 5.7 \\
\hline 14. Decor & $5.3^{* * *}$ & $4.7^{* * *}$ & 4.8 & $5.1 \bullet$ & 5.5 \\
\hline
\end{tabular}

Note: statistical significance levels are shown as $0.10 \geq p$-value $>0.05(\bullet), 0.05 \geq p$-value $>0.01\left(^{*}\right), 0.01 \geq p$-value $>$ $0.001\left(^{* *}\right), p$-value $\leq 0.001\left(^{* *}\right)$. Significance levels shown indicate statistical significance in two-proportions $z$-tests between the proportion of a give topic in a subset and proportion of the same topic in all other subsets.

Chi-square goodness of fit tests were applied to assess whether the distributions of topics within each trait (e.g., Urban, Rural, Hanok, etc.) were distributed equally. Wilcoxon Signed Rank tests were applied to assess whether there is a difference between the distribution of topics in one trait with all corresponding traits within the same characteristic (i.e., Urbanization, or Accommodation Type). Two proportions z-tests were applied to test whether a statistically significant difference exists 
between the proportion of each topic for each trait compared to the proportion of each topic in the corresponding traits.

\subsubsection{Urbanization Comparison}

The chi-square goodness of fit tests reveal that topics are not equally distributed in reviews of urban locations $\left(\chi^{2}=3339.40, p\right.$-value $\left.<0.001\right)$ and rural locations $\left(\chi^{2}=591.92, p\right.$-value $\left.<0.001\right)$, thereby showing a difference in the importance of topics among customers staying in both urban and rural locations. Wilcoxon Signed Rank tests demonstrate that the distribution of the prominence of topics between urban versus rural locations are not the same ( $p$-value $<0.001)$. Specifically, the most prominent topics for urban accommodations are evaluation, staff professionalism, and reception. Whereas, rural accommodations observe the highest proportion of reviews regarding both value and evaluation, followed by staff professionalism and hospitality. Comparisons between urban and rural accommodations via the proportions for each topic are visualized in Figure 1.

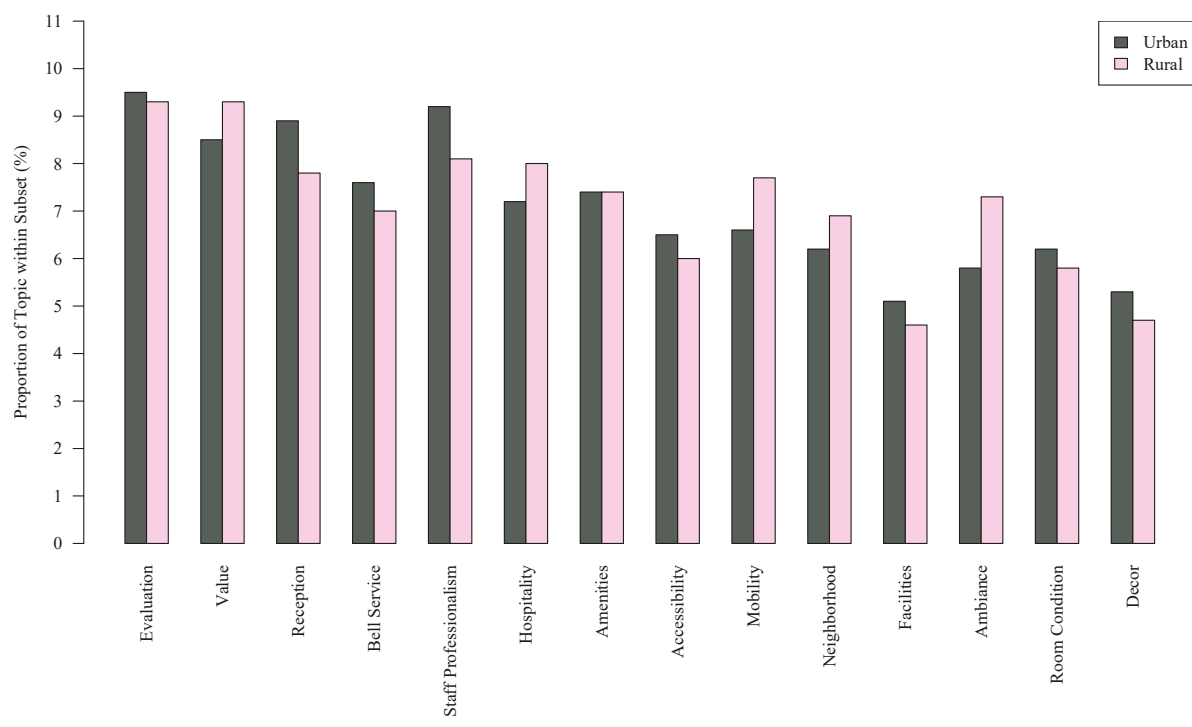

Figure 1. Proportions of topics by urbanization.

Two proportions z-tests determine whether a statistically significant difference exists between the proportion of a given topic between the urban and rural locations and their statistical significance is reported in Table 3. Reviews of rural accommodations relative to urban accommodations have a statistically higher focus on value, hospitality, ambiance, mobility, and neighborhood. We submit that the emphasis on warm hospitality in a location with easy access to the local area and sights is a response of guests wanting to connect with people and the local environment at a low price. The relatively lower emphasis on topics such as reception, room size, staff professionalism, facilities, room condition, and décor reinforce the image of customers staying in the countryside in order to escape the higher luxury and social rigidity of the metropolitan areas, in favor of stronger connections with the local people and the area.

Likewise, the higher focus on reception, room size, staff professionalism, facilities, room condition, and décor in urban accommodations relative to rural locations supports the idea that metropolitan guests tend towards professional service and chic, comfortable facilities with greater access to the airport compared to their rural counterparts. The lower emphasis on value indicates that the metropolitan 
clientele are less concerned about the price and more concerned with their overall satisfaction from higher service quality compared to overnight travelers to countryside towns.

\subsubsection{Accommodation Type Comparison}

The chi-square goodness of fit tests reveal that topics are not equally distributed in reviews of hotels $\left(\chi^{2}=901.02, p\right.$-value $\left.<0.001\right)$, commercial home enterprises $\left(\chi^{2}=296.12, p\right.$-value $\left.<0.001\right)$, and hanoks $\left(\chi^{2}=80.00, p\right.$-value $\left.<0.001\right)$, thereby showing that some topics are more prominent than other topics for each accommodation type. The rank order of topics was compared using dependent two-group Wilcoxon signed rank tests between all pairs of accommodation types. All comparisons show a statistically different rank order between accommodation types at $p<0.001$, meaning that the importance of topics differs between customers staying overnight at hotels, CHEs, and hanok accommodation. Specifically, hotels experience most reviews for evaluation, value, and professionalism. The most prominent topics for CHEs are reception, evaluation, and amenities. While similarly, hanok reviews are most prominent regarding reception, evaluation, and mobility.

Finally, two proportions $z$-tests were performed for each topic within each accommodation type and significance is reported in Table 3 as whether the topic within the type of accommodation is significantly different from the proportion of the same topic in all other accommodation types. The comparative proportions for each topic by accommodation type are visualized in Figure 2.

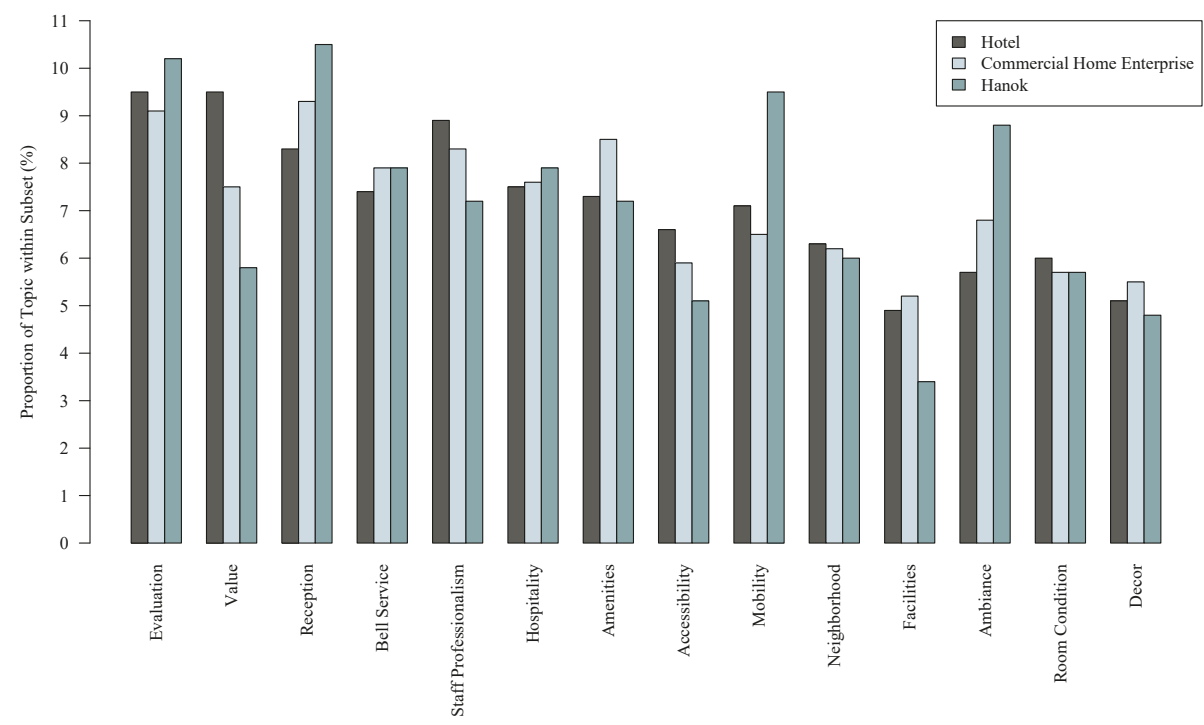

Figure 2. Proportions of topics by accommodation type.

Points of competitiveness and uniqueness tend to be discussed topics with significantly higher proportions, while points that are often standardized or that have less variation are observed with much lower emphasis between accommodation types. A point of particular interest is the high proportion of ambiance in the traditional Korean hanok reviews relative to the lower proportions in CHEs and even lower in hotels. Hanoks are unique in that the building design virtually always has a heated 'ondol' floor system and guests often sleep on mats on the floor, which, from foreign travelers' perspectives are a very unique point to overnight stays in hanoks. This ondol system is sometimes used in commercial home enterprises as well, but is much rarer in hotels. In fact, ambiance is one of the lowest ranking topics in hotels, which may be due to precisely the opposite effect-standardized 
control of air temperature and other ambient factors in hotels make the topic much less variable, and therefore less prominent as a topic of discussion for customers.

Another notable point of competitiveness is high price competition in hotels. The topic of value indicates that a relatively high proportion of reviews are dedicated to discussing the value of the hotel stay, while lesser so in CHEs. Commercial home enterprises, while also competitive on price, are much less so, since CHEs are comparatively less variable in price and already relatively cheap. We expect overnight travelers staying in hanoks are more interested in the experience than the price and are therefore less price sensitive.

While hanoks and hotels sometimes offer breakfast, it is not a considerably strong selling point. However, in CHEs, breakfast and other amenities are a strong selling point since many travelers staying in guesthouses, bed and breakfasts, and hostels are traveling on a budget and most CHEs offer some breakfast service, whether paid or otherwise. As might be expected, due to the competitive nature of amenities in guesthouses, it is of higher concern for reviewers, while much less so in both hotels and hanoks.

What makes each accommodation unique relative to other accommodation types is also a source for statistical differences in topic prevalence. Hotels highly prioritize professional service and, therefore, staff professionalism is notably more prevalent in hotel reviews. However, both hanoks and CHEs are considerably smaller than hotels, so without as many resources for staff service training, hanoks and CHEs instead focus on creating a more intimate and less formal relationship with customers. As such, we see a higher prominence of reception (and while not a significant difference, it may be notable that hospitality is also higher), rather than staff professionalism in CHEs and hanoks relative to hotels.

Another difference between accommodation types is the importance of location. While there are no significant differences between the prominence of the topic of neighborhood, there do exist differences in getting to and from the accommodation locations. Specifically, accessibility to the hotel from long distances is of more importance than accessibility to CHEs and hanoks. We propose that the reason is based on the purpose of stay. While access to the airport is often an important trait for hotels, the main reason of stay in a commercial home enterprise is generally price or connection with the locals, while hanok guests are generally most interested in the experience. Similarly, while mobility to and from the accommodation to the local area is also important in hotels, it is less important in CHEs, since the purpose of stay in guesthouses is price and also connecting with other people. For hanoks, however, mobility is important since hanoks are often built in clusters in close proximity to other hanoks in old neighborhoods. As such, traveling out of the old neighborhoods into the city centers and to other attractions might be an important concern.

\section{Discussion}

\subsection{Conclusions}

Latent Dirichlet allocation provides an inductive, data-driven approach that validates and extends current theory regarding the dimensions that affect customers in the hospitality industry by utilizing large, unstructured text directly coming from the consumers themselves. More precisely, important dimensions seen in literature such as service quality, location, value, etc., are corroborated by the LDA approach. However, LDA extends the theory by offering more precise distinctions between the dimensions. Whereas extant literature identifies the location as important [39,54], this study extracted three dimensions related to the location of the accommodation-its accessibility to and from out-of-town, the mobility in the local area, and the attractions and centrality of the neighborhood. LDA contributes further by distinguishing a statistical difference between different aspects of service quality in the topics of staff professionalism, hospitality, and reception as separate topics, as opposed to different items within the same dimension [24,30,37,39,40,42].

Evaluating the extracted topics by their proportionality within different characteristics of the accommodations reveals that points of competitiveness and uniqueness are the topics of higher relative 
prominence. Competitive points such as value in hotels, and staff professionalism in metropolitan cities are of statistically more prominence relative to the same topics in other accommodation types or rural towns. Points of uniqueness also see higher relative prominence, such as the ambient temperature in hanoks which are heated by traditional floor heating systems, or the neighborhood attractions in rural areas which generally offer more unique attractions than those in metropolitan areas.

\subsection{Implications}

Similarly to other studies using LDA [54], this study identifies more dimensions than traditional studies have identified. Since topics are determined statistically and inductively based on the consumers' perspectives, the results should guide future researchers in developing more precise measurement tools that consider a holistic approach to customer determinants of satisfaction. The results also demonstrate that the importance of topics differs by accommodation characteristics, showing which topics are more important by urbanization of the city or accommodation type. We recommend researchers consider such factors when developing measurement tools, in that, all topics do not have equal weighting for shaping customer experience and those weightings differ for accommodations with different characteristics.

Results indicate topic areas that customers willingly choose to discuss and therefore the results bring the attention of industry practitioners to the topics that are relevant. However, customer reviews should be viewed with caution since they do not indicate valence. Therefore, it is of utmost importance to take the individual characteristics and preferences of the customers into account but to use the recommendations of this research to focus on which areas of interest those customers may focus on. This study helps hotel managers develop those areas of concern within their operations from the consumers perspective, not the providers perspective.

\subsection{Limitations and Future Research}

As the digital world, along with technologies of Tourism 4.0 such as big data and artificial intelligence, are being harnessed more efficiently and effectively in the hospitality industry [12], areas of future research are rapidly opening up. The new technologies in this paradigm shift are fundamental toward better understandings and predictions of tourist and consumer behaviors. Particularly, we hope to see future research applying topic modeling and other techniques in order to better serve the individualized preferences of customers and progress towards more sustainable business practices in the tourism industry. For example, research shows that better prediction and understanding of tourists' behaviors can better help inform decisions on how to mitigate environmental impacts from tourism, such as cruise tourism [59], or with tourists renting cars [60]. We expect that better understanding of visitors' behavior in the hospitality industry will better inform policymakers and managers for better environmental practices as well as business practices.

As with all research, several important limitations should be noted in the study. Using a sample of English reviews of Korean accommodations allows for a more precise understanding of the differences between accommodation types and locations since it is not confounded by the different characteristics that exist between countries within the same accommodation types. It also allows for a clearer distinction between countryside and metropolitan accommodations since, for example, there is more commonality between Korean metropolitan cities than there is between Korean metropolitan cities and other countries' metropolitan cities. However, the inherent weakness of a single-country analysis means a lack of generalizability of the results to other countries. Therefore, we urge that further research be carried out to reveal the distinctions between accommodation characteristics in other countries as well.

Secondly, the use of online reviews may be biased in terms of the topics of interest being discussed. We expect selection bias in terms of which types of customers post reviews may exist, particularly in terms of the extremity of reviews. For example, customers with a particularly good or extremely bad experience are more likely to post an online review to recommend or warn others about the 
accommodation. While this would likely help to distinguish the topics of interest, it may bias the prominence of which topics are represented in terms of tending toward extremity.

Finally, due to limitations in the data available, many accommodation types are unlisted, and some are relatively broad categorizations, so more precision should reveal more distinctions between accommodation type categories. While star-rated hotels were used for the hotel categorization, lower rated hotels are often dubbed motels, and were not distinguished in the data used. Furthermore, different types of commercial home enterprises, such as hostels, guesthouses, pensions, and bed and breakfasts could also not be distinguished between. We expect more differences exist within the three broad categories of accommodation types that were used in this study, and hope that further research will shed light on those differences in the future.

Author Contributions: Conceptualization, I.S., K.K., S.K.L. and Y.S.; data collection, Y.S. and S.K.L., analysis, I.S., K.K., S.K.L. and J.B.; writing, I.S.; editing, I.S., K.K., S.K.L., J.B. and Y.S.; supervision, S.K.L. and J.B. All authors have read and agreed to the published version of the manuscript.

Funding: This research received no external funding

Acknowledgments: We would like to thank the Tourism Industry Data Analytics Lab members who helped support the research with helpful discussion-Youngjoo Ahn, Namho Lee, Young Sun Baek, Nahyoung Kim, and Cheolwoo Park.

Conflicts of Interest: The authors declare no conflict of interest.

\section{References}

1. Kotler, P.; Keller, K.L. Marketing Management, 13th ed.; Pearson Education Inc.: Upper Saddle River, NJ, USA, 2009.

2. Chen, S.-C. The customer satisfaction-loyalty relation in an interactive e-service setting: The mediators. J. Retail. Consum. Serv. 2012, 19, 202-210. [CrossRef]

3. Kumar, V.; Pozza, I.D.; Ganesh, J. Revisiting the Satisfaction-Loyalty Relationship: Empirical Generalizations and Directions for Future Research. J. Retail. 2013, 89, 246-262. [CrossRef]

4. Suh, J.-C.; Youjae, Y. When Brand Attitudes Affect the Customer Satisfaction-Loyalty Relation: The Moderating Role of Product Involvement. J. Consum. Psychol. 2006, 16, 145-155. [CrossRef]

5. Bowen, J.T.; Chen McCain, S.-L. Transitioning loyalty programs: A commentary on "the relationship between customer loyalty and customer satisfaction". Int. J. Contemp. Hosp. Manag. 2015, 27, 415-430. [CrossRef]

6. Lee, H.S. Major Moderators Influencing the Relationships of Service Quality, Customer Satisfaction and Customer Loyalty. Asian Soci. Sci. 2013, 9, 2. [CrossRef]

7. Zhou, X.; Tang, C.; Lv, X.; Xing, B. Visitor Engagement, Relationship Quality, and Environmentally Responsible Behavior. Int. J. Environ. Res. Public. Health 2020, 17, 1151. [CrossRef]

8. Lasi, H.; Fettke, P.; Kemper, H.-G.; Feld, T.; Hoffmann, M. Industry 4.0. Bus. Inf. Syst. Eng. 2014, 6, $239-242$. [CrossRef]

9. Forum, W.E. The future of jobs: Employment, skills and workforce strategy for the fourth industrial revolution. In Proceedings of the Global Challenge Insight Report, World Economic Forum, Geneva, Switzerland, 22 January 2016.

10. Peceny, U.S.; Urbančič, J.; Mokorel, S.; Kuralt, V.; Ilijaš, T. Tourism 4.0: Challenges in Marketing a Paradigm Shift. In Consumer Behavior and Marketing.; IntechOpen: London, UK, 2019.

11. Alexis, P. R-Tourism: Introducing the Potential Impact of Robotics and Service Automation in Tourism. Ovidius Univ. Ann. Ser. Econ. Sci. 2017, 17, 211-216.

12. Żukowska, M.; Szawluk, J.; Mańko, M.; Wodzyńska, A.; Panfiluk, E. TOURISM 4.0—DEVELOPMENT TRENDS OF THE HOTEL INDUSTRY. J. Szydlo Danuta Szpilko 2019, 87. [CrossRef]

13. Urbančič, J.; Kuralt, V.; Ratkajec, H.; Straus, M.; Vavroš, A.; Mokorel, S.; Peceny, U.S.; Ilijaš, T. Expansion of Technology Utilization Through Tourism 4.0 in Slovenia. In Handbook of Research on Smart Technology Applications in the Tourism Industry; IGI Global: Hershey, PA, USA, 2020; pp. 229-253.

14. Carman, J.M. Consumer perceptions of service quality: An assessment of T. J. Retail. 1990, 66, 33.

15. Getty, J.M.; Thompson, K.N. A procedure for scaling perceptions of lodging quality. Hosp. Res. J. 1994, 18, 75-96. [CrossRef] 
16. Saleh, F.; Ryan, C. Analysing service quality in the hospitality industry using the SERVQUAL model. Serv. Ind. J. 1991, 11, 324-345. [CrossRef]

17. Mei, A.W.O.; Dean, A.M.; White, C.J. Analysing service quality in the hospitality industry. Manag. Serv. Q. Int. J. 1999, 11, 324-345.

18. Sureshchandar, G.S.; Rajendran, C.; Anantharaman, R.N. Determinants of customer-perceived service quality: A confirmatory factor analysis approach. J. Serv. Mark. 2002, 16, 9-34. [CrossRef]

19. Verhoef, P.C.; Langerak, F.; Donkers, B. Understanding brand and dealer retention in the new car market: The moderating role of brand tier. J. Retail. 2007, 83, 97-113. [CrossRef]

20. Maklan, S.; Klaus, P. Customer experience: Are we measuring the right things? Int. J. Mark. Res. 2011, 53, 771-772. [CrossRef]

21. Klaus, P. 'Phil'; Maklan, S. Towards a better measure of customer experience. Int. J. Mark. Res. 2013, 55, 227-246. [CrossRef]

22. Milman, A.; Zehrer, A.; Tasci, A.D. Measuring the components of visitor experience on a mountain attraction: The case of the Nordkette, Tyrol, Austria. Tour. Rev. 2017, 72, 429-477. [CrossRef]

23. Parasuraman, A.; Zeithaml, V.A.; Berry, L.L. Servqual: A multiple-item scale for measuring consumer perc. J. Retail. 1988, 64, 12.

24. Al Khattab, S.A.; Aldehayyat, J.S. Perceptions of service quality in Jordanian hotels. Int. J. Bus. Manag. 2011, 6, 226. [CrossRef]

25. BLEŠIĆ, I.; Ivkov-Džigurski, A.; Stankov, U.; Stamenković, I.; Bradić, M. Research of expected and perceived service quality in hotel management. Rev. Tur.-Stud. Si Cercet. Tur. 2011, 11, 6-14.

26. Chang, H.-S. Increasing hotel customer value through service quality cues in Taiwan. Serv. Ind. J. 2008, 28, 73-84. [CrossRef]

27. Nicod, P.; Mungall, A.; Henwood, J. Self-catering accommodation in Switzerland. Int. J. Hosp. Manag. 2007, 26, 244-262. [CrossRef]

28. Morrison, A.M.; Pearce, P.L.; Moscardo, G.; Nadkarni, N.; O’Leary, J.T. Specialist accommodation: Definition, markets served, and roles in tourism development. J. Travel Res. 1996, 35, 18-26. [CrossRef]

29. Pearce, P.L.; Moscardo, G.M. The boutique/specialist accommodation sector: Perceived government needs and policy initiatives. Qld. Small Bus. Res. J. 1992, 1, 34-41.

30. Wang, S.; Hung, K.; Li, M. Development of measurement scale for functional congruity in guest houses. Tour. Manag. 2018, 68, 23-31. [CrossRef]

31. Lynch, P.A. The commercial home enterprise and host: A United Kingdom perspective. Int. J. Hosp. Manag. 2005, 24, 533-553. [CrossRef]

32. McIntosh, A.J.; Lynch, P.; Sweeney, M. "My Home Is My Castle" Defiance of the Commercial Homestay Host in Tourism. J. Travel Res. 2011, 50, 509-519. [CrossRef]

33. Ye, S.; Xiao, H.; Zhou, L. Commodification and perceived authenticity in commercial homes. Ann. Tour. Res. 2018, 71, 39-53. [CrossRef]

34. Choi, Y.; Buzinde, C.N.; Lee, C.-K. Visitor books and guest-generated discourses of hospitality: The case of the Hanok. Asia Pac. J. Tour. Res. 2015, 20, 114-132. [CrossRef]

35. Zane, B. The B \& B guest: A comprehensive view. Cornell Hotel Restaur. Adm. Q. 1997, 38, 69-75.

36. Radder, L.; Wang, Y. Dimensions of guest house service. Int. J. Contemp. Hosp. Manag. 2006, 18, 554. [CrossRef]

37. Wu, W.-C.; Yang, C.-Y. An Empirical Study on the Impact of Differences in Bed and Breakfast Service Quality Attributes on Customers' Revisiting Desires. Int. J. Organ. Innov. Online 2010, 2, 223.

38. Chen, C.M.; Chen, S.H.; Lee, H.T. Interrelationships between physical environment quality, personal interaction quality, satisfaction and behavioural intentions in relation to customer loyalty: The case of Kinmen's bed and breakfast industry. Asia Pac. J. Tour. Res. 2013, 18, 262-287. [CrossRef]

39. Wang, S.; Hung, K. Customer perceptions of critical success factors for guest houses. Int. J. Hosp. Manag. 2015, 48, 92-101. [CrossRef]

40. Amoah, F.; Radder, L.; van Eyk, M. Perceived experience value, satisfaction and behavioural intentions: A guesthouse experience. Afr. J. Econ. Manag. Stud. 2016, 7, 419-433. [CrossRef]

41. Hwang, D.J. Hanok Is Back; Seoul Gong Gan-Sa: Seoul, Korea, 2006. 
42. Behavioral Intentions of International Visitors to the Korean Hanok Guest Houses: Quality, Value and Satisfaction. Available online: https://www.cabdirect.org/cabdirect/abstract/20143249593 (accessed on 17 February 2020).

43. Kharrazi, A.; Qin, H.; Zhang, Y. Urban big data and sustainable development goals: Challenges and opportunities. Sustainability 2016, 8, 1293. [CrossRef]

44. Stoicescu, C. Big Data, the perfect instrument to study today's consumer behavior. Database Syst. J. 2016, 6, 28-42.

45. Mudambi, S.M.; Schuff, D. Research note: What makes a helpful online review? A study of customer reviews on Amazon. com. MIS Q. 2010, 34, 185-200. [CrossRef]

46. Duan, W.; Gu, B.; Whinston, A.B. Do online reviews matter? An empirical investigation of panel data. Decis. Support Syst. 2008, 45, 1007-1016. [CrossRef]

47. Chen, P.-Y.; Wu, S.; Yoon, J. The impact of online recommendations and consumer feedback on sales. ICIS 2004 Proc. 2004, 58.

48. Liu, Y. Word of mouth for movies: Its dynamics and impact on box office revenue. J. Mark. 2006, 70, 74-89. [CrossRef]

49. Chevalier, J.A.; Mayzlin, D. The effect of word of mouth on sales: Online book reviews. J. Mark. Res. 2006, 43, 345-354. [CrossRef]

50. Gu, B.; Park, J.; Konana, P. Research note-the impact of external word-of-mouth sources on retailer sales of high-involvement products. Inf. Syst. Res. 2012, 23, 182-196. [CrossRef]

51. Park, J.; Jeong, E. Service quality in tourism: A systematic literature review and keyword network analysis. Sustainability 2019, 11, 3665. [CrossRef]

52. Park, S.; Nicolau, J.L. Asymmetric effects of online consumer reviews. Ann. Tour. Res. 2015, 50, 67-83. [CrossRef]

53. Li, H.; Ye, Q.; Law, R. Determinants of customer satisfaction in the hotel industry: An application of online review analysis. Asia Pac. J. Tour. Res. 2013, 18, 784-802. [CrossRef]

54. Guo, Y.; Barnes, S.J.; Jia, Q. Mining meaning from online ratings and reviews: Tourist satisfaction analysis using latent dirichlet allocation. Tour. Manag. 2017, 59, 467-483. [CrossRef]

55. Griffiths, T.L.; Steyvers, M. Finding scientific topics. Proc. Natl. Acad. Sci. USA 2004, 101, 5228-5235. [CrossRef]

56. Cao, J.; Xia, T.; Li, J.; Zhang, Y.; Tang, S. A density-based method for adaptive LDA model selection. Neurocomputing 2009, 72, 1775-1781. [CrossRef]

57. Arun, R.; Suresh, V.; Madhavan, C.V.; Murthy, M.N. On finding the natural number of topics with latent dirichlet allocation: Some observations. In Proceedings of the Pacific-Asia Conference on Knowledge Discovery and Data Mining; Springer: Berlin/Heidelberg, Germany, 2010; pp. 391-402.

58. Deveaud, R.; SanJuan, E.; Bellot, P. Accurate and effective latent concept modeling for ad hoc information retrieval. Doc. Numér. 2014, 17,61-84. [CrossRef]

59. Ruiz-Guerra, I.; Molina-Moreno, V.; Cortés-García, F.J.; Núñez-Cacho, P. Prediction of the impact on air quality of the cities receiving cruise tourism: The case of the Port of Barcelona. Heliyon 2019, 5, e01280. [CrossRef] [PubMed]

60. Martín Martín, J.M.; Guaita Martínez, J.M.; Molina Moreno, V.; Sartal Rodríguez, A. An Analysis of the Tourist Mobility in the Island of Lanzarote: Car Rental Versus More Sustainable Transportation Alternatives. Sustainability 2019, 11, 739. [CrossRef]

(C) 2020 by the authors. Licensee MDPI, Basel, Switzerland. This article is an open access article distributed under the terms and conditions of the Creative Commons Attribution (CC BY) license (http://creativecommons.org/licenses/by/4.0/). 

Article

\title{
Shared Short-Term Rentals for Sustainable Tourism in the Social-Network Age: The Impact of Online Reviews on Users' Purchase Decisions
}

\author{
Jie Zhao * and Zhixiang Peng \\ School of Business, Anhui University, Hefei 230601, China \\ * Correspondence: zhaojie@ahu.edu.cn
}

Received: 9 July 2019; Accepted: 26 July 2019; Published: 28 July 2019

\begin{abstract}
With the development of social networks and the Internet-based sharing economy, shared short-term rentals are emerging as a new kind of service that provides a convenient way for people to buy short-term rental services in cities through social-network-enabled platforms. However, like other social-network-based services, shared short-term rental is also likely to be impacted by online reviews. This paper aims to investigate the impact of online reviews on users' purchase decisions toward shared short-term rentals, and further to provide optimization suggestions for the future advance of shared short-term rentals. The contributions of this paper are many-fold. First, we introduce the Stimuli-Organism-Response (SOR) model into the study and propose new variables for the model, including stimulus variables, organism variables, response variable, and moderating variables. Second, we propose eight hypotheses to evaluate the impact of online reviews on users' purchase decisions toward shared short-term rentals. Finally, we collect data through a questionnaire survey and present comprehensive results on many aspects. Based on the data analysis, we find out that the quality of online reviews impacts users' perceived value and perceived risk, which in turn impacts users' purchase decisions toward shared short-term rentals. In addition, the cognitive needs of users can adjust the impact of online reviews on the perceived risk of users but have no explicit adjusting effect for users' perceived value. Further, we present some research implications as well as suggestions for rental platforms to advance shared short-term rentals in the Internet age.
\end{abstract}

Keywords: shared short-term rental; sustainable tourism; online reviews; purchase decisions; social networks

\section{Introduction}

The popularity of the Internet and social networks have made the sharing economy develop rapidly in modern city life [1]. Internet-based sharing economy, such as shared rentals, shared cars, and shared bicycles, offers many kinds of smart services to cities, becoming an intrinsic feature of smart cities [2,3]. Among all sharing economy products, shared short-term rentals have become a recent focus, because of the rapid development of the sustainable-tourism industry [4,5]. An Internet-based platform for shared short-term rentals provides a convenient way for people to sell or buy short-term rental services in cities through the Internet. This is especially helpful for tourists. As a result, many people consider shared short-term rentals as their first choice of accommodation during a travel.

Shared short-term rental is a product of sharing economy in the field of hotel accommodation [6]. It means that the owners of houses can share their rooms or houses to get economic returns within a certain time period. This new type of leasing mode develops rapidly due to its diversified leasing style, simple operation, and low cost. However, due to the inequality of information, consumers usually do not trust shared rentals and are eager to know necessary information about shared rentals before they buy the service [7]. Therefore, online reviews, probably provided by social-network-enabled rentals 
platforms, become a major information source for people to make decisions [8]. Online reviews are popular in social networks; they can reflect the real experiences of consumers, which are more reliable than the advertisements or other information offered by service providers. At the same time, online reviews cover a wide range of contents and diverse forms of expressions, which can effectively stimulate consumers' psychological perception and influence users' decisions on shared short-term rentals.

So far, the related research on shared short-term rentals mainly focused on the operation modes of shared short-term rentals, factors influencing shared short-term rentals, and the impact of shared short-term rentals on traditional hotel industries $[7,9,10]$. Factors affecting shared short-term rental decision include the owner's features, reputation, listing status and location, and rental rules. However, most of the existing explorations of influencing factors are based on websites or tenants, and the results of such explorations cannot well explain the impact of shared customer reviews on consumer decision on shared short-term rentals. At present, there is little work focusing on the internal influencing mechanism of online reviews on users' decisions toward shared short-term rentals. Thus, this paper builds a research model to study the impact of online reviews of shared short-term rentals on users' purchase decisions under the shared economic environment. In particular, this study aims to answer the following questions:

(1) How do online reviews affect users' decisions on shared short-term rentals? What are the internal mechanisms?

(2) Is the impact of online reviews on users' decisions on shared short-term rentals affected by the cognitive needs of users?

Briefly, we make the following contributions in this paper:

(1) We propose a research model that is inspired by the Stimuli-Organism-Response (SOR) model. We set up the quality of online reviews as a stimulus, use perceived sensory and perceived values as organism variables, and let users' decisions on shared short-term rentals be a response variable. In addition, we add the cognitive needs of users as a control variable.

(2) We evaluate users' decisions on Internet-based shared short-term rentals based on the proposed research model and questionnaire data, and present a number of results. The results show that the quality of online reviews influences consumers' perceived value and perceived risk, which in turn impacts users' decisions on shared short-term rentals. In addition, the cognitive needs of consumers can adjust the impact of online reviews on the perceived risk of users. However, it has no explicit adjusting effects between the quality of online reviews and consumers' perceived values.

The remainder of this paper is organized as follows: Section 2 reviews related work. Section 3 presents the theoretical research model and the research hypotheses. Section 4 details the data collection and analysis, and Section 5 discusses research implications and proposes some suggestions for the future development of shared short-tern rentals. Finally, Section 6 concludes the whole paper.

\section{Background and Related Work}

\subsection{Sharing Economy and Shared Short-Term Rentals}

Felson and Speath first proposed the concept of sharing economy [11]. They argued that sharing economy is an information-technology-based marketplace for platforms created by third parties. Individuals can achieve point-to-point direct commodity and service transactions through third-party market platforms. Sharing economy offers users the opportunity to enjoy products without ownership. The widespread use of mobile Internet and online marketing has made sharing economy a sustainable profitable area [1]. Generally, the sharing economy can be divided into three stages. The first stage is code sharing, initiated by Linux. The second stage is content sharing on YouTube or life event sharing on Facebook, which is boosted by Web 2.0 and social networks. The third stage is the sharing of offline 
resources [12]. Houses as a kind of offline resource began to be a sustainable and profitable resource with the rapid development of the sharing economy, because the sharing economy offers house owners opportunities to get economic benefits.

Shared short-term rentals developed with the sharing moving of offline resources. Zervas et al. took the American Airbnb company as an example to study the impact of the short-term rentals on the tourism and accommodation industry [9], which indicated that Airbnb's short-term rentals highly impacted the local hotel industry. As a result, it decreased the revenue of the local traditional hotel industry by 8 to $10 \%$. In order to exploit users' willingness to accept shared short-term rentals, Botsman et al. conducted a study from the perspective of economic motivation and social motivation [12]. They found that the appearing of shared short-term rentals was highly associated with the global economic recession, which caused economic and social pressure and made people consider the cost-effective shared short-term rentals for accommodation. In addition, Liu et al. empirically studied the strategy of online advertising [13]. They found that the attractiveness of shared short-term rental advertisements and the sense of user power interacted with the willingness to click and the willingness to purchase. In recent years, scholars have been keen to explore the impact of landlord photos on users' decisions on shared short-term rentals: Ert et al. used the experimental method to study Airbnb and found that compared with the landlord's reputation, the landlord's photos can influence consumers' shared short-term rental behavior [14]. Zhang et al. and others studied the impact of the source and quality of landlord's photos on the use of housing resources [15]. They found that the platform professionals took more convincing pictures, and high-quality pictures would also increase landlord's income. In addition, they argued the influence of the source and quality of the landlord on the use of the property. They also found that the high-quality images taken by the platform professionals were more convincing which could increase the income of the landlord.

With the rapid development of the Internet, more and more users start to experience online hotel booking on Internet-based hotel reservation platforms such as Booking.com and Ctrip.com. According to a recent study [16], a ten-percent increasing of online reviews on hotel reservation platforms would lead to five-percent increasing of online booking orders. This shows that online reviews are influential to hotel booking. Moreover, a study of Swiss hotels revealed that the number of positive online reviews had a significant positive impact on hotel sales revenue $[16,17]$. Other researchers $[18,19]$ considered the users' professionalism when quantifying the impact of online reviews on users' decision-making toward hotel booking. In addition, other features like user characteristics that might impact hotel-booking intention were analyzed [20]. In summary, previous studies have shown that online reviews have a significant impact on the sales of the hotel industry. However, differing from existing studies that focused on hotel booking, this paper concentrates on shared short-term rentals. Most of short-term rentals are private apartments. As the features of apartments surely differ from those of hotels, online reviews toward shared short-rentals are also quite different from those toward hotels. To this end, it is reasonable that users purchasing shared short-rentals have varying needs compared to users in the traditional hotel-booking market.

\subsection{Perceived Value and Perceived Risk}

The concept of perceived value was formally proposed by Porter [21]. Perceived value refers to the competitive advantage of a company compared with other competitors. Since then, perceived value has been applied to the research of e-commerce, network shopping, and market development [22,23]. Zeithaml et al. studied perceptual value from a psychological perspective [24]. He believed that perceived value was the overall evaluation of a customer toward a service or a product of a particular company. He also claimed that value was superior to other factors. Further, value can be perceived and was influenced by personal knowledge. To this end, perceived value is subjective.

Perceived value was divided into functional value and emotional value [25]. Later in the field of brand research, Sweeney et al. measured the perceived value of customers of high-end durable goods from the four dimensions of emotion, society, quality, and currency [26]. Based on 
Sweeney's work, Petrick et al. further studied the measurement of perceived value and proposed five dimensions, including quality perception, emotional value perception, currency value, industry cost, and reputation [27].

Another factor that has been extensively studied in the field of e-commerce is perceived risk. Since Bauer proposed the concept of perceived risk of consumers [28], many scholars have discussed the issue and have presented numerous extended definitions. Perceived risk is a possibly previous measure of consumer perceived usefulness and perceived ease of use towards purchasing on the internet. Bauer believed that human behavior was highly uncertain; thus so people's behavior was risking. Due to the inequality of information, users' decisions on shared short-term rentals are also highly uncertain. Cox et al. believed that perceived risk can be divided into two dimensions [29]: one is that consumers may be suspicious of bad consequences before making consumption decisions; the other is the extent to which an accident affects them. People are used to reducing perceived risk before making decisions. For sharing this emerging thing and model, the user's uncertainty may be higher compared to the traditional consumption model, and perceived risk is more valuable for studying user behavior.

So far, there have been a lot of studies toward perceived risk. Peter and Ryan pointed out that people will experience perceived risks from a social, economic and functional perspective [30]. Jacoby et al. argued that the perspective of perception should include five aspects [31]: body (whether user behavior will cause physical damage), property (whether user behavior will cause loss of property), society (whether user behavior will threaten user position), function (whether user behavior will satisfy user's own needs), and psychology (whether use behavior will impact user's emotion). Peter and Tarpey proposed the dimension of time based on the above research [32]. In the shopping environment of the Internet era, Jarvenpaa et al. argued that consumers' perceived risk needs further exploration [33]. They recalibrated the six dimensions to five, which include efficacy level, economic level, security level, privacy level and social level. The level of privacy is based on the new environment. He points out that when consumers consume in the network environment, their personal data will be leaked and used. This perspective is also the product of the new environment. In short, the uncertainty of the consequences of user behavior creates a hesitant state of mind that affects the generation of behavior. Although there are a few existing research on shared services and economy [34], consumer uncertainty may be more serious in the Internet age. The high quality information provided by online reviews may affect the perceived risk of consumers, and further affect users' decisions on shared short-term rentals.

\subsection{Users' Purchase Decisions}

Users' purchase decisions are always an important issue in the field of user behavior [35]. Online reviews are also regarded as a stimulus for consumers to buy share short-term rentals. Positive online reviews will make consumers feel that they can benefit from share short-term rentals, which will guide the purchase behavior of users. User behavior is an act or activity. Nicosica et al. believed that user behavior is a purchase, not a resale [36]. Other researchers claimed that users' purchase-decision behavior is the activity of consumers to choose, use, and process products or services [37]. This includes not only purchase, but also ideas, advice, and actions related to the purchase behavior of users. In this paper, users' decisions on shared short-term rentals are regarded as one kind of purchase behavior. Feeny argued that user behavior is an activity in which users choose, use, or process products or services [38]. Users have temporary use rights for idle houses by booking shared short-term rentals; this behavioral user decision on shared short-term rentals can be regarded as a process. In addition, user behavior can also be a process that encompasses all activities for a product or service [39]. The essence of user behavior is an activity in which consumers satisfy their needs by purchasing products or services. In this paper, the main concern is whether users are engaged in bookings when they are shared short-term rentals.

Users' purchase decisions refer to the optimal purchase plan that is analyzed, evaluated, selected, and implemented among two or more purchase plans that are available for selection in order to meet a 
certain demand under the control of certain purchase motivation as well as post-purchase evaluation activities. User purchase decision has been mostly studied in the area of online shopping and social networks [40]. Olbrich et al. analyzed certain social commerce data, and found that products with labels and praise had better sales [41]. In addition, Moody et al. revealed that the seller's reputation, size, amount of information, transaction security, communication, and word-of-mouth had a positive impact on user information and purchase intention [42]. Previous research results showed that the collection and processing of information was an important factor affecting users' purchase intension. In the field of online shopping, users' online reviews posted on platforms are often one of the main sources for user to get information about products. Godes et al. used online reviews as antecedents for decision-making based on users' past experiences [43]. Online review has turned traditional interpersonal communication into online communication. Online reviews spread more widely and faster than traditional product information, and have a deeper impact on sales. In short, there are many factors affecting user behavior, and online review has an important impact on users' purchase intension [44]. In this study, we attempt to reveal the impact as well as the internal mechanism of online reviews on users' decisions on shared short-term rentals.

\section{Research Model and Hypotheses}

\subsection{Research Model}

In 1974, Mehrabian and Russwell proposed the Stimuli-Organism-Response (SOR) model based on environmental psychology [45]. The model is used to explain the relationship between the physical environment and human behavior, consisting of an antecedent variable (Stimulus), a mediator variable (Organism), and a result variable (Response). In the SOR model, S represents the external environmental stimulus that causes individual response, $\mathrm{O}$ represents the evaluation of the stimulus by the organism or the subject of the response, and $\mathrm{R}$ represents the behavioral response after the evaluation of the stimulus. The SOR model implies that external stimuli affect an individual's behavioral response by affecting the individual's mental state. Eroglu et al. (2003) improved the SOR model for external online shopping scenarios by using online product display as an external stimulus to explore customer online buying behavior from the perspective of online user sentiment and cognitive changes [46].

In this study, we adopt the SOR model as the fundamental research model. We believe that online review on the shared short-term rentals platforms is the external environmental stimulus that causes individual reactions, which affects the individual's psychological perception, i.e., the internal evaluation of shared short-term rentals. Then, users can make purchase decisions. We also use online review as an independent variable to explore the internal mechanism of users' decision behavior of shared short-term rentals through the mediating role of perceived risk and perceived value of users. In addition, this paper attempts to explore the moderating effect of online reviews on users' psychological perception.

Figure 1 shows the research model of this study, which mainly measures the relationship between the quality of online reviews and users' decisions on shared short-term rentals. The higher the quality of online reviews, the more complete the information provided, and the more effective it is to stimulate users' behavior. For the intermediary variables, we mainly measure two aspects of user perception, namely perceived value and perceived risk. Shared short-term rentals, when compared with traditional city hotels, have higher uncertainty because of the less understanding of users on shared short-term rentals.

We also introduce a new moderating variable called cognitive needs of users (shown as cognitive need in Figure 1). That is because the personality characteristics of users are the main adjustment factors to shared short-term rentals. In general, if the information obtained by users is more complete, the perceived value is higher and the perceived risk is lower. However, this depends on users' personal cognitive needs. 


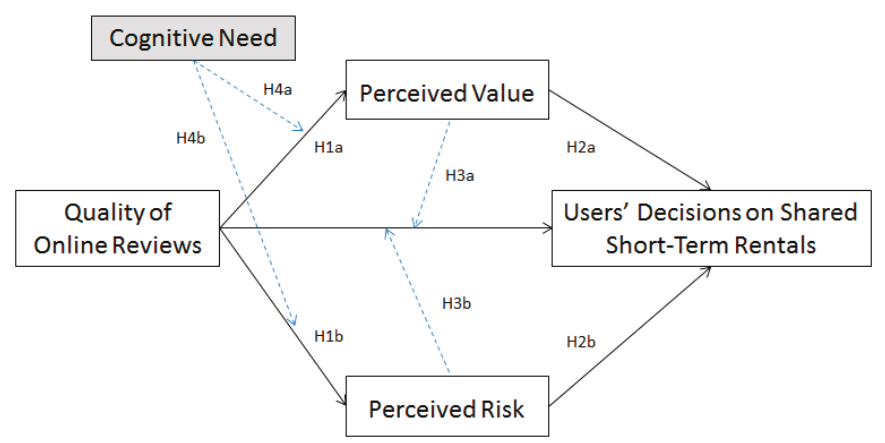

Figure 1. The research model.

Cognitive needs refer to the process in which users participate in thinking and enjoying thinking in the process of understanding things. Users with high cognitive needs are eager to get more information about product attributes, which can help them make decisions. Table 1 summarizes the variables of this research model.

Table 1. Variables in the research model.

\begin{tabular}{ccc}
\hline Role & Variable & Description \\
\hline Independent Variable & Quality of Online Reviews & $\begin{array}{c}\text { Users can find the information they need in high-quality } \\
\text { online reviews. }\end{array}$ \\
\hline Mediating Variables & Perceived Value & $\begin{array}{c}\text { High-quality online reviews can perceive the value of } \\
\text { choosing to share short rents. }\end{array}$ \\
\cline { 2 - 3 } Dependent Variable & Perceived Risk & $\begin{array}{c}\text { High-quality online reviews can reduce } \\
\text { user uncertainty. }\end{array}$ \\
\hline Moderating Variable & Short-Term Rentals & $\begin{array}{c}\text { When choosing hotels, users make short-rent } \\
\text { reservation on shared short-rental platform. }\end{array}$ \\
\hline
\end{tabular}

\subsection{Research Hypothesis}

Users' decisions on shared short-term rentals have a major impact on the development of shared short-term rental platforms. Therefore, it is necessary to explore the factors that influence users' decisions on shared short-term rentals. Online reviews are one of the important factors that affect users' online shopping behavior. Previous work has demonstrated the impact of online reviews on hotel accommodation sales and users' decision-making [47,48].

According to the research model shown in Figure 1, the quality of online reviews as an independent variable affects users' decisions on shared short-term rentals by affecting users' perceived value and perceived risk. In order to explain the internal mechanism of online reviews on the impact of users' decisions on shared short-term rentals, we first set up the following questions, as shown in Table 2 . The Q1 question is to find out the influential relationship between independent variables and the mediating variable. The Q2 question is to find out the influential relationship between the mediating variables and the dependent variable. The Q3 question aims to find out the intermediate relationship among all the factors in the research model. Finally, the Q4 question is to find out what effect the moderating variable has on the relationship between independent variables and mediating variables. 
Table 2. Research questions and corresponding hypotheses.

\begin{tabular}{clc}
\hline Question Number & \multicolumn{1}{c}{ Research Question } & Corresponding Hypotheses \\
\hline Q1 & $\begin{array}{l}\text { What influences exist between independent } \\
\text { variables and the mediating variable (perceived } \\
\text { value and perceived risk)? }\end{array}$ & $\mathrm{H} 1 \mathrm{a}, \mathrm{H} 1 \mathrm{~b}$ \\
\hline Q2 & $\begin{array}{l}\text { How does the mediating variable (perceived } \\
\text { value and perceived risk) impact the dependent } \\
\text { variable (users' decisions on shared } \\
\text { short-term rentals)? }\end{array}$ & $\mathrm{H} 2 \mathrm{a}, \mathrm{H} 2 \mathrm{~b}$ \\
\hline $\mathrm{Q} 3$ & $\begin{array}{l}\text { How does a factor in the research model play } \\
\text { intermediate role between other factors? }\end{array}$ & $\mathrm{H} 3 \mathrm{a}, \mathrm{H} 3 \mathrm{~b}$ \\
\hline $\mathrm{Q} 4$ & $\begin{array}{l}\text { What effect does the moderating variable } \\
\text { (cognitive need) have on the relationship } \\
\text { between independent variables (quality of } \\
\text { online reviews) and intermediate variables } \\
\text { (perceived value and perceived risk)? }\end{array}$ & $\mathrm{H} 4 \mathrm{a}, \mathrm{H} 4 \mathrm{~b}$ \\
\hline
\end{tabular}

Previous studies have pointed out that perceived value is the ratio between the total revenue of a product or service users get from their purchase and the total cost they pay for it [24]. The results showed that the high information quality provided by websites leads to lowness of users' perception on costs as well as the increasing of the perceived value of users. According to these studies, in the sharing economy environment, the high quality of online reviews displaying on shared short-term rentals platforms indicates that platforms can offer high quality information, which can therefore lower the perceived cost of users and increase the perceived value of users.

On the other hand, perceived risk is also the subjective feeling of users, referring to the uncertainties and negative consequences that users perceive subjectively in purchase activities. Murray et al. showed that when potential users perceived high risk in the shopping process [49], they tended to use Internet word-of-mouth to search for relevant products or services. Through summarizing the research literature on the influencing factors of perceived risk of online shopping at home and abroad, we found that perceived risk of consumers mainly comes from three aspects: individual factors (gender, involvement degree, etc.), product factors (product type, brand, etc.) and environmental factors (economy, culture, etc.) Since online reviews reflect users' experiences in the real world, we can reasonably assume that online reviews are more reliable than the information provided by shared short-term rentals platforms. Thus, users are likely to refer to online reviews when they try to make a decision.

In order to answer these questions, we set up several hypotheses:

Hypothesis (H1a): In shared short-term rentals, the high quality of online reviews will increase the perceived value of users.

Hypothesis (H1b): In shared short-term rentals, the high quality of online reviews will lower the perceived risk of users.

Users' decisions on shared short-term rentals also follow the general process of user behavior, meaning that users undertake complex psychological activities under the influence of external stimuli and finally make behavioral decisions. In the study of the relationship between online reviews and user behavior, some scholars believed that online reviews had an impact on user behavior. What really work are not online reviews themselves, but the subjective psychological perception that users form after reading online reviews, including perceived value and perceived risk. Specifically, when choosing to use shared short-term rentals, users' psychological perception directly affects users' decisions on shared short-term rentals. Thus, we make the following hypotheses. 
Hypothesis (H2a): User perceived value has a significant impact on users' decisions on shared short-term rentals.

Hypothesis (H2b): User perceived risk has a significant impact on users' decisions on shared short-term rentals.

In addition, Sussman et al. integrated the elaboration likelihood model (ELM) and the technology acceptance model (TAP) and proposed an online review information adoption model [50]. This model mainly analyzes the influence of users' adoption of online review information on their consumption decision-making behavior in the network environment. They also proposed to use perceptual value as a mediating factor in the relationship between online review quality and user behavior of travel decision-making. In addition, Miyazaki et al. pointed out that the perceived risk is negatively correlated with user consumption behavior through the study of perceived risk antecedent variables [51]. Users' psychological perception is the subjective attitude of people. When reading the same online reviews, users' psychological perceptions may be different, which leads to different decision-making behavior. In other words, in the context of sharing economy, users' psychological perception may play a mediating role in the relationship between online reviews and users' decisions on shared short-term rentals. Thus, we make the following hypotheses:

Hypothesis (H3a): The perceived value of users plays a mediating role between online reviews and users' decisions on shared short-term rentals.

Hypothesis (H3b): The perceived risk of users plays a mediating role between online reviews and users' decisions on shared short-term rentals.

Next, we consider that the influence of online reviews on users' psychological perception. We guess that users' psychological perception depends not only on the characteristics of online reviews, but also on the characteristics of recipients. This is because users may have different personality characteristics and attitudes towards online reviews, which may lead to different user purchase decision. Cognitive needs refer to the process in which an individual participates in and enjoys thinking in the process of recognizing things. Users with high cognitive needs are eager to get more information about product attributes that can help them make decisions. Cacioppo et al. carried out experiments to measure the level of cognitive needs [52]. They found that people can be divided into two categories: a group of people with low cognitive needs, who think that the situation should be orderly and regular, and another group of people with high cognitive needs who think that the situation is not clear and they will analyze the situation according to personal experience to understand the situation. Cacioppo et al. also pointed out that people with high cognitive needs prefer to think and form a brand attitude through thinking. On the contrary, people with low cognitive needs are susceptible to the side-effects of advertising. That is to say, people with different cognitive needs have different ways of processing information. In general, the information about the products with high-quality online reviews is more about post-consumer experiences and feelings, which can meet the needs of users with low cognitive needs and fasten decision-making processes. In summary, because of high-quality online reviews, users with low cognitive needs may get high perceived value and lower perceived risk. Thus, we make the following hypotheses:

Hypothesis (H4a): Cognitive needs play a moderating role during the process where online reviews impact perceived value.

Hypothesis (H4b): Cognitive needs play a moderating role during the process where online reviews impact perceived risk. 


\section{Data Collection and Analysis}

\subsection{Questionnaire Design}

We used a questionnaire to test the hypotheses. Specially, we design the questionnaire according to the general principles and steps of the literature. Firstly, the indicators of each variable were extracted through a great number of literature studies, and the designed questionnaires were tested on a small scale among 28 college students who had rented a house on the Internet shared short-term rental platforms. Some of the items were revised according to the results of the small sample analysis, and the formal research questionnaire was finally formed through repeated revisions. The questionnaire consists of three parts:

(1) Information about user travelling experiences. This section focuses on general information about user travelling, including the frequency of experiencing shared short-term rentals, the frequency of travelling, and the type of accommodation.

(2) Basic information about users. This part of questionnaire includes the gender, age, and educational background of the surveyed users.

(3) Questionnaire on independent variable, mediating variable, dependent variable and moderating variable, which is the core of the whole questionnaire. The sources of variables are as follows. The online review quality scale comes from the previous studies $[53,54]$. The risk-aware scale was designed according to the literature $[55,56]$. The perceptual value scale was developed according to the literature [57]. The scale of user shared short-term rentals is based on the scale developed by Fishbein and Bansal $[58,59]$. The scale of cognitive needs was modified according to the scale designed by Cacioppo [52].

The questionnaire was designed according to the Likert five-point scale, and the respondent was asked to choose a score ranging from 1 (totally disagree) to 5 (totally agree) to answer each question. The details of the questionnaire are shown in Table 3.

\subsection{Data Collection}

The data collection of this study was conducted by an online questionnaire, mainly through the WeChat circle of friends. The product introduction of the shared short-term rental platforms is detailed on the homepage of the questionnaire. The participants are required to have an Internet accommodation booking experience. After screening the questionnaire and eliminating the items that do not have online accommodation booking experiences, we finally obtained 232 valid questionnaires. Baumgartner and Homburg proposed that the number of samples required for a structural equation analysis is at least five times the estimated parameter [60]. Since the research model in this study involves 18 parameters, we need at least 90 samples. Thus, the number of collected samples can satisfy the data analysis. The descriptive statistics of the samples are as follows: 121 males and 111 females, whose age is mostly within the range from 20 to 25 years (85.7\%). In addition, $77.5 \%$ of participants hold a college degree. 
Table 3. Indicators of the questionnaire.

\begin{tabular}{|c|c|c|}
\hline Construct & Question Code & Measurement Problem \\
\hline \multirow{4}{*}{$\begin{array}{l}\text { Quality of Online } \\
\text { Reviews }\end{array}$} & QU1 & Those online reviews are reliable. \\
\hline & QU2 & These online reviews are detailed and detailed. \\
\hline & QU3 & $\begin{array}{l}\text { These online reviews are closely related to the } \\
\text { characteristics of shared short rental itself. }\end{array}$ \\
\hline & QU4 & Overall, online reviews are of high quality and useful. \\
\hline \multirow{3}{*}{ Perceived value } & VA1 & $\begin{array}{c}\text { Online reviews accurately reflect the true quality of } \\
\text { shared short rentals. }\end{array}$ \\
\hline & VA2 & $\begin{array}{l}\text { Online reviews can make me feel that the introduction of } \\
\text { shared short rentals is true. }\end{array}$ \\
\hline & VA3 & Online reviews can help me better perceive products. \\
\hline \multirow{3}{*}{ Perceived risk } & RI1 & $\begin{array}{l}\text { These comments are a good way to assess the economic } \\
\text { risks of purchases. }\end{array}$ \\
\hline & RI2 & $\begin{array}{l}\text { These reviews are a good way to assess whether the } \\
\text { product description is true or not. }\end{array}$ \\
\hline & RI3 & $\begin{array}{l}\text { These comments are a good way to avoid the risk that I } \\
\text { would be laughed at for making a mistake. }\end{array}$ \\
\hline \multirow{4}{*}{ Cognitive needs } & KN1 & $\begin{array}{l}\text { I have a strong interest in the shared short-term rental I } \\
\text { want to book. }\end{array}$ \\
\hline & KN2 & I like to do things that require a lot of thinking. \\
\hline & KN3 & I like to do complicated things instead of simple things. \\
\hline & KN4 & $\begin{array}{l}\text { I will know some housing conditions and the } \\
\text { surrounding environment before buying. }\end{array}$ \\
\hline \multirow{4}{*}{$\begin{array}{l}\text { Users' Decisions on } \\
\text { Shared Short-Term } \\
\quad \text { Rentals }\end{array}$} & SR1 & $\begin{array}{l}\text { These online reviews are helpful for my purchase } \\
\text { decision. }\end{array}$ \\
\hline & SR2 & $\begin{array}{l}\text { I will refer to these comments if I choose to share } \\
\text { short-term rentals. }\end{array}$ \\
\hline & SR3 & $\begin{array}{c}\text { Online review makes me more confident when I am } \\
\text { determined to book a short-term rental }\end{array}$ \\
\hline & SR4 & $\begin{array}{c}\text { Online review have an impact on when I booked a shared } \\
\text { short-term rental }\end{array}$ \\
\hline
\end{tabular}

\subsection{Factor Analysis}

In this section, we use the confirmatory factor analysis (CFA) to analyze the reliability and validity of factors. This paper first uses the SPSS 22.0 software (IBM, Armonk, NY, USA) to conduct the reliability analysis. The results are shown in Table 4. The value of KMO (Kaiser-Meyer-Olkin) is 0.874 , and the Bartlett spheroid test results are significant (sig. $=0.000$ ). The Bahrain alpha coefficient (Cronbach's $\alpha$ ) and the composite reliability are mostly greater than 0.8 , indicating that the scale has high reliability.

The normalized factor load of the seven explicit variables of the model is higher than 0.8 and reaches a significant level. The CR value is greater than 0.7 , and the AVE rate is greater than 0.5. Overall, the model meets the requirements for convergence and validity.

Next, we perform the discriminant validity test. Discriminant validity is proposed to examine whether a measurement is a reflection of any other measurement. As shown in Table 5, the correlation coefficient of each variable is smaller than the square root of the mean variance extraction rate of its corresponding variable, indicating that the discriminant validity is satisfied. 
Table 4. Reliability and validity analysis on variables.

\begin{tabular}{|c|c|c|c|c|c|}
\hline Variable & $\begin{array}{c}\text { Observation } \\
\text { Variable }\end{array}$ & Factor load & Cronbach's $\alpha$ & $\begin{array}{c}\text { Composite } \\
\text { Reliability (CR) }\end{array}$ & $\begin{array}{c}\text { Average Variance } \\
\text { Extracted (AVE) }\end{array}$ \\
\hline \multirow{4}{*}{$\begin{array}{c}\text { Quality of } \\
\text { Online Reviews }\end{array}$} & QU1 & 0.73 & \multirow{4}{*}{0.878} & \multirow{4}{*}{0.876} & \multirow{4}{*}{0.639} \\
\hline & QU2 & 0.76 & & & \\
\hline & QU3 & 0.88 & & & \\
\hline & QU4 & 0.82 & & & \\
\hline \multirow{3}{*}{ Perceived value } & VA1 & 0.80 & \multirow{3}{*}{0.818} & \multirow{3}{*}{0.832} & \multirow{3}{*}{0.609} \\
\hline & VA2 & 0.76 & & & \\
\hline & VA3 & 0.78 & & & \\
\hline \multirow{3}{*}{ Perceived risk } & RI1 & 0.76 & \multirow{3}{*}{0.737} & \multirow{3}{*}{0.774} & \multirow{3}{*}{0.533} \\
\hline & RI2 & 0.71 & & & \\
\hline & RI3 & 0.72 & & & \\
\hline \multirow{4}{*}{$\begin{array}{l}\text { Cognitive } \\
\text { needs }\end{array}$} & KN1 & 0.80 & \multirow{4}{*}{0.842} & \multirow{4}{*}{0.852} & \multirow{4}{*}{0.592} \\
\hline & KN2 & 0.84 & & & \\
\hline & KN3 & 0.72 & & & \\
\hline & KN4 & 0.71 & & & \\
\hline \multirow{4}{*}{$\begin{array}{c}\text { Shared } \\
\text { Short-Term } \\
\text { Rental Decision }\end{array}$} & SR1 & 0.85 & \multirow{4}{*}{0.886} & \multirow{4}{*}{0.881} & \multirow{4}{*}{0.649} \\
\hline & SR2 & 0.80 & & & \\
\hline & SR3 & 0.81 & & & \\
\hline & SR4 & 0.76 & & & \\
\hline
\end{tabular}

Table 5. Discriminant validity test.

\begin{tabular}{cccccc}
\hline & $\begin{array}{c}\text { Quality of Online } \\
\text { Reviews }\end{array}$ & $\begin{array}{c}\text { Perceived } \\
\text { Value }\end{array}$ & $\begin{array}{c}\text { Perceived } \\
\text { Risk }\end{array}$ & $\begin{array}{c}\text { Cognitive } \\
\text { Needs }\end{array}$ & $\begin{array}{c}\text { Users' Decisions on } \\
\text { Shared Short-Term } \\
\text { Rentals }\end{array}$ \\
\hline $\begin{array}{c}\text { Quality of Online } \\
\text { Reviews }\end{array}$ & 0.799 & & & \\
\hline Perceived Value & 0.717 & 0.780 & & \\
\hline Perceived Risk & -0.443 & -0.424 & 0.730 & 0.769 & 0.805 \\
\hline Cognitive Needs & 0.647 & 0.681 & -0.467 & 0.735 & \\
\hline $\begin{array}{c}\text { Users' Decisions on } \\
\text { Shared Short-Term } \\
\text { Rentals }\end{array}$ & 0.690 & 0.749 & -0.517 & & \\
\hline
\end{tabular}

Note: each value along the diagonal line is the square root of the corresponding AVE.

\subsection{Structural Model Evaluation}

The structural model measures the relationship between latent variables. The path coefficients between the latent variables in the study model were verified using AMOS 23.0 (IBM, Armonk, NY, USA). The path coefficient values between the variables are shown in Figure 2.

When users subscribe to shared short-term rentals, the quality of online reviews significantly affects users' perceived value ( $\beta=0.84, p<0.01$ ), assuming H1a is verified. This shows that the quality of online reviews can significantly improve the perceived value of users when users subscribe to shared short-term rentals. When users subscribe to shared short-term rentals, the quality of online reviews significantly affects visitors' perceived risk ( $\beta=-0.55, p<0.01)$, assuming $\mathrm{H} 2 \mathrm{~b}$ is verified. This shows that the quality of online reviews can reduce the perceived risk of users. This may be due to the fact that when visitors receive higher quality online reviews, users get more information with more value, less uncertainty, perceived cost of decline, and thus higher perceived value and perceived risk. 


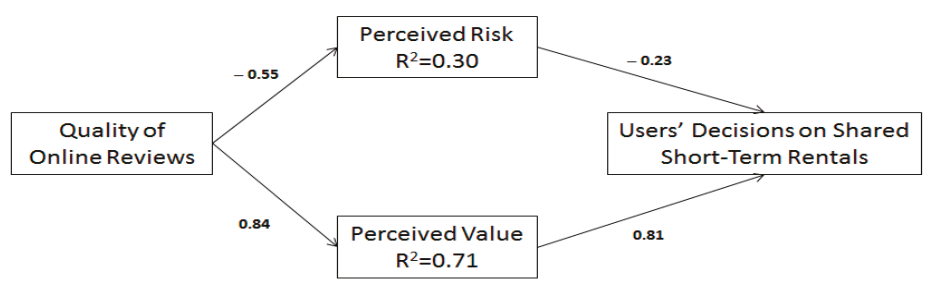

Figure 2. The path coefficient values between the variables for structural model evaluation.

The perceived value of users has a significant impact on users' decisions on shared short-term rentals ( $\beta=0.81, p<0.01$ ), assuming $\mathrm{H} 2 \mathrm{a}$ is verified. This shows that the perceived value of users is positively related to users' decisions on shared short-term rentals. In addition, the perceived risk of users has a significant impact on users' decisions on shared short-term rentals $(\beta=-0.23, p<0.01)$. This shows that users' perceived risk is negatively correlated with users' decisions on shared short-term rentals. Perceived value and perceived risk are subjective feelings of people, which means that users may be affected by various stimulating factors, resulting in the change of perceived value and perceived risk, and in turn the decision behavior of users.

In addition, the permissible coefficients of perceived value and perceived risk are 0.71 and 0.30 , respectively. This indicates that the quality of online reviews explains the variance variation of $71 \%$ of the perceived value generated by users' online bookings for shared short-term rentals. The quality of online reviews explains the perceived risk by $30 \%$ variance variation. As a result, the model explains the $84 \%$ variance variation of users' decisions on shared short-term rentals, showing that the degree of interpretation is satisfied. Table 6 shows the model fitting results. The descriptions of the symbols shown on the first row are provided in Table 7.

Table 6. Model fitting results.

\begin{tabular}{cccccccccccc}
\hline Statistical Test & $X^{2} / d f$ & SMRM & RMSEA & AGFI & NFI & RFI & CFI & IFI & PGFI & PNFI & PCFI \\
\hline Ideal Standard Value & $<2.00$ & $<0.08$ & $<0.05$ & $>0.80$ & $>0.90$ & $>0.90$ & $>0.90$ & $>0.90$ & $>0.50$ & $>0.50$ & $>0.50$ \\
\hline Acceptable Standard & $<3.00$ & $<0.1$ & $<0.08$ & $>0.70$ & $>0.80$ & $>0.80$ & $>0.80$ & $>0.80$ & & & \\
\hline Results of This Study & 1.712 & 0.056 & 0.056 & 0.893 & 0.938 & 0.922 & 0.973 & 0.973 & 0.700 & 0.752 & 0.780 \\
\hline
\end{tabular}

Table 7. Explanations of the symbols used in Table 6.

\begin{tabular}{cc}
\hline Symbol & Description \\
\hline$X^{2}$ & Chi square statistic \\
\hline$d f$ & Degrees of freedom \\
\hline SMRM & Standardized root mean squared \\
\hline RMSEA & Root-mean-square error of approximation \\
\hline AGFI & Adjusted goodness-of-fit index \\
\hline NFI & Normed fit index \\
\hline RFI & Relative fit index \\
\hline CFI & Comparative fit index \\
\hline IFI & Incremental fit index \\
\hline PGFI & Parsimonious goodness-of-fit index \\
\hline PNFI & Parsimonious normed fit index \\
\hline PCFI & Parsimonious comparative fit index \\
\hline
\end{tabular}




\subsection{Mediating Effect Test}

In order to verify the intrinsic mechanism of the impact of online-review quality on users' decisions on shared short-term rentals, we use the bootstrap method (Bootstrap) for mediation analysis (sample size is set to 2000) [61]. Thus, it is judged whether the mediating effects of perceived value and perceived risk exist. The results are shown in Table 8. We can see that under the 95\% confidence interval (CI), the bias-corrected, percentile, and PRODCLIN2 testing results show that perceive value and perceived risk have a mediating effect in the research model.

Table 8. Coefficient products and bootstrapping checking results.

\begin{tabular}{|c|c|c|c|c|c|c|c|c|c|}
\hline \multirow{4}{*}{ Mediation Path } & \multirow{4}{*}{ Effect } & \multirow{4}{*}{$\begin{array}{l}\text { Point } \\
\text { Estimation }\end{array}$} & \multicolumn{4}{|c|}{ Bootstrapping (2000 Sample) } & \multirow{3}{*}{\multicolumn{2}{|c|}{$\begin{array}{c}\text { PRODCLIN2 } \\
95 \% \mathrm{CI}\end{array}$}} & \multirow{4}{*}{ Conclusion } \\
\hline & & & \multirow{2}{*}{\multicolumn{2}{|c|}{$\begin{array}{c}\text { Bias-Corrected } \\
95 \% \mathrm{CI}\end{array}$}} & \multirow{2}{*}{\multicolumn{2}{|c|}{$\begin{array}{c}\text { Percentile } \\
95 \% \mathrm{CI}\end{array}$}} & & & \\
\hline & & & & & & & & & \\
\hline & & & Lower & Upper & Lower & Upper & Lower & Upper & \\
\hline \multirow{5}{*}{$\begin{array}{l}\text { Online-review quality } \\
\text { Perceived } \\
\text { value/Perceived risk } \\
\text { Users' decisions on } \\
\text { shared short-term } \\
\text { rentals }\end{array}$} & Total Effect & 0.733 & 0.591 & 0.875 & 0.591 & 0.877 & - & - & Exist \\
\hline & Indirect Effect & 0.864 & 0.614 & 0.888 & 0.613 & 0.887 & - & - & Exist \\
\hline & Direct Effect & -0.132 & -0.762 & 0.136 & -0.736 & 0.143 & - & - & Not exist \\
\hline & \multicolumn{6}{|c|}{$\begin{array}{l}\text { Perceived value plays a full mediating role between online-review } \\
\text { quality and users' decisions on shared short-term rentals. }\end{array}$} & 0.460 & 0.822 & Exist \\
\hline & \multicolumn{6}{|c|}{$\begin{array}{l}\text { Perceived risk plays a full mediating role between online-review } \\
\text { quality and users' decisions on shared short-term rentals. }\end{array}$} & 0.066 & 0.193 & Exist \\
\hline
\end{tabular}

Note: All the bootstrapping values are non-standardized values.

In summary, there is a complete mediation between perceived value and perceived risk in the decision-making path of online-review quality affecting users' decisions on shared short-term rentals. This shows that the quality of online reviews mainly affects the perceived value and perceived risk of users' psychology, and further affect users' decisions on shared short-term rentals. Both perceived value and perceived risk have a dual intermediary role between online-review quality and users' decisions on shared short-term rentals, demonstrating the validity of $\mathrm{H3a}$ and $\mathrm{H} 3 \mathrm{~b}$.

\subsection{Moderating Effect Test}

In this section, we test the moderating effect of the moderating variable. Here, SPSS 20 is used to conduct the test by using the hierarchical regression method (HRM). The results show that the variance inflation factors (VIF) of the related variables are all less than 2, indicating that there is less information overlap between explanatory variables and there is no serious multicollinearity problem between variables.

Table 9 shows the moderating effect of user cognitive needs. We can see that the moderating effect of users' cognitive needs between online-review quality and perceived value does not exist. Meanwhile, user cognitive need has a moderating effect between online-review quality and perceived risk. Therefore, the hypothesis $\mathrm{H} 4 \mathrm{a}$ is not valid, but the hypothesis $\mathrm{H} 4 \mathrm{~b}$ is verified. For people with different cognitive needs, the valuable information provided is the same, so the change of perceived value is not significant. On the other hand, the quality of online reviews indirectly proves the authenticity of information provided by platforms, which can reduce the perception of users' uncertainty. The reduction of users' uncertainty leads to the reduction of users' perceived risk. Compared with users with low cognitive needs who are eager to make decisions as soon as possible, users with high cognitive needs still need more information to help them verify the authenticity of their information and make decisions. Therefore, the perceived risk of users with high cognitive needs decreases at a slow rate with the improvement of online-review quality, which means that cognitive needs have a significant moderating effect between online-review quality and perceived risk. 
Table 9. Moderating effect testing results of cognitive needs.

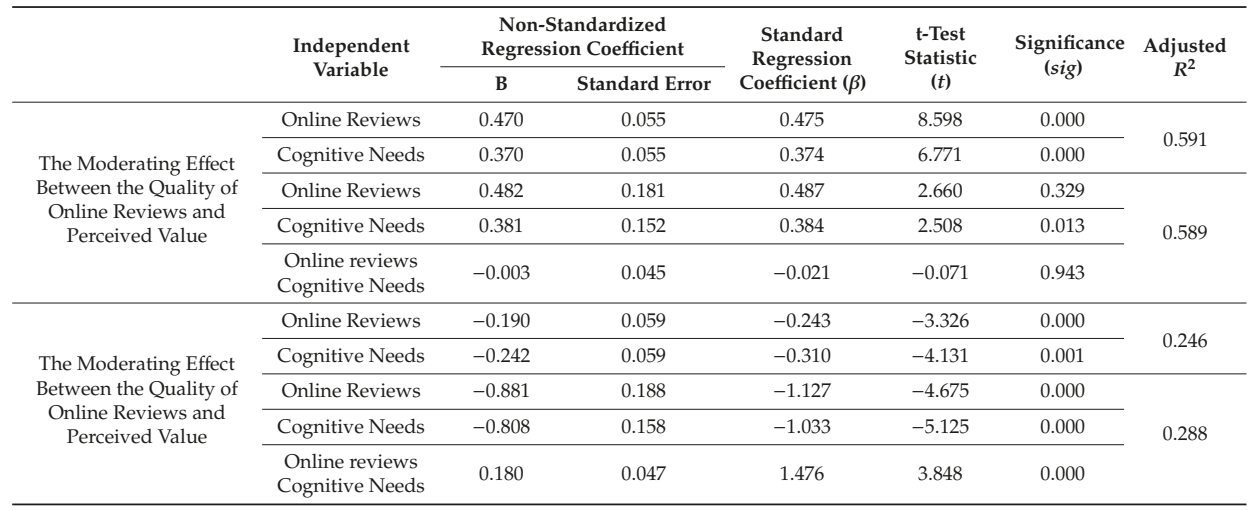

\subsection{Summary of Hypotheses Validation}

Based on the results described in Sections 4.1-4.6, we present the summary of the hypotheses validation in Table 10. The validation-result column in Table 9 shows the final validation results of each hypothesis. The hypotheses $\mathrm{H} 1 \mathrm{a}, \mathrm{H} 1 \mathrm{~b}, \mathrm{H} 2 \mathrm{a}$, and $\mathrm{H} 2 \mathrm{~b}$ are all established. These assumptions correspond to our Q1 and Q2. This means that the quality of online reviews, which is the independent variable in our research model, has a positive impact on users' decisions on shared short-term rentals. $\mathrm{H} 4 \mathrm{~b}$ has also been verified, and H4a has not been verified, which corresponds to our Q3. This means that the personality characteristics of users can influence users' internal perception of online reviews. The main impact is the perceived risk of users, and its moderating effect on the perceived value is not significant. Platforms should pay more attention to the personality characteristics of users and adopt relevant marketing strategies to reduce users' perceived risks. Regarding the hypotheses of H3a, $\mathrm{H} 3 \mathrm{~b}$, which aim to answer the research question of Q4, the results show that both perceived value and perceived risk play a full intermediary role between online-review quality and users' decisions on shared short-term rentals. Therefore, platforms should focus on user psychological perception and promote users' decisions on shared short-term rentals.

Table 10. Summary of hypotheses validation.

\begin{tabular}{|c|c|c|}
\hline Number & Hypothesis & Validation Result \\
\hline H1a & $\begin{array}{l}\text { In shared short-term rentals, the high quality of online reviews } \\
\text { will increase the perceived value of users. }\end{array}$ & Established \\
\hline $\mathrm{H} 1 \mathrm{~b}$ & $\begin{array}{l}\text { In shared short-term rentals, the high quality of online reviews } \\
\text { will lower the perceived risk of users. }\end{array}$ & Established \\
\hline $\mathrm{H} 2 \mathrm{a}$ & $\begin{array}{l}\text { User perceived value has a significant impact on users' decisions } \\
\text { on shared short-term rentals. }\end{array}$ & Established \\
\hline $\mathrm{H} 2 \mathrm{~b}$ & $\begin{array}{l}\text { User perceived risk has a significant impact on users' decisions } \\
\text { on shared short-term rentals. }\end{array}$ & Established \\
\hline $\mathrm{H} 3 \mathrm{a}$ & $\begin{array}{l}\text { The perceived value of users plays a mediating role between } \\
\text { online reviews and users' decisions on shared short-term rentals. }\end{array}$ & Established \\
\hline $\mathrm{H} 3 \mathrm{~b}$ & $\begin{array}{l}\text { The perceived risk of users plays a mediating role between } \\
\text { online reviews and users' decisions on shared short-term rentals. }\end{array}$ & Established \\
\hline $\mathrm{H} 4 \mathrm{a}$ & $\begin{array}{l}\text { Cognitive needs play a moderating role during the process } \\
\text { where online reviews impact perceived value. }\end{array}$ & Not established \\
\hline $\mathrm{H} 4 \mathrm{~b}$ & $\begin{array}{l}\text { Cognitive needs play a moderating role during the process } \\
\text { where online reviews impact perceived risk. }\end{array}$ & Established \\
\hline
\end{tabular}




\section{Discussion}

\subsection{Research Implications}

First, in this study, we employ the SOR model to explore the internal mechanism of online reviews on users' decisions on shared short-term rentals. This model expands the application scope of the SOR model and provides referential values for other related research. In addition, we also presented empirical results that reflecting the impact of online reviews on users' decisions on shared short-term rentals, which can provide a new perspective for promoting shared short-term rentals and new marketing mechanisms.

Second, this paper studied the factors impacting shared short-term rental decisions, and provided referential values for enterprises to increase users' willingness to be engaged in today's mobile internet enabled sharing economy. Our study reveals that online reviews can inspire users' decisions on shared short-term rentals.

Third, we used perceived value and perceived risk as a mediator between online reviews and users' decisions on shared short-term rentals. The empirical results show that perceived value and perceived risk can influence users' decisions on shared short-term rentals. In addition, both perceived value and perceived risk play a full intermediary role between online reviews and users' decisions on shared short-term rentals. Therefore, the sharing of short-term rental platforms needs to pay attention to the psychological perception of users. Moreover, platforms need to reduce the perceived risk of users and increase the perceived value of users.

Fourth, regarding the measurement of users' personality characteristics, this paper mainly focused on users' cognitive needs. The results show that cognitive needs play a moderating role in the relationship between online reviews and perceived risk. Users with high cognitive needs need better information to reduce their perceived risk. Thus, users need more information from the Internet to reduce their uncertainty. Therefore, shared short-term rental platforms should provide users with more opportunities to communicate on the basis of correctly guiding online reviews.

Fifth, the data used in this paper are mainly from surveys of young people. Our research shows that young people can quickly accept a new type of city accommodation such as shared short-term rentals. Therefore, how to improve young people's choice of shared short-term rentals is what a shared short-term rental platform should consider. Many people are struggling to find a hotel around their destination when they have a temporarily traveling. Due to geographical locations and other issues, the traffic around the hotel may be inconvenient. Based on the wide range of sources of tenants on the shared short-term rental platform, when users post accommodation requirements or choose accommodation, they can easily choose the right place to stay. To this end, shared short-term rental platforms still have spaces and potentials for further development.

In summary, the main conclusions of this study, i.e., online reviews are highly influential to users' decision-making behavior, are coherent with previous works $[17,19]$. However, in the traditional hotel industry, the studies about online reviews and users' purchasing intention mainly focused on the characteristics of hotels and users. On the contrary, this paper is mainly toward online reviews on shared short-term rentals, which typically refer to private apartments advertised by owners on Internet-based rental platforms. Apartments have different features from hotels, and users engaged in shared short-term rentals are also much different from those choosing hotel booking. For example, many young students are likely to try shared short-term rentals, while most business people prefer high-standard hotels. Based on these differences, we set up new variables for the SOR research model. Specially, we focus on the mediating effect of users' perceived value and perceived risk, because users choosing shared short-term rentals have to balance their perceived values and possible risks. On the other hand, although shared short-term rentals as a new kind of shared economy have received much attention in recent years, there are few previous studies that focus on the impact of online reviews on users' purchasing decision on shared short-term rentals. We believe that the main conclusions of this paper can provide referential values for further studies in this field. 


\subsection{Suggestions}

Based on the empirical analysis of this study, we provide the following suggestions for better implementation of shared short-term rental platforms.

(1) Guide users to provide effective online reviews.

The quality of online reviews significantly affects users' decisions on shared short-term rentals. On the one hand, the higher the quality of online reviews, the richer the review content, the higher the perceived value of users, and the lower the perceived risk. On the other hand, platforms should appropriately encourage purchased users to comment on the shared rentals, so that users with low cognitive needs can make up useful suggestive strategies. At the same time, platforms should guide users to provide product information that the website has not yet paid attention to, so as to help users with high cognitive needs to make decisions.

(2) Create topics and provide material rewards for online reviews.

By creating topics and rewarding material prizes, platforms can effectively guide the review content, direction, and quantity of online reviews. According to previous research, the number of online reviews positively affects users' willingness to purchase. More online reviews imply that more potential users will be able to learn more product-related information and be able to better perceive products. If there are many online reviews with high quality, shared rental platforms will attract more attention of users. In order to do so, platforms are suggested rewarding users with little gifts to encourage users to share short-term rental photos or experiences, so that the quality and quantity of online reviews can be improved.

(3) Provide better qualified rental products and services.

We suggest that shared short-term rental platforms provide qualified rental products and services to ensure that users can experience shared short-term rentals with their psychological expectations. The products and services provided by platforms can directly affect the perceived value of users. This is because shared rental industry is actually a service-oriented industry. So shared rental platforms should not only provide shared short-term housing, but also provide services related to the products, such as route planning, traffic information, and information about surrounding sightseeing places. This will increase the perceived value of users and has a significant positive impact on users' decisions on shared short-term rentals.

\section{Conclusions and Future Work}

In this paper, we empirically investigated the impact of online reviews on users' decisions toward shared short-term rentals in the context of social networks, sustainable tourism, and sharing economy. Our study found that the quality of online reviews significantly affects users' perceived value, and negatively affects perceived risk. In addition, both perceived value and perceived risk have a dual intermediary role between online-review quality and users' decisions on shared short-term rentals. We also studied the moderating effect of the cognitive needs of users. We found that the moderating effect of user cognitive needs between online-review quality and perceived value does not exist. Meanwhile, user cognitive need has a moderating effect between online-review quality and perceived risk.

Some limitations of this study can be summarized as follows. First, although hundreds of valid questionnaires are theoretically enough to conduct data analysis, in this big data era, this number is relatively small to draw reliable and robust conclusions. Second, the results of this study have shown that online reviews have positive impact on users' decisions on shared short-term rentals. However, the inherent theoretical basis has not been revealed yet.

Thus, in the future there are some research issues that are worth further investigating. First, in terms of the impact of review quality on the purchase intention of short-term rentals, secondary 
data collected from home sharing platforms like Airbnb could be a better choice. One possible way is to conduct text mining of the reviews of each property to measure review quality monthly, and to use the review volume to predict the purchase intention of each property. Second, because users' decision-making behavior could be impacted by other factors, future work can be focused on other possible factors, such as users' income and education background, review volume or variance, and users' online experience. Finally, it is also worth to study other research models [62] on user acceptance and consider online reviews within the community context [63].

Author Contributions: J.Z:: Conceptualization, Project administration, Supervision, and Writing-original draft, Z.P.: Data curation, Formal analysis, Investigation, and Software.

Funding: This paper is partially supported by the National Science Foundation of China (No. 71273010) and the Innovation Team Construction Project of Anhui University.

Acknowledgments: We would like to thank the editors and anonymous reviewers for their suggestions and comments to improve the quality of the paper.

Conflicts of Interest: The authors to declare no conflict of interest.

\section{References}

1. Sutherland, W.; Jarrahi, M. The Sharing Economy and Digital Platforms: A Review and Research Agenda. Int. J. Inf. Manag. 2018, 43, 328-341. [CrossRef]

2. Ganapati, S.; Reddick, C.G. Prospects and challenges of sharing economy for the public sector. Gov. Inf. Q. 2018, 35, 77-87. [CrossRef]

3. Jin, S.; Kong, H.; Wu, R.; Sui, D. Ridesourcing, the Sharing Economy, and the Future of Cities. Cities 2018, 76, 96-104. [CrossRef]

4. Martín, J.M.; Martínez, J.M.; Fernández, J.A. An analysis of the factors behind the citizen's attitude of rejection towards tourism in a context of over-tourism and economic dependence on this activity. Sustainability 2018, 10, 2851. [CrossRef]

5. Martín, J.M.; Martín, J.A.; Mejía, K.A.; Fernández, J.A. Effects of vacation rental websites on the concentration of tourists-Potential environmental impacts. An application to the Balearic Islands in Spain. Int. J. Envron. Res. Public Health. 2018, 15, 347. [CrossRef] [PubMed]

6. Wu, J.; Ma, P.; Xie, K. In Sharing Economy We Trust: The Effects of Host Attributes on Short-Term Rental Purchases. Int. J. Contemp. Hosp. M. 2017, 29, 2962-2976. [CrossRef]

7. Hu, L.Q.; Liu, H.; Zou, X. An Evaluation of the User Experience on C2C Online Short-Term Rentals. Iop Conf. Ser. Earth Env. Sci. 2018, 199, 032001.

8. Andrey, F.; Elena, G.; David, H. The Determinants of Online Review Informativeness: Evidence from Field Experiments on Airbnb. SSRN Electron. J. 2018. Available online: https://ssrn.com/abstract=2939064 (accessed on 1 July 2019).

9. Zervas, G.; Proserpio, D.; Byers, J. The Rise of the Sharing Economy: Estimating the Impact of Airbnb on the Hotel Industry. SSRN Electron. J. 2013. Available online: https://ssrn.com/abstract=2366898 (accessed on 1 July 2019).

10. Yang, Y.; Mao, E.Z. Welcome to My Home! An Empirical Analysis of Airbnb Supply in US Cities. J. Travel Res. 2018, 53. [CrossRef]

11. Felson, M.; Spaeth, J.L. Community Structure and Collaborative Consumption: A Routine Activity Approach. Am. Behav. Sci. 1978, 21, 614-624. [CrossRef]

12. Botsman, R.; Rogers, R. What's Mine Is Yours: The Rise of Collaborative Consumption; Harper Business: New York, NY, USA, 2010; pp. 30-59.

13. Liu, S.Q.; Mattila, A.S. Airbnb: Online targeted advertising, sense of power, and consumer decisions. Int. J. Hosp. Manag. 2017, 60, 33-41. [CrossRef]

14. Ert, E.; Fleischer, A.; Magen, N. Trust and reputation in the sharing economy: The role of personal photos in Airbnb. Tour. Manag. 2016, 55, 62-73. [CrossRef]

15. Zhang, S.; Lee, D.; Singh, P.V.; Srinivasan, K. How Much Is an Image Worth? Airbnb Property Demand Estimation Leveraging Large Scale Image Analytics. SSRN Electron. J. 2017. Available online: https: //www.ssrn.com/abstract=2976021 (accessed on 1 July 2019). 
16. Ye, Q.; Law, R.; Gu, B.; Chen, W. The influence of user-generated content on traveler behavior: An empirical investigation on the effects of e-word-of-mouth to hotel online bookings. Comput. Hum. Behav. 2011, 27, 634-639. [CrossRef]

17. Phillips, P.; Zigan, K.; Silva, M. The interactive effects of online reviews on the determinants of Swiss hotel performance: A neural network analysis. Tour. Manag. 2015, 50, 130-141. [CrossRef]

18. Zhao, R.X.; Wang, L.; Guo, X.; Law, C.H.R. The influence of online reviews to online hotel booking intentions. Int. J. Contemp. Hosp. Manag. 2015, 27, 1343-1364. [CrossRef]

19. Ladhari, R.; Michaud, M. eWOM effects on hotel booking intentions, attitudes, trust, and website perceptions. Int. J. Contemp. Hosp. Manag. 2015, 46, 36-45. [CrossRef]

20. Tsao, W.C.; Hsieh, M.T.; Shih, L.W.; Lin, T.M. Compliance with eWOM: The influence of hotel reviews on booking intention from the perspective of consumer conformity. Int. J. Hosp. Manag. 2015, 46, 99-111. [CrossRef]

21. Porter, M. Competitive Advantage; The Free Press: New York, NY, USA, 1985.

22. Wang, H.W.; Wang, H.; Chang, H.H. The moderating effect of customer perceived value on online shopping behaviour. Online Inf. Rev. 2011, 35, 333-359.

23. Peng, L.; Zhang, W.; Wang, X.; Liang, S. Moderating effects of time pressure on the relationship between perceived value and purchase intention in social E-commerce sales promotion: Considering the impact of product involvement. Inf. Manag. 2019, 56, 317-328. [CrossRef]

24. Zeithaml, V.A. Consumer Perceptions of Price, Quality, and Value: A Means-End Model and Synthesis of Evidence. J. Mark. 1988, 52, 2. [CrossRef]

25. Darden, W.R.; Griffin, M.; Babin, B.J. Work and/or Fun: Measuring Hedonic and Utilitarian Shopping Value. J. Consum. Res. 1994, 20,644.

26. Sweeney, J.C.; Soutar, G.N. Consumer perceived value: The development of a multiple item scale. J. Retail. 2001, 77, 203-220. [CrossRef]

27. Petrick, J.F. Development of a Multi-Dimensional Scale for Measuring the Perceived Value of a Service. J. Leis. Res. 2002, 34, 119-134. [CrossRef]

28. Bauer, R. Consumer Behavior as Risk Taking. In Proceedings of the 43rd Conference of the American Marketing Association, Chicago, IL, USA, 15-17 June 1960; pp. 389-398.

29. Cox, D.; Rich, S. Perceived Risk in Consumer Decision Making-The Case of Telephone Shopping. J. Mark. Res. 1964, 1, 32-39.

30. Peter, J.P.; Ryan, M.J. An Investigation of Perceived Risk at the Brand Level. J. Mark. Res. 1976, $13,184$. [CrossRef]

31. Jacoby, J.; Kaplan, L.; Szybillo, G. Components of Perceived Risk in Product Purchase: A Cross-Validation. J. Appl. Psychol. 1974, 59, 287-291.

32. Peter, J.P.; Tarpey, L.X. A Comparative Analysis of Three Consumer Decision Strategies. J. Consum. Res. 1975, 2, 29-37. [CrossRef]

33. Jarvenpaa, S.L.; Todd, P.A. Consumer Reactions to Electronic Shopping on the World Wide Web. Int. J. Electron. Commer. 1996, 1, 59-88. [CrossRef]

34. Jordan, E.; Moore, J. An In-Depth Exploration of Residents' Perceived Impacts of Transient Vacation Rentals. J. Travel Tour. Mark. 2018, 35, 90-101. [CrossRef]

35. Akar, E.; Dalgic, T. Understanding online consumers' purchase intentions: A contribution from social network theory. Behav. Inf. Technol. 2018, 37, 473-487. [CrossRef]

36. Nicosica, F. Consumer Decision Process-Marketing and Advertising Implications; Prentice-Hall: Englewood Cliffs, NJ, USA, 1966.

37. Mowen, J. Consumer Behaviour; Macmillan Coll Div: New York, NY, USA, 1990.

38. Feeny, P. Seasonal Changes in Oak Leaf Tannins and Nutrients as a Cause of Spring Feeding by Winter Moth Cater. Ecology 1970, 51, 565-581. [CrossRef]

39. Engel, P. Facilitating Innovation: An Action-Oriented Approach and Participatory Methodology to Improve Innovative Social Practice in Agriculture. Ph.D. Thesis, Wageningen University, Wageningen, The Netherland, 1995.

40. Chen, A.; Lu, Y.; Wang, B. Customers' purchase decision-making process in social commerce: A social learning perspective. Int. J. Inf. Manag. 2017, 37, 627-638. [CrossRef] 
41. Olbrich, R.; Holsing, C. Modeling Consumer Purchasing Behavior in Social Shopping Communities with Clickstream Data. Int. J. Electron. Commer. 2011, 16, 15-40. [CrossRef]

42. Moody, G.D.; Galletta, D.F.; Lowry, P.B. When trust and distrust collide online: The engenderment and role of consumer ambivalence in online consumer behavior. Electron. Commer. Res. Appl. 2014, 13, $266-282$. [CrossRef]

43. Godes, D.; Mayzlin, D. Using Online Conversations to Study Word-of-Mouth Communication. Mark. Sci. 2004, 23, 545-560. [CrossRef]

44. Xu, X.; Wang, X.; Li, Y.; Haghighi, M. Business Intelligence in Online Customer Textual Reviews: Understanding Consumer Perceptions and Influential Factors. Int. J. Inf. Manag. 2007, 37, 673-683. [CrossRef]

45. Mehrabian, A.; Russell, J. An Approach to Environmental Psychology; MIT Press: Cambridge, MA, USA, 1974.

46. Eroglu, S.A.; Machleit, K.A.; Davis, L.M. Empirical testing of a model of online store atmospherics and shopper responses. Psychol. Mark. 2003, 20, 139-150. [CrossRef]

47. Vermeulen, I.E.; Seegers, D. Tried and tested: The impact of online hotel reviews on consumer consideration. Tour. Manag. 2009, 30, 123-127. [CrossRef]

48. Sotiriadis, M.; Zyl, C. Electronic Word-of-Mouth and Online Reviews in Tourism Services: The Use of Twitter by Tourists. J. Electron. Commer. Re. 2017, 13, 103-124. [CrossRef]

49. Murray, K.B.; Schlacter, J.L. The impact of services versus goods on consumers' assessment of perceived risk and variability. J. Acad. Mark. Sci. 1990, 18, 51-65. [CrossRef]

50. Mhembere, D.; Roncal, W.R.; Sussman, D.L.; Priebe, C.E.; Jung, R.E.; Ryman, S.; Vogelstein, R.J.; Vogelstein, J.T; Burns, R.C. Computing scalable multivariate global invariants of large (brain-) graphs. In Proceedings of the Global Conference on Signal \& Information Processing, Austin, TX, USA, 3-5 December 2013.

51. Featherman, M.S.; Miyazaki, A.D.; Sprott, D.E. Reducing online privacy risk to facilitate e-service adoption: The influence of perceived ease of use and corporate credibility. J. Serv. Mark. 2010, 24, 219-229. [CrossRef]

52. Cacioppo, J.; Petty, R. The Need for Cognition. J. Pers. Soc. Psychol. 1982, 42, 116-131. [CrossRef]

53. Ariff, M.S.M.; Sylvester, M.; Zakuan, N.; Ismail, K.; Ali, K.M. Consumer Perceived Risk, Attitude and Online Shopping Behaviour; Empirical Evidence from Malaysia. Iop Conf. Ser. Mater. Sci. Eng. 2014, 58, 012007. [CrossRef]

54. Chatterjee, P. Online Reviews: Do Consumers Use Them? Adv. Consum. Res. 2001, 28.

55. Poon, W.C.; Poon, W. Users' adoption of e-banking services: The Malaysian perspective. J. Bus. Ind. Mark. 2007, 23, 59-69. [CrossRef]

56. Miley, P. Perceived Risk for Shopping via the Internet among United States Consumers. Ph.D. Thesis, Texas Woman's University, Denton, TX, USA, 2001.

57. Duman, T.; Mattila, A.S. The role of affective factors on perceived cruise vacation value. Tour. Manag. 2005, 26, 311-323. [CrossRef]

58. Fishbein, M.; Ajzen, I. Belief, Attitude, Intention, and Behavior: An Introduction to Theory and Research; Addison-Wesley: Reading, MA, USA, 1975.

59. Bansal, H.S.; Voyer, P.A. Word-of-Mouth Processes within a Services Purchase Decision Context. J. Serv. Res. 2000, 3, 166-177. [CrossRef]

60. Baumgartner, H.; Homburg, C. Applications of structural equation modeling in marketing and consumer research: A review. Int. J. Res. Mark. 1996, 13, 139-161. [CrossRef]

61. Hayes, A.; Scharkow, M. The Relative Trustworthiness of Inferential Tests of the Indirect Effect in Statistical Mediation Analysis: Does Method Really Matter? Psychol. Sci. 2013, 24, 1918-1927. [CrossRef]

62. Zhao, J.; Fang, S.; Jin, P. Modeling and Quantifying User Acceptance of Personalized Business Modes Based on TAM, Trust and Attitude. Sustainability 2018, 10, 356. [CrossRef]

63. Zhao, J.; Wang, J.; Fang, S.; Jin, P. Towards Sustainable Development of Online Communities in the Big Data Era: A Study of the Causes and Possible Consequence of Voting on User Reviews. Sustainability 2018, 10, 3156. [CrossRef]

(C) 2019 by the authors. Licensee MDPI, Basel, Switzerland. This article is an open access article distributed under the terms and conditions of the Creative Commons Attribution (CC BY) license (http://creativecommons.org/licenses/by/4.0/). 

Article

\title{
An Analysis of the Sustainable Tourism Value of Graffiti Tours through Social Media: Focusing on TripAdvisor Reviews of Graffiti Tours in Bogota, Colombia
}

\author{
Hwayoon Seok, Yeajin Joo and Yoonjae Nam * \\ Department of Culture, Tourism and Content, Kyung Hee University, South 130-701, Korea; \\ vipshy5734@khu.ac.kr (H.S.); jyj6241@khu.ac.kr (Y.J.) \\ * Correspondence: ynam@khu.ac.kr
}

Received: 1 May 2020; Accepted: 26 May 2020; Published: 29 May 2020

\begin{abstract}
This study attempts to analyze the value of graffiti tours from the perspective of sustainable tourism by examining actual reviews by social media users using text mining and social network analysis. The text mining technique indicates that "artist," "history," "political," "culture," "social," "city," "background," "great," "recommend," "excellent", and "worth," are frequently used keywords for the reviews. When comparing word frequencies per review between 2013-2016 and 2017-2019, the results show that the words, "history," "political," "culture," and "social," have been more frequently used over time. Furthermore, the network visualization shows that those words connoting socio-cultural sustainability are mutually connected. Therefore, the study suggests that graffiti tours can have potential for assuming the role of sustainable tourism, since the keywords from reviews are associated with the perspective of socio-cultural sustainability.
\end{abstract}

Keywords: sustainable tourism; graffiti; social media; text mining; social network analysis

\section{Introduction and Research Backgrounds}

Visual image is a medium containing a clear reality or truth. This visual communication is an important theoretical concept as it emphasizes various characteristics of the cultural process [1]. The mural, which was the first visual communication, is an example of a work that consists not only of an artistic aspect, but also depicts the culture and way of life at the time it was created. Graffiti has existed as a social phenomenon since the beginning of cave paintings. Graffiti is defined as writing or drawing on walls or other surfaces of public places by uneven scratching or spraying [2]. This rather unregulated form of artwork is often misinterpreted as an expression of crime and disorder, since "in its purest form," street art is "artwork created without authorization, usually illegally, on either private or public property" [3]. In tackling vandalism, graffiti has been the subject of discussion among policymakers around the world. However, there is a certain complexity to the culture of graffiti; it is not a simple matter of acceptance of art or vandalism [4]. Recent urban policies, along with increasing discourse on the 'creative city,' have led to changes by encouraging communication among people to promote urban development [5]. In the same context, the importance of creativity in economies after industrialization has pushed urban managers to reevaluate graffiti [6]. Likewise, graffiti arts in some cities including Bogota, Colombia has been regarded as a tool for tourism development, heritage conservation, socio-economic regeneration and cultural empowerment.

For example, in 2007, graffiti was legalized in Colombia's capital, Bogota, by the power of civil society. The city has since changed through the cooperation of the government, graffiti artists, and citizens. The first project involving graffiti was the 26 th road environment improvement project, 
which extended to Bogota's buildings, shops, and homes. Such efforts contribute to the transformation of the image of Colombia as a crime-ridden and depressed country [7]. Jose Andres Duarte, the head of the Research Institution of Local Tourism of Bogota, stated, "We have improved the competitiveness of Bogota through the development of tourism wherein local residents are put first and improvement of environment of internet connection." This was in line with its policy of prioritizing local residents, which it sought to implement by creating a new environment for the city and boosting tourism According to the UNWTO's (United Nations World Tourism Organization) [8] country-specific inbound tourism data, Colombia had 4,282,000 people enter the country in 2018. The average increase rate of inbound tourists over the past 10 years was $9.7 \%$; this is significantly higher than the $5.4 \%$ average growth rate of other 101 countries. Furthermore, in the Travel and Tourism Competitiveness Index of 136 countries in 2017, Colombia scored 3.37 in the Cultural Resources and Business Travel sector (average of 2.32 points in the whole country) [9]. In urban planning, resilience has become a complex interaction between society, nature, land use management, and policy. This dynamic ensures meaningful participation as the different arms prepare for the collapse of socio-economic change, thus achieving equality and meaningful stakeholder involvement. A policy-adaptable social infrastructure must be built to ensure participation of these sectors [10,11]. In other words, urban regeneration combines sustainability with economic, social, cultural, and environmental perspectives. Under the goal of urban regeneration, graffiti tours that interact with tourists through works containing cultural messages, along with the cooperation of local residents, are found to be linked to sustainability.

In a situation where concepts of sustainable and responsible tourism are gaining more attention, it is necessary to understand the role of graffiti tours as sustainable tourism. Studies on graffiti and graffiti tours mainly present graffiti's various social phenomena, roles, and utilization plans through case studies, spatial analysis, and in-depth interviews with local residents, graffiti artists, and policy-related individuals $[4,6,12-15]$. However, still there have been only few researches on the perspectives of tourists who have actually experienced graffiti tours and evaluated them. In particular, tourism is a major source of information for many tourists. Many tend to search online-based reviews of various users in evaluating experiential products [16]. These opinions spread to potential tourists through various social media platforms; as such, these data also serve as a tool for understanding the opinions of numerous tourists from diverse backgrounds.

Thus, this study assumes that graffiti tourists' online reviews may reflect economic, socio-cultural, and environmental aspects of sustainability beyond showing their satisfactions from travel experiences. Therefore, this study examines how sustainable issues have been discussed among actual tourists by examining their reviews on social media. In 2017, the United Nations General Assembly declared 2017 to be "the International Year of Sustainable Tourism for Development," and it was also the year that the UNWTO proclaimed the "2030 Agenda for Sustainable Development." For these reasons, the year 2017 is a pivotal period for sustainable tourism and was determined to be a reference point when analyzing the shift in views on graffiti tours.

Social media platforms are an important resource of big data, as they have become diversified by generalized use. They are suitable for text mining analysis because they consist mainly of unstructured texts [17-19]. For the above purposes, the study employed the technique of text mining in order to extract useful information in big data to analyze and interpret texts posted on social media. In addition, the structure of the network of words was examined based on the results of the analysis for the social network analysis. Through this analysis, we confirm the role of sustainable tourism in graffiti tours from a tourist's perspective, instead of in the indiscriminate development of graffiti tours, and draw implications accordingly.

\subsection{Function of Sustainable Tourism}

The concept of sustainability originates in the report "Our Common Future," published by the WCED (World Commission on Environment and Development) in 1987. It defines sustainable development as the ability of humanity to make development sustainable to ensure that it meets 
the needs of the present without compromising the ability of future generations to meet their own needs [20]. Ever since the report was published, sustainable development has received extensive public attention and provided many challenges worldwide [21]. There are several factors that explain why the tourism industry shows such an interest in sustainable development: Tourism has great economic significance, as it creates employment and generates income, it impacts various industries, and it depends on the cultural heritage and landscape of a place. Tourism also has negative and positive effects on the natural environment and tourist locations [22]. The UNEP (United Nations Environment Program) and UNWTO [23] also mentioned two relationships between tourism and sustainable development. First, tourism is a dynamic, growing industry that makes key contributions to many countries. Second, tourism is a set of activities that includes special relations among visitors, industries, the environment, and communities. Therefore, sustainable tourism is defined as tourism that protects and promotes the opportunities of future generations, while simultaneously meeting the present needs of tourists and communities by not only preserving and maintaining culture, essential ecological processes, biodiversity, and life support systems but also managing resources to meet economic, social, and aesthetic needs.

Donyadide [24] presented “The Magic Pentagon of Sustainable Tourism," which describes five elements of sustainable tourism, from an ethical standpoint, in order to maintain a high level of balance with tourists in terms of the social and ecological characteristics of travel sites. The five elements are: economic health, the satisfaction of guests, a healthy culture, protection of resources, and the well-being of locals. Recently, the approach has been subdivided into more categories to emphasize the relevance to tourism of social values, such as population, peace, prosperity, pollution, and protection [25]. Tosun [26] mentioned that the operation of sustainable tourism development principles requires tough political and economic choices and decisions based on complicated socioeconomic and environmental transactions. Crouch and Ritchie [27] argue that not considering sustainability in discussing the competitiveness of travel destinations is an unsubstantial concept, and that it is necessary to reflect not only on the economic resources but also on the ecological, social, cultural, and political resources. Tourism competitiveness is the capacity to accumulate and create value-added products that make the value of resources sustainable, while maintaining the current market position [28]. Sustainable tourism is in line with tourism competitiveness-it creates and integrates product values and maintains resources.

As numbers of tourists increase each year, so do issues such as greenhouse gas emissions, financial leakage, resource management, or effects on communities and cultural heritage, which require strong partnership among all stakeholders of tourism in implementing critical measures through the "2030 Agenda for Sustainable Development" [29]. These efforts for sustainable tourism require an integrated policy that addresses multiple aspects. It is desirable to understand sustainable tourism not as a substitute for conventional mass tourism, but as a process to improve it and solve the current issues tourism faces, while meeting the needs of all the stakeholders of sustainable tourism, such as governments, local governments, residents, communities, tourism business operators, and tourists [30]. In other words, sustainable tourism is a concept that considers and promotes positive interactions among all residents, tourists, organizations, and business operations related to tourist sites. From the relationship-based perspective, the key components of the paradigm for sustainable tourism are enhancing the competencies of a community and building relationships and alliances to steer the regional economy in a sustainable direction that is beneficial for the environment [28]. In other words, sustainable tourism requires development in consideration of both the community and tourists. The indicators of sustainable tourism, according to the three perspectives proposed by UNEP and UNWTO [23], are presented in Table 1. 
Table 1. Sustainable development of tourism [23]

\begin{tabular}{cr}
\hline Sustainability Perspective & Contents \\
\hline Economic sustainability & $\begin{array}{c}\text { long-term economic operations, stable employment, income-earning } \\
\text { opportunities, social services to host communities, poverty alleviation }\end{array}$ \\
\hline Socio-cultural sustainability & $\begin{array}{r}\text { socio-cultural authenticity, cultural heritage, traditional values, } \\
\text { inter-cultural understanding and tolerance }\end{array}$ \\
\hline Environmental sustainability & essential ecological processes, conserve natural heritage and biodiversity \\
\hline
\end{tabular}

Richins [31] and Choi and Turk [32] summarized the details of each subcategory of sustainability in addition to the contents of previous research and viewpoints of international organizations. This information is shown in Table 2.

Table 2. Sub-categories according to sustainable tourism perspective [31,32].

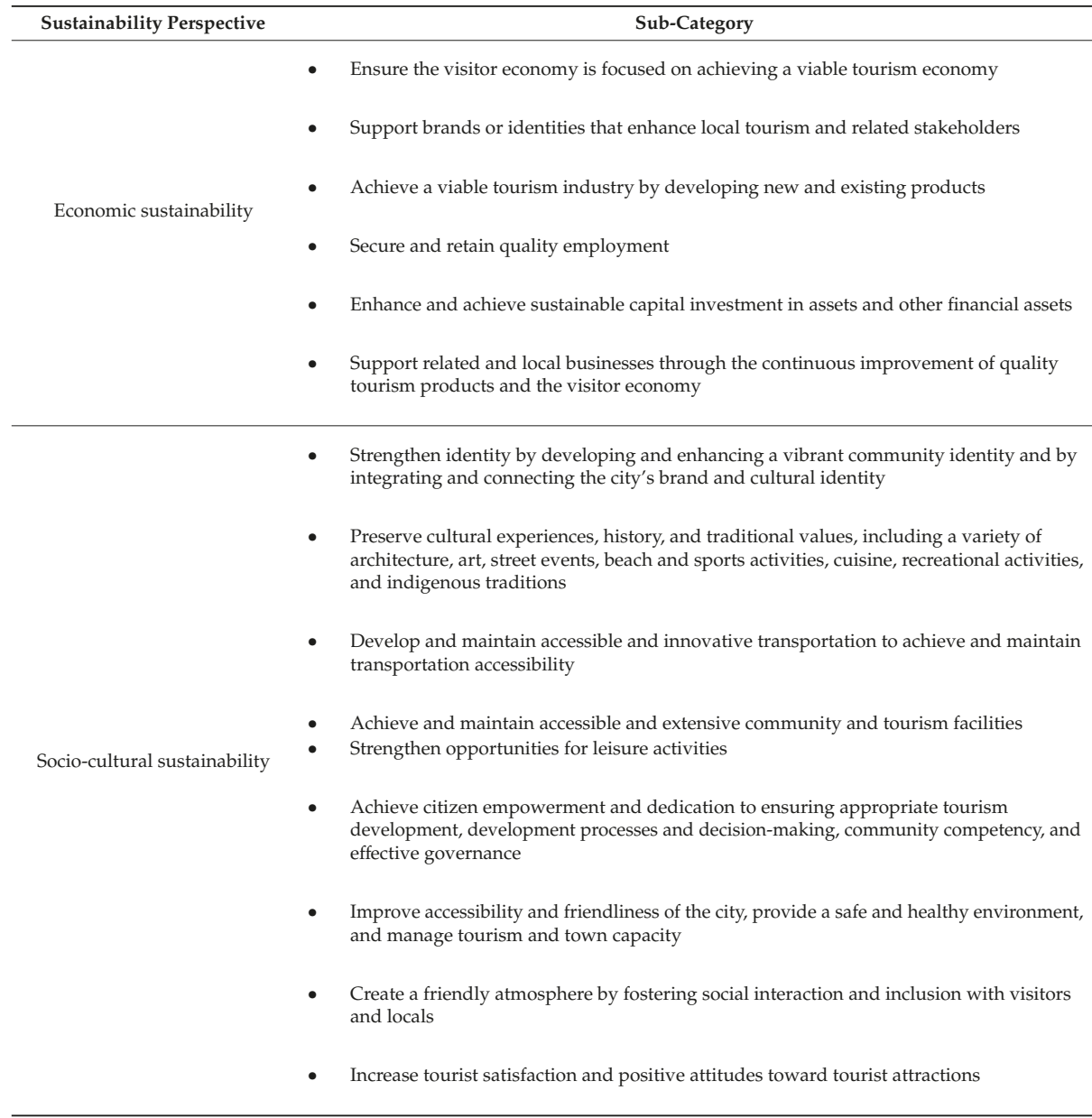


Table 2. Cont.

\begin{tabular}{cc}
\hline Sustainability Perspective & \multicolumn{1}{c}{ Sub-Category } \\
\hline Environmental sustainability & $\begin{array}{l}\text { Provide sustainable technologies, systems, and requirements to new and existing } \\
\text { buildings, and minimize environmental impact through regional improvements }\end{array}$ \\
& $\begin{array}{l}\text { Manage, maintain, and enhance natural assets; protect flora and fauna; minimize loss of } \\
\text { habitat and biodiversity; and use minimal resources to attract visitors }\end{array}$ \\
\hline
\end{tabular}

In this study, economic, socio-cultural, and environmental sustainability, along with the subcategories of sustainability, form the framework for the analysis of the perspectives of Bogota graffiti tours that are reflected in sustainability as values of sustainable tourism

\subsection{Graffiti Tour}

Practical visual communication like the Altamira and Lascaux cave paintings are able to show the culture of an era. Their main purpose is the outcome of the image, which was the first visualization work [33]. Cave paintings were the first attempt to construct an effective visual layout system through the arrangement of visual elements and replace abstract concepts with visual forms. In the same vein, Jordan's 5th century BC mural includes geometric designs and hierarchical, complex social depictions, which help viewers understand the role of art in early visual communication systems and preliterate societies [34]. This allows art not to be appreciated simply as an aesthetic element, but also as an innovative creation that maximizes the use of natural objects and explains art from a semiotic perspective. In modern times, the visual communication of various artefacts, designs, social media platforms, etc., is ingrained in our lives and spreads different ideologies by controlling the time and space they create $[35,36]$. Visual image is a medium containing a clear reality or truth, and visual communication is an important theoretical concept as it emphasizes various characteristics of cultural processes [1].

The mural — the first visual communication and the origin of art-again emerged in the present and developed into a genre of art that conveys social messages and expresses the artist's values and personality. Graffiti, which can be seen as a representative model of cave murals, is defined as a writing or painting that is irregularly scratched or sprayed upon walls or other surfaces in a public place [2]. Graffiti has existed for as long as mankind. Cave paintings can be considered to be an example of graffiti [2]. In the 1970s, graffiti was derived from hip-hop culture, which played a significant part in representing the culture of the young generation through the expression of a new form of music, dance, and art [6]. From the 1970s to the 1980s, graffiti was regarded negatively, as it was characterized as a key factor in profit loss, depreciation, and decrease in retail sales in certain regions or communities [37]. However, recent urban policies, along with increasing discourse on the "creative city," have led to changes by encouraging communication among people to promote urban development [5]. In the same context, the importance of creativity in economies after industrialization has pushed urban managers to reevaluate graffiti [6]. The urban environment has countless surfaces and objects for expression and, at the same time, provides an audience with an opportunity to see these expressions. The aesthetic image of graffiti is being magnified in galleries, on the internet, and via social media [38]. This suggests that the aforementioned visual communication is directly connected to graffiti.

Gomez [39] distinguished art in the form of graffiti as "graffiti art" and stated that it is characterized as "high art" and "folk art" in which the desire for artistic creation has become its motivation. This art also plays an important role in tourism. All of the cultural elements experienced by tourists are included in the category of cultural tourism, which is expanding in an artistic sense. As seen in Table 3, art tourism refers to a set of tourism-related activities of consuming contemporary culture. These include participation in the fine arts, performing and exhibiting arts, artistic events, festivals, and entertainment. 
Table 3. Definition of art tourism.

\begin{tabular}{cr}
\hline Researcher & Definition of Art Tourism \\
\hline Weiler and Hall [40] & Tourism activities in which one participates in performing arts and exhibiting arts \\
\hline Zeppel [41] & $\begin{array}{c}\text { Experiential art in which one participates and is motivated by art practices, } \\
\text { visual arts, and art festivals }\end{array}$ \\
\hline Gratton and Taylor [42] & Tourism activities consuming contemporary culture \\
\hline Hughes [43] & $\begin{array}{r}\text { Tourism activities that include participation in the fine arts, performing arts, } \\
\text { cultural events, as well as festivals and entertainment }\end{array}$ \\
\hline
\end{tabular}

From the commercial aspect, art invigorates tourism products, increases market attractiveness, provides new value to a region, and performs an important role in sales and promotion, while also improving product quality and appeal [41]. Graffiti has become an art tourism space that contributes not only to the creation of new tourism assets, but also to its revitalization [15]. For example, through the promotion of graffiti tours, graffiti has now come to be perceived as valuable rather than negative in terms of urban aesthetics, and as contributing to place-making by performing a key role that gives an identity to a city $[4,38]$. Graffiti can be linked to urban morphologies and the social identity of a place, [13] leading to it ultimately becoming a tourist attraction. In other words, a city's graffiti can be promoted as a new tourist spot and become a communication tool that leads to interaction between residents and tourists.

\subsection{Graffiti Tours in Bogota, Colombia}

Initially, graffiti art in Bogota was not recognized as art and was seen as spoiling the cityscape. Following an action brought by a civic organization to legalize graffiti-art activities in 2007, the city began to view graffiti art as an important activity of art and culture. According to the "Bogota graffiti tour", a representative cooperative and commercial site related to Bogota graffiti tours [44], graffiti tours in Bogota began with Austrian and Canadian graffiti artists who wanted to show the unique and diverse aspects of the work of local artists to international tourists. Since then, graffiti art in Bogota has been growing, and art sites have become famous sightseeing spots. Graffiti art painted on commercial places or on the walls of private houses covers culture, history, and messages related to society, such as the indigenous people of Colombia, female aborigines, racial discrimination, preservation of life, and feminism. The Bogota Graffiti Tour provides tourists with an introduction to graffiti tours in Bogota, free or private tours led by guides, bike tours, city tours, and workshops. The Bogota Graffiti Tour is guided by a graffiti promoter, manager, artist, anthropologist, and designer. They guide tourists throughout the city, providing noteworthy information and key facts about the Bogota graffiti scene to facilitate appreciation of the artwork. The bike tour is significant as it can help reduce environmental issues related to traffic. Graffiti artists and associated creators have become members of a team that plans the best alternatives to help artists' projects. This differentiated approach to the cultural development of Bogota is "Responsible Graffiti Practice." The "Responsible Graffiti Practice" is a participative process aiming to develop new cultural policies for graffiti art, wherein graffiti is recognized as a form of art in which the participation of citizens in making decisions on public spaces is encouraged. Such efforts contribute to the transformation of the image of Colombia as a crime-ridden and depressed country [7]. As examined earlier in the theoretical background, sustainable tourism coincides with the process of solving problems in current tourism with satisfying the needs of relevant stakeholders. As such, graffiti in Bogota incorporates the virtuous cycle.

According to UNWTO statistics [8] on the number of inbound tourists to countries each year, the rate of increase in the number of tourists to Colombia from 2008 to $2018(4,282,000)$ exhibited a $9.7 \%$ annual average increase, whereas the annual average in the other 112 countries over the same period was 5.4\%. In particular, the number of inbound tourists to Colombia in 2010 and 2011 were 1,719,000 and 3,324,000-the rate of increase was $93.4 \%$. According to UNWTO's October 2010 report, tourism in 
Colombia showed a trend of recovery through new investment and active promotion, despite disasters, such as flooding from the end of 2010 to the middle of 2011. Tourism was concentrated in business tours, and domestic sightseeing in Bogotá, Cali, Medellin, and Cartagena increased [45]. Figure 1 presents graffiti in Bogota, Colombia.
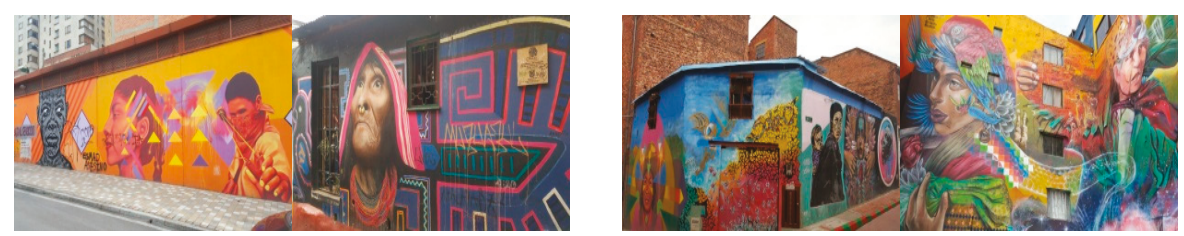

Figure 1. Graffiti in Bogota, Colombia. Source: Photo: Hwayoon Seok.

\subsection{Value of Sustainability of Graffiti Tours}

In general, the behaviors associated with environmental conservation are frequently counted as those of sustainability. Therefore, a survey of tourists' perception on sustainable tourism in the Netherlands and the differences between the types of tourists and the perception of sustainability in certain places were analyzed. Particular importance was given to the ecological view of sustainability, followed by socio-cultural and economic views [46]. The ecological perspective is considered an environmental perspective, which measures the perception of sustainable tourism by classifying it into three categories of sustainability. In general, it can be assumed that the tourists' perception of sustainability focuses on the environment. The economic perception is given the lowest weight because it affects the locals more than the tourists. Currently, it is the environment that inhabits the recognition of consumers and takes precedence to everything else, along with the efforts made to secure sustainability in diverse industries. Thus, as for sustainable tourism, alternative tourism comprising ecotourism, responsible tourism, and fair tourism, is often characterized by recognition of environment [47]. It is the form of positive tourism insisting conservation of current sites of tourism for the sustainable tourism. However, there exist aspects wherein the educational element rather than entertainment ones are spotlighted by tourist conscious of morality and regulations on tourism. From the standpoint of traditional socio-tourism, tourists experience the 'communitas' wherein the morality and regulations set forth in the space of ordinary life are ignored through migration into sites of tourism $[48,49]$. Undoubtedly, though the introspection and moral norm are taken seriously in contemporary tourism along with the changes in ages, the evidence implies that sustainable tourism needs newness and to be interesting if it is to be presented to tourists.

As examined previously, sustainability comprises environmental sustainability together with economic and socio-cultural sustainability. By taking this viewpoint into account, the preservation of socio-cultural resources, based on the conservation of environmental resources, posits the economic value thereof. In the field of tourism, the concept of sustainability was defined by Inskeep [50] as the sustainment of a life conservation system comprising cultural preservation, the sustainment of necessary ecological processes, and biodiversity together with all resources that can fulfill economic, social, and aesthetic needs to improve opportunities for future tourism. This is compatible with the thinking intending to protect resources for tourism and contemporary tourists. UNEP and UNWTO [23] also mentioned sustainable tourism as the promotion and protection of the opportunities of tourism for future generations, balanced with the fulfillment of the needs of contemporary tourists and local communities to maintain cultural integrity, ecological processes, biodiversity, and systems for sustainable life, as well as to control all resources for the fulfillment of economic, social, and aesthetic needs. Graffiti tours, which construct a new environment by creating mural paintings of stories of society and culture, can be regarded as a rearrangement of resources and the composition of a new environment to attract the interest of tourists. Moreover, culture is the way that members respond to specific political situations facing the city [7]. The proliferation of cultural planning and public art policies, along with 
the right of a large city to promote creativity, provides an opportunity to symbolize graffiti as a productive creative practice [6]. In the current trend of increasing tourism, the balanced development of cities can offer a solution to the concentration of tourists visiting famous sights. The importance of the effectiveness of local tourism and its sustainable competitiveness, through improving attitudes to accommodate tourists, has been growing gradually $[28,51]$. Graffiti tours align with sustainable tourism in how they develop socio-cultural functions based on their aesthetic functions, environmental functions by relocating the urban environment, and the revitalization of local economic functions due to job creation and tourism through the cooperation of citizens and graffiti artists. Therefore, it is necessary to conduct an empirical analysis to confirm whether graffiti tours reflect the value of sustainability from the perspective of sustainable tourism. Recently, with the increasing popularity of social media, reviews by consumers enable them to exchange product information more easily and be affected by other people's choices [52-54]. As tourism products—experiential products—cannot be evaluated before the actual experience, consumers find useful information about tourist spots, activities, and experiences on a real-time basis through online reviews, which influence decision-making and behaviors when traveling [55,56]. For these reasons, many previous studies [57-61] used online reviews of hotels, Airbnb, restaurants, and other tourist attractions to examine customer satisfaction attributes, causes, perception, and destination characteristics. Therefore, we intend to measure the sustainability of graffiti tours by analyzing the online reviews on social media based on the three perspectives of sustainable tourism. In 2017, the United Nations General Assembly declared the international year of sustainable tourism for development, and UNWTO promulgated the '2030 Agenda' for sustainable development. Given that this is a major starting point for the development of sustainable tourism, it is necessary to look at the changing issues by cycle. Based on this theoretical background, the following research questions were identified:

Research question 1: How are perspectives on sustainability reflected in the online reviews about graffiti tours in Bogota?

Research question 1-1: Which keywords in the online reviews about graffiti tours in Bogota are related to perspectives on sustainability?

Research question 1-2: How have perspectives on sustainability in the online reviews about graffiti tours changed over time?

Research question 1-3: What are the structural characteristics, meanings, and roles of the key words network from the online reviews about graffiti tours for reflecting sustainability over time?

Research question 1-4: How are keywords in the online reviews about graffiti tours associated with the perspectives of sustainability?

\section{Data and Methods}

\subsection{Data}

Online travel communities that share tourist information include TripAdvisor, Lonely Planet, Travellerspoint, VirtualTourist, and Yahoo! Travel. Many studies have analyzed the utility of tourist information by reviewers and its effects on the decision-making of other tourists to assess the reliability of information on the largest online travel community, TripAdvisor. The results proved that such information had significant effects, indicating that there is high utility value in terms of information delivery $[62,63]$. TripAdvisor provides countless reviews and feedback on accommodation, restaurants, experiences, airline tickets, and cruises, and has the ability to influence approximately 500 million travelers. Travelers compare prices of hotels, flights, and cruises; book popular tours and attractions; and make restaurant reservations. Currently, TripAdvisor offers its services across 49 markets in 28 languages [64].

Accordingly, this study examines 1991 online reviews in English for "The Original Bogota Graffiti Tour" on tripadvisor.com to analyze the direct perception of tourists who experienced this tour. As there were only seven reviews in 2012, this study analyzed a total of 1984 reviews from January 2013 to July 
2019. Moreover, to examine changes in the issues of each period, an analysis was conducted on online reviews from 2013 to 2016 and from 2017 to 2019 on the basis of the year 2017, which was declared the International Year of Sustainable Tourism for Development by the United Nations General Assembly, and in which the "2030 Agenda" was introduced for sustainable development by UNWTO.

For traveler ratings, TripAdvisor's "The Original Bogota Graffiti Tour" was rated excellent in 1843 reviews (92.9\%) and terrible in six reviews $(0.3 \%)$. On the basis of the date of experience, there were 764 reviews from January 2013 to December 2016 and 1220 reviews from January 2017 to July 2019. Examined by month, most travel experiences were in December, followed by similar numbers in February, March, July, and August. December is the month in which the dry season begins in Colombia, with most Colombians taking a vacation between December and mid-January (see Figures 2 and 3).

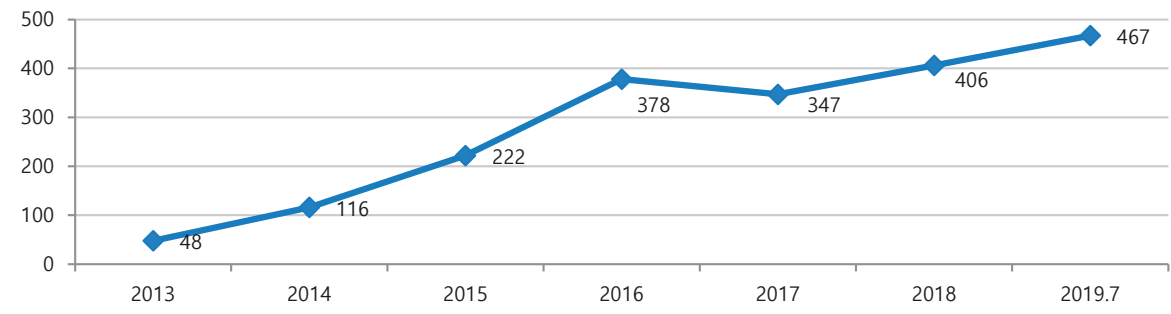

Figure 2. Bogota Graffiti Tour annual trend (No. of reviews based on experience date).

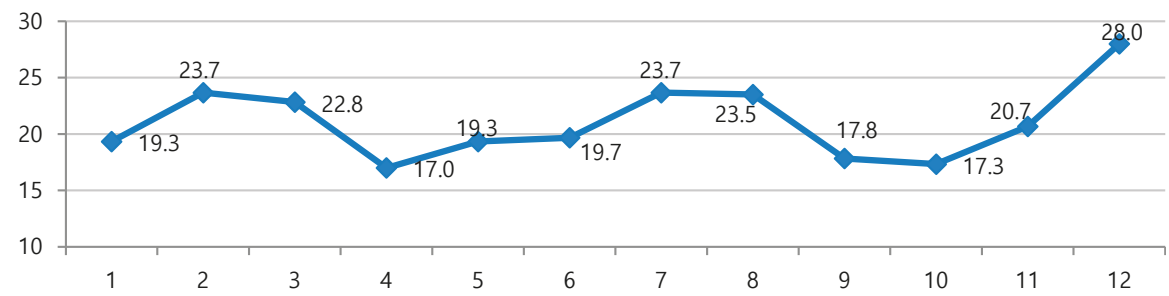

Figure 3. Bogota Graffiti Tour monthly average (No. of reviews based on experience date in 2013-2018).

\subsection{Analysis}

Recently, big data has been employed as a means of analyzing trends and customers, predicting sales, and developing marketing strategy in diverse industries. Social media has contributed to the rapid emergence of big data [17]. Social media platforms are an important resource of big data, as they have become diversified by generalized use. They are suitable for text mining analysis because they consist mainly of unstructured texts [17-19]. Text mining was introduced initially as a concept of knowledge discovery in a textual database [65]. It is a process of extracting relevant information automatically through the interaction of interest between language (text) and a mechanical algorithm (analysis) for the purposes of finding useful patterns and knowledge based on the technology of natural language processing (NLP) from text-based data. It can, therefore, be seen as an extraction of interesting and non-ordinary knowledge from unstructured language [66-68]. Thus, text mining is employed as a theoretical approach and a corresponding method in diverse fields, as well as a means of searching for information in disciplines associated with information retrieval [69].

Therefore, this study analyzed the online reviews available on TripAdvisor using text mining to address the research questions established earlier. In the present study, the program R (Version 3.5.0, RStudio Inc., Boston, MA, USA) was used to analyze source codes of the homepage TripAdvisor to examine online reviews posted there, by text mining. Upon acquisition of the data of 'online reviews' in 'TripAdvisor,' the following jobs were carried out: the preprocessing of data, the extraction of keywords, the identification of the relationship between the extracted words, and the visualization 
of the extracted words based on frequencies through word cloud [69]. Then, the structure of the network of words was examined based on the results of the analysis for the social network analysis. In addition, unnecessary or inappropriate words for this study were removed through the general refinement procedures like tokenization, normalization, stop words, strip whitespace, tolower, and stemming. By those procedures, special symbols, spaces, numbers, English punctuation marks, and stop words were eliminated; capital and lower letters were differentiated; word roots were extracted from phrases. Furthermore, some words which could imply graffiti tours, such as graffiti, mural, tour, trip, travel, and art were excluded, since the issue of 'nested patterns' could occur during the text mining procedure. Next, the structure of the network of words was examined based on the results of social network analysis. To analyze the network structure between words, a co-occurrence matrix based on the frequency of occurrence between words was generated through text mining. Each word is a node in the network based on $\mathrm{N} \times \mathrm{N}$ matrix structure (S). From this network, degree centrality is calculated. Here, each cell, Sij, indicates the intensity between word $i$ and word $j[70]$. Therefore, the word network structure based on the frequency of co-occurrence between words was presented as visualized data. Such an approach to network analysis can be regarded as appropriate to examine and compare relationships inside a network [71]. These matrices were utilized as inputs for the social network analysis software package UCINET (Version 6.624, Analytic Technologies, Lexington, KY, USA). The word networks were analyzed and visualized by NetDraw (Version 2.160. Analytic Technologies, Lexington, KY, USA).

\section{Results}

RQ1-1. The Table 4 shows the results of keywords analysis of "The Original Bogota Graffiti Tour" reviews.

Table 4. Review data set word frequencies results (2013 2019).

\begin{tabular}{cccccc}
\hline Rank & Word & Frequency & Rank & Word & Frequency \\
\hline 1 & artist & 1024 & 26 & worth & 214 \\
2 & great & 959 & 27 & love & 209 \\
3 & history & 849 & 28 & area & 208 \\
4 & recommend & 815 & 29 & local & 206 \\
5 & city & 780 & 30 & excellent & 197 \\
6 & political & 608 & 31 & experience & 189 \\
7 & knowledge & 588 & 32 & like & 187 \\
8 & interesting & 550 & 33 & nice & 176 \\
9 & informative & 541 & 34 & beautiful & 165 \\
10 & learn & 494 & 35 & understand & 157 \\
11 & way & 465 & 36 & social & 152 \\
12 & well & 426 & 37 & background & 148 \\
13 & culture & 424 & 38 & fantastic & 142 \\
14 & amazing & 383 & 39 & incredible & 134 \\
15 & highly & 379 & 40 & friendly & 133 \\
16 & know & 352 & 41 & meaning & 133 \\
17 & good & 326 & 42 & want & 129 \\
18 & work & 320 & 43 & place & 122 \\
19 & different & 270 & 44 & first & 121 \\
20 & best & 248 & 45 & suggest & 120 \\
21 & insight & 236 & 46 & style & 119 \\
22 & behind & 231 & 47 & highlight & 113 \\
23 & definitely & 231 & 48 & miss & 112 \\
24 & story & 224 & 49 & awesome & 111 \\
25 & enjoy & 221 & 50 & country & 111 \\
\hline
\end{tabular}

The top 50 words from the keywords analysis show that "artist" had the highest frequency with 1024 appearances, followed by "great." Excluding sentiment words, words such as "history," "city," "political," "culture," "work," "story," "social," and "background" showed high frequency. 
Moreover, people used words related to knowledge acquisition and influence, such as "informative," "learn," "know," "insight," "worth," "understand," and "meaning." The results showed that these words played an important role in tourists' perceptions of the Bogota Graffiti Tour. Figure 4 presents a word cloud of word frequencies results.

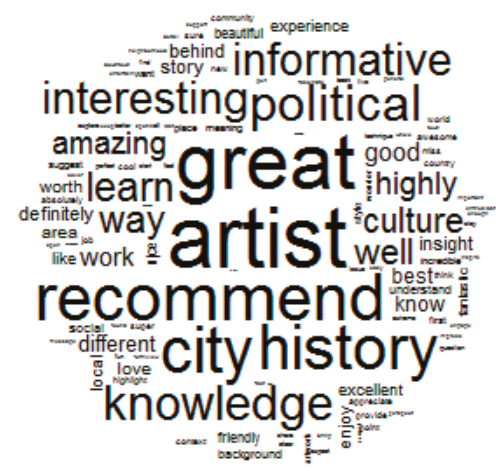

Figure 4. Online review word frequencies results in word cloud format (2013 2019).

RQ1-2. In 2017, the United Nations General Assembly declared "the International Year of Sustainable Tourism for Development," and UNWTO promulgated the "2030 Agenda for Sustainable Development." On the basis of date of experience, there are 764 reviews from January 2013 to December 2016 and 1220 reviews from January 2017 to July 2019. Then, frequency of each word per the number of total reviews were calculated, and the differences of those scores between the two time periods of 2013-2016 and 2017-2019 were presented in Table 5. In a comparison of the results of the analyses done between 2013-2016 and 2017-2019, words such as "artist," "great," "knowledge," and "city" appeared with decreased frequency per number of total reviews. However, the words that show an increase, excluding sentiment ones, are "history," "political," "culture," "behind," "story," "social," "background," "context," "artwork," and "community." Considering the rank of word frequency, there are new words ranked the top 50 for 2017 to 2019, such as "context," "artwork," and "community." New words also appeared, such as "fantastic," "wonderful," "super," and "perfect" signifying more intense, positive feelings.

Table 5. Review data set word frequencies results.

\begin{tabular}{|c|c|c|c|c|c|c|c|c|}
\hline \multirow[b]{2}{*}{ No. } & \multirow[b]{2}{*}{ Word } & \multicolumn{3}{|c|}{$2013 \sim 2016$} & \multicolumn{3}{|c|}{ 2017 2019 } & \multirow[b]{2}{*}{ (2) - (1) } \\
\hline & & Rank & $\begin{array}{c}\text { Word } \\
\text { Frequency }\end{array}$ & $\begin{array}{l}\text { Frequency/The } \\
\text { Number of Total } \\
\text { Reviews (1) }\end{array}$ & Rank & $\begin{array}{c}\text { Word } \\
\text { Frequency }\end{array}$ & $\begin{array}{l}\text { Frequency/the } \\
\text { Number of Total } \\
\text { Reviews (2) }\end{array}$ & \\
\hline 1 & artist & 1 & 440 & 0.58 & 1 & 584 & 0.48 & -0.10 \\
\hline 2 & great & 2 & 412 & 0.54 & 2 & 547 & 0.45 & -0.09 \\
\hline 3 & history & 5 & 307 & 0.40 & 3 & 542 & 0.44 & 0.04 \\
\hline 4 & recommend & 4 & 316 & 0.41 & 4 & 500 & 0.41 & - \\
\hline 5 & city & 3 & 330 & 0.43 & 5 & 450 & 0.37 & -0.06 \\
\hline 6 & political & 11 & 205 & 0.27 & 6 & 394 & 0.32 & 0.05 \\
\hline 7 & knowledge & 6 & 257 & 0.34 & 7 & 331 & 0.27 & -0.07 \\
\hline 8 & informative & 8 & 231 & 0.30 & 8 & 310 & 0.25 & -0.05 \\
\hline 9 & learn & 10 & 220 & 0.29 & 9 & 306 & 0.25 & -0.04 \\
\hline 10 & interesting & 7 & 245 & 0.32 & 10 & 304 & 0.25 & -0.07 \\
\hline 11 & culture & 14 & 152 & 0.20 & 11 & 272 & 0.22 & 0.02 \\
\hline 12 & amazing & 18 & 128 & 0.17 & 12 & 255 & 0.21 & 0.04 \\
\hline 13 & way & 9 & 222 & 0.29 & 13 & 244 & 0.20 & -0.09 \\
\hline 14 & highly & 16 & 144 & 0.19 & 14 & 233 & 0.19 & - \\
\hline 15 & well & 12 & 201 & 0.26 & 15 & 224 & 0.18 & -0.08 \\
\hline
\end{tabular}


Table 5. Cont.

\begin{tabular}{|c|c|c|c|c|c|c|c|c|}
\hline \multirow[b]{2}{*}{ No. } & \multirow[b]{2}{*}{ Word } & \multicolumn{3}{|c|}{$2013 \sim 2016$} & \multicolumn{3}{|c|}{ 2017 2019 } & \multirow[b]{2}{*}{ (2) - (1) } \\
\hline & & Rank & $\begin{array}{l}\text { Word } \\
\text { Frequency }\end{array}$ & $\begin{array}{l}\text { Frequency/The } \\
\text { Number of Total } \\
\text { Reviews (1) }\end{array}$ & Rank & $\begin{array}{c}\text { Word } \\
\text { Frequency }\end{array}$ & $\begin{array}{c}\text { Frequency/the } \\
\text { Number of Total } \\
\text { Reviews (2) }\end{array}$ & \\
\hline 16 & good & 13 & 155 & 0.20 & 16 & 171 & 0.14 & -0.06 \\
\hline 17 & work & 15 & 152 & 0.20 & 17 & 168 & 0.14 & -0.06 \\
\hline 18 & behind & 29 & 75 & 0.10 & 18 & 157 & 0.13 & 0.03 \\
\hline 19 & story & 32 & 75 & 0.10 & 19 & 149 & 0.12 & 0.02 \\
\hline 20 & definitely & 26 & 93 & 0.12 & 20 & 138 & 0.11 & -0.01 \\
\hline 21 & different & 19 & 133 & 0.17 & 21 & 137 & 0.11 & -0.06 \\
\hline 22 & know & 17 & 126 & 0.16 & 22 & 137 & 0.11 & -0.05 \\
\hline 23 & insight & 24 & 100 & 0.13 & 23 & 136 & 0.11 & -0.02 \\
\hline 24 & best & 20 & 113 & 0.15 & 24 & 135 & 0.11 & -0.04 \\
\hline 25 & excellent & 38 & 64 & 0.08 & 25 & 133 & 0.11 & 0.03 \\
\hline 26 & love & 27 & 87 & 0.11 & 26 & 121 & 0.10 & -0.01 \\
\hline 27 & experience & 30 & 75 & 0.10 & 27 & 114 & 0.09 & -0.01 \\
\hline 28 & enjoy & 21 & 108 & 0.14 & 28 & 113 & 0.09 & -0.05 \\
\hline 29 & beautiful & 43 & 57 & 0.07 & 29 & 108 & 0.09 & 0.02 \\
\hline 30 & local & 25 & 99 & 0.13 & 30 & 107 & 0.09 & -0.04 \\
\hline 31 & area & 23 & 102 & 0.13 & 31 & 106 & 0.09 & -0.04 \\
\hline 32 & worth & 22 & 108 & 0.14 & 32 & 106 & 0.09 & -0.05 \\
\hline 33 & nice & 34 & 71 & 0.09 & 33 & 105 & 0.09 & - \\
\hline 34 & fantastic & 67 & 39 & 0.05 & 34 & 103 & 0.08 & 0.03 \\
\hline 35 & like & 28 & 85 & 0.11 & 35 & 102 & 0.08 & -0.03 \\
\hline 36 & social & 46 & 55 & 0.07 & 36 & 97 & 0.08 & 0.01 \\
\hline 37 & understand & 40 & 64 & 0.08 & 37 & 94 & 0.08 & 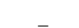 \\
\hline 38 & background & 45 & 55 & 0.07 & 38 & 93 & 0.08 & 0.01 \\
\hline 39 & meaning & 49 & 48 & 0.06 & 39 & 85 & 0.07 & 0.01 \\
\hline 40 & context & 85 & 29 & 0.04 & 40 & 79 & 0.06 & 0.02 \\
\hline 41 & friendly & 41 & 59 & 0.08 & 41 & 74 & 0.06 & -0.02 \\
\hline 42 & highlight & 60 & 41 & 0.05 & 42 & 72 & 0.06 & 0.01 \\
\hline 43 & miss & 63 & 40 & 0.05 & 43 & 72 & 0.06 & 0.01 \\
\hline 44 & artwork & 70 & 36 & 0.05 & 44 & 71 & 0.06 & 0.01 \\
\hline 45 & absolutely & 65 & 40 & 0.05 & 45 & 70 & 0.06 & 0.01 \\
\hline 46 & community & 79 & 32 & 0.04 & 46 & 68 & 0.06 & 0.02 \\
\hline 47 & super & 78 & 32 & 0.04 & 47 & 68 & 0.06 & 0.02 \\
\hline 48 & wonderful & 64 & 40 & 0.05 & 48 & 68 & 0.06 & 0.01 \\
\hline 49 & awesome & 50 & 46 & 0.06 & 49 & 65 & 0.05 & -0.01 \\
\hline 50 & perfect & 83 & 30 & 0.04 & 50 & 65 & 0.05 & 0.01 \\
\hline
\end{tabular}

RQ1-3. Figures 5 and 6 show the results of the structural characteristics of the network formed by the words' co-occurrence. These figures are visualized images of a network based on words with a stronger connection. "Artist," which was ranked the highest, was strongly connected to "great," "history," "culture," "city," "recommend," and "political," indicating that they frequently appeared together. This shows a common feature in each period. In other words, people tend to refer to "artist," while associating it with history, politics, culture, and society, and they feel that it is "great," or "amazing," so they "recommend" it to others. This result is similar to that of the previously conducted keywords analysis. The words with the second-highest connectivity vary across the different periods. While words such as "style," "technique," "amazing," and "suggest" are observed between 2013 and 2016, "community," "context," "mean," and "fantastic" are observed between 2017 and 2019. That is, these words appeared with the words that play a key role in understanding graffiti in line with concepts such as society, community, and context. 


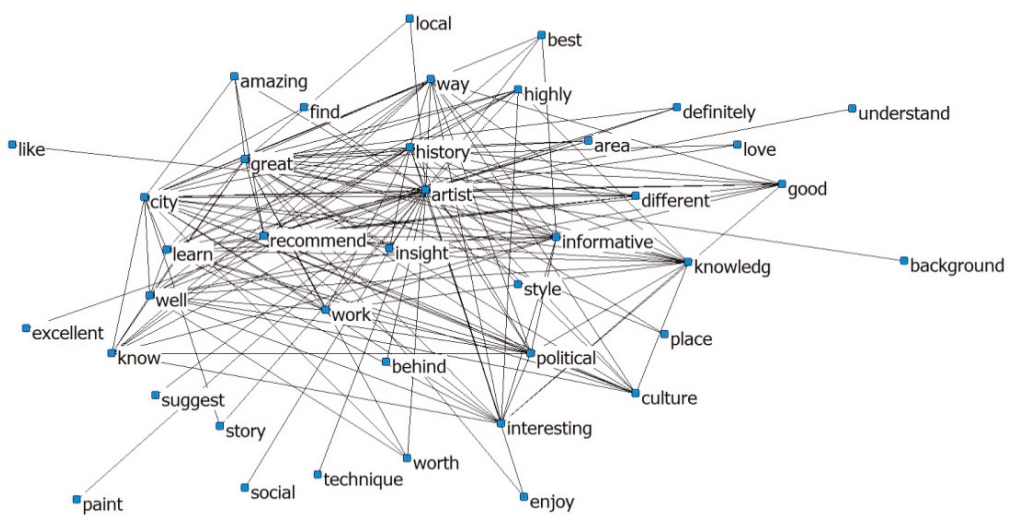

Figure 5. Word co-occurrence network (2013-2016).

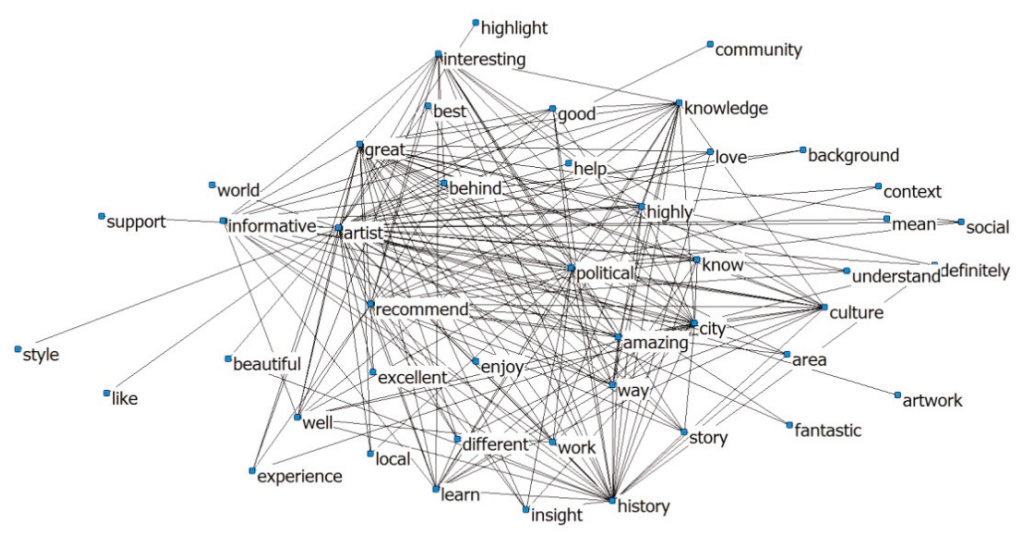

Figure 6. Word co-occurrence network (2017-2019).

RQ1-4. The words of high rank, such as "artist," "history," "city," "political," "culture," "work," "story," "social," and "background," which are associated with societal and cultural aspects were found from the keywords. There are also words associated with satisfaction and an experience of significance felt by tourists, including, "great," "recommend," "interesting," "informative," "learn," "amazing," "insight," "worth," "excellent," "incredible," and "awesome" etc. The significance of these words can be understood in the context associated with socio-cultural sustainability among viewpoints of economic, socio-cultural, and environmental sustainability. The actual reviews from tourists that include the words that show increased frequencies per total reviews between 2017 and 2019 are highlighted in Table 6. 
Table 6. Samples of tourist's reviews.

\begin{tabular}{|c|c|c|}
\hline No. & Word & Reviews \\
\hline 1 & political & $\begin{array}{c}\text { "Great tour of Bogota's graffiti, really insightful analysis of the political undertones and } \\
\text { history that is incorporated into the art." } \\
\text { "The art work may seem just pretty but if you take a closer look, it's very political with } \\
\text { Strong messages." }\end{array}$ \\
\hline 2 & history & $\begin{array}{l}\text { "The artists styles, their political views, plus history of the native Colombians and their } \\
\text { struggle to keep their identity." } \\
\text { "Learned a lot about the history of people here and how changes in their socio-economic } \\
\text { environment leads to freedom of expression through art." }\end{array}$ \\
\hline 3 & behind & $\begin{array}{c}\text { "The art is impressive and the stories behind it are captivating." } \\
\text { "It was great to have a description of what the political and historical meanings were } \\
\text { behind the art and artists." }\end{array}$ \\
\hline 4 & culture & $\begin{array}{c}\text { "The graffiti tour was an excellent way to explore Bogota's neighborhoods through the } \\
\text { artistic and cultural lense of street art." } \\
\text { "Street art is a significant part of Colombian culture so you can see it everywhere - to } \\
\text { understand the context is great." }\end{array}$ \\
\hline 5 & story & $\begin{array}{c}\text { "As well as the art work being visually beautiful it was backed up with history, politics } \\
\text { and stories of the artists." } \\
\text { "The Bogota Graffiti Tour is a brilliant way to explore the city and understand some of its } \\
\text { political background and the stories behind Bogotas street art and it's artists." }\end{array}$ \\
\hline 6 & context & $\begin{array}{l}\text { "I've been living in Bogota for a while now and this greatly enriched my understanding } \\
\text { of local street art as well as its social and political contexts in Colombia." } \\
\text { "Good knowledge shown of the street art and context social and political and the } \\
\text { importance of this art in Bogota reflecting some of the indigenous people and political } \\
\text { struggles as well as the legalities." }\end{array}$ \\
\hline 7 & community & $\begin{array}{c}\text { "It was interesting and eye opening to hear the history and politics/political discord of } \\
\text { Colombia through the artists' eyes and how these artworks have worked with the } \\
\text { community as well to strengthen them." } \\
\text { "I've learned a lot about graffiti artists and how graffiti can really make a difference for } \\
\text { some troubled communities." }\end{array}$ \\
\hline 8 & artwork & $\begin{array}{l}\text { "The tour was excellent, really informative, gave me a sense of the history and current } \\
\text { issues of Bogota in such a short stay as well as seeing some amazing artwork." } \\
\text { "The tour captures not only the artists and how they create the work but also what the } \\
\text { artwork represents which in turn informs you of Colombian society." }\end{array}$ \\
\hline 9 & background & $\begin{array}{l}\text { "A complete different view on the city, but with many details on the history of the town, } \\
\text { background stories on the art scene and as well as some political stuff." } \\
\text { "The tour not only gave an in-depth look at graffiti in the neighbourhood of the walk, but } \\
\text { also a background of graffiti/street art in Colombia along with a recent history of the } \\
\text { political state of the city of Bogota, and the country in general." }\end{array}$ \\
\hline 10 & social & $\begin{array}{l}\text { "Pretty much the best thing to do in Bogota, not just for the art, but also to understand } \\
\text { some of the social and political issues facing Bogota." } \\
\text { "Since the street art movement in Bogota began pretty much in } 2011 \text { most of the } \\
\text { information is recent and relevant to the current social and political climate in Bogota." }\end{array}$ \\
\hline
\end{tabular}

The highlight of tourist's reviews is "The art work may seem just pretty but if you take a closer look, it's very political with Strong messages," "The artists styles, their political views, plus history of the native Colombians and their struggle to keep their identity," "The art is impressive and the stories behind it are captivating," "The graffiti tour was an excellent way to explore Bogota's neighborhoods through the artistic and cultural lense of street art," and "Good knowledge shown of the street art and context social and political and the importance of this art in Bogota reflecting some of the indigenous people and political struggles as well as the legalities." This can be interpreted as socio-cultural sustainability in the Bogota graffiti tour. 


\section{Discussion and Conclusions}

The purpose of this study was to analyze the value of graffiti tours from the perspective of sustainable tourism by examining actual reviews by social media users using text mining and social network analysis. The text mining technique indicated that "artist," "history," "political," "culture," "social," "city," "background," "great," "recommend," "excellent", and "worth," were frequently used keywords for the reviews. When comparing word frequencies per review between 2013-2016 and 2017-2019, the results showed that the words, "history," "political," "culture," and "social," had been more frequently used over time. Furthermore, the network visualization showed that those words were mutually connected. From the results of contents analysis of reviews, it is suggested that tourists have gradually focused on not only artistic aspects, but also social, cultural, historical and political aspects of graffiti in Bogota. In addition, the results imply that tourists have been aware of the function of graffiti for establishing and preserving the identity of the community. Thus, this study may suggest that graffiti tours can have potential for assuming the role of sustainable tourism, since the keywords from reviews are associated with the perspective of socio-cultural sustainability, which comprises the appreciation of socio-cultural authenticity, conservation of cultural heritage and traditional values, and intercultural understanding and tolerance [23]. Based on these results, three more implications can be made.

First, the mentions of history, politics, culture, and society in graffiti tours are connected with positive emotions, interest, and the insight of tourists. The results of the network analysis on words, and their frequency of use, comprise words like the above. The factors that have the most influence over tourists' positive recognition of sightseeing spots are complex ones, comprising history, politics, culture, and society, together with the artistic perspective of graffiti. As discussed earlier, Zeppel [41] stated art invigorates tourism products, increases market attractiveness, provides new value to a region, and performs an important role in sales and promotion, while also improving product quality and appeal. Graffiti art can be a way to inspire tourists' sympathy through content associated with politics, culture, and society. This should be considered in the development or improvement of graffiti tours.

Second, tourists' viewpoint of the sustainability of graffiti tours is expanding over time. In a comparison of the results of the analyses done between 2013-2016 and 2017-2019, words such as "history," "political," "culture," "behind," "story," "social," "background," "context," "artwork," and "community" appeared with increased frequency per number of total reviews. This suggests a gradual increase in the recognition of the importance of sustainability in the consciousness of tourists confronted with graffiti tours. Expressions of more intensified positive feelings demonstrate that consequent satisfaction of tourists growing. This is in accordance with the study by Parker et al. [38] and Haworth et al. [4] that states graffiti has now come to be perceived as valuable rather than negative in terms of urban aesthetics, and as contributing to place-making by performing a key role that gives an identity to a city. Moreover, Dovey et al. [13] stated graffiti can be linked to urban morphologies and the social identity of a place. Therefore, areas where graffiti appears should be encouraged to be evolving spaces for the art through the creation of additional space to reflect contemporary issues.

Third, observing the keywords of the review, there was more content mainly related to socio-cultural sustainability, which also proves that tourists mainly perceive graffiti tours through the socio-cultural sustainability lens. This also supports Richins [31] and Choi and Turk's [32] study that explained how socio-cultural sustainability includes preserving cultural experiences, history, and traditional values, including a variety of architecture, street scenes, and art. In addition, socio-cultural sustainability's subcategory consists of tourist satisfaction/attitude toward tourist attractions, strengthening identity by developing and enhancing a vibrant community identity, and integrating and connecting brand and cultural identity. Given that the arts are at the center of elaborate visual discourse on neoliberalism, democracy, and the battle against public space, researchers can continue to examine how street artists inscribe social justice in, on, and around the streets [72]. The frequent mention of socio-cultural sustainability in the 'online review,' compared to economic or environmental sustainability - which is more relevant to local residents-is attributable to the 
viewpoints of tourists. However, greater recognition of the significance of environmental sustainability is necessary, as it is important for both tourists and local residents. This also supports the idea presented in the literature review that Cottrell et al. [46] stated environmental sustainability is the most important perspective in tourists' perception of sustainable tourism. Therefore, the stories of the role of graffiti art in environmental improvement and sustainability, as well as socio-cultural sustainability in the places in which graffiti appears, should be researched.

\section{Limitations and Future Studies}

This study assumes that graffiti tourists' online reviews may reflect the economic, socio-cultural, and environmental aspects of sustainability, beyond showing their satisfactions from travel experiences. Nevertheless, since only the case of Bogota graffiti tours was analyzed in this study, it would be prudent to gain a deeper understanding of the topic through a comparative analysis of various cases. Through this, it would be possible to draw the direction and policy implications for graffiti tours as sustainable tourism. This study analyzed only English reviews, despite the subject of the study being a Hispanic country. By analyzing reviews in other languages such as Spanish, the diversity and practicality of opinion would be better represented. Furthermore, the meaning and significance of the relationship between each word in diverse contexts can be concretized through emotional analysis on the results of network analysis.

Author Contributions: Data curation, Y.J.; Project administration, Y.N.; Supervision, Y.N.; Writing-original draft, H.S.; Writing-review and editing, Y.N. All authors have read and agreed to the published version of the manuscript.

Funding: This work was supported by a grant from KyungHee University in 2019 (KHU-20191216).

Conflicts of Interest: The authors declare no conflict of interest.

\section{References}

1. Messaris, P. Visual communication: Theory and research. J. Commun. 2003, 53, 551-556. [CrossRef]

2. Megler, V.; Banis, D.; Chang, H. Spatial analysis of graffiti in San Francisco. Appl. Geogr. 2014, 54, 63-73. [CrossRef]

3. Smith, C.Y. Street Art: An Analysis under US Intellectual Property Law and Intellectual Property's Negative Space Theory. DePaul J. Art Tech. Intell. Prop. L 2013, 24, 259.

4. Haworth, B.; Bruce, E.; Iveson, K. Spatio-temporal analysis of graffiti occurrence in an inner-city urban environment. Appl. Geogr. 2013, 38, 53-63. [CrossRef]

5. Landry, C. The Creative City: A Toolkit for Urban Innovators; Earthscan: London, UK, 2012.

6. McAuliffe, C. Graffiti or street art? Negotiating the moral geographies of the creative city. J. Urban Aff. 2012, 34, 189-206. [CrossRef]

7. World Cities Culture Forum. Available online: http://www.worldcitiescultureforum.com/cities/bogota (accessed on 7 July 2019).

8. UNWTO. Tourism Statistics Inbound Tourism: Compendium of Tourism Statistics Dataset; UNWTO: Madrid, Spain, 2020.

9. World Economic Forum. The Travel \& Tourism Competitiveness Report 2017; World Economic Forum: Cologny, Switzerland, 2017.

10. Ahern, J. From fail-safe to safe-to-fail: Sustainability and resilience in the new urban world. Landsc. Urban Plan. 2011, 100, 341-343. [CrossRef]

11. Wilson, G.A. Community resilience, policy corridors and the policy challenge. Land Use Policy 2013, 31, 298-310. [CrossRef]

12. Bowen, T. Graffiti as spatializing practice and performance. Rhizomes Cult. Stud. Emerg. Knowl. 2013, 25, 1-8.

13. Dovey, K.; Wollan, S.; Woodcock, I. Placing graffiti: Creating and contesting character in inner-city Melbourne. J. Urban Des. 2012, 17, 21-41. [CrossRef]

14. Halsey, M.; Young, A. 'Our desires are ungovernable' Writing graffiti in urban space. Theor. Criminol. 2006, 10, 275-306. [CrossRef] 
15. Mokras-Grabowska, J. Art-tourism space in Łódź: The example of the Urban Forms Gallery. Turyzm 2014, 24, 23-30. [CrossRef]

16. Chevalier, J.A.; Mayzlin, D. The effect of word of mouth on sales: Online book reviews. J. Mark. Res. 2006, 43, 345-354. [CrossRef]

17. Chae, B.K. Insights from hashtag\# supplychain and Twitter Analytics: Considering Twitter and Twitter data for supply chain practice and research. Int. J. Prod. Econ. 2015, 165, 247-259.

18. Chen, H.; Chiang, R.H.; Storey, V.C. Business intelligence and analytics: From big data to big impact. MIS Q. 2012, 36, 1165-1188. [CrossRef]

19. Russell, M.A. Mining the Social Web: Data Mining Facebook, Twitter, LinkedIn, Google+, GitHub, and More; O'Reilly Media, Inc.: Sebastopol, CA, USA, 2013.

20. WCED. Our Common Future; Oxford University Press: New York, NY, USA, 1987.

21. Hall, C.M.; Lew, A.A. The geography of sustainable tourism development: An introduction. In Sustainable Tourism: Geographical Perspectives; Addison Wesley Longman Ltd.: Harlow, UK, 1998; pp. 1-24.

22. Wight, P. Tools for Sustainable Analysis in Planning and Managing Tourism and Recreation in the Destination, Dalam Suatainable Tourism, a Geographical Perspective; Hall, C.M., Lew, A.A., Eds.; Longman: Harlow, UK, 2008.

23. UNEP, UNWTO. Making Tourism More Sustainable - A Guide for Policy Makers; UNWTO: Madrid, Spain, 2005.

24. Donyadide, A. Ethics in tourism. Eur. J. Soc. Sci. 2010, 17, 426-433.

25. Buckley, R. Sustainable tourism: Research and reality. Ann. Tour. Res. 2012, 39, 528-546. [CrossRef]

26. Tosun, C. Challenges of sustainable tourism development in the developing world: The case of Turkey. Tour. Manag. 2001, 22, 289-303. [CrossRef]

27. Crouch, G.I.; Ritchie, J.B. Tourism, competitiveness, and societal prosperity. J. Bus. Res. 1999, 44, 137-152. [CrossRef]

28. Hassan, S.S. Determinants of market competitiveness in an environmentally sustainable tourism industry. J. Travel Res. 2000, 38, 239-245. [CrossRef]

29. UNWTO. Annual Report 2017; UNWTO: Madrid, Spain, 2018.

30. Butler, R. Sustainable tourism-looking backwards in order to progress? In Sustainable Tourism: A Geographical Perspective; Addison Wesley Longman Ltd.: Harlow, UK, 1998; pp. 25-34.

31. Richins, H. Environmental, cultural, economic and socio-community sustainability: A framework for sustainable tourism in resort destinations. Environ. Dev. Sustain. 2009, 11, 785-800. [CrossRef]

32. Choi, H.C.; Turk, E.S. Sustainability indicators for managing community tourism. In Quality-of-Life Community Indicators for Parks, Recreation and Tourism Management; Springer: Dordrecht, The Netherlands, 2011; pp. 115-140.

33. Huppatz, D. The Cave and the Retrospective Construction of Design. AMD Writing Intersections Conference Proceedings. 2009. Available online: https://researchbank.swinburne.edu.au/file/ad3a1aa9-a7bc-4f87-85913f08b0ddf6dc/1/PDF\%20\%28Published\%20version\%29.pdf (accessed on 29 May 2020).

34. Drabsch, B.; Bourke, S. Early visual communication: Introducing the 6000-year-old Buon Frescoes from Teleilat Ghassul, Jordan. Arts 2019, 8, 79. [CrossRef]

35. Hepworth, K. History, power and visual communication artefacts. Rethink. Hist. 2016, 20, 280-302. [CrossRef]

36. Schreiber, M. Audiences, aesthetics and affordances: Analysing practices of visual communication on social media. Digit. Cult. Soc. 2017, 3, 143-163. [CrossRef]

37. Weisel, D.L. Graffiti; Office of Community-oriented Policing Services: Washington, DC, USA, 2009.

38. Parker, A.; Khanyile, S.; Joseph, K. Where Do We Draw the Line?: Graffiti in Maboneng, Johannesburg; Gauteng City Region Observatory (GCRO): Johannesburg, South Africa, 2019; Volume 13.

39. Gómez, M.A. The writing on our walls: Finding solutions through distinguishing graffiti art from graffiti vandalism. U. Mich. JL Reform 1992, 26, 633.

40. Weiler, B.; Hall, C.M. Special interest tourism: In search of an alternative. In Special Interest Tourism: In Search of an Alternative; Belhaven Press: London, UK, 1992; pp. 199-204.

41. Zeppel, H. Review: Arts and Heritage Tourism. In Special Interest Tourism; Weiler, B., Hall, C.M., Eds.; Belhaven Press: London, UK, 1992.

42. Gratton, C.; Taylor, P. Impacts of festival events: A case study of Edinburgh. In Tourism and Spatial Transformations; CAB International: Wallingford, UK, 1995; pp. 225-238.

43. Hughes, H. Arts, Entertainment and Tourism; Taylor \& Francis: Milton Park, UK, 2013.

44. The Original Bogota Graffiti Tour. Available online: http://bogotagraffiti.com (accessed on 2 February 2019). 
45. UNWTO. World Tourism Barometer; UNWTO: Madrid, Spain, 2011.

46. Cottrell, S.; Van der Duim, R.; Ankersmid, P.; Kelder, L. Measuring the sustainability of tourism in Manuel Antonio and Texel: A tourist perspective. J. Sustain. Tour. 2004, 12, 409-431. [CrossRef]

47. Lerario, A.; Di Turi, S. Sustainable urban tourism: Reflections on the need for building-related indicators. Sustainability 2018, 10, 1981. [CrossRef]

48. Cohen, E. Traditions in the qualitative sociology of tourism. Ann. Tour. Res. 1988, 15, 29-46. [CrossRef]

49. Turner, V.; Abrahams, R.D.; Harris, A. The Ritual Process: Structure and Anti-Structure; Routledge: Abingdon-on-Thames, UK, 2017.

50. Inskeep, E. Tourism Planning: An Integrated and Sustainable Development Approach; Van Nostrand Reinhold: New York, NY, USA, 1991.

51. Dwyer, L.; Kim, C. Destination competitiveness: Determinants and indicators. Curr. Issues Tour. 2003, 6, 369-414. [CrossRef]

52. Buttle, F.A. Word of mouth: Understanding and managing referral marketing. J. Strateg. Mark. 1998, 6, $241-254$. [CrossRef]

53. Gretzel, U.; Yoo, K. Use and Impact of Online Travel Reviews Information and Communication Technologies in Tourism. In Proceedings of the International Information and Communication Technologies in Tourism Conference, Innsbruck, Austria, 23-25 January 2008.

54. Xiang, Z.; Gretzel, U. Role of social media in online travel information search. Tour. Manag. 2010, 31, $179-188$. [CrossRef]

55. Lewis, R.C.; Chambers, R.E. Marketing Leadership in Hospitality. Foundations and Practices; Van Nostrand Reinhold: New York, NY, USA, 1989.

56. Liu, Y.; Teichert, T.; Hu, F.; Li, H. How do tourists evaluate Chinese hotels at different cities? Mining online tourist reviewers for new insights. In Proceedings of the WHICEB, Wuhan, China, 27 May 2016; p. 67.

57. Berezina, K.; Bilgihan, A.; Cobanoglu, C.; Okumus, F. Understanding satisfied and dissatisfied hotel customers: Text mining of online hotel reviews. J. Hosp. Mark. Manag. 2016, 25, 1-24. [CrossRef]

58. Cheng, M.; Jin, X. What do Airbnb users care about? An analysis of online review comments. Int. J. Hosp. Manag. 2019, 76, 58-70.

59. Choi, S.; Lehto, X.Y.; Morrison, A.M. Destination image representation on the web: Content analysis of Macau travel related websites. Tour. Manag. 2007, 28, 118-129. [CrossRef]

60. Dickinger, A.; Lalicic, L.; Mazanec, J. Exploring the generalizability of discriminant word items and latent topics in online tourist reviews. Int. J. Contemp. Hosp. Manag. 2017, 29, 803-816. [CrossRef]

61. Shin, S.-H.; Yang, S.-B.; Nam, K.; Koo, C. Conceptual foundations of a landmark personality scale based on a destination personality scale: Text mining of online reviews. Inf. Syst. Front. 2017, 19, 743-752. [CrossRef]

62. Crotts, J.C.; Mason, P.R.; Davis, B. Measuring guest satisfaction and competitive position in the hospitality and tourism industry: An application of stance-shift analysis to travel blog narratives. J. Travel Res. 2009, 48, 139-151.

63. Wang, Y.; Chan, S.C.F.; Ngai, G.; Leong, H.-V. Quantifying reviewer credibility in online tourism. In Proceedings of the International Conference on Database and Expert Systems Applications, Prague, Czech Republic, 26-29 August 2013; pp. 381-395.

64. Tripadvisor. Available online: http://tripadvisor.com (accessed on 2 March 2019).

65. Feldman, R.; Dagan, I. Knowledge Discovery in Textual Databases (KDT). In Proceedings of the KDD, Montreal, QC, Canada, 20-21 August 1995; pp. 112-117.

66. Chau, M.; Xu, J. Business intelligence in blogs: Understanding consumer interactions and communities. MIS Q. 2012, 36, 1189-1216. [CrossRef]

67. Hotho, A.; Nürnberger, A.; Paaß, G. A brief survey of text mining. In Proceedings of the Ldv Forum-GLDV J. Comput. Linguistics Lang. Technol., 13 May 2005; pp. 19-62.

68. Kao, A.; Poteet, S.R. Natural Language Processing and Text Mining; Springer Science \& Business Media: Berlin/Heidelberg, Germany, 2007.

69. Meyer, D.; Hornik, K.; Feinerer, I. Text mining infrastructure in R. J. Stat. Softw. 2008, 25, 1-54.

70. Nam, Y. Institutional network structure of corporate stakeholders regarding global corporate social responsibility issues. Qual. Quant. 2015, 49, 1063-1080. [CrossRef] 
71. Nam, Y.; Barnett, G.A. Globalization of technology: Network analysis of global patents and trademarks. Technol. Forecast. Soc. Chang. 2011, 78, 1471-1485. [CrossRef]

72. Ulmer, J.B. Writing urban space: Street art, democracy, and photographic cartography. Cult. Stud. Crit. Methodol. 2017, 17, 491-502.

(C) (1) BY

(C) 2020 by the authors. Licensee MDPI, Basel, Switzerland. This article is an open access article distributed under the terms and conditions of the Creative Commons Attribution (CC BY) license (http://creativecommons.org/licenses/by/4.0/). 



\title{
Considering Cultural Consonance in Trustworthiness of Online Hotel Reviews among Generation Y for Sustainable Tourism: An Extended TAM Model
}

\author{
So Young Bae ${ }^{1}$ and Ju Hyoung Han ${ }^{2, *}$ \\ 1 Department of Culture, Tourism \& Content, College of Hotel \& Tourism Management, \\ Kyung Hee University, 26 Kyungheedae-ro, Dongdaemun-gu, Seoul 02447, Korea; sybae@khu.ac.kr \\ 2 Department of Tourism Administration, College of Business Administration, Kangwon National University, \\ 1 Kangwondaehak-gil, Chuncheon 24341, Korea \\ * Correspondence: juhyounghan@kangwon.ac.kr
}

Received: 17 March 2020; Accepted: 4 April 2020; Published: 7 April 2020

\begin{abstract}
This study aims to predict Generation Y customers' acceptance of user-generated content (UGC) websites by integrating an extended technology acceptance model (TAM), trustworthiness of online reviews, and cultural consensus and cultural consonance theory. To collect data, a free listing method was first used to recognize our ad hoc domain, i.e., the factors that determine the trustworthiness of online hotel reviews among Generation $Y$ in South Korea, from an emic perspective. Based on the free listing results from 39 individuals, an online survey was conducted with 273 samples using a self-administered questionnaire. Cultural consensus analysis was conducted to determine whether there is a shared cultural model in trustworthiness factors among participants, and cultural consonance values were calculated. Then, a structural equation modeling technique was used to estimate how the proposed model explains the collected data. Results indicate that an agreed-upon cultural model of trustworthiness of online hotel reviews exists among sample members. Cultural consonance of trustworthiness was found to constitute a significant antecedent of perceived ease of use and attitude towards websites in the extended TAM model. This study can contribute to predict Generation Y customers' acceptance of UGC websites and offer meaningful implications for sustainable tourism management, particularly when cultural variables are considered.
\end{abstract}

Keywords: cultural consensus; cultural consonance; online hotel reviews; trustworthiness; technology acceptance model; Generation Y

\section{Introduction}

Online social media has become remarkably widespread in the hospitality and tourism industry. The emergence of Web 2.0 has led to manifold user-generated content (UGC) websites, which serve as powerful platforms for customers to spread information via word-of-mouth (WOM), seek other travelers' experiences, compare alternative products and services, and make informed purchase decisions regarding their travel planning, such as hotel selection [1]. As a result, many hospitality and tourism businesses have been utilizing UGC websites in their online business strategies.

Cost considerations constitute important elements of travel planning and decisions. Such costs include not only monetary costs, but also personal effort and time consumed in accessing necessary information and making reservations [2]. For example, individuals may perceive increased psychological barriers and costs when using UGC websites if the process of accessing useful information is cumbersome or the reservation system is not easy to use. Studies on technology adoption have primarily applied Davis' [3] Technology Acceptance Model (TAM) as a key theoretical framework, that holds that individuals' technology acceptance is determined by two cognitive constructs of a 
system, i.e., perceived usefulness and perceived ease of use. TAM has been widely applied in various disciplines, including hospitality and tourism [4-6]. Previous investigations have confirmed that the two major constructs of TAM are significant contributors to explaining customers' UGC websites adoption for travel planning $[7,8]$.

The UGC websites could be successful when customers accept Internet technologies as viable transaction methods and consider review contents as trustworthy and acceptable. Consequently, explaining decision-making processes of UGC website adoption may be highly relevant to both technology-acceptance and information-acceptance constructs. Despite the extensive applicability of TAM, a majority of the extant literature has attempted to extend existing TAM models by including additional technology-acceptance constructs, such as security [9], trust in website developers [7,10], and perceived risk [11]. However, few researchers have attempted to integrate information-acceptance constructs in their extended TAM models, particularly when explaining the adoption of UGC websites for hotel bookings.

With regard to information-acceptance constructs, the topic of trustworthiness of online review messages has attracted substantial attention in industry and academia over time. In addition, some controversial issues exist, such as deceptive online review postings in hospitality industries, e.g., some managers post promotional reviews about their business or offer benefits to customers in exchange for positive review contents [10]. The topic of trustworthiness of online reviews in travel-related UGC websites has also received a great deal of academic interest. Previous studies demonstrated that trustworthiness plays a significant role in influencing customers' attitudes and behavioral intentions toward UGC utilization in their travel-related decisions [12,13]. Despite a rising interest in trustworthiness of online reviews, however, there is limited understanding of how information-acceptance constructs (e.g., trustworthiness of online review messages) are associated with technology-acceptance constructs (e.g., perceived usefulness and perceived ease of use) that predict individuals' adoption behaviors.

Also, while existing research on trustworthiness of online reviews has examined trustworthiness as an antecedent to predict individuals' attitudes and behaviors, previous studies adopted pre-determined items to assess trustworthiness. However, such a concept of trustworthiness toward newly developed technology could have different meaning structures depending on the customer-groups examined, such as generational cohorts or cultural groups. In understanding individuals' behaviors, culture has long constituted a popular research construct. Researchers have utilized different definitions of culture, such as mental phenomena, behavioral patterns, and tangible artifacts. Cognitive anthropologists have regarded culture as information shared and taught to influence individuals' behaviors [14]. Based on this approach, Romney et al. [15] developed the theory of cultural consensus to determine whether a shared cultural model exists for a particular cultural domain. Later, the concept of cultural consonance was established to demonstrate the link between an agreed-upon cultural model and individuals' actual behaviors [16-18].

Even though the concept of cultural consonance has proven useful in understanding the relationship between a particular culture and individuals' actual behaviors, it has rarely been applied in hospitality and tourism research. Moreover, although the concept of culture has been frequently utilized, it has been primarily equated with the concept of nationality or ethnicity, and employed as a moderator to segment a group of people [19]. The trustworthiness of online reviews is certainly highly relevant to all customer groups, however, the present study focuses on Generation Y. Generation Y, referring to individuals born in between 1978 and 1994 [20], is generally technologically savvy and involved in online purchase behaviors [21]. Also, this cohort tends to travel frequently and to adopt UGC in their travel-related decision-making [22]. The advent of online social networks and their significant role in the travel industry have greatly accelerated the utilization of UGC websites among Generation $\mathrm{Y}$ as both consumers and producers of information. Indeed, establishing long-term relationships with customers has become critical in an increasing competitive online travel business, particularly for sustainable tourism management [23]. Consequently, it is imperative that hospitality 
and tourism businesses utilizing UGC websites as their fundamental online marketing strategies understand Generation Y, and develop optimal marketing strategies to target this cohort successfully.

In the present study, we adopted the concept of cultural consonance for factors used to determine the trustworthiness of online hotel reviews. The cultural consonance theory allows researchers to construct the trustworthiness of online hotel reviews with an emic approach or, from respondents' instead of researchers' perspective. This study aims to predict Generation Y customers' acceptance of UGC websites by integrating the theoretical framework of TAM, trustworthiness of online reviews, and cultural consensus and cultural consonance theory. The purpose of this study is: (1) to determine whether there is a shared cultural model of the trustworthiness of online reviews among Generation $Y$ using cultural consensus analysis (CCA) [15]; and (2) to integrate a concept of cultural consonance in trustworthiness to predict Generation Y customers' behavioral intention to adopt UGC websites for their travel planning using an extended TAM model.

\section{Literature Review}

\subsection{Generation $Y$ and Online Travel Websites}

According to the cohort theory [24], generational cohorts facilitate further understanding of individuals' behaviors, as each cohort involves people born and grew up during the same specific period of time. Generational cohorts share similar experiences and contexts in life, which prompt each generational cohort to attain comparable attitudes, beliefs, and behaviors that remain relatively stable during one's life [24]. Brosdahl and Carpenter [25] classified generational cohorts as Baby Boomers (born between 1946 and 1960), Generation X (born between 1961 and 1981), and Generation Y (born between 1981 and 1996). Recently, researchers also used the cohort of Generation Z (born in 1997 and after) to indicate a newer generation.

While older cohorts are considered as digital immigrants, Generation Y members are innately digital natives [26]. Among the generational cohorts, Generation $Y$ is characterized as technologically savvy and more absorbed in online purchase behaviors [27]. This cohort consumes as well as produces content on social media. That is, they are simultaneously both producers and consumers of information, and tend to engage energetically in conversations via social media, such as UGC websites [28]. Due to the unique feature of social media which allows for two-way communication between users and businesses (and/or other users) [29], understanding Generation Y in a social media context has become increasingly significant. In achieving this understanding, it is reasonable to understand that Generation Y's perceptions and attitudes, especially with regard to their online activities on social media and UGC websites, differ from those of other generational cohorts.

The emergence of online social media and the significant role that Generation Y plays in the hospitality and tourism industries are closely connected to the distinctive characteristics of Generation Y. Generation Y prefers to spend money on experiences, travels frequently, and reviews UGC websites prior to making travel purchases [30]. Generation $Y$ is also characterized as being economically robust, and has emerged as an influential consumer group with growing consumption power. Indeed, they spend USD 200 billion annually [31], and their global travel spending totals USD 136 billion annually [32]. They also constitute more than $25 \%$ of the world's population, and they are highly sociable and tech-literate [33,34]. Due to these characteristics, it is a paramount goal for marketers and managers of online travel websites to thoroughly understand this cohort member's travel-related online customer behaviors [21,35].

Nusair et al. [21] explains how Generation $Y$ in the U.S. develops commitment to an online travel website vendor, and shows that commitment is a key factor in developing and maintaining long-term relationships with Generation Y. Bilgihan [35] reports that the features of hotel booking websites and customer trust are primary antecedents of e-loyalty for Generation Y customers. Bento et al. [36] showed that Generation Y, compared to Generation X, in Portugal, consumed more content on social media pages and were more likely to possess an e-WOM intention. Indeed, the extant literature has 
demonstrated that Generation $Y$ and the use of social media are inseparable [21,35]. To consider the extensive influence of digital technology environments on digital natives' behaviors, the current study discusses online travel website adoption behaviors targeting Generation $\mathrm{Y}$ in South Korea.

\subsection{Online Review Trustworthiness}

When individuals assess information, they judge the credibility of the information depending on beliefs about the source, the message, the medium, and the information providers [37,38]. Trustworthiness is one of the most important dimensions (e.g., authenticity, transparency, fairness, accuracy) that information-seekers use to evaluate information credibility [13,38]. Unlike offline WOM, which is based on face-to-face communication, e-WOM communication on UGC websites exhibits no direct or prior relationships with information providers [39]. This may obstruct individuals in relying upon the trustworthiness of online review messages. Therefore, in order to accurately assess trustworthiness, customers attempt to utilize other cues, such as characteristics of messages, and reviewers' geographical locations, demographics, and previous experiences [40].

Previous investigations have attempted to identify how customers evaluate online reviews as being trustworthy. Cheung et al. [41] showed that source credibility, confirmation of prior beliefs, recommendation consistency, recommendation ratings, and argument strength influence the trustworthiness of online review messages. In addition, Qiu et al. [42] found that a conflicting aggregated rating decreased review trustworthiness. Kusumasondjaja et al. [43] empirically demonstrated that a negative review is perceived as more trustworthy than a positive review when the posting reveals the identity of the reviewer. Nevertheless, when the reviewer's identity was not disclosed, there was no significant difference in trustworthiness between positive and negative reviews [43].

The findings of the abovementioned investigations imply that customers' strategies to appraise the level of trustworthiness of messages vary, and individuals do not assign equal evaluations of trustworthiness to online review messages. Nevertheless, online review trustworthiness in previous literature is studied with pre-existing scales or universally operationalized constructs, which may differ depending on cultural customer groups or generational cohorts. Little research has considered the existence of a cultural disposition towards trustworthiness and its effects on online travel website adoption behavior. From an information-seeker's perspective, culturally shared beliefs about online review trustworthiness provide an important cue for them to evaluate UGC websites in their travel-related decision-making process. The present investigation seeks to examine cultural consonance in online review trustworthiness among Generation $Y$ members, and then assess whether it plays a significant role in UGC website adoption for their travel planning.

\subsection{Cultural Consensus and Cultural Cononance}

Culture is defined as "whatever it is one has to know and believe to operate in a manner acceptable to members" [44] (p. 167). In cognitive anthropology, culture has often been considered as a set of information that shared and taught in a society which affects human behaviors [14,45]. In this context, anthropologists have explored cultural domains, indicating "a set of items that are all of the same type" which exist in line with culture [46] (p. 1).

Among cultural domains, there are explicit domains, such as "animals" or "flowers", as well as implicit domains, also called ad hoc domains, such as "the things to use for baking" or "qualifications to become a competitive researcher" [47]. Ad hoc domains are goal-derived in nature and often comprise highly specified contexts. Moreover, researchers analyze a particular cultural domain from cultural insiders' perspectives rather than their own [48]. Free listing is one of the preferred methods to determine the content of a domain [46]. It requires respondents to list all of the items that they believe fit into a given domain.

The idea of cultural consensus was developed as a theory and a method to assess intracultural differences in a single cultural domain [15]. CCA is employed to determine the degree to which individuals have agreement on the substance of specific knowledge domains. It offers "culturally 
correct answers" to questions about a given domain [49] (p. 339), and indicates the existing level of variability in cultural sharing among cultural insiders $[15,50]$. Although CCA originated in cultural anthropology, it has been frequently employed in other disciplines, such as medicine [51], sociology [52], and gerontology [53].

Researchers in tourism and leisure studies have also applied CCA in their research. For example, Ribeiro and Yarnal [54] investigated whether there was an agreed cultural model concerning perceptions of spring breakers among college students in the U.S. Kerstetter et al. [55] also discussed the sense of place among Fijian highlanders. Recently, Bae and Chick [56] studied a domestic rail travel phenomenon among the young generation in Korea, called Rail-ro. In this study, Rail-ro experiences were determined as a coherent culture among travelers who undergo Rail-ro experiences.

Dressler and colleagues later attempted to extend CCA [16-18]. Specifically, they included the concept of cultural consonance, which refers to the degree to which individual behavior is in line with the existing cultural model for that particular behavior [16]. Cultural consonance analysis (CCoA) deals with the association between the agreed-upon cultural model and individual beliefs and behaviors [17]. In their research, they mainly examined the influence of cultural consonance with regards to individuals' lifestyle, social support, family life, and national identity on psychological and physical health outcomes (e.g., stress level, depressive symptoms, blood pressure) $[17,18]$.

CCoA has considerable merit in understanding the nature and effects of a particular cultural domain in a specific cultural context [57]. Chick and colleagues applied this concept in their investigation of leisure behaviors. The results of their study, using the concept of cultural consonance in leisure, indicated that cultural consonance with respect to leisure activities to a good leisure life positively influenced leisure satisfaction, life satisfaction, and self-reported health $[58,59]$. In their subsequent study, they also determined that cultural consonance mediates the relationship between leisure constraints and leisure satisfaction [57].

Despite the utility of the concept of cultural consonance in elucidating the link between a particular culture and individuals' actual behaviors, it has rarely been implemented in the field of hospitality and tourism. The primary focus of the present study is to examine the following ad hoc cultural domain: "the factors that determine the trustworthiness of online hotel reviews among Generation $\mathrm{Y}$ in South Korea." Cultural consonance for this domain indicates the degree to which individuals behave in accordance with a cultural model of the importance of a set of elements to determine the trustworthiness of online hotel reviews. In this study, cultural consonance was considered as a possible antecedent to other meaningful variables in analyzing attitudes or behaviors of a particular cultural group.

\subsection{Extended TAM}

In 1985, Fred Davis initially introduced TAM, which postulates that: (1) behavioral intention to adopt new technology is affected by attitude; and (2) attitude is influenced by two technology-related constructs, i.e., perceived ease of use and perceived usefulness. This model was developed based on the Theory of Reasoned Action [60]. In TAM, perceived ease of use and perceived usefulness are included as belief constructs in predicting attitude and behavioral intention. TAM stands out as a parsimonious and effective model for explaining users' technology adoption behavior [7,61], and assists researchers and practitioners to determine the acceptance of a particular technology or system.

Studies employing TAM have demonstrated substantial empirical support for explaining individuals' acceptance of technology, such as smart phones, technology-based services, and new media $[4,62]$. In tourism and hospitality literature, TAM has also been applied to explore employees' acceptance of technology, such as hotel front office systems [5], travel agency computerized reservation systems [6], biometric system adoption in hotels [63], and restaurant computing systems [64].

While this model was initially applied to system usage in the workplace, researchers have used it to understand individual customers' website use [8,11] and intention to shop travel online [65]. The findings of these previous studies consistently showed that perceived ease of use, perceived usefulness, and 
attitude are significant determinants of users' acceptance of technology-related applications. For example, previous findings supported significant correlations between trustworthiness and the aforementioned determinants. Ayeh [61] demonstrated that trustworthiness has a positive effect on the usefulness of, and attitudes toward, consumer-generated media adoption for travel planning. Pavlou [8] showed that, in a B2C e-commerce context, trustworthiness exerts a positive effect on perceived ease of use and perceived usefulness. Furthermore, Xie et al. [66] and Chircu et al. [67] agreed that perceived ease of use and perceived usefulness are positively affected by trustworthiness. The findings of these empirical studies supported the existence of positive associations between trustworthiness, perceived ease of use, perceived usefulness, and attitude.

Researchers have found that trustworthiness increases perceived ease of technology usage because trustworthiness assists the customer to understand, monitor, and control situations or web retailer's actions, and thus facilitates the transaction and makes the process effortless [8,67]. Researchers also demonstrated that trustworthiness is an important determinant of perceived usefulness [8]. This is partly because customers would expect to obtain usefulness from a website if they believe the people behind the website or trust the information provided via the website. In other words, if a website's contents are not trusted to behave in line with the customers' beliefs and expectations, then customers will not anticipate obtaining any utility from using the website or develop positive attitudes toward it.

Based on the wide applicability of TAM, researchers have attempted to extend the model by adding more determinant factors in order to augment the model's predictive power [68]. In the hospitality and tourism context, a popular area has been understanding website acceptance for customer decision-making. For example, Ayeh [61] conducted an online survey in Singapore, and found that perceived ease of use and perceive usefulness, other than source credibility, in an extended TAM model, play significant roles in explaining attitude and intention to use TripAdvisor. Agag and El-Masry [11] developed an extended TAM model by including a website's characteristics (e.g., perceived ease of use and perceived usefulness), company's characteristics (e.g., website reputation), and customer's characteristics (e.g., customer experience), and showed that the additional variables further help to explain users' attitudes toward online travel websites in Egypt. In the present study, we attempt to develop an extended TAM model by adding the variable of cultural consonance in online review trustworthiness to further understand adoption behavior of UGC websites for travel planning.

\subsection{Research Model}

The value of TAM in a technology-acceptance context has been widely demonstrated, and employing it in the context of UGC website adoption behavior in travel-related decision-making processes is theoretically and empirically supported. Considering the importance of understanding the link between a particular culture and individuals' actual behaviors, it is also reasonable to add a cultural variable in the information-acceptance context, i.e., cultural consonance in online review trustworthiness, in an extended TAM model. Drawing upon the findings and theories of the previous literature reviewed above, this paper attempts to theoretically develop and empirically validate a research model that predicts travelers' acceptance of UGC websites among Generation Y in South Korea. The following is a proposed research model with 10 research hypotheses (Figure 1): 


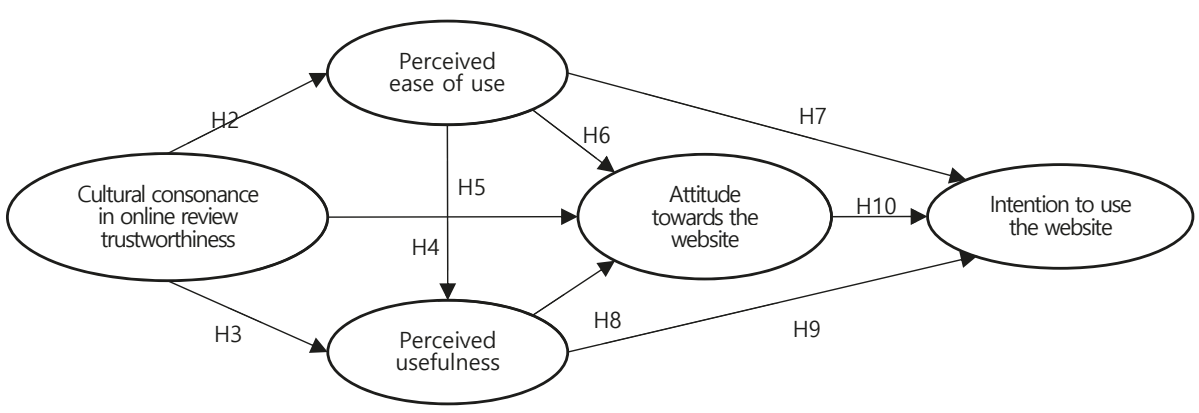

Figure 1. The proposed research model.

Hypothesis 1 (H1). An agreed-upon cultural model of online hotel review trustworthiness exists among Generation Y in Korea, i.e., a cultural consensus exists with regard to the degree of importance of elements for online hotel review trustworthiness.

Hypothesis 2 (H2). Cultural consonance in online hotel review trustworthiness positively influences perceived ease of use.

Hypothesis 3 (H3). Cultural consonance in online hotel review trustworthiness positively influences perceived usefulness.

Hypothesis 4 (H4). Cultural consonance in online hotel review trustworthiness positively influences attitude towards the website.

Hypothesis 5 (H5). Perceived ease of use positively influences perceived usefulness.

Hypothesis 6 (H6). Perceived ease of use positively influences attitude towards the website.

Hypothesis 7 (H7). Perceived ease of use positively influences intention to use the website.

Hypothesis 8 (H8). Perceived usefulness positively influences attitude towards the website.

Hypothesis 9 (H9). Perceived usefulness positively influences intention to use the website.

Hypothesis 10 (H10). Attitude towards the website positively influences intention to use the website.

\section{Methods}

\subsection{Data Collection}

We collected the data in two phases in this study. We first used a free listing method to recognize our ad hoc domain, i.e., the factors that determine the trustworthiness of online hotel reviews among Generation $\mathrm{Y}$ in South Korea, from an emic perspective. Based on the free listing results, an online questionnaire was distributed to a larger sample.

First, the free listing method was applied. The free listing method is a useful ethnographic method to collect possible items which can be members of a particular cultural domain by asking respondents to provide as many items as possible that they would include in the given cultural domain $[46,69]$. At this stage, we administered a free listing that targeted young Korean domestic travelers who have made a purchase decision based on online hotel reviews from hotel reservation websites or applications. 
We asked respondents to make a list of all of the factors which they believe were important to consider when determining whether or not online hotel reviews were trustworthy. We also included a minimal level of demographic information, i.e., such as gender and age.

Considering the acceptable sample size of 30 for free listing in general [70], our lists from 39 individuals were sufficient. Our lists provided a total of 32 items, but we finally used 21 items to measure cultural consonance after rewording, clarifying, or removing items (Table 1). For example, we combined items which included the same meaning, but in a different wording. We also split items into two, which included more than two meaning constructs within a single item.

Table 1. Final items for the trustworthiness of online hotel reviews.

\begin{tabular}{|c|c|}
\hline \multicolumn{2}{|r|}{ Is Important for Trustworthy Online Hotel Reviews. } \\
\hline 1. & a reviewer is the person who actually stayed at a hotel \\
\hline 2. & a review was written frankly \\
\hline 3. & a review includes points in which I am interested (e.g., cleanliness, accessibility) \\
\hline 4. & a review was written objectively \\
\hline 5. & a review includes a detailed explanation about facilities \\
\hline 6. & a review was written recently \\
\hline 7. & there are consistent comments about a particular accommodation \\
\hline 8. & a review includes negative aspects \\
\hline 9. & there are a number of reviews for a particular accommodation \\
\hline 10. & a review was written sincerely \\
\hline 11. & a review includes photos taken by the reviewer \\
\hline 12. & review comments are aligned with the current reputation of the accommodation \\
\hline 13. & a review was written from a balanced perspective \\
\hline 14. & review comments are aligned with a current rating of the accommodation \\
\hline 15. & the website manager had consistent and interactive communication with users \\
\hline 16. & the website has a good reputation \\
\hline 17. & a review was written in an expected length \\
\hline 18. & a review displays personal information about reviewers (e.g., age, gender) \\
\hline 19. & a reviewer's purpose of his or her trip is similar to mine \\
\hline 20. & a review includes personal travel stories \\
\hline 21. & a reviewer has rich travel experiences \\
\hline
\end{tabular}

Second, an online questionnaire was developed based on the free listing result. It was then distributed to online panels of one of the major online survey companies in Korea from August 3, 2017 to August 21, 2017. The study population included: (1) Korean college students; (2) who currently reside in Seoul or neighboring areas (i.e., Kyunggi and Incheon); and (3) have reserved a room based on online hotel reviews from hotel reservation websites or applications for a domestic trip. A total of 400 responses were received, and 273 responses were used for analysis after removing missing and irrelevant data for CCA.

To measure our first construct, cultural consonance, we included two sets of questions with regards to our 21 items derived from the free listing: (1) how important each item is to produce trustworthiness of online reviews (importance); and (2) how much each item was referred to in their recent decision-making concerning a hotel reservation (frequency). Next, we developed measurements for the four constructs in TAM based on Davis [3]. Perceived ease of use was defined as the degree to which an individual believes that using a hotel reservation website or application will be effort-free, and was measured using four items (e.g., It is easy to explore this website). Perceived usefulness was defined as the degree to which an individual believes that using a hotel reservation website or application will improve one's performance, and was assessed using four items (e.g., This website helps me save time in booking accommodations). Attitude towards the website was defined as an individual's preposition toward the website, and was measured using four items (e.g., I felt comfortable in exploring the website). Intention to use the website was defined as the level of satisfaction with the website and intention to revisit it, and was assessed using four items (e.g., I am satisfied with the 
service provided by this website). All of the items for the five constructs above were measured using a 5-point Likert-type scale (1: extremely disagree; 5: extremely agree).

Prior to asking a series of questions about these five constructs, we asked respondents to name a hotel reservation website or application that they have used recently in order to remind them of their purchase experience. At the conclusion of the survey, we requested certain demographic information, such as gender, age, school year, education, and frequency of using online hotel reviews. Prior to distributing the survey, two professionals reviewed the questionnaire for clarity and completeness. Then, we administered a pilot survey targeting 100 respondents in order to receive feedback concerning the length and flow of the questionnaire, as well as to identify and correct errors.

\subsection{Data Analysis}

A series of data analyses were conducted using SPSS 23.0 and Amos 20. First, to confirm whether a shared cultural model exists for the ad hoc domain, "factors that determine the trustworthiness of online hotel reviews among Generation Y in South Korea," we used a CCA based on the guideline in Chick et al. [57]. Using SPSS 23.0, we first randomized the data with regards to the importance question (i.e., how important each item is to produce trustworthiness of online reviews), and transposed the data matrix from rows (cases) to columns (variables), and from columns to rows. We then ran multiple factor analyses using principal axis factoring to obtain a stable solution, since CCA inherently includes a much greater number of columns than rows when the sample size is large. In this study, we ran factor analysis 39 times using seven columns at a time. We also calculated culturally correct answers (i.e., cultural answer keys) by weighting the answers of each informant by their competence scores and averaging the values [49]. Second, upon the existence of the agreed-upon cultural model of online hotel review trust, we calculated cultural consonance scores. We summed all respondents' importance ratings for each of the 21 items, and created sample level importance values. Next, we correlated the frequency values of each respondent with the agreed-upon importance scores. When a respondent reported a frequent consideration of the particular trustworthiness item that was agreed-upon as being important, the result exhibited higher positive correlations, representing high cultural consonance. Third, we ran structural equation modeling using Amos 20 to analyze the data based on the five variables, including cultural consonance calculated in the earlier step. Based on the two-step analysis [71], confirmatory factor analysis (CFA) was conducted first to confirm the measurement of each scale and fit the measurement model. We then ran SEM to test hypothesized relationships among latent variables in the research model.

\section{Results}

\subsection{Sampmle Characteristics}

Table 2 presents the profile of the samples. Of all 273 respondents, $53.8 \%$ were females. Their age ranged from 19 to 26 , with slightly higher proportions of $22(19.0 \%)$ and $23(23.1 \%)$ than the other ages. The sample included more juniors and seniors than freshmen and sophomores. A majority of them (91.4\%) were four-year university students. All respondents answered that they refer to online reviews when planning to travel, while the frequency varied from sometimes to always. Approximately half of the respondents $(47.6 \%)$ responded that they always refer to online reviews in their travel planning.

\subsection{Cultural Consensus and Cultural Consonance Analysis}

We ran CCA on our ad hoc domain, "factors that determine the trustworthiness of online hotel reviews among Generation $\mathrm{Y}$ in South Korea." The ratio of the average value for the first (mean = 3.69) and second eigenvalues (mean $=1.12$ ) was 3.29, which is above the threshold (three-to-one ratio). The first factors represented an average of $52.83 \%$ of the variance, and the average of the first factor loading was 0.66 . None of the 39 analyses contained negative factor loadings. According to Romney et al. [15], these values met the guidelines for a single factor solution. Therefore, the result indicates that 
sample members share a cultural model of the relative importance of online hotel review trustworthiness. This result supports $\mathrm{H} 1$ - that an agreed-upon cultural model of trustworthiness in online hotel reviews exists among Generation Y in South Korea. Cultural answer keys are listed in Table 3.

Table 2. Socio-demographic characteristics of respondents.

\begin{tabular}{|c|c|c|c|c|c|c|c|}
\hline \multicolumn{2}{|c|}{ Characteristics } & \multirow{3}{*}{$\begin{array}{c}\mathbf{N} \\
126 \\
147\end{array}$} & \multirow{3}{*}{\begin{tabular}{c|}
$\%$ \\
46.2 \\
53.8 \\
\end{tabular}} & \multicolumn{2}{|c|}{ Characteristics } & \multirow{2}{*}{$\begin{array}{l}\mathbf{N} \\
27\end{array}$} & \multirow{2}{*}{$\begin{array}{c}\% \\
9.9\end{array}$} \\
\hline & Male & & & \multirow{4}{*}{ Year } & Freshman & & \\
\hline Gender & Female & & & & Sophomore & 55 & 20.1 \\
\hline \multirow{8}{*}{ Age } & 19 & 20 & 7.3 & & Junior & 88 & 32.2 \\
\hline & 20 & 21 & 7.7 & & Senior & 103 & 37.7 \\
\hline & 21 & 40 & 14.7 & \multirow{2}{*}{ Education } & Two-year college & 23 & 8.6 \\
\hline & 22 & 52 & 19.0 & & Four-year university & 246 & 91.4 \\
\hline & 23 & 63 & 23.1 & \multirow{3}{*}{$\begin{array}{l}\text { Frequency of } \\
\text { referring to online } \\
\text { reviews }\end{array}$} & Sometimes & 29 & 10.8 \\
\hline & 24 & 31 & 11.4 & & Often & 111 & 41.3 \\
\hline & 25 & 31 & 11.4 & & Always & 128 & 47.6 \\
\hline & 26 & 14 & 5.5 & Total & & 273 & 100.0 \\
\hline
\end{tabular}

Table 3. Cultural answer keys of each item.

\begin{tabular}{|c|c|c|c|}
\hline & Items & Score & Rank \\
\hline \multicolumn{4}{|c|}{ Whether __ is important for trustworthy online hotel reviews. } \\
\hline 1. & a reviewer is the person who actually stayed at a hotel & 4.54 & 1 \\
\hline 2. & a review was written frankly & 4.49 & 2 \\
\hline 3. & $\begin{array}{l}\text { a review includes the points in which I am interested } \\
\text { (e.g., cleanliness, accessibility) }\end{array}$ & 4.46 & 3 \\
\hline 4. & a review was written objectively & 4.37 & 4 \\
\hline 5. & a review includes a detailed explanation about facilities & 4.25 & 5 \\
\hline 6. & a review was written recently & 4.16 & 6 \\
\hline 7. & $\begin{array}{l}\text { there are consistent comments about a } \\
\text { particular accommodation }\end{array}$ & 4.15 & 7 \\
\hline 8. & a review includes negative aspects & 4.15 & 8 \\
\hline 9. & there are a number of reviews for a particular accommodation & 4.04 & 9 \\
\hline 10.. & a review was written sincerely & 4.04 & 10 \\
\hline 11. & a review includes photos taken by the reviewer & 3.93 & 11 \\
\hline 12. & $\begin{array}{l}\text { review comments are aligned with the current reputation of } \\
\text { the accommodation }\end{array}$ & 3.92 & 12 \\
\hline 13. & a review was written from a balanced perspective & 3.84 & 13 \\
\hline 14. & $\begin{array}{l}\text { review comments are aligned with the current } \\
\text { rating of the accommodation }\end{array}$ & 3.82 & 14 \\
\hline 15. & $\begin{array}{l}\text { the website manager had consistent and interactive } \\
\text { communication with users }\end{array}$ & 3.69 & 15 \\
\hline 16. & the website has a good reputation & 3.50 & 16 \\
\hline 17. & a review was written in an expected length & 2.96 & 17 \\
\hline 18. & $\begin{array}{l}\text { a review displays personal information about the reviewers } \\
\text { (e.g., age, gender) }\end{array}$ & 2.95 & 18 \\
\hline 19. & a reviewer's purpose of his or her trip is similar to mine & 2.71 & 19 \\
\hline 20. & a review includes personal travel stories & 2.66 & 20 \\
\hline 21. & a reviewer has rich travel experiences & 2.33 & 21 \\
\hline
\end{tabular}

Since the results demonstrated that there is cultural consensus for our ad hoc domain, we proceeded to calculate cultural consonance values. We ran a correlation analysis with the two sets of data, i.e., importance and frequency values for the 21 items in our ad hoc domain. Then, the calculated value was added to the extended TAM as a new variable. 


\subsection{Measurement Model}

To assess the overall measurement model, CFA was conducted (Table 4$)$. The measurement model test showed a good fit to the data $\left(\chi^{2}=199.538, \mathrm{df}=105, p=0.000, \mathrm{CFI}=0.955, \mathrm{NFI}=0.911\right.$, IFI $=$ $0.956, \mathrm{SRMR}=0.049, \mathrm{RMSEA}=0.058)$. The Cronbach's alpha values of the four constructs were above 0.7 , which confirms the reliability of internal consistency [72]. The values for composite reliability (CR) and average variance extracted (AVE) were above the threshold of 0.7 and 0.5 , respectively, which demonstrates construct reliability and convergent validity [73]. Discriminant validity was also confirmed since the square root of the AVE was greater than each correlation coefficient [74] (Table 5).

Table 4. Confirmatory factor analysis of constructs and indicators.

\begin{tabular}{|c|c|c|c|c|}
\hline Constructs and Indicators & Loading & t-Value & AVE & CR \\
\hline Perceived ease of use $(\alpha=0.815)$ & & & 0.705 & 0.903 \\
\hline PEOU1: It is easy to explore this website. & 0.598 & - & & \\
\hline $\begin{array}{l}\text { PEOU2: It is easy to find the accommodation } \\
\text { that I want from this website. }\end{array}$ & 0.679 & 11.116 & & \\
\hline $\begin{array}{l}\text { PEOU3: It is not difficult to make reservations } \\
\text { and payments on this website. }\end{array}$ & 0.708 & 8.983 & & \\
\hline PEOU4: Overall, this website is easy to use. & 0.880 & 10.046 & & \\
\hline Perceived usefulness $(\alpha=0.746)$ & & & 0.670 & 0.890 \\
\hline $\begin{array}{l}\text { PU1: This website helps me to save time to } \\
\text { book accommodation. }\end{array}$ & 0.652 & - & & \\
\hline $\begin{array}{l}\text { PU2: This website helps me save money to } \\
\text { book accommodation. }\end{array}$ & 0.574 & 7.716 & & \\
\hline $\begin{array}{l}\text { PU3: This website helps me to lessen the } \\
\text { difficulties of booking accommodation. }\end{array}$ & 0.644 & 8.445 & & \\
\hline $\begin{array}{l}\text { PU4: Overall, this website is useful to } \\
\text { book accommodation. }\end{array}$ & 0.711 & 9.076 & & \\
\hline Attitude towards the website $(\alpha=0.833)$ & & & 0.702 & 0.903 \\
\hline Att1: I felt comfortable exploring the website. & 0.850 & - & & \\
\hline Att2: I had a good time exploring the website. & 0.779 & 14.094 & & \\
\hline $\begin{array}{l}\text { Att3: This website helped me to become } \\
\text { familiar with this online booking company. }\end{array}$ & 0.648 & 11.095 & & \\
\hline $\begin{array}{l}\text { Att4: Compared to other websites, this website } \\
\text { is excellent. }\end{array}$ & 0.660 & 11.503 & & \\
\hline Intention to use the website $(\alpha=0.851)$ & & & 0.745 & 0.921 \\
\hline $\begin{array}{l}\text { Int1: I am satisfied with the service provided } \\
\text { by this website. }\end{array}$ & 0.688 & - & & \\
\hline $\begin{array}{l}\text { Int2: I would like to visit this website again in } \\
\text { the future. }\end{array}$ & 0.701 & 10.045 & & \\
\hline Int3: I will use this website with no hesitation. & 0.831 & 11.746 & & \\
\hline $\begin{array}{l}\text { Int4: I will consider this website first for future } \\
\text { accommodation booking. }\end{array}$ & 0.799 & 11.483 & & \\
\hline
\end{tabular}

Table 5. Correlations among latent constructs, means, and standard deviations (SD).

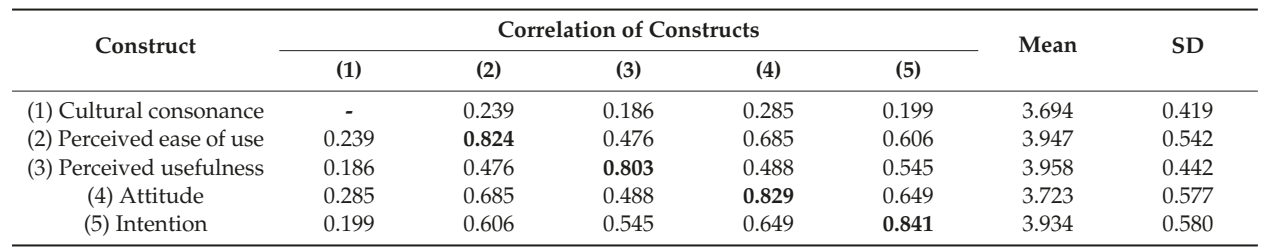

Note: Values on the diagonal are the square roots of average variance extracted. For adequate discriminant validity, diagonal elements should be greater than corresponding off-diagonal elements. $p<0.05$. 


\subsection{Structural Model}

The results indicate that the data are sufficiently explained by the proposed structural model $\left(\chi^{2}=199.509, \mathrm{df}=106, p=0.000, \mathrm{CFI}=0.956, \mathrm{NFI}=0.911, \mathrm{IFI}=0.956, \mathrm{SRMR}=0.048, \mathrm{RMSEA}=0.057\right)$. As shown in Figure 2, cultural consonance indicated significant positive influence on perceived ease of use $(\beta=0.20, p<0.001)$ and attitude towards the website $(\beta=0.12, p<0.001)$, supporting $\mathrm{H} 2$ and H4. However, there was no significant influence between cultural consonance and usefulness, which rejects H3. Perceived ease of use showed a significant positive influence on perceived usefulness ( $\beta=0.60, p<0.001)$, which supports H5. Both perceived ease of use and perceived usefulness were significant antecedents for attitude towards the website $(\beta=0.69, p<0.001 ; \beta=0.17, p<0.001)$, which supports $\mathrm{H} 6$ and H8. Finally, intention to use the website was significantly affected by perceived ease of use $(\beta=0.22, p<0.001)$, perceived usefulness $(\beta=0.37, p<0.001)$, and attitude towards the website $(\beta=0.35, p<0.001)$, which supports $\mathrm{H} 7, \mathrm{H} 9$, and $\mathrm{H} 10$.

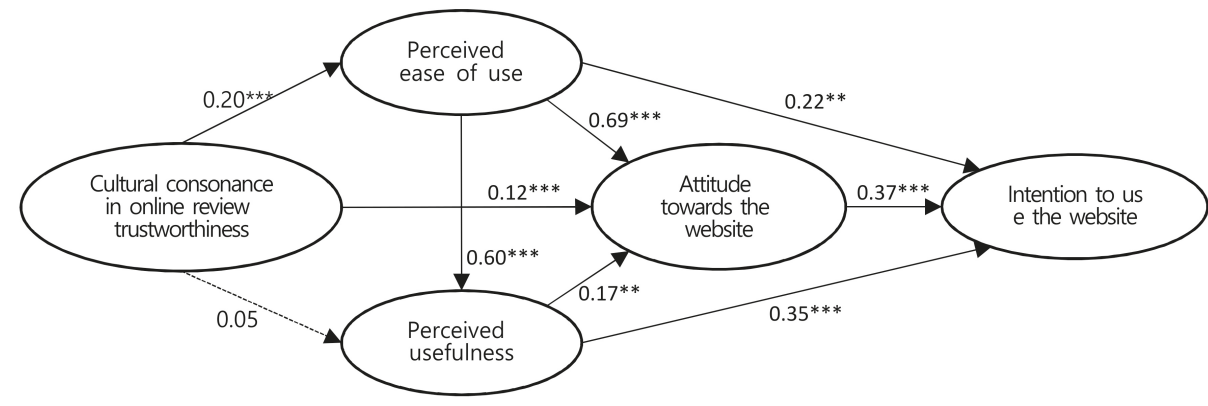

Figure 2. The estimated structural model. Notes: $\chi^{2}=199.509, \mathrm{df}=106, p=0.000, \mathrm{CFI}=0.956, \mathrm{NFI}=$ 0.911, IFI $=0.956$, SRMR $=0.048$, RMSEA $=0.057 ;{ }^{* *} p<0.01 ; * * *<0.001$; two-tailed test, standardized coefficient; solid line: significant path; dotted line: non-significant path.

\section{Discussion and Conclusions}

In order to consider the substantial influence of Generation $\mathrm{Y}$ on the hospitality and tourism sectors, this study's results help to predict Generation Y customers' acceptance of UGC websites and offer meaningful implications, particularly when cultural consensus and cultural consonance are taken into account.

According to the CCA, the result indicates that a cultural model of the importance of trustworthiness toward online hotel reviews did exist among the sample members, which supports H1. In other words, there was an agreed-upon cultural model for our ad hoc domain (i.e., the importance of trustworthiness in online hotel reviews) among Generation Y. While previous studies have reported various antecedents of trustworthiness of online hotel reviews (e.g., the reviewer's identity, review valence, and reader-reviewer similarity), this study offered the culturally correct answers (i.e., cultural answer keys) for trustworthiness among Generation Y using the CCA. The results demonstrated that reviews which were written honestly and objectively were ranked high, whereas reviewers' travel career or personal travel stories were not considered important. It is plausible to conclude that reviewers' travel history did not strongly appeal to Generation $\mathrm{Y}$ because travel has now become universal and de-exoticized, i.e., travel is not perceived as being an activity that is exclusive to a limited population [75]. In addition, considering the abundance of experience and information, sometimes being commercialized, respondents might want to seek multi-criteria and multi-person information from objectively written reviews to obtain more robust information [76].

Based on the CCA, this investigation assessed the link between the agreed-upon cultural model and individual behaviors using the concept of cultural consonance. When cultural consonance was added as an extended variable in the TAM model, the results indicate that cultural consonance exerts a 
significant positive influence on perceived ease of use, which supports $\mathrm{H} 2$. Individuals use various trustworthiness cues to evaluate websites [40]. Specifically, when Generation Y in South Korea referred to particular trustworthiness factors which were considered to be important by other members in the cultural group, he or she perceived that it was easy to navigate the website and find his or her desired accommodation. In this study, culture is assumed to be highly important in an individual's evaluation of the website. This is partly in line with the finding in Sohaib and Kang [77] that opinions from social media cues are more strongly referred to in building trust towards a website in a collectivistic culture than in an individualistic society. However, cultural consonance did not significantly affect perceived usefulness, which led us to reject H3. Although an individual considered certain trustworthiness factors which were deemed important by his or her peers in the cultural group, it was not necessarily related to saving time or money when reserving a room. It is possible that consumers might evaluate whether the reviews are trustworthy and the usefulness of the website differently. In other words, despite the trustworthiness of the reviews shared among cultural group members, individuals could consider the website as either useful or useless when making a final reservation and payment decision on the UGC websites.

Cultural consonance positively affected attitude towards the website, which confirms $\mathrm{H} 4$. When respondents referred to particular trustworthiness factors considered important in their cultural in-group, they felt comfortable, enjoyed navigating the website, and perceived that the website is excellent. It has been shown that attitude towards the website is improved when perceived risk becomes minimal [78]. In this study, culture matters in determining attitude towards the website. Specifically, it could be possible that the congruence between culturally shared beliefs and individual behaviors (i.e., cultural consonance) helps people to minimize perceived risk. While previous studies have confirmed that trustworthiness of online reviews boosts attitude towards the website, culture per se (i.e., cultural consonance of trustworthiness) also contributes to predict people's behaviors [79].

All of the relationships among the existing variables in the TAM model received strong support. Specifically, perceived ease of use of a website exerted a significant positive influence on perceived usefulness, attitudes towards the website, and intention to use the website, which supports H5, H6, and H7. Perceived usefulness was a significant antecedent of attitude towards the website and intention to use the website, which supports $\mathrm{H} 8$ and $\mathrm{H} 9$. Attitude also predicted the intention to use the website, which supports H10. This study result is in accordance with those of previous studies in which attitude towards the website was tested based on TAM, such as Agag and El-Masry [11], Pavlou [8], and Wu et al. [80].

This study has contributed to expanding the understanding of Generation Y customers' acceptance of UGC websites. It is meaningful that, in this study, the trustworthiness factor was examined from an emic perspective (i.e., informants' perspective) instead of an etic perspective (i.e., predetermined factors by researchers). Ethnographic tools, such as the free listing method, CCA and CCoA were found to be useful in elucidating consumer-oriented trustworthiness factors. In addition, this study offered baseline insights for utilizing a cultural variable to extend an existing TAM model. The result confirmed that the link between a particular culture and individuals' actual behaviors constituted a significant antecedent to attitude towards the website, demonstrating the usefulness of cultural consonance, which is a concept that originated in cognitive anthropology.

This study offers practical implications to managerial teams for UGC websites. Overall, is important to make an effort to enhance the trustworthiness of a website. However, it would be more meaningful to segment their target consumers, and achieve a thorough understanding of culturally favored and disfavored trustworthiness factors for each subgroup. To do so, UGC website managers could administer a regular assessment of perceived trustworthiness factors, particularly among the segmented cultural group. Then, managers would need to support appropriate formats and functions to realize important trustworthiness factors, highlight them in writing, and arrange reviews. For example, one of the highly-ranked trustworthiness factors was whether a review includes points in which an individual is interested (e.g., cleanliness, accessibility). It would be desirable to ask reviewers to mark the key satisfaction points among the given multiple options and, at the same time, allow customers to filter 
the reviews based on their primary points of interest in accommodation selection. Such efforts would assist to enhance consumers' positive attitudes, as well as their continued use of a website.

Despite the merits of this study, it is not without limitations. First, since the sample was not randomly selected, the results might not be representative of all members in Generation Y. Moreover, small cities or rural areas were excluded in this study, although Internet use is nationwide and universal. Therefore, researchers should expand the target population to increase the level of representativeness in future research. Second, in this study, we examined cultural consensus of the importance of trustworthiness toward online hotel reviews for only one group: Generation Y. In future research, it would be useful to demonstrate cultural models of multiple groups by subculture, such as age, gender, or nationality, and make cross-cultural comparisons among them. Third, cultural consonance was calculated as a single value, indicating whether individual beliefs were in accordance with one's behaviors. It would be meaningful to segment items into multiple groups by their level of importance [57,59]. For example, if items were specified as items rated highest in importance, items rated next-highest in importance, and items rated lowest in importance, cultural consonance for each group may exert different influences on outcome variables.

Author Contributions: Conceptualization, S.Y.B. and J.H.H.; data collection, S.Y.B. and J.H.H.; data analysis, S.Y.B; writing - original draft preparation, S.Y.B. and J.H.H; writing—review and editing, S.Y.B. and J.H.H. All authors have read and agreed to the published version of the manuscript.

Funding: This research received no external funding.

Conflicts of Interest: The authors declare no conflict of interest.

\section{References}

1. Filieri, R.; McLeay, F. E-WOM and accommodation: An analysis of the factors that influence travelers' adoption of information from online reviews. J. Travel Res. 2014, 53, 44-57. [CrossRef]

2. Parra-López, E.; Bulchand-Gidumal, J.; Gutiérrez-Taño, D.; Díaz-Armas, R. Intentions to use social media in organizing and taking vacation trips. Comput. Hum. Behav. 2011, 27, 640-654. [CrossRef]

3. Davis, F.D. A Technology Acceptance Model for Empirically Testing New End-User Information Systems: Theory and Results. Ph.D. Thesis, Sloan School of Management, Massachusetts Institute of Technology, Cambridge, MA, USA, 1985.

4. Joo, J.; Sang, Y. Exploring Koreans' smartphone usage: An integrated model of the technology acceptance model and uses and gratifications theory. Comput. Hum. Behav. 2013, 29, 2512-2518. [CrossRef]

5. Kim, T.G.; Lee, J.H.; Law, R. An empirical examination of the acceptance behaviour of hotel front office systems: An extended technology acceptance model. Tour. Manag. 2008, 29, 500-513. [CrossRef]

6. Lee, H.Y.; Kim, W.G.; Lee, Y. Testing the determinants of computerized reservation system users' intention to use via a structural equation model. J. Hosp. Tour. Res. 2006, 30, 246-266. [CrossRef]

7. Beldad, A.D.; Hegner, S.M. Expanding the technology acceptance model with the inclusion of trust, social influence, and health valuation to determine the predictors of German users' willingness to continue using a fitness app: A structural equation modeling approach. Int. J. Hum. Comput. Int. 2018, 34, 882-893. [CrossRef]

8. Pavlou, P.A. Consumer acceptance of electronic commerce: Integrating trust and risk with the technology acceptance model. Int. J. Electron Comm. 2003, 7, 101-134.

9. Lai, P. Security as an extension to TAM model: Consumers' intention to use a single platform E-Payment. Asia Pac. J. Manag. Res. Innov. 2017, 13, 110-119. [CrossRef]

10. Filieri, R.; Alguezaui, S.; McLeay, F. Why do travelers trust TripAdvisor? Antecedents of trust towards consumer-generated media and its influence on recommendation adoption and word of mouth. Tour. Manag. 2015, 51, 174-185. [CrossRef]

11. Agag, G.M.; El-Masry, A.A. Why do consumers trust online travel websites? Drivers and outcomes of consumer trust toward online travel websites. J. Travel Res. 2017, 56, 347-369. [CrossRef]

12. Ayeh, J.K.; Au, N.; Law, R. Do we believe in TripAdvisor? Examining credibility perceptions and online travelers' attitude toward using user-generated content. J. Travel Res. 2013, 52, 437-452. [CrossRef] 
13. Casalo, L.V.; Flavian, C.; Guinaliu, M.; Ekinci, Y. Do online hotel rating schemes influence booking behaviors? Int. J. Hosp. Manag. 2015, 49, 28-36. [CrossRef]

14. Roberts, J.M. The self-management of cultures. In Explorations in Cultural Anthropology; Goodenough, W., Ed.; MacGraw-Hill: New York, NY, USA, 1964; pp. 433-454.

15. Romney, A.K.; Weller, S.C.; Batchelder, W.H. Culture as consensus: A theory of culture and informant accuracy. Am. Anthropol. 1986, 88, 313-338. [CrossRef]

16. Dressler, W.W. Cultural consonance: Linking culture, the individual and health. Prev. Med. 2012, 55, 390-393. [CrossRef] [PubMed]

17. Dressler, W.W.; Balieiro, M.C.; dos Santos, J.E. Cultural consonance in life goals and depressive symptoms in urban Brazil. J. Anthropol. Res. 2017, 73, 43-65. [CrossRef]

18. Dressler, W.W.; Balieiro, M.C.; Ribeiro, R.P.; Dos Santos, J.E. Cultural consonance and psychological distress: Examining the associations in multiple cultural domains. Cult. Med. Psychiatry 2007, 31, 195-224. [CrossRef]

19. Chick, G. Culture as a variable in the study of leisure. Leis. Sci. 2009, 31, 305-310. [CrossRef]

20. Sheahan, P. Generation Y: Thriving and Surviving with Generation Y at Work; Hardie Grant Books Prahran: Victoria, Australia, 2005.

21. Nusair, K.K.; Parsa, H.G.; Cobanoglu, C. Building a model of commitment for Generation Y: An empirical study on e-travel retailers. Tour. Manag. 2012, 32, 833-843. [CrossRef]

22. Benckendorff, P.; Moscardo, G.; Pendergast, D. Tourism and Generation Y; CAB International: Oxfordshire, UK, 2010.

23. Boyer, K.K.; Hult, G.T.M. Customer behavior in an online ordering application: A decision scoring model. Decis. Sci. 2005, 36, 569-598. [CrossRef]

24. De Pelsmacker, P.; Geuens, M.; Van den Bergh, J. Marketing Communicatie; Pearson Education Benelux: Amsterdam, The Netherlands, 2005.

25. Brosdahl, D.J.; Carpenter, J.M. Shopping orientations of US males: A generational cohort comparison. J. Retail. Consum. Serv. 2011, 18, 548-554. [CrossRef]

26. Prensky, M. Digital natives, digital immigrants. In Crosscurrents: Cultures, Communities, Technologies; Blair, K.L., Almjeld, J., Murphy, R.M., Eds.; Wadsworth Cengage Learning: Boston, MA, USA, 2001; pp. 45-51.

27. Lester, D.H.; Forman, A.M.; Loyd, D. Internet shopping and buying behavior of college students. Serv. Mark. Q. 2006, 27, 123-138. [CrossRef]

28. Sago, B. The influence of social media message sources on millennial generation consumers. Int. J. Integr. Mark. Commun. 2010, 2, 7-18.

29. Rapp, A.; Beitelspacher, L.S.; Grewal, D.; Hughes, D.E. Understanding social media effects across seller, retailer, and consumer interactions. J. Acad. Market. Sci. 2013, 41, 547-566. [CrossRef]

30. Mettler, L. Millennial Appeal: 5 Ways Hotels Are Angaging Generation Y. US News. Available online: http://travel.usnews.com/features/Millennial-Appeal-5-wayshotels-are-engaging-Gen-Y (accessed on 15 September 2015).

31. Djamasbi, S.; Siegel, M.; Tullis, T. Generation Y, web design, and eye tracking. Int. J. Hum-Comput. St. 2010, 68, 307-323. [CrossRef]

32. Petrak, N. Generation Y: Soon-to-be Your Next Best Customers. Available online: https://www. adventuretravelnews.com/generation-y-soon-to-be-your-next-best-customers. (accessed on 12 July 2011).

33. Farris, R.; Chong, F.; Danning, D. Generation Y: Purchasing power and implications for marketing. Acad. Mark. Stud. J. 2002, 6, 89-101.

34. Nusair, K.K.; Bilgihan, A.; Okumus, F.; Cobanoglu, C. Generation Y travelers' commitment to online social network websites. Tour. Manag. 2013, 35, 13-22. [CrossRef]

35. Bilgihan, A. Gen Y customer loyalty in online shopping: An integrated model of trust, user experience and branding. Comput. Hum. Behav. 2016, 61, 103-113. [CrossRef]

36. Bento, M.; Martinez, L.M.; Martinez, L.F. Brand engagement and search for brands on social media: Comparing Generations X and Y in Portugal. J. Retail. Consum. Serv. 2018, 43, 234-241. [CrossRef]

37. Johnson, T.J.; Kaye, B.K. In blog we trust? Deciphering credibility of components of the internet among politically interested internet users. Comput. Hum. Behav. 2009, 25, 175-182. [CrossRef]

38. Sarabia-Sánchez, FJ.; Rodríguez-Sánchez, C.; Hyder, A. The role of personal involvement, credibility and efficacy of conduct in reported water conservation behaviour. J. Environ. Psychol. 2014, 38, 206-216. [CrossRef] 
39. Abarbanel, B.; Bernhard, B.; Singh, A.K.; Lucas, A. Impact of virtual atmospherics and functional qualities on the online gambler's experience. Behav. Inform. Technol. 2015, 34, 1005-1021. [CrossRef]

40. Filieri, R. What makes an online consumer review trustworthy? Ann. Tour.Res. 2016, 58, 46-64. [CrossRef]

41. Cheung, M.Y.; Luo, C.; Sia, C.L.; Chen, H. Credibility of electronic word-of-mouth: Informational and normative determinants of on-line consumer recommendations. Int. J. Electron. Comm. 2009, 13, 9-38. [CrossRef]

42. Qiu, L.; Pang, J.; Lim, K.H. Effects of conflicting aggregated rating on eWOM review credibility and diagnosticity: The moderating role of review valence. Decis. Support Syst. 2012, 54, 631-643. [CrossRef]

43. Kusumasondjaja, S.; Shanka, T.; Marchegiani, C. Credibility of online reviews and initial trust: The roles of reviewer's identity and review valence. J. Vacat. Mark. 2012, 18, 185-195. [CrossRef]

44. Goodenough, W.H. Cultural Anthropology and Linguistics; Bobbs-Merrill: Indianapolis, IN, USA, 1957.

45. Boyd, R.; Richerson, P.J. The Origin and Evolution of Cultures; Oxford University Press: New York, NY, USA, 2005.

46. Borgatti, S.P.; Halgin, D.S. Elicitation techniques for cultural domain analysis. Ethnogr. Toolkit 1999, 3, $115-151$.

47. Barsalou, L.W. Ad hoc categories. Mem. Cognit. 1983, 11, 211-227. [CrossRef]

48. Collins, C.C.; Dressler, W.W. Cultural consensus and cultural diversity: A mixed methods investigation of human service providers' models of domestic violence. J. Mix. Method Res. 2008, 2, 362-387. [CrossRef]

49. Weller, S.C. Cultural consensus theory: Applications and frequently asked questions. Field Methods 2007, 19, 339-368. [CrossRef]

50. Caulkins, D.; Hyatt, S.B. Using consensus analysis to measure cultural diversity in organizations and social movements. Field Methods 1999, 11, 5-26. [CrossRef]

51. Snodgrass, J.G.; Dengah, H.F., II; Lacy, M.G.; Bagwell, A.; Van Oostenburg, M.; Lende, D. Online gaming involvement and its positive and negative consequences: A cognitive anthropological "cultural consensus" approach to psychiatric measurement and assessment. Comput. Hum. Behav. 2017, 66, 291-302. [CrossRef]

52. Fielding-Miller, R.; Dunkle, K.L.; Cooper, H.L.; Windle, M.; Hadley, C. Cultural consensus modeling to measure transactional sex in Swaziland: Scale building and validation. Soc. Sci. Med. 2016, 148, 25-33. [CrossRef] [PubMed]

53. Schrauf, R.W.; Sanchez, J. Using freelisting to identify, assess, and characterize age differences in shared cultural domains. J. Gerontol. Ser. B Psychol. Sci. Soc. 2008, 63, S385-S393. [CrossRef]

54. Ribeiro, N.F.; Yarnal, C.M. 'It wasn't my sole purpose for going down there'-An inquiry into the Spring Break experience and its relation to risky behaviors and alcohol consumption. Ann. Leis. Res. 2008, 11, 351-367. [CrossRef]

55. Kerstetter, D.L.; Bricker, K.S.; Li, H. Vanua and the people of the Fijian Highlands: Understanding sense of place in the context of nature-based tourism development. Tour. Anal. 2010, 15, 31-44. [CrossRef]

56. Bae, S.Y.; Chick, G. An emerging Korean youth culture, Rail-ro: The application of cultural consensus analysis to domestic rail travel experiences. Curr. Issues Tour. 2017, 20, 363-368. [CrossRef]

57. Chick, G.; Hsu, Y.C.; Yeh, C.K.; Hsieh, C.M.; Ramer, S.; Bae, S.Y.; Xue, L.; Dong, E. Cultural consonance mediates the effects of leisure constraints on leisure satisfaction: A reconceptualization and replication. Leis. Sci. 2018, 1-20. [CrossRef]

58. Chick, G.; Dong, E.; Iarmolenko, S. Cultural consonance in leisure activities and self-rated health in six cities in China. World Leis. J. 2014, 56, 110-119. [CrossRef]

59. Chick, G.; Hsu, Y.C.; Yeh, C.K.; Hsieh, C.M.; Bae, S.Y.; Iarmolenko, S. Cultural consonance in leisure, leisure satisfaction, life satisfaction, and self-rated health in urban Taiwan. Leis. Sci. 2016, 38, 402-423. [CrossRef]

60. Ajzen, I.; Fishbein, M. Understanding Attitudes and Predicting Social Behavior; Prentice Hall: Englewood Cliffs, NJ, USA, 1980.

61. Ayeh, J.K. Travellers' acceptance of consumer-generated media: An integrated model of technology acceptance and source credibility theories. Comput. Hum. Behav. 2015, 48, 173-180. [CrossRef]

62. Persico, D.; Manca, S.; Pozzi, F. Adapting the technology acceptance model to evaluate the innovative potential of e-learning systems. Comput. Hum. Behav. 2014, 30, 614-622. [CrossRef]

63. Morosan, C. Theoretical and empirical considerations of guests' perceptions of biometric systems in hotels: Extending the technology acceptance model. J. Hosp. Tour. Res. 2012, 36, 52-84. [CrossRef]

64. Ham, S.; Kim, W.G.; Forsythe, H.W. Restaurant employees' technology use intention: Validating technology acceptance model with external factors. J. Hosp. Leis. Mark. 2008, 17, 78-98. [CrossRef]

65. Amaro, S.; Duarte, P. An integrative model of consumers' intentions to purchase travel online. Tour. Manag. 2015, 46, 64-79. [CrossRef] 
66. Xie, Q.; Song, W.; Peng, X.; Shabbir, M. Predictors for e-government adoption: Integrating TAM, TPB, trust and perceived risk. Electron. Libr. 2017, 35, 2-20. [CrossRef]

67. Chircu, A.M.; Davis, G.B.; Kauffman, R.J. Trust, expertise and ecommerce intermediary adoption. In Proceedings of the Sixth Americas Conference on Information Systems, Long Beach, CA, USA, 10-13 August 2000; DeGross, J., Ed.; ACM: New York, NY, USA, 2000; pp. 710-716.

68. King, W.R.; He, J. A meta-analysis of the technology acceptance model. Inform. Manag. 2006, 43, 740-755. [CrossRef]

69. Weller, S.C.; Romney, A.K. Systematic Data Collection; Sage Publications: Newbury Park, CA, USA, 1988.

70. Schrauf, R.W.; Sanchez, J. Age effects and sample size in free listing. Field Methods 2010, 22, 70-87. [CrossRef]

71. Anderson, J.C.; Gerbing, D.W. Structural equation modeling in practice: A review and recommended two-step approach. Psychol. Bull. 1988, 103, 411-423. [CrossRef]

72. Campbell, D.T.; Donald, W.F. Convergent and discriminant validation by the multitrait-multimethod matrix. Psychol. Bull. 1959, 56, 81-105. [CrossRef]

73. Hair, J.F.; Black, W.C.; Babin, B.J.; Anderson, R.E. Multivariate Data Analysis; Pearson Prentice Hall: Upper Saddle River, NJ, USA, 2010.

74. Fornell, C.; Larcker, D.F. Evaluating structural equation models with unobservable variables and measurement error. J. Market. Res. 1981, 18, 39-50. [CrossRef]

75. Larsen, J. De-exoticizing tourist travel: Everyday life and sociality on the move. Leis. Stud. 2008, 7, 21-34. [CrossRef]

76. Merigo, J.M.; Palacios-Marques, D.; Zeng, S. Subjective and objective information in linguistic multi-criteria group decision making. Eur. J. Oper. Res. 2016, 248, 522-531. [CrossRef]

77. Sohaib, O.; Kang, K. Individual level culture influence on online consumer iTrust aspects towards purchase intention across cultures: A SOR model. Int. J. Electron. Bus. 2015, 12, 142-161. [CrossRef]

78. Thongpapanl, N.; Ashraf, A.R. Enhancing online performance through website content and personalization. J. Comput. Inform. Syst. 2011, 52, 3-13.

79. De Mooij, M.; Hofstede, G. The Hofstede model: Applications to global branding and advertising strategy and research. Int. J. Advert. 2010, 29, 85-110. [CrossRef]

80. Wu, M.Y.; Chou, H.P.; Weng, Y.C.; Huang, Y.H. TAM-2 based study of website user behavior-using web 2.0 websites as an example. WSEAS Trans. Bus. Econ. 2011, 4, 133-151.

(C) 2020 by the authors. Licensee MDPI, Basel, Switzerland. This article is an open access article distributed under the terms and conditions of the Creative Commons Attribution (CC BY) license (http://creativecommons.org/licenses/by/4.0/). 

MDPI

St. Alban-Anlage 66

4052 Basel

Switzerland

Tel. +41616837734

Fax +41 613028918

www.mdpi.com

Sustainability Editorial Office

E-mail: sustainability@mdpi.com

www.mdpi.com/journal/sustainability

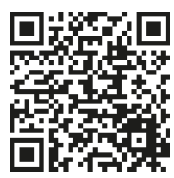



MDPI

St. Alban-Anlage 66

4052 Basel

Switzerland

Tel: +41 616837734

Fax: +41 613028918 